\title{
Ordnungs-/Unordnungsphänomene in korrelierten Perowskitschichten anhand von fortgeschrittener Raman-Spektroskopie
}

\section{Dissertation}

zur Erlangung des mathematisch-naturwissenschaftlichen Doktorgrades

„Doctor rerum naturalium“

der Georg-August-Universität Göttingen

im Promotionsprogramm PROPHYS

der Georg-August University School of Science (GAUSS)

vorgelegt von

Christoph Meyer

aus Alfeld (Leine)

Göttingen, 2018 


\section{Betreuungsausschuss}

Prof. Dr. Vasily Moshnyaga,

I. Physikalisches Institut, Georg-August-Universität Göttingen

Prof. Dr. Claus Ropers

IV. Physikalisches Institut, Georg-August-Universität Göttingen

\section{Mitglieder der Prüfungskomission}

\section{Referent:}

Prof. Dr. Vasily Moshnyaga

I. Physikalisches Institut, Georg-August-Universität Göttingen

\section{Korreferent:}

Prof. Dr. Peter Lemmens

Institut für Physik der Kondensierten Materie, Technische Universität Braunschweig

\section{Weitere Mitglieder der Prüfungskomission}

Prof. Dr. Michael Seibt

IV. Physikalisches Institut, Georg-August-Universität Göttingen

PD Dr. Martin Wenderoth

IV. Physikalisches Institut, Georg-August-Universität Göttingen

Prof. Dr. Christian Joos

Institut für Materialphysik, Georg-August-Universität Göttingen

Prof. Dr. Hans-Christian Hofsäss

II. Physikalisches Institut, Georg-August-Universität Göttingen

\section{Tag der mündlichen Prüfung: 18.07.2018}




\section{Inhaltsverzeichnis}

\begin{tabular}{lll}
\hline 1 & Einleitung & 1
\end{tabular}

2 Theoretischer Hintergrund 3

2.1 Perowskit-Kristallstruktur . . . . . . . . . . . . . . . . . . . . . . . . . . 3

2.2 Perowskitische Manganate . . . . . . . . . . . . . . . . . . . 5

$2.2 .1 \quad$ Elektronische Struktur und Jahn-Teller-Effekt . . . . . . . . . . . . 5

2.2 .2 Austauschmechanismen . . . . . . . . . . . . . . . . . 7

$2.2 .3 \quad$ Phasendiagramm von moderat dotiertem $\mathrm{La}_{1-\mathrm{x}} \mathrm{Sr}_{\mathrm{x}} \mathrm{MnO}_{3} \ldots \ldots$

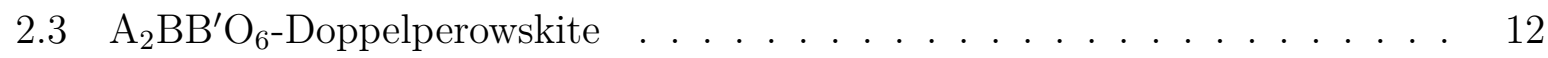

2.3 .1 Aufbau, Kristallstruktur und Kationenordnung. . . . . . . . . . . . 12

2.3 .2 Kobalt-Mangan-basierte $\mathrm{A}_{2} \mathrm{CoMnO}_{6}$-Doppelperowskite . . . . . . . 16

$2.3 .3 \quad$ Nickel-Mangan-basierte $\mathrm{A}_{2} \mathrm{NiMnO}_{6}$-Doppelperowskite . . . . . . . . 18

3 Experimentelle Methoden 19

3.1 Probenherstellung . . . . . . . . . . . . . . . . . . . . . . . . . . . . . . . . 19

$3.1 .1 \quad$ Prinzip der metallorganischen Aerosol Deposition (MAD) . . . . . . 19

3.1 .2 Herstellung der Dünnschicht-Oxide mithilfe der MAD . . . . . . . . 21

3.2 Standard-Probencharakterisierung . . . . . . . . . . . . . . . . . . 23

3.2 .1 Röntgendiffraktometrie (XRD) . . . . . . . . . . . . . . . . . 23

3.2 .2 Röntgenreflektometrie (XRR) . . . . . . . . . . . . . . . . . . . . 29

3.2 .3 Rasterkraftmikroskopie (AFM) . . . . . . . . . . . . . . . 32

3.2 .4 Rastertunnelmikroskopie (STM) . . . . . . . . . . . . . . . . 35

3.2 .5 SQUID-Magnetometrie . . . . . . . . . . . . . . . . . . . . 39

3.2 .6 Elektrische Transportmessungen . . . . . . . . . . . . . . . . . . . 43

3.2 .7 Rasterelektronenmikroskopie (REM) . . . . . . . . . . . . . . . 44

3.2 .8 Transmissionselektronenmikroskopie (TEM) . . . . . . . . . . . . 45

3.3 Raman-Spektroskopie . . . . . . . . . . . . . . . . . . . 47

$3.3 .1 \quad$ Einführung in die Raman-Spektroskopie . . . . . . . . . . . . . . . 47

3.3 .2 Theoretische Beschreibung der Raman-Streuung . . . . . . . . . . . 48

3.3 .3 Verwendeter Raman-Messaufbau . . . . . . . . . . . . . . . 56

3.3 .4 Polarisationsabhängige Raman-Spektroskopie . . . . . . . . . . . . 60 
$3.4 \quad$ Spitzenverstärkte Raman-Spektroskopie (TERS) . . . . . . . . . . . . . . . 62

3.4.1 Motivation und Prinzip der TERS-Technik . . . . . . . . . . . . . . 63

$3.4 .2 \quad$ Plasmonische Nahfeldanregung und Feldverstärkung . . . . . . . . . . 64

3.4.3 Signalverstärkung, Kontrast und Oberflächensensitivität . . . . . . 66

3.4 .4 Verwendeter TERS-Messaufbau . . . . . . . . . . . . . . . . . . . . 68

$3.4 .5 \quad$ Polarisationsabhängige TERS-Messungen . . . . . . . . . . . . . . . 71

$3.4 .6 \quad$ Spitzeneffekte und Nahfeldeffekte . . . . . . . . . . . . . . . . . . . 75

$\begin{array}{lll}4 \text { Auswertung und Ergebnisse } & \mathbf{8 1}\end{array}$

4.1 B-Platz geordnete $\mathrm{A}_{2} \mathrm{BMnO}_{6}$-Doppelperowskite . . . . . . . . . . . . . 81

4.1.1 Schichtwachstum und Optimierung der Wachstumsparameter . . . . 81

$4.1 .2 \quad$ Einfluss des A-Platz- und des B-Platz-Kations . . . . . . . . . . . . 86

$4.1 .3 \quad$ B-Platz-Ordnung und der Einfluss der Verspannung . . . . . . . . . 99

4.1 .4 Temperaturabhängige Raman-Messungen . . . . . . . . . . . . . . . 113

$4.1 .5 \quad$ Spin-Phonon-Kopplung . . . . . . . . . . . . . . . . . 125

4.2 Tip-Enhanced Raman Spectroscopy (TERS) ～. . . . . . . . . . . . . . . . 136

$4.2 .1 \quad$ Erste Testmessungen an Azobenzol und Malachit-Grün . . . . . . . 136

$4.2 .2 \quad$ Polarisationsabhängige TERS-Messungen an Silizium . . . . . . . . 140

4.2 .3 Polarisationsabhängige TERS-Messungen an $\mathrm{La}_{2} \mathrm{CoMnO}_{6}$. . . . . . . 144

4.2.4 Einfluss der STM-Parameter und TERS-Oberflächensensitivität . . 159

4.2 .5 TERS an weiteren Doppelperowskit-Dünnschichten . . . . . . . . . 166

$4.3 \quad \mathrm{La}_{1-\mathrm{x}} \mathrm{Sr}_{\mathrm{x}} \mathrm{MnO}_{3}$-Schichten $(0 \leq x \leq 0,3) \ldots \ldots \ldots \ldots \ldots$

$4.3 .1 \quad$ Schichtwachstum und allgemeine Charakterisierung . . . . . . . . . 173

4.3 .2 Dotierungs- und temperaturabhängige Raman-Messungen . . . . . . 183

4.3 .3 Laserleistungsabhängige Raman-Messungen und TERS . . . . . . . 191

5 Zusammenfassung und Ausblick 201

5.1 Zusammenfassung der Ergebnisse . . . . . . . . . . . . . . . . . . . 201

5.2 Ausblick . . . . . . . . . . . . . . . . . . . . 207

\begin{tabular}{ll}
\hline Literaturverzeichnis & 209
\end{tabular}

\begin{tabular}{lr}
\hline Publikationen & 227
\end{tabular}

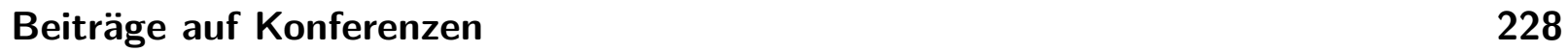

\begin{tabular}{lr}
\hline Danksagung & 229
\end{tabular} 


\section{Abbildungsverzeichnis}

2.1.1 Einheitszelle der idealen kubischen $\mathrm{ABO}_{3}$-Perowskit-Struktur. . . . . . . . . 3

2.1.2 Lage der Sauerstoffoktaeder in der idealen und verspannten Perowskit-Struktur 4

$2.2 .13 \mathrm{~d}$-Orbitale des $\mathrm{Mn}^{3+}$-Kations im Kristallfeld und der Jahn-Teller-Effekt . . 6

2.2 .2 Magnetische Kopplungen in Manganaten aufgrund des Superaustauschs . . . 9

$2.2 .3 \quad$ Prinzip des Doppelaustauschs bei gemischtvalenten Manganaten . . . . . . 10

$2.2 .4 \quad T$ - $x$-Phasendiagramm von $\mathrm{La}_{1-\mathrm{x}} \mathrm{Sr}_{\mathrm{x}} \mathrm{MnO}_{3}$ für $0 \leq x \leq 0,35 \ldots \ldots \ldots . . \ldots 11$

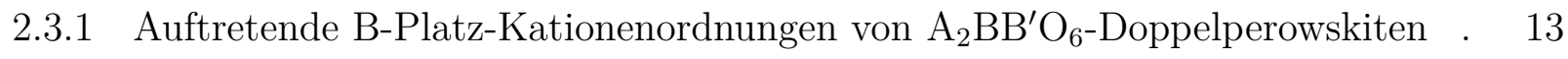

2.3 .2 Reduzierung der B-Platz-Ordnung durch Gitterfehler und Antiphasengrenzen 15

2.3 .3 Einheitszelle und B-Platz-Ordnung der $\mathrm{A}_{2} \mathrm{CoMnO}_{6}$-Doppelperowskite . . . . 16

3.1 .1 Schematischer Aufbau der MAD-Anlage $\ldots \ldots \ldots$

3.1 .2 Struktur der metallorganischen Acetylacetonat-Präkursoren und von $\mathrm{Mn}(\mathrm{acac})_{2} 21$

$3.2 .1 \quad$ Schematischer Aufbau eines Röntgendiffraktometers . . . . . . . . . . . 23

$3.2 .2 \quad$ Beugungsgeometrie zur Herleitung von Bragg- und Laue-Bedingung . . . . . 24

$3.2 .3 \quad$ Prinzip der Röntgenreflektometrie mit zwei Beispielmessungen . . . . . . . . 30

3.2 .4 Schematischer Aufbau eines Rasterkraftmikroskops mit Lichtzeigerprinzip. . 32

$3.2 .5 \quad$ Charakteristische Kraft-Abstands-Kurve des Rasterkraftmikroskops . . . . . 34

3.2 .6 Schematischer Aufbau und die Betriebsarten eines Rastertunnelmikroskops . 35

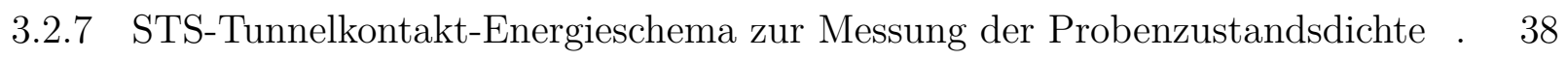

3.2 .8 SQUID-Magnetisierungsreferenzmessungen der verwendeten Substrate. . . . 40

3.2 .9 Beispiele für die Schichtmagnetisierungskorrektur um den Substratbeitrag. . 41

3.2 .10 PPMS-Probenhalter und Kontaktierung für die Vier-Punkt-Methode . . . . 43

3.2 .11 REM-Aufnahmen einer TERS-STM-Goldspitze $\ldots \ldots \ldots \ldots$. . . . . 45

3.3 .1 Jablonski-Diagramm für die Raman-Streuung und das resultierende Spektrum 48

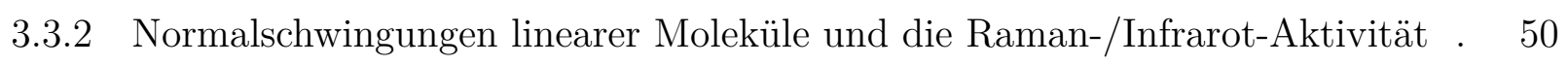

\begin{tabular}{|lll}
\hline 3.3 .3 & Übersicht der aus den Raman-Spektren zu gewinnenden Probeninformationen 54
\end{tabular}

$\begin{array}{lll}3.3 .4 & \text { Raman-Spektrometer mit konfokalem Raman-Mikroskop und CCD-Detektor } 56\end{array}$

$3.3 .5 \quad$ Schematische Übersicht des Strahlengangs für die Raman-Messungen . . . . 57

$3.3 .6 \quad$ Aufbau eines Raman-Spektrometers mit Czerny-Turner-Konfiguration . . . . 58

$3.3 .7 \quad$ Verwendete Kühlzelle für die temperaturabhängigen Raman-Messungen . . . 60

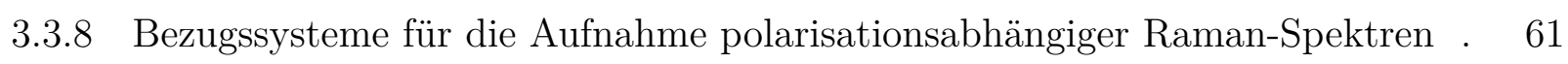

3.4 .1 TERS-Nahfelderzeugung durch Oberflächenplasmon-Polariton-Anregungen . 63 
3.4.2 $\quad$ Prinzip der LSP-Anregung an einem kugelförmigen Metallnanopartikel . . 65

3.4 .3 TERS-Spitze in seitlicher Einstrahlung und die Modellierung der Spitze . . . 67

3.4 .4 TERS-Aufbau mit seitlicher Einstrahlung in Rückstreugeometrie . . . . . . . 69

3.4 .5 TERS- und STM-Kamerabilder einer TERS-Goldspitze in Tunnelposition . . 70

3.4.6 Bezugssysteme für die Aufnahme polarisationsabhängiger TERS-Messungen 71

3.4.7 TERS-Bezugsystemüberführung von der (100)- in die (111)-Orientierung . . 73

3.4 .8 TERS-Nahfeldpolarisation und quantenmechanisches TERS-Modell . . . . . 76

3.4 .9 Wechselwirkung des einfallenden und gestreuten Lichts mit der TERS-Spitze 79

4.1.1 AFM-Aufnahmen an zwei unterschiedlich dicken LCMO/STO (100)-Schichten 82

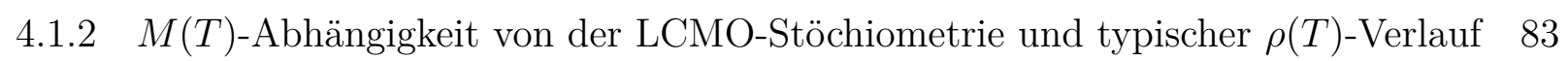

$4.1 .3 \quad$ Polarisierte Raman-Spektren einer $85 \mathrm{~nm}$ LCMO/STO (100)-Schicht . . . . 84

$4.1 .4 \quad$ XRD-Spektren der $\mathrm{A}_{2} \mathrm{CoMnO}_{6} / \mathrm{STO}$ (111)-Schichtserie . . . . . . . . . . . 86

$4.1 .5 \quad$ XRD-Spektren der $\mathrm{A}_{2} \mathrm{NiMnO}_{6} / \mathrm{STO}(111)$-Schichtserie $\ldots . . . . .87$

4.1 .6 Ordnungsparameter und Gitterkonstanten der $\mathrm{A}_{2} \mathrm{BMnO}_{6} / \mathrm{STO}$ (111)-Schichten 88

$4.1 .7 \quad M(T)$-Magnetisierungskurven der $\mathrm{A}_{2} \mathrm{CoMnO}_{6} / \mathrm{STO}(111)$-Schichten . . . . . 90

$4.1 .8 \quad M(H)$-Hysteresekurven der $\mathrm{A}_{2} \mathrm{CoMnO}_{6} / \mathrm{STO}(111)$-Schichten $\ldots . . . .91$

$4.1 .9 \quad M(T)-/ M(H)$-Magnetisierungskurven der $\mathrm{A}_{2} \mathrm{NiMnO}_{6} / \mathrm{STO}(111)$-Schichten . 94

4.1 .10 Polarisierte Raman-Spektren der $\mathrm{A}_{2}(\mathrm{Co} / \mathrm{Ni}) \mathrm{MnO}_{6} / \mathrm{STO}(111)$-Schichten . . 97

4.1 .11 A-Kationeneinfluss auf die $\mathrm{A}_{2} \mathrm{BMnO}_{6}$-Raman-Spektren und der $T_{C^{-} \text {-Verlauf }} .98$

4.1 .12 Gitterfehlanpassung verschiedener Substrate zu Bulk-LCMO . . . . . . . . . 99

4.1 .13 XRD-Spektren von LCMO-Schichten auf unterschiedlichen Substraten. . . . 100

$4.1 .14 M(T)$-Kurven von LCMO-Schichten auf unterschiedlichen Substraten . . . . 102

$4.1 .15 M(H)$-Kurven von LCMO-Schichten auf unterschiedlichen Substraten . . . . 103

4.1 .16 STEM-/EELS-Bilder der B-Platz geordneten LCMO/LAO (111)-Schicht . . 105

4.1 .17 Polarisierte Raman-Spektren der LCMO-Schichten im Vergleich . . . . . . . 106

4.1.18 XRD-Spektren unterschiedlich geordneter LCMO/ $\mathrm{Al}_{2} \mathrm{O}_{3}(0001)$-Schichten . . 107

$4.1 .19 M(T)$-Kurven unterschiedlich geordneter LCMO $/ \mathrm{Al}_{2} \mathrm{O}_{3}(0001)$-Schichten . . 109

$4.1 .20 M(H)$-Kurven unterschiedlich geordneter LCMO/ $\mathrm{Al}_{2} \mathrm{O}_{3}(0001)$-Schichten . . 110

4.1.21 STEM-/EELS-Bilder der B-Platz geordneten LCMO/ $\mathrm{Al}_{2} \mathrm{O} 3$ (0001)-Schicht . 111

4.1 .22 Polarisierte Raman-Spektren der LCMO/ $\mathrm{Al}_{2} \mathrm{O}_{3}(0001)$-Schichten. . . . . . . . 112

$4.1 .23 \omega(T)$-Vergleich der $A_{g}$ Breathing Mode der $\mathrm{A}_{2} \mathrm{CoMnO}_{6} / \mathrm{STO}(111)$-Schichten 114

4.1.24 $\omega(T)$-Vergleich der $A_{g}$ Breathing Mode der $\mathrm{A}_{2} \mathrm{NiMnO}_{6} / \mathrm{STO}(111)$-Schichten 115

4.1 .25 Temperaturentwicklung $\Gamma(T)$ der Linienbreite der $A_{g}$ Breathing Mode der $\mathrm{A}_{2} \mathrm{CoMnO}_{6} / \mathrm{STO}(111)$ - und $\mathrm{A}_{2} \mathrm{NiMnO}_{6} /$ STO (111)-Schichten im Vergleich. 116

4.1 .26 Temperaturentwicklung $\omega(T)$ der Linienposition der $A_{g}$ Breathing Mode geordneter LCMO-Schichten auf verschiedenen Substraten im Vergleich . . . . 117

4.1.27 Unpolarisierte Raman-Spektren der LCMO-Schichten auf verschiedenen Substraten für kleine Wellenzahlen zum Nachweis der R⿳亠丷⿵-Hochtemperaturphase

119 
4.1.28 Temperaturentwicklung $\Gamma(T)$ der Linienbreite der $A_{g}$ Breathing Mode geordneter LCMO-Schichten auf verschiedenen Substraten im Vergleich . . . . . . 120

4.1.29 Anharmonische Fitparameter für die Linienbreite der $A_{g}$ Breathing Mode der LCMO-Schichten auf unterschiedlichen Substraten im Vergleich . . . . . . . 122

$4.1 .30 \omega(T)$ und $\Gamma(T)$ der $A_{g}$ Breathing Mode zweier LCMO/ $/ \mathrm{Al}_{2} \mathrm{O}_{3}(0001)$-Schichten mit B-Platz-Ordnung/-Teilordnung und R $\overline{3}$-Hochtemperaturphasennachweis 123

4.1.31 Entwicklung der Phonon-Renormierung $\Delta \omega(T)$ der $A_{g}$ Breathing Mode und der Magnetisierung $M^{2}(T) / M_{\text {max. }}^{2}$ der $\mathrm{A}_{2} \mathrm{CoMnO}_{6} / \mathrm{STO}(111)$-Schichten . . . 126

4.1.32 Lineare Abhängigkeit der Phonon-Renormierung $\Delta \omega\left(M^{2}(T) / M_{\max }^{2}\right) \operatorname{der} A_{g}$ Breathing Mode der $\mathrm{A}_{2} \mathrm{CoMnO}_{6}$-Schichten auf STO (111) . . . . . . . . . . 126

4.1.33 Entwicklung der Phonon-Renormierung $\Delta \omega(T)$ der $A_{g}$ Breathing Mode und

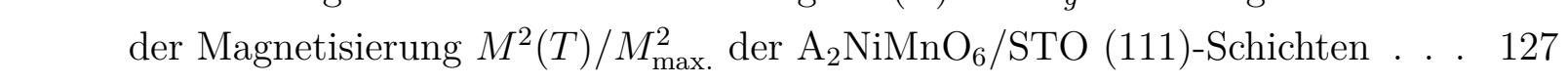

4.1.34 Lineare Abhängigkeit der Phonon-Renormierung $\Delta \omega\left(M^{2}(T) / M_{\text {max. }}^{2}\right) \operatorname{der} A_{g}$ Breathing Mode der $\mathrm{A}_{2} \mathrm{NiMnO}_{6}$-Schichten auf STO (111) . . . . . . . . . . . 128

4.1.35 Spin-Phonon-Kopplungsstärke der B-Platz geordneten $\mathrm{A}_{2} \mathrm{CoMnO}_{6} / \mathrm{STO}$ (111)und $\mathrm{A}_{2} \mathrm{NiMnO}_{6} / \mathrm{STO}$ (111)-Doppelperowskit-Schichten im Vergleich . . . . . 129

4.1.36 Phonon-Renormierung $\Delta \omega(T) \operatorname{der} A_{g}$ Breathing Mode und $M^{2}(T) / M_{\max }^{2}$ von (111)-orientierten $\mathrm{La}_{2} \mathrm{CoMnO}_{6}$-Schichten auf verschiedenen Substraten . . . 130

4.1.37 Lineare Abhängigkeit $\Delta \omega\left(M^{2}(T) / M_{\text {max. }}^{2}\right)$ der $A_{g}$ Breathing Mode der (111)orientierten $\mathrm{La}_{2} \mathrm{CoMnO}_{6}$-Schichten auf verschiedenen Substraten . . . . . . . 131

4.1 .38 Spin-Phonon-Kopplungsstärke (111)-orientierter $\mathrm{La}_{2} \mathrm{CoMnO}_{6}$-Schichten in Abhängigkeit des Substrats und der in-plane Gitterfehlanpassung . . . . . . . . 132

4.1.39 Entwicklung der Phonon-Renormierung $\Delta \omega(T)$ der $A_{g}$ Breathing Mode und der Magnetisierung $M^{2}(T) / M_{\text {max. }}^{2}$ der $\mathrm{La}_{2} \mathrm{CoMnO}_{6} / \mathrm{MgO}(100)$-Schicht . . . 133

4.1 .40 Phonon-Renormierung $\Delta \omega(T)$ der $A_{g}$ Breathing Mode und $M^{2}(T) / M_{\text {max. }}^{2}$ der zwei $\mathrm{La}_{2} \mathrm{CoMnO}_{6} / \mathrm{Al}_{2} \mathrm{O}_{3}$ (0001)-Schichten mit B-Platz-Ordnung/-Teilordnung 135

4.2.1 Strukturformeldarstellung von Azobenzol und Malachitgrün Isothiocynat . . 137

4.2 .2 TERS- und Fernfeld-Raman-Spektrum von Azobenzol . . . . . . . . . . . . . 137

4.2 .3 TERS- und Fernfeld-Raman-Spektrum von Malachitgrün Isothiocynat. . . . 139

4.2 .4 Diamantstruktur und Phononen-Dispersionskurven von Silizium . . . . . . . 140

4.2.5 Polarisierte TERS- und Fernfeld-Raman-Spektren eines Si (100)-Substrats . 142

4.2.6 Kennlinien einer TERS-STM-Goldspitze, gemessen an LCMO/STO (100) . . 144

4.2.7 Polarisierte TERS-Messungen an der $25 \mathrm{~nm} \mathrm{La}_{2} \mathrm{CoMnO}_{6} / \mathrm{STO}$ (100)-Schicht 145

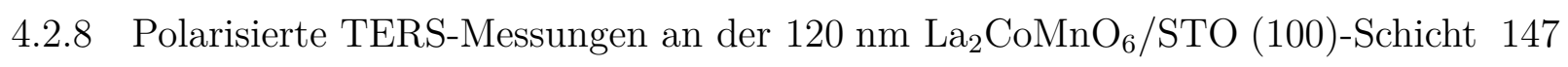

4.2.9 Polarisationsabhängige TERS- und Fernfeld-Raman-Intensität $\left(A_{g}\right.$ Breathing Mode) der $25 \mathrm{~nm} \mathrm{La} \mathrm{LoMnO}_{6} / \mathrm{STO}(100)$-Schicht . . . . . . . . . . . . . . . 150

4.2 .10 TERS-Kontrast der $A_{g}$ Breathing Mode der $25 \mathrm{~nm} \mathrm{La}{ }_{2} \mathrm{CoMnO}_{6} / \mathrm{STO}(100)-$

Schicht in Abhängigkeit der Polarisationskonfiguration . . . . . . . . . . . . 154 
4.2.11 Polarisationsabhängige TERS- und Fernfeld-Raman-Intensität ( $A_{g}$ Breathing Mode und $B_{g}$ Mischmode) der $120 \mathrm{~nm} \mathrm{La}_{2} \mathrm{CoMnO}_{6} / \mathrm{STO}(100)$-Schicht . . . 156 4.2 .12 TERS-Kontrast der $120 \mathrm{~nm} \mathrm{La}_{2} \mathrm{CoMnO}_{6} / \mathrm{STO}(100)$-Schicht $\left(A_{g}\right.$ Breathing Mode und $B_{g}$ Mischmode) in Abhängigkeit der Polarisationskonfiguration. . 158 4.2 .13 TERS-Spitzencharakterisierung an der $25 \mathrm{~nm}$ LCMO/STO (100)-Schicht . . 159

4.2 .14 Einfluss der TERS-Spitze auf die LCMO-TERS-Messungen . . . . . . . . . . 160 4.2 .15 Einfluss der STM-Parameter auf die LCMO-TERS-Messungen . . . . . . . . 161

4.2 .16 TERS-Messungen an unterschiedlich dicken LCMO/STO (100)-Schichten . . 163

4.2 .17 Schichtdickenabhängigkeit des TERS-Kontrastes und des Nahfeldes . . . . . 164

4.2.18 Polarisierte TERS-Messungen an einer 65 nm PCMO/STO (111)-Schicht . . 166

4.2.19 Polarisierte TERS-Messungen an einer $100 \mathrm{~nm} \mathrm{LNMO/MgO} \mathrm{(100)-Schicht} \mathrm{.} 168$

4.2 .20 Polarisierte TERS-Messungen an einer $12 \mathrm{~nm}$ LCMO/LAO (100)-Schicht . . 170

4.2 .21 TERS-Messung an einer $35 \mathrm{~nm}$ LNMO/LAO (100)-Schicht . . . . . . . . . . 172

4.3 .1 XRD-Spektren der $30 \mathrm{~nm} \mathrm{La} \mathrm{La}_{1-\mathrm{x}} \mathrm{Sr}_{\mathrm{x}} \mathrm{MnO}_{3} / \mathrm{MgO}(100)$-Schichten . . . . . . . 174

4.3.2 LSMO-Gitterkonstanten $c_{\mathrm{pc}}$ auf $\mathrm{MgO}(100)$ als Funktion der Sr-Dotierung 175

4.3 .3 XRD-Spektren der $30 \mathrm{~nm} \mathrm{La}{ }_{1-\mathrm{x}} \mathrm{Sr}_{\mathrm{x}} \mathrm{MnO}_{3} / \mathrm{LAO}(100)$-Schichten . . . . . . . 176

4.3 .4 Laue-Oszillationen in den XRD-Spektren der LSMO/LAO (100)-Schichten . 177

4.3.5 AFM-Bilder der LSMO-Schichten $(x=0,05)$ auf MgO (100) und LAO (100) 178

$4.3 .6 \quad$ Magnetische Charakterisierung der $30 \mathrm{~nm}$ LSMO-Schichten . . . . . . . . . . 179

4.3.7 Widerstandscharakterisierung $\rho(T)$ der $30 \mathrm{~nm}$ LSMO/MgO (100)-Schichten . 181

4.3.8 Widerstandscharakterisierung $\rho(T)$ der $30 \mathrm{~nm}$ LSMO/LAO (100)-Schichten . 182

4.3 .9 Raman-Spektren der LSMO/MgO (100)-Schichten mit $x=0$ und $x=0,30$. 184

4.3 .10 Raman-Spektren der LSMO-Schichten in Abhängigkeit der Sr-Dotierung . . 186

4.3 .11 Charakterisierung des Dotierungseinflusses auf die LSMO-Raman-Spektren . 187

4.3 .12 Temperaturabhängige LSMO/LAO (100)-Raman-Spektren $(x=0,10)$. . . . 188

$4.3 .13 \omega(T)$ der SS-JT-Mode und das $B_{2 g}(3) /$ SS-JT-Verhältnis für $T \leq 300 \mathrm{~K}$. . . 189

4.3.14 Laserleistungsabhängige LSMO/LAO (100)-Raman-Spektren $(x=0,10)$. . . 191

$4.3 .15 \Gamma(T)$ - und $\Gamma(P)$-Entwicklung der $E_{g}$-Biegemode des LAO (100)-Substrats . . 193

4.3.16 Charakterisierung des Laserleistungseinflusses auf die LSMO-Raman-Spektren 194

4.3 .17 Schematische Darstellung lichtinduzierter Übergänge in LSMO . . . . . . . . 196

4.3 .18 TERS-Messungen an der $30 \mathrm{~nm}$ LSMO/LAO (100)-Schicht mit $x=0.10$. . 197 


\section{Tabellenverzeichnis}

3.3.1 Mulliken-Symbole in der Raman-Spektroskopie und ihre Bedeutung . . . . . . 61

4.1.1 Magnetische Kenngrößen der $\mathrm{A}_{2} \mathrm{CoMnO}_{6} / \mathrm{STO}$ (111)-Schichten im Vergleich . 92 4.1.2 Magnetische Kenngrößen der $\mathrm{A}_{2} \mathrm{NiMnO}_{6} / \mathrm{STO}$ (111)-Schichten im Vergleich . 96 4.1 .3 Strukturelle XRD-Daten der LCMO-Schichten auf verschiedenen Substraten . 101 4.1.4 Fitparameter für die Temperaturentwicklung $\omega_{\text {anh. }}(T)$ der Linienposition der $A_{g}$ Breathing Mode der LCMO-Schichten auf verschiedenen Substraten. . . . 118 4.1 .5 Fitparameter für die Temperaturentwicklung $\Gamma_{\text {anh. }}(T)$ der Linienbreite der $A_{g}$ Breathing Mode der LCMO-Schichten auf verschiedenen Substraten . . . . . . 121 4.1.6 Spin-Phonon-Kopplungsstärke $\lambda$ der $\mathrm{A}_{2} \mathrm{CoMnO}_{6} / \mathrm{STO}$ (111)-Schichten . . . . 127 4.1.7 Spin-Phonon-Kopplungsstärke $\lambda$ der $\mathrm{A}_{2} \mathrm{NiMnO}_{6} / \mathrm{STO}$ (111)-Schichten . . . . 129 4.1.8 Spin-Phonon-Kopplungsstärke $\lambda$ der (111)-orientierten $\mathrm{La}_{2} \mathrm{CoMnO}_{6}$-Schichten 132 4.3.1 Position und Breite der JT-Moden in den TERS- und Fernfeld-Raman-Spektren der $30 \mathrm{~nm}$ LSMO/LAO (100)-Schicht mit $x=0,10(\lambda=532 \mathrm{~nm})] \ldots . . . \quad 198$

4.3.2 Position und Breite der JT-Moden in den TERS- und Fernfeld-Raman-Spektren der $30 \mathrm{~nm}$ LSMO/LAO (100)-Schicht mit $x=0,10(\lambda=632,8 \mathrm{~nm})$. . . . . . 198

4.3 .3 Übersicht der TERS-Kontrast-Werte verschiedener Raman-Moden der $30 \mathrm{~nm}$ LSMO/LAO (100)-Schicht $(x=0,10)$ für die zwei verfügbaren Raman-Laser . 199

4.3.4 Relative Intensitäten verschiedener Raman-Moden im TERS-Gesamtfeld und Fernfeld der $30 \mathrm{~nm}$ LSMO/LAO (100)-Schicht mit $x=0,10$. . . . . . . . . . 200 


\section{Einleitung}

Mit der Entdeckung des Minerals Perowskit mit der chemischen Zusammensetzung $\mathrm{CaTiO}_{3}$ im Jahr 1839 im Ural-Gebirge durch den deutschen Mineralogen Gustav Rose, der dieses nach dem russischen Mineralogen Graf Lev Aleksevich von Perovski benannte [1], rückte mit der dazugehörigen Perowskit-Struktur $\mathrm{ABX}_{3}$ ein Strukturtyp in den Fokus der Forschung, der heute zu einem der häufigsten anzutreffenden und vielseitigsten Strukturtypen in der Festkörperphysik und anorganischen Chemie überhaupt zählt [1-4]. Insbesondere das Bekanntwerden von perowskitischen Ferroelektrika wie $\left.\mathrm{BaTiO}_{3}[1] 3\right]$ und Piezoelektrika wie $\mathrm{PbZr}_{1-\mathrm{x}} \mathrm{Ti}_{\mathrm{x}} \mathrm{O}_{3}(0 \leq x \leq 1)[3]$ sowie die Entdeckung des kolossalen Magnetowiderstands (engl.: colossal magnetoresistance, CMR) in gemischtvalenten Manganaten wie $\mathrm{La}_{1-\mathrm{x}} \mathrm{Sr}_{\mathrm{x}} \mathrm{MnO}_{3}$ oder $\mathrm{La}_{1-\mathrm{x}} \mathrm{Ca}_{\mathrm{x}} \mathrm{MnO}_{3}(0 \leq x \leq 1)$ [5, 6] führt seit der Mitte des 20. Jahrhunderts zu einer verstärkten Erforschung perowskitischer Materialsysteme [1].

Aufgrund der Flexibilität der Perowskit-Struktur mit den Kationen der meisten metallischen Elemente auf den A- und B-Gitterplätzen und verschiedenen Anionen kristallisieren zu können sowie eine systematische Dotierung der A-, B- und X-Plätze zu ermöglichen, zeigen perowskitische Materialien eine große Vielfalt an elektrischen und magnetischen Eigenschaften mit technologischen Anwendungen, die sich von der HochtemperaturSupraleitung, der Sensortechnologie, der Verwendung als Festelektrolyt bis hin zu den Entwicklung von Perowskit-Solarzellen erstrecken [1 4]. Eine wichtige Grundlage für diese Vielfalt sind dabei strukturelle Verzerrungen, die sowohl durch die unterschiedliche Größe der Kationen als auch durch den Dotierungsgrad entstehen und zu einer signifikanten Abweichung von dem kubischen Perowskit-Aristotyp führen [1,3]. Diese werden für $80-90 \%$ aller Perowskite beobachtet und bieten einen direkten Weg für die Beeinflussung der strukturellen, elektrischen, magnetischen und optischen Eigenschaften [3 8]. Zusätzlich können Effekte wie Jahn-Teller-Verzerrungen und orbitale Ordnungsphänomene 3, 5, 7, 8], die für Kationen mit geeigneten Elektronenkonfiguration entstehen, oder spezifische Kationenordnungen, die für zwei oder mehrere unterschiedliche Kationen in bestimmten Verhältnissen auf den A- oder B-Gitterplätzen auftreten [3,4,9], die charakteristischen Eigenschaften der Perowskite drastisch beeinflussen. Entsprechend wichtig ist es daher für eine technologische Anwendung sowohl das Wachstum, die Dotierung und die strukturelle Stabilität der perowskitischen Materialien zu optimieren [1, 2, 9] als auch den Einfluss der spezifischen Ordnungs- und Unordnungsphänomene zu bestimmen und diese zu kontrollieren [4,9]. 


\section{Einleitung}

In dieser Arbeit werden nun Ordnungs- und Unordnungsphänomene dünner epitaktischer $\mathrm{A}_{2} \mathrm{BMnO}_{6}$-Doppelperowskit-Schichten ( $\left.\mathrm{A}=\mathrm{La}, \mathrm{Pr}, \mathrm{Nd}, \mathrm{Sm}, \mathrm{Gd}, \mathrm{Y} ; \mathrm{B}=\mathrm{Co}, \mathrm{Ni}\right)$ und von niedrig-dotierten $\mathrm{La}_{1-\mathrm{x}} \mathrm{Sr}_{\mathrm{x}} \mathrm{MnO}_{3}$-Dünnschichten (LSMO) $(0 \leq x \leq 0,3)$, die mithilfe der metallorganischen Aerosol Deposition (MAD) auf verschiedenen Substraten aufgewachsen wurden, anhand ihrer strukturellen, magnetischen und phononischen Eigenschaften untersucht. Dabei wird sowohl der Einfluss unterschiedlicher externer und interner Parameter (chemische Verspannung, Substrat, Temperatur, Dotierung, Laserleistung) auf die strukturelle Ordnung diskutiert als auch verschiedene Ansätze zur Abschätzung der jeweiligen Ordnung/Unordnung vorgestellt. In diesem Zusammenhang nimmt die Raman-Spektroskopie aufgrund ihrer großen Sensitivität für die Kristallinität, Struktur, chemische Zusammensetzung und Verspannung von Perowskit-Materialien eine zentrale Stellung ein.

Die Arbeit ist dabei so aufgebaut, dass zunächst mit einer theoretischen Einführung in die Perowskit-Struktur, die Manganate und die $\mathrm{A}_{2} \mathrm{BMnO}_{6}$-Doppelperowskite begonnen wird, bevor die experimentellen Methoden zur Herstellung und Charakterisierung der Dünnschichten mit dem Schwerpunkt auf der Raman-Spektroskopie und der spitzenverstärkten Raman-Spektroskopie (TERS, engl.: tip-enhanced Raman spectroscopy) beschrieben werden. Anschließend folgen die Ergebnisse dieser Arbeit. Der erste Teil befasst sich dabei mit den B-Platz geordneten $\mathrm{A}_{2} \mathrm{BMnO}_{6}$-Doppelperowskit-Schichten, wobei zunächst auf die Herstellung und die Optimierung des Schichtwachstums eingegangen wird, bevor anschließend die Einflüsse des A-Kations und des B-Kations auf die strukturellen, magnetischen und phononischen Eigenschaften untersucht werden. In diesem Zusammenhang werden unterschiedliche Methoden zur Bestimmung des B-Platz-Ordnungsgrades vorgestellt und für $\mathrm{La}_{2} \mathrm{CoMnO}_{6}$ das Wachstum auf verschiedenen Substraten diskutiert. Der zweite Teil befasst sich wiederum mit der TERS-Technik, die im Zuge dieser Arbeit erstmalig an den Doppelperowskit-Dünnschichten angewendet wird. Hierfür wird zunächst einführend mit Messungen an bekannten TERS-Testmaterialien begonnen, bevor im zweiten Schritt polarisationsabhängige TERS-Messungen an den Doppelperowskiten durchgeführt und die Ergebnisse in Abhängigkeit der Polarisationskonfiguration mit theoretischen Berechnungen unter der Berücksichtigung eines kombinierten Verstärkungs- und Depolarisationsmodells verglichen werden. Zusätzlich wird der Einfluss verschiedener Parameter des verwendeten TERS-Systems und der Schichtdicke der LCMO-Schichten untersucht. Im dritten Teil werden schließlich die niedrig-dotierten $\mathrm{La}_{1-\mathrm{x}} \mathrm{Sr}_{\mathrm{x}} \mathrm{MnO}_{3}$-Schichten $(0 \leq x \leq 0,3)$ hinsichtlich der Vielzahl an strukturellen, elektrischen und magnetischen Phasen in diesem Bereich des $T$ - $x$-Phasendiagramms betrachtet. Neben der Entwicklung der Raman-Spektren mit der Temperatur $T$ und der Sr-Dotierung $x$ wird dabei der Einfluss der Leistung des eingestrahlten Laserlichts auf die strukturelle Ordnung von LSMO diskutiert. Am Ende der Arbeit erfolgt dann eine Zusammenfassung der Ergebnisse und ein Ausblick auf mögliche Anwendungsgebiete und Ansätze für Messungen an weiteren Perowskitsystemen. 


\section{Theoretischer Hintergrund}

In diesem Kapitel sollen nun zunächst in einem Überblick die strukturellen, elektrischen und magnetischen Eigenschaften der in dieser Arbeit untersuchten Dünnschicht-PerowskitOxide vorgestellt werden. Hierfür wird zunächst mit der Kristallstruktur und den strukturellen Eigenschaften begonnen, bevor auf die elektronische Struktur sowie die auftretenden Austauschwechselwirkungen eingegangen und die Auswirkungen auf die elektrischen und magnetischen Eigenschaften besprochen werden. Im Anschluss daran wird schließlich die Kationenordnung in den Perowskiten vorgestellt und der damit einhergehende Einfluss der Ordnung auf die Eigenschaften der untersuchten Materialsysteme diskutiert.

\subsection{Perowskit-Kristallstruktur}

Die ideale oxidische Perowskit-Struktur mit der chemischen Formel $\mathrm{ABO}_{3}$ kristallisiert dabei in der Grundform in einer der kubischen Kristallstruktur mit der dazugehörigen Raumgruppe Pm̄̄m (Raumgruppe 221) [2,3]. Sie beschreibt damit den unverzerrten Aristotyp der Struktur mit kubischer Einheitszelle, bei der die kleineren B-Platz-Kationen jeweils von sechs Sauerstoff-Anionen (Koordinationszahl $\mathrm{KZ}=6$ ) in Form von Oktaedern umgeben werden, die über ihre gemeinsamen Ecken ein dreidimensionales Netzwerk bilden, in dessen Lücken $(\mathrm{KZ}=12)$ die größeren A-Platz-Kationen platziert sind [2 4$]$. Eine Darstellung der kubischen Perowskit-Einheitszelle ist in der Abbildung 2.1.1 gegeben.

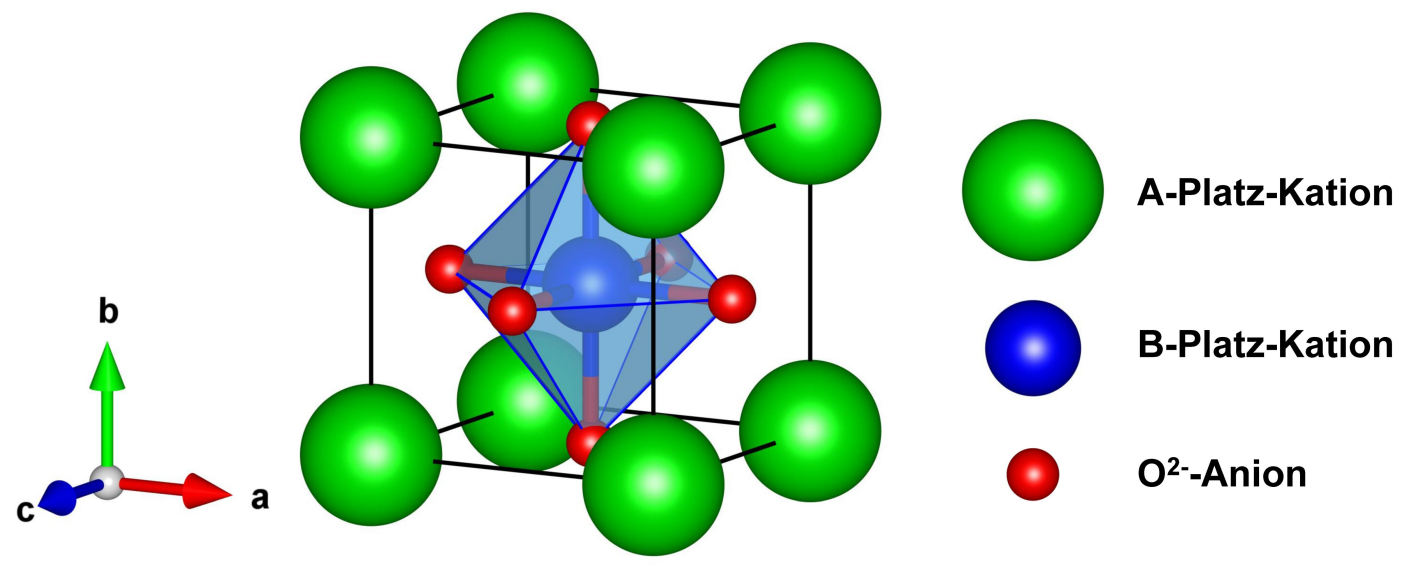

Abbildung 2.1.1: Einheitszelle der idealen kubischen $\mathrm{ABO}_{3}$-Perowskit-Struktur mit dem im Zentrum von einem Sauerstoff-Oktaeder umgebenen B-Platz-Kation und den A-Platz-Kationen auf den Zellenecken (mit VESTA 10 gezeichnet). 


\section{Theoretischer Hintergrund}

Aufgrund der Geometrie ist für die ideale kubische Perowskit-Struktur für die Bindungslängen zwischen den A-Platz-Kationen und den $\mathrm{O}^{2-}$-Anionen $d_{\mathrm{A}, \mathrm{O}}$ und zwischen den B-PlatzKationen und den $\mathrm{O}^{2-}$-Anionen $d_{\mathrm{B}, \mathrm{O}}$ die Relation $d_{\mathrm{A}, \mathrm{O}}=d_{\mathrm{B}, \mathrm{O}} \sqrt{2}$ vorausgesetzt 4,11 . In Bezug auf die Gitterkonstante $a$ der kubischen Perowskit-Struktur ergibt sich daraus die Bedingung $r_{\mathrm{A}}+r_{\mathrm{O}}=\sqrt{2}\left(r_{\mathrm{B}}+r_{\mathrm{O}}\right)$, wobei $r_{\mathrm{A}}, r_{\mathrm{B}}$ und $r_{\mathrm{O}}$ die Ionenradien der A- und B-PlatzKationen sowie des $\mathrm{O}^{2-}$-Anions bezeichnen. Folglich ist das Kristallisieren in der idealen kubischen $\mathrm{ABO}_{3}$-Perowskit-Struktur nur für ein passendes Größenverhältnis der A-Platzund B-Platz-Kationen möglich. Eine Abweichung von diesem unverzerrten Idealfall kann mithilfe des Goldschmidtschen Toleranzfaktors $t$ angegeben werden $[12$ :

$$
t=\frac{1}{\sqrt{2}} \frac{d_{\mathrm{A}-\mathrm{O}}}{d_{\mathrm{B}-\mathrm{O}}}=\frac{1}{\sqrt{2}} \frac{\left\langle r_{\mathrm{A}}\right\rangle+r_{\mathrm{O}}}{\left\langle r_{\mathrm{B}}\right\rangle+r_{\mathrm{O}}}
$$

Hierbei bezeichnen $\left\langle r_{\mathrm{A}}\right\rangle$ und $\left\langle r_{\mathrm{B}}\right\rangle$ die mittleren Ionenradien der A- und B-Platz-Kationen, die für mehrere unterschiedliche Kationen auf den A- und B-Plätzen aus dem jeweiligen Mittelwert der auftretenden Ionenradien ermittelt werden [4,5]. Für die kubische PerowskitStruktur nimmt der Toleranzfaktor entsprechend den Wert $t=1$ an. Die PerowskitStruktur erlaubt jedoch eine gewisse Fehlanpassung bei zu kleinen A-Kationen $(t<1)$. Diese werden durch Verdrehungen und Verkippungen der $\mathrm{BO}_{6}$-Oktaeder kompensiert und führen zu einer Verzerrung der Struktur und zu einer Reduzierung der Symmetrie 11. Dieses äußert sich in einer Änderung der Gitterstruktur durch eine Vergrößerung der PerowskitEinheitszelle und führt zu einer Verkleinerung des B-O-B-Bindungswinkels zwischen den B-Platz-Kationen und den $\mathrm{O}^{2-}$-Anionen. Der Einfluss von $t$ auf den Bindungswinkel ist schematisch in der Abbildungen 2.1.2 (a) und (b) (nach [5]) für $t=1$ und $t<1$ dargestellt.
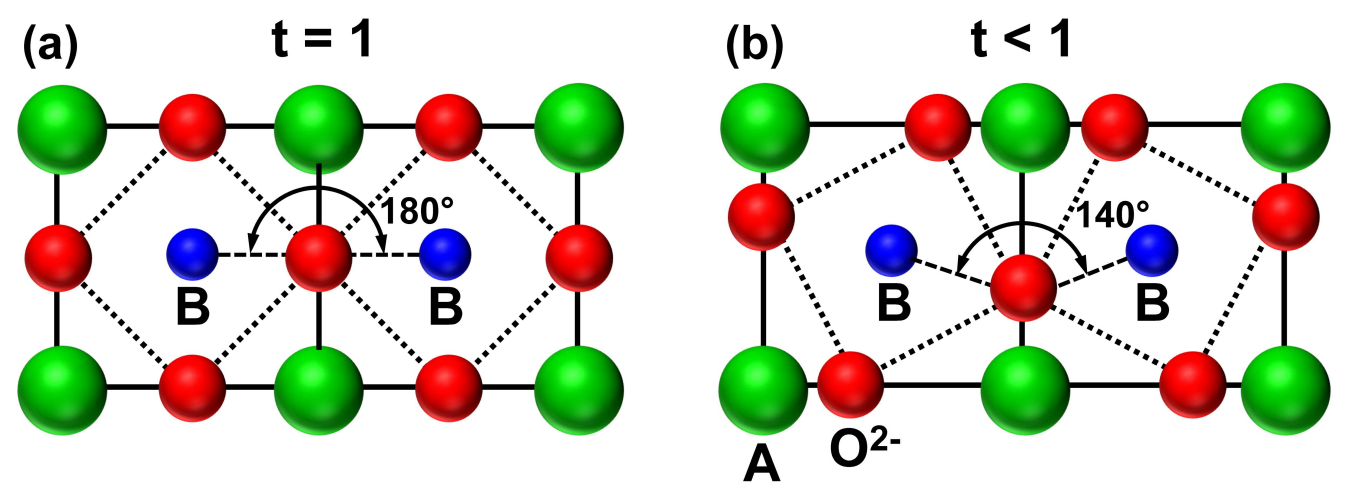

Abbildung 2.1.2: $\mathrm{B}-\mathrm{O}-\mathrm{B}-\mathrm{Bindungswinkel} \mathrm{und} \mathrm{die} \mathrm{jeweilige} \mathrm{Lage} \mathrm{der} \mathrm{BO}_{6}$-Oktaeder in der idealen $(t=1)$ (a) und verspannten $(t<1)$ (b) Perowskit-Struktur (nach [5]).

Empirische Untersuchungen zeigen eine Stabilität der Perowskit-Struktur für Toleranzfaktoren im Bereich von etwa $0,75 \lesssim t \lesssim 1,02$ [5,11]. Die Verzerrung der Gitterstruktur verstärkt sich hierbei für $t<1$ mit abnehmenden Toleranzfaktor beginnend bei einer rhomboedrische Struktur bis hin zu einer monoklinen Struktur, bevor die Perowskit-Struktur 
instabil wird und sich andere Strukturtypen wie Korund oder Ilmenit ausbilden [11, 12]. Die meisten Perowskit-Materialien besitzen daher eine entsprechend verzerrte Gitterstruktur. Für zu große A-Kationen (Toleranzfaktor $t>1$ ) kann die resultierende Verspannung hingegen nicht durch Verdrehungen und Verkippungen der Sauerstoffoktaeder ausgeglichen werden, sodass die Perowskit-Struktur für die meisten Materialien bereits für $t>1,02$ instabil wird und sich sich stattdessen eine hexagonale Struktur ausbildet [4,11].

\subsection{Perowskitische Manganate}

Die in dieser Arbeit untersuchten gemischtvalenten Manganate $\mathrm{A}_{1-\mathrm{x}} \mathrm{A}_{\mathrm{x}}^{\prime} \mathrm{MnO}_{3}$ (wobei die A-Plätze durch trivalente Kationen der Seltenen Erden oder je nach Substitution $x$ durch divalente $\mathrm{A}^{\prime}$-Kationen der Erdalkalimetalle eingenommen werden, sodass die B-Plätze von $\mathrm{Mn}^{3+}$ - und $\mathrm{Mn}^{4+}$-Ionen besetzt werden) kristallisieren ebenfalls in unterschiedlich verzerrten Arten der $\mathrm{ABO}_{3}$-Perowskit-Struktur. Hierbei wird mit Abnahme des Toleranzfaktors $t$ zunächst eine Änderung von der kubischen in eine rhomboedrische $(0,96<t<1)$ und darüber hinaus in eine orthorhombische Gitterstruktur $(t<0,96)$ beobachtet [5,7]. Die damit einhergehende Verkleinerung des Mn-O-Mn-Bindungswinkels hat dabei wie das Verhältnis der Mn-Valenzen $\mathrm{Mn}^{3+} / \mathrm{Mn}^{4+}=(1-\mathrm{x}) / \mathrm{x}$ auf den B-Plätzen und die Anordnung der Kationen aufgrund ihrer unterschiedlichen Radien einen großen Einfluss auf die elektrischen und magnetischen Eigenschaften [5,8]. Die für die Manganate auftretenden Wechselwirkungen und Austauschmechanismen werden nun in den folgenden Abschnitten vorgestellt.

\subsubsection{Elektronische Struktur und Jahn-Teller-Effekt}

Daher soll nun zunächst die elektronische Struktur der Manganate betrachtet werden. Die $\mathrm{Mn}^{3+}$-Kationen besitzen hierbei eine $[\mathrm{Ar}] 3 \mathrm{~d}^{4}$-Elektronenkonfiguration und damit eine Argon-Edelgaskonfiguration mit vier zusätzlichen Elektronen auf der 3d-Schale, während die $\mathrm{Mn}^{4+}$-Kationen entsprechend über ein Elektron weniger und somit über eine $[\mathrm{Ar}] 3 \mathrm{~d}^{3}$ Elektronenkonfiguration verfügen. Für ein freies Mangan-Ion sind dabei die fünf Orbitale der 3d-Schale energetisch entartet und die Besetzung erfolgt nach den Hundschen Regeln in einer paralleler Ausrichtung mit maximalem Spin [5]. Im Manganat befinden sich die Mangan-Ionen hingegen auf den B-Plätzen in der Perowskit-Struktur umgeben von den Sauerstoff-Oktaedern. Die resultierende Coulomb-Wechselwirkung mit dem oktaedrischem Kristallfeld führt daher aufgrund der unterschiedlichen Ausrichtung der Orbitale zu einer Aufspaltung der Orbitale in drei energetisch abgesenkte $t_{2 g}$-Orbitale, die sich aus den $d_{x y^{-}}$, $d_{x z^{-}}$und $d_{y z^{-}}$Orbitalen zusammensetzen, und zwei energetisch erhöhte $e_{g^{-}}$Orbitale, die aus den $d_{x^{2}-y 2^{-}}$und $d_{3 z^{2}-r^{2}}$ Orbitalen gebildet werden. Für Manganate liegt die Kristallfeldenergie $\Delta_{\mathrm{CF}}$, die die Aufspaltung der $t_{2 g^{-}}$und $e_{g^{-}}$Orbitale quantifiziert, in der Größenordnung von $\Delta_{\mathrm{CF}} \approx 1-2 \mathrm{eV}$ [5,7,8]. Eine Darstellung der Aufspaltung und Besetzung der 3d-Orbitale ist für das $\mathrm{Mn}^{3+}$-Kation in der Abbildung 2.2.1 (a) (nach [5]) dargestellt. 


\section{Theoretischer Hintergrund}

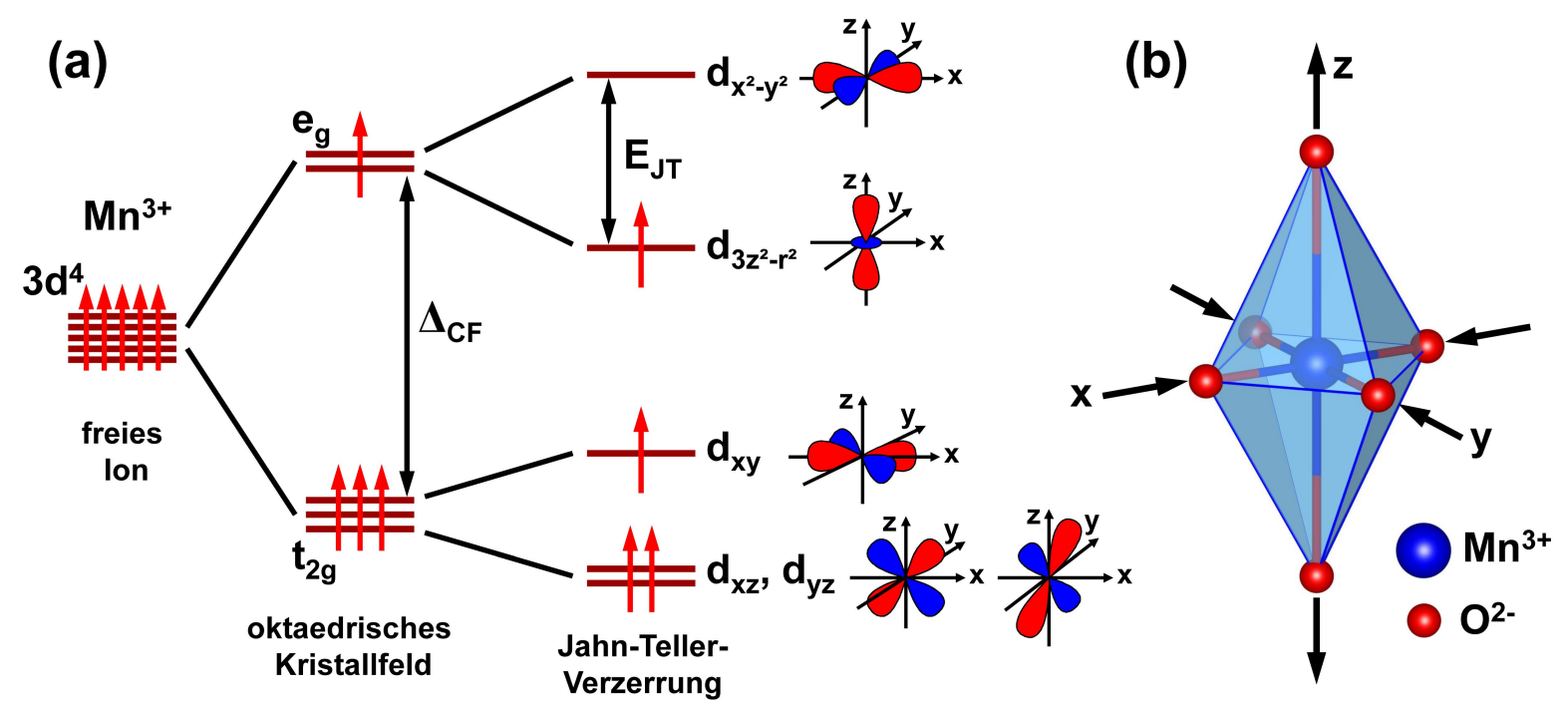

Abbildung 2.2.1: Aufspaltung der 3d-Orbitale eines $\mathrm{Mn}^{3+}$-Kations durch das Kristallfeld und den Jahn-Teller-Effekt in der Perowskit-Struktur (a) (nach [5]) und Prinzip der Jahn-Teller-Verzerrung des $\mathrm{MnO}_{6}$-Oktaeders (b). Die Vorzeichen der Wellenfunktionen der 3d-Orbitale sind hierbei jeweils durch die Farben mit rot (positives Vorzeichen) und blau (negatives Vorzeichen) gekennzeichnet.

Aufgrund der Hundschen Regeln und der gegenüber der Kristallfeldenergie $\Delta_{\mathrm{CF}}$ größeren Hundschen Kopplungsenergie $J_{H} \approx 2-3 \mathrm{eV}>\Delta_{\mathrm{CF}}$ für Manganate [5,7] besetzen drei der vier $3 \mathrm{~d}$-Elektronen des $\mathrm{Mn}^{3+}$-Kations die drei $t_{2 g}$-Orbitale und das vierte Elektron eines der $e_{g}$-Orbitale mit parallelem Spin (High-Spin-Zustand, Gesamtspin $S=2$ ). Der High-Spin-Zustand (HS) ist für viele 3d-Übergangsmetalloxide charakteristisch [5, 11. Ausnahmen sind Nickelate $\left(3 \mathrm{~d}^{7}\right.$-Konfiguration für $\left.\mathrm{Ni}^{3+}\right)$, für die sich formal ein Low-SpinZustand (LS) ergibt $\left(J_{H}<\Delta_{\mathrm{CF}}\right) \sqrt{13}$, und Cobaltate, bei denen sich für das $\mathrm{Co}^{3+}$-Kation (3d $\mathrm{d}^{6}$-Konfiguration) neben dem HS- und LS- ein Intermediate-Spin-Zustand (IS) ausbilden kann $\left(J_{H} \approx \Delta_{\mathrm{CF}}\right)$ 11, 14. Für ein $\mathrm{Mn}^{4+}$-Kation werden die drei 3d-Elektronen den Hundschen Regeln folgend mit parallelem Spin $\left(S=\frac{3}{2}\right)$ in die drei $t_{2 g}$-Orbitale eingebaut.

Die energetische Entartung der zwei $e_{g}$-Orbitale für die Besetzung des vierten 3d-Elektrons des $\mathrm{Mn}^{3+}$-Kations wird hingegen nach dem Jahn-Teller-Theorem [15] durch eine zusätzliche Verzerrung des Gitters aufgehoben. Für die $\mathrm{Mn}^{3+}$-Kationen in der Perowskit-Struktur wird dieses durch die Elongation des umgebenden Sauerstoffoktaeders in die z-Richtung bei einer gleichzeitigen Kompression in die dazu senkrechten $\mathrm{x}$ - und $\mathrm{y}$-Richtungen erreicht. Dieser Prozess ist für das $\mathrm{MnO}_{6}$-Oktaeder in der Abbildung 2.2.1 (b) dargestellt. Die Aufspaltung der Energieniveaus der Orbitale entsteht dabei wie bei dem Kristallfeld aus einer unterschiedlich starken Coulomb-Wechselwirkung zwischen den Elektronen in den Orbitalen und den $\mathrm{O}^{2-}$-Anionen des Oktaeder. Folglich kommt es zu einer energetischen Absenkung des $d_{3 z^{2}-r^{2}}$ und einer energetischen Erhöhung des $d_{x^{2}-y 2^{2}}$ Orbitals. In gleicher Weise wird bei den $t_{2 g}$-Orbitalen durch den Jahn-Teller-Effekt das $d_{x y}$-Orbital energetisch erhöht und die $d_{x z^{-}}$und $d_{y z}$-Orbitale energetisch abgesenkt (Abbildung 2.2.1(a)). Es ergibt 
sich ein energetisches Minimum zwischen der aufzuwendenden elastischen Energie und der Orbitalaufspaltung. Die Aufspaltung der $e_{g}$-Orbitale liegt für die $\mathrm{Mn}^{3+}$-Kationen hierbei in einer Größenordnung von $E_{\mathrm{JT}} \approx 0,5-1,5 \mathrm{eV}[8]$, während diese aufgrund des fehlenden $e_{g}$-Elektrons für die $\mathrm{Mn}^{4+}$-Kationen entsprechend entfällt. Die Jahn-Teller-Verzerrung der $\mathrm{MnO}_{6}$-Oktaeder führt zudem zu einer Verspannung des gesamten Kristallgitters, die für eine große Anzahl an $\mathrm{Mn}^{3+}$-Kationen wie für undotierte Manganate $(x=0)$ in einer kollektiven Anordnung der Oktaeder in Form einer kooperativen Jahn-Teller-Verzerrung resultiert, die mit einem strukturellen Phasenübergang des Kristallsystems einhergeht. Dieses lässt sich beispielsweise für $\mathrm{LaMnO}_{3}$ (LMO) beobachten, das bis etwa $T \approx 800 \mathrm{~K}$ einen kooperativen Jahn-Teller Effekt mit einer orthorhombischen Gitterstruktur zeigt, bevor dieser darüber hinaus in einen unkoordinierten Zustand übergeht und sich für $T \approx 1000 \mathrm{~K}$ schließlich sogar eine rhomboedrische Struktur ausbildet [16, 17]. Der kooperative Zustand der Jahn-Teller-Verzerrungen mit jeweils einem Elektron im $e_{g}$-Orbital wird hierbei auch als Jahn-Teller-Polaron bezeichnet 5,8. Durch ein Einbringen von $\mathrm{Mn}^{4+}$-Kationen durch eine Substitution von $\mathrm{La}^{3+}$ etwa durch $\mathrm{Sr}^{2+}$ wird der Phasenübergang mit zunehmenden Dotierung zu niedrigeren Temperaturen verschoben, da die $\mathrm{Mn}^{4+}$-Kationen aufgrund des fehlenden $e_{g}$-Elektrons nicht zu einer kooperativen Jahn-Teller-Verzerrung beitragen [5].

Die strukturellen Eigenschaften und die elektronische Struktur wirken sich daher sehr stark auf die elektrischen und magnetischen Eigenschaften der Manganate aus. So sind für die gemischtvalenten Manganate $\mathrm{A}_{1-\mathrm{x}} \mathrm{A}_{\mathrm{x}}^{\prime} \mathrm{MnO}_{3}$ in Abhängigkeit der Dotierung und des $\mathrm{Mn}^{3+} / \mathrm{Mn}^{4+}$-Verhältnisses verschiedene magnetische Phasen zu beobachten. Das Sättigungsmoment von $\mathrm{La}_{1-\mathrm{x}} \mathrm{Sr}_{\mathrm{x}} \mathrm{MnO}_{3}$ (LSMO) ist dabei aufgrund des im Kristallfeld der $\mathrm{MnO}_{6}$-Oktaeder verschwindenden Bahnmoments und der Edelgaskonfigurationen der Kationen der Erdalkalimetalle, der $\mathrm{O}^{2-}$-Anionen und des $\mathrm{La}^{3+}$-Kations nur durch die SpinBeiträge der $\mathrm{Mn}^{3+}$ - und die $\mathrm{Mn}^{4+}$-Kationen bestimmt [5]. Für das Sättigungsmoment folgt somit $m_{S}=2\left(2(1-x)+\frac{3}{2} x\right) \mu_{B}=(4-x) \mu_{B}$. Für andere Seltenen Erden auf dem A-Platz muss zudem ein möglicher Beitrag der Elektronen der 4f-Schale berücksichtigen werden.

\subsubsection{Austauschmechanismen}

Die elektrischen und magnetischen Eigenschaften in Übergangsmetalloxiden werden über verschiedene Austauschmechanismen vermittelt. Diese zeichnen sich dadurch aus, dass Elektronen mit einer gewissen Wahrscheinlichkeit zu den Übergangsmetall-Kationen auf benachbarten Gitterplätzen hüpfen können und dabei die Energie des Systems abgesenkt wird. Die zwei für die Manganate relevanten Prozesse sind der Superaustausch [18, 19] und der Doppelaustausch 20,21. Da der Überlapp benachbarter 3d-Orbitale bei Manganaten jedoch nur sehr gering ist, wird der Austauschprozess bei beiden Mechanismen indirekt über den großen Überlapp und die Hybridisierung mit den 2p-Orbitalen des $\mathrm{O}^{2-}$-Anions zwischen zwei benachbarten Mangan-Kationen im Kristallgitter vermittelt. 


\section{Theoretischer Hintergrund}

\section{Superaustausch}

Für den Superaustausch kann zunächst eine Vereinfachung nach dem Hubbard-Modell für nicht-entartete Niveaus betrachtet werden, in dem sowohl das Hüpfen der Elektronen zwischen den Mangan-Gitterplätzen als auch ihre Coulomb-Wechselwirkung berücksichtigt wird und sich auf den Mangan-Gitterplätzen jeweils nur ein relevantes Elektron mit einem verfügbaren Niveau befindet. Der Transfer eines Elektrons zu einem benachbarten Gitterplatz ist daher mit der Aufbringung einer Coulomb-Energie $U$ verbunden, während die Delokalisierung der Elektronen durch den Hüpfprozess zu einem Gewinn an kinetischer Energie führt. Dieses wird mit der Hüpfamplitude $t_{0}$ charakterisiert. Ist nun $U \gg t_{0}$, so ist es energetisch günstiger jeden Gitterplatz mit nur einem Elektron zu besetzen. Die Elektronen sind in diesem Fall lokalisiert und es stellt sich mit dem Mott-Hubbard-Isolator ein elektrisch isolierender Zustand ein. Anhand von Störungstheorie zweiter Ordnung ergibt sich ein Energiegewinn in der Größenordnung von $t_{0}^{2} / U$ für virtuelle Hüpfprozesse der lokalisierten Elektronen [5]. Die Spins der Elektronen auf benachbarten Gitterplätzen bevorzugen dabei aufgrund des Pauli-Prinzips eine antiferromagnetische Spin-Anordnung, die mit einer Energieabsenkung um $\Delta E=-2 t_{0}^{2} / U$ einhergeht 22 . Hierbei sind bei den Manganaten nur die $e_{g}$-Orbitale beteiligt, da die stärker lokalisierten $t_{2 g}$-Orbitale einen geringeren Überlapp mit den 2 p-Orbitalen des $\mathrm{O}^{2-}$-Anionen, die gegenüber den $e_{g}$-Orbitalen um die Ladungstransferenergie $\Delta$ abgesenkt sind, aufweisen 22. Der Überlapp ist zudem mit $\propto|\cos (\theta)|$ vom Mn-O-Mn-Bindungswinkel $\theta$ abhängig [5]. Folglich nimmt die Stärke der Austauschs mit Zunahme der Verzerrung der Perowskit-Struktur systematisch ab.

Je nach Konfiguration der beteiligten Orbitale der Übergangsmetallionen im Kristallgitter kann sich nun jedoch sowohl eine antiferromagnetische (AFM) als auch eine ferromagnetische (FM) Kopplung zwischen den Spins der d-Elektronen einstellen. Für Manganate wie $\mathrm{LMO}$, das nur über $\mathrm{Mn}^{3+}$-Kationen verfügt und ein $e_{g}^{1}-\mathrm{O}^{2-}-e_{g}^{1}$-System repräsentiert, können die drei in den Abbildungen 2.2.2 (a)-(c) (nach [5]) dargestellten Fälle betrachtet werden. Die Orbitale sind dabei so ausgerichtet, dass die $d_{3 z^{2}-r^{2}}$-Orbitale der $\mathrm{Mn}^{3+}$-Kationen auf die z-Komponente der 2p-Orbitale der $\mathrm{O}^{2-}$-Anionen zeigen und die $d_{x^{2}-y^{2}}$-Orbitale dazu senkrecht stehen. Die stärker lokalisierten $t_{2 g}$-Elektronen nehmen zudem aufgrund der Hundschen Kopplung eine parallele Spinausrichtung zum $e_{g}$-Elektron ein. Im ersten Fall (a) befindet sich nun das jeweilige $e_{g}$-Elektron der beiden $\mathrm{Mn}^{3+}$-Kationen im $d_{3 z^{2}-r^{2}}$-Orbital und es stellt sich eine antiferromagnetische Kopplung der $e_{g}$-Elektronen ein. Eine ebenso antiferromagnetische Kopplung ergibt sich im zweiten Fall (b), bei dem sich das jeweilige $e_{g}$-Elektron der $\mathrm{Mn}^{3+}$-Kationen im $d_{x^{2}-y^{2}}$-Orbital befindet und die $d_{3 z^{2}-r^{2} \text {-Orbitale }}$ unbesetzt sind. Im dritten Fall (c) besetzt nun das $e_{g}$-Elektron des einen $\mathrm{Mn}^{3+}$-Kations das $d_{3 z^{2}-r^{2}}$-Orbital, während sich das $e_{g}$-Elektron des anderen $\mathrm{Mn}^{3+}$-Kations im $d_{x^{2}-y^{2-}}$ Orbital befindet. Folglich stellt sich in diesem Fall aufgrund der Hundschen Kopplung eine ferromagnetische Anordnung der Spins der $e_{g}$-Elektronen der beiden $\mathrm{Mn}^{3+}$-Kationen ein. 


\subsection{Perowskitische Manganate}

(a)
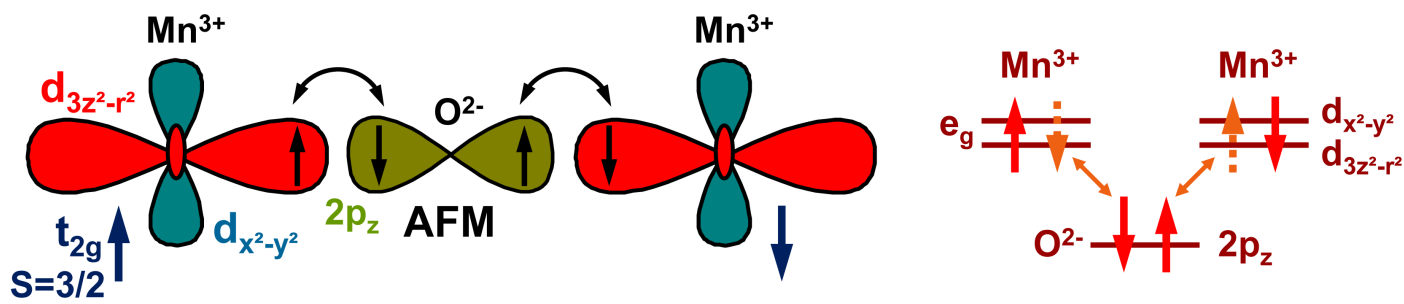

(b)
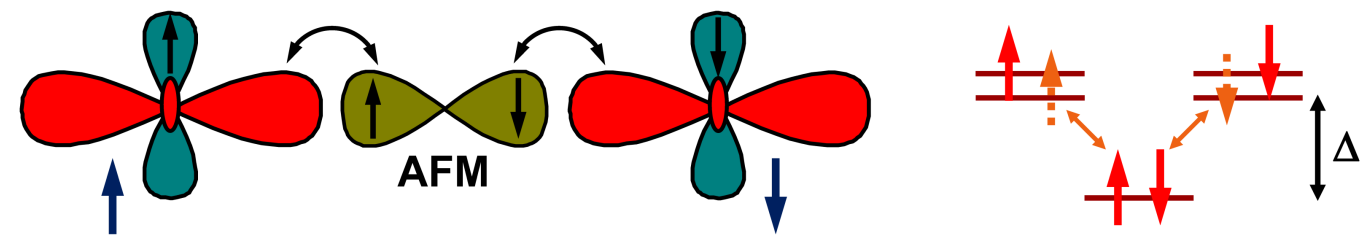

(c)
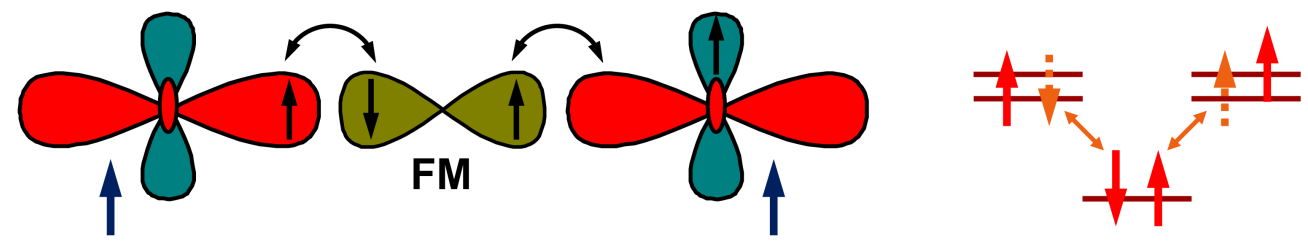

Abbildung 2.2.2: Magnetische Austauschwechselwirkungen zwischen zwei $\mathrm{Mn}^{3+}$-Kationen eines Manganats wie LMO aufgrund des Superaustauschs mit einer antiferromagnetischen Kopplung für besetzte (a) und unbesetzte (b) $d_{3 z^{2}-r^{2} \text {-Orbitale }}$ sowie einer entsprechend ferromagnetischen Kopplung für ein besetztes und ein unbesetztes $d_{3 z^{2}-r^{2}}$-Orbital (c) im Vergleich (nach [5]).

Die zu erwartende magnetische Kopplung lässt sich zudem mithilfe der GoodenoughKanamori-Anderson-Regeln (GKA) [19,23, 24] abschätzen, in die auch die oben gezeigten Fälle des Superaustauschs für die $\mathrm{Mn}^{3+}$-Kationen von LMO eingeordnet werden können (5):

1. $180^{\circ}$-Austausch zwischen gefüllten oder leeren Orbitalen $\Rightarrow$ AFM (stark)

2. $180^{\circ}$-Austausch zwischen einem gefüllten und einem leeren Orbitalen $\Rightarrow \mathrm{FM}$ (schwach)

3. $90^{\circ}$-Austausch zwischen gefüllten Orbitalen $\Rightarrow$ FM (schwach)

Demnach sind die in den Abbildungen 2.2.2 (a) und (b) dargestellten Fälle der 1. GKARegel zuzuordnen, während der Fall in der Abbildung 2.2.2 (c) der 2. GKA-Regel entspricht. Die 3. GKA-Regel mit einem $90^{\circ}$-Austausch tritt hingegen für Manganate mit Perowskit-Struktur aufgrund der damit einhergehenden Orbital-Anordnung nicht auf.

\section{Doppelaustausch}

Während beim Superaustausch die Besetzung der d-Orbitale der Übergangsmetallionen gleich ist oder sich um zwei unterscheidet, die 3d-Elektronen entsprechend lokalisiert sind und der Hüpfprozess zum benachbarten Gitterplatz nur von virtueller Art ist, wird beim Doppelaustausch die Austauschwechselwirkung zwischen Übergangsmetallionen mit gemischter Valenz, delokalisierten d-Elektronen und reellen Hüpfprozessen betrachtet [5.22]. Für LMO wird dieses etwa durch die Dotierung mit Strontium (LSMO) erreicht, sodass 


\section{Theoretischer Hintergrund}

sowohl $\mathrm{Mn}^{3+}$ - als auch $\mathrm{Mn}^{4+}$-Kationen vorhanden sind. Anschließend kann wieder eine Ausrichtung der $d_{3 z^{2}-r^{2}}$-Orbitale der $\mathrm{Mn}^{3+}$ - und $\mathrm{Mn}^{4+}$-Kationen auf die z-Komponente der 2p-Orbitale der $\mathrm{O}^{2-}$-Anionen, über die die Kopplung vermittelt wird, und dazu senkrecht orientierte $d_{x^{2}-y^{2}}$-Orbitale betrachtet werden. Dieses ist in der Abbildung 2.2.3 (nach [5]) dargestellt. Dabei ist das $d_{3 z^{2}-r^{2}}$-Orbital des $\mathrm{Mn}^{3+}$-Kations mit einem $e_{g^{-}}$Elektron besetzt, während es für das $\mathrm{Mn}^{4+}$-Kation aufgrund des fehlenden $e_{g}$-Elektrons unbesetzt bleibt. Das $e_{g}$-Elektron des $\mathrm{Mn}^{3+}$-Kations kann nun über das dazwischen liegende $\mathrm{O}^{2-}$-Anion bei einer parallelen Spin-Ausrichtung der dazugehörigen $t_{2 g}$-Elektronen ohne ein zusätzliches Aufwenden von Coulomb-Energie $U$ oder Hundscher Kopplungsenergie $J_{H}$ in das leere $d_{3 z^{2}-r^{2}}$-Orbital des $\mathrm{Mn}^{4+}$-Kations hüpfen. Der Doppelaustauschs wird hierbei über das gleichzeitige Hüpfen des $e_{g}$-Elektrons in das $2 \mathrm{p}_{\mathrm{z}}$-Orbital des $\mathrm{O}^{2-}$-Anions und des Elektrons im $2 \mathrm{p}_{\mathrm{z}}$-Orbital mit zum $e_{g}$-Elektron parallelem Spin in das leere $d_{3 z^{2}-r^{2}}$-Orbital des $\mathrm{Mn}^{4+}$-Kations vermittelt 8]. Das 3d-Elektron des $\mathrm{Mn}^{3+}$-Kations ist somit delokalisiert und gegenüber dem $\mathrm{Mn}^{4+}$-Kation beweglich, sodass sich für die ferromagnetische Kopplung der gemischtvalenten Mangan-Ionen ein metallischer Zustand einstellt. Die effektive Hüpfamplitude $t_{\text {eff. }}$ und damit die Beweglichkeit der 3d-Elektronen ist folglich von der relativen Orientierung der benachbarten $t_{2 g}$-Spins zueinander abhängig, da für eine antiparallele Ausrichtung zusätzlich die Hundsche Kopplungsenergie $J_{H}$ aufgebracht werden muss und der Hüpfprozess stark eingeschränkt wird. Es ergibt sich die Relation $t_{\text {eff. }}=t_{0} \cos (\theta / 2)$ [8,11], wobei $\theta$ den Winkel zwischen den lokalisierten Spins der 3d-Elektronen auf benachbarten Gitterplätzen und $t_{0}$ die Hüpfamplitude des $e_{g}$-Elektrons bezeichnet. Entsprechend bevorzugt ist somit eine ferromagnetische Kopplung $\left(\theta=0^{\circ}\right)$ der gemischtvalenten ManganIonen, die gleichzeitig mit einer hohen Beweglichkeit der $e_{g}$-Elektronen einhergeht.
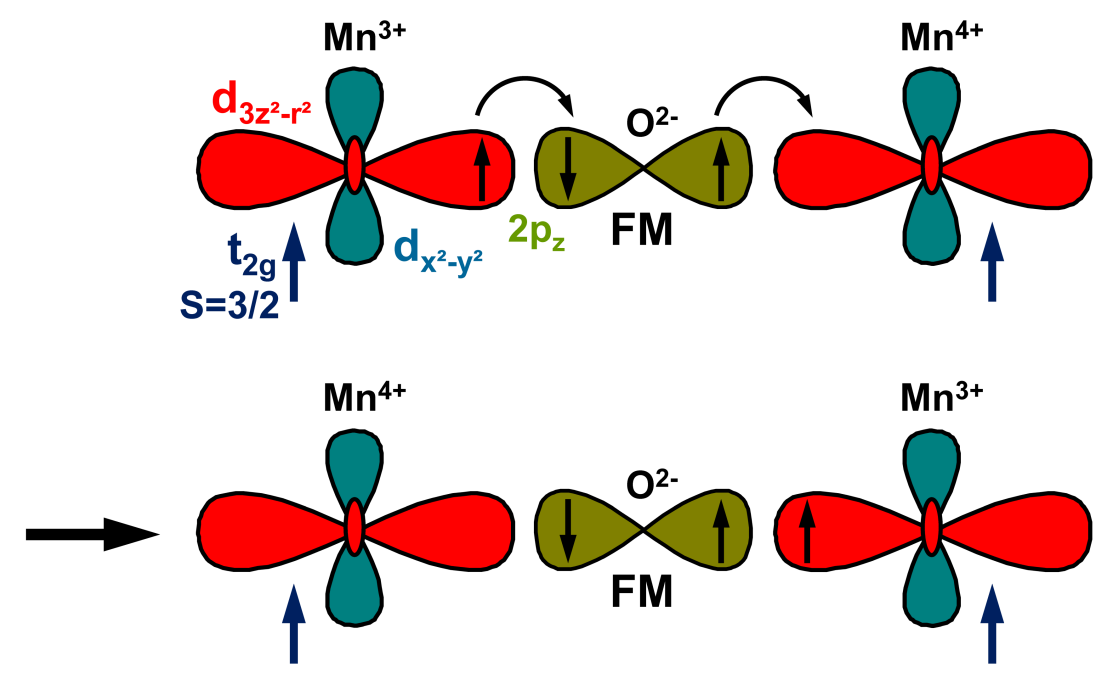

Abbildung 2.2.3: Doppelaustausch zwischen einem $\mathrm{Mn}^{3+}$ - und einem $\mathrm{Mn}^{4+}$-Kation eines gemischtvalenten Manganats wie LSMO für $180^{\circ}$-Bindungen entlang der $d_{3 z^{2}-r^{2}}$-Orbitale, vermittelt über das $2 \mathrm{p}_{\mathrm{z}}$-Orbital eines $\mathrm{O}^{2-}$-Anions durch einen Hüpfprozess des $e_{g}$-Elektrons vom $\mathrm{Mn}^{3+}$ - zum $\mathrm{Mn}^{4+}$-Kation bei ferromagnetischer Kopplung. Es ergibt sich ein metallischer Zustand (nach [5]). 


\subsubsection{Phasendiagramm von moderat dotiertem $\mathrm{La}_{1-\mathrm{x}} \mathrm{Sr}_{\mathrm{x}} \mathrm{MnO}_{3}$}

Anhand des Superaustauschs und des Doppelaustauschs lassen sich nun die komplexen Phasendiagramme der gemischtvalenten Manganate $\mathrm{A}_{1-\mathrm{x}} \mathrm{A}_{\mathrm{x}}^{\prime} \mathrm{MnO}_{3}$ in Abhängigkeit der Dotierung $x$ nachvollziehen. In der Abbildung 2.2.4 ist beispielhaft das kombinierte strukturelle, elektronische und magnetische $T$ - $x$-Phasendiagramm für Bulk-La ${ }_{1-x} \mathrm{Sr}_{\mathrm{x}} \mathrm{MnO}_{3}$ (LSMO) im Bereich einer moderaten Sr-Dotierung $0 \leq x \leq 0,35$ (nach [16, 17, 25 27]) dargestellt.

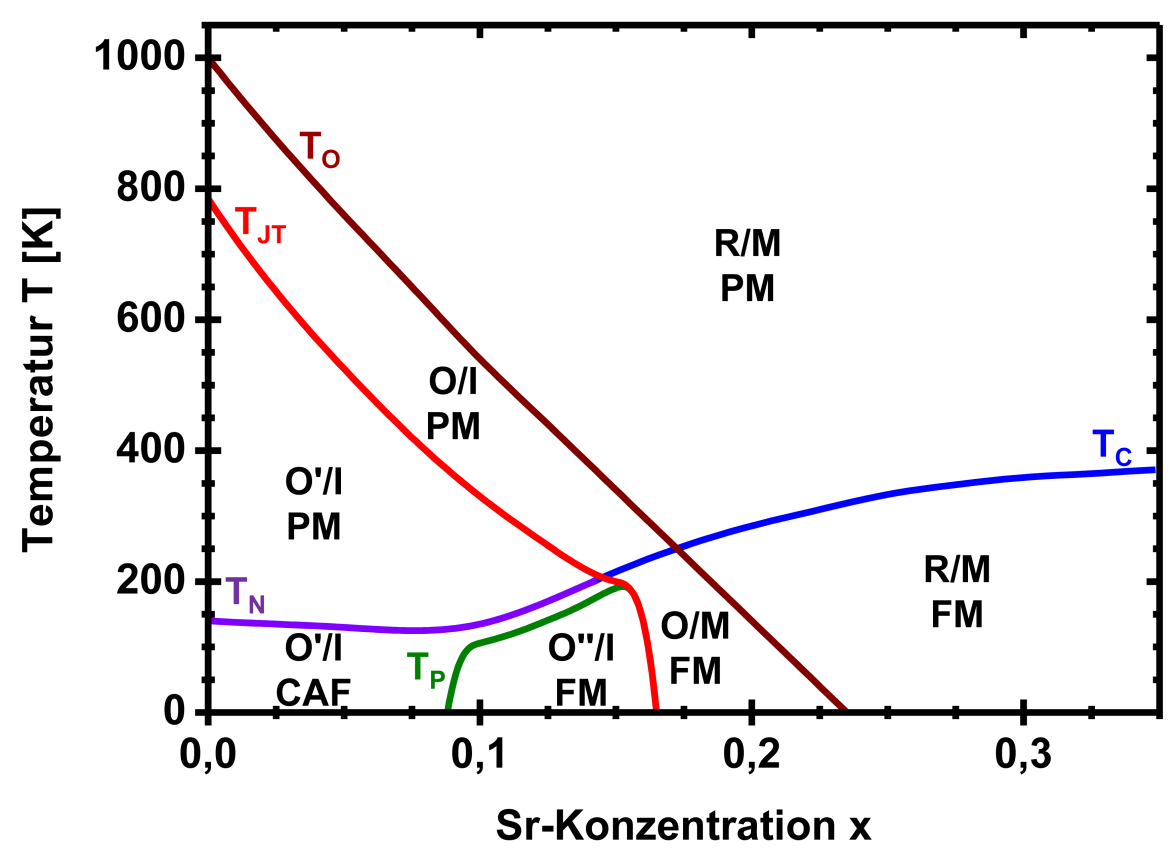

Abbildung 2.2.4: Strukturelles, elektronisches und magnetisches $T$ - $x$-Phasendiagramm für $\mathrm{La}_{1-\mathrm{x}} \mathrm{Sr}_{\mathrm{x}} \mathrm{MnO}_{3}$ (LSMO) im Bereich einer moderaten Strontium-Dotierung von $0 \leq x \leq 0,35$ (nach [16, 17, 25 27]). R, O, O' und O" kennzeichnen hierbei eine rhomboedrische, orthorhombische, Jahn-Teller-verzerrte orthorhombische und die orbital-geordnet orthorhombische Kristallstruktur, während PM, FM und CAF eine paramagnetische, ferromagnetische und gekantet-antiferromagnetische (A-Typ) Phase sowie I und M eine entsprechend elektrisch-isolierende und metallische Phase bezeichnen.

Für niedrige Temperaturen und Dotierungen $x<0,1$ lässt sich eine elektrisch-isolierende Phase mit kooperativ Jahn-Teller-verzerrter orthorhombischer Perowskit-Struktur $\left(\mathrm{O}^{\prime}\right)$ beobachten, die mit einem gekantet-antiferromagnetischen Verhalten (CAF) unterhalb der Néel-Temperatur $T_{N} \approx 140 \mathrm{~K}$ (für $x=0$ ) einhergeht [16,26]. Dieses ist auf die bevorzugte antiferromagnetische Kopplung des Superaustauschs zurückführen, die in diesem Bereich gegenüber des Doppelaustauschs aufgrund der nur kleinen Sr-Dotierung überwiegt. Die $e_{g}$-Orbitale ordnen dabei in der Art, dass sich nach den GKA-Regeln entlang der c-Achse eine antiferromagnetische und in der Ebene senkrecht dazu eine ferromagnetische Kopplung ergibt, die insgesamt zur Ausbildung eines Antiferromagnets vom Typ-A führt [5]. Die leichte Abnahme der Néel-Temperatur $T_{N}$ mit steigender Sr-Konzentration für $x<0,1$ ist dabei auf die fortschreitende Störung der kooperativen Jahn-Teller-Verzerrung und auf 


\section{Theoretischer Hintergrund}

eine beginnende Abschwächung des Superaustauschs gegenüber dem einsetzenden Doppelaustausch zurückzuführen [17, 25]. Diese zunehmende Instabilität äußert sich auch in der starken Abnahme der Übergangstemperaturen $T_{\mathrm{JT}}$ und $T_{O}$, oberhalb derer sich zunächst eine unkoordinierte orthorhombische $(\mathrm{O})$ und schließlich eine rhomboedrische $(\mathrm{R})$ Perowskit-Struktur ausbildet, bis es zu einem völligen Ausbleiben der O'-Phase für SrKonzentrationen $x>0,15$ und der O-Phase für $x>0,20$ kommt [16, 17, 25. Da LMO zudem ein Mott-Hubbard-Isolator ist und die $e_{g}$-Elektronen durch den überwiegenden Superaustausch stark lokalisiert sind, ergibt sich im Bereich von $x<0,1$ ein elektrischisolierendes Verhalten. Für Sr-Konzentrationen darüber hinaus gewinnt der Doppelaustausch zwischen den $\mathrm{Mn}^{3+}$ - zum $\mathrm{Mn}^{4+}$-Kationen verstärkt an Wirkung und es stellt sich für $0,10 \leq x \leq 0,15$ ein ferromagnetischer Grundzustand ein, der jedoch durch die Konkurrenzsituation von Super- und Doppelaustausch mit einem Übergang in eine neue lokal orbital-geordnete Phase für Temperaturen $T \leq T_{P}<T_{N}$ beeinflusst wird und somit weiterhin einen elektrisch-isolierenden Charakter aufweist [16,25]. Der Anstieg von $T_{N}$ und $T_{P}$ mit Zunahme der Sr-Konzentration zeigt jedoch bereits einen Zugewinn der DoppelaustauschWechselwirkung an, bis die $e_{g}$-Elektronen schließlich soweit delokalisiert sind, dass sich für $x>0,17$ ein ferromagnetisch-metallischer Charakter einstellt [17, 25, 26], der mit weiter zunehmender Sr-Konzentration mit einem kontinuierlichen Anstieg der Curie-Temperatur $T_{C}$ einhergeht, bis sich für $0,3 \leq x \leq 0,4$ mit $T_{C} \approx 370 \mathrm{~K}$ ein Maximum einstellt [16, 27.

\section{$2.3 \mathrm{~A}_{2} \mathrm{BB}^{\prime} \mathrm{O}_{6}$-Doppelperowskite}

Anhand der gemischtvalenten Manganate $\mathrm{A}_{1-\mathrm{x}} \mathrm{A}_{\mathrm{x}}^{\prime} \mathrm{MnO}_{3}$ zeigt sich somit die durch die Dotierung des A-Platz-Kations durch ein A'-Kation mit unterschiedlicher Größe und Valenz entstehende große Vielfalt an strukturellen, elektrischen und magnetischen Phasen, über die Materialien in der Perowskit-Struktur $\mathrm{ABO}_{3}$ verfügen. Neben dem A-Platz-Kation lässt sich auch das B-Platz-Kation partiell ersetzen, um die Eigenschaften des Perowskits systematisch zu beeinflussen. Die Kationen auf den B-Plätzen des resultierenden Perowskits $\mathrm{AB}_{1-\mathrm{x}} \mathrm{B}_{\mathrm{x}}^{\prime} \mathrm{O}_{3}$ können hierbei chemisch ordnen. Im geordneten Fall gleicher Mengen an B- und B'-Kationen $(x=0,5)$ kann dieses zu der Entstehung sogenannter $\mathrm{A}_{2} \mathrm{BB}^{\prime} \mathrm{O}_{6^{-}}$ Doppelperowskit-Oxide führen, die in dieser Arbeit ein zentrales Materialsystem darstellen und in den folgenden Abschnitten dieses Kapitels ausführlich vorgestellt werden sollen.

\subsubsection{Aufbau, Kristallstruktur und Kationenordnung}

$\mathrm{A}_{2} \mathrm{BB}^{\prime} \mathrm{O}_{6}$-Doppelperowskite bezeichnen somit Materialien in der Perowskit-Struktur $\mathrm{ABO}_{3}$, bei der eine spezifisch geordnete Verteilung der in gleicher Anzahl (1:1-Verhältnis) vorhandenen, unterschiedlichen B- und B'-Kationen vorliegt, die über eine völlig zufällige Anordnung $\left(\mathrm{AB}_{0,5} \mathrm{~B}_{0,5}^{\prime} \mathrm{O}_{3}\right)$ hinausgeht und in dem am häufigsten zu beobachtenden Ordnungstyp der NaCl-Struktur in einer in alle drei Raumrichtungen abwechselnden B-Platz-Besetzung 
der B/B'-Platz-Kationen resultiert. Da diese NaCl-Anordnung der B/B'-Platz-Kationen in Abwesenheit einer oktaedrischen Verkippung (Toleranzfaktor $t=1$ ) mit einer Verdopplung der kubischen Einheitszelle (mit der doppelten Gitterkonstante $a_{\mathrm{DP}}=2 a$ ) in der Raumgruppe Fm $\overline{3} \mathrm{~m}$ (Raumgruppe 225) einhergeht 9,28, werden derart ordnende Systeme Doppelperowskite genannt. Wie bei den gewöhnlichen Perowskiten führt eine Abweichung vom idealen Größenverhältnis der A- und B-Platz-Kationen zu einer Verzerrung der kubischen Struktur durch Verdrehungen und Verkippungen der $\mathrm{BO}_{6} / \mathrm{B}^{\prime} \mathrm{O}_{6}$-Oktaeder, mit Toleranzfaktoren $0,85 \lesssim t \lesssim 1,07$ [4, 29]. Häufig ist daher neben der unverzerrten Fm $\overline{3}$ m-Struktur eine Kristallisation in der tetragonalen $\mathrm{I} 4 / \mathrm{m}$ - und in der monoklinen P2 $1 / \mathrm{n}$-Raumgruppe zu beobachten, während ungeordnete Doppelperowskite zumeist die orthorhombische PnmaRaumgruppe favorisieren [4]. Neben der B-Platz-Ordnung in der NaCl-Struktur existieren mit der kolumnaren und lagenweisen Ordnung zwei weitere sehr viel seltener auftretende Doppelperowskit-B-Platz-Ordnungsarten [3, 4]. Die drei Typen der B-Platz-Ordnung sind nachfolgend für einen Vergleich in den Abbildungen 2.3.1 (a)-(c) (nach [3, 4]) dargestellt.

(a)

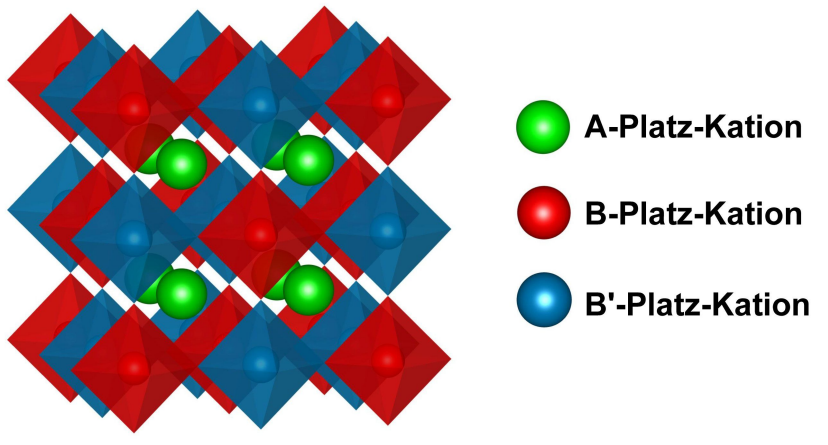

(b)

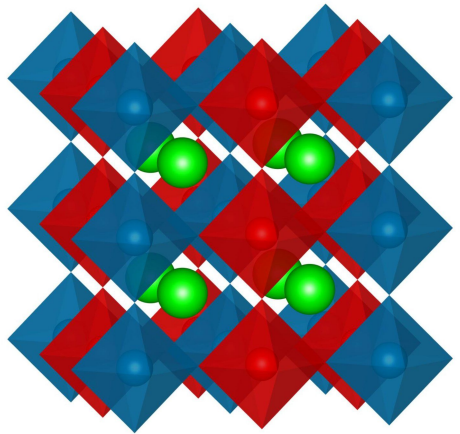

(c)

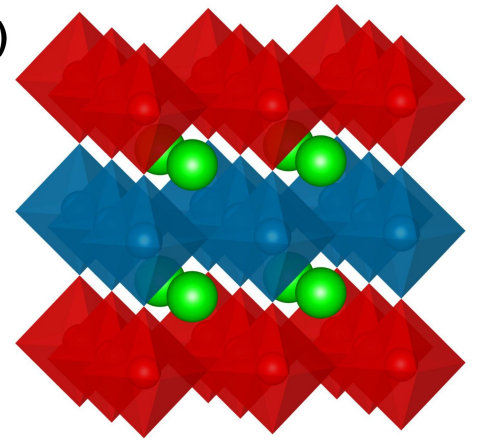

Abbildung 2.3.1: B-Platz-Kationenordnungen von $\mathrm{A}_{2} \mathrm{BB}^{\prime} \mathrm{O}_{6}$-Doppelperowskiten mit der Ordnung in der NaCl-Struktur (a) sowie den selteneren kolumnaren (b) und lagenweisen (c) Ordnungen (mit VESTA [10] gezeichnet, nach [3],4]).

Während die Ordnungsabfolge der B/B'-Platz-Kationen in der NaCl-Struktur einer nulldimensionalen Anordnung entspricht, da jedes $\mathrm{BO}_{6}$-Oktaeder durch sechs $\mathrm{B}^{\prime} \mathrm{O}_{6}$-Oktaeder von den anderen $\mathrm{BO}_{6}$-Oktaedern getrennt ist und umgekehrt, stellt die kolumnare B-PlatzOrdnung eine eindimensionale Ordnungsform dar, bei der $\mathrm{BO}_{6} / \mathrm{B}^{\prime} \mathrm{O}_{6}$-Oktaeder gleichen Typs in jeweils eine Raumrichtung aneinander gereiht sind [3. Folglich ergibt sich in der lagenweisen B-Platz-Ordnung eine zweidimensionale Ordnungsform. Werden nun jeweils die Ebenen betrachtet, in denen sich auf den B/B'-Plätzen nur die gleiche Kationenart 


\section{Theoretischer Hintergrund}

befindet, so lassen sich die drei Ordnungstypen auch anhand der Orientierung der Abfolge dieser Ebenen mit einer Überstruktur von $\mathrm{ABO}_{3}$ - und $\mathrm{AB}^{\prime} \mathrm{O}_{3}$-Perowskiten in der (111)-Orientierung für die B-Platz-Ordnung in der NaCl-Anordnung, einer Anordnung in (110)-Orientierung für die kolumnare B-Platz-Ordnung und einer (100)-Orientierung für die lagenweise B-Platz-Ordnung klassifizieren [3, 9]. Anhand dieser Orientierungen wird direkt der Doppelperowskit-Charakter ersichtlich. Während die B-Platz-Ordnung in der NaCl-Struktur der gewöhnlich zu beobachtende Doppelperowskit-Ordnungstyp ist, treten die anderen zwei Ordnungen nur sehr selten unter speziellen Voraussetzungen wie einer starken Jahn-Teller-Verzerrung oder bei einer Teildotierung des A-Platzes auf [3,4].

Die treibende Kraft bei der Realisierung der B-Platz-Ordnung ist nun einerseits ein großer Unterschied $\Delta Z_{B}=\left|Z_{B}-Z_{B^{\prime}}\right|$ in der Oxidationszahl der B/B'-Platz-Kationen, wodurch eine Anordnung in der NaCl-Struktur gegenüber den anderen Ordnungstypen aufgrund des größeren Abstandes der stärker geladenen Kationen voneinander aus elektrostatischen Gründen energetisch favorisiert wird [3, 4]. So hat in der NaCl-Struktur jedes B/B'-PlatzKation sechs B/B'-Kationen des anderen Typs als nächste Nachbarn, während jedes B/B'Platz-Kation bei der kolumnaren Ordnung vier nächste Nachbarn des anderen und zwei des gleichen Typs besitzt und bei der lagenweise Ordnung jedes B/B'-Platz-Kation sogar nur zwei Kationen des anderen und vier des gleichen Typs als nächste Nachbarn hat [3]. Empirische Untersuchungen zeigen, dass Doppelperowskite mit $\Delta Z_{B}>2$ zumeist geordnet und mit $\Delta Z_{B}<2$ sehr häufig ungeordnet sind, während für $\Delta Z_{B}=2$ sowohl B-Platz geordnete als auch ungeordnete Doppelperowskite nachgewiesen werden können [4]. Daneben hat die Differenz der Ionenradien $\Delta r_{B}=\left|r_{B}-r_{B^{\prime}}\right|$ der B/B'-Platz-Kationen einen großen Einfluss auf die B-Platz-Ordnung. Hierbei zeigt sich, dass mit dem Anstieg von $\Delta r_{B}$ die Tendenz der Kationen zur Anordnung in einer geordneten Struktur für viele Doppelperowskite aufgrund der entstehenden Verspannung signifikant zunimmt [4]. Eine einfache Quantifizierung der jeweils resultierenden B-Platz-Ordnung kann dabei mithilfe der Definition eines langreichweitigen Ordnungsparameters $S$ abgeschätzt werden [4]:

$$
S=2 g_{B}-1
$$

Hierbei bezeichnet $g_{B}$ die Besetzung eines B/B'-Kations auf dem richtigen Gitterplatz. Für ein vollständig B-Platz geordnetes $\mathrm{A}_{2} \mathrm{BB}^{\prime} \mathrm{O}_{6}$-Doppelperowskit nimmt der Parameter den Wert $S=1$, während sich für eine vollständige Unordnung $S=0$ ergibt [4]. Dazwischen weisen Doppelperowskite eine B-Platz-Teilordnung mit einem Ordnungsgrad $S$ auf. Mögliche B-Platz-Ordnungsfehler können in vielfältiger Art auftreten. Neben einer kristallinen Unordnung, die durch fehlerhafte Kristallstrukturen aufgrund von Versetzungen, Korngrenzen, Fehlstellen oder Fremdphasen entsteht, treten insbesondere Gitterfehlbesetzungen (in Form von Punktdefekten) auf, bei denen die B- und B'-Kationen jeweils auf 
vertauschten Plätzen positioniert sind. Ein weiterer häufiger Ordnungsfehler sind Antiphasengrenzen (APG, zweidimensionaler Gitterfehler), welche zwei B-Platz geordnete Domänen mit entsprechend umgekehrter Besetzung der B- und B'-Kationen voneinander trennen und sich demnach wie komplette Gitterfehlbesetzungsebenen im Material verhalten. In den Abbildungen 2.3.2 (a)-(c) (nach [4]) sind nachfolgend die drei Fälle einer perfekten B-Platz-Kationenordnung in der $\mathrm{NaCl}-$ Struktur sowie einer Ordnungsreduzierung sowohl durch Gitterfehlbesetzungen als auch durch Antiphasengrenzen im Vergleich dargestellt.

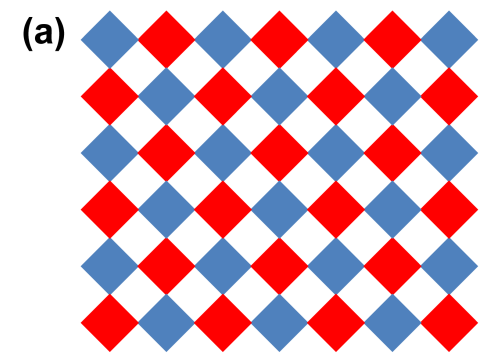

(b)

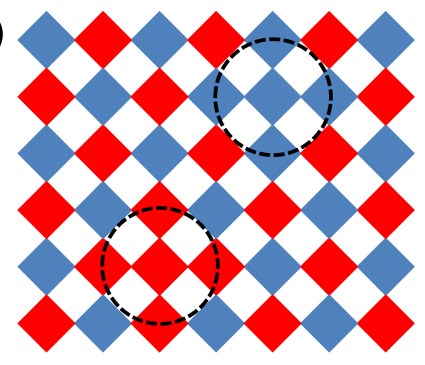

(c)

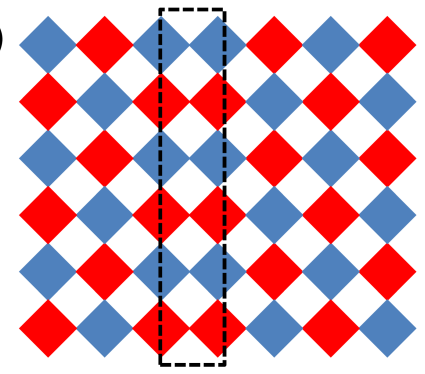

Abbildung 2.3.2: Perfekte B-Platz-Kationenordnung in der NaCl-Struktur (a) und Reduzierung der B-Platz-Ordnung durch einzelne B-Platz-Gitterfehlbesetzungen (Punktdefekte) (b) und Antiphasengrenzen (APG) (c) (nach [4]). Rot und blau kennzeichnen jeweils die unterschiedlichen $\mathrm{BO}_{6^{-}}$und $\mathrm{B}^{\prime} \mathrm{O}_{6}$-Oktaeder.

Die roten und blauen Quadrate repräsentieren jeweils die unterschiedlichen $\mathrm{BO}_{6^{-}}$und $\mathrm{B}^{\prime} \mathrm{O}_{6^{-}}$ Oktaeder in der schematischen Seitenansicht. Der Ordnungsgrad $S$ der Doppelperowskite lässt sich demnach auch umgekehrt über die Fehlbesetzungen $g_{F}$ der B/B'-Platz-Kationen auf ihren Gitterplätzen mit $S=1-2 g_{F}$ definieren [30], wobei $S=1$ erneut ein perfekt B-Platz geordnetes und $S=0$ ein vollständig B-Platz ungeordnetes Doppelperowskit beschreibt. Während die einzelnen Gitterfehler die B-Platz-Ordnung lokal im Umkreis des Besetzungsfehlers stören, führen die APG zu einer Reduzierung der langreichweitigen Ordnung bei gleichzeitig B-Platz geordneten Domänen auf einer kurzreichweitigeren Skala zwischen den APG. Folglich muss die Abschätzung der B-Platz-Ordnung mithilfe eines langreichweitigen Ordnungsparameters mit Vorsicht behandelt werden, da Doppelperowskite trotz APG in den jeweiligen Domänen mit entgegengesetzter Orientierung eine sehr hohe B-Platz-Ordnung aufweisen können [4,31. Zu berücksichtigen ist zudem der große Einfluss der Ordnung auf die Austauschwechselwirkungen zwischen den B/B'-PlatzKationen und die magnetischen Eigenschaften der Doppelperowskite [4, 30 32]. Dieses soll nun an den in dieser Arbeit untersuchten Doppelperowskit-Reihen diskutiert werden. 


\section{Theoretischer Hintergrund}

\subsubsection{Kobalt-Mangan-basierte $\mathrm{A}_{2} \mathrm{CoMnO}_{6}$-Doppelperowskite}

Hierbei wird nun zunächst mit den $\mathrm{A}_{2} \mathrm{CoMnO}_{6}$-Doppelperowskiten begonnen, bei denen der A-Platz jeweils durch ein trivalentes Kation der Seltenen Erden besetzt wird. Diese kristallisieren im Fall einer vorhandenen B-Platz-Ordnung der Cobalt- und Mangan-Ionen allesamt in einer monoklinen Kristallstruktur mit der dazugehörigen Raumgruppe P $2_{1} / \mathrm{n}$ (Raumgruppe 14 mit $\beta \approx 90^{\circ}$, in der Form P121/n1) 31,33 35], während für entsprechend B-Platz ungeordnete Systeme eine orthorhombische Kristallstruktur in der Raumgruppe Pnma (Raumgruppe 62, zumeist in der Alternativform Pbnm angegeben) resultiert [31, 33. Eine Darstellung der Einheitszelle der monoklinen P12 1 n1-Struktur der B-Platz geordneten $\mathrm{A}_{2} \mathrm{CoMnO}_{6}$-Doppelperowskite ist nachfolgend in der Abbildung 2.3 .3 (a) gegeben.
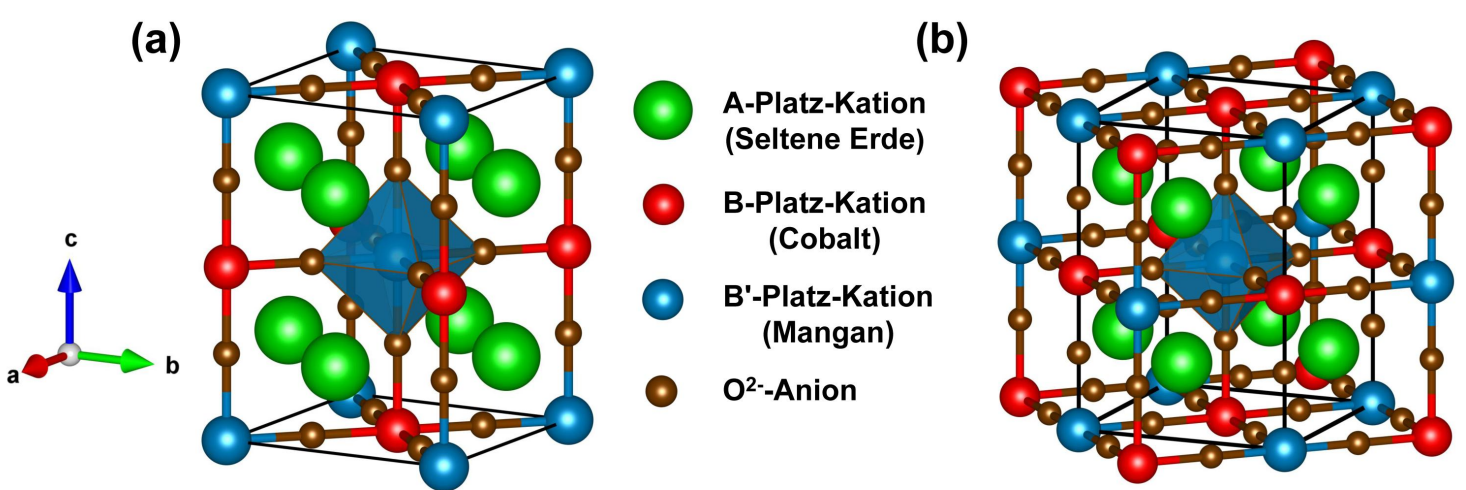

(c)

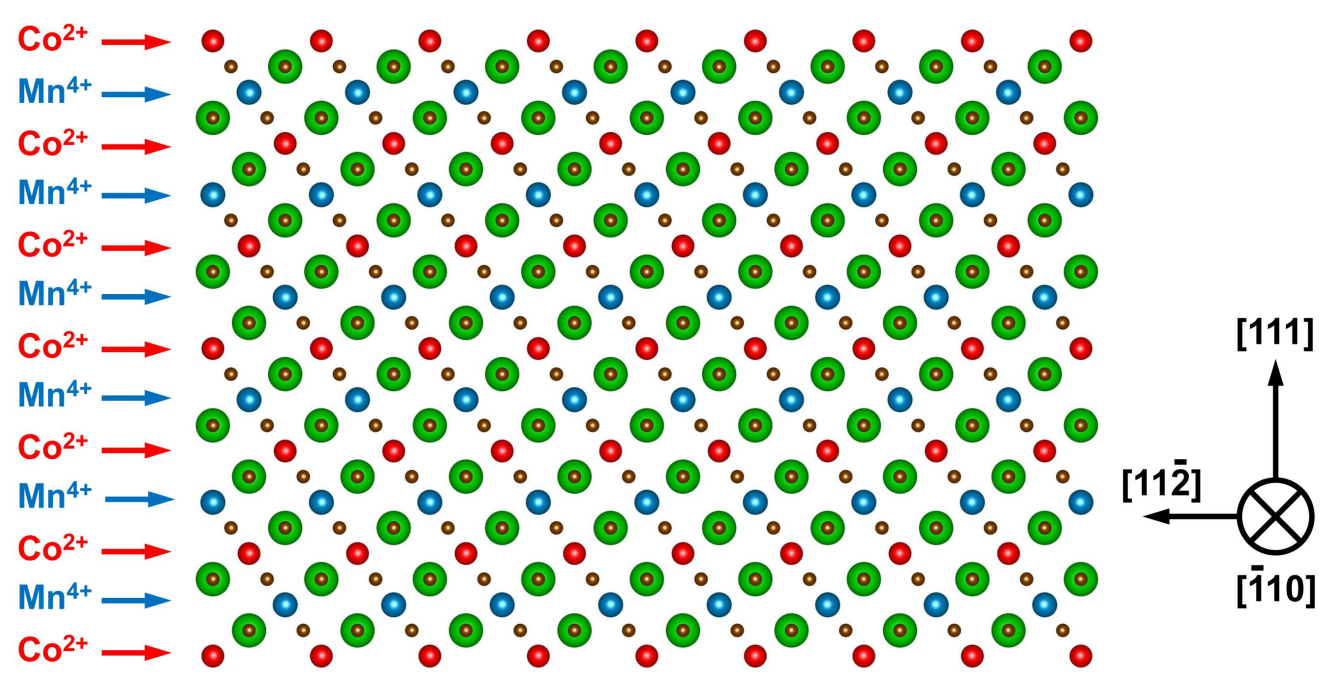

Abbildung 2.3.3: Einheitszelle der monoklinen P12/n1-Struktur der B-Platz geordneten $\mathrm{A}_{2} \mathrm{CoMnO}_{6}$-Doppelperowskite (a), die Einheitszellenlage in der ordnenden $\mathrm{NaCl}-$ Struktur (b) und die Ordnungsüberstruktur aus $\mathrm{AMnO}_{3^{-}}$und $\mathrm{ACoO}_{3^{-}}$ Perowskit-Lagen in der [111]-Richtung (c) (mit VESTA [10] gezeichnet).

Die B/B'-Kationenordnung ist für die $\mathrm{A}_{2} \mathrm{CoMnO}_{6}$-Doppelperowskite über eine Anordnung in der NaCl-Struktur realisiert, sodass die Cobalt- und Mangan-Ionen abwechselnd die B/B'-Gitterplätze besetzen. Die Lage der monoklinen P12/n1-Einheitszelle in der NaClStruktur ist in der Abbildung 2.3.3 (b) dargestellt. Hierbei ergeben sich die Gitterkonstanten $a=\sqrt{2} a_{\mathrm{pc}}, b=\sqrt{2} a_{\mathrm{pc}}, c=2 a_{\mathrm{pc}}$, wobei $a_{\mathrm{pc}}$ die dazugehörige Gitterkonstante der 
pseudokubischen Einheitszelle der idealen $\mathrm{ABO}_{3}$-Perowskit-Struktur bezeichnet. Aufgrund der NaCl-B-Platz-Anordnung lässt sich zudem für die (111)-Ebenen eine Ordnungsüberstruktur beobachten, die sich aus Lagen von $\mathrm{AMnO}_{3^{-}}$und $\mathrm{ACoO}_{3}$-Perowskiten in der [111]-Richtung zusammensetzt und in der Abbildung 2.3.3 (c) dargestellt ist. Der Ordnungsgrad der $\mathrm{A}_{2} \mathrm{CoMnO}_{6}$-Doppelperowskite hat dabei einen großen Einfluss auf die elektronische Struktur der B/B'-Platz-Kationen und die Austauschwechselwirkungen. So werden im Fall der B-Platz-Ordnung in der NaCl-Anordnung die B/B'-Plätze abwechselnd von $\mathrm{Co}^{2+}$-Kationen im HS-Zustand ([Ar] $3 \mathrm{~d}^{7}$ mit $\mathrm{t}_{2 \mathrm{~g}}^{5} \mathrm{e}_{\mathrm{g}}^{2}$ und $\left.S=\frac{3}{2}\right)$ und $\mathrm{Mn}^{4+}$-Kationen im HS-Zustand ([Ar] $] 3 \mathrm{~d}^{3}$ mit t $_{2 \mathrm{~g}}^{3} \mathrm{e}_{\mathrm{g}}^{0}$ und $S=\frac{3}{2}$ ) besetzt [31]. Nach der 2. GKA-Regel führt diese Konfiguration bei einer entsprechenden Vermittlung über die 2p-Orbitale der $\mathrm{O}^{2-}$-Anionen $\left(\mathrm{Co}^{2+}-\mathrm{O}^{2-}-\mathrm{Mn}^{4+}-\right.$ Kopplung) zu einem ferromagnetischen Superaustausch 31,36], der sich aufgrund der Ordnung langreichweitig über die gesamte Gitterstruktur erstreckt und für $\mathrm{La}_{2} \mathrm{CoMnO}_{6}$ in einer Sättigungsmagnetisierung der Spin-Beiträge von $M_{S}=6 \mu_{\mathrm{B}} /$ f.u. resultiert. Für andere Seltenen Erden auf dem A-Platz muss gegebenenfalls noch ein Beitrag der Elektronen der 4f-Schale berücksichtigt werden [34]. Für Ordnungsfehler auf den B/B'Plätzen durch Gitterfehlstellen oder APG und die dadurch entstehenden $\mathrm{Co}^{2+}{ }_{-} \mathrm{O}^{2-}{ }_{-} \mathrm{Co}^{2+}{ }_{-}$ und $\mathrm{Mn}^{4+}-\mathrm{O}^{2-}-\mathrm{Mn}^{4+}$-Konfigurationen ergeben sich hingegen nach der 1. GKA-Regel antiferromagnetische Superaustauschwechselwirkungen [31]. Folglich trennen die APG nicht nur B-Platz geordnete Domänen mit umgekehrter Orientierung, sondern führen auch zu einer antiferromagnetischen Kopplung der ferromagnetischen Domänen untereinander, die mit einer Reduzierung der Remanenz $M_{R}$ einhergeht [31,36]. In genügend starken Magnetfeldern lassen sich die einzelnen Domänen aber wieder in einen ferromagnetischen Gesamtzustand ausrichten, während einzelne Gitterfehlbesetzungen auch dann einen antiferromagnetischen Beitrag beibehalten und damit die Sättigungsmagnetisierung verringern. Für tiefe Temperaturen und kleine Magnetfelder kann die konkurrierende Existenz beider Wechselwirkungen zudem in einer Art Spin-Glas-Verhalten resultieren [34,36,37.

Für ungeordnete $\mathrm{A}_{2} \mathrm{CoMnO}_{6}$-Doppelperowskite wiederum sind die B/B'-Plätze völlig zufällig von $\mathrm{Co}^{3+}$-Kationen im IS-Zustand ([Ar] $3 \mathrm{~d}^{6}$ mit $\mathrm{t}_{2 \mathrm{~g}}^{5} \mathrm{e}_{\mathrm{g}}^{1}$ und $S=1$ ) und $\mathrm{Mn}^{3+}$-Kationen im HS-Zustand ( $[\mathrm{Ar}] 3 \mathrm{~d}^{4}$ mit t $\mathrm{t}_{2 \mathrm{~g}}^{3} \mathrm{e}_{\mathrm{g}}^{1}$ und $S=2$ ) besetzt, indem je ein $e_{g^{-}}$Elektron von den $\mathrm{Co}^{2+}{ }_{-}$ auf die $\mathrm{Mn}^{4+}$-Kationen übertragen und die IS-Co ${ }^{3+}$-Kationen durch lokale Jahn-TellerVerzerrungen sowie einem Sauerstoffdefizit $\delta\left(\mathrm{A}_{2} \mathrm{CoMnO}_{6-\delta}\right)$ stabilisiert werden [31, 37. Die Austauschwechselwirkung dieser $\mathrm{Co}^{3+}-\mathrm{O}^{2-}-\mathrm{Mn}^{3+}$-Konfiguration kann hierbei durch einen dreidimensionalen, vibronischen Superaustausch beschrieben werden, der durch ein kooperatives Zusammenspiel der lokalen Jahn-Teller-Verzerrungen langreichweitig ist und entsprechend der vibronischen Besetzung der $e_{g}$-Orbitale nach der 2. GKA-Regel mit einer ferromagnetischen Kopplung einhergeht [31, 37]. Im Vergleich zur B-Platz geordneten Phase mit der statischen $\mathrm{Co}^{2+}-\mathrm{O}^{2-}-\mathrm{Mn}^{4+}-$ Kopplung ist der vibronische Austausch jedoch weniger stabil, sodass sich eine im Vergleich dazu signifikante Reduzierung der 


\section{Theoretischer Hintergrund}

Curie-Temperatur $T_{C}$ und der Sättigungsmagnetisierung $M_{S}$ ergibt (für $\mathrm{La}_{2} \mathrm{CoMnO}_{6}$ beispielsweise von $T_{C} \approx 230 \mathrm{~K}$ und $M_{S}=6 \mu_{\mathrm{B}} /$ f.u. in der B-Platz geordneten Phase auf $T_{C} \approx 80-130 \mathrm{~K}$ und $M_{S} \approx 3,5-4,5 \mu_{\mathrm{B}} /$ f.u. in der ungeordneten Phase) 31, 36, 37. Die Austauschstärke nimmt dabei mit der Verkleinerung des B-O-B'-Winkels und des damit einhergehenden orbitalen Überlapps ab. Für die $\mathrm{A}_{2} \mathrm{CoMnO}_{6}$-Doppelperowskite lässt sich dieses direkt anhand einer systematischen Abnahme der Curie-Temperatur $T_{C}$ mit der Verringerung des Ionenradius der Seltenen Erde auf dem A-Platz beobachten 34,35. Unabhängig von der Ordnung weisen die $\mathrm{A}_{2} \mathrm{CoMnO}_{6}$-Doppelperowskite zudem aufgrund des fehlenden Doppelaustauschprozesses stets einen elektrisch isolierenden Charakter auf [31].

\subsubsection{Nickel-Mangan-basierte $\mathrm{A}_{2} \mathrm{NiMnO}_{6}$-Doppelperowskite}

Anschließend sollen noch die $\mathrm{A}_{2} \mathrm{NiMnO}_{6}$-Doppelperowskite, bei denen der A-Platz ebenfalls durch ein trivalentes Kation der Seltenen Erden besetzt wird, betrachtet werden. Diese kristallisieren wie die $\mathrm{A}_{2} \mathrm{CoMnO}_{6}$-Doppelperowskite bei einer B-Platz-Ordnung der Nickel- und Mangan-Ionen in einer monoklinen P12/n1-Kristallstruktur und im ungeordneten Fall in einer orthorhombischen Pbnm-Kristallstruktur [32, 35, 38, 39]. Ein Unterschied ist jedoch, dass die $\mathrm{Ni}^{2+}$-Kationen im HS-Zustand ([Ar] $3 \mathrm{~d}^{8}$ mit $\mathrm{t}_{2 \mathrm{~g}}^{6} \mathrm{e}_{\mathrm{g}}^{2}, S=1$ ) ein $e_{g}$-Elektron mehr als die $\mathrm{Co}^{2+}-$ Kationen aufweisen, sodass die Magnetisierung der SpinBeiträge der ferromagnetischen $\mathrm{Ni}^{2+}-\mathrm{O}^{2-}-\mathrm{Mn}^{4+}$-Kopplung der B-Platz geordneten Phase maximal $M_{S}=5 \mu_{\mathrm{B}} /$ f.u. beträgt. Die weiteren Superaustauschwechselwirkungen mit antiferromagnetischer $\mathrm{Ni}^{2+}-\mathrm{O}^{2-}{ }_{-} \mathrm{Ni}^{2+}$ - und $\mathrm{Mn}^{4+}{ }_{-} \mathrm{O}^{2-}{ }_{-} \mathrm{Mn}^{4+}{ }_{-}$Kopplung durch APG und Gitterfehlstellen sowie der im ungeordneten Fall weniger stabile vibronische $\mathrm{Ni}^{3+} \mathrm{O}^{2-}{ }_{-} \mathrm{Mn}^{3+}{ }_{-}$ Ferromagnetismus mit LS-Ni ${ }^{3+}$-Kationen ([Ar] $3 \mathrm{~d}^{7}$ mit $\mathrm{t}_{2 \mathrm{~g}}^{6} \mathrm{e}_{\mathrm{g}}^{1}$ und $S=\frac{1}{2}$ ) erfolgen wie bei den $\mathrm{A}_{2} \mathrm{CoMnO}_{6}$-Doppelperowskiten nach den gleichen Mechanismen 32, 39]. Es zeigt sich jedoch, dass die vibronische Kationenkonfiguration bei den $\mathrm{A}_{2} \mathrm{NiMnO}_{6}$-Doppelperowskiten durch einen Sauerstoffüberschuss $\delta\left(\mathrm{A}_{2} \mathrm{NiMnO}_{6+\delta}\right)$ und der damit einhergehenden Bildung von A-Platz-Kation-Leerstellen stabilisiert wird [32]. Die Curie-Temperatur der ungeordneten Phase ist dabei wie für die $\mathrm{A}_{2} \mathrm{CoMnO}_{6}$-Doppelperowskite gegenüber der der B-Platz geordneten Phase signifikant reduziert (für $\mathrm{La}_{2} \mathrm{NiMnO}_{6}$ von $T_{C} \approx 280 \mathrm{~K}$ in der B-Platz geordneten Phase auf $T_{C} \approx 130-140 \mathrm{~K}$ in der ungeordneten Phase) 40,41. Die ferromagnetische Austauschstärke nimmt zudem mit der Verkleinerung des B-O-B'-Winkels ab. Dieses äußert sich in einer systematischen Abnahme der Curie-Temperatur $T_{C}$ mit der Verringerung des Ionenradius der Seltenen Erde auf dem A-Platz 35, 38. Im Vergleich zu den $\mathrm{A}_{2} \mathrm{CoMnO}_{6}$-Doppelperowskiten sind die jeweiligen Curie-Temperaturen $T_{C}$ bei gleichen APlatz-Kationen jedoch erhöht, wobei der Unterschied für kleinere A-Kationenradius wieder abnimmt. Dieses ist auf einen leicht größeren orbitalen Überlapp der $\mathrm{Ni}^{2+}-\mathrm{O}^{2-}-\mathrm{Mn}^{4+}$ - gegenüber der $\mathrm{Co}^{2+}-\mathrm{O}^{2-}-\mathrm{Mn}^{4+}-$ Kopplung zurückzuführen 35. Aufgrund des fehlenden Doppelaustauschprozesses besitzen dabei auch die $\mathrm{A}_{2} \mathrm{NiMnO}_{6}$-Doppelperowskite unabhängig von der vorhandenen B-Platz-Ordnung stets einen elektrisch isolierenden Charakter. 


\section{Experimentelle Methoden}

\subsection{Probenherstellung}

\subsubsection{Prinzip der metallorganischen Aerosol Deposition (MAD)}

Die Herstellung der in dieser Arbeit untersuchten Dünnschichten geschieht mithilfe der sogenannten metallorganischen Aersol Deposition (MAD) 42, 43, mit der bereits verschiedenste Oxidschichten wie gemischtvalente Manganate [42 44], Titanate [45], Hochtemperatur-Supraleiter-Cuprate [46], ZnO [47] oder Doppelperowskite [36, 48, 49] in äußerst hoher Qualität erzeugt werden konnten. Grundlage dieses chemischen Depositionsverfahrens sind metallorganische Präkursoren, die in dem gewünschten stöchiometrischen Verhältnis und in gewünschter Konzentration in einem polaren, organischem Lösungsmittel (hier Dimethylformamid (DMF); chemische Formel: $\mathrm{C}_{3} \mathrm{H}_{7} \mathrm{NO}$ ) gelöst und anschließend unter Umgebungsluft über ein computergesteuertes Dosier-Spritzensystem (Typ SyrDos 2 von HiTec Zang) mithilfe von Druckluft ( $p \approx 5$ bar) und einer pneumatischen Düse als Aerosol auf ein geheiztes, kristallines Substrat gesprüht werden. Der dazugehörige Aufbau der verwendeten MAD-Anlage ist schematisch in der nachfolgenden Abbildung 3.1.1 dargestellt.

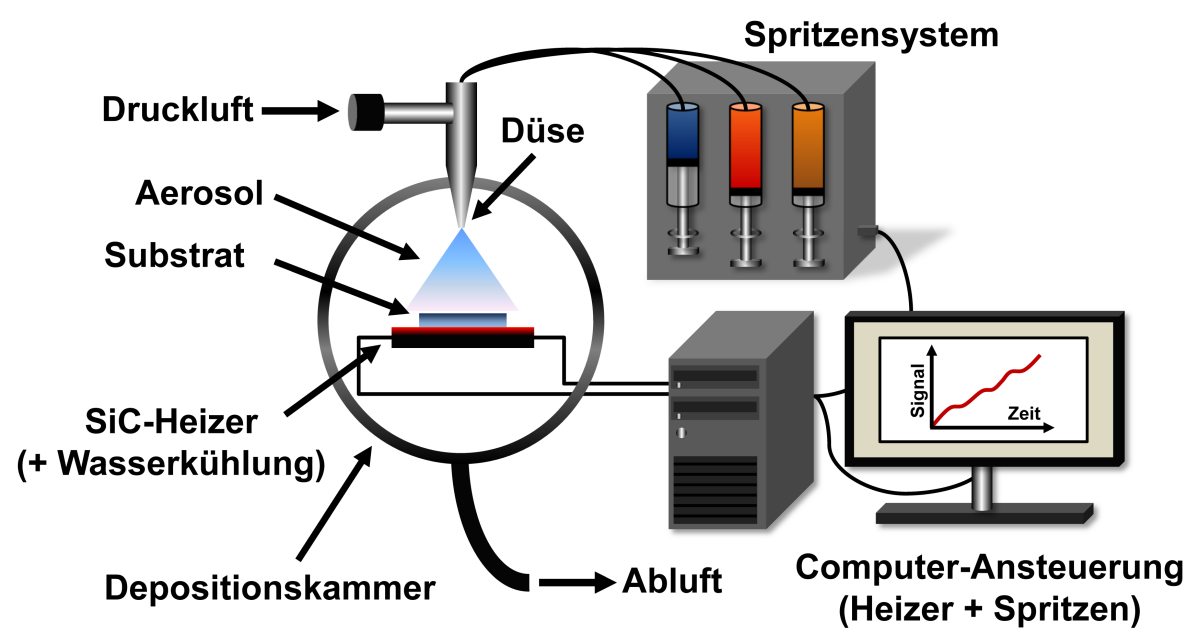

Abbildung 3.1.1: Schematischer Aufbau der MAD-Anlage mit der Depositionskammer und der Anordnung von Heizer, pneumatischer Düse und Spritzensystem.

Das Substrat selbst wird zentriert vor der Düse auf einem SiC-Heizer, dessen Temperatur sich resistiv über eine Stromquelle regeln lässt, platziert. Somit können Schichten bei unterschiedlichen Substrattemperaturen hergestellt werden. Diese liegen je nach Oxid in 


\section{Experimentelle Methoden}

einem Bereich von $T=500-1200{ }^{\circ} \mathrm{C}$. Eine Wasserkühlung, die Druckluft und die Steuerung der Stromstärke über einen Computer schützen den Heizer und das Substrat vor zu schneller Erhitzung vor und zu schnellem Erkalten nach der Deposition. Das Spritzensystem lässt sich dabei in unterschiedlicher Geschwindigkeit betreiben (Schritte pro Sekunde), wodurch es möglich ist, die Depositionsrate (in $\mu \mathrm{l} / \mathrm{s}$; typischerweise $\approx 1-100 \mu \mathrm{l} / \mathrm{s}$ ) und damit die Wachstumsgeschwindigkeit der Schichten (in $\mathrm{nm} / \mathrm{s}$; hier etwa $0,1-3 \mathrm{~nm} / \mathrm{s}$ ) anzupassen. Das Oxidschichtwachstum auf dem Substrat geschieht nach dem Prinzip der chemischen Gasphasenabscheidung durch eine Pyrolysereaktion, bei der die Metallionen der gelösten Präkursoren aus den Tröpfchen des Aerosols (einige $\mu \mathrm{m}$ Tröpfchengröße) mit dem Sauerstoff der Druckluft die Metalloxid-Schicht bilden, während die restlichen Lösungsbestandteile (das verbliebene Lösungsmittel und organische Reste des Präkursors) zu $\mathrm{CO}_{2}, \mathrm{H}_{2} \mathrm{O}$ und weiteren Bestandteilen verbrennen und mit der Abluft aus der Depositionskammer abgeführt werden. Über die proportionale Abhängigkeit der Oxidschichtdicke vom Lösungsvolumen lässt sich die Lösung für eine atomare Monolage (ML) des Oxids auf $V_{M L} \approx 5-10 \mu \mathrm{l}$ je nach Oxid, Substrat, Temperatur und Lösungskonzentration bestimmen. Über die Präzision der Spritzen von $\Delta V \approx 10 \mathrm{nl}$ [51 (das Spritzenvolumen beträgt $V=500 \mu \mathrm{l}$ pro Komplettfüllung) kann die Schichtdicke bis auf 1/100 einer Monolage kontrolliert werden. Zudem kann bei Übergitter-Schichten eine optionale Wachstumskontrolle mithilfe eines integrierten Ellipsometrie-Messaufbaus verwendet werden $46,48,50,52$.

Gegenüber der Molekularstrahlepitaxie (engl.: molecular beam epitaxy, MBE), der gepulsten Laserdeposition (engl:: pulsed laser deposition, PLD), Sputtern oder thermischer Verdampfung bietet die MAD dabei den Vorteil, dass für das Schichtwachstum kein Ultrahochvakuum benötigt wird. Weiterhin lassen sich die Wachstumsparameter wie die Stöchiometrie in der Lösung, die Wachstumsgeschwindigkeit, die Schichtdicke und die Wachstumstemperatur flexibel für jede Schicht variieren [42,43]. Durch das Wachstum unter Umgebungsluft (Sauerstoffpartialdruck $p_{\mathrm{O}_{2}} \approx 0,21$ bar) wird zudem ein stöchiometrisches Sauerstoffdefizit durch Sauerstoffleerstellen in der Oxidschicht vermieden [43]. Eine nachbehandelndes Ausheizen unter Sauerstoffatmosphäre ist daher nicht notwendig. Beachtet werden muss jedoch, dass für die Pyrolysereaktion und das Verbrennen des Lösungsmittels eine gewisse Temperatur benötigt wird, sodass eine Deposition etwa bei Raumtemperatur nicht möglich ist. Für Metalle mit mehreren Oxidationsstufen muss außerdem das Zusammenspiel von Temperatur und Sauerstoffpartialdruck berücksichtigt werden, um bei der MAD-Standardtemperatur $\left(T \approx 1000{ }^{\circ} \mathrm{C}\right)$ das gewünschte Oxid zu erhalten [48,53. Daher ist es nötig die Anlage so zu erweitern, den Sauerstoffpartialdruck während der Deposition beeinflussen zu können. Dieses ermöglicht das Konzept der sauerstoffreduzierten MAD (LOMAD, Low-Oxygen-MAD) 48,53,54. Eine andere Erweiterung stellt zudem das Wachstum von Mikrostrukturen mit Linienbreiten unter einem Mikrometer durch lokale Erhitzung des Substrats mithilfe eines in der Position steuerbaren Lasers dar [55]. 


\subsubsection{Herstellung der Dünnschicht-Oxide mithilfe der MAD}

Die Schichtherstellung in dieser Arbeit erfolgt mit der Standard-MAD (Abbildung 3.1.1) unter Umgebungsluft auf einkristallinen Substraten aus industrieller Fertigung (Crystal, CrysTec, MaTecK und Surface.net) mit einer polierten Oberfläche $(10 \mathrm{~mm} \times 5 \mathrm{~mm})$ und einer Dicke von 0,5 mm. Verwendet werden $\mathrm{Al}_{2} \mathrm{O}_{3}$ in (0001)-Orientierung (hexagonal), $\mathrm{MgO}$ in (100)-Orientierung, LAO in (100)- und (111)-Orientierung, LSAT in (111)-Orientierung und STO in (100)- und (111)-Orientierung. Bis zur Verwendung sind die Substrate dabei trocken in einem Vakuumexsikkator gelagert. Die STO (100)-Substrate wurden jedoch aufgrund des Kristallaufbaus aus Lagen von $\mathrm{SrO}$ und $\mathrm{TiO}_{2}$ entlang der [100]-Richtung und der zufälligen Mischterminierung an der Oberfläche mit einer speziellen Behandlungsabfolge auf eine einheitliche $\mathrm{TiO}_{2}$-Oberfläche terminiert $[56$. Hierzu werden die Substrate in deionisiertem Wasser gereinigt und das $\mathrm{SrO}$ an der Oberfläche anschließend durch ein selektives Ätzen mit gepufferter Flusssäure (BHF) abgelöst. Danach erfolgt ein weiteres Spülen mit deionisiertem Wasser, bevor die STO-Substrate auf dem SiC-Heizer der MAD für eine Stunde bei $T \approx 1000{ }^{\circ} \mathrm{C}$ ausgelagert werden [48, 50, damit sich eine definierte Terrassenstruktur mit $\mathrm{TiO}_{2}$-Terminierung an der Substratoberfläche ergibt. Die anderen Substrate wurden hingegen nicht terminiert oder vorbehandelt. Die metallorganischen Präkursoren sind Metallacetylacetonate und stammen sowohl aus kommerziell-industrieller Serienfertigung von Merck Sigma-Aldrich $\left(\mathrm{Mn}(\mathrm{acac})_{2}, \mathrm{Co}(\mathrm{acac})_{3}, \operatorname{Pr}(\mathrm{acac})_{3}, \mathrm{Nd}(\mathrm{acac})_{3}\right.$, $\mathrm{Sm}(\mathrm{acac})_{3}, \mathrm{Gd}(\mathrm{acac})_{3}, \mathrm{Y}(\mathrm{acac})_{3}$ und $\left.\mathrm{Ca}(\mathrm{acac})_{2}\right)$ und Alfa Aesar $\left(\mathrm{Ni}(\mathrm{acac})_{3}\right)$ als auch aus individueller Fertigung $\left(\mathrm{La}(\mathrm{acac})_{3}\right.$ und $\left.\mathrm{Sr}(\mathrm{acac})_{2}\right)$ der Fakultät für Chemie der GeorgAugust-Universität Göttingen (Serhiy Demeshko). Für die Eigenschaften der industriell bezogenen Präkursoren sei auf die Herstellerangaben verwiesen. Eine Darstellung der Präkursorstruktur auf Acetylacetonat-Basis und der Strukturformel anhand des Beispiels von Mangan(II)-Acetylacetonat $\left(\mathrm{Mn}(\mathrm{acac})_{2}\right)$ ist in den Abbildungen 3.1.2 (a) und (b) gegeben.

(a)

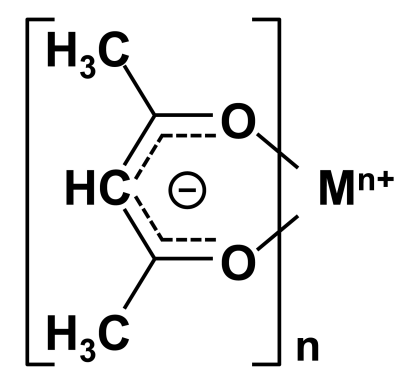

(b)

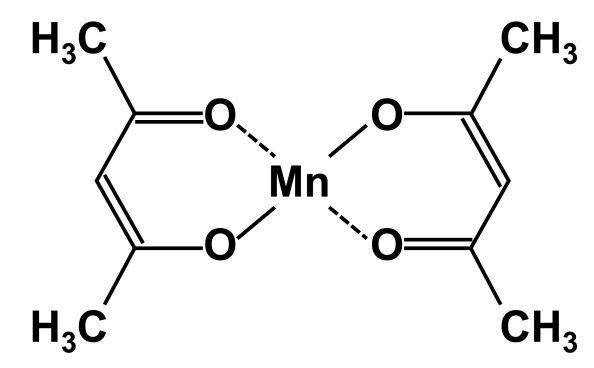

Abbildung 3.1.2: Allgemeine Übersicht der Struktur der metallorganischen Präkursoren auf Acetylacetonat-Basis (a) und die dazugehörige Strukturformeldarstellung anhand des Beispiels von Mangan(II)-Acetylacetonat $\left(\mathrm{Mn}(\mathrm{acac})_{2}\right)(\mathrm{b})$.

Die Präkursoren werden dabei in der benötigten Menge in einem Becherglas abgewogen und mit einem Ultraschallbad bei einer Temperatur von $T=30{ }^{\circ} \mathrm{C}$ für etwa 5 Minuten in DMF gelöst. Die fertige Lösung kann danach in das MAD-Spritzensystem gezogen und 


\section{Experimentelle Methoden}

anschließend die Deposition gestartet werden. Die Pyrolysereaktionen für die Präkursoren der drei Übergangsmetalle (Mn, Co und Ni), mit denen alle Schichten in dieser Arbeit aus dem versprühten Aerosol unter Verbrennung des organischen Rests auf den Substraten gewachsen werden, sind in den Gleichungen (3.1.1), (3.1.2) und (3.1.3) dargestellt:

$$
\begin{array}{r}
6 \mathrm{Mn}\left(\mathrm{C}_{5} \mathrm{H}_{7} \mathrm{O}_{2}\right)_{2}+73 \mathrm{O}_{2} \longrightarrow 2 \mathrm{Mn}_{3} \mathrm{O}_{4}+60 \mathrm{CO}_{2}+42 \mathrm{H}_{2} \mathrm{O} \\
12 \mathrm{Co}\left(\mathrm{C}_{5} \mathrm{H}_{7} \mathrm{O}_{2}\right)_{3}+215 \mathrm{O}_{2} \longrightarrow 4 \mathrm{Co}_{3} \mathrm{O}_{4}+180 \mathrm{CO}_{2}+126 \mathrm{H}_{2} \mathrm{O} \\
6 \mathrm{Ni}\left(\mathrm{C}_{5} \mathrm{H}_{7} \mathrm{O}_{2}\right)_{2}+73 \mathrm{O}_{2} \longrightarrow 2 \mathrm{Ni}_{3} \mathrm{O}_{4}+60 \mathrm{CO}_{2}+42 \mathrm{H}_{2} \mathrm{O} .
\end{array}
$$

Für die anderen Präkursoren lassen sich die Pyrolysereaktionen analog aufstellen. Die abzuwiegende Masse $m_{i}$ des Präkursors $i$ wird mithilfe der molaren Präkursormasse $M_{i}$, der gewünschten Stoffmengenkonzentration $c_{i}$ und des DMF-Lösungsvolumens $V$ bestimmt:

$$
m_{i}=M_{i} \cdot c_{i} \cdot V
$$

Bei mehrkomponentigen Systemen ist zu beachten, dass die Reaktionen der Präkursoren unterschiedlich effektiv ablaufen können, sodass das Verhältnis der Präkursoren von der Stöchiometrie der Metallionen in der gewachsen Schicht abweicht. Dieses ist in der Komplexität der kinematischen und thermodynamischen Prozesse während des Schichtwachstums begründet. Zudem variieren die Präkursoren in ihrer Löslichkeit und reagieren unterschiedlich auf Restfeuchtigkeit bei der Lagerung. Für eine korrekte Stöchiometrie muss daher das Verhältnis der Präkursormassen zueinander angepasst werden. Folglich werden Korrekturfaktoren zwischen den einzelnen Präkursoren eingeführt, die sich empirisch über die Optimierung der strukturellen, elektrischen oder magnetischen Schichteigenschaften ergeben, da diese zumeist stark von der stöchiometrischen Zusammensetzung abhängen [48,50]. Für die korrigierte Masse $m_{i}^{\prime}$ eines Präkursors der Komponente $i$ bezogen auf den Präkursor der Komponente $j$ ergibt sich in einem System mit den Komponenten $i$ und $j$ somit:

$$
m_{i}^{\prime}=x_{i / j} \cdot k_{i / j} \cdot m_{i}
$$

Hierbei bezeichnet $m_{i}$ die unkorrigierte Masse des Präkursors $i, x_{i / j}$ das gewünschte stöchiometrische Verhältnis der beiden Schichtkomponenten und $k_{i / j}$ den Korrekturfaktor zwischen den zwei Präkursoren. Dieser ist so definiert, dass für $k_{i / j}=1$ keine Korrektur benötigt wird und sich die Stöchiometrie direkt über $x_{i / j}$ kontrollieren lässt. Oft weichen die empirisch ermittelten Werte für $k_{i / j}$ für die meisten Präkursoren jedoch davon ab. 


\subsection{Standard-Probencharakterisierung}

\subsubsection{Röntgendiffraktometrie (XRD)}

Zur Bestimmung der Kristallstruktur der hergestellten Schichten werden diese mithilfe der Röntgendiffraktometrie (XRD, engl.: X-ray diffraction) untersucht. Bei dem verwendeten Röntgendiffraktometer D8 Advance von Bruker AXS trifft durch einen Göbelspiegel monochromatisierte, in Form eines Primärstrahls kollimierte $K_{\alpha}$-Strahlung der Röntgenröhre (Kupferanode mit einer mittleren $K_{\alpha}$-Wellenlänge $\bar{\lambda}_{\mathrm{K}_{\alpha}}=\frac{2 \lambda_{K_{\alpha_{1}}}+\lambda_{K_{\alpha_{2}}}}{3}=1,54184 \AA$, gewichteter Mittelwert aus der $\mathrm{Cu}-\mathrm{K}_{\alpha_{1}}$ - und der $\mathrm{Cu}-\mathrm{K}_{\alpha_{2}}$-Linie mit $\lambda_{K_{\alpha_{1}}}=1,54056 \AA$ und $\lambda_{K_{\alpha_{2}}}=1,54439 \AA$ ) unter einem Winkel $\theta$ auf die zwischen der Röntgenröhre und einem Detektor platzierte Probe. Die an der Probe reflektierte Strahlung wird anschließend unter dem Winkel $2 \theta$ zur ursprünglichen Einfallsrichtung vom Detektor registriert. Die Probe ist dabei im Zentrum des Führungskreises von Röntgenröhre und Detektor platziert und wird mit der halben Winkelgeschwindigkeit $\omega=\theta$ des Detektors gedreht. In diesem gekoppelten Modus mit Winkelgeschwindigkeiten im Verhältnis von $\theta$ zu $2 \theta$ in Relation zur Röntgenröhre (die Anordnung wird als Bragg-Brentano-Geometrie bezeichnet; zusätzlich lässt sich eine Abweichung $\Delta$ zwischen $\theta$ und $2 \theta$ mit $\theta=\frac{1}{2} \cdot 2 \theta+\Delta$ durch einen Substratfehlschnitt oder eine Probenverkippung berücksichtigen) kann nun die Intensität $I$ der Strahlung als Funktion des Winkels $2 \theta$ zur Röntgenröhre gemessen werden. Eine schematische Darstellung der Anordnung in der Bragg-Brentano-Geometrie ist in der Abbildung 3.2.1 gegeben.

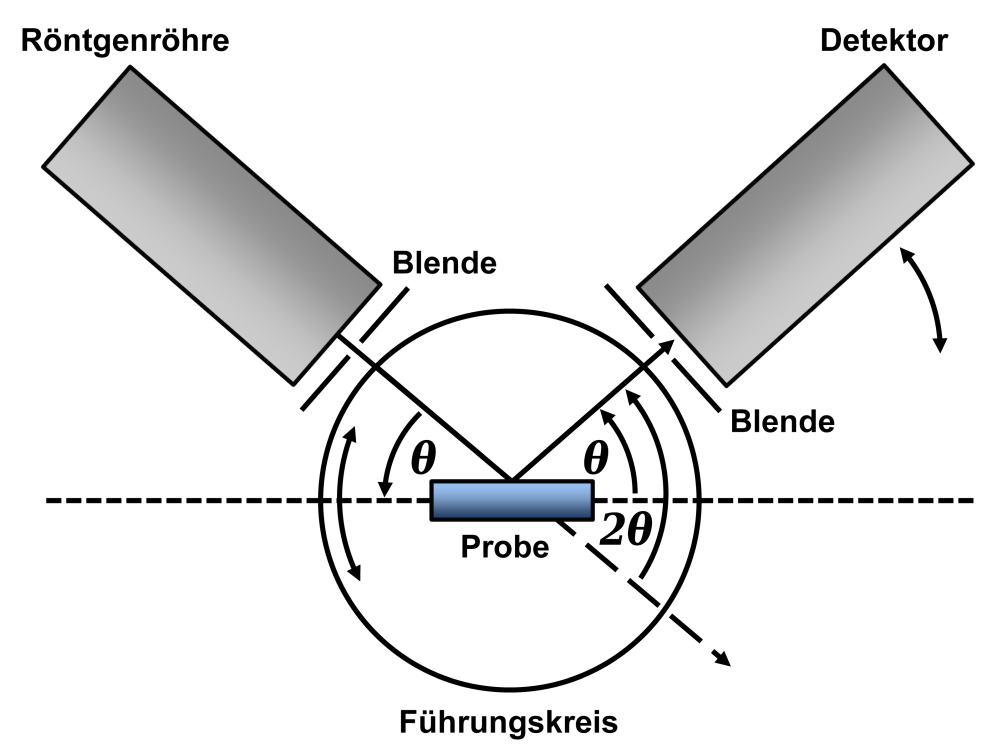

Abbildung 3.2.1: Schematischer Aufbau eines Röntgendiffraktometers mit Anordnung von Röntgenröhre, Probe und Detektor in der Bragg-Brentano-Geometrie.

Die $\mathrm{Cu}-\mathrm{K}_{\alpha}$-Röntgenstrahlung der Kupfer-Anode ist jedoch aufgrund von Verunreinigungen nicht völlig monochromatisch, sodass sich schwache Linien, die sich der $\mathrm{Cu}-\mathrm{K}_{\beta^{-}}, \mathrm{W}-\mathrm{L}_{\alpha^{-}}$ und $\mathrm{W}-\mathrm{L}_{\beta}$-Strahlung zuordnen lassen, sowie ein Hintergrundbeitrag durch die Bremsstrahlung in den XRD-Spektren nachweisen lassen [48,50]. Aufgrund der geringen Intensität in 


\section{Experimentelle Methoden}

Relation zu den $\mathrm{Cu}-\mathrm{K}_{\alpha}$-Hauptlinien sind diese nur für die Substrate relevant und können für dünne Schichten aufgrund des deutlich reduzierten Streuvolumens vernachlässigt werden. Zur Identifizierung von Substrat- und Schichtreflexen sind die Schichtspektren daher stets mit einem Referenzspektrum des Substrats dargestellt. Für die kristalline XRDStrukturbestimmung wird nun die Beugung von Röntgenstrahlung an einem dreidimensionalen Kristallgitter betrachtet. Da Beugung dann auftritt, wenn der Abstand innerhalb der Gitterstruktur (mit Atomabständen im Bereich einiger $10^{-10} \mathrm{~m}=1 \AA$ [57,58]) in der Größenordnung der einfallenden Wellenlänge liegt, hat $\mathrm{K}_{\alpha}$-Röntgenstrahlung mit Wellenlängen je nach Anode von etwa $1-10 \AA$ eine geeignete Größenordnung. Die Röntgenstrahlung wird nun an den Elektronenhüllen der Gitteratome gestreut, in denen die Elektronen klassisch zu Schwingungen angeregt werden, wodurch Strahlung in Form elektromagnetischer Sekundärwellen emittiert wird [59]. Die gebeugte Strahlung interferiert und liefert je nach Atomabstand, Wellenlänge der Röntgenstrahlung und Einfallswinkel der Strahlung die typischen Beugungserscheinungen konstruktiver und destruktiver Überlagerung. Zur Erklärung der Röntgenbeugung werden mit der Bragg-Bedingung und der Laue-Bedingung zwei zueinander äquivalente Beschreibungen verwendet. Als Ausgangspunkt dient jeweils die Annahme, dass Kristalle zunächst als eine starre Anordnung von Gitteratomen in Form von punktförmigen Streuzentren mit periodischen Abständen in einem dreidimensionalem Gitter betrachtet werden, an denen die Röntgenstrahlung elastisch ohne Energieverlust und einfach, das heißt ohne Mehrfachstreuung (kinematische Streuung), gestreut wird 60,61.

(a)

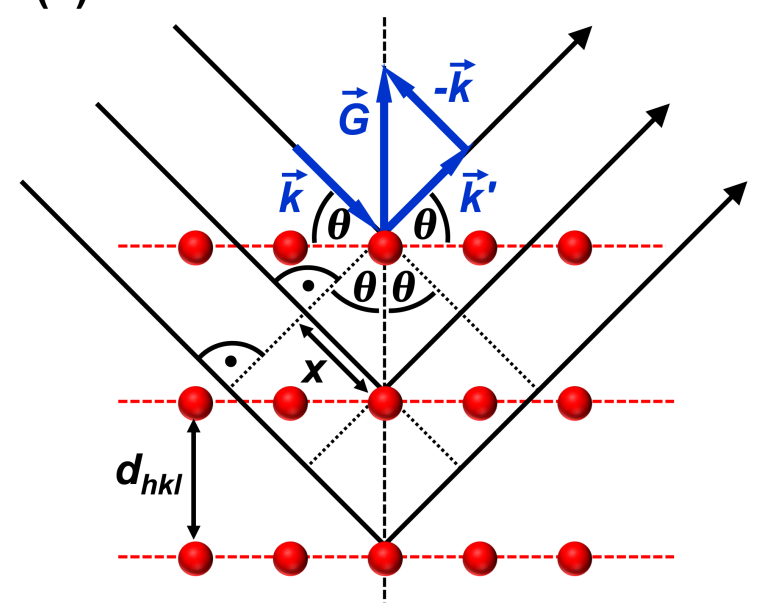

(b)

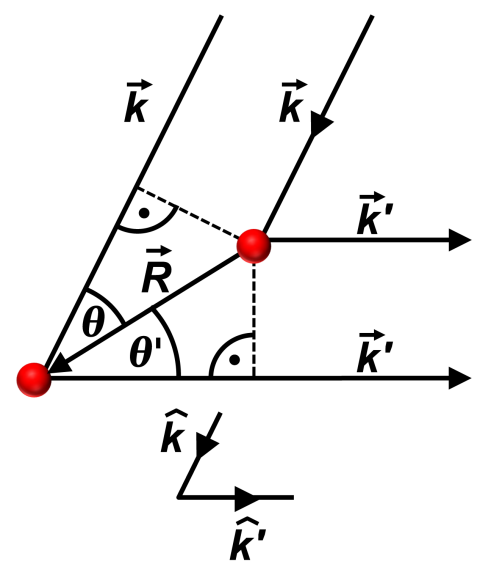

Abbildung 3.2.2: Schematische Darstellung der Beugungsgeometrie zur Herleitung der BraggBedingung (a) und der Laue-Bedingung (b) (nach [57]) für konstruktive Interferenz bei der Beugung von Röntgenstrahlung. Die Äquivalenz der beiden Bedingungen ist mithilfe der Wellenvektoren direkt in (a) ersichtlich.

In der Bragg-Bedingung werden die Atome im Kristall in parallele Gitterebenen mit konstantem Abstand zueinander (Netzebenen) angeordnet, an denen die mit dem Winkel $\theta$ einfallende Röntgenstrahlung reflektiert wird und zu Beugungsreflexen in den Richtungen führt, für die die reflektierten Röntgenstrahlen konstruktiv interferieren. Eine Darstellung 


\subsection{Standard-Probencharakterisierung}

dieser Anordnung ist in der Abbildung 3.2.2 (a) gegeben. Konstruktive Interferenz tritt auf, wenn der Gangunterschied zwischen den Strahlen, die an unterschiedlichen Netzebenen gestreut werden, ein ganzzahliges Vielfaches der Wellenlänge $\lambda$ der Röntgenstrahlung ist. Mit dem Gangunterschied $2 x=2 d_{h k l} \sin (\theta)$ zwischen Röntgenwellen, die an benachbarten Netzebenen reflektiert werden, folgt für die Bragg-Bedingung konstruktiver Interferenz [60,62]:

$$
2 d_{h k l} \sin (\theta)=n \lambda
$$

Hierbei bezeichnet $d_{h k l}$ den Abstand der Netzebenen $(h k l)$, während die ganze Zahl $n$ die Reflexordnung angibt. Der Abstand zweier Ebenen $(h k l)$ lässt sich über die Beziehung $d_{h k l}=2 \pi /\left|\vec{G}_{h k l}\right|$ mithilfe des reziproken Gittervektors $\vec{G}=h \vec{b}_{1}+k \vec{b}_{2}+l \vec{b}_{3}$, welcher senkrecht auf der durch die Millerschen Indizes $(h k l)$ definierten Gitterebene steht, bestimmen [59]. Die Vektoren $\vec{b}_{1}, \vec{b}_{2}$ und $\vec{b}_{3}$ stellen die Basisvektoren des reziproken Gitters dar, die aus den Basis-Vektoren $\vec{a}_{1}, \vec{a}_{2}$ und $\vec{a}_{3}$ des Bravais-Kristallgitters ermittelt werden. Für ein kubisches Kristallsystem mit der Gitterkonstante $a$ folgt daher $d_{h k l}=a / \sqrt{h^{2}+k^{2}+l^{2}}$. Mit der Bragg-Bedingung aus der Gleichung (3.2.1) lässt sich eine Beziehung zwischen der Gitterkonstante $a$ des kubischen Kristallsystems, des Reflexes $(h k l)$, des Einfallswinkel $\theta$ und der Wellenlänge $\lambda$ der Röntgenstrahlung herstellen. Für $n=1$ ergibt sich:

$$
a=\frac{\lambda}{2 \sin (\theta)} \sqrt{h^{2}+k^{2}+l^{2}}
$$

Mit der Gleichung (3.2.2) können nun die Reflexe $(h k l)$ für Schichten mit kubischer Kristallstruktur im $I(2 \theta)$-Röntgenspektrum identifiziert werden. Für andere Kristallsysteme müssen die jeweiligen Gittervektoren und Gitterkonstanten berücksichtigt werden 62]. Bei dem D8-Röntgendiffraktometer beträgt der maximale Winkel $2 \theta=99^{\circ}$. Somit können auch noch Reflexe höherer Ordnung beobachtet werden. Aus der Bragg-Bedingung wird zudem die Notwendigkeit von Röntgenstrahlung ersichtlich. Da für den Einfallswinkel stets $\sin (\theta)<1$ gilt, folgt für $n=1$ die Bedingung $\lambda<2 d_{h k l}$ und für $\lambda$ eine zwingende Größenordnung einiger $10^{-10} \mathrm{~m}[62]$. Für das hexagonale Kristallsystem wird zudem eine Reflexbeschreibung mit vier Achsen und vier Millerschen Indizes (hkil) verwendet. Hierbei sind die Indizes $(h k l)$ identisch zu der gewohnten Definition, während sich für den vierten Index $i=-(h+k)$ ergibt. Der Vorteil liegt nun darin, dass auch im hexagonalen Kristallsystem kristallographisch gleichwertige Ebenen mit einer äquivalenten Reflexindizierung bezeichnet werden können, ohne dass diese grundlegend geändert werden muss $[59,62]$.

Für die Laue-Bedingung hingegen wird der Kristall als ein Bravais-Gitter mit auf den Gitterplätzen sitzenden Atomen angenommen, an denen die Röntgenstrahlung mit dem Wellenvektor $\vec{k}$ gestreut wird. Eine Darstellung ist in der Abbildung 3.2 .2 (b) (nach [57]) 


\section{Experimentelle Methoden}

gegeben. Für eine konstruktive Interferenz muss nun der Gangunterschied $\Delta$ der gestreuten Röntgenstrahlen mit dem Wellenvektor $\overrightarrow{k^{\prime}}$ ein ganzzahliges Vielfaches der Wellenlänge $\lambda$ der einfallenden Strahlung sein. In einer geometrischen Betrachtung folgt für den Gangunterschied konstruktiver Interferenz für eine elastische Streuung $|\vec{k}|=\left|\overrightarrow{k^{\prime}}\right||57,60|$ :

$$
\Delta=R\left(\cos (\theta)+\cos \left(\theta^{\prime}\right)\right)=\vec{R} \cdot\left(\hat{k}-\hat{k}^{\prime}\right)=m \lambda
$$

Hierbei bezeichnen $\hat{k}=\vec{k} /|\vec{k}|$ und $\hat{k}^{\prime}=\overrightarrow{k^{\prime}} /|\vec{k}|$ die Einheitsvektoren in Richtung der einfallenden und gestreuten Röntgenstrahlung, $m$ eine ganze Zahl und $\vec{R}$ den Gittervektor im Bravais-Gitter des Kristalls zwischen zwei Streuzentren. Über den Betrag des Wellenvektors $|\vec{k}|=\frac{2 \pi}{\lambda}$ ergibt sich daraus die Relation $\vec{R} \cdot\left(\vec{k}-\overrightarrow{k^{\prime}}\right)=2 \pi m$, die wiederum äquivalent zu der Bedingung $\mathrm{e}^{i \vec{R}\left(\overrightarrow{k^{\prime}}-\vec{k}\right)}=1$ ist. Dieser Ausdruck entspricht der Definition des reziproken Gitters $\mathrm{e}^{i \vec{R} \cdot \vec{G}_{h k l}}=1$. Somit folgt für die Laue-Bedingung konstruktiver Interferenz 57 :

$$
\vec{K}=\overrightarrow{k^{\prime}}-\vec{k}=\vec{G}_{h k l}
$$

Nach der Laue-Bedingung in der Gleichung (3.2.4) ist konstruktive Interferenz genau dann zu beobachten, wenn die Wellenvektoränderung $\vec{K}=\overrightarrow{k^{\prime}}-\vec{k}$ während der Beugung einem reziproken Gittervektor $\vec{G}_{h k l}$ entspricht. Die Laue-Bedingung ist dabei äquivalent zu der Bragg-Bedingung. Dieses ist in der Abbildung 3.2.2 (a) veranschaulicht. Es ergibt sich nach der Laue-Bedingung bei konstruktiver Interferenz beliebiger Ordnung für den reziproken Gittervektor $\left|\vec{G}_{h k l}\right|=2|\vec{k}| \sin (\theta)=n \frac{2 \pi}{d_{h k l}}$, woraus mit $|\vec{k}|=\frac{2 \pi}{\lambda}$ direkt die Bragg-Bedingung für konstruktive Interferenz aus der Gleichung (3.2.1) folgt. Zu berücksichtigen ist, dass mit der Bragg- und der Laue-Bedingung die Richtungen der Reflexe $(h k l)$ mit konstruktiver Interferenz am Kristallgitter gebeugter Röntgenstrahlung beschrieben werden. Die relative Intensität der Reflexe ist von der Kristallstruktur und der Verteilung der Atome anhand der Basis und dem Streuvermögen der Basisatome (aufgrund der Streuung an den Elektronen der Atome) abhängig [59] und kann mit dem Strukturfaktor $F_{h k l}$ berechnet werden. Dieser stellt die Fouriertransformation der Elektronendichte in der Elementarzelle der Kristallstruktur dar und ergibt sich durch Aufsummierung der atomaren Streufaktoren $f_{i}(\vec{K})$ der Basisatome $i$ bezüglich ihrer Verteilung in der Elementarzelle der Kristallstruktur [57,59]:

$$
F_{h k l}=\sum_{i=1}^{n} f_{i}(\vec{K}) \mathrm{e}^{i \vec{K} \cdot \overrightarrow{r_{i}}}=\sum_{i=1}^{n} f_{i}(\vec{K}) \mathrm{e}^{i \vec{G} \cdot \overrightarrow{r_{i}}}=\sum_{i=1}^{n} f_{i}(\vec{K}) \mathrm{e}^{2 \pi i\left(h \cdot u_{i}+k \cdot v_{i}+l \cdot w_{i}\right)}
$$

Hier bezeichnet $\vec{K}=\overrightarrow{k^{\prime}}-\vec{k}=\vec{G}$ den Streuvektor der Reflexe, der nach der Laue-Bedingung für die Beobachtung konstruktiver Interferenz ein Vektor des reziproken Gitters sein muss, $\vec{r}_{i}=u_{i} \vec{a}_{1}+v_{i} \vec{a}_{2}+w_{i} \vec{a}_{3}$ die Ortsvektoren der Atome $i$ der realen Basis des Bravais-Gitters 


\subsection{Standard-Probencharakterisierung}

der Kristallstruktur und $f_{i}(\vec{K})$ die atomaren Streufaktoren der Basisatome. Diese sind ein Maß für das Streuvermögen der Atome und Ionen in der Einheitszelle und hängen von der inneren Struktur und Elektronenzahl des jeweiligen Atomrumpfes oder Ions ab [59,61,63]:

$$
f_{i}(\vec{K})=\int_{V_{i}} \rho_{i}\left(\overrightarrow{r^{\prime}}\right) \mathrm{e}^{i \vec{K} \cdot \vec{r}^{\prime}} \mathrm{d}^{3} r^{\prime}
$$

Der atomare Formfaktor ist damit die Fouriertransformation der Elektronendichte $\rho$ in den Atomen und Ionen und der Vektor $\overrightarrow{r^{\prime}}$ ist der Anteil des Ortsvektors $\vec{r}=\overrightarrow{r_{i}}+\overrightarrow{r^{\prime}}$, der vom Kern des Atoms $i$ zum jeweiligen Volumenelement der Elektronenhülle des Atoms zeigt [59]. Die Reflexintensität $I_{h k l}$ in einer Kristallelementarzelle ist mit $I \propto\left|F_{h k l}\right|^{2}=F_{h k l} \cdot \bar{F}_{h k l}$ proportional zum Betragsquadrat des Strukturfaktors [62] aus der Gleichung (3.2.5). Folglich lassen sich die Reflexe vorhersagen, die durch konstruktive Interferenz beobachtbar sind und die durch destruktive Auslöschung fehlen. Weitere Einflüsse auf die Reflexintensitäten sind kristallographisch begründet (der Multiplizitätsfaktor $p$, der die Anzahl äquivalenter Ebenen zu der Reflexebene beschreibt), berücksichtigen aber auch die Winkel- und Polarisationsabhängigkeit der gestreuten Röntgenstrahlung (Lorentz-Polarisationsfaktor LP) und die Probeneigenschaften (Absorptionsfaktor $A$ und Probenvolumen $V$ ). Für dünne Schichten können die Probeneigenschaften für alle Reflexe als konstant angenommen und mit der Reflexmultiplizität in einem Skalierungsfaktor $K$ zusammengefasst werden [62]. Oft werden relative Reflexintensitäten betrachtet, sodass die einfallende Intensität $I_{0}$ der Röntgenstrahlung nicht einbezogen, sondern eine Normierung auf den stärksten Reflex durchgeführt wird 64. Es verbleibt der LP-Faktor, der die Polarisation der Röntgenstrahlung und die Probenbewegung beim Streuprozess (Thomson-Streuung) als Funktion des Einfallswinkels $\theta$ berücksichtigt. Da die Röntgenstrahlung des verwendeten D8-Diffraktometers unpolarisiert ist [50], folgt für die relative integrale Reflexintensität $I_{\text {rel. }}(h k l)$ 62:

$$
I_{\text {rel. }}(h k l)=K \cdot L P \cdot\left|F_{h k l}\right|^{2}=K \cdot\left|F_{h k l}\right|^{2} \cdot\left(\frac{1+\cos ^{2}(2 \theta)}{\sin ^{2}(\theta) \cos (\theta)}\right) \text {. }
$$

In der kinematischen Beugungstheorie ist zudem eine Abhängigkeit der elastisch gestreuten Reflexintensität $I(h k l)$ von der Temperatur durch thermisch angeregte Schwingungen der Atome im Kristallgitter zu beobachten. Der Einfluss der Gitterschwingungen auf die Beugungsreflexe wird mit dem Debye-Waller-Faktor $D_{h k l}$ beschrieben 65, 66. Es ergibt sich eine exponentielle Abnahme der Reflexintensität für ansteigende Temperaturen $T$ und größer werdende Indizierung $(h k l)$ der Reflexe bei gleichbleibender Reflexbreite 60, 61]:

$$
I(h k l)=I_{0} \cdot \exp \left(-\frac{k_{B} T}{M \omega^{2}} G^{2}\right)=I_{0} \cdot D_{h k l} .
$$




\section{Experimentelle Methoden}

Hier bezeichnet $I_{0}$ die Reflexintensität für ein starres Gitter, $M$ die Atommasse, $\vec{G}$ den reziproken Gittervektor des Reflexes $(h k l)$ und $\omega$ die Kreisfrequenz der harmonisch genäherten Gitterschwingung. Der Debye-Waller Faktor $D_{h k l}$ ist somit für Reflexe höherer Indizierung kleiner und die Abnahme dieser Reflexe mit der Temperatur größer [58 61,65]. Da die Gitterschwingungen durch die Unabhängigkeit ihrer Bewegung im Mittel die Gitterstruktur und ihre Periodizität behalten, verändert sich die Reflexbreite nicht [58, 60, 65]. Aufgrund des klimatisierten D8-Röntgendiffraktometerlabors können Temperaturschwankungen vernachlässigt werden. Da aber Reflexe höherer Ordnung nach der Gleichung (3.2.8) auch bei konstanter Temperatur einen kleineren Debye-Waller-Faktor gegenüber Reflexen niedrigerer Indizierung 60, 61 aufweisen, muss die Gleichung 3.2.7 um den Debye-WallerFaktor ergänzt werden. In der Bragg-Brentano-Geometrie ist der Streuvektor senkrecht zur Probenoberfläche orientiert. Somit lässt sich für eine Schicht mit einer out-of-plane $\langle 100\rangle$-Orientierung mit der Gitterkonstante $c_{\perp}$ senkrecht zur Probenoberfläche und der unverspannten Gitterkonstante $c_{0}$ die out-of-plane Verspannung $\varepsilon_{\perp}$ bestimmen 67]:

$$
\varepsilon_{\perp}=\frac{c_{\perp}-c_{0}}{c_{0}}
$$

Für Schichten in einer [hkl]-Orientierung, die nicht den $\langle 100\rangle$-Kristallachsen entspricht, ergibt sich die Verspannung $\varepsilon_{h k l}=\left(a_{h k l}-a_{0_{h k l}}\right) / a_{0_{h k l}}$. Die Verspannung $\varepsilon=\Delta d / d$ ist dabei direkt anhand der Verschiebung $\triangle \theta$ der Reflexe $(h k l)$ im XRD-Spektrum in Relation zur unverspannten Kristallstruktur beobachtbar. Es folgt durch Differenzierung der BraggBedingung aus der Gleichung (3.2.1 bezüglich des Gitterebenenabstandes $d$ [68]:

$$
\triangle \theta=-\varepsilon \tan (\theta)
$$

Durch das negative Vorzeichen führt ein größerer Gitterebenenabstand somit zu einem kleineren Winkel $\theta$ für den Reflex $(h k l)$. Für kubische Kristalle ergibt sich $\triangle \theta=-\frac{\triangle a}{a_{0}} \tan (\theta)$. Somit lässt sich für eine Zugspannung $\left(a>a_{0}\right)$ eine Reflexverschiebung zu kleineren Winkeln $\theta$ und für eine Druckspannung $\left(a<a_{0}\right)$ eine Reflexverschiebung zu größeren Winkeln beobachten. Zusätzlich kann eine nicht einheitliche Verteilung der Verspannung in der Schicht die Form der Reflexe beeinflussen [68. Eine Qualitätsmerkmal der Schichten ist zudem die Kristallitgröße. Hierfür kann die Scherrer-Gleichung verwendet werden, die einen Zusammenhang zwischen der Breite $b_{h k l}$ des Schichtreflexes und der Kristallitgröße $D$ in der Schicht senkrecht zu der jeweils betrachteten Reflexgitterebene herstellt 69 71]:

$$
D_{h k l}=\frac{K \lambda}{b_{h k l} \cos (\theta)} .
$$


Hier bezeichnet $\lambda$ die Wellenlänge der Röntgenstrahlung, während $\theta$ den Winkel beschreibt, bei dem sich der Reflex $(h k l)$ beobachten lässt. Hinzu kommt der Scherrer-Formfaktor $K$, dessen Größe von der Reflexbreitendefinition, der Kristallitform und der Verteilung der Kristallitgröße beeinflusst ist 70,71 . So ergibt sich für Pulver mit kubischen Kristalliten $K=2 \cdot(\ln 2 / \pi)^{1 / 2} \approx 0,94$ für eine Reflexlinienbreite $b_{\text {FwHм }}$ in Höhe der halben Reflexintensität (FWHM, engl.: full width at half maximum) [69,72]. Allgemein ist $K$ jedoch nur numerisch zugänglich 70,71. Neben der FWHM-Halbwertsbreite $b_{\text {FWHM }}$ wird oft die integrale Linienbreite $b_{\text {ILW }}$ (ILW, engl.: integral line width) verwendet. Diese ergibt sich aus dem Verhältnis des Integrals des Reflexes zum Intensitätsmaximum. Üblicherweise wird für eine einfache Abschätzung der Kristallitgröße eine kubische Kristallitform mit $K_{\text {FWHM }} \approx 0,9$ oder $K_{\text {ILW }} \approx 1$ verwendet $62,71,73$. In dieser Arbeit wird die FWHM-Reflexbreite und $K_{\mathrm{FWHM}}=0,9$ benutzt. Die Scherrer-Gleichung ist dabei nur für Kristallitgrößen von etwa 10 - $1000 \AA$ sinnvoll anzuwenden, da die Reflexe für kleinere Kristallite stark verbreitert und abgeschwächt sind, während sie für zu große Kristallite so schmal werden, dass die Breite von instrumentellen Einflüssen des Röntgendiffraktometers überlagert ist 71 73. Die Halbwertsbreite $b_{h k l}$ kann aus dem Intensitätsverlauf $I\left(2 \theta_{h k l}\right)$ einer Rocking-Kurve (XRC, engl:: X-ray rocking curve) ermittelt werden, indem der Winkel $2 \theta_{h k l}$, bei dem die Bragg-Bedingung erfüllt ist, eingestellt und $\theta$ um den Idealwinkel $\theta_{h k l}$ herum variiert wird.

\subsubsection{Röntgenreflektometrie (XRR)}

Die Schichtdicke lässt sich wiederum mit einer abgewandelten XRD-Methode, der Röntgenreflektometrie (XRR, engl.: X-ray reflection) bestimmen [67,74 76]. Hierzu wird erneut das Bruker AXS D8 Advance in der Bragg-Brentano-Geometrie verwendet, nur dass die Röntgenstrahlung unter einem kleinen Einfallswinkel von $\theta \approx 0,5^{\circ}-5^{\circ}$ auf die Probe trifft. Das Prinzip basiert auf der Reflexion von Röntgenstrahlung an den Grenzflächen von Medien mit unterschiedlichen Brechungsindizes $n_{i}$. Für eine dünne Schicht mit dem Brechungsindex $n$ auf einem Substrat mit dem Brechungsindex $n_{S}$ treten Reflexionen sowohl an der Schichtoberfläche als auch an der Grenzfläche von Schicht und Substrat auf 74, 75]. Bis $\mathrm{zu}$ einem kritischen Winkel von $\theta<\theta_{c} \approx 0,2-0,6^{\circ}$ (abhängig von der Dichte und der Elektronendichte des Materials) 67, 77 liegt dabei eine Totalreflexion an der Schichtoberfläche vor, da der Brechungsindex der Schicht für Röntgenstrahlung $n<1$ ist 67,74 77], sodass die Reflexion beim Übergang von einem optisch dichteren Medium (hier Luft mit $n_{L} \approx 1$ ) auf ein optisch dünneres Medium (Schicht) stattfindet. Entsprechend hoch ist die am Detektor registrierte Intensität. Wird der kritische Winkel $\theta_{c}$ überschritten, so wird ein Teil der Röntgenstrahlung gemäß des Snelliusschen Brechungsgesetzes und der Fresnelschen Gleichungen in die Schicht hinein gebrochen und an der Grenzfläche von Schicht und Substrat (total-)reflektiert. Es kommt in Abhängigkeit von $\theta$ zu einer konstruktiven oder destruktiven Überlagerung 67, 76. Der Strahlengang ist für die Näherung, dass Schicht und Substrat über eine ebene Oberfläche verfügen, in der Abbildung 3.2 .3 (a) dargestellt. 


\section{Experimentelle Methoden}

(a)

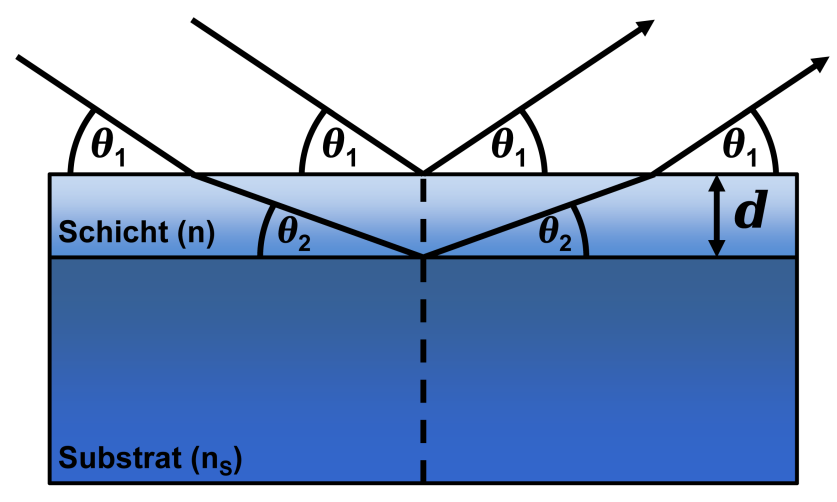

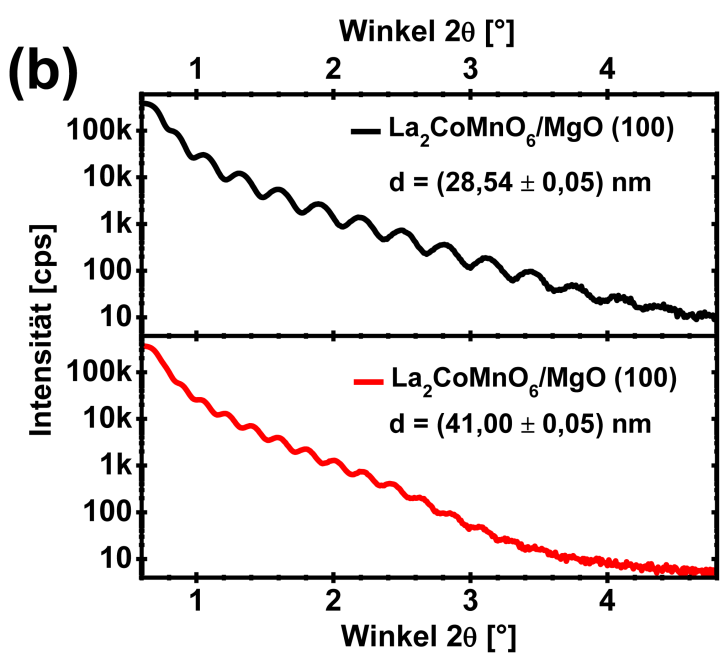

Abbildung 3.2.3: Prinzip der Röntgenreflektometrie mit der Reflexion an der Schichtoberfläche und der Schicht-Substrat-Grenzfläche (a) und Beispielmessungen an zwei $\mathrm{La}_{2} \mathrm{CoMnO}_{6}$-Schichten mit unterschiedlicher Schichtdicke (b).

Nach der Bragg-Bedingung kommt es zur konstruktiven Interferenz, wenn der optische Weg in der Schicht $m \lambda=2 n d \sin \left(\theta_{2}\right)$ ein ganzzahliges Vielfaches der Wellenlänge $\lambda$ der Röntgenstrahlung beträgt [76, und zur destruktiven Interferenz, wenn der Gangunterschied ein ungerades Vielfaches der halben Wellenlänge einnimmt. Der Winkel $\theta_{2}$ in der Schicht unterscheidet sich vom Einfallswinkel $\theta_{1}$ außerhalb, die Abweichung ist jedoch aufgrund der ähnlichen Brechungsindizes $n_{L}-n<10^{-4}$ vernachlässigbar 76. Zudem sind Phasensprünge von $\pi$ (halbe Wellenlänge) bei der Reflexion an optisch dichteren Medien zu beachten, sodass sich die Bedingungen für Minima und Maxima umkehren 75,76]. Für die Intensität $I(2 \theta)$ lässt sich nun aufgrund der Winkelabhängigkeit der Interferenz ein Wechsel von Maxima und Minima (sogenannte Kiessig-Oszillation [67]) beobachten. Die XRR-Messungen zweier $\mathrm{La}_{2} \mathrm{CoMnO}_{6}$-Schichten auf $\mathrm{MgO}$ (100) mit unterschiedlicher Schichtdicke sind in der Abbildung 3.2.3 (b) dargestellt. Die Abnahme der Intensität und der Oszillationen für größere Winkel resultiert aus der Rauigkeit an der Schichtoberfläche und an der Grenzfläche zwischen Schicht und Substrat (durch chemische Durchmischung) [50], wodurch die Röntgenstrahlung für größere Winkel diffuser reflektiert wird. Die Oszillationszahl und der Intensitätsverlauf stellen daher ein Maß für die Reflektivität und die Grenzflächenrauigkeiten des Schichtsystems dar. Der Verlauf und die Schichtdicke $d$ können mit den Fresnelschen Formeln ermittelt werden, indem die Schichtdicke und die Ober- und Grenzflächenrauigkeit als Parameter angepasst werden [50, 67, 76. Die Schichtdicke $d$ lässt sich zudem mit der Bragg-Bedingung für kleine Winkel über den Abstand benachbarter Interferenzmaxima (bzw. Minima) $\triangle \theta_{1,(m+1)-m}=\theta_{1, m+1}-\theta_{1, m}$ in der XRR-Kurve bestimmen 67, 75, 76,

$$
\sin ^{2}\left(\theta_{1, m}\right)=\frac{\lambda^{2}}{4 d^{2}} m^{2}+\theta_{c}^{2} \quad \Rightarrow d \approx \frac{\lambda}{2} \cdot \frac{1}{\triangle \theta_{1,(m+1)-m}} .
$$




\subsection{Standard-Probencharakterisierung}

Für Röntgenstrahlung wird der Brechungsindex mit $n=1-\delta<1$ beschrieben 67,74 76, wobei das sogenannte Brechzahldekrement $\delta$ dabei einen dispersiven Korrekturfaktor darstellt und mit dem kritischen Winkel für Totalreflexion über $\theta_{c}=\sqrt{2 \delta}$ 74, 77, in Relation steht. Aus der Bragg-Bedingung ergibt sich für die Schichtdicke $d$ für kleine Winkel $\sin \left(\theta_{1, m}\right) \approx \theta_{1, m}$ und großer Ordnungen $m$ (d.h. es gilt $\theta_{1, m} \gg \theta_{c}$ ) ein reziprokes Verhalten $d \sim 1 / \triangle \theta_{1,(m+1)-m}$ zum Winkelabstand benachbarter Maxima 67, 75]. Folglich liegen die Interferenzmaxima für die dickere der in der Abbildung 3.2.3 (b) gezeigten Schichten dichter zusammen. Die geringere Anzahl an Oszillationen und die schnellere Intensitätsabnahme für die dickere Schicht sind auf die größere Grenzflächenrauigkeit an der Schichtoberfläche und der Grenzfläche zum Substrat sowie der resultierenden Verringerung der Reflektivität zurückführen. Da für größere Schichtdicken die Absorption für den in der Schicht verlaufenden Teil des Röntgenstrahls zunimmt [75], sich der Reflexionsbeitrag an der Grenzfläche von Schicht und Substrat weiter verringert, und die Oberflächenrauigkeit tendenziell mit der Schichtdicke weiter anwächst, ist die Interferenz für dickere Schichten weniger scharf und die Oszillationen weniger ausgeprägt, bis von einer gewissen Schichtdicke an (typischerweise > $150 \mathrm{~nm}$ ) keine Interferenzen mehr registriert werden können 67, 75].

Daneben kann auch mit der XRD-Methode die Schichtdicke bestimmt werden, wenn in der Umgebung der Schichtreflexe sogenannte Laue-Oszillationen beobachtet werden, die vergleichbar wie bei den Kiessig-Oszillationen aufgrund von Interferenzeffekten auftreten. Während die Kiessig-Oszillationen jedoch durch die Reflexion und Interferenz an zwei oder mehreren Grenzflächen entstehen und folglich eine starke Sensitivität bezüglich der Grenzflächenrauigkeiten aufweisen, ist das Auftreten der Laue-Oszillationen von der Ordnung in der kristallinen Struktur abhängig, sodass diese nur für eine hohe Kristallqualität zu beobachten sind. Aus dem Abstand zweier Laue-Oszillationen $\triangle \theta_{(n+1)-n}$ folgt durch Differenzierung der Bragg-Bedingung bezüglich des Winkels $\theta$ für die Schichtdicke $d$ 67]:

$$
d=\frac{\lambda}{\triangle \theta_{(n+1)-n}} \cdot \frac{1}{2 \cos (\theta)}
$$

$\mathrm{Zu}$ beachten ist, dass die mit der Gleichung (3.2.13) bestimmte Schichtdicke den Teil der Schicht mit kristalliner Ordnung beschreibt und mit der nach Gleichung 3.2.12 ermittelten Schichtdicke daher nur für einkristalline Schichten übereinstimmt. Durch Blenden unterschiedlicher Breite $(0,05-0,6 \mathrm{~mm})$ vor der Röntgenröhre und dem Detektor können die Intensität (breitere Blenden, XRD) und die Auflösung (schmalere Blenden, XRR) beeinflusst werden. Über einen Keil im Abstand weniger $\mu \mathrm{m}$ vor der Probe (nur bei XRR) wird zudem sichergestellt, dass nur Strahlung nahe des Drehpunkts in der Bragg-BrentanoGeometrie bei genau definiertem Winkel $\theta$ bzw. $2 \theta$ bei der Messung erfasst wird. 


\section{Experimentelle Methoden}

\subsubsection{Rasterkraftmikroskopie (AFM)}

Die Oberflächenmorphologie und die Rauigkeit der hergestellten Schichten werden mithilfe eines Rastkraftmikroskops (AFM, engl.: atomic force microscope) [78] untersucht. Hierfür besitzen Rasterkraftmikroskope wie das verwendete Innova-IRIS [79] von Bruker eine am Ende einer dünnen balkenförmigen Blattfeder (Cantilever) befestigte nanoskopisch kleine Spitze mit einem Radius weniger Nanometer [80, 81], die in einem Raster über die Probenoberfläche bewegt wird. Der Aufbau ist schematisch in der Abbildung 3.2.4 (a) dargestellt. Das Messprinzip basiert auf der Wechselwirkung zwischen Spitze und Probe und die auf den Cantilever übertragene Kraft als Funktion des Spitzenabstandes zur Probe. Dieses resultiert in einer spezifischen Verbiegung des Cantilevers in Abhängigkeit der Probenoberflächenstruktur, welche mithilfe des Lichtzeigerprinzips gemessen wird, indem ein Laser, der auf die Cantilever-Oberseite fokussiert ist, auf eine positionssensitive Photodiode reflektiert wird. Zur Signalerhöhung ist die Cantilever-Oberseite mit einer reflektierenden Metallschicht bedampft. Die Photodiode besteht aus vier gleichgroßen Quadranten (Vierfeld-Photodiode) in rechteckiger Anordnung, die je nach Cantilever-Verbiegung unterschiedlich beleuchtet werden und die Intensität $\left(I_{1}, I_{2}, I_{3}\right.$ und $\left.I_{4}\right)$ in ein Spannungs- oder Stromsignal umsetzen 82. Das Prinzip ist in der Abbildung 3.2.4 (b) (nach [82]) gezeigt.

(a)

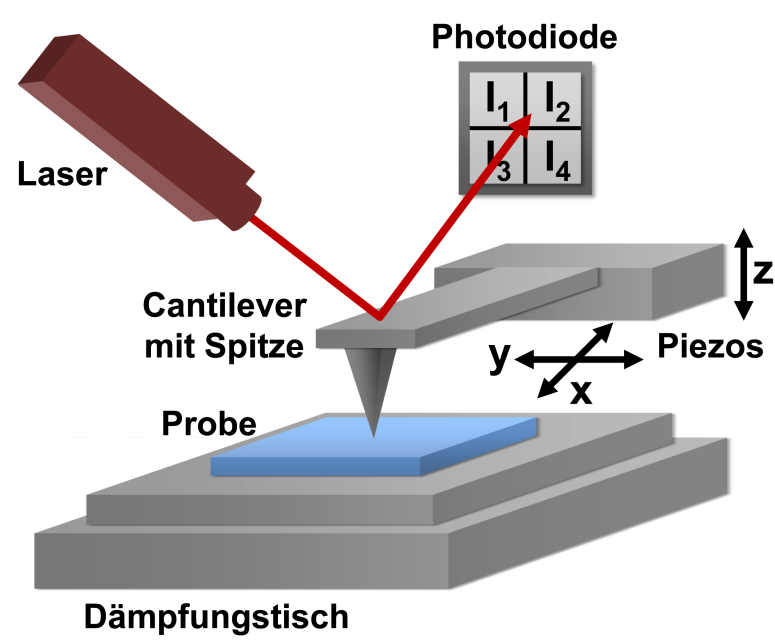

(b)

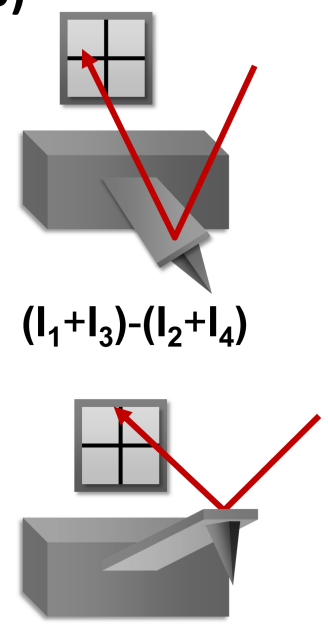

$\left(I_{1}+I_{2}\right)-\left(I_{3}+I_{4}\right)$

Abbildung 3.2.4: Schematischer Aufbau eines Rasterkraftmikroskops (a) und Aufzeichnung der horizontalen und vertikalen Cantilever-Verbiegung mithilfe einer VierfeldPhotodiode durch das Lichtzeigerprinzip (b) (nach [82]).

Im Ausgangszustand (gerader Cantilever; Spitze nicht in Wechselwirkung mit der Probe) sind Laser und Vierfeld-Photodiode so ausgerichtet, dass der Laser alle vier Quadranten gleichmäßig beleuchtet (Nulllage). Wird die Spitze angenähert und über die Probe bewegt, kommt es zu Normalkräften, die den Cantilever vertikal verbiegen, und zu lateralen Kräften, die den Cantillever horizontal tordieren [80,82,84], und die den Laser ablenken. Durch einen Signalvergleich kann die Cantilever-Verbiegung und damit die Oberflächengeometrie 


\subsection{Standard-Probencharakterisierung}

für jeden Rasterpunkt bestimmt werden. Die horizontale Komponente ergibt sich aus der Differenz $\left(I_{1}+I_{3}\right)-\left(I_{2}+I_{4}\right)$ der Quadrantensignale links und rechts, während für eine vertikale Verbiegung die Signaldifferenz $\left(I_{1}+I_{2}\right)-\left(I_{3}+I_{4}\right)$ der oberen und unteren Segmente betrachtet wird 80,82,84. Die Daten können dann mit einem Programm wie Gwyddion 85 abgebildet werden. Das Rastern wird über eine elektrische Spannung $U_{\text {Piezo }}$ durch Piezoelemente gesteuert, die den Cantilever horizontal (x-y-Ebene) und vertikal (z-Richtung) mit einer Empfindlichkeit von etwa $0,1-10 \mathrm{~nm} / \mathrm{V}$ bewegen 80,82 , sodass vertikale Auflösungen bis $<1 \AA$ erreichbar sind $[78,82,86$. Die Annäherung bis in Mikrometerbereich erfolgt mit einem Stellmotor. Die laterale Auflösung hängt von der Spitzenqualität ab und erreicht etwa $30 \AA$ [8]. Zur Entkopplung von Gebäudeschwingungen und Trittschall ist das AFM auf einem aktiv gedämpften Vibrationsausgleichstisch (Accurion Halcyonics_i4) installiert, der sich auf einem massiven optischen Tisch (Thorlabs) befindet.

Ein AFM verfügt dabei über verschiedene Betriebsarten. Im Kontakt-Modus (engl.: contact mode, CM-AFM) befindet sich die Spitze während des Rasterns in Kontakt mit der Probenoberfläche. Der Betrieb kann in konstanter Höhe (engl.: constant height mode) oder mit konstanter Kraft (engl.: constant force mode) erfolgen [82], indem die Höhe des Cantilevers über der Probe fest eingehalten (die Verbiegung des Cantilevers gibt die Topographie der Probenoberfläche wieder) oder die Cantilever-Verbiegung und die dabei wirkende Kraft konstant gehalten wird (die Oberfläche lässt sich aus der Piezoelementansteuerung rekonstruieren). Zudem gibt es den Nicht-Kontakt-Modus (engl.: non-contact mode, NC-AFM) und den intermittierenden Modus (engl.: intermittent contact or tapping mode, TM-AFM), bei denen die Spitze die Probenoberfläche nicht (Nicht-Kontakt-Modus) oder nur zeitweise berührt 82, 83, 86. Die Bildgebung erfolgt, indem der Cantilever auf ein Piezoelement angebracht wird, das ihn in eine Schwingung nahe der Resonanzfrequenz versetzt 80. Abweichungen von der Amplitude und der Frequenz bei kurzer Berührung der Probenoberfläche (tapping mode) oder der Annäherung durch wechselwirkende Kräfte mit der Probe (Nicht-Kontakt-Modus) werden registriert und aus der Anregungskorrektur zur Aufrechterhaltung der Schwingung kann die Oberflächentopographie rekonstruiert werden 80].

Die Kräfte zwischen der AFM-Spitze und der Probe sind sowohl attraktiv (van-der-WaalsKraft, Kapillar- und Adhäsisonskräfte) als auch repulsiv (elektrostatische Kräfte, Abstoßung aufgrund des Pauli-Prinzips) [80,83, 84]. Während die van-der-Waals-Kraft, Kapillarkräfte und elektrostatische Kräfte langreichweitig sind und für Abstände $\gg 5 \AA$ überwiegen (die Kapillarkräfte entstehen durch den Wasserfilm auf der Probenoberfläche, der durch die Luftfeuchtigkeit hervorgerufen wird [83,84]), ist die Abstoßung aufgrund des Pauli-Prinzips für kleine Abstände $<1 \AA$ vorherrschend [80]. Die Wechselwirkung zwischen Spitze und Probe in Abhängigkeit des Abstandes kann mit einer Kraft-Abstands-Kurve vermessen werden. Diese ist schematisch in der Abbildung 3.2.5 (a) (nach [81]) dargestellt. 


\section{Experimentelle Methoden}

(a)

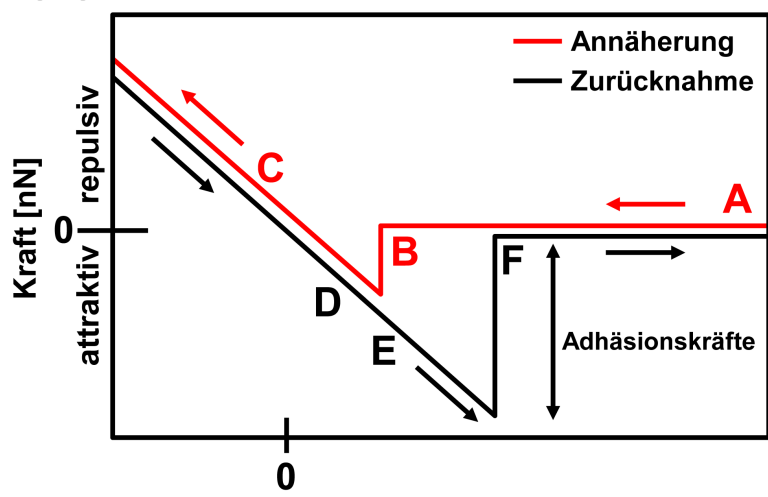

Abstand Spitze-Probe [nm] (Cantilver unverbogen)
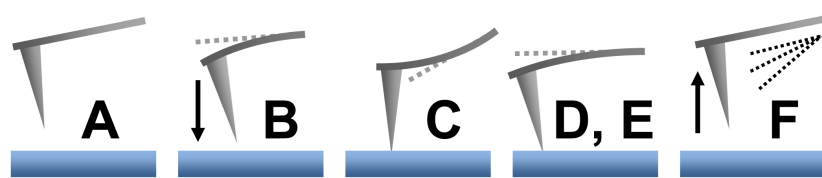

(b)

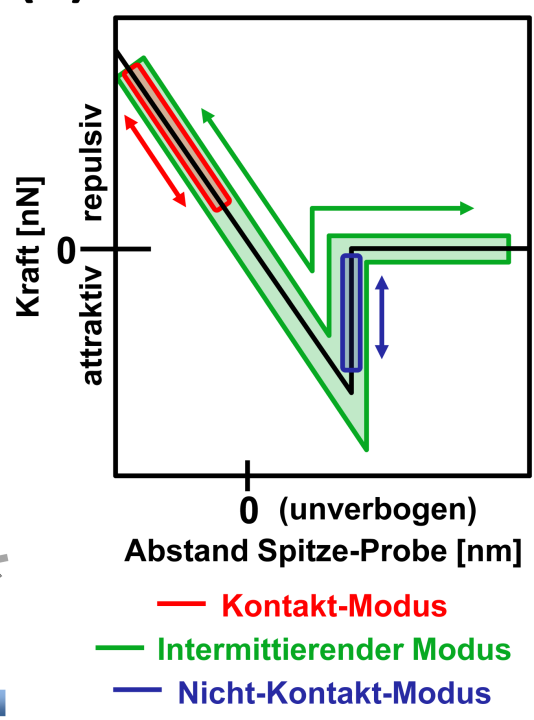

Abbildung 3.2.5: Charakteristische Kraft-Abstands-Kurve zwischen AFM-Spitze und Probe bei der Annäherung und anschließender Zurücknahme (a) (nach 81]) und der Arbeitsbereich im Kontakt-Modus, intermittierenden Modus und NichtKontakt-Modus in der Kraft-Abstands-Kurve (b) (nach 82]).

Wird die Spitze aus großer Distanz angenähert (Punkt A), so wirken zunächst keine Kräfte auf die Spitze (gerader Cantilever), bis der Wirkungsbereich attraktiver Kräfte erreicht wird und die Spitze schlagartig an die Probenoberfläche gezogen wird (Punkt B; nach unten gebogener Cantilever). Ein weiteres Annähern gleicht diese aus bis sich eine repulsive Kraft auf die Spitze einstellt, die nach dem Hookeschen Gesetz linear mit der Abstandsverringerung zur Probe zunimmt und den Cantilever nach oben verbiegt (Punkt C). Bei einer anschließenden Zurücknahme des Cantilever entspricht der Verlauf in der Kraft-AbstandsKurve zunächst dem der Annäherung (Punkt D). Aufgrund attraktiver Adhäsionskräfte bleibt der Kontakt zwischen Probe und Spitze jedoch über den Ablösepunkt hinaus erhalten (Punkt E; weiterhin nach unten verbogener Cantilever) bis die Rückstellkraft überwiegt und der Cantilever in seine gerade Form zurückkehrt (Punkt F). Die Hysterese ist ein Maß für die Adhäsionskräfte, während über die Steigung des linearen Teils der Kraft-AbstandsKurve die elastischen Eigenschaften der Probe charakterisiert werden können [82, 83]. In der Abbildung 3.2.5 (b) (nach [82]) sind zudem die Arbeitsbereiche der AFM-Betriebsarten in der Kraft-Abstands-Kurve dargestellt. Im Kontakt-Modus ist die Spitze permanent mit der Probe in Kontakt und übt auf diese eine Kraft aus, sodass der lineare Teil mit repulsiver Kraftwirkung genutzt wird (roter Bereich). In dieser Arbeit wurden alle Messungen in diesem Bereich bei konstanter Kraft durchgeführt. Im Nicht-Kontakt-Modus berührt die Spitze des schwingenden Cantilevers die Probe nicht und der Betrieb erfolgt bei einer attraktiven Kraft ohne Probenkontakt (blauer Bereich). Im intermittierenden Modus berührt die Spitze des schwingenden Cantilevers zeitweise die Probe, sodass sich der Arbeitsbereich entsprechend auf die gesamte Kraft-Abstands-Kurve erstreckt (grüner Bereich). 
Aus den AFM-Bildern lässt sich zudem für eine Probencharakterisierung die Oberflächenrauigkeit statistisch bestimmen. Hierfür gibt es verschiedene Größen, mit denen sich die Oberflächenrauigkeit angeben lässt. Zumeist wird jedoch die quadratische Rauigkeit $R_{q}$ (RMS-Rauigkeit; engl.: root mean squared roughness, RMS roughness) verwendet 86, 87]:

$$
R_{q}=\mathrm{RMS}=\sqrt{\frac{\sum_{i=1}^{N}\left(Z_{i}-\bar{Z}\right)^{2}}{N}} .
$$

Hier bezeichnet $\bar{Z}$ den mittleren Höhenwert, $Z_{i}$ die Höhenwerte und $N$ die Rasterpunktanzahl. Der RMS-Wert $R_{q}$ ist dabei umso größer, je höher die Oberflächenrauigkeit ist (positive Korrelation). Andere Rauigkeitskenngrößen sind $R_{q}$ in der Güte im Allgemeinen unterlegen 82,87]. Folglich wird in dieser Arbeit nur der RMS-Wert verwendet.

\subsubsection{Rastertunnelmikroskopie (STM)}

Eine weitere Untersuchungsmöglichkeit von Oberflächen ist die Verwendung eines Rastertunnelmikroskops (STM, engl.: scanning tunneling microscope) 88]. Das Prinzip basiert auch hier darauf, dass die Probenoberfläche mit einer feinen nanoskopischen Spitze abgerastert wird. Im Fall des Rastertunnelmikroskops erfolgt die Bildgebung, indem bei einem angelegten elektrischen Potential $U_{\text {Bias }}$ zwischen einer metallischen Spitze und der Probe der vom Abstand $z$ zwischen Probenoberfläche und Spitze abhängige Tunnelstrom $I$ für jeden Rasterpunkt registriert und in ein Höhenprofil umgesetzt wird. Folglich dient der quantenmechanische Tunneleffekt als wechselwirkender Prozess zwischen Probe und Spitze. Eine schematische Übersicht des Prinzips ist in der Abbildung 3.2.6 (a) dargestellt.

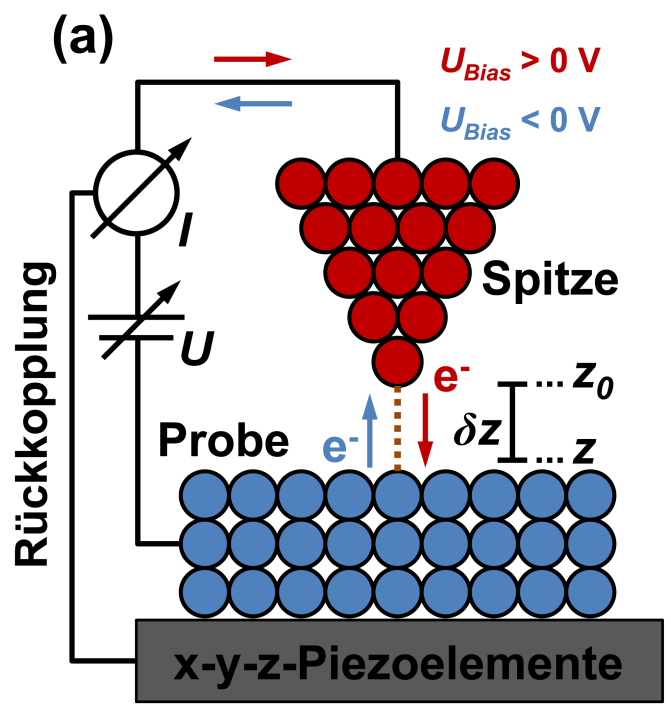

(b)

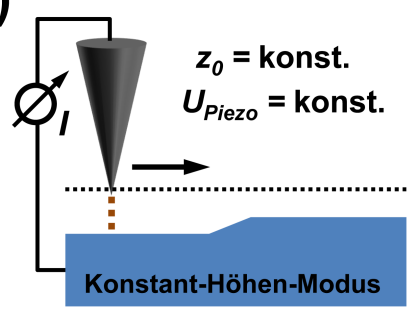

(c)

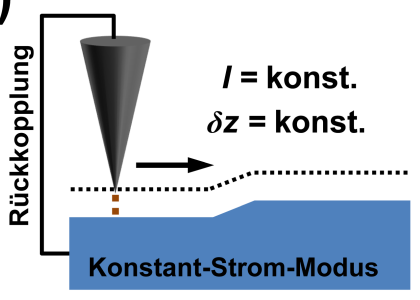

Abbildung 3.2.6: Schematische Darstellung des Aufbaus eines Rastertunnelmikroskops (a) sowie das jeweilige Messprinzip für den Betrieb der Abrasterung der Probe im Konstant-Höhen-Modus (b) und im Konstant-Strom-Modus (c). 


\section{Experimentelle Methoden}

Der Abstand und die Relativbewegung zwischen Spitze und Probe werden mit Piezoelementen gesteuert. Beim Bruker Innova [79] befinden sich diese unter dem Probenhalter. Somit ist die Spitzenposition in fester Position, während die Probe relativ zur Spitze bewegt wird. Dieses ist äquivalent zu einer Spitzenbewegung relativ zur Probe, bietet für die spitzenverstärkte Raman-Spektroskopie (TERS) jedoch den Vorteil, dass der RamanLaser beim Rastern auf der Spitze fokussiert bleibt (Kapitel 3.4). Da die Schichten auf elektrisch isolierenden Substraten aufgewachsen werden, müssen diese mit Leitsilber kontaktiert werden. Dieses dient zudem der Fixierung. Es ist ein Betrieb mit konstantem Strom (Konstant-Strom-Modus), bei dem der Tunnelstrom $I$ zwischen Spitze und Probe durch Nachkorrigieren des Abstandes $z=z_{0}+\delta z$ zwischen Probe und Spitze mit dem Probenoberflächenprofil über einen Rückkopplungskreis konstant gehalten wird (Piezoelement in z-Richtung), und ein Betrieb in konstanter Höhe (Konstant-Höhen-Modus), bei dem ein Anfangsabstand zwischen Spitze und Probenoberfläche mit dem Tunnelstrom $I$ eingestellt wird und dieser während des Rasterns nicht nachkorrigiert wird (deaktivierte Rückkopplung), möglich 89, 90. Im Konstant-Strom-Betrieb wird über die Tunnelstromkontrolle durch die daran gekoppelte Vertikalbewegung der Spitze das Höhenprofil der Probenoberfläche nachgezeichnet, während dieses im Konstant-Höhen-Betrieb über die Tunnelstromvariation an den Rasterpunkten rekonstruiert wird. Das jeweilige Prinzip ist für den Fall der Bewegung der Spitze über die Probe in den Abbildungen 3.2.6 (b) und (c) dargestellt.

Die Spannung $U_{\text {Bias }}$ bestimmt die am Tunnelstrom beteiligten Zustände und die Tunnelstromrichtung 83 (Abbildung 3.2.6 (a)). So führt eine Spannung $U_{\text {Bias }}>0 \mathrm{~V}$ zu einem Tunneln der Elektronen aus besetzten Spitzenzuständen in unbesetzte Zustände der Probenoberfläche und eine Spannung $U_{\text {Bias }}<0 \mathrm{~V}$ zu einem Tunnelstrom aus besetzten Probenoberflächenzuständen in unbesetzte Spitzenzustände 80, 83. Die beteiligten Elektronenzustände liegen in dem Energieintervall $\left|e U_{\text {Bias }}\right|$ zwischen den Fermi-Energien $E_{F}$ von Spitze und Probe 90,91. Daneben wird der Abstand zwischen Spitze und Probenoberfläche beeinflusst. Wird im Konstant-Strom-Modus mit aktiver Rückkopplung die Spannung $U_{\text {Bias }}$ erhöht, führt dieses zu einem Anstieg des Stroms, der durch eine Abstandsvergrößerung wieder auf den Sollwert reduziert wird, sodass der Spitzenabstand z zur Probenoberfläche über die Spannung indirekt angepasst werden kann. In einer Vereinfachung kann von einem Tunneln der Elektronen durch einen planaren Potentialwall ausgegangen werden. Mit der Wentzel-Kramers-Brillouin-Näherung (WKB-Näherung), in der ein trapezförmiger Potentialverlauf zwischen der Spitze und der Probenoberfläche im Abstand z angenommen wird, folgt für die Tunnelwahrscheinlichkeit $T$ der Elektronen mit der Energie $E$ [61, 80, 90]:

$$
T(E, e U, \phi, z)=\exp \left(-\frac{2 z \sqrt{2 m_{e}}}{\hbar} \sqrt{\frac{\phi_{P}+\phi_{S}+e U_{\mathrm{Bias}}}{2}-E}\right) .
$$




\subsection{Standard-Probencharakterisierung}

Hierbei bezeichnet $m_{e}$ die Masse des freien Elektrons, während $\phi_{P}$ und $\phi_{S}$ die Austrittsarbeiten von Probe und Spitze beschreiben, aus denen sich in dem vereinfachten Modell die Größe $\phi=\left(\phi_{P}+\phi_{S}\right) / 2$ ableiten lässt. Nach Gleichung (3.2.15) ist die Tunnelwahrscheinlichkeit $T$ je nach angelegter Spannung $U_{\text {Bias }}$ maximal für Elektronen bei der Fermi-Energie der Spitze (entspricht einer Energie $E=e U_{\text {Bias }}$ für $U_{\text {Bias }}>0 \mathrm{~V}$ ) oder der Probenoberfläche (entspricht $E=0 \mathrm{eV}$ für $U_{\text {Bias }}<0 \mathrm{~V}$ ). Für den Fall kleiner Spannungen $|e U| \ll \phi$ und kleiner Elektronenenergien kann damit die Abhängigkeit des Tunnelstroms von der Abstandsänderung $\delta z$ zwischen der Probenoberfläche und der Spitze angegeben werden [80]:

$$
I(\delta z)=I\left(z_{0}\right) \exp \left(-\frac{2 \sqrt{2 m_{e} \phi}}{\hbar} \delta z\right)=I\left(z_{0}\right) \exp (-\alpha \delta z) .
$$

Es ist eine exponentielle Abhängigkeit des Tunnelstroms $I$ vom Abstand der Spitze z zur Probe zu beobachten, sodass die hohe vertikale STM-Auflösung direkt ersichtlich wird. Entsprechend stark ist der Tunnelstrom auf die vordersten Spitzenatome konzentriert. Die Größe $\alpha=2 \sqrt{2 m_{e} \phi} / \hbar \approx 10,25 \mathrm{~nm}^{-1}(\phi / \mathrm{eV})^{-1 / 2} 80$ in der Gleichung (3.2.16) stellt die Abklinglänge des Tunnelstroms dar, die von der Höhe der Tunnelbarriere $\phi$ abhängt. Für eine genaue STM-Beschreibung ist allerdings die Kenntnis der atomaren und elektronischen Struktur von der Spitze und der Probenoberfläche notwendig [92]. Eine Vereinfachung ist die Tersoff-Haman-Theorie [93, 94, bei der der Einfluss der atomaren und elektronischen Struktur der Spitze vernachlässigt und der vorderste Teil der Spitze mit einem sphärischen Potential mit festem Radius, einer linearen elektronischen Zustandsdichte und kugelsymmetrischen s-Wellenfunktionen modelliert wird. In der Folge lassen sich Aussagen über die elektronische Struktur und die lokale Zustandsdichte an der Probenoberfläche sowie über die Abhängigkeit des Tunnelstroms von der Zustandsdichte treffen [83, 91, 94].

Ein spezieller STM-Modus ist die Rastertunnelspektroskopie (engl.: scanning tunneling spectroscopy, STS), bei der die Abhängigkeit des Tunnelstroms $I$ von der angelegten Spannung $U$ lokal als Funktion der Position der Spitze über der Probe vermessen wird. Hierfür wird die Spitze an einer Stelle $(x, y)$ an die Probenoberfläche angenähert und ein fester Abstand $z$ bei entsprechend eingestellten Sollwerten $I_{0}$ und $U_{0}$ vorgegeben. Anschließend wird die Rückkopplung deaktiviert und das Rastertunnelmikroskop im Konstant-HöhenModus betrieben. Die Messung der $I\left(x, y, z\left(I_{0}, U_{0}\right), U\right)$-Kennlinie erfolgt, indem die Spannung in diskreten Schritten in einem vorgegebenen Intervall durchgefahren wird und der Tunnelstrom aufgezeichnet wird. Wird der Vorgang mit dem Rastern der Probe kombiniert, kann gleichzeitig die Probenoberfläche topographisch abgebildet werden 80,83 . Die $I(U)$-Kennlinie ist charakteristisch für die lokale elektrische Leitfähigkeit $G=I / V$ und die elektronische Struktur des von der Spitze und der Probenoberfläche gebildeten Tunnelkontakts. Da die elektronische Struktur der Spitze allgemein nicht unmittelbar zugänglich 


\section{Experimentelle Methoden}

ist, wird zumeist ein Ansatz nach dem Tersoff-Haman-Modell [93, 94 für den Tunnelstrom verwendet, wobei die Tunnelwahrscheinlichkeit $T(E, U, z)$ nach der Gleichung (3.2.15) in WKB-Näherung beschrieben wird. Für den Tunnelstrom $I$ ergibt sich damit 80,90,91]:

$$
I(x, y, z, U) \propto \int_{0}^{e U} \rho_{S}(E-e U) \rho_{P}(x, y, E) T(E, U, z) \mathrm{d} E .
$$

Dabei bezeichnet $\rho_{S}(E-e U)$ die Zustandsdichte der Spitze und $\rho_{P}(x, y, E)$ die lokale Zustandsdichte der Probe unter der Spitze für den Fall, dass diese durch eine positive Spannung $U>0 \mathrm{~V}$ um die Energie $e U$ bezüglich der Fermi-Energie der Probe zueinander verschoben sind. Für eine negative Spannung $U<0 \mathrm{~V}$ ist die Verschiebung umgekehrt. Um die Abhängigkeit von der lokalen Probenzustandsdichte zu untersuchen, kann die differentielle elektrische Leitfähigkeit $\mathrm{d} I / \mathrm{d} U$ betrachtet werden. Für eine metallische Spitze mit in Näherung konstanter Zustandsdichte und kleine Spannungen $|e U| \ll \phi$, sodass die Tunnelwahrscheinlichkeit näherungsweise unabhängig von der Spannung ist, folgt für die differentielle elektrische Leitfähigkeit durch partielles Ableiten der Gleichung (3.2.17):

$$
\frac{\partial I(x, y, z, U)}{\partial U} \propto e T\left(e U, U, z_{0}\right) \rho_{S}(0) \rho_{P}(x, y, e U)
$$

Somit ist die differentielle elektrische Leitfähigkeit proportional zur Probenzustandsdichte bei $E=E_{F}+e U$ und ist durch die $I(U)$-Kennlinie direkt charakterisiert. Dieses ist in der Abbildung 3.2.7 (nach 80 ) für die Spannungen $U>0 \mathrm{~V}$ (a) und $U<0 \mathrm{~V}$ (b) dargestellt.
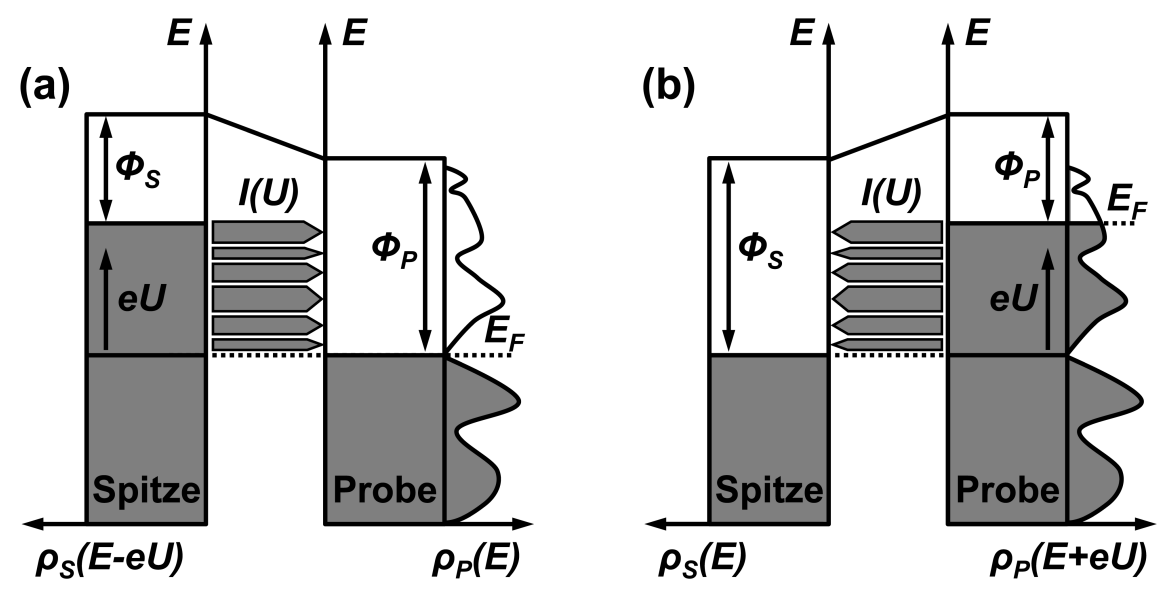
Abbildung 3.2.7: Energieschema des STS-Tunnelkontakts für Spannungen $U>0 \mathrm{~V}$ (a) und $U<0 \mathrm{~V}$ (b) mit einer konstanten Spitzenzustandsdichte zur Messung der lokalen Probenzustandsdichte aus der $I(U)$-Tunnelkennlinie (nach $[80]$ ).

Für eine metallische Spitze und eine metallische Probe ergibt sich eine lineare Abhängigkeit des Tunnelstroms $I$ von der Spannung $U(|e U| \ll e \phi)$, während für Halbleiter und Isolatoren aus der $I(U)$-Kennlinie die Bandlücke $E_{g}$ bestimmt werden kann $83,90,91$. 


\subsection{Standard-Probencharakterisierung}

\subsubsection{SQUID-Magnetometrie}

Die magnetischen Eigenschaften der Schichten werden mit einem MPMS (engl.: magnetic property measurement system) von Quantum Design [95,96 untersucht. Grundlage dieses Magnetometers ist eine Kombination aus einem Detektionsspulensystem zur induktiven Erfassung der Probenmagnetisierung, das von einem supraleitendem Magneten zur Messung der Magnetfeldabhängigkeit umgeben ist, und einer supraleitenden Quanteninterferenzeinheit SQUID (engl.: superconducting quantum interference device). Im MPMS wird ein rf-SQUID (engl.: radio frequency) verwendet, das aus einem supraleitendem Ring, der an einer Stelle durch einen Normalleiter (Josephon-Kontakt) unterbrochen ist, besteht und induktiv an den supraleitenden Stromkreis der Detektionsspulen gekoppelt ist [97,98]. Für die Magnetisierungsmessung wird die Probe im Magnetfeld oszillierend an einem motorgetriebenen Halter durch die Detektionsspulen auf und ab bewegt (RSO, engl.: reciprocating sample option), wodurch in den Spulen ein zur Probenmagnetisierung proportionaler Strom induziert wird [95, 99]. Dieser Wechselstrom wird über eine induktive Kopplung auf den SQUID-Sensor übertragen und durch Ausnutzung der magnetischen Flussquantisierung $\phi_{0}=\frac{h}{2 e}$ und des Josephon-Effekts in der nachfolgenden Elektronik als eine zur Probenmagnetisierung proportionalen Spannung aufgezeichnet, sodass die Magnetisierung indirekt über die magnetische Flussänderung in den Detektionsspulen gemessen wird, wobei das SQUID als Strom-Spannung-Signal-Konverter agiert 95, 96. Für eine messtechnische Beschreibung sei entsprechend auf die weiterführende Literatur verwiesen 95 98].

Das Detektionsspulensystem ist ein Gradiometer 2. Ordnung [95, 96, bei dem die Spulen auf zwei äußere Einzelwindungen gleicher Windungsrichtung und eine zentrale Zweifachwindung mit entgegengesetzter Windungsrichtung aufgeteilt sind. Dieser Aufbau bietet den Vorteil, dass Störungen durch Fluktuationen im supraleitenden Magneten und äußere Magnetfelder durch einen Ausgleich in den äußeren und inneren Spulen stark verringert und eine hohe Empfindlichkeit bezüglich der Probenposition beibehalten wird [95 98]. Es wird eine Sensitivität in der Magnetisierung von $\sim 10^{-7}-10^{-8}$ emu [95, 96, 99] erreicht, während der SQUID-Sensor magnetische Flussänderungen von $\lesssim 10^{-3} \phi_{0}$ detektieren kann 95, 99, 100. Das System ist in einem mit flüssigem Helium gekühlten Kryostaten eingebaut, wobei der SQUID-Sensor, die Elektronik und die induktive Kopplung mit einem supraleitenden Schild umgeben sind, um den supraleitenden Magnet und äußere Magnetfelder abzuschirmen. Die Temperatur in der Probenkammer kann in einem Bereich $T=1,9-400 \mathrm{~K}$ variiert werden, während mit dem supraleitenden Magneten Magnetfeldstärken bis zu $H_{\text {max. }}= \pm 50 \mathrm{kOe}$ angelegt werden können. Das Magnetfeld ist dabei parallel zur Schichtoberfläche ausgerichtet. Folglich ergibt sich für Schichten auf einem (100)-Substrat eine Magnetfeldorientierung in [001]-Richtung und auf einem (111)-Substrat

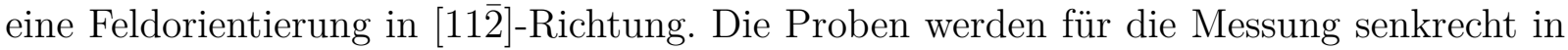
einer Hartgelatine-Kapsel fixiert, in einem Kunststoffstrohhalm am Probenhalter befestigt 


\section{Experimentelle Methoden}

und symmetrisch zwischen den zwei inneren Detektionsspulen platziert. Zu beachten ist, dass die Kapseln und Strohhalme schwach diamagnetisch sind und die Magnetisierung von dem Substratbeitrag überlagert ist. Folglich muss für die reine Probenmagnetisierung der Anteil vom Substrat und dem Probenhalter abgezogen werden, indem Leermessungen mit einem frischen Substrat unter gleichen Bedingungen durchgeführt werden. Die Messungen sind in Abhängigkeit der Temperatur und des Magnetfeldes für alle in dieser Arbeit zum Einsatz kommenden Substrate in den Abbildungen 3.2.8 (a), (b), (c) und (d) dargestellt.
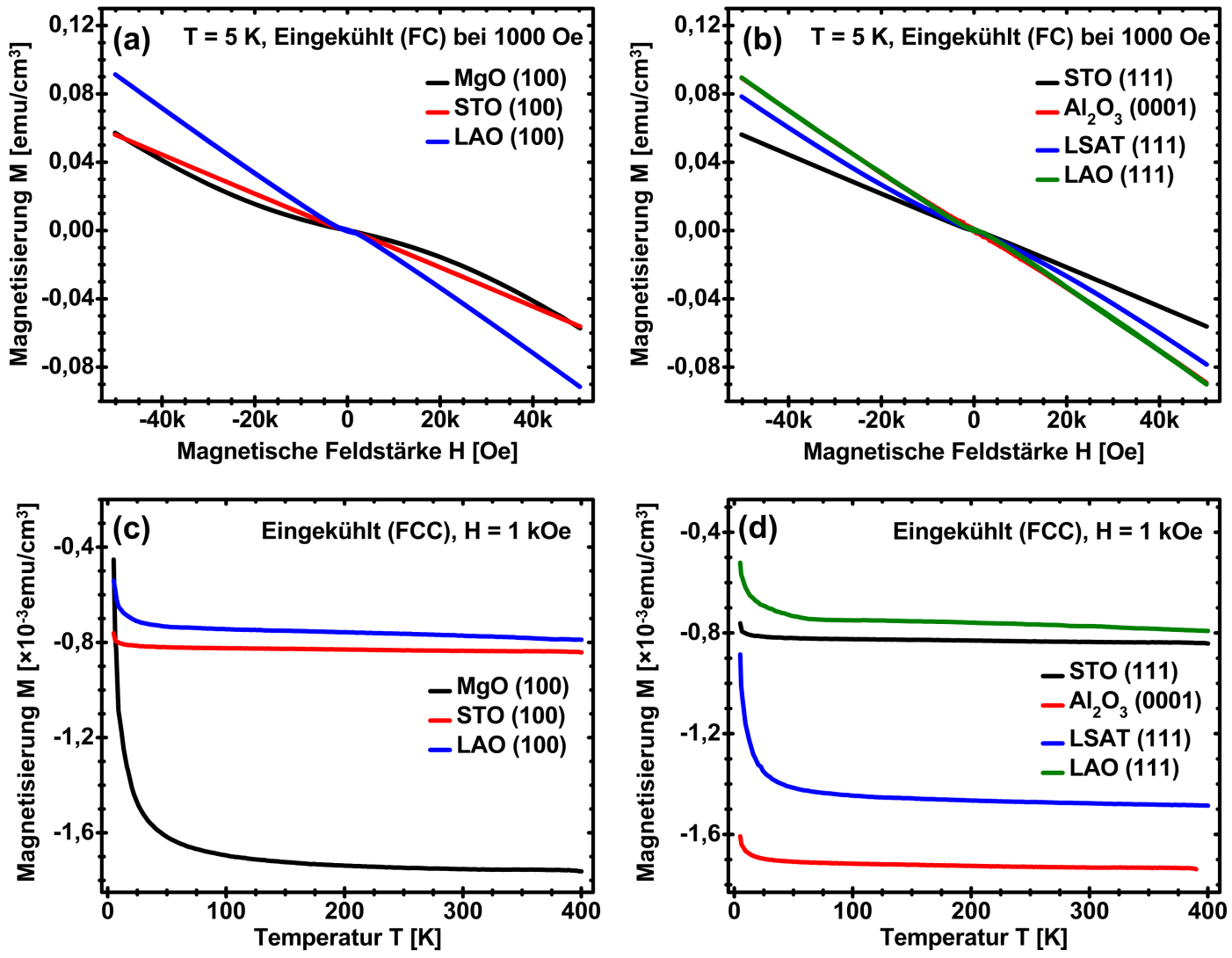

Abbildung 3.2.8: Magnetfeldabhängigkeit der Substrate mit einer (100)-Orientierung (a) und einer (111)-Orientierung (b) sowie die Temperaturabhängigkeit der Magnetisierung der Substrate mit einer (100)-Orientierung (c) und einer (111)Orientierung (d) (Substratmaße $10 \mathrm{~mm} \times 5 \mathrm{~mm} \times 0,5 \mathrm{~mm}$ ).

Es zeigt sich ein vorwiegend diamagnetisches Substratverhalten. So weisen die $M(H)$ Kurven (außer für MgO) in den Abbildungen 3.2.8 (a) und (b) eine nahezu lineare Abhängigkeit der Magnetisierung $M$ von der Magnetfeldstärke $H$ mit der für Diamagneten negativen Steigung, der magnetischen Suszeptibilität $\chi=M / H<0$, auf [95, 100]. Da die Magnetisierung zudem nach den Abbildungen 3.2.8 (c) und (d) für alle Substrate eine Temperaturunabhängigkeit bis zu tiefen Temperaturen zeigt, kann der magnetische Hauptbeitrag der Substrate als diamagnetisch bezeichnet werden. Der schwache paramagnetische Anstieg bei tiefen Temperaturen wird durch Verunreinigungen mit Übergangsmetallionen hervorgerufen 101 103. Hierbei ist für MgO eine erhöhte Konzentration nachweisbar 101,102, der sich in den Abbildungen 3.2.8 (a)-(d) bei kleinen Feldern und Temperaturen durch 
ein überlagertes paramagnetisches Verhalten zeigt, das für größere Felder durch Sättigung oder bei höheren Temperaturen in das diamagnetische Verhalten übergeht. Zudem können die Substrate einen schwachen ferromagnetischen Beitrag aufweisen, der als Artefakt eingebracht wird oder intrinsisch vom Substrat stammt 101 104. Beispiele für externe Quellen sind eisenhaltige Pinzetten, Kleber- und Leitsilberpartikel, deformierte Probenhalterstrohhalme, die Substratherstellung oder im SQUID verbliebene Restfelder 101 104. Ursache eines intrinsischen Beitrags ist der $d^{0}$-Ferromagnetismus 102, 105. Vermutete Quellen sind oberflächennahe Sauerstoff- und Kationenleerstellen, Oberflächenreaktionen, Wasserstoffdotierungen oder die Oberflächenpolarität der Substrate 101,102. Allgemein wird nun die Magnetisierung der Schichten vermessen und um den Substrat- und Probenhalterhintergrund korrigiert. Dieses ist in der Abbildung 3.2.9 (a) für die $M(H)$-Kurve $(T=5 \mathrm{~K}, \mathrm{FC}$ mit $H=1000$ Oe) einer $\mathrm{La}_{2} \mathrm{CoMnO}_{6} / \mathrm{STO}$ (111)-Schicht und in der Abbildung 3.2.9 (b) für die $M(T)$-Kurve (FCC bei $H=1000 \mathrm{Oe}$ ) einer $\mathrm{LaCoO}_{3} / \mathrm{MgO}$ (100)-Schicht gezeigt.
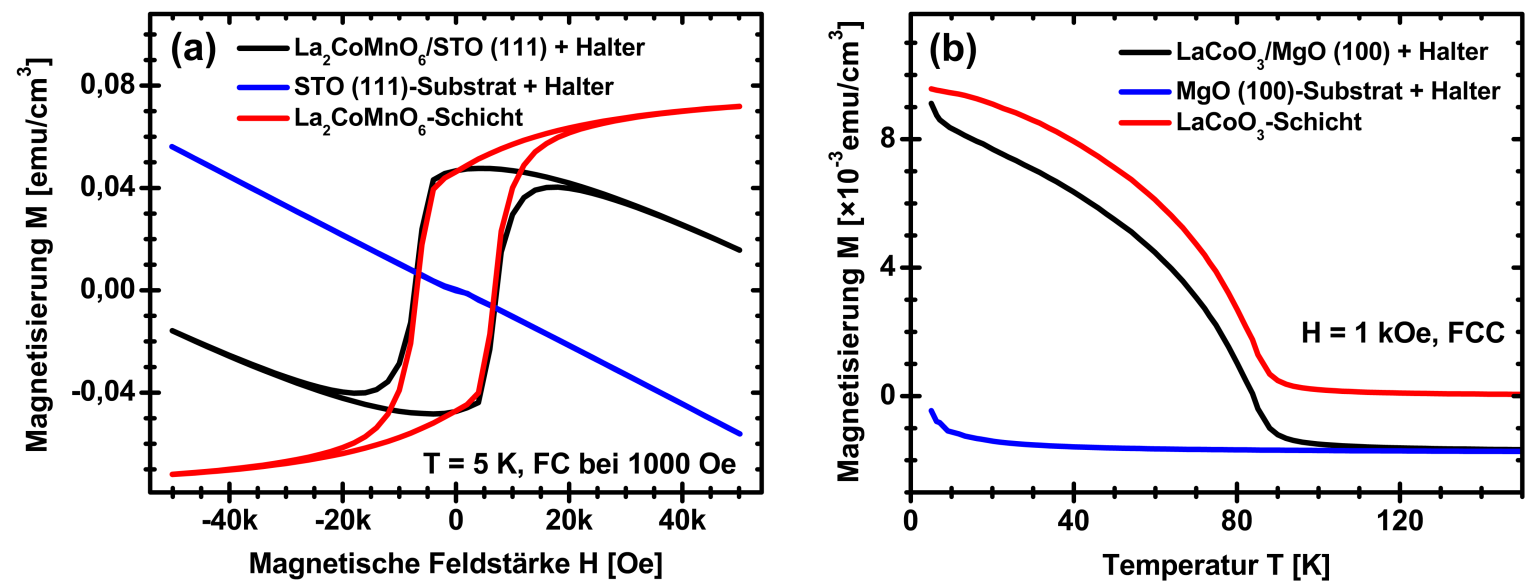

Abbildung 3.2.9: Beispiel für die entsprechende Korrektur einer magnetfeldabhängigen Hysteresekurve einer $\mathrm{La}_{2} \mathrm{CoMnO}_{6}$-Schicht auf einem STO (111)-Substrat (a) und einer temperaturabhängigen Magnetisierungsmessung einer $\mathrm{LaCoO}_{3}$-Schicht auf einem MgO (100)-Substrat (b) um den Beitrag von Substrat und Probenhalter durch Differenzbildung zur Leermessung ohne Schicht.

Folglich müsste jedes Substrat im SQUID vermessen werden. Für die diamagnetischen Substrate STO, Saphir, LSAT und LAO, die nur einen kleinen paramagnetischen Anteil bei tiefen Temperaturen aufweisen, ist dieses bei Schichten mit einem großen magnetischen Moment jedoch nicht notwendig. Zudem kann sich der Magnetisierungsbeitrag des Substrats während der weiteren Verwendung verändern und zu Korrekturfehlern führen. Ein Spezialfall sind die MgO-Substrate, die neben einem intrinsischen Diamagnetismus einen stark variierenden paramagnetischen Beitrag bei tiefen Temperaturen zeigen [48, 101, 102, 104. $\mathrm{MgO}$ ist zudem aufgrund seines hygroskopischen Charakters sehr empfindlich gegenüber Luftfeuchtigkeit 106. Dieses führt zu der Bildung von Hydroxiden und Kohlenwasserstoffverbindungen an der Oberfläche [106] und nimmt Einfluss auf die Substratqualität und das Schichtwachstum, sodass ein Vermessen der Magnetisierung jedes MgO-Substrats 


\section{Experimentelle Methoden}

nicht empfehlenswert ist. Stattdessen wurde ein MgO-Substrat aus jeder Substratlieferung magnetisch vermessen und für die Korrektur aller weiteren MgO-Substrate verwendet. Hierfür wurden die $M(T)$ - und die $M(H)$-Messdaten der Schichten um den Beitrag des MgO-Referenzsubstrats korrigiert und die $M(H)$-Kurven je nach diamagnetischem Restbeitrag um einen weiteren linearen Hintergrund reduziert. Mögliche Fehler können nur schwer abgeschätzt werden und hängen von dem MgO-Substrat ab. Die $M(T)$-Kurven können beim Abkühlen mit Magnetfeld (FCC, engl.: field-cooled cooling), bei der Erwärmung mit Magnetfeld nach einer vorherigen Abkühlung mit Magnetfeld (FCW, engl.: field-cooled warming) oder bei der Erwärmung mit Magnetfeld nach einer vorherigen Einkühlung ohne Magnetfeld (ZFC, engl.: zero-field cooling) aufgenommen werden. Da die Vorgeschichte einen Einfluss auf den $M(T)$-Verlauf nehmen kann (wie beim Vergleich von reinen Ferromagneten und Spin-Gläsern unterhalb der Übergangstemperatur $T_{C}$ 100]), ist diese für alle Magnetisierungsmessungen entsprechend angegeben. Die $M(H)$-Kurven wurden stets im Anschluss an eine FCC- $M(T)$-Messung (FC, im Feld eingekühlt) aufgenommen.

Für die Sättigungsmagnetisierung pro Einheitszelle oder Übergangsmetallion wird zudem das Schichtvolumen $V$ benötigt. Die Schichtdicke $d$ kann mit der XRR-Methode ermittelt werden, während die Schichtoberfläche $A$ mit einem digitalen Messschieber anhand der Bedeckung auf dem Substrat abgeschätzt wird. Hierbei sind zwei Ränder an der Substratlängsseite mit einer Breite von ungefähr 0,5-1 mm abzuziehen, die bei der Schichtherstellung durch die Befestigung auf dem MAD-Heizer abgeschattet sind. Untersuchungen von Markus Jungbauer [50] zeigen, dass MAD-Schichten in Richtung der Halterung leicht abflachen und ein diffuser Randbereich von etwa 0,1 mm Breite zu beobachten ist. Der Volumenfehler kann auf etwa $5 \%$ abgeschätzt werden, wobei das Volumen durch die Randeffekte systematisch überschätzt wird. Folglich wird die Sättigungsmagnetisierung pro Einheitszelle oder Übergangsmetallion systematisch um mindestens $5 \%$ unterschätzt. Zudem beeinflusst die horizontale Probenposition im SQUID die Magnetisierung [103, 104]. So führt eine horizontale Abweichung von der Gradiometerachse zu einem größerem magnetischen Fluss durch die Detektionsspulen [104]. Mit dem Standardprobenhalter ist dieser Effekt allerdings vernachlässigbar. Jedoch hat eine verkippte Ausrichtung durch magnetische Anisotropieeffekte einen Einfluss auf die Magnetisierung, da das Magnetfeld nicht parallel zur Probenoberfläche ausgerichtet ist und neben der in-plane Magnetisierung ein Teil der out-of-plane Komponente gemessen wird. Für dünne Schichten, die zumeist eine in-plane Anisotropie zeigen [100], führt dieses zu einer Verringerung der gemessenen Magnetisierung parallel zum Magnetfeld und damit zu einem kleineren Strom $I$, der in den Detektionsspulen induziert wird. Es ergibt sich eine Abhängigkeit $I \sim \cos (\varphi)$ vom Verkippungswinkel $\varphi$ der Schicht entlang der Gradiometerachse 48. Für eine Verkippung von $\varphi_{\text {max. }}=10^{\circ}$ folgt daraus eine Abnahme der registrierten Magnetisierung von etwa $2 \%$. 


\subsubsection{Elektrische Transportmessungen}

Die elektrischen Transporteigenschaften der Schichten werden mithilfe eines PPMS-Gerätes (engl.: physical property measurement system) von Quantum Design gemessen. Dieses Gerät bezeichnet ein in ein Kryostat integriertes multifunktionales System, wodurch verschiedene physikalische Größen automatisiert als Funktion der Temperatur und des Magnetfeldes gemessen werden können. Das PPMS wurde für die Messung des Gleichstromwiderstandes mit der Gleichstrom-Widerstandsoption betrieben. Die Proben werden hierfür auf einem speziellen Probenhalter befestigt, der in der Abbildung 3.2.10 (a) 107 dargestellt ist, kontaktiert und in den mit flüssigem Helium gekühlten Kryostaten eingebaut.

(a)

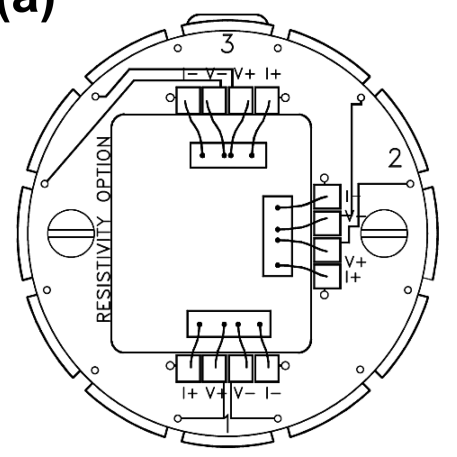

(b)

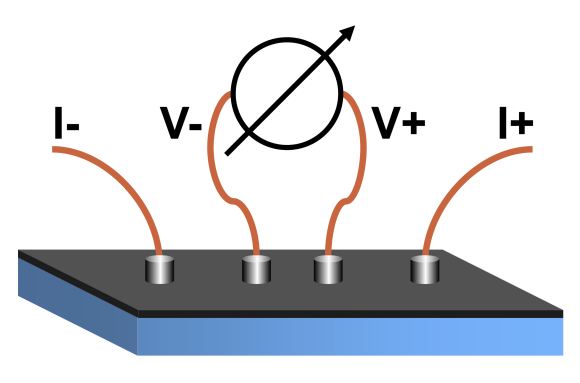

Abbildung 3.2.10: PPMS-Probenhalter für die Messung des Probenwiderstands mithilfe der Gleichstromwiderstandsoption (a) 107 und schematische Übersicht der Anordnung der Kontaktierung bei der Vier-Punkt-Methode (b).

Die Temperaturen können in einem Bereich von $T=1,9-400 \mathrm{~K}$ variiert werden. Zusätzlich besteht mithilfe eines supraleitenden Magnets die Möglichkeit ein Magnetfeld mit einer Stärke von $H=0-90 \mathrm{kOe}$ in der Probenkammer anzulegen. Die Probenkontaktierung erfolgt mit dünnen Kupferdrähten und Leitsilber in der Vier-Punkt-Methode. Die Anordnung dieser Kontaktierung ist schematisch in der Abbildung 3.2.10 (b) dargestellt. Hierbei wird der Strom über die beiden äußeren Kontakte der vier in einer Reihe auf der Probenoberfläche gesetzten Messpunkte angelegt, während die an dem Widerstand der Probe abfallende Spannung getrennt davon über die beiden inneren Kontakte gemessen wird. Dieses hat den Vorteil, dass die Messung der Spannung aufgrund des hohen Innenwiderstands des Spannungsmessgeräts nahezu stromlos erfolgt, der Strom nur durch die Probe fließt und die Spannungsmessung nicht durch die Leitungswiederstände und durch die Kontaktwiderstände an den Zuleitungen beeinflusst wird. Durch die Vorgabe des Stroms und die Messung der an der Probe abfallenden Spannung kann somit der elektrische Widerstand der Probe mit sehr hoher Genauigkeit über das Ohmsche Gesetz bestimmt werden [107]. Über die spezifische Probengeometrie mit der Querschnittsfläche $A=d \cdot b$ (mit der Schichtdicke $d$ und der Schichtbreite $b$ ) und dem Abstand $l$ der beiden Spannungskontakte kann darüber hinaus der spezifische Widerstand der Schicht $\rho=R \cdot \frac{A}{l}=R \cdot \frac{d \cdot b}{l}$ ermittelt werden. 


\section{Experimentelle Methoden}

\subsubsection{Rasterelektronenmikroskopie (REM)}

Eine weitere bildgebende Methode stellt die Verwendung eines Rasterelektronenmikroskops (REM; oder engl.: scanning electron microscope, SEM) dar. Hierbei wird ein fokussierter Elektronenstrahl systematisch in einem Raster über die Probenoberfläche bewegt und die Wechselwirkung der Elektronen mit der Probe durch elastische und inelastische Streuung zur Bilderzeugung genutzt 108 110]. Das verwendete Leo Supra 35 von Zeiss verfügt über eine thermische Feldemissionskathode (Schottky-Kathode), die aus einer feinen mit $\mathrm{ZrO}_{2}$ beschichteten Wolfram-Spitze besteht 108,111]. Zur Erzeugung des Elektronenstrahls wird diese geheizt und ein elektrisches Feld zwischen Spitze und einer ersten Anode angelegt, wodurch Elektronen durch Glühemission und Feldemission aus der Kathode freigesetzt werden. Durch ein elektrisches Feld zwischen Spitze und einer weiter entfernten zweiten Anode mit einer einstellbaren Beschleunigungsspannung $(U=0,1-30 \mathrm{kV})$ wird der Elektronenstrahl erzeugt. Mit elektromagnetischen Linsen (stromdurchflossene Spulen) kann dieser zur zweidimensionalen Abrasterung der Probe abgelenkt und fokussiert werden. Die Schottky-Kathode verbindet dabei die hohe Stromdichte, Kohärenz und Feinheit eines Feldemission-Elektronenstrahls mit dem hohen Elektronenstrom (Elektronenausbeute) und der hohen Strahlstabilität einer thermischen Kathode [108, 111]. Dieses führt zu einer verbesserten Intensität, Auflösung und Bildqualität [108, 109]. Ein Hochvakuum dient der Vermeidung von Wechselwirkungen des Elektronenstrahls mit Gasmolekülen und von elektrischen Überschlägen zwischen Kathode und Anode durch Ionisation sowie dem Schutz der Kathodenspitze und der $\mathrm{ZrO}_{2}$-Beschichtung [108 110]. Folglich müssen die Proben im Vakuum stabil sein oder mit einem Metall beschichtet werden. Dieses ist auch bei elektrisch isolierenden Materialien notwendig, um eine elektrische Aufladung durch den Elektronenstrahl, die zu Störungen in der Bildgebung führen kann, zu verhindern 108 110.

Die Wechselwirkungen der Elektronen mit der Probe werden von Detektoren innerhalb der Probenkammer für die Bildgebung der Probenoberfläche als Signal registriert. Es kommt zur Elektronenrückstreuung, der Erzeugung von Sekundär- und Auger-Elektronen, charakteristischer Röntgenstrahlung, Bremsstrahlung und Kathodolumineszenz [110]. Für eine Beschreibung der Prozesse für die Bildgebung, den Kontrast und die chemische Analyse der Probenoberfläche sei auf die Literatur verwiesen 108 110. Über die Beschleunigungsspannung wird die Eindringtiefe des Elektronenstrahls beeinflusst, während über den Arbeitsabstand (im Bereich einiger Milimeter) zwischen Probe und fokussierendem Objektiv die Aufösung und Schärfentiefe angepasst werden kann 108, 109. Das Bild wird durch Rasterung über die Probenoberfläche und die Aufzeichnung der Signalintensität für jeden Rasterpunkt rekonstruiert. Hierfür wird das Signal Punkt für Punkt in eine Grauskala umgewandelt und auf einem Bildschirm angezeigt. Die Vergrößerung des Bildes ist über das Verhältnis der Bildgröße auf dem Bildschirm zur real abgerasterten Probenoberfläche gegeben. Es werden Auflösungen von $\leq 1 \mathrm{~nm}$ bei bis zu $10^{5} \times$ Vergrößerung erreicht 108, 109]. 


\subsection{Standard-Probencharakterisierung}
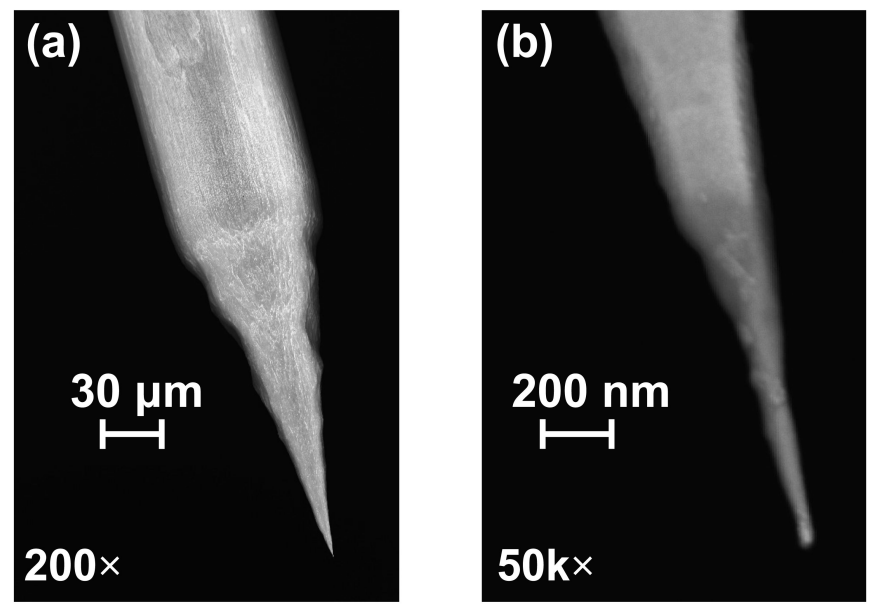

Abbildung 3.2.11: REM-Aufnahmen einer TERS-STM-Goldspitze für eine $200 \times$ (a) und 50k× Vergrößerung (b). Der Spitzenradius beträgt etwa $R \approx 20 \mathrm{~nm}$.

In dieser Arbeit dient das REM der Abbildung von STM-Goldspitzen für die spitzenverstärkte Raman-Spektroskopie (TERS; Kapitel 3.4). Hierzu werden die Spitzen auf ihre Oberfläche und Form untersucht und der Spitzenradius bestimmt. Eine Beispielaufnahme für eine der TERS-Spitzen ist in der Abbildung 3.2.11 mit einer $200 \times$ (a) und 50k $\times$ Vergrößerung (b) $(U=5 \mathrm{keV})$ dargestellt. Der Spitzenradius beträgt etwa $R \approx 20 \mathrm{~nm}$.

\subsubsection{Transmissionselektronenmikroskopie (TEM)}

Eine weitere Methode der Elektronenmikroskopie ist die Verwendung eines Transmissionselektronenmikroskops (TEM, engl.: transmission electron microscope). Die in dieser Arbeit mit dieser Technik aufgenommenen Bilder stammen von einem FEI Titan 80-300 G2 mit einer Beschleunigungsspannung von bis zu $U=300 \mathrm{kV}$ und wurden von Vladimir Roddatis vom Institut für Materialphysik der Georg-August-Universität Göttingen erstellt. Wie bei dem Rasterelektronenmikroskop erfolgt die Bildgebung durch einen fokussierten Elektronenstrahl unter Verwendung einer elektromagnetischen Optik. Der Unterschied besteht darin, dass die Proben durchstrahlt werden, sodass dünne Proben und eine höhere Beschleunigungsspannung notwendig sind. Daher werden Beschleunigungsspannungen von $100-500 \mathrm{kV}$ benötigt [108, 112] und dünne Lamellen in Querschnittsrichtung aus den Proben mit einer Dicke von etwa $10-100 \mathrm{~nm}$ 68,108 ausgeschnitten. In dieser Arbeit wurde dieses mithilfe eines fokussierten Ionenstrahls (FIB, engl.: focused ion beam) realisiert (unter Verwendung eines FEI Nova 600 NanoLab Systems). Die erreichbare TEM-Auflösung liegt im Bereich von < 0,2 nm 61, 108, 112 und kann durch Korrektoren für die sphärische und chromatische Aberration der elektromagnetische Linsen weiter verbessert werden. Die Wechselwirkung der Elektronen des Elektronenstrahls mit der Probenlamelle erfolgt sowohl elastisch (Rutherford-Streuung) an den Atomkernen als auch durch inelastische Streuprozesse an den Elektronen der Atome [108]. Die gestreuten Elektronen werden anschließend von Detektoren hinter der Probe registriert. Abhängig von den Ablenkungswinkeln werden 


\section{Experimentelle Methoden}

die zum Bildsignal beitragenden Elektronen klassifiziert. Die nicht oder nur um einen kleinen Winkel abgelenkten Elektronen werden als Hellfeldelektronen (BF, engl.: bright field) bezeichnet und von Detektoren auf und nahe der optischen Achse des Mikroskops aufgefangen (Hellfeld). Konzentrisch darum positioniert finden sich Detektoren für die stärker abgelenkten Dunkelfeldelektronen (DF, engl.: dark field). Dieses Signal wird als annulares Dunkelfeld (ADF, engl.: annular dark field) bezeichnet und kann durch das Einbringen einer Apertur vom Hellfeld getrennt werden. Die Detektion besonders stark abgelenkter Elektronen wird unter dem Begriff HAADF (engl.: high-angle annular dark field) zusammengefasst. Charakteristisch für Hellfeld und Dunkelfeld ist der invertierte Kontrast. Erscheinen für Elektronen besonders dichte Bereiche im Hellfeld sehr dunkel, so weisen sie im Dunkelfeld eine besonders hohe Helligkeit auf [108. Insbesondere das HAADF-Signal kann aufgrund der Skalierung der Signalintensität (hauptsächlich durch Rutherford-Streuung) mit dem Quadrat der Ordnungszahl $Z$ auch zur Differenzierung der chemischen Elemente in der atomaren Probenstruktur verwendet werden [68]. Hierbei kann der Elektronenstrahl wie beim REM in einem Raster über die Probenoberfläche bewegt werden. Folglich wird diese Methode als Rastertransmissionselektronenmikroskopie (STEM, engl.: scanning transmission electron microscopy) bezeichnet 68. Eine TEM-Besonderheit bei kristallinen Proben stellt die Kombination von der Abbildung der Struktur und der Aufnahme von Beugungsbildern selektiver Probenbereiche (SAD, engl.: selected area diffraction) mit einem Durchmesser von 0,1-1 $\mathrm{mm}$ dar. Hierfür kann durch Änderungen an der Fokussierung des Linsensystems zwischen Probenabbildung und Beugungsbild gewechselt werden 112. Die Beugung der Elektronen am kristallinen Gitter der Probe kann wie die Röntgendiffraktometrie für die Identifizierung der Kristallstruktur und der kristallinen Ordnung verwendet werden. Während die Röntgendiffraktometrie von globaler Art ist, da die Röntgenstrahlung in einer Breite einiger Zentimeter an der Probe gebeugt wird, ist die SAD-Methode der Transmissionselektronenmikroskopie jedoch lokal auf einige hundert Nanometer begrenzt.

Zusätzlich existieren analytische Betriebsarten des Transmissionselektronenmikroskops, um chemische Informationen über die Probe aus der elektronischen Anregung zu erhalten. Die zwei wichtigsten Verfahren sind die energiedispersive Röntgenspektroskopie (EDX, engl.: energy dispersive X-ray spectroscopy), bei der die durch die Wechselwirkung der Probe mit dem Elektronenstrahl erzeugte charakteristische Röntgenstrahlung für die Bestimmung der Zusammensetzung und Konzentration der sich im betrachteten Probenbereich befindlichen Elemente analysiert wird, und die Elektronenenergieverlustspektroskopie (EELS, engl.: electron energy loss spectroscopy), bei der die Verteilung des Energieverlusts der Elektronen im Elektronenstrahl nach Wechselwirkung durch inelastische Streuung mit der Probe spektral ausgewertet und aus dem Energieverlustspektrum (durch Streuung an Außenhüllen- und Kernelektronen und Plasmonen-Anregung) Informationen über die lokale chemische Zusammensetzung und Struktur der Probe ermittelt werden können 68, 112]. 


\subsection{Raman-Spektroskopie}

Im Anschluss soll nun mit der Raman-Spektroskopie, mit der die nach dem indischen Physiker Sir Chandrasekhara Venkata Raman benannte spektrale Untersuchung inelastischer Streuung von Licht an Molekülen und Festkörpern 113 116 beschrieben wird, die zentrale Messmethode dieser Arbeit vorgestellt werden. Hierfür wird zunächst mit einer Einführung begonnen sowie eine theoretische Beschreibung der Raman-Streuung und der in dieser Arbeit verwendete Messaufbau für die Raman-Spektroskopie-Messungen vorgestellt. Anschließend wird mit der polarisationsabhängigen Raman-Spektroskopie eine für kristalline Proben wichtige Variante der Raman-Streuung aufgezeigt, mit der sich die strukturellen Eigenschaften von Kristallen untersuchen und Informationen zur kristallinen Qualität und zu kationischen Ordnungsphänomenen in den dünnen Schichten gewinnen lassen.

\subsubsection{Einführung in die Raman-Spektroskopie}

Die Raman-Spektroskopie beschreibt eine Methode der inelastischen Streuung von Licht an Molekülen und Festkörpern. Hierzu wird die Probe mit monochromatischem Laserlicht bestrahlt und das gestreute Licht spektral analysiert. Im Vergleich zur Infrarot-Spektroskopie muss die Wellenlänge nicht zu den Abständen bestimmter Energiezustände passen, da relative Energiedifferenzen betrachtet werden [117]. Neben einer hauptsächlich elastischen Streuung an der Probe, die als Rayleigh-Streuung bezeichnet wird und die die gleiche Frequenz $\nu_{0}$ wie der Anregungslaser besitzt, ist ein kleiner inelastischer Anteil im Streusignal zu beobachten (Raman-Streuung), der eine Frequenzverschiebung $\Delta \nu=\nu_{n}$ aufweist (ein inelastisch gestreutes Photon bei $10^{6}-10^{8}$ elastisch gestreuten Photonen [117,118]). Hierbei kommt es sowohl zu einer Verschiebung zu kleineren Frequenzen $\nu_{\mathrm{S}}=\nu_{0}-\nu_{n}$ durch eine Energieübertragung der eingestrahlten Photonen an die Probenmoleküle und -Atome (Stokes-Streuung), die zu einer Anregung von Molekülschwingungen und Phononen führt, als auch zu einer Verschiebung zu größeren Frequenzen $\nu_{\mathrm{AS}}=\nu_{0}+\nu_{n}$ und zu einer Energieübertragung bereits angeregter Moleküle und Phononen auf die gestreuten Photonen (Anti-Stokes-Streuung), wodurch die Moleküle in einen energetisch niedrigeren Schwingungszustand übergehen. Für die Veranschaulichung kann das in der Abbildung 3.3.1 (a) dargestellte Jablonski-Diagramm betrachtet werden. Die Vermittlung der Streuprozesse erfolgt über virtuelle Zustände, die nicht-stationäre Zustände zwischen dem energetischen Schwingungsgrundzustand und dem ersten elektronischen Zustand darüber darstellen, in einem Zwei-Photonen-Prozess [117- 119]. Für die elastische Rayleigh-Streuung wird das Molekül durch ein Photon in einen virtuellen Zustand gehoben, von dem es sofort wieder in den Schwingungsgrundzustand zurückfällt. Im Fall der inelastischen Stokes-Streuung verbleibt das angeregte Molekül in einem Schwingungszustand oberhalb des Grundzustandes. Ist das Molekül bereits angeregt, kann es durch die inelastische Anti-Stokes-Streuung entsprechend in den energetisch niedrigeren Schwingungsgrundzustand übergehen. 
(a)

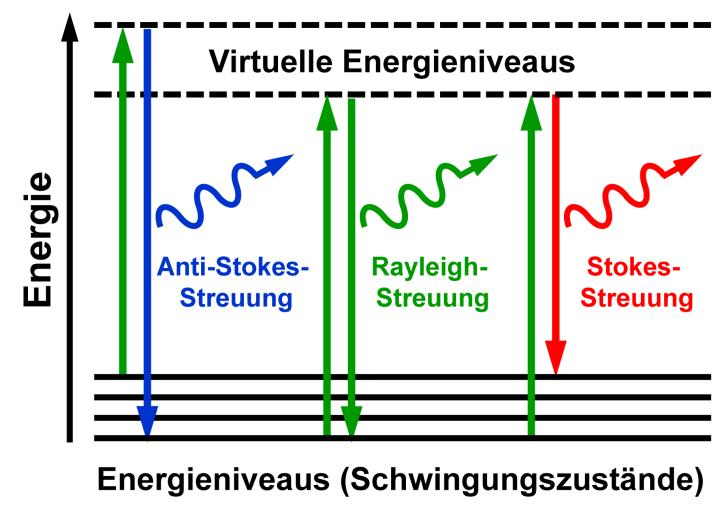

(b)

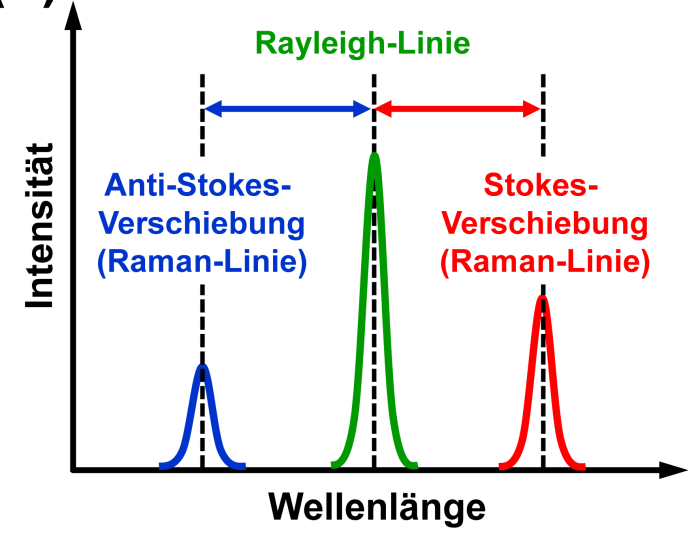

Abbildung 3.3.1: Jablonski-Diagramm zur Beschreibung der Rayleigh-Streuung und des Raman-Streuprozesses mit der Stokes- und Anti-Stokes-Streuung (a) und die schematische Darstellung des dazugehörigen Spektrums mit den einzelnen Linienpositionen in Abhängigkeit der Wellenlänge (b) (nach [118] $)$.

Im Raman-Spektrum wird die Intensität in Abhängigkeit der energetischen Raman-Verschiebung der Photonen aufgetragen. Eine vereinfachte Darstellung mit den Linien in Abhängigkeit der Wellenlänge ist in der Abbildung 3.3.1 (b) (nach [118]) gegeben. Die Raman-Verschiebung wird jedoch aus spektroskopischen Gründen üblicherweise über die Wellenzahl $\bar{\nu}_{n}=\frac{\nu_{n}}{c}=\frac{\omega_{n}}{2 \pi c}$ in der Einheit $\left[\mathrm{cm}^{-1}\right]$ angegeben. Hierbei wird die RayleighLaserlinie auf Null gesetzt, sodass die Raman-Verschiebungen der angeregten Schwingungsmoden in Relation zum Laserlicht direkt im Spektrum ersichtlich werden. Typische Raman-Verschiebungen liegen im Bereich bis etwa $1000 \mathrm{~cm}^{-1}$ 58. Da die Stokes-Linien bei Raumtemperatur aufgrund der höheren Besetzungsdichte des Schwingungsgrundzustands gegenüber den angeregten Schwingungsniveaus viel intensiver als die Anti-Stokes-Linien sind, wird oft nur der Stokes-Bereich vermessen und den Stokes-Linien positive Wellenzahlen und den Anti-Stokes-Linien stattdessen negative Wellenzahlen zugeordnet [117,120.

\subsubsection{Theoretische Beschreibung der Raman-Streuung}

Eine Beschreibung des Raman-Effekts der inelastischen Streuung von Licht an Materie kann zunächst klassisch erfolgen, indem die Wechselwirkung zwischen dem oszillierenden elektromagnetischen Feld des einfallenden Lichts und der Moleküle betrachtet wird. Die resultierende Störung der molekularen Ladungsverteilung induziert ein oszillierendes Dipolmoment $\vec{p}(t)$ in den Molekülen und regt diese zu spezifischen Normalschwingungen um ihre Ruhelage $q_{0}$ herum an, wobei die Emission von Sekundärstrahlung das gestreute Licht repräsentiert. In einem externen elektrischen Feld $\vec{E}=\vec{E}_{0} \cdot \cos \left(\omega_{0} t\right)$ mit der Amplitude $\vec{E}_{0}$, das mit der Kreisfrequenz $\omega_{0}$ oszilliert, folgt für das Gesamtdipolmoment $\vec{p}(t)$ 121,122:

$$
\vec{p}(t)=\vec{\mu}_{0}+\tilde{\alpha} \vec{E}(t)
$$


Hierbei bezeichnet $\vec{\mu}_{0}$ ein im Molekül vorhandenes permanentes Dipolmoment und $\tilde{a}=\alpha_{i j}$ die Polarisierbarkeit des Moleküls, die im Allgemeinen die Form eines Tensors 2. Stufe mit von der Molekülsymmetrie abhängigen Komponenten annimmt und die Verschiebbarkeit der molekularen Ladungsverteilung in einem externen elektrischen Feldes charakterisiert. Folglich sind die Polarisierbarkeit und das Dipolmoment Funktionen der Elektronen- und Kernkoordinaten des Moleküls [121] und die angeregten Schwingungen können mit einem Satz von Normalkoordinaten $q_{n}$ beschrieben werden. Für kleine Auslenkungen der Amplitude $q_{n 0}$ aus der Ruhelage $q_{0}$ des Moleküls mit der Resonanzkreisfrequenz $\omega_{n}$ können diese in der Näherung eines harmonischen Oszillators mit $q_{n}(t)=q_{n 0} \cdot \cos \left(\omega_{N} t\right)$ beschrieben werden. Die Polarisierbarkeit $\alpha_{i j}$ und das molekulare Dipolmoment $\vec{\mu}$ lassen sich anschließend in den Normalkoordinaten $q_{n}$ mithilfe einer Taylor-Reihe um $q_{0}$ herum entwickeln [121, 122]:

$$
\begin{array}{r}
\alpha_{i j}(q)=\alpha_{i j}\left(q_{0}\right)+\sum_{n}^{3 Q-f}\left(\frac{\partial \alpha_{i j}}{\partial q_{n}}\right)_{q_{0}} q_{n}+\mathcal{O}\left(q_{n}^{2}\right), \\
\vec{\mu}(q)=\vec{\mu}\left(q_{0}\right)+\sum_{n}^{3 Q-f}\left(\frac{\partial \vec{\mu}}{\partial q_{n}}\right)_{q_{0}} q_{n}+\mathcal{O}\left(q_{n}^{2}\right) .
\end{array}
$$

Hierbei ist $Q$ die Atom- oder Atomkernanzahl des Moleküls und $3 Q-f$ die mögliche Normalschwingungsanzahl mit $f=5$ für lineare und $f=6$ für nichtlinearer Moleküle, während $\alpha_{i j}\left(q_{0}\right)$ die Polarisierbarkeit des Moleküls in Ruhelage beschreibt [121]. In der elektrischharmonischen Näherung werden die Taylor-Reihen nach dem linearen Term abgebrochen, da nur kleine Auslenkungen aus der Ruhelage betrachtet werden. Folglich sind die Normalschwingungen aufgrund fehlender Kreuzterme unabhängig voneinander 120,121,123. Werden nun die beiden Gleichungen 3.3.2 und 3.3.3 in die Gleichung 3.3.1 eingesetzt, und das trigonometrische Additionstheorem $\cos (\theta) \cos (\phi)=\frac{1}{2}(\cos (\theta+\phi)+\cos (\theta-\phi))$ für das Produkt zweier Kosinusfunktionen verwendet, so folgt für das zeitabhängige Gesamtdipolmoment $\vec{p}(t)$ des Moleküls bei Einstrahlung von Licht mit der Frequenz $\nu_{0}=\frac{\omega_{0}}{2 \pi}$ 121, 122,

$$
\begin{aligned}
\vec{p}(t)= & \vec{\mu}\left(q_{0}\right)+\sum_{n}\left(\frac{\partial \vec{\mu}}{\partial q_{n}}\right)_{q_{0}} q_{n 0} \cos \left(2 \pi \nu_{n} t\right)+\alpha_{i j}\left(q_{0}\right) \vec{E}_{0} \cos \left(2 \pi \nu_{0} t\right) \\
& +\left(\sum_{n}\left(\frac{\partial \alpha_{i j}}{\partial q_{n}}\right)_{q_{0}} q_{n 0}\left[\cos \left(2 \pi\left(\nu_{0}+\nu_{n}\right) t\right)+\cos \left(2 \pi\left(\nu_{0}-\nu_{n}\right) t\right)\right]\right) \frac{\vec{E}_{0}}{2}+\mathcal{O}\left(q_{n}^{2}\right) .
\end{aligned}
$$

Die einzelnen Terme der Gleichung (3.3.4) geben nun mit Ausnahme des ersten Terms, der das permanente Dipolmoment der Moleküle in der Ruhelage darstellt, direkt die Wechselwirkungen der Probe mit dem einfallenden Licht wieder. Während der zweite Term die 


\section{Experimentelle Methoden}

Molekülschwingungen im Infrarot-Bereich (IR) des Spektrums beschreibt und eine Abhängigkeit von der Änderung des molekularen Dipolmoments $\vec{\mu}$ bezüglich der Ruhelage $q_{0}$ mit den normalen Kernkoordinaten $q_{n}$ aufweist, kennzeichnet der von der Polarisierbarkeit des Moleküls in Ruhelage abhängige dritte Term des oszillierenden Dipolmoments den Lichtanteil, der elastisch (Rayleigh-Streuung) mit der Einfallfrequenz $\nu_{0}$ an der Probe gestreut wird. Der vierte Term, der von der Änderung der Polarisierbarkeit $\alpha_{i j}\left(q_{0}\right)$ in der Ruhelage $q_{0}$ mit den normalen Kernkoordinaten $q_{n}$ abhängt, beschreibt schließlich den Anteil des Lichts, der inelastisch (Raman-Streuung) mit einer um $\Delta \nu=\nu_{n}$ vergrößerten Frequenz $\nu_{\mathrm{AS}}=\nu_{0}+\nu_{n}$ (Anti-Stokes-Streuung) sowie mit einer um $\Delta \nu=\nu_{n}$ verkleinerten Frequenz $\nu_{\mathrm{S}}=\nu_{0}-\nu_{n}$ (Stokes-Streuung) an den Molekülen der Probe gestreut wird 121,122. Zudem ergibt sich aus der Gleichung (3.3.4) auch die jeweilige Bedingung, für die eine Schwingung in der IR-Spektroskopie auftritt (IR-aktiv) und für die sich eine Schwingung in der RamanSpektroskopie beobachten lässt (Raman-aktiv). Für diese Auswahlregeln folgt 121 123:

$$
\left(\frac{\partial \alpha_{i j}}{\partial q_{n}}\right)_{q_{0}} \neq 0 \quad(\Rightarrow \text { Raman-aktiv }), \quad\left(\frac{\partial \vec{\mu}}{\partial q_{n}}\right)_{q_{0}} \neq 0 \quad(\Rightarrow \text { IR-aktiv })
$$

In den Abbildungen 3.3.2 (a)-(d) (nach [123]) sind nun zur Verdeutlichung der Folgen dieser Auswahlregeln die Streckschwingung eines linearen zweiatomigen $\mathrm{A}_{2}$-Moleküls und die Normalschwingungen eines linearen dreiatomigen $\mathrm{AB}_{2}$-Moleküls mit dem Verlauf des Dipolmoments und der Polarisierbarkeit nach der Anregung aus der Ruhelage dargestellt.

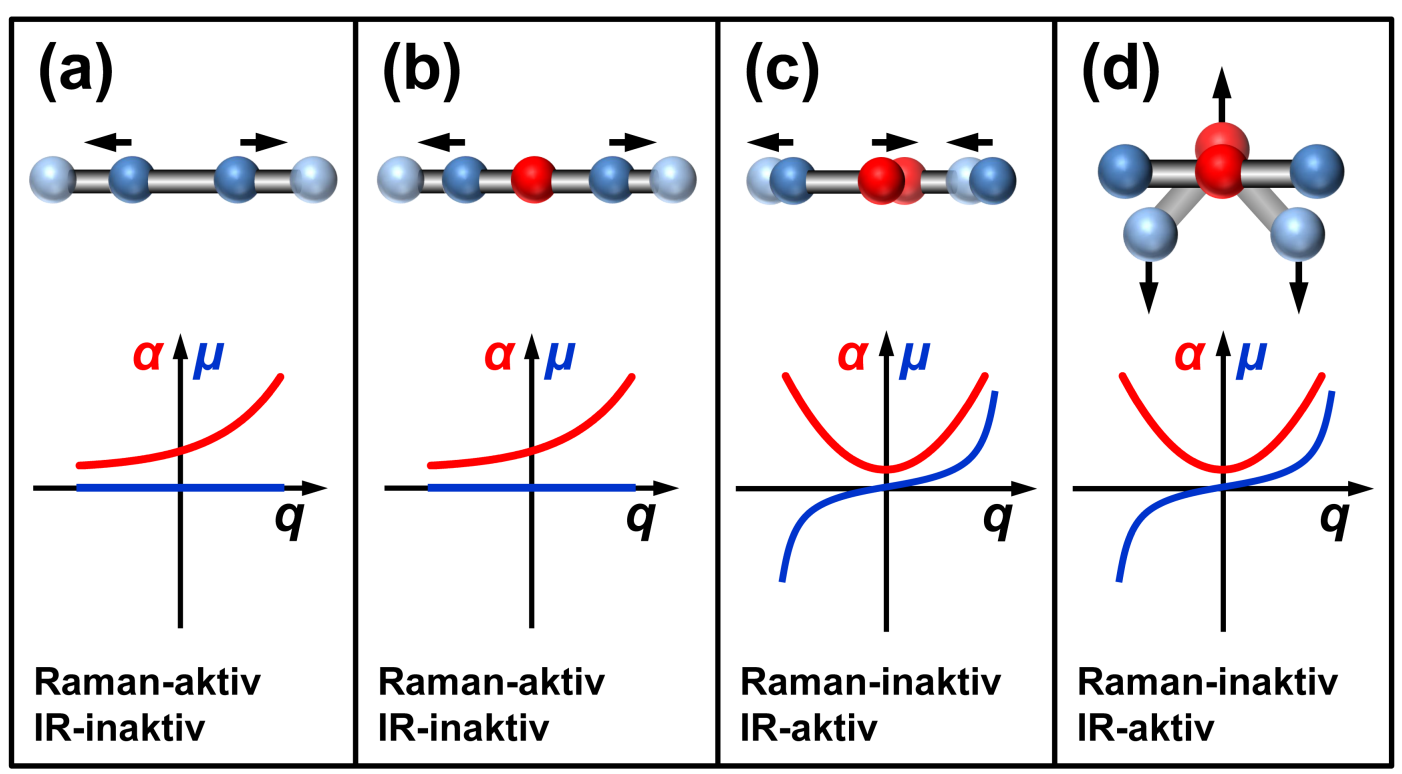

Abbildung 3.3.2: Streckschwingung eines linearen zweiatomigen $A_{2}$-Moleküls (a) und die verschiedenen Normalschwingungen eines linearen dreiatomigen $\mathrm{AB}_{2}$-Moleküls mit einer symmetrischen Streckschwingung (b), einer antisymmetrischen Streckschwingung (c) und einer Biegeschwingung in der Vertikalebene (d). Angegeben ist zudem der Verlauf des Dipolmoments und der Polarisierbarkeit sowie die resultierende Raman- und Infrarot-Aktivität (nach [123]). 
Hierbei lässt sich für die Normalschwingungen mit den Gleichungen (3.3.4) und 3.3.5 folgern, dass diese in harmonischer Betrachtung entweder nur Raman-aktiv oder nur IRaktiv sind. Diese Eindeutigkeit wird Alternativverbot genannt und gilt für Moleküle mit Inversionszentrum 117, 120, 124, 125]. Eine Betrachtung der Schwingungssymmetrie zeigt, dass die Raman-aktiven Schwingungen symmetrisch zum Inversionszentrum erfolgen, während die IR-aktiven Schwingungen antisymmetrisch sind [117, 123,124]. Die symmetrischen Schwingungsmoden werden auch als gerade (g) und die antisymmetrischen Schwingungen als ungerade (u) bezeichnet 117, 125. Für komplexe Moleküle ohne Inversionszentrum und reduzierter Symmetrie ist das Alternativverbot nicht gültig. Je nach Symmetrie kann es für diese Moleküle auch Schwingungen geben, die gleichzeitig Raman- und IR-aktiv sind [121]. Daneben können auch stille Moden existieren, die weder Raman- noch IR-aktiv sind [124].

Die Abhängigkeit der Intensität $I_{\text {Raman }}=A \cdot \beta$ einer Raman-aktiven Mode kann in eine Gruppe $A$ der Probeneigenschaften und in eine Gruppe $\beta$ für den instrumentellen Einfluss unterteilt werden [120,123, 126], die sich sich mit $\beta=B \cdot V \cdot F_{\text {Instrument }}$ in Abhängigkeit der Intensität $B$ des Lasers, des Probenvolumens $V$ und einer instrumenteller Parametergruppe $F_{\text {Instrument }}$ des Spektrometers und des Mikroskops angeben lässt 120, 126]. Eine Abschätzung der Raman-Linienintensität lässt sich mithilfe der Gleichung (3.3.4) ableiten. Hierzu wird angenommen, dass jeder oszillierende Dipol, der durch das einfallende Licht induziert wird, in der klassischen Betrachtung als Hertzscher Dipol agiert und das Streulicht in Form elektromagnetischer Sekundärwellen aussendet. Die Raman-Intensität $I_{\text {Stokes }}$ der Stokes-Streuung ist dann proportional zur insgesamt emittierten Strahlungsleistung des in Wechselwirkung mit dem einfallenden Licht induzierten Dipolmoments [123, 127]:

$$
I_{\text {Stokes }} \propto\left(\frac{\partial \alpha_{i j}}{\partial q_{n}}\right)_{q_{0}}^{2}\left(\nu_{0}-\nu_{n}\right)^{4} E_{0}^{2} .
$$

Für die Intensität der Anti-Stokes-Streuung folgt $I_{\text {Anti-Stokes }} \propto\left(\nu_{0}+\nu_{n}\right)^{4}$. Es zeigt sich sowohl eine molekulare Abhängigkeit über das Quadrat der Polarisierbarkeitsänderung bei einer Schwingungsanregung aus der Ruhelage und von der Frequenzverschiebung $\Delta \nu=\nu_{n}$ als auch ein experimentelle Abhängigkeit von der Frequenz $\nu_{0}$ und der Intensität $I_{0} \propto E_{0}^{2}$ des einfallenden Lichts. So führt eine Erhöhung der Laserleistung wie auch der Frequenz zu einer Intensitätserhöhung der Raman-Linien [123. Die Änderung der Polarisierbarkeit ist der sogenannte Raman-Tensor $\mathcal{R}$, der die materielle Antwort bezüglich einer möglichen Anregung von Raman-Linien durch das einfallende Licht beschreibt 119, 122]:

$$
\mathcal{R}=\left(\frac{\partial \alpha_{i j}}{\partial q_{n}}\right)_{q_{0}}
$$




\section{Experimentelle Methoden}

Für das Dipolmoment ergibt sich $\vec{p}=\mathcal{R} \vec{E}_{0}$. Für kristalline Proben ist der Raman-Tensor durch die Kristallstruktur gegeben. Die klassische Behandlung der Raman-Streuung hat jedoch Einschränkungen. So lassen sich weder Rotationen beschreiben, noch ein Zusammenhang zwischen dem Raman-Tensor, dem Dipolmoment und der Anregungsfrequenz angeben, sodass für die resonante Raman-Spektroskopie, bei der der Laser an einen elektronischen Übergang der Probe angepasst ist, und für die oberflächenverstärkte RamanSpektroskopie (SERS, engl.: surface-enhanced Raman spectroscopy), bei der das Signal elektromagnetisch und chemisch verstärkt wird, eine quantenmechanische Beschreibung benötigt wird 120,123. Zudem impliziert die Gleichung (3.3.6) eine fast identische Intensität der Stokes- und Anti-Stokes-Linien. Dieses widerspricht Messungen mit einer zumeist viel stärkeren Intensität der Stokes-Linien 127. Die quantenmechanische Behandlung basiert auf einem zeitabhängigen störungstheoretischen Ansatz und Gruppentheorie 120,123, 124, kann jedoch in harmonischer Näherung mit diskreten Energieniveaus für die Schwingungsmoden der Moleküle erfolgen. Für ein $\mathrm{A}_{2}$-Molekül (Abbildung 3.3.2 (a)) mit den Energieniveaus $E_{0}$ in Ruhelage und $E_{1}$ in Schwingung beschreibt die Stokes-Streuung den Übergang $E_{0} \rightarrow E_{1}$ und die Anti-Stokes-Streuung den Übergang $E_{1} \rightarrow E_{0}$ 127. Die relative Stärke der Stokes- und Anti-Stokes-Linien lässt sich im thermodynamischen Gleichgewicht mit der Boltzmann-Verteilung über das Verhältnis der jeweiligen Besetzungsdichten $N_{1}$ und $N_{0}$ der dazugehörigen Energieniveaus $E_{0}$ und $E_{1}$ des Moleküls angeben [117, 127]:

$$
\frac{N_{1}}{N_{0}}=\mathrm{e}^{-\hbar \omega_{n} / k_{B} T}
$$

Hierbei ist $k_{B}$ die Boltzmann-Konstante, $T$ die Probentemperatur und $\omega_{n}$ die Kreisfrequenz der Raman-Linie. Gegebenenfalls muss je nach Entartungsgrad noch ein Faktor $g_{i}$ berücksichtigt werden [117]. Bei Raumtemperatur ist $N_{1} \ll N_{0}$ und die Intensität der AntiStokes-Linien deutlich gegenüber der Intensität der Stokes-Linien verringert. Für sehr hohe Temperaturen nähern sich beide Intensitäten jedoch an. Dieses entspricht experimentellen Beobachtungen [127]. Im Gegensatz zu Molekülen, die räumlich lokalisierte Schwingungszustände mit diskreten Energieniveaus aufweisen, sind in Kristallen die Atome in einem Gitter in einer für den Kristall spezifischen Struktur und Energiebändern eingebunden. Folglich sind Schwingungsanregungen nicht mehr isoliert zu betrachten, sondern es werden kollektive Gitterschwingungen in Form von Phononen betrachtet [122, 127]. Diese verfügen im Allgemeinen über eine wohldefinierte Dispersionsrelation $\omega(k)$ zwischen der Kreisfrequenz und der Wellenzahl und folgen als bosonische Quasiteilchen der Bose-Einstein-Verteilung, die die mittlere Besetzungsdichte $n_{\mathrm{BE}}\left(\omega_{n}\right)$ im thermischen Gleichgewicht beschreibt 119, 127]:

$$
n_{\mathrm{BE}}\left(\omega_{n}\right)=\frac{1}{\exp \left(\hbar \omega_{n} / k_{B} T\right)-1}
$$


Die von der Umgebungstemperatur $T$ und der Kreisfrequenz $\omega_{n}$ der angeregten Schwingungsmode abhängige Besetzungsdichte, die für Phononen unabhängig von dem chemischen Potential $\mu$ ist, da die Phononenzahl keine Erhaltungsgröße des Systems ist, kann nun dazu verwendet werden, das Intensitätsverhältnis der Anti-Stokes-Linien zu den jeweiligen Stokes-Linien im Raman-Spektrum zu bestimmen. Es ergibt sich [119, 120, 127]:

$$
\frac{I_{\text {Anti-Stokes }}}{I_{\text {Stokes }}}=\frac{n_{\mathrm{BE}}\left(\omega_{n}\right)}{\left(n_{\mathrm{BE}}\left(\omega_{n}\right)+1\right)} \frac{\left(\omega_{0}+\omega_{n}\right)^{4}}{\left(\omega_{0}-\omega_{n}\right)^{4}}=\frac{\left(\omega_{0}+\omega_{n}\right)^{4}}{\left(\omega_{0}-\omega_{n}\right)^{4}} \exp \left(-\hbar \omega_{n} / k_{B} T\right) .
$$

Das Verhältnis der Besetzungsdichten für den Stokes- und Anti-Stokes-Streuprozess entspricht mit $n_{\mathrm{BE}}\left(\omega_{n}\right) /\left(n_{\mathrm{BE}}\left(\omega_{n}\right)+1\right)=\exp \left(-\hbar \omega_{n} / k_{B} T\right)$ dem Boltzmann-Faktor $N_{1} / N_{0}$ aus der Gleichung (3.3.8), ist jedoch eine Folge der Bose-Einstein-Statistik 119, 127]. So tritt die Anti-Stokes-Streuung nur für bereits angeregte Schwingungszustände auf, deren Besetzungswahrscheinlichkeit durch $n_{\mathrm{BE}}\left(\omega_{n}\right)$ gegeben ist, während die Stokes-Streuung sowohl durch stimulierte als auch durch spontane Emission mit einer Gesamtproportionalität von $\left(n_{\mathrm{BE}}\left(\omega_{n}\right)+1\right)$ zu beobachten ist [119. Folglich sind die Anti-Stokes- und Stokes-Linien stark von der Temperatur $T$ beeinflusst, wobei sich für die Anti-Stokes-Streuung durch den Proportionalitätsfaktor $n_{\mathrm{BE}}\left(\omega_{n}\right)$ eine stärkere Intensitätsänderung mit der Temperatur im Vergleich zur Stokes-Streuung ergibt und das Intensitätsverhältnis $\frac{I_{\text {Anti-Stokes }}}{I_{\text {Stokes }}}$ somit charakteristisch für die Probentemperatur ist. Durch das Messen der Stokes- und AntiStokes-Linien kann daher die lokale Temperatur der Probe bestimmt werden [119,120,127].

Weitere wichtige Größen sind die Raman-Linienposition (Frequenzverschiebung $\nu_{n}$ ) und die Linienbreite $\Gamma$. In der harmonischen Näherung ist die Schwingungsfrequenz für ein $\mathrm{A}_{2^{-}}$ Molekül über die Relation $\omega_{n}=\sqrt{\frac{K}{m_{\text {red. }}}}$ durch die Kraftkonstante $K$ und die reduzierte Masse $m_{\text {red. }}=\frac{M_{1} M_{2}}{M_{1}+M_{2}}$ gegeben. Folglich ist die Kreisfrequenz $\omega_{n}$ der Schwingungsmode von den Atommassen, den Bindungslängen und den Bindungswinkeln abhängig [117, 120, 128]. Dieses lässt sich für kristalline Festkörper auf die Anregung von Phononen und die Dispersionsrelation übertragen. Auch die Raman-Linienbreite wird beeinflusst. Neben einer natürlichen Linienbreite, die durch die Heisenbergsche Unschärferelation gegeben ist, und einer instrumentellen Abhängigkeit von der Auflösung des Spektrometers oder der Wellenlängenstabilität des Lasers, ist die Linienbreite für kristalline Festkörper durch die Kristallinität und den Grad der chemischen Ordnung bestimmt 120, 123, 128. So führen Abweichungen bei den Atomassen und den Bindungen zu kleinen Verschiebungen der Raman-Linien, sodass sich in Superposition eine Linienverbreiterung ergibt [120, 124]. Für dünne Schichten hat die von dem Substrat induzierte Verspannung einen großen Effekt auf die Form und Position der Linien. Eine Übersicht möglicher Einflüsse auf die Raman-Linien und die daraus zu gewinnenden Informationen ist in der Abbildung 3.3.3 (nach [128]) dargestellt. 


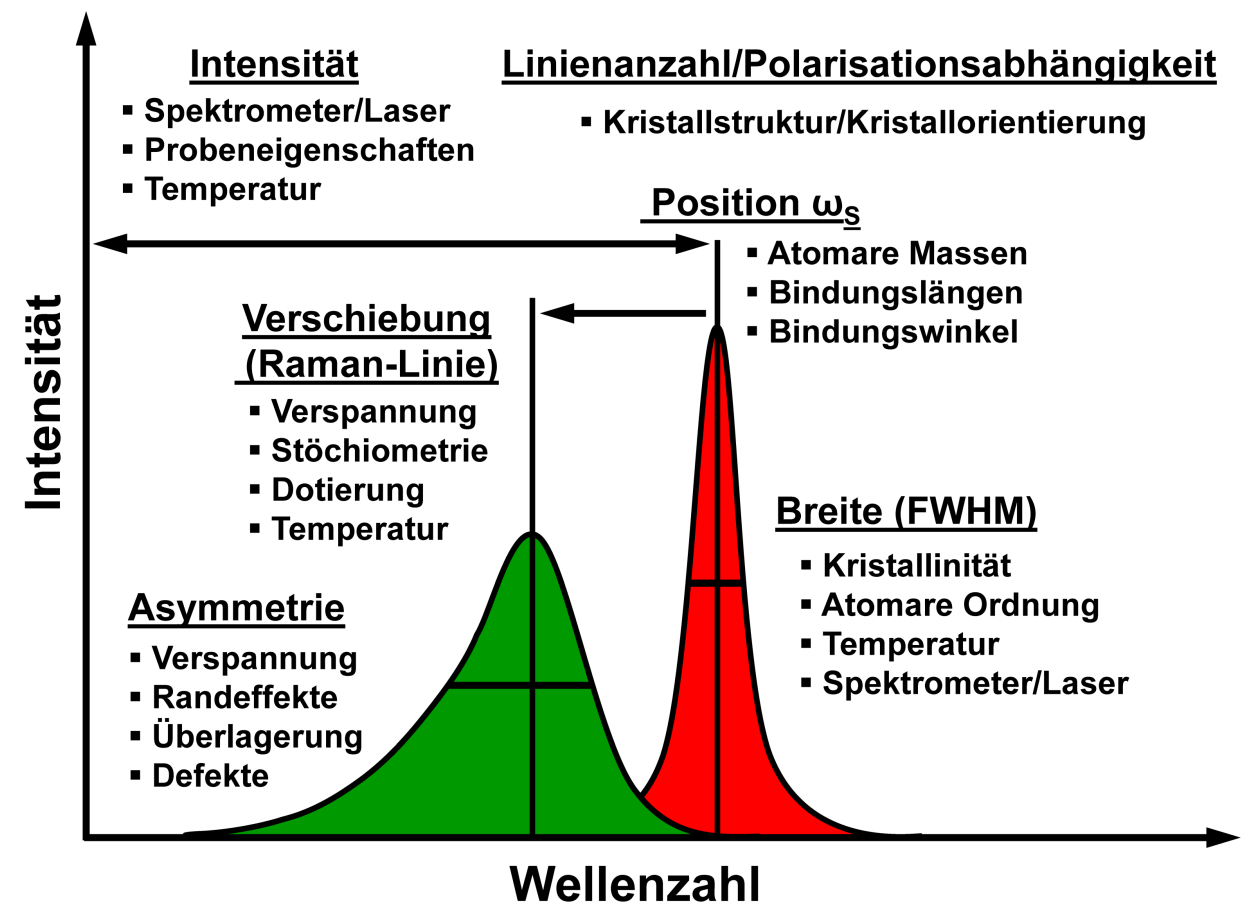

Abbildung 3.3.3: Zusammenfassende Übersicht der aus der Intensität, Position und Halbwertsbreite beobachtbarer Raman-Linien zu gewinnenden Probeninformationen in Abhängigkeit verschiedener Einflussfaktoren (nach [128]).

$\mathrm{Zu}$ beachten ist, dass die Schwingungen bisher in harmonischer Näherung betrachtet wurden, die Gitteratome aber in Wechselwirkung treten, das Gitter nicht beliebig gedehnt und gestaucht werden kann und die Phononen miteinander koppeln. Diese Effekte können mit einem anharmonischen Potential beschrieben werden, indem für die Potentialentwicklung um die Gleichgewichtsposition der Atome auch Terme höher der quadratischen Ordnung berücksichtigt werden [59,60]. Dabei wird zwischen mechanischer und elektrischer Anharmonizität unterschieden. So beeinflusst die mechanische Anharmonizität die Normalkoordinaten für die Anregung der Atome zu Gitterschwingungen in der Art, dass neben den fundamentalen Anregungen mit $q_{n} \propto \cos \left(\omega_{n} t\right)$ auch Terme mit $\cos \left(2 \omega_{n} t\right), \cos \left(3 \omega_{n} t\right)$ usw., die Oberschwingungen (engl.: overtone) repräsentieren, und Terme mit $\cos \left(\omega_{n} t\right) \cos \left(\omega_{m} t\right)$, die Kombinationsschwingungen verschiedener Phononen darstellen und eine Streuung höherer Ordnung beschreiben, möglich sind [124]. Da der Polarisationstensor unverändert bleibt, ergeben sich die gleichen Auswahlregeln wie für die Fundamentalschwingungen. Die elektrische Anharmonizität berücksichtigt die höheren Terme $\mathcal{O}\left(q_{n}^{2}\right)$ in den TaylorEntwicklungen für den Polarisationstensor $\alpha_{i j}$ (Gleichung (3.3.2), die ebenfalls zu Oberund Kombinationsschwingungen führen, aber aufgrund der Abhängigkeit des modifizierten Polarisationstensors $\alpha_{i j}^{\prime}$ von Termen wie $\left(\partial^{2} \alpha_{i j}^{\prime} / \partial q_{n}^{2}\right)$ und $\left(\partial^{2} \alpha_{i j}^{\prime} / \partial q_{n} \partial q_{m}\right)$ neuen RamanAuswahlregeln folgen, sodass diese unabhängig von nicht Raman-aktiven Fundamentalschwingungen selbst Raman-aktiv sind und umgekehrt [119,124. Da die anharmonischen Anteile zumeist nur klein sind, ist die Intensität der Ober- und Kombinationsschwingungen im Vergleich zu den Fundamentalschwingungen deutlich reduziert [119]. Daneben führt 
die Anharmonizität auch zu einer Temperaturabhängigkeit der Linienposition und der Linienbreite im Raman-Spektrum. Hierbei kann für den Raman-Streuprozess angenommen werden, dass ein einfallendes Photon der Energie $\hbar \omega_{i}$ absorbiert und ein Photon der Energie $\hbar \omega_{s}$ emittiert wird, während gleichzeitig ein Phonon der Energie $\hbar \omega_{n}=\hbar\left(\omega_{i}-\omega_{s}\right)$ erzeugt wird, das aufgrund der Anharmonizität in $n$ Phononen gleicher Frequenz $\omega_{n} / n$ zerfällt [129]. Im einfachsten Fall kann ein Dreiphononen-Prozess betrachtet werden, bei dem das angeregte Phonon in zwei Phononen mit der Frequenz $\omega_{1}=\omega_{2}=\omega_{n} / 2$ zerfällt 129,130 . Für die Temperaturabhängigkeit der Linienposition $\omega_{\text {anh. }}(T)$ der Raman-Moden folgt dann für ein anharmonisches Potential bis zu den Termen kubischer Ordnung 129 131:

$$
\omega_{\text {anh. }}(T)=\omega_{0}+C\left(1+\frac{2}{\exp \left(\hbar \omega_{0} / 2 k_{B} T\right)-1}\right)
$$

Hierbei kennzeichnet $\omega_{0}$ die Linienposition der betrachteten Mode im Raman-Spektrum bei $T=0 \mathrm{~K}$, während $C$ eine anharmonische Konstante darstellt. In gleicher Weise lässt sich die Entwicklung der Linienbreite $\Gamma(T)$ einer Schwingungsmode mit der Temperatur $T$ im Raman-Spektrum in anharmonischer Betrachtung beschreiben 39,129 131]:

$$
\Gamma(T)=\gamma+\Gamma_{0}\left(1+\frac{2}{\mathrm{e}^{\hbar \omega_{0} / 2 \mathrm{k}_{\mathrm{B}} \mathrm{T}}-1}\right)
$$

In diesen Zusammenhang bezeichnet $\omega_{0}$ wieder die Linienposition und $\Gamma_{0}$ entsprechend die Linienbreite der Mode bei $T=0 \mathrm{~K}$, während über die Größe $\gamma$ ein zusätzlicher Beitrag zur Linienbreite durch Randeffekte und Verspannungen insbesondere bei nanokristallinen Materialien und epitaktischen Dünnschichten berücksichtigt wird [131]. Daneben lässt sich die Lebensdauer der Phononen direkt aus der Linienbreite $\Gamma$ der angeregten Moden im Raman-Spektrum mithilfe der Heisenbergschen Unschärferelation bestimmen [132]:

$$
\frac{1}{\tau}=\frac{\Delta E}{\hbar}=2 \pi c \Gamma
$$

Die Lebensdauer der Phononen $\tau$ ist hauptsächlich durch zwei Mechanismen bestimmt. Neben dem anharmonischen Zerfall des Phonons mit einer charakteristischen Zerfallszeit $\tau_{\text {anh. }}$ in zwei oder mehrere Phononen in der Art, dass der Impuls erhalten bleibt, beeinflusst insbesondere die Störung der kristallinen Translationssymmetrie durch Verunreinigungen, Defekte und chemischer Durchmischung im Kristall mit einer Zerfallszeit $\tau_{\text {krist. }}$ die Lebensdauer der Phononen, sodass sich insgesamt die Relation $\frac{1}{\tau}=\frac{1}{\tau_{\text {anh. }}}+\frac{1}{\tau_{\text {krist. }}}$ ergibt 132 . 


\section{Experimentelle Methoden}

\subsubsection{Verwendeter Raman-Messaufbau}

Anschließend soll nun der Messaufbau für die Aufnahme der Raman-Spektren beschrieben werden. Hierbei wird mit dem LabRAM HR Evolution Raman-Spektrometer ein Komplettsystem des Herstellers Horiba [133] verwendet, mit dem alle in dieser Arbeit gezeigten Raman-Spektren aufgenommen wurden. Dieses besteht in den Kernkomponenten aus einem konfokalen Raman-Mikroskop mit integrierter Kamera, einem motorisiertem Mikrometertisch, auf dem die zu untersuchenden Proben platziert und systematisch verfahren werden können, einer Optik für die Kopplung von Lichtquelle und Probe, einem speziellen Raman-Filter für die Trennung des Raman-Signals vom einfallenden Licht und einem Detektor, mit dem das Raman-Signal entsprechend spektral analysiert werden kann. Ein Foto des Raman-Spektrometers im Labor ist nachfolgend in der Abbildung 3.3.4 gezeigt.

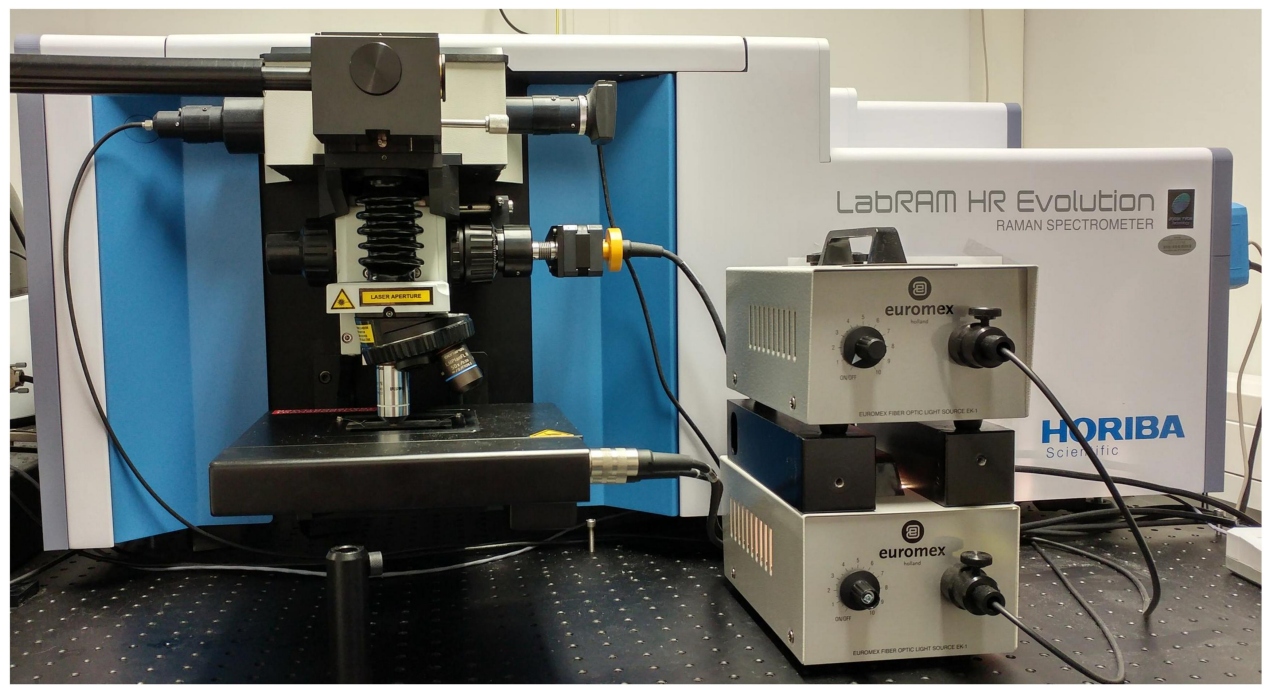

Abbildung 3.3.4: Das verwendete Raman-Spektrometer (Horiba LabRAM HR Evolution) mit dem CCD-Detektor, dem konfokalem Raman-Mikroskop, einem motorisierten Mikrometertisch, einer Kamera und einer Halogenlampenbeleuchtung.

Eine Übersicht des Strahlengangs im Raman-Spektrometer ist in der Abbildung 3.3.5 dargestellt. Für die optische Anregung der Raman-Streuung stehen ein roter HeNe-Laser von Melles-Griot mit einer Wellenlänge von $\lambda=632,8 \mathrm{~nm}$ (Ne-Übergang $3 \mathrm{~s}_{2} \rightarrow 2 \mathrm{p}_{4}[134$ ), linearer Polarisation und einer maximalen Ausgangsleistung von $P_{0}=22 \mathrm{~mW}$ sowie ein grüner Nd:YAG-Laser (Neodym-dotiertes Yttrium-Aluminium-Granat) von Laser Quantum (torus 532) mit einer Wellenlänge von $\lambda=532 \mathrm{~nm}\left(\mathrm{Nd}^{3+}\right.$-Übergang ${ }^{4} F_{3 / 2} \rightarrow{ }^{4} I_{11 / 2} \mathrm{im}$ YAG-Kristall und Frequenzverdopplung [135]), linearer Polarisation und einer maximalen Ausgangsleistung von $P_{0}=100 \mathrm{~mW}$ zur Verfügung. Während der rote Laser mit einer optischen Faser in das LabRAM-Spektrometer gelangt (rote Linie; Abbildung 3.3.5), wird der grüne Laser über ein Spiegelsystem eingekoppelt (grüne Linie; Abbildung 3.3.5). Das System lässt sich zwischen beiden Lasern umschalten, indem der Strahlengang im Gerät mithilfe eines Stellmotors verfahren wird und der andere Laser entsprechend geblockt wird. 


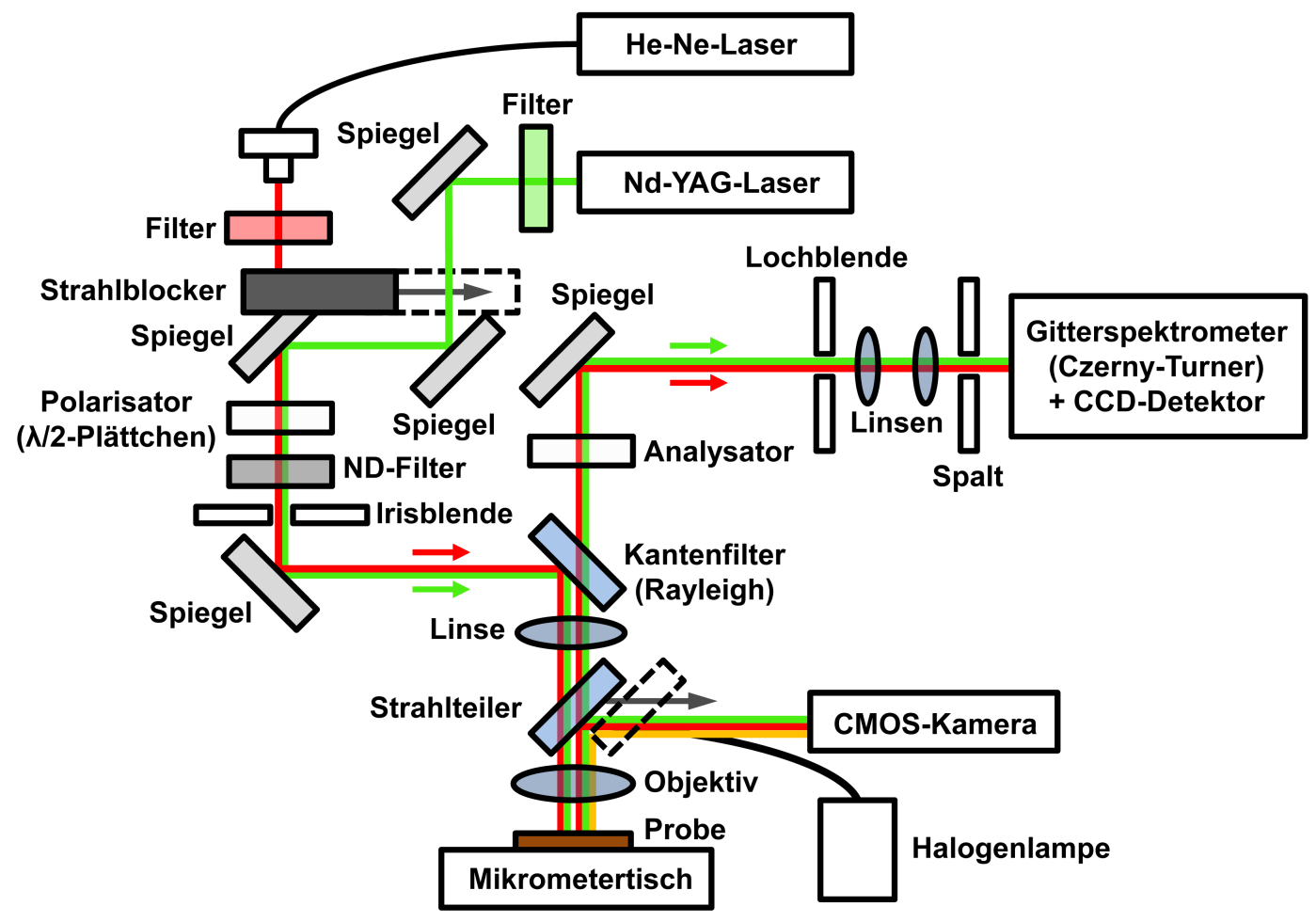

Abbildung 3.3.5: Schematische Übersicht des Strahlengangs im Raman-Spektrometer für die Anregung der Raman-Streuung mit vorheriger Aufbereitung des Laserlichts und nachfolgender Detektion des Raman-Signals im Detektor.

Mit einem Neutraldichtefilter (ND-Filter) kann die Laserintensität auf 0,01-100\% der Ausgangsintensität variiert werden. Eine Irisblende schirmt Streulicht ab. Anschließend trifft der Laserstrahl auf den Kantenfilter für die spätere Trennung des Raman-Signals vom Anregungssignal, wird dort reflektiert und mit einem Objektiv auf die Probe fokussiert. Hierfür stehen drei Objektive von Olympus mit 10× Vergrößerung (numerische Apertur $\mathrm{NA}=0,25), 50 \times$ Vergrößerung $(\mathrm{NA}=0,5)$ und $100 \times$ Vergrößerung $(\mathrm{NA}=0,9)$ mit unterschiedlichen Arbeitsabständen zur Verfügung. Zudem lässt sich eine CMOS-Kamera mit Halogenlampenbeleuchtung (gelbe Linie; Abbildung 3.3.5) zur Bildgebung und Fokussierung mit einem Strahlteiler in den Strahlengang integrieren. Der Fokuspunkt kann bis auf eine Fläche von $A \approx 1 \mu^{2}$ verringert werden. Mit dem motorisierten Mikrometertisch des Mikroskops (Märzhäuser Scan $100 \times 100$ [136]) lassen sich Kartierungen des RamanSignals (Raman-Mappings) der Probenoberfläche bis zu einer Größe von $100 \mathrm{~mm} \times 100 \mathrm{~mm}$ und einer lateralen Auflösung von bis zu 1 um aufzeichnen. Die Aufnahme der RamanSpektren erfolgt in senkrechter Rückstreugeometrie, bei der der Laser senkrecht auf die Probe trifft und das zurückgestreute Raman-Signal über das gleiche Objektiv aufgesammelt wird. Dieses passiert den Kantenfilter, der den elastisch mit der Wellenlänge $\lambda_{\text {Laser }}$ gestreuten Anteil (Rayleigh-Streuung) der im Vergleich zum Raman-Signal sehr viel intensiveren Laser-Linie herausfiltert. Für die eingesetzten Kantenfilter wird ein Spektralbereich bis zu einer Wellenzahl von $100 \mathrm{~cm}^{-1}$ oberhalb der Laserwellenlänge absorbiert, sodass die Anti-Stokes-Streuung nicht erfasst werden kann. Für die beiden Laser entspricht dieses 


\section{Experimentelle Methoden}

einer Absorptionskante bei $\lambda_{\text {Filter }} \approx 534,9 \mathrm{~nm}$ (grün) und bei $\lambda_{\text {Filter }} \approx 636,8 \mathrm{~nm}$ (rot). Der weitere Weg des Raman-Signals ist von einer Lochblende und einem Spalt gekennzeichnet. Beide können mit einem Schrittmotor in ihrem Durchmesser (Lochblende), beziehungsweise in ihrer Breite (Spalt), im Bereich einiger Hundert Mikrometern variiert werden. Anschließend wird das Raman-Signal in ein Czerny-Turner-Gitterspektrometer eingekoppelt, dessen grundlegender Aufbau in der Abbildung 3.3.6 (nach [118]) dargestellt ist.

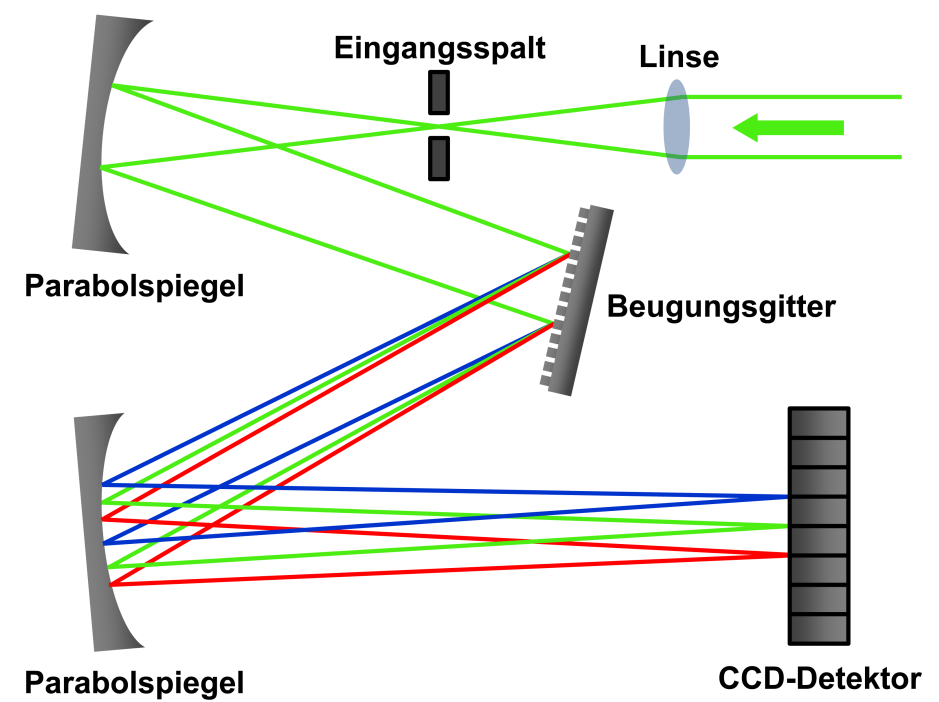

Abbildung 3.3.6: Aufbau eines Czerny-Turner-Spektrometers mit der Einkopplung des Lichts durch einen Eingangsspalt, Parabolspiegeln, einem Beugungsgitter zur Auffächerung des Lichts in Abhängigkeit der Wellenlänge und der spektralen Erfassung mithilfe eines CCD-Detektors (nach 118).

Das fokussiert durch den Spektrometer-Eingangsspalt einfallende Licht trifft auf einen Parabolspiegel, der das Licht parallelisiert auf ein optisches Gitter reflektiert, an dem dieses durch Beugung spektral aufgetrennt und in Abhängigkeit der Wellenlänge unterschiedlich stark abgelenkt wird. Mit einem zweiten Parabolspiegel werden die einzelnen Anteile dann derart auf den Mehrfachzellendetektor des Spektrometers fokussiert, dass jede Zelle mit einer bestimmten Wellenlänge (Raman-Verschiebung) beleuchtet und die dazugehörige Intensität registriert wird [137]. Die spektrale Auflösung erreicht $\approx 1-2 \mathrm{~cm}^{-1}$ und ist vom optischen Gitter, der Detektor-Pixelgröße, der Laserwellenlänge, der Fokallänge (Abstand zwischen Gitter und Detektor) und der Eingangsspaltbreite abhängig [137, 138. Hierbei führen feinere Gitter, eine größere Wellenlänge und Fokallänge sowie kleinere Detektorpixel und Spaltbreiten zu einer höheren spektralen Auflösung, während umgekehrt gröbere Gitter und ein breiterer Eingangsspalt in einer höheren Intensität resultieren. Das verwendete Spektrometer verfügt über eine Fokallänge von $800 \mathrm{~mm}$ und zwei optische Gitter mit Strukturbreiten von $600 \mathrm{gr} / \mathrm{mm}$ und $1800 \mathrm{gr} / \mathrm{mm}$, zwischen denen gewechselt werden kann. Die Konfokalität ist durch die numerische Apertur, die Laserwellenlänge und die Lochblendengröße bestimmt, wobei eine kleinerer Durchmesser der Lochblende zu einer höheren Konfokalität, jedoch auch zu einer geringeren Raman-Intensität führt 138. 
Der Detektor besteht aus einem zweidimensionalen Pixelfeld von CCD-Halbleiter-Chips (engl.: charged-coupled device) mit einer Pixelzahl von $1024 \times 256$ bei einer Pixelgröße von $26 \mu \mathrm{m} \times 26 \mu \mathrm{m}$ und wird für eine höhere Effizienz rückwärtig beleuchtet. Mit einer Antireflex-Beschichtung lässt sich eine Quanteneffizienz > 85 \% im sichtbaren Wellenlängenbereich erreichen. Eine detaillierte Beschreibung findet sich in der Literatur [120, 123]. Die hohe Pixelanzahl und die kleine Pixelgröße ermöglichen es, große Spektralbereiche in einem Schritt aufzunehmen. Die Fenstergröße hängt vom optischen Gitter und der Laserwellenlänge ab. Für eine Verbesserung des Signal-Rausch-Verhältnisses wird der Detektor mit einem Peltier-Element auf eine Temperatur von etwa $-70{ }^{\circ} \mathrm{C}$ herunter gekühlt, wodurch der Anteil thermisch generierter Ladungsträger drastisch reduziert wird [123. Wichtig ist zudem die Vermeidung von Umgebungslichtartefakten. Ein weiteres Artefakt sind Spikes, die durch kosmische Strahlung hervorgerufen werden, daher zufällig auftreten und in den Spektren als scharfe Linien mit hoher Intensität beobachtbar sind 120,123, 128, sich durch eine geeignete Analyse jedoch aus den Raman-Spektren herausfiltern lassen 123, 128. Zudem muss das Spektrometer an einem Referenz-Material kalibriert werden, um die korrekte Position der Raman-Linien im Spektrum und eine Vergleichbarkeit zwischen den Messungen zu ermöglichen. Im Fall des LabRAM HR Evolution wird kristallines Silizium verwendet, dessen Raman-Spektrum von einer scharfen Linie hoher Intensität bei $520 \mathrm{~cm}^{-1}$ dominiert ist, die eine dreifach entartete Raman-Mode erster Ordnung am $\Gamma$-Punkt der Brillouin-Zone repräsentiert [58, 139, 140]. Die Kalibrierung wird mit einer automatisierten Routine für beide Laser und Gitter durchgeführt. Da sich mit der Zeit durch äußere Einflüsse Verschiebungen ergeben können [141], wurde die Linienposition nach jeder längeren Messung überprüft und das Spektrometer gegebenenfalls erneut kalibriert.

Für die Untersuchung der Temperaturabhängigkeit der Raman-Spektren steht zudem mit der THMS350EV eine mit flüssigem Stickstoff $\left(T_{\mathrm{N}_{2}}=77 \mathrm{~K}\right)$ gekühlte Kühlzelle von Linkam zur Verfügung [142], mit der sich die Raman-Spektren in einem Temperaturbereich von $T=80-620 \mathrm{~K}$ aufnehmen lassen. Hierzu werden die Proben auf einem Halter in der Kühlzelle platziert und die Probenkammer mit einer Drehschieberpumpe bis auf einen Druck von etwa $p \approx 10^{-3}$ mbar evakuiert, um Vereisungen an der Probe und dem Einkopplungsfenster beim Herunterkühlen zu vermeiden. Zudem kann das optische Fenster von außen zur Vermeidung von Kondensation mit Luft angeblasen werden. Mit einem resistiven Heizer im Probenhalter und der Anpassung der Durchflussrate des flüssigen Stickstoffs kann die Temperatur bis auf die Genauigkeit von $\pm 0,1 \mathrm{~K}$ angefahren werden. Die optische Kopplung mit dem Raman-Spektrometer erfolgt mit einem LWD-Objektiv (engl.: long working distance) mit 50× Vergrößerung und einer numerischen Apertur NA $=0,5$ von Olympus in senkrechter Rückstreuung, wobei die Kühlzelle direkt in den Mikrometertisch des Raman-Mikroskops integriert wird. Fotos der Kühlzelle im Betrieb und die Kopplung mit dem Raman-Spektrometer sind in den Abbildungen 3.3.7 (a) und (b) gezeigt. 


\section{Experimentelle Methoden}
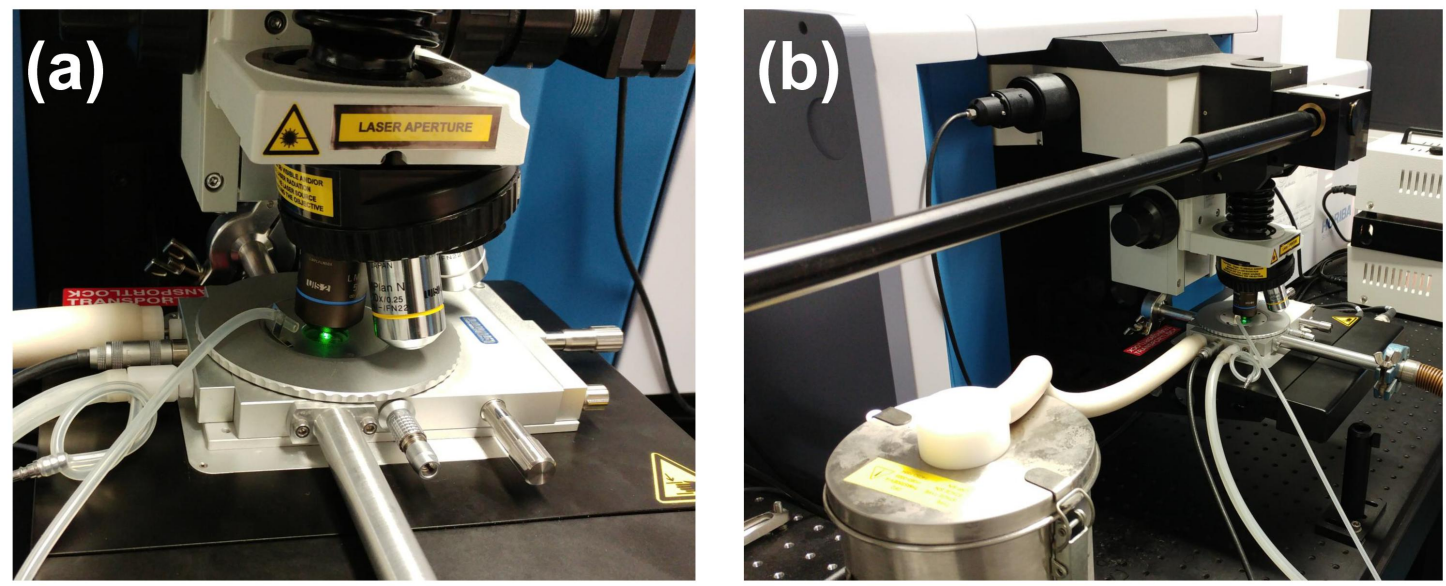

Abbildung 3.3.7: Die Kühlzelle für die temperaturabhängigen Raman-Messungen (a) und die Integration in das Raman-Spektrometer (b) mit optischer Kopplung durch ein LWD-Objektiv mit 50× Vergrößerung $(\mathrm{NA}=0,5)$. Die Temperatursteuerung erfolgt mithilfe eines Heizers und flüssigem Stickstoff.

\subsubsection{Polarisationsabhängige Raman-Spektroskopie}

Die Schichten können außerdem mithilfe der polarisationsabhängigen Raman-Spektroskopie analysiert werden. Hierzu werden die Raman-Spektren in spezifischen Polarisationskonfigurationen aufgenommen, bei denen die lineare Polarisation des Laserlichts systematisch durch einen Polarisator vorgegeben und die Polarisationskomponenten des gestreuten Lichts mithilfe eines Analysators betrachtet werden. In Abhängigkeit der Polarisator- und Analysator-Stellung ergeben sich unterschiedliche Intensitäten der beobachteten RamanModen. Die Änderung der Raman-Intensität mit der Polarisationskonfiguration wird dabei von der Kristallstruktur, der Raumgruppe, der Symmetrie der Moden und damit vom jeweiligen Raman-Tensor $\mathcal{R}$ (Gleichung (3.3.7)) vorgegeben. Es ergibt sich die Relation [143]:

$$
I_{\text {Raman }} \propto\left|\sum_{k=1}^{n} \hat{e}_{s}^{T} \mathcal{R}_{k} \hat{e}_{i}\right|^{2}
$$

Hierbei sind $\hat{e}_{i}$ und $\hat{e}_{s}$ die Einheitsvektoren der Polarisation des einfallenden und gestreuten elektrischen Feldes (mit der transponierten Form $\hat{e}_{s}^{T}$ von $\hat{e}_{s}$ ). Für n-fach entartete Moden muss die Summe der einzelnen Beiträge addiert werden. Die Form des Raman-Tensors ergibt sich anhand der Raumgruppe der Kristallstruktur und kann in Tabellen [143] und Datenbanken [144] nachgeschlagen werden. Die Raman-Tensoren $\mathcal{R}_{k}$ sind im Allgemeinen symmetrisch, für die Tensoreinträge folgt somit $\alpha_{i j}^{\prime}=\alpha_{j i}^{\prime}$ 119,143], und werden anhand der Symmetrie der Schwingung und der Verteilung der Einträge $\alpha_{i j}^{\prime} \neq 0$ mithilfe der MullikenSymbole eingeordnet. Die in der Raman- und IR-Spektroskopie vorkommenden MullikenSymbole [145] und die Bedeutung sind in der Tabelle 3.3.1 (nach 145, 146]) dargestellt. 


\begin{tabular}{|c|c|}
\hline Mulliken-Symbol & Bedeutung \\
\hline \hline A & nicht entartet (1D), symmetrisch zur Hauptachse \\
B & nicht entartet (1D), antisymmetrisch zur Hauptachse \\
E & zweifach entartet (2D) \\
T & dreifach entartet (3D) \\
\hline g (gerade) & symmetrisch zum Symmetriezentrum (Raman-aktiv) \\
u (ungerade) & antisymmetrisch zum Symmetriezentrum (IR-aktiv) \\
\hline 1 & symmetrisch zur Achse senkrecht zur Hauptachse \\
2 & antisymmetrisch zur Achse senkrecht zur Hauptachse \\
antisym. zur zweiten Achse senkrecht zur Hauptachse \\
\hline
\end{tabular}

Tabelle 3.3.1: Bezeichnung der Raman-Moden mithilfe der Mulliken-Symbole und die dazugehörige Bedeutung. Hierbei werden die Schwingungen anhand des Grades der Entartung (Dimension) und der Symmetrie eingeordnet (nach [145, 146]).

Die Raman-Spektren werden unter dem Mikroskop in senkrechter Rückstreugeometrie aufgenommen. Für die Wellenvektoren des einfallenden und gestreuten Lichts folgt demnach $\hat{k}_{i}=-\hat{k}_{s}$. Die Porto-Notation kann daher verkürzt mit $\left(\hat{e}_{i} \hat{e}_{s}\right)$ angegeben werden (allgemein wird die Porto-Notation in der Form $\hat{k}_{i}\left(\hat{e}_{i} \hat{e}_{s}\right) \hat{k}_{s}$ angegeben 147]). Das verwendete Probenbezugssystem, das in senkrechter Rückstreuung dem Laborbezugssystem entspricht, und die Polarisationskonfigurationen in Abhängigkeit der Polarisator- und Analysator-Stellung sowie einer in-plane Probenorientierung sind in der Abbildung 3.3 .8 dargestellt.

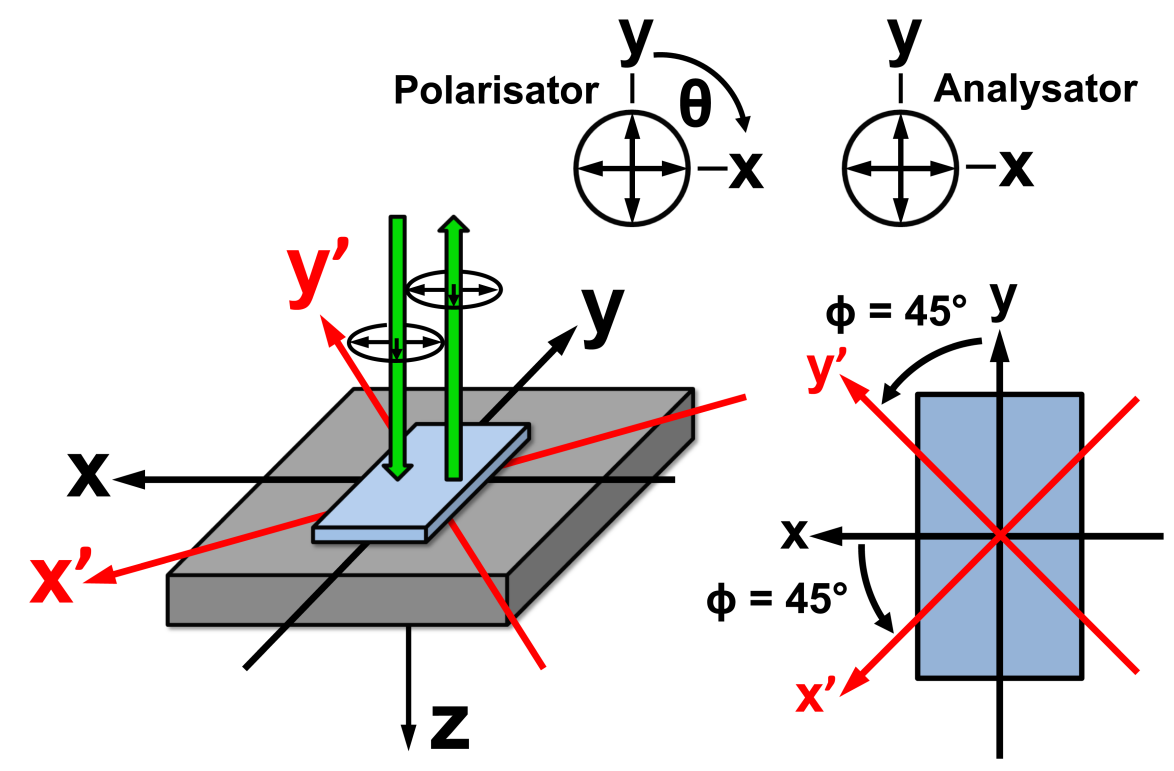

Abbildung 3.3.8: Definition des Proben-Bezugssystems zur Aufnahme polarisationsabhängiger Raman-Spektren in der senkrechten Rückstreugeometrie und die dazugehörigen Polarisationskonfigurationen in Abhängigkeit der Einstellung von Polarisator und Analysator sowie der in-plane Probenorientierung. 


\section{Experimentelle Methoden}

Das Probenbezugssystem ist kartesisch entlang der $\langle 100\rangle$-Kristallhauptachsen ausgerichtet, da die Raman-Tensoren $\mathcal{R}_{k}$ für diese Geometrie angegeben werden. Für Proben mit anderer Orientierung müssen die Raman-Tensoren zunächst in ein angepasstes Probenbezugssystem überführt werden. Eine Beschreibung findet sich in dem Abschnitt 3.4.5. Bei polarisationsabhängigen Raman-Messungen wird zwischen einer parallelen Polarisationskonfiguration, für die die einfallende Laserpolarisation parallel zur Analysator-Stellung orientiert ist $\left(\hat{e}_{i} \| \hat{e}_{s}\right)$, und einer gekreuzten Polarisationskonfiguration, für die die Laserpolarisation senkrecht zur Analysator-Stellung orientiert ist $\left(\hat{e}_{i} \perp \hat{e}_{s}\right)$, unterschieden. Für das verwendete Bezugssystem sind nach der Porto-Notation in der Rückstreugeometrie die yy- und die xx-Konfigurationen parallele Polarisationskonfigurationen und die yx- und die xy-Konfigurationen gekreuzte Polarisationskonfigurationen. Zusätzlich kann die Probe in-plane gedreht werden. Für eine Drehung um $\phi=45^{\circ}$ ergibt sich eine spezielle Konstellation (Abbildung 3.3.8), die mit einem Strichindex markiert wird. Die $y^{\prime} y^{\prime}-$ und die $\mathrm{x}^{\prime} \mathrm{x}^{\prime}$-Konfigurationen beschreiben wie zuvor parallele Polarisationskonfigurationen $\left(\hat{e}_{i} \| \hat{e}_{s}\right)$ und die $\mathrm{y}^{\prime} \mathrm{x}^{\prime}$ - und die $\mathrm{x}^{\prime} \mathrm{y}^{\prime}$-Konfigurationen gekreuzte Polarisationskonfigurationen $\left(\hat{e}_{i} \perp \hat{e}_{s}\right)$. Die Drehung der linearen Polarisation des Laserlichts erfolgt mit einem $\lambda / 2$-Plättchen als Polarisator, indem dessen optische Achse um einen Winkel $\theta$ in Relation zur einfallenden Laserpolarisation gedreht wird, wodurch die lineare Polarisation um den Winkel $2 \theta$ rotiert wird. Im definierten Probenbezugssystem weist der Laser im Ausgangszustand eine lineare y-Polarisation auf (entspricht einer Stellung des $\lambda / 2$-Polarisators mit $\theta=0^{\circ}$ ). Folglich ergibt sich eine dazu vertikale x-Polarisation $\left(2 \theta=90^{\circ}\right)$ durch eine Drehung des $\lambda / 2$ Polarisators um $\theta=45^{\circ}$. Der Analysator ist hingegen ein Polarisationsfilter, der in zwei Stellungen (x-Polarisation und y-Polarisation) vor den Detektor gesteckt werden kann.

\subsection{Spitzenverstärkte Raman-Spektroskopie (TERS)}

Als abschließende Messmethode soll nun mit der spitzenverstärkten Raman-Spektroskopie (TERS, engl.: tip-enhanced Raman spectroscopy) eine spezielle Erweiterung der RamanSpektroskopie vorgestellt werden, die die Signalverstärkung der oberflächenverstärkten Raman-Spektroskopie (SERS, engl.: surface-enhanced Raman spectroscopy) mit der lokalen Auflösung eines Rastersondenmikroskops kombiniert. Hierfür wird in den nachfolgenden Abschnitten zunächst mit einer Einführung und Motivation in die TERS-Methodik begonnen und auf wesentliche Aspekte wie das Verstärkungsprinzip des Raman-Signals, den TERS-Kontrast und die Oberflächensensitivität eingegangen, bevor anschließend der verwendete TERS-Messaufbau vorgestellt und mit der Beschreibung polarisationsabhängiger TERS-Messungen eine weitere wichtige Variante der TERS-Technik aufgezeigt wird. Im Anschluss wird dann noch in einem Überblick auf verschiedene Spitzen- und Nahfeldeffekte, die während der TERS-Messungen auftreten können, eingegangen. 


\title{
3.4.1 Motivation und Prinzip der TERS-Technik
}

Die Untersuchung von Nanomaterialien mithilfe der Raman-Spektroskopie ist im Allgemeinen sowohl durch den geringen Wirkungsquerschnitt des Raman-Streuprozesses von nur $\sigma_{\text {Raman }} \approx 10^{-30} \mathrm{~cm}^{2} \ll \sigma_{\text {Fluoreszenz }} \approx 10^{-14}-10^{-16} \mathrm{~cm}^{2}$ 148, 149 im Vergleich zur Fluoreszenz als auch durch die Beugungsbegrenzung der räumlichen Auflösung der optischen Raman-Mikroskope von $d_{\text {min. }} \approx 200 \mathrm{~nm}[118,150$ im sichtbaren Wellenlängenbereich stark eingeschränkt. Während ein konfokales Raman-Mikroskop und eine angepasste Laserwellenlänge zu einer starken Reduzierung des Fluoreszenz beitragen, bleibt die geringe Raman-Intensität und die Auflösungsbegrenzung bei Nanomaterialien auch für die resonante Raman-Spektroskopie (RRS) mit einer Erhöhung des Wirkungsquerschnitts auf $\sigma_{\mathrm{RRS}} \approx 10^{-27} \mathrm{~cm}^{2} 119,148$ ein Problem. Stattdessen wird eine signifikante Signalverstärkung bei einer räumlichen Aufösung im Bereich weniger Nanometern benötigt. Hier setzt die TERS-Technik an, die die SERS-Methodik eines in der Umgebung von Metallpartikeln oder aufgerauten Metallsubstraten plasmonisch um mehrere Größenordnungen verstärkten Raman-Signals $\left(\sigma_{\text {SERS }} \approx 10^{-18}-10^{-25} \mathrm{~cm}^{2}[119,151)\right.$ mit einem optischen Rasternahfeldmikroskop (SNOM, engl.: scanning near-field optical microscope) kombiniert [150,152 154]. Durch die Beleuchtung des Spitzenbereichs einer elektrisch leitfähigen STM- oder AFMSpitze wird ein stark konzentriertes elektrisches Nahfeld erzeugt, durch das das RamanSignal lokal mit einer räumlichen Auflösung $<20 \mathrm{~nm}$ verstärkt wird. Das Prinzip der TERS-Nahfeldverstärkung ist schematisch in der Abbildung 3.4.1 (nach [155]) dargestellt.

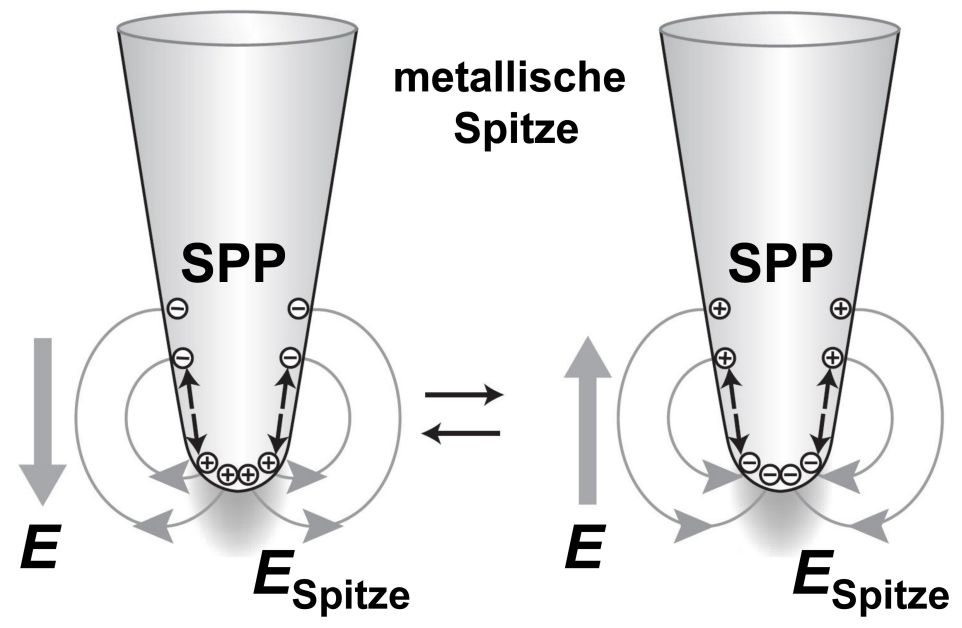

\begin{abstract}
Abbildung 3.4.1: Prinzip der TERS-Signalverstärkung durch ein aufgrund der Anregung von Oberflächenplasmon-Polaritonen (SPP) erzeugtes Spitzennahfeld, das sowohl das elektrische Feld des einfallenden Laserlichts als auch das inelastisch an der Probe gestreute Raman-Signal verstärkt (nach 155).
\end{abstract}

Der Hauptbeitrag zur TERS-Nahfelderzeugung ist plasmonischen Ursprungs und resultiert aus der durch das einfallende Laserlicht induzierten Anregung an der Spitzenoberfläche propagierender Oberflächenplasmon-Polaritonen (SPP, engl.. surface plasmon polariton) und lokalisierter Oberflächenplasmonen (LSP, engl.: localized surface plasmons), die kollektive 


\section{Experimentelle Methoden}

Ladungsschwingungen von Elektronen an der Oberfläche der Metallspitze bezeichnen und zu einem stark lokalisierten Nahfeld am vordersten Spitzenbereich führen [148, 150, 155]. Zudem treten mit dem lightning rod Effekt, der eine durch die geometrische Form der TERS-Spitze verursachte Verdichtung von Oberflächenladungen in der Spitze beschreibt und zu einer Konzentration des elektrischen Nahfeldes im Spitzenbereich führt 150, 156], und Antennenresonanzen, die eine Nahfeldverstärkung hervorrufen, wenn die Ausmaße der Spitze ein Vielfaches der halben Wellenlänge des eingestrahlten Lichts betragen [150], weitere Mechanismen auf, die zur TERS-Nahfeldverstärkung beitragen. Folglich ergibt sich die TERS-Gesamtverstärkung aus der Kombination dieser Effekte und Prozesse [49, 150]. Weitere TERS-Artefakte und -Nebeneffekte sind in dem Abschnitt 3.4.6 aufgeführt.

\subsubsection{Plasmonische Nahfeldanregung und Feldverstärkung}

Während der lightning rod Effekt durch die geometrischen Eigenschaften der Spitze bestimmt ist und für die Antennenresonanzen zusätzlich die Wellenlänge des eingestrahlten Laserlichts berücksichtigt werden muss, sind die Bedingungen für die plasmonische Anregung des Spitzennahfeldes komplexer. Der Grund hierfür ist der resonante Charakter. So müssen Spitzenmaterial, Spitzenform, Spitzengeometrie und Wellenlänge des eingestrahlten Lichts aufeinander abgestimmt werden, um eine signifikante Verstärkung des Nahfeldes zu erzielen, wobei die Spitze selbst mit dem Radius $R_{\text {Spitze }}$ als Nanostruktur zur Anregung der SPP und LSP dient. Die Charakteristik dieser lokalisierten Oberflächenplasmonenresonanz (LSPR, engl.: localized surface plasmon resonance) ist im Allgemeinen sehr stark von der Form, der Oberfläche, dem Material und dem Radius der TERS-Spitze abhängig. Aufgrund dieser Komplexität wird die Plasmonenresonanzbedingung oft numerisch anhand verschiedener SERS-Partikel- und TERS-Spitzenformen simuliert 122, 157 161. Eine ausführliche Beschreibung der plamonischen Anregung findet sich in der Literatur 118, 119. In einer Vereinfachung kann die metallische Spitze im vordersten Bereich als Kugel mit einem Radius $r$ angenommen werden, die aus dem Spitzenmaterial mit der von der eingestrahlten Frequenz des Lichts abhängigen Permittivität $\varepsilon_{M}(\nu)$ besteht. Das in einem externen elektrischen Feld $\vec{E}$ induzierte Dipolmoment $\vec{p}=\alpha \vec{E}$ lässt sich in Abhängigkeit der Clausius-Mossotti-Gleichung angeben. Für die Polarisierbarkeit der Kugel folgt 118,122]:

$$
\alpha=4 \pi \varepsilon_{0} r^{3} \frac{\varepsilon_{\mathrm{M}}(\nu)-\varepsilon_{\mathrm{U}}}{\varepsilon_{\mathrm{M}}(\nu)+2 \varepsilon_{\mathrm{U}}}=4 \pi \varepsilon_{0} r^{3} g
$$

Hier bezeichnet $\varepsilon_{U}$ die Permittivität der Umgebung (mit $\varepsilon_{U} \approx 1$ für Luft). Die Polarisierbarkeit $\alpha$ ist dabei für eine freistehende Kugel isotrop und damit ein Skalar. In einer realen Spitze ist dieses allgemein nicht gültig. Stattdessen wird eine Abhängigkeit von der Polarisation des eingestrahlten Laserlichts beobachtet 122,161]. Der Faktor $g$ ist hierbei der Verstärkungsfaktor des elektrischen Nahfeldes $\vec{E}_{\mathrm{nf}}$ der Kugel gegenüber des externen 
elektrischen Feldes $\vec{E}$. Für das Verhältnis der elektrischen Feldstärke des Spitzennahfeldes $E_{\text {Spitze }}$ zur elektrischen Feldstärke des Laserlichts $E_{0}$ folgt somit $g=E_{\text {Spitze }} / E_{0}[150,162]$. Nach der Gleichung (3.4.1) ist die Resonanzbedingung der größten Nahfeldverstärkung durch $\varepsilon_{\mathrm{M}}(\nu)=-2 \varepsilon_{\mathrm{U}}\left(\operatorname{mit} \operatorname{Re}\left(\varepsilon_{\mathrm{M}}(\nu)\right)=-2\right.$ und $\left.\operatorname{Im}\left(\varepsilon_{\mathrm{M}}(\nu)\right) \ll 1\right)$ gegeben 118, 122, 123, 163. Das Prinzip der Anregung eines LSP einer Metallkugel in Resonanz (LSPR) durch ein externes elektrisches Feld ist schematisch in der Abbildung 3.4.2 (nach [163]) dargestellt.

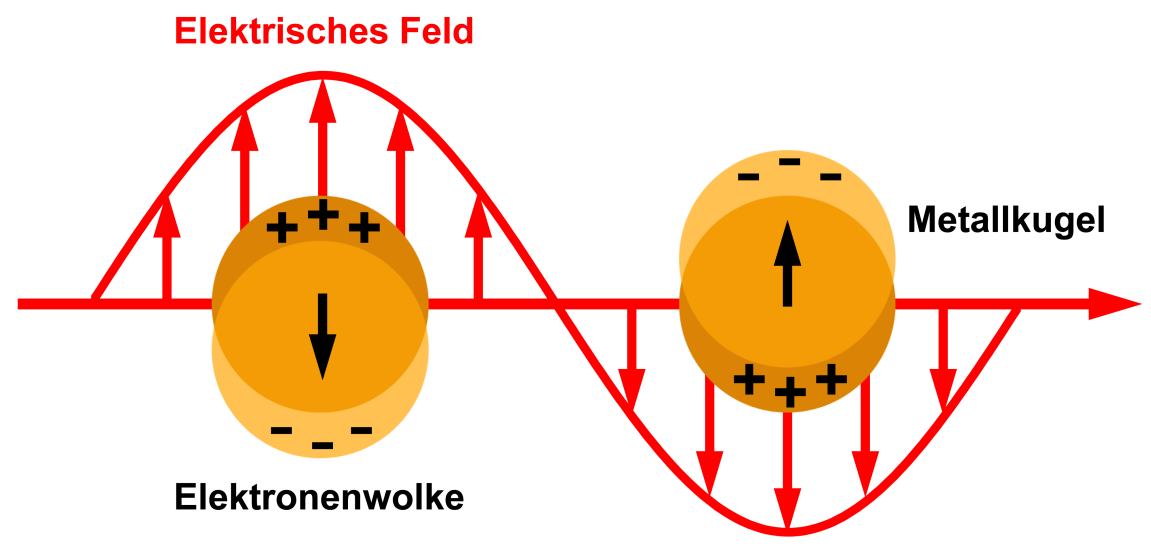

Abbildung 3.4.2: Prinzip der LSP-Anregung an einem kugelförmigen Metallnanopartikel in Resonanz (LSPR) durch ein externes elektrisches Feld (nach $163 \mid$ ).

Folglich wird für eine plasmonische Anregung eine metallische TERS-Spitze mit freien Elektronen und negativer Permittivität benötigt. Alternativ werden TERS-Spitzen oft auch durch ellipsenförmige Metallpartikel simuliert [118, 122, 161]. Als Spitzenmaterial wird zumeist Gold oder Silber verwendet, da diese Metalle eine Plasmonenresonanz im sichtbaren Wellenlängenbereich aufweisen 164 166]. Für Silber ist die Plasmonenresonanz im Vergleich zu Gold zu höheren Frequenzen verschoben [118. Aufgrund der Abhängigkeit der Intensität $I \propto E^{2}$ vom Quadrat der elektrischen Feldstärke ist die Verstärkung der Intensität des mit der Wellenlänge $\lambda_{i}$ eingestrahlten Laserlichts mit $I\left(\lambda_{i}\right) \propto g_{\lambda_{i}}{ }^{2}$ proportional zum Quadrat des Verstärkungsfaktors $g_{\lambda_{i}}$ der Laserwellenlänge, der mit dem Plasmonenresonanzspektrum der Spitze in Beziehung steht und damit wellenlängenabhängig ist 118, 150. Das durch inelastische Streuung an der Probe erzeugte Raman-Signal der Wellenlänge $\lambda_{s}$ wird mit $I\left(\lambda_{s}\right) \propto g_{\lambda_{s}}^{2}$ in gleicher Weise vom Spitzennahfeld verstärkt. Die TERS-Intensitätsverstärkung ist somit eine Kombination der Verstärkung des Anregungssignals und des gestreuten Raman-Signals. Für Raman-Moden mit Wellenlängenverschiebungen, die klein $\left(\lambda_{i} \approx \lambda_{s}\right)$ gegenüber dem Bereich hoher Resonanz im Plasmonenresonanzspektrum der Spitze sind, kann in Näherung mit $g_{\lambda_{i}} \approx g_{\lambda_{s}}=g$ [118, 150, 167] ein gemeinsamer Verstärkungsfaktor für die einfallende und die gestreute Strahlung betrachtet

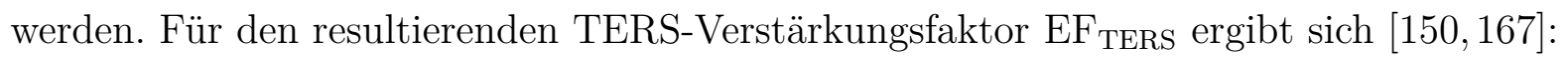

$$
\mathrm{EF}_{\mathrm{TERS}}=\mathrm{g}_{\lambda_{\mathrm{i}}}^{2} \cdot \mathrm{g}_{\lambda_{\mathrm{s}}}^{2} \approx \mathrm{g}^{4}
$$




\subsubsection{Signalverstärkung, Kontrast und Oberflächensensitivität}

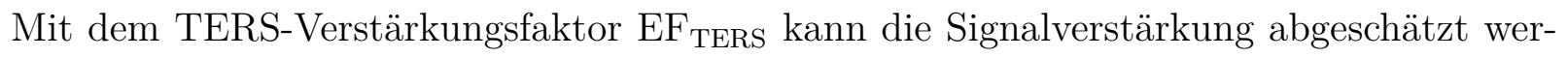
den. Da das Verhältnis der elektrischen Feldstärken von Nahfeld und Fernfeld nicht direkt bestimmt werden kann, sondern das verstärkte Raman-Signal mit an die Probenoberfläche angenäherter Spitze sowie das Fernfeld ohne Spitze gemessen werden, bietet sich für eine Verstärkungsquantifizierung ein Vergleich der Gesamtfeldintensität $I_{\text {tef }}=I_{\mathrm{nf}}+I_{\mathrm{ff}}$ (die Summe aus der Nahfeldintensität $I_{\mathrm{nf}}$ und der Fernfeldintensität $I_{\mathrm{ff}}$ ) bei angenäherter Spitze, mit der Fernfeldintensität $I_{\mathrm{ff}}$ ohne Spitze an. Der sogenannte TERS-Kontrast $C_{\text {TERS }}$ ergibt sich aus dem Intensitätsverhältnis von Gesamtfeld und Fernfeld [150, 168]:

$$
C_{\mathrm{TERS}}=\frac{I_{\mathrm{tef}}}{I_{\mathrm{ff}}}=\frac{I_{\mathrm{nf}}+I_{\mathrm{ff}}}{I_{\mathrm{ff}}} .
$$

Neben $C_{\text {TERS }}$ wird mit $C^{\prime}=I_{\text {nf }} / I_{\text {nf }}$ eine weitere Definition verwendet 150,168. Für große Werte ist der Unterschied wegen $C_{\text {TERS }}=C^{\prime}+1$ vernachlässigbar. In der Literatur finden sich beide Definitionen 118, 150,168 172]. Der Kontrast $C_{\text {TERS }}$ ist dabei praktikabler, da die Gesamtfeldintensität $I_{\text {tef }}$ und die Fernfeldintensität $I_{\text {ff }}$ experimentell gemessen werden und der Kontrast sich somit direkt abschätzen lässt 150, 168. Die TERS-Kontrastwerte in dieser Arbeit sind daher nach der Gleichung (3.4.3) bestimmt. Die Kontrast $C^{\prime}$ bietet sich an, wenn ein Vergleich mit Simulationen oder SERS-Messungen erfolgen soll [150,168, wobei die Nahfeldintensität $I_{\mathrm{nf}}$ nur indirekt zugänglich ist. Wird das Anregungsvolumen von Nahfeld und Fernfeld berücksichtigt, kann der TERS-Verstärkungsfaktor EF TERS $_{\text {ab- }}$ geschätzt werden, indem das Verhältnis der Nahfeldintensität $I_{\text {nf }}$ bezogen auf das durch das Nahfeld angeregte Probenvolumen $V_{\text {nf }}$ zur Fernfeldintensität $I_{\text {nf }}$ in Bezug auf das durch das Fernfeld angeregte Probenvolumen $V_{\mathrm{ff}}$ betrachtet wird [150,167]:

$$
\mathrm{EF}_{\mathrm{TERS}}=\frac{\mathrm{I}_{\mathrm{nf}}}{\mathrm{I}_{\mathrm{ff}}} \cdot \frac{\mathrm{V}_{\mathrm{ff}}}{\mathrm{V}_{\mathrm{nf}}}=\frac{\mathrm{I}_{\mathrm{tef}}-\mathrm{I}_{\mathrm{ff}}}{\mathrm{I}_{\mathrm{ff}}} \cdot \frac{\mathrm{V}_{\mathrm{ff}}}{\mathrm{V}_{\mathrm{nf}}}=\left(\mathrm{C}_{\mathrm{TERS}}-1\right) \cdot \frac{\mathrm{V}_{\mathrm{ff}}}{\mathrm{V}_{\mathrm{nf}}} .
$$

Das Fernfeld-Volumen $V_{\mathrm{ff}}=A_{\text {Fokus }} \cdot L_{\mathrm{ff}}=\pi R_{\text {Fokus }}^{2} L_{\mathrm{ff}}$ kann über die Fläche $A_{\text {Fokus }}$ des Laserpunkts und die Laser-Eindringtiefe $L_{\mathrm{ff}}$, die für dünne Proben durch die Schichtdicke $d$ und für dicke Proben durch die Fokaltiefe der Mikroskops begrenzt ist 168, 172, abgeschätzt werden. Die Evaluierung des Nahfeld-Volumens $V_{\text {nf }}$ ist komplexer, da die Größen nicht direkt messbar sind. Üblicherweise wird die vom Nahfeld angeregte Fläche mit dem Spitzenradius bestimmt. Untersuchungen zeigen jedoch, dass das räumliche TERS-Auflösungsvermögen mit einem effektiven TERS-Radius $R_{\text {TERS }}$, der kleiner als der Radius $R_{\text {Spitze }}$ der Spitze ist, darüber hinausgeht 173,174. In einer Näherung wird oft $R_{\text {TERS }} \approx \frac{1}{2} R_{\text {Spitze }} 167,175$ verwendet. Das vom Nahfeld angeregte Probenvolumen $V_{\mathrm{nf}}=A_{\mathrm{Nahfeld}} \cdot L_{\mathrm{nf}} \approx\left(\frac{1}{2} R_{\mathrm{TERS}}\right)^{2} \pi L_{\mathrm{ff}}$ kann dann über die Fläche $A_{\text {Nahfeld }}$ unter der Spitze 
und die Eindringtiefe $L_{\mathrm{nf}}$ des Nahfeldes in die Probe abgeschätzt werden. Diese lässt sich für dünne Schichten über die Entwicklung der TERS-Verstärkung mit der Schichtdicke bestimmen 49, 168, 172]. Zudem ist es über eine Abstandsvariation der Spitze zur Probe möglich die Nahfeldausdehnung in der Spitzenachsenrichtung zu bestimmen. Hierbei zeigt sich eine schnelle Abnahme des Nahfeldes bei einer Vergrößerung des Spitzenabstandes von der Probenoberfläche 162,174, 176 179. Wird zudem eine seitliche Einstrahlung wie in der Abbildung 3.4 .3 (a) verwendet, bei der der Laserstrahl und die Normale der Probenoberfläche den Winkel $\beta$ bilden, ist der Laserpunkt auf der Probe elliptisch.

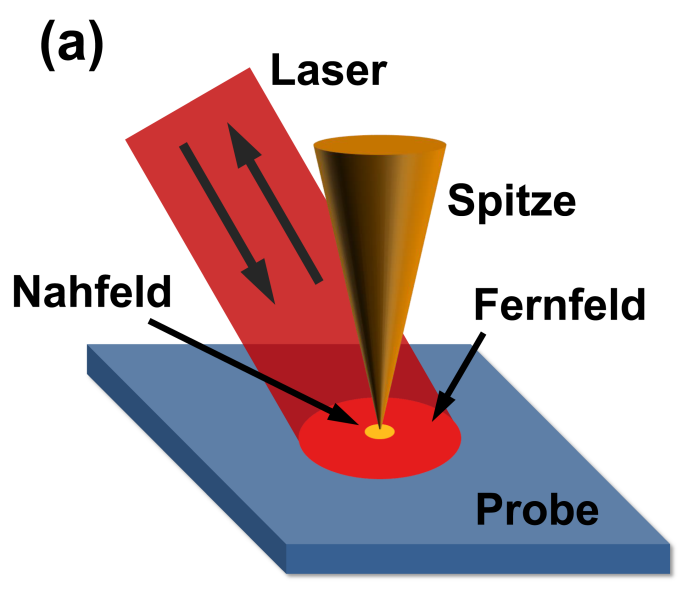

(b)

\begin{abstract}
Abbildung 3.4.3: TERS-Spitze in Seiteneinstrahlung mit Fernfeld und Nahfeld (a) sowie die Spitzenmodellierung mit kegelförmigem Schaft und kugelförmigem Spitzenbereich (Radius $R_{\text {Spitze }}$ ) im Abstand $z$ von der Probe (b) (nach [177]).
\end{abstract}

Dieses führt zu einer reduzierten fokalen Intensität für die Nahfeldanregung, während die geringere Intensitätsdichte beim Fernfeld durch die größere Anzahl an Molekülen im Fokus kompensiert wird 167]. Es ergibt sich daher die Relation $\mathrm{EF}_{\mathrm{TERS}} \propto \cos (\beta)$, sodass mit der Gleichung (3.4.4) für den Verstärkungsfaktor $\mathrm{EF}_{\text {TERS }}$ in seitlicher Einstrahlung folgt [167]:

$$
\mathrm{EF}_{\mathrm{TERS}} \approx\left(\frac{\mathrm{I}_{\text {tef }}}{\mathrm{I}_{\mathrm{ff}}}-1\right)\left(\frac{\mathrm{R}_{\text {Fokus }}}{\frac{1}{2} \mathrm{R}_{\text {Spitze }}}\right)^{2} \frac{\mathrm{L}_{\mathrm{ff}}}{\mathrm{L}_{\mathrm{nf}}} \cos (\beta) .
$$

Bei sehr dünnen Schichten ist $L_{\mathrm{ff}} \approx L_{\mathrm{nf}}$. Typische TERS-Verstärkungsfaktoren liegen je nach Messaufbau in einer Größenordnung $\mathrm{EF}_{\text {TERS }} \approx 10^{2}-10^{7}$ mit TERS-Kontrasten $C_{\text {TERS }} \approx 1,5-10000$ 150, 167, 168. Ein Vergleich von STM-TERS- und AFM-TERSSystemen zeigt, dass größere Verstärkungsfaktoren $\mathrm{EF}_{\mathrm{TERS}} \approx 10^{4}-10^{7}$ für STM-Systeme erzielt werden, während AFM-Systeme nur $\mathrm{EF}_{\text {TERS }} \approx 10^{2}-10^{4}$ erreichen $150,167,168$. Dieses wird hauptsächlich auf die Spitzen zurückgeführt 150,180. So sind TERS-STMSpitzen im Gegensatz zu beschichteten TERS-AFM-Spitzen vollständig aus Metall und verfügen über ein höheres Aspektverhältnis bei kleineren Öffnungswinkeln, was eine effizientere Kopplung mit dem Laserlicht und größere TERS-Verstärkungen ermöglicht [150,180]. Gleichzeitig ist der anregende Raman-Laser einfacher auf die STM-Spitze zu fokussieren 


\section{Experimentelle Methoden}

und das Einstellen eines geringen Arbeitsabstandes zur Probe $(z \approx 1 \mathrm{~nm})$ über den Tunnelstrom präziser 150,180]. Zudem besteht bei STM-TERS-Systemen die Möglichkeit, die Spitze mit einem metallischen Substrat plasmonisch zu koppeln (engl.: gap-mode, bezogen auf die Lücke zwischen Spitze und Substrat), indem der durch das Laserlicht in der Spitze induzierte Dipol über die angeregten SPP einen weiteren Dipol im metallischen Substrat induziert. Dieses führt bei passenden Plasmonenresonanzfrequenzen von Spitze und Substrat dazu, dass sich ein starkes Nahfeld in der Lücke zwischen Spitze und Substrat ausbildet und ein besonders hoher TERS-Verstärkungsfaktor für das Raman-Signal einzelner Moleküle, die in diesen Bereich gebracht werden, erreicht wird [118, 150, 180].

Experimentell lässt sich für die TERS-Nahfeldintensität $I_{\mathrm{nf}}$ eine schnelle Abnahme bei Vergrößerung des Abstands $z=z_{0}+\delta z$ von der Spitze beobachten, die oft als exponentiell beschrieben wird 162,174, 177, 179]. Theoretische Berechnungen in einem gekoppelten System von Spitze und Substrat, bei dem die Spitze wie in Abbildung 3.4.3 (b) (nach 177) durch einen kegelförmigen Schaft mit kugelförmigen Dipol (Radius $R_{\text {Spitze }}$ ) im Spitzenbe-

reich modelliert wird, zeigen jedoch, dass $\frac{I_{\mathrm{nf}}(z)}{I_{\mathrm{nf}}\left(z_{0}\right)}$ einem Potenzgesetz folgt $176,181,184$ :

$$
\frac{I_{\mathrm{nf}}(z)}{I_{\mathrm{nf}}\left(z_{0}\right)} \approx\left(1+\frac{z}{R_{\text {Spitze }}}\right)^{-p}
$$

Für eine vollständig inkohärente Raman-Streuung ergibt sich dabei ein Exponent $p=10$ und damit eine Abhängigkeit $I_{\mathrm{nf}} \propto\left(R_{\text {Spitze }}+z\right)^{-10}$ vom Abstand $z$ der Spitze 176, 181 184, sodass direkt die hohe Oberflächensensitivität, die durch das TERS-Nahfeld der Spitze lokal an der Probenoberfläche erreicht werden kann, ersichtlich wird 118, 150, 176, 181].

\subsubsection{Verwendeter TERS-Messaufbau}

Die TERS-Messungen erfolgen in dieser Arbeit mit einem STM-TERS-System, bei dem das Horiba LabRAM HR Evolution Raman-Spektrometer [133] aus dem Abschnitt 3.3.3 optisch mit dem Bruker Innova Rastertunnelmikroskop 79] aus dem Abschnitt 3.2.4 als integriertes Raman-Bildgebungssystem gekoppelt wird (Bruker Innova-IRIS, engl.: integrated Raman imaging system). Die optische Kopplung wird mithilfe eines LWD-Objektivs mit $50 \times$ Vergrößerung $(\mathrm{NA}=0,5)$ von Olympus in einer seitlichen Einstrahlung mit einem Winkel $\alpha=30^{\circ}$ zwischen dem einfallenden Laserstrahl und der Probenoberfläche in Rückstreugeometrie (Aufsammeln des TERS-Signals über den gleichen optischen Strahlengang) realisiert. Der Laserfokuspunkt des Objektivs kann dabei bis auf eine Fläche $A \approx 1 \mu^{2}$ (elliptische Form) verringert werden. Mithilfe eines Spiegels, der sich vor das Raman-Mikroskop schieben lässt, ist der TERS-Strahlengang an das Spektrometer koppelbar und ein Umschalten zwischen beiden Systemen möglich. Eine Übersicht des TERSAufbaus ist anhand von Fotos in den Abbildungen 3.4.4 (a), (b), (c) und (d) gezeigt. 


\subsection{Spitzenverstärkte Raman-Spektroskopie (TERS)}
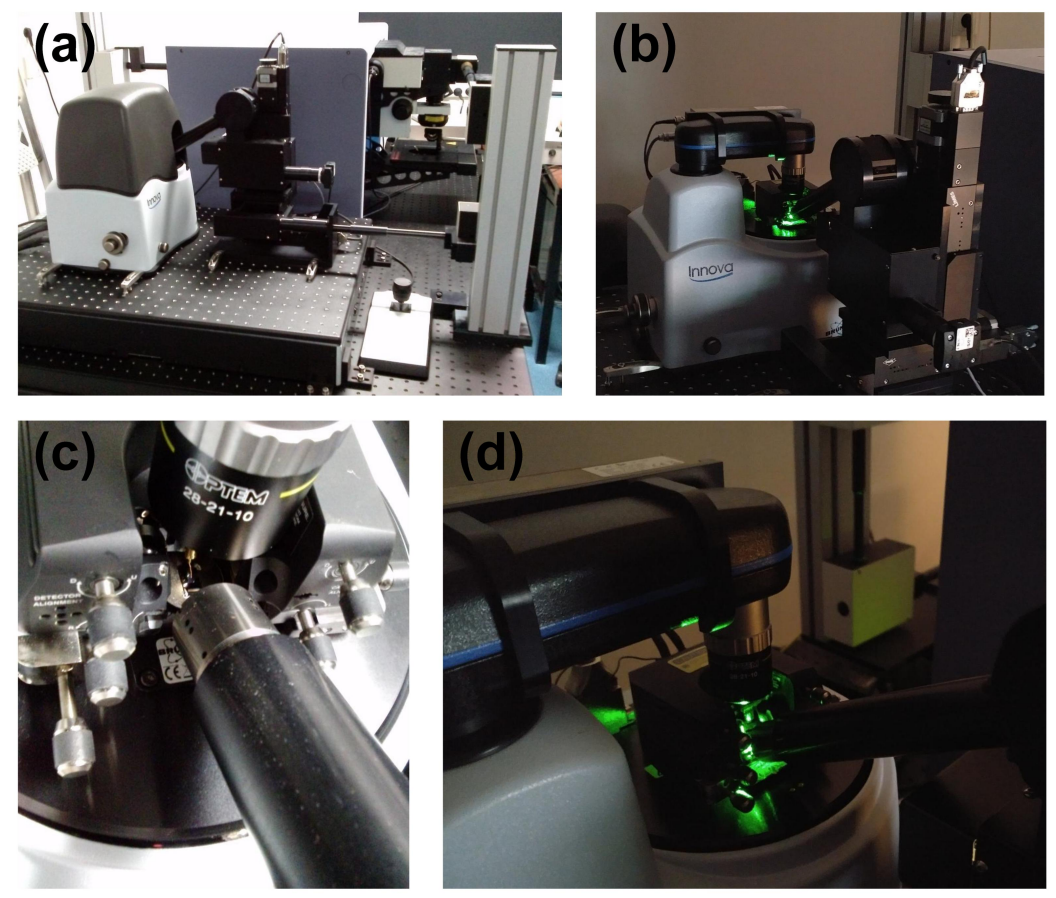

Abbildung 3.4.4: STM-TERS-Aufbau mit der Kopplung an das Raman-Spektrometer (a), die Rückstreugeometrie mit Seiteneinstrahlung bei eingekoppeltem grünen Laser (mit $10 \%$ Laserleistung) (b), der STM-Kopf mit der Goldspitze, dem LWD-Objektiv und der STM-Kamera (c) und die Ansicht bei eingekoppeltem grünen Laser mit 10 \% Laserleistung während einer TERS-Messung (d).

Das STM wird für die TERS-Messungen im Konstant-Strom-Modus (Abschnitt 3.2.4 mit aktiver Rückkopplung betrieben. Der Tunnelstrom $j$ und die Spannung $U_{\text {Bias }}$ sind von der elektrischen Leitfähigkeit und der $j\left(U_{\text {Bias }}\right)$-Kennlinie des Tunnelkontakts aus Probe und Spitze bestimmt 49, 176, 184. Daneben beeinflussen der Spitzenradius 93, 94 und die materialspezifischen Eigenschaften von Spitze und Probe [80, 176, 184, 185] den Tunnelstrom. Tunnelstrom und Spannung haben zudem einen großen Einfluss auf den Spitzenabstand $\delta z$ zur Probe und die TERS-Verstärkung [49, 184 187]. Typisch sind Tunnelströme $j \approx 0,01-2 \mathrm{nA}$ und Spannungen $U_{\text {Bias }} \approx \pm(0,01-2) \mathrm{V}$ [4, 171, 176, 177, 184,187 . Um eine Verwechselung mit der Raman-Intensität $I$ und dem Probenbezugssystem $(x, y, z)$ zu vermeiden, wird der Tunnelstrom im Folgenden mit $j$ und der Spitzenabstand von der Probenoberfläche mit $\delta z$ bezeichnet. Den größten Einfluss hat jedoch die Spitze selbst, da diese abhängig von Material, Form und Radius die Plasmonenresonanz, die Nahfeldeigenschaften und das TERS-Auflösungsvermögen bestimmt [150]. In dieser Arbeit kommen elektrochemisch geätzte Goldspitzen von Bruker zum Einsatz [188], die mit dem REM auf ihre Form und den Radius untersucht wurden. Mit der $j(\delta z)$-Kennlinie kann der Abstand $\delta z$ der Spitze von der Probe in Abhängigkeit des Tunnelstroms $j$ bestimmt werden (Gleichung (3.2.16)). Um Spitzenschäden zu vermeiden, wird der Tunnelstrom in kleinen Schritten erhöht. Danach werden $j$ und $U_{\text {Bias }}$ so angepasst, dass sich ein stabiler Tunnelstrom bei einem Spitzenabstand $\delta z \approx 1 \mathrm{~nm}$ zur Probe einstellt. Je nach Spitze und Probe ist hierfür eine Mindestspannung erforderlich, die sich aus der $j\left(U_{\text {Bias }}\right)$-Kennlinie ergibt. 


\section{Experimentelle Methoden}

Ist die Spitze in einer stabilen Tunnelposition, muss der Laser zwischen Spitze und Probenoberfläche fokussiert werden. Hierfür ist der Teil des TERS-Strahlengangs mit dem LWD-Objektiv auf einem motorisierten Tisch installiert, um das Objektiv horizontal und vertikal zum STM zu verschieben und den Laserfokuspunkt zu variieren. Über einen Strahlteiler kann eine CMOS-Kamera mit Halogenbeleuchtung dazu geschaltet werden, die eine seitliche Betrachtung ermöglicht. Zudem kann die STM-Kamera mit 10× Vergrößerung in senkrechter Perspektive verwendet werden. Mit den zwei Kameras wird nun der Laserfokuspunkt zuerst auf die Spitze und dann zwischen Spitze und Probenoberfläche gebracht. Diese Position ist für eine der Spitzen in den Abbildungen 3.4.5 (a)-(e) dargestellt.
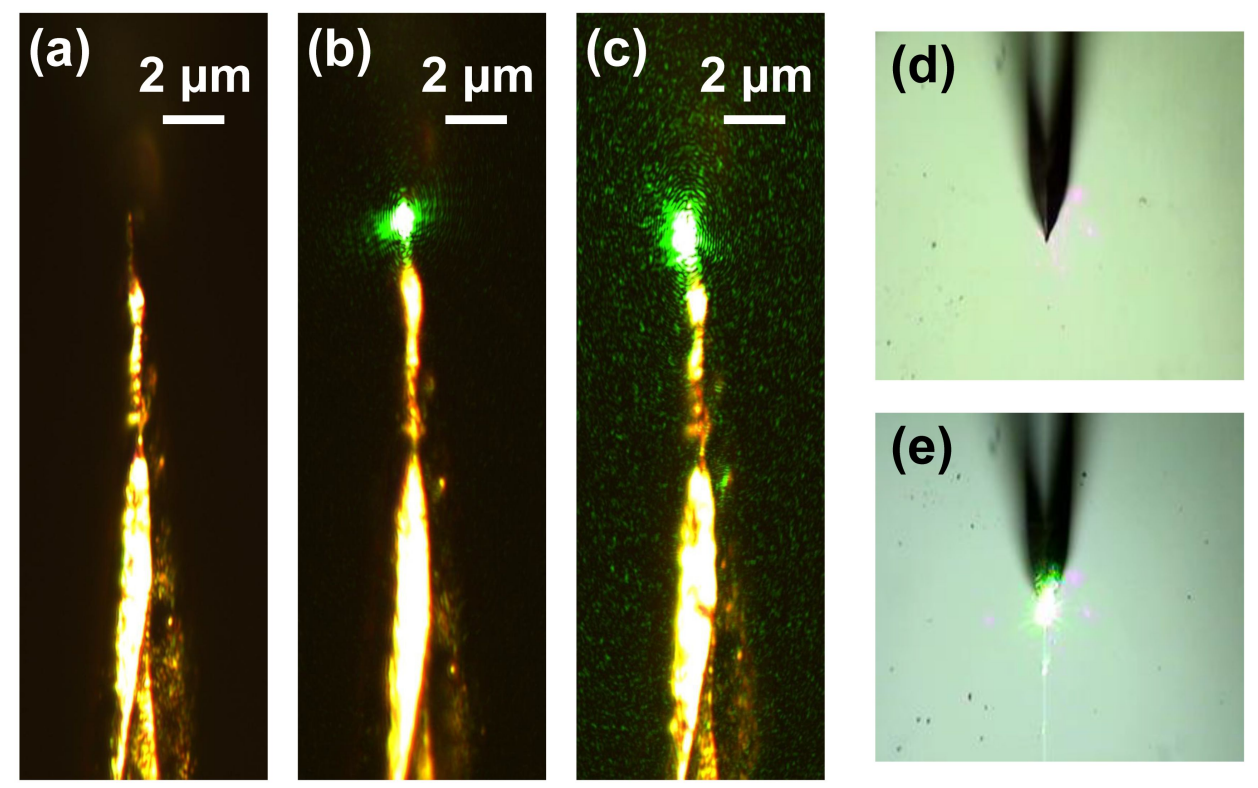

Abbildung 3.4.5: Bilder einer an eine Probe angenäherte TERS-Goldspitze, aufgenommen mit der seitlichen TERS-Kamera und Halogenlampenbeleuchtung ohne Laser (a) und mit dem grünen Laser auf die Spitze fokussiert (1 \% Laserleistung (b) und $5 \%$ Laserleistung (c)). Die Bilder zeigen die Spitze aufgrund des Strahlengangs der Kamera verkehrt herum nach oben deutend. Zusätzlich ist die Situation mithilfe der STM-Kamera von oben ohne Laser (d) und mit dem grünen Laser bei einer Laserleistung von $5 \%$ (e) dargestellt. Zu erkennen ist dabei neben der Spitze aus fast senkrechter Perspektive auch ihr Schatten.

Die TERS-Messungen erfolgen bei Raumtemperatur unter klimatisierter Umgebungsluft. Durch die Luftfeuchtigkeit ist die Probenoberfläche daher von einer einige Nanometer dünnen Wasserschicht bedeckt, mit der die Spitze in Wechselwirkung tritt [83, 189]. Ein entsprechenden Einfluss kann diese damit auf den Abstand der Spitze zur Probenoberfläche, die STM-Parameter und die TERS-Verstärkung haben [186]. Für eine Entkopplung von mechanischen Schwingungen befindet sich das TERS-System wie im normalen STMund AFM-Betrieb auf einem aktiv gedämpften Vibrationsausgleichstisch. 


\title{
3.4.5 Polarisationsabhängige TERS-Messungen
}

Die TERS-Messungen können auch polarisationsabhängig durchgeführt werden. Hierfür stehen mit dem $\lambda / 2$-Plättchen (Polarisator) für die eingestrahlte Laserpolarisation und dem Polarisationsfilter mit zwei Stellungen für den vertikalen und den horizontalen Polarisationsanteil als Analysator vor dem Detektor die gleichen Komponenten wie für den Raman-Aufbau zur Verfügung. Die TERS-Messungen werden jedoch in Rückstreugeometrie mit seitlicher Einstrahlung durchgeführt. Entsprechend verschieden sind das Probenund das Laborbezugssystem orientiert. Während das Laborbezugssystem $\left(x_{0}, y_{0}, z_{0}\right)$ über die Richtung des einfallenden Laserstrahls und den dazu senkrechten Richtungen linearer Polarisation festgelegt wird und dem System für die Raman-Messungen entspricht, bietet es sich an das Probenbezugssystem $(x, y, z)$ in Relation zur Spitzenachse und der Kristallorientierung der Probe zu definieren und die Polarisation des Laserlichts in einen Anteil linearer Polarisation parallel zur Spitzenachse und einen Anteil senkrecht zur Spitzenachse zu trennen. In der Literatur hat sich die Bezeichnung P für parallel zur Spitzenachse und S für senkrecht zur Spitzenachse etabliert $49,169,170,190,194$. Eine Übersicht des Strahlengangs, der Definition der Bezugssysteme und der Polarisationskonfigurationen in Relation zur TERS-Spitze sowie der Probenorientierung ist in der Abbildung 3.4.6 dargestellt.
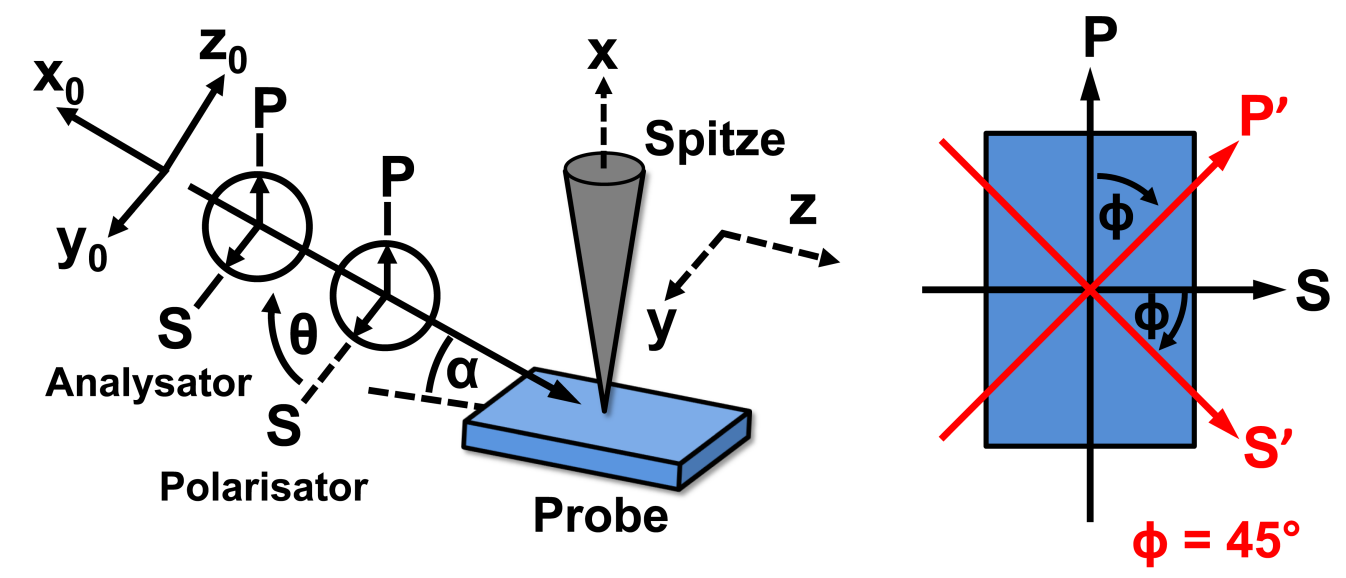

\begin{abstract}
Abbildung 3.4.6: Definition des Proben- und Laborbezugssystems für die Durchführung polarisationsabhängiger TERS-Messungen bei Einstrahlung von der Seite (mit $\alpha=30^{\circ}$ ) und die jeweiligen Polarisationskonfigurationen in Relation zur TERS-Spitzenachse und der Probenorientierung (nach [49]).
\end{abstract}

Die PP- und die SS-Konfigurationen bezeichnen in der Porto-Notation parallele Polarisationskonfigurationen, für die die Analysator-Stellung parallel zur Laserpolarisation orientiert ist $\left(\hat{e}_{i} \| \hat{e}_{s}\right)$, während die PS- und die SP-Konfigurationen gekreuzte Polarisationskonfigurationen beschreiben, bei denen die Analysator-Stellung senkrecht zur Laserpolarisation orientiert ist $\left(\hat{e}_{i} \perp \hat{e}_{s}\right)$. Da der Laser ohne Polarisator durch die Spiegelanordnung im Strahlengang eine horizontale Polarisation und in Relation zur Spitzenachse eine S-Polarisation aufweist $\left(\theta=0^{\circ}\right.$ für das $\lambda / 2$-Plättchen), ergibt sich die vertikale P-Polarisation $\left(2 \theta=90^{\circ}\right)$ durch Drehen des $\lambda / 2$-Polarisators um $\theta=45^{\circ}$. Gegenüber dem Raman-Aufbau ist diese im 


\section{Experimentelle Methoden}

TERS-System daher um $90^{\circ}$ gedreht. Zudem kann die Probe in-plane gedreht werden. Ist das Probenbezugssystem out-of-plane entlang der TERS-Spitzenachse senkrecht zur Probenoberfläche sowie in-plane entlang der Kristallachsen der Probe ausgerichtet, ergibt sich für eine Drehung um einen Winkel $\phi=45^{\circ}$ um die Spitzenachse eine Konfiguration, die mit einem Strichindex markiert wird $33,39,40,49$. Eine $\mathrm{P}^{\prime}$-Polarisation bezeichnet eine Polarisation parallel zur Spitzenachse und eine $\mathrm{S}^{\prime}$-Polarisation eine Polarisation senkrecht zur Spitzenachse. Folglich sind die $\mathrm{P}^{\prime} \mathrm{P}^{\prime}$ - und die $\mathrm{S}^{\prime} \mathrm{S}^{\prime}$-Konfigurationen parallele $\left(\hat{e}_{i} \| \hat{e}_{s}\right)$ und die $\mathrm{P}^{\prime} \mathrm{S}^{\prime}$ - und die $\mathrm{S}^{\prime} \mathrm{P}^{\prime}$-Konfigurationen gekreuzte Polarisationskonfigurationen $\left(\hat{e}_{i} \perp \hat{e}_{s}\right)$. Für den TERS-Messaufbau müssen die Raman-Tensoren $\mathcal{R}_{k}$ der Kristallstruktur der Probe dabei sowohl für eine in-plane Drehung als auch für die Überführung aus dem Proben- in das Laborbezugssystem auf die Streugeometrie mit Seiteneinstrahlung und das Laborbezugssystem des Raman-Spektrometers angepasst werden. Dieses ist nötig, da die RamanTensoren $\mathcal{R}_{k}$ in der üblichen Form für eine senkrechte Rückstreugeometrie mit identischer Proben- und Laborbezugssystemorientierung entlang der $\langle 100\rangle$-Kristallhauptachsen angegeben sind. Dieses wird mit einem Transformationstensor $T$ realisiert. Da Raman-Tensoren stets Tensoren 2. Stufe sind, folgt für den vom Probenbezugssystem $(x, y, z)$ in das Laborbezugssystem $\left(x_{0}, y_{0}, z_{0}\right)$ überführten Raman-Tensor $\mathcal{R}_{k}^{\prime} 49,169,194$ :

$$
\mathcal{R}_{k}^{\prime}=T^{T} \mathcal{R}_{k} T
$$

Hierbei ist $T^{T}$ die transponierte Form des Transformationstensors $T$. Da beide Bezugssysteme in einem kartesischen Koordinatensystem beschrieben werden, erfolgt die Überführung durch eine Rotation im Raum. Die Transformationstensoren sind damit Drehmatrizen (orthogonale Matrizen mit der Eigenschaft $T^{T}=T^{-1}[195]$ ). Eine Rücktransformation vom Labor- $\left(x_{0}, y_{0}, z_{0}\right)$ in das Probenbezugssystem $(x, y, z)$ ist daher durch $\mathcal{R}_{k}=T \mathcal{R}_{k}^{\prime} T^{T}$ gegeben. Für das Probenbezugssystem $(x, y, z)$ mit der TERS-Spitzenachse in x-Richtung senkrecht über der Probe (Abbildung 3.4.6) ergibt sich für die modifizierten Raman-Tensoren $\mathcal{R}_{k}^{\prime}$ für eine Probenrotation um den Winkel $\phi$ um die Spitzenachse herum 169, 194]:

$$
\mathcal{R}_{k}^{\prime}=T_{x}^{T}(\phi) \mathcal{R}_{k} T_{x}(\phi)
$$

Anschließend muss das Probenbezugssystem $(x, y, z)$ in der Rückstreugeometrie mit Seiteneinstrahlung (hier $\alpha=30^{\circ}$ ) in das Laborbezugssystem $\left(x_{0}, y_{0}, z_{0}\right)$ überführt werden, indem dieses um den Winkel $\beta=90^{\circ}-\alpha$ zwischen Lasereinfallsrichtung und Spitzenachse um die eigene y-Achse gedreht wird. Für die überführten Raman-Tensoren $\mathcal{R}_{k}^{\prime \prime}$ folgt $49,169,194$ :

$$
\mathcal{R}_{k}^{\prime \prime}=T_{y}^{T}(\beta) \mathcal{R}_{k}^{\prime} T_{y}(\beta) .
$$


Dabei ist $T_{x}(\phi)$ die Rotationsmatrix für die Drehung der Probe um den Winkel $\phi$ um die in x-Richtung orientierte Spitzenachse und $T_{y}(\beta)$ die Rotationsmatrix für die Drehung des Probenbezugssystems $(x, y, z)$ im TERS-Messaufbau um den Winkel $\beta=90^{\circ}-\alpha$ um die y-Achse (Abbildung 3.4.6 für die Überführung in das Laborbezugssystem $\left(x_{0}, y_{0}, z_{0}\right)$ [195]:

$$
T_{x}(\phi)=\left(\begin{array}{ccc}
1 & 0 & 0 \\
0 & \cos (\phi) & -\sin (\phi) \\
0 & \sin (\phi) & \cos (\phi)
\end{array}\right), \quad T_{y}(\beta)=\left(\begin{array}{ccc}
\cos (\beta) & 0 & \sin (\beta) \\
0 & 1 & 0 \\
-\sin (\beta) & 0 & \cos (\beta)
\end{array}\right)
$$

In einem Rechtssystem sind die Drehwinkel dabei positiv für Drehungen im mathematisch positiven Sinn gegen dem Uhrzeigersinn 195. Für die in der Abbildung 3.4.6 dargestellte in-plane Drehung der Probe im Uhrzeigersinn ist daher $\phi<0^{\circ}$. Für entlang der $\langle 100\rangle$ Hauptachsen orientierte Kristalle können die Polarisationsvektoren $\hat{e}_{i}$ und $\hat{e}_{s}$ alternativ bei Beibehaltung der Raman-Tensoren $\mathcal{R}_{k}$ mit $T_{x}(\phi)$ multipliziert werden 49]. Für entlang der $\langle 100\rangle$-Hauptkristallachsen orientierte Schichten werden die an den TERS-Messaufbau angepassten Raman-Tensoren $\mathcal{R}_{k}^{\prime \prime}$ mit den Gleichungen 3.4.8) und 3.4.9) bestimmt. Für Schichten, deren Kristallachsen nicht entlang der $\langle 100\rangle$-Hauptachsen ausgerichtet sind, müssen die Raman-Tensoren in ein an die Orientierung angepasstes Probenbezugssystem überführt werden. Dieses kann durch eine mehrschrittige Rotation realisiert werden. Dieser Prozess ist für Schichten mit einer (111)-Orientierung in der Abbildung 3.4.7 dargestellt.
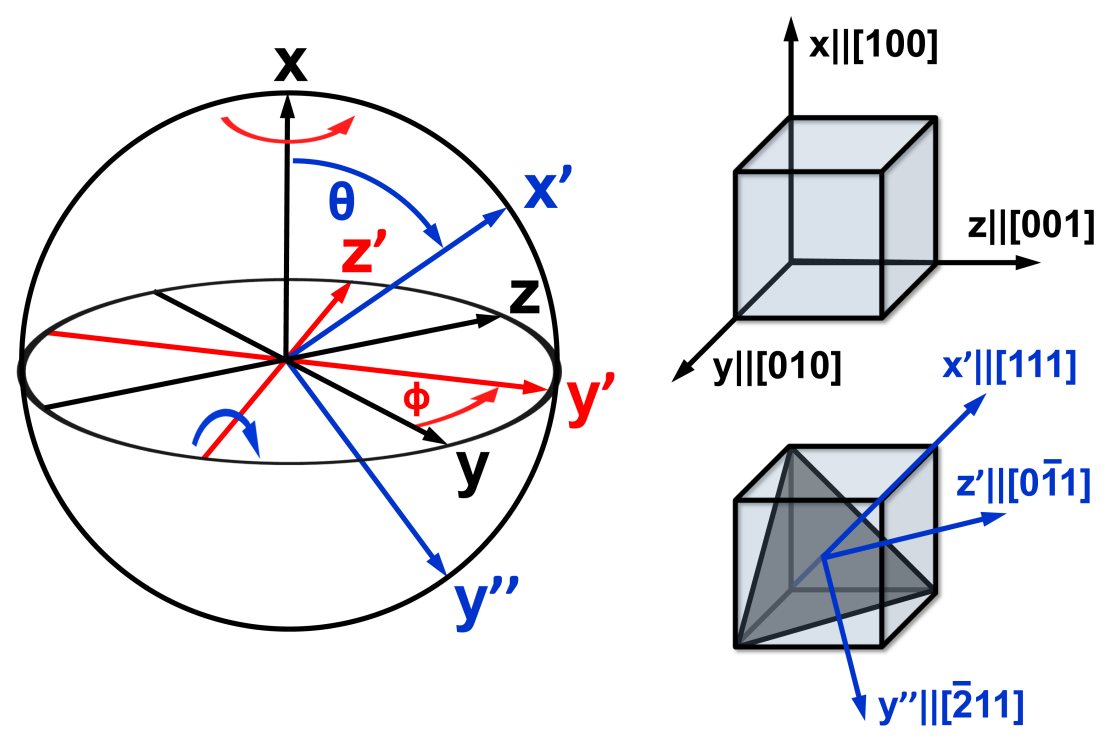

Abbildung 3.4.7: Rotationsabfolge für die Überführung des TERS-Probenbezugssystems von der (100)- in die (111)-Orientierung und die Lage der beiden Bezugssysteme für kubische Kristalle mit (100)- und (111)-Orientierung.

Ausgangspunkt ist das Bezugssystem entlang der $\langle 100\rangle$-Hauptachsen mit einer out-of-plane Orientierung $x \|$ [100] entlang der Spitzenachse und den in-plane Orientierungen $y \|$ [010] und $z \|[001]$. Zunächst wird dieses mit der Gleichung 3.4.8 um einen Winkel $\phi=45^{\circ}$ 


\section{Experimentelle Methoden}

um die Spitzenachse herum gedreht, sodass sich die Richtungen $y^{\prime} \|$ [011] und $z^{\prime} \|[0 \overline{1} 1]$ ergeben. Anschließend wird das System um einen Winkel $\theta$ um die $z^{\prime}$-Achse herum gedreht. Der Drehwinkel $\theta=\arccos (1 / \sqrt{3}) \approx 54,736^{\circ}$ zwischen der $\mathrm{x}$-Achse und der $\mathrm{x}^{\prime}$-Achse $\left(x^{\prime} \|\right.$ [111]) ergibt sich aus dem Skalarprodukt der Richtungsvektoren. Da die Drehachse mit dem Richtungsvektor $\hat{a}^{T}=\left(0, \frac{-1}{\sqrt{2}}, \frac{1}{\sqrt{2}}\right)$ durch den Koordinatenursprung verläuft, kann die Matrixform der Rodrigues-Formel verwendet werden, mit der sich eine Drehung um eine durch den Ursprung verlaufene Drehachse mit Richtung $\hat{a}$ beschreiben lässt [195, 196]:

$$
T(\hat{a}, \theta)=I \cos (\theta)+\sin (\theta)[\hat{a}]_{\times}+(1-\cos (\theta)) \hat{a} \otimes \hat{a} .
$$

Hierbei bezeichnet $I$ die Einheitsmatrix, $\hat{a}$ den Einheitsvektor der Drehachsenorientierung, $[\hat{a}]_{\times}$die Kreuzproduktmatrix des Vektors $\hat{a}$ und $\hat{a} \otimes \hat{a}$ das dyadische Produkt des Vektors $\hat{a}$ mit sich selbst. Die Gesamtmatrix $T$ zur Überführung der Raman-Tensoren $\mathcal{R}_{k}$ von der (100)-Orientierung in die (111)-Orientierung ergibt sich schließlich aus dem Produkt $T_{(111)}(\theta, \hat{a}, \phi)=T_{z^{\prime}}(\theta, \hat{a}) \cdot T_{x}(\phi)$ der beiden Teilrotation in entsprechender Reihenfolge:

$$
T_{(111)}(\theta, \hat{a}, \phi)=T_{z^{\prime}}\left(\hat{z}^{\prime}, \theta=\arccos (1 / \sqrt{3})\right) \cdot T_{x}\left(\phi=45^{\circ}\right)=\left(\begin{array}{ccc}
\frac{1}{\sqrt{3}} & \frac{-\sqrt{2}}{\sqrt{3}} & 0 \\
\frac{1}{\sqrt{3}} & \frac{1}{\sqrt{6}} & \frac{-1}{\sqrt{2}} \\
\frac{1}{\sqrt{3}} & \frac{1}{\sqrt{6}} & \frac{1}{\sqrt{2}}
\end{array}\right)
$$

Die Spalten von $T_{(111)}(\theta, \hat{a}, \phi)$ repräsentieren dabei die Achsen des Probenbezugssystems mit $x^{\prime} \|$ [111], $y^{\prime \prime} \|$ [211] und $z^{\prime} \|$ [011] (Abbildung 3.4.7). Für die Raman-Tensoren $\mathcal{R}_{k,(111)}^{\prime}$ der (111)-Probenorientierung folgt $\mathcal{R}_{k,(111)}^{\prime}=T_{(111)}^{T} \mathcal{R}_{k} T_{(111)}$. Diese müssen dann mit der Gleichung (3.4.9) in das Laborbezugssystem überführt werden. Eine Abschätzung der Reflexion und Transmission des Laserlichts an der Schichtoberfläche und der Grenzfläche von Schicht und Substrat in der seitlichen Einstrahlung in Abhängigkeit der Polarisation, des Einfallwinkels $\beta$ und der Brechungsindizes von Schicht und Substrat ist mit dem Snelliusschen Brechungsgesetz und der Fresnelschen Formeln möglich [169, 194, 197]. Für die TERS-Messungen muss zudem der Einfluss der Spitze miteinbezogen werden. Hierzu kann ein phänomenologisches Modell verwendet werden, das die Signalverstärkung und die Polarisationsabhängigkeit mit einem Spitzenverstärkungstensor $A$ beschreibt [169, 198, 199]. Unter der Voraussetzung, dass die TERS-Spitze senkrecht zur Probenoberfläche orientiert ist, hat dieser die vereinfachte Form einer diagonalisierten Matrix [169, 198, 199]:

$$
A=\left(\begin{array}{ccc}
a_{1} & 0 & 0 \\
0 & a_{2} & 0 \\
0 & 0 & a_{3}
\end{array}\right)
$$


Hierbei sind die Koeffizienten $a_{1}, a_{2}$ und $a_{3}$ die phänomenologischen Spitzenverstärkungsfaktoren, deren Größe von der geometrischen Charakteristik der Spitze (Form, Spitzenradius, Aspektverhältnis, Oberfläche) und materialspezifischen Spitzeneigenschaften (Material, Beschichtung, Plasmonenresonanzfrequenz, Permittivität) beeinflusst ist 169,198. Ihr Verhältnis bestimmt zudem die Affinität der Spitze elektrische Feldkomponenten des Lichts mit verschiedener Polarisation unterschiedlich zu verstärken, mit einer experimentell und theoretisch beobachteten Bevorzugung einer Verstärkung für eine Polarisation parallel zur Spitzenachse $169,170,190,191,199,200]$. Für eine rotationssymmetrische Spitze in xRichtung folgt daher $a_{1} \gtrsim a_{2} \approx a_{3}$. Die Überführung der Raman-Tensoren $\mathcal{R}_{k}$ in den Nahfeldeinfluss der angenäherten Spitze wird mithilfe des Spitzenverstärkungstensors $A$ und der Gleichung (3.4.7) realisiert. Für die TERS-Raman-Tensoren $\mathcal{R}_{k, \text { TERS }}$ folgt 169, 199]:

$$
\mathcal{R}_{k, \mathrm{TERS}}=A^{T} \mathcal{R}_{k} A
$$

Der Spitzenverstärkungstensor $A$ muss dabei mit der Gleichung (3.4.9) wie die RamanTensoren $\mathcal{R}_{k}$ in das Laborbezugssystem überführt werden. Die Vor- und Nach-Multiplikation der Raman-Tensoren $\mathcal{R}_{k}$ mit dem Spitzenverstärkungstensor $A$ in der Gleichung 3.4.14 charakterisiert die Wechselwirkung der Spitze mit dem elektrischen Feld der einfallenden Laserstrahlung und dem gestreuten Feld. Die TERS-Raman-Tensoren $\mathcal{R}_{k, \text { TERS }}$ beschreiben somit die effektiven Raman-Tensoren des Systems aus Spitze und Probe 169, 199. Im Allgemeinen ist der Spitzenverstärkungstensor $A$ zusätzlich von der Frequenz des Laserlichts abhängig, da die Stärke des Spitzennahfeldes von der Plasmonenresonanzfrequenz der Spitze und der Resonanzkurvenform abhängt. Für Silberspitzen, für die die Nahfeldstärke bereits für kleine Abweichungen von der Resonanzfrequenz sehr schnell abfällt [157, 158, 164, 174], und für Raman-Moden $>1000 \mathrm{~cm}^{-1}$ (eine Wellenlängenverschiebung einiger $10^{1} \mathrm{~nm}$ ) muss daher der Spitzenverstärkungstensor $A$ für Anregung und Streuung gesondert mit einem Tensor $A_{i}(\omega)$ für das Laserlicht mit der Frequenz $\omega$ und mit einem Tensor $A_{w}\left(\omega^{\prime}\right)$ für das mit der Frequenz $\omega^{\prime}$ gestreute Licht $\left(\operatorname{mit}\left(A_{i}(\omega) \neq A_{s}\left(\omega^{\prime}\right)\right)\right)$ betrachtet werden [169, 199]. Für Goldspitzen (wie hier), die üblicherweise über eine gleichmäßigeres Plasmonenresonanzverhalten und einen breiten Resonanzfrequenzbereich verfügen [158 160, 164, kann für Raman-Moden mit Wellenzahlen, die klein gegenüber der charakteristischen Plasmonenresonanzbreite der Spitze sind, in Näherung ein identischer Spitzenverstärkungstensor $A_{i} \approx A_{s}=A$ für die einfallende und gestreute Strahlung verwendet werden [169,199].

\subsubsection{Spitzeneffekte und Nahfeldeffekte}

Experimentelle Beobachtungen zeigen jedoch, dass die Wechselwirkung von Spitze, Nahfeld und Probe und die Zusammensetzung des TERS-Signals sehr komplex ist, da neben der plasmonischen Verstärkung des Raman-Signals durch das Spitzennahfeld weitere durch das 


\title{
3 Experimentelle Methoden
}

Nahfeld und die Spitze initiierte Effekte auftreten können [161, 169, 190 194, 199, 201, 209], die zum TERS-Signal beitragen und dieses beeinflussen. Entsprechend wichtig ist die Identifizierung und Separierung dieser Effekte, um den Einfluss auf das TERS-Signal zu bestimmen. Ein Hauptaspekt stellt die Nahfeldwirkung auf die Raman-Moden in polarisationsabhängigen TERS-Untersuchungen dar. Wie im phänomenologischen Modell mit dem Spitzenverstärkungstensor $A$ berücksichtigt, zeigt sich für TERS-Spitzen mit großem Aspektverhältnis und kleinem Öffnungswinkel eine starke Anisotropie in der Polarisierbarkeit mit einer bevorzugten Nahfeldpolarisation entlang der Spitzenachse [169, 170, 190, 191, 199,200 . Dieses Polarisationsaffinität des Nahfeldes folgt aus der Spitzensymmetrie und ist schematisch anhand der Feldlinien im Dipol-Modell für ein STM-TERS-System im gap mode in der Abbildung 3.4.8 (a) (nach 122]) dargestellt. Es ergibt sich eine mehrheitliche Feldkomponente parallel zur Spitzenachse, die jedoch nur direkt unterhalb der Spitze eine reine P-Polarisation aufweist, während sich abseits von der Spitzenachse durch die Feldlinienkrümmung eine gemischte Nahfeldpolarisation (Depolarisation) mit Komponenten sowohl parallel (P) als auch senkrecht (S) zur TERS-Spitzenachse einstellt [199].
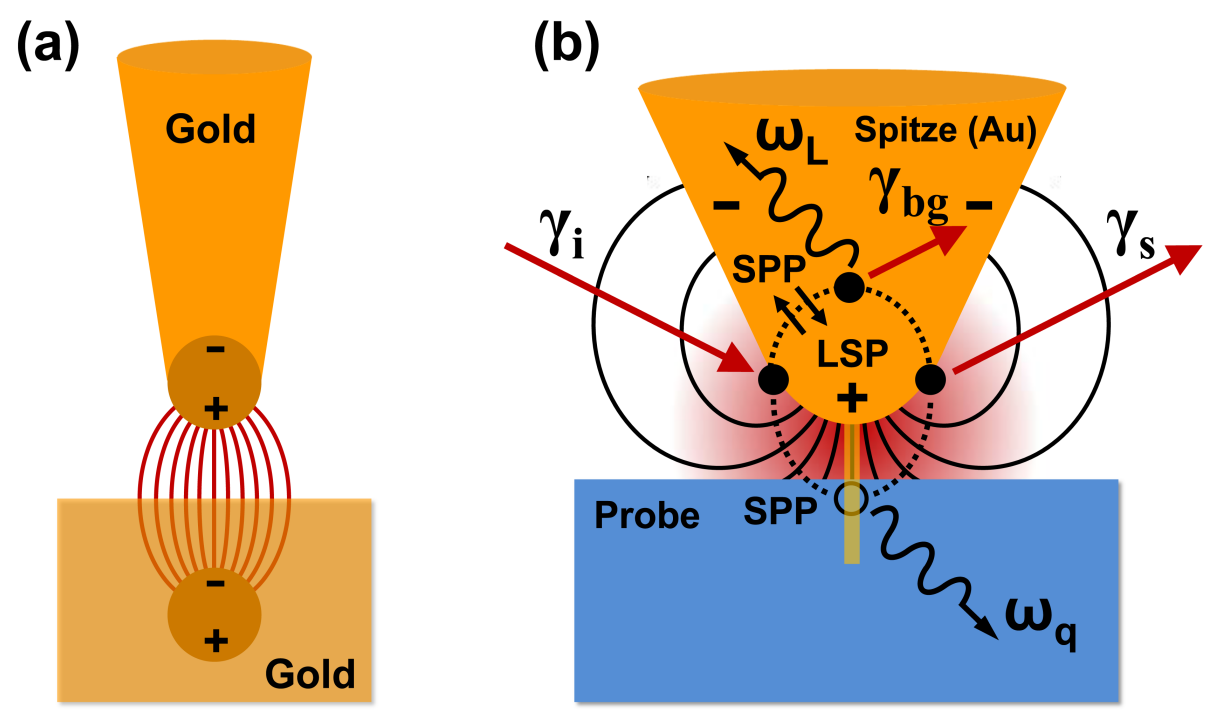

\begin{abstract}
Abbildung 3.4.8: Effektive Nahfeldpolarisation eines gekoppelten TERS-Systems aus Spitze und Probe im Modell eines kugelförmigen Spitzendipols (a) (nach [122]) und schematische Darstellung der Wechselwirkungen zwischen Licht, Spitze, Nahfeld und Probe im Zuge eines quantenmechanischen Modells zur Erklärung der Depolarisation des TERS-Spitzennahfeldes (b) (nach [209]).
\end{abstract}

Dieses führt dazu, dass die Nahfeldpolarisation von der Fernfeldpolarisation und damit die Raman-Auswahlregeln für Nah- und Fernfeld zu einem bestimmten Teil voneinander abweichen. Experimentelle Beobachtungen bei polarisationsabhängigen TERS-Messungen an kristallinen Proben wie Silizium bestätigen eine im Allgemeinen höhere Verstärkung und Nahfeldintensität für eine Laserpolarisation parallel (P) zur Spitzenachse, zeigen jedoch, dass sich ein besonders hoher TERS-Kontrast für gekreuzte Polarisationskonfigurationen oder Polarisationskonfigurationen, für die das Fernfeld durch die Raman-Auswahlregeln 
stark verringert ist, einstellt $148,169,170,172,192,193,210]$. Dieses von der Depolarisation des Nahfeldes verursachte Verhalten lässt sich auch gezielt nutzen, um ein starkes Hintergrundsignal durch das Fernfeld aus dem TERS-Signal zu filtern 122, 148, 170, 172, 194. Wird nun das phänomenologische TERS-Modell mit dem Spitzenverstärkungstensor $A$ betrachtet, so beschreibt dieses zunächst eine unendlich dünne Spitze, die senkrecht zur Probenoberfläche ausgerichtet ist, ohne dass die Form, die Ausrichtung und die Oberflächenbeschaffenheit realer Spitzen einbezogen wird [169,199]. Um die reale Spitze und die Nahfelddepolarisation zu berücksichtigen, muss daher die tensorielle Antwort über die finiten Öffnungswinkel der Spitze gemittelt werden [169]. Alternativ kann der diagonalisierte Spitzenverstärkungstensor $A$ um Nichtdiagonaleinträge, die eine Nahfeldepolarisation in gekreuzten Polarisationskonfigurationen berücksichtigen [190,199, ergänzt werden:

$$
A_{\mathrm{TERS}}=\left(\begin{array}{ccc}
a_{x x} & a_{x y} & a_{x z} \\
a_{y x} & a_{y y} & a_{y z} \\
a_{z x} & a_{z y} & a_{z z}
\end{array}\right)
$$

Hierbei sind die Koeffizienten $a_{i j}$ von der TERS-Spitze und der Orientierung des Probenbezugssystems abhängig. Ein darauf aufbauender Ansatz, der den TERS-Tensor in einen Spitzenverstärkungstensor $A$ für die Nahfeldverstärkung und einen Depolarisationstensor $B$ für die Nahfelddepolarisation aufteilt und die Berechnung beider Anteile am Nahfeld ermöglicht, ist im Zuge dieser Arbeit entstanden und wird in [49] sowie im Abschnitt 4.2.3 am Beispiel von TERS-Messungen an dünnen $\mathrm{La}_{2} \mathrm{CoMnO}_{6}$-Schichten dargestellt.

Die Wechselwirkung von Licht, Spitze und Probe lässt sich zudem in einem quantenmechanischen Modell beschreiben [209], das über den klassischen Ansatz der Spitzenmodellierung als punktförmiger Dipol hinausgeht und den TERS-Prozess und die Nahfeldpolarisation über die plasmonische Kopplung des Lichts im Einfluss von Spitze und Probe beschreibt. Die Prozessabfolge dieses Modellvorschlags ist in der Abbildung 3.4.8 (b) (nach [209]) gezeigt. Zunächst werden durch die eingestrahlten Photonen $\left(\gamma_{i}\right)$ durch Interaktion mit der TERS-Goldpsitze SPP und LSP angeregt, die miteinander agieren und wechselseitige Zerfallskanäle ineinander besitzen, wobei der Zerfall der LSP in SPP nichtstrahlend und der Zerfall der SPP in LSP strahlend ist. Daneben besitzen auch die LSP einen strahlenden Kanal, der durch Energieverluste an der Spitzenoberfläche entsteht $\left(\omega_{L}\right)$ und zum TERSHintergrundsignal $\left(\gamma_{b g}\right)$ beiträgt. Wird nun die Probe in die Nähe der Spitze gebracht, so wirkt diese als Störung für die Ausbreitung des von den SPP generierten evaneszenten Nahfeldes und eröffnet neue Zerfallskanäle für die SPP und LSP in Form eines Tunnelprozesses von Photonen von der Spitze in die Probenoberfläche 209,211 213. Hierbei entkoppeln die Photonen von den SPP, wodurch diese ausgelöscht werden, die Polarisation jedoch weiterhin von den plasmonischen Ladungsoszillationen durch eine imaginäre Komponente im 


\section{Experimentelle Methoden}

evaneszenten Nahfeld bestimmt bleibt, sodass das mit der Probe wechselwirkende lokale Nahfeld eine Polarisation völlig unabhängig von der des einfallenden Lichts aufweist [209]. Wie beim STM ergibt sich eine räumliche Aufösung, die kleiner als der Spitzenradius ist. In der Probe kommt es anschließend zur Raman-Streuung (Phononenanregung, $\omega_{q}$ ), und die gestreuten Photonen werden als Nahfeldbeitrag im Raman-Signal $\left(\gamma_{s}\right)$ registriert. Die Signalverstärkung ist dabei von der Plasmonenresonanz des Systems aus Spitze, Probe und Licht abhängig. Die gestreuten Photonen können außerdem erneut mit LSP koppeln (Antenneneffekt). Wird das TERS-System im gap mode betrieben, erfolgt das Tunneln in Resonanz und die LSP können am Tunnelprozess beteiligt werden, wobei die Anzahl verfügbarer LSP von der Geometrie der Spitze abhängt. Experimentelle Beobachtungen in [209] zeigen anhand von gleichbleibenden Intensitätsverhältnissen von Raman-Moden unterschiedlicher Symmetrie für verschiedene Fernfeldpolarisationskonfigurationen eine völlige Unabhängigkeit (Depolarisation) der Nahfeldpolarisation von der Polarisation des einfallenden Lichts im Fernfeld und vom Fernfeld abweichende, neue TERS-Auswahlregeln.

Das TERS-Nahfeld kann empfindliche Proben jedoch auch negativ beeinflussen. Da das Nahfeld zwischen der Spitze und der Probenoberfläche konzentriert ist und mehrere Größenordnungen gegenüber dem Fernfeld verstärkt ist, wird auch der durch den Laser induzierte Temperaturanstieg lokal an der Probenoberfläche verstärkt 201-203]. Dieses kann bei organischen oder biologischen Materialien zu Schäden und Heizeffekten führen 203]. Beispiele für zu hohe Laserleistungen oder zu lange Belichtungszeiten sind Photobleichungsprozesse bei Farbmolekülen [167, 171, 173, 198, 204, chemische Photodegenerationen bei organischen und biologischen Proben 167,203 oder Strukturänderungen an den Probenoberflächen durch Diffusions- und Desorptionsprozesse 202,203. Andererseits bietet die Temperaturerhöhung jedoch die Möglichkeit lokal experimentelle Bedingungen zu erzeugen, die das Auslösen von Katalysereaktionen bewirken und simultan mit der TERSTechnik beobachtet werden könnten [205]. Zudem ist das Zusammenspiel von Spitze, Umgebungsmedium, Probe und Laserwellenlänge, das den Temperaturanstieg im Bereich des Nahfeldes ebenfalls beeinflusst 201 203, zu berücksichtigen. Für reproduzierbare TERSMessungen und eine Beibehaltung der experimentellen Bedingungen muss daher sowohl die eingestrahlte Laserleistung als auch die Belichtungszeit an die Probe angepasst werden.

Weiterhin ist die Spitze ein Streukörper, an dem Teile des Laserlichts sowohl direkt als auch durch Interaktion mit der Probe gestreut werden. Hierbei kommt es durch Reflexionen und Mehrfachstreuungen zu Verstärkungsartefakten unabhängig des plasmonischen Nahfeldes, die das reale TERS-Signal überlagern [180,206,207]. Für eine theoretische Analyse der Streubeiträge wird in der optischen Rasternahfeldmikroskopie (SNOM) ein Greenscher Tensor-Formalismus verwendet, bei dem das elektrische Gesamtfeld nach der Streuung an Spitze und Probe in Relation zum einfallenden elektrischen Feld bestimmt wird 206, 208]. 
Dieses lässt sich auch auf das TERS-Prinzip anwenden, mit dem Unterschied, dass Spitzen ohne Apertur betrachtet werden und zwischen dem Laserlicht und der Raman-Strahlung eine Frequenzverschiebung auftritt. Das Gesamtsignal aus Nahfeld, Fernfeld und Artefakten ergibt sich dann als Summe der jeweils auftretenden Streuprozesse [206]:

$$
E^{s}=(S+T+T S+S T+T S T+S T S+\ldots) E^{i}
$$

Hierbei bezeichnet $E^{i}$ das einfallende und $E^{s}$ das gestreute elektrische Feld, während $S$ und $T$ Streuoperatoren der Probe und der TERS-Spitze sind, die mit $S=S_{\text {Probe }}+S_{\text {Substrat }}$ für die Probe und $T=T_{\mathrm{NF}}+T_{\text {Schaft }}$ für die Spitze in die einzelnen Streubeiträge zerlegt werden können 206, 208. Für dünne Schichten, die großflächig und homogen das Substrat bedecken, kann die Trennung in $S_{\text {Schicht }}$ und $S_{\text {Substrat }}$ vernachlässigt werden, da im Fernfeld beide stets zusammen erfasst werden. Die einzelnen Terme in der Gleichung 3.4.16 repräsentieren somit die Streuinteraktionen, die während der TERS-Messung auftreten können, wobei die Einzelterme $T$ und $S$ die direkte Streuung an der Spitze und die direkte Fernfeld-Anregung des Raman-Signals der Probe beschreiben und folglich den Hintergrund des TERS-Signals darstellen. Eine Übersicht weiterer wichtiger Interaktionen während einer TERS-Messung ist schematisch in den Abbildungen 3.4.9 (a)-(c) (nach 208]) gegeben.

(a)

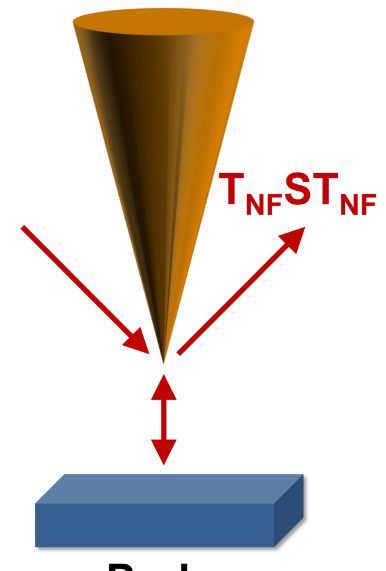

Probe (b)

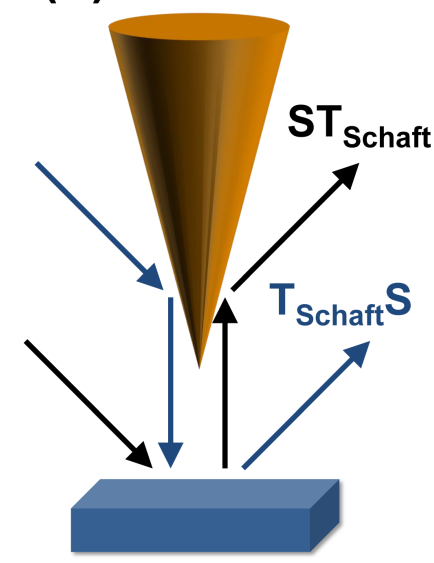

Probe (c)

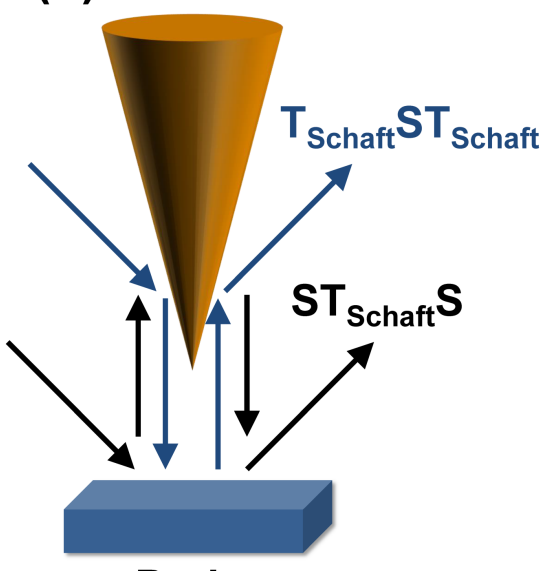

Probe

Abbildung 3.4.9: Auftretende Interaktionen des einfallenden Lichts und des gestreuten RamanSignals mit der TERS-Spitze mit der Signalverstärkung durch das TERSNahfeld (a), der einfachen Streuung am Spitzenschaft (b) und der Mehrfachstreuung an der Spitze und der Probe (c) (nach 208.).

Das einfallende Licht, die Streurichtung sowie das registrierte Signal sind durch Pfeile symbolisiert, während die Pfeilfarbe Teilprozesse der Interaktion des Lichts mit der TERSSpitze und der Probe kennzeichnet. Die plasmonische TERS-Verstärkung durch das Spitzennahfeld ist durch den Term $T_{\mathrm{NF}} S T_{\mathrm{NF}}$ in der Gleichung 3.4.16 gegeben und in der Abbildung 3.4 .9 (a) dargestellt. Folglich sind nicht-plasmonische Verstärkungsartefakte im 


\section{Experimentelle Methoden}

TERS-Signal durch die Terme repräsentiert, die eine Interaktion mit der TERS-Spitze nur in Form einer Streuung am Spitzenschaft $T_{\text {Schaft }}$ beinhalten. Neben einer einfachen Streuung am Spitzenschaft vor oder nach der Raman-Anregung in der Abbildung 3.4.9 (b), welche zu einer erhöhten Ablenkung des Raman-Signals in Objektivrichtung führen kann, sind dieses die in der Abbildung 3.4 .9 (c) dargestellten Mehrfachstreuungen am Spitzenschaft und der Probe, die das Raman-Signal durch Mehrfachanregung und Spiegelungen erhöhen können. Eine Separierung der plasmonischen Verstärkung ist über die lokale Begrenztheit des Nahfeldes möglich [162, 174, 176, 179]. Zudem können die Artefakte durch eine konfokale Optik, glatte TERS-Spitzen mit großem Aspektverhältnis und durch einen steileren Einfallswinkel $\alpha$ der Laserlichts reduziert werden [180,206,207]. Eine Teilstreuung des Laserlichts an der Spitze kann jedoch nicht vermieden werden. Es entsteht der charakteristische kontinuierliche TERS-Hintergrund (im gap mode tritt ein zusätzlicher Substrathintergrundbeitrag auf) 118, 177, 193. Ein Vergleich mit den plasmonisch verstärkten Raman-Moden zeigt, dass der TERS-Hintergrund oft über eine größere integrale Intensität als alle diskreten Raman-Linien zusammen verfügt, sodass TERS-Messungen oft ohne den Hintergrund dargestellt werden 177, 181. Die Position des Hintergrundmaximums und der spektrale Verlauf lassen sich der Plasmonenresonanz der TERS-Spitze in Wechselwirkung mit der Probe zuordnen [118,185]. Zusätzlich lässt sich eine Abhängigkeit der TERS-Hintergrundintensität vom Spitzenabstand beobachten, die dem Abklingen der plasmonischen Verstärkung der Raman-Linien mit dem Spitzenabstand gleicht 177, 181, 185.

In der seitlichen Einstrahlung wird zudem das TERS-Gesamtfeld $I_{\text {tef }}=I_{\mathrm{nf}}+I_{\mathrm{ff}}$ durch einen von der TERS-Spitze verursachten Abschattungseffekt beeinflusst, der das Fernfeld $I_{\mathrm{ff}}$ für eine angenäherte Spitze leicht verringert. Infolgedessen wird der TERS-Kontrast $C_{\text {TERS }}$ unterschätzt, da das Fernfeld für eine zurückgezogene Spitze nicht abgeschattet wird. Eine Quantifizierung des Effekts ist jedoch schwierig, da experimentell nur die Intensitäten des TERS-Gesamtfeldes bei einer angenäherten Spitze und des Fernfeldes für eine zurückgefahrene Spitze zugänglich sind. Folglich kann die Abschattung nur abgeschätzt werden. Für eine geometrische Betrachtung, die sowohl die Form der Spitze als auch den in Näherung kreisförmigen Laserpunkt mit $A_{\text {Fokus }} \approx 1 \mu^{2}$ berücksichtigt, lässt sich die Intensitätsverringerung des Fernfeldes im TERS-Gesamtfeld auf maximal $5 \%$ abschätzen. Demnach ist die Abschattung für ein STM-TERS-System nur gering, kann bei AFM-TERS-Systemen jedoch größer sein. Eine Diskussion der Abschattung findet sich auch in der Literatur, wo diese als klein bezeichnet und zumeist vernachlässigt wird [49, 169, 170, 172, 194]. Eine Schwierigkeit ist die Polarisationsabhängigkeit des Abschattungseffekts aufgrund unterschiedlicher Fernfeldbeiträge zur Gesamtfeldintensität in Abhängigkeit der Polarisationskonfiguration. Durch spitzeninduzierte Depolarisationseffekte kann der Beitrag des Fernfeldes zum Gesamtfeld sehr verschieden sein, sodass die Abschattung durch die TERS-Spitze einen unterschiedlichen Einfluss auf das Gesamtfeld und den TERS-Kontrast hat [49]. 


\section{Auswertung und Ergebnisse}

In diesem Kapitel sollen die Ergebnisse der Arbeit präsentiert werden. Der erste Teil befasst sich mit den B-Platz geordneten $\mathrm{A}_{2} \mathrm{BMnO}_{6}$-Doppelperowskiten. Dabei wird mit der Herstellung und Optimierung der Doppelperowskit-Schichten begonnen, um anschließend die Einflüsse des A-Kations und des B-Kations auf die strukturellen, magnetischen und phononischen Eigenschaften der Schichten zu untersuchen. Hierbei nimmt die RamanSpektroskopie eine zentrale Stellung ein. Für das $\mathrm{La}_{2} \mathrm{CoMnO}_{6}$-Doppelperowskit werden zudem Methoden zur Bestimmung der B-Platz-Ordnung vorgestellt sowie das Wachstum auf verschiedenen Substraten zur Verbesserung der B-Platz-Ordnung diskutiert. Der zweite Teil befasst sich mit der spitzenverstärkten Raman-Spektroskopie (TERS), die im Zuge dieser Arbeit erstmalig auf den vorgestellten Doppelperowskiten als Modellmaterialsystem angewandt wird. Hierfür wird zunächst mit Messungen an bekannten TERS-Testmaterialien begonnen, um darauf aufbauend die Polarisationsabhängigkeit der TERS-Messungen an dünnen Doppelperowskit-Schichten zu untersuchen und die Beiträge des TERS-Nahfeldes zu charakterisieren. Im dritten Teil werden schließlich dünne $\mathrm{La}_{1-\mathrm{x}} \mathrm{Sr}_{\mathrm{x}} \mathrm{MnO}_{3}$-Schichten hinsichtlich der Vielzahl der strukturellen Phasen für Sr-Dotierungen von $0 \leq x \leq 0,3$ diskutiert. Neben der Entwicklung der Raman-Spektren mit der Dotierung und der Temperatur wird dabei der Einfluss von Licht auf die strukturelle Ordnung der Schichten untersucht.

\subsection{B-Platz geordnete $\mathrm{A}_{2} \mathrm{BMnO}_{6}$-Doppelperowskite}

Im Folgenden sollen nun die Ergebnisse zu den dünnen $\mathrm{A}_{2} \mathrm{BMnO}_{6}$-Doppelperowskit-Schichten (mit B = Co, Ni und A = La, Pr, Nd, Sm, Gd, Y) vorgestellt werden, die mithilfe der metallorganischen Aerosol Deposition bei einem Sauerstoffpartialdruck $p \approx 0,21$ bar und einer Temperatur $T \approx 1000{ }^{\circ} \mathrm{C}$ auf verschiedenen Substraten aufgewachsen wurden.

\subsubsection{Schichtwachstum und Optimierung der Wachstumsparameter}

Die Grundlage für das Wachstum der Doppelperowskit-Schichten mit B-Platz-Ordnung ist das Einstellen und die Optimierung der MAD-Wachstumsparameter. Dieses soll hier kurz für $\mathrm{La}_{2} \mathrm{CoMnO}_{6}$ (LCMO) beschrieben werden. Das Vorgehen lässt sich dann auf die anderen $\mathrm{A}_{2} \mathrm{CoMnO}_{6}$-Doppelperowskite übertragen. Für ein stöchiometrisches Schichtwachstum müssen zunächst die richtigen Präkursorverhältnisse ermittelt werden (Abschnitt 3.1.2). Im ersten Schritt wird das Wachstumsverhalten der Perowskite $\mathrm{LaMnO}_{3}$ (LMO) und 


\section{Auswertung und Ergebnisse}

$\mathrm{LaCoO}_{3}$ (LCO), aus denen sich das LCMO-Doppelperowskit später zusammensetzt, betrachtet. Hierfür werden LMO- und LCO-Schichten mit unterschiedlichen La/Mn- und La/Co-Präkursorverhältnissen hergestellt, die Schichteigenschaften in Abhängigkeit der Lösungszusammensetzung analysiert und so die Korrekturfaktoren $k_{\mathrm{La} / \mathrm{Mn}}$ und $k_{\mathrm{La} / \mathrm{Co}}$ des La-Präkursors in Relation zu den Mn- und Co-Präkursoren für ein stöchiometrisches Wachstum ermittelt. Mit einer LMO- und LCO-Schichtdickenserie kann dann das Lösungsvolumen für das Wachstum einer Einheitszelle LMO und LCO und damit das Co/MnPräkursorverhältnis für stöchiometrisches $\mathrm{La}_{2} \mathrm{CoMnO}_{6}$ bestimmt werden. Die resultierenden La/(Co+Mn)- und Co/Mn-Präkursorverhältnisse können anschließend je nach Substrat weiter angepasst werden. Eine Orientierung geben AFM-Aufnahmen der Schichtoberfläche, die für eine Schicht von hoher Qualität durch eine glatte Oberflächenmorphologie gekennzeichnet sind. Hierbei können STO (100)-Substrate mit $\mathrm{TiO}_{2}$-terminierter Oberfläche verwendet werden, deren Stufenstruktur an der Oberfläche sich für Schichten hoher Qualität fortsetzt. In den Abbildungen 4.1.1 (a) und (b) sind die AFM-Aufnahmen zweier LCMO/STO (100)-Schichten mit den Schichtdicken $d=25 \mathrm{~nm}$ und $d=85 \mathrm{~nm}$ dargestellt. Es ist ein Schichtwachstum an den Substratstufen zu beobachten, das für die dickere der zwei Schichten zu breiteren Terrassen führt. Beide Schichten sind sehr glatt mit RMSRauigkeiten RMS =0,10 nm (25 nm LCMO) und RMS =0,13 nm (85 nm LCMO).

(a)

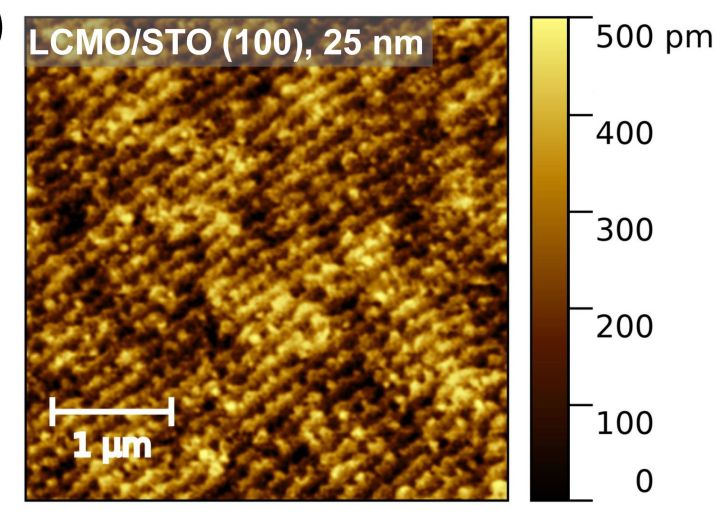

(b)

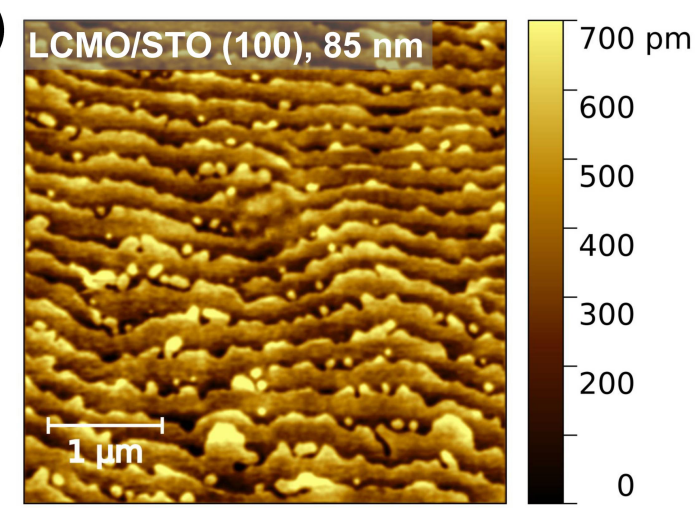

Abbildung 4.1.1: AFM-Oberflächenaufnahmen zweier unter Optimalbedingungen gewachsener LCMO-Schichten auf STO (100)-Substraten mit $\mathrm{TiO}_{2}$-terminierter Oberfläche mit den Schichtdicken $d=25 \mathrm{~nm}$ (a) und $d=85 \mathrm{~nm}$ (b).

Für die weiteren $\mathrm{A}_{2} \mathrm{CoMnO}_{6}$-Doppelperowskite wird das jeweilige $\mathrm{A} /(\mathrm{Co}+\mathrm{Mn})$-Verhältnis eingestellt. Der Unterschied zu LCMO ist aufgrund des ähnlichen Verhaltens der Präkursoren der Seltenen Erden nur gering, sodass die optimalen Präkursorverhältnisse alle in einem Bereich $\mathrm{A} /(\mathrm{Co}+\mathrm{Mn}) \approx 1,28-1,4$ und $\mathrm{Co} / \mathrm{Mn} \approx 0,89-0,97 \mathrm{zu}$ finden sind. Das gleiche Verfahren wird dann für $\mathrm{La}_{2} \mathrm{NiMnO}_{6}$ (LNMO) und die $\mathrm{A}_{2} \mathrm{NiMnO}_{6}$-Doppelperowskite angewandt. Dabei wird zunächst das Wachstum von $\mathrm{LaNiO}_{3}$ (LNO) betrachtet und die La/(Ni+Mn)- und Ni/Mn-Präkursorverhältnisse für stöchiometisches LNMO bestimmt. Diese können dann für die anderen $\mathrm{A}_{2} \mathrm{NiMnO}_{6}$-Doppelperowskite angepasst werden und liegen alle in einem Bereich $\mathrm{A} /(\mathrm{Ni}+\mathrm{Mn}) \approx 1,25-1,36$ und $\mathrm{Ni} / \mathrm{Mn} \approx 1,00-1,10$. 
Das Präkursorverhältnis beeinflusst zudem die magnetischen Eigenschaften der Doppelperowskite. In der Abbildung 4.1.2 (a) sind die $M(T)$-Magnetisierungskurven (SQUID, FCC mit $H=1 \mathrm{KOe}$ ) von je drei LCMO/STO (111)-Schichten, für die das La/(Co+Mn)oder das Co/Mn-Präkursorverhältnis variiert wurde, dargestellt. Während die stöchiometrische LCMO-Schicht die für eine hohe B-Platz-Kationenordnung zu erwartende ferromagnetische Phase mit einer Curie-Temperatur $T_{C}=227(2) \mathrm{K}$ zeigt, ist für die Schicht mit $+8 \%$ höherem La/(Co+Mn)-Verhältnis eine Abnahme der Curie-Temperatur auf $T_{C 1}=208(2) \mathrm{K}$ und ein zweiter Übergang bei $T_{C 2} \approx 127(2) \mathrm{K}$ zu beobachten, der charakteristisch für B-Platz ungeordnetes LCMO ist (Abschnitt 2.3.2). Ein $-8 \%$ geringeres $\mathrm{La} /(\mathrm{Co}+\mathrm{Mn})$-Verhältnis ändert die $M(T)$-Kurve im Vergleich zum Optimum nur wenig. Dieses kann durch isolierte La-defizitäre Ausscheidungen begründet werden, die die BPlatz-Ordnung nicht beeinträchtigen 48 . Ein um $\pm 2,5 \%$ verändertes $\mathrm{Co} / \mathrm{Mn}$-Verhältnis führt wiederum nur zu einem Abflachen der Magnetisierungskurve im Temperaturbereich $T \approx 100-150 \mathrm{~K}$. In der Abbildung 4.1.2 (b) sind zudem die $\rho(T)$-Kurven des spezifischen Widerstands einer $25 \mathrm{~nm}$ LCMO-Schicht auf STO (100) und einer $100 \mathrm{~nm}$ LCMO-Schicht auf LAO (111) dargestellt, die stöchiometrisch unter Optimalbedingungen hergestellt wurden. Beide Schichten zeigen ein zu erwartendes, elektrisch isolierendes Verhalten mit einem hohen spezifischen elektrischen Widerstand. Die mit einer Arrhenius-Auftragung (eingebettete Grafik) bestimmten Aktivierungsenergien für einen thermisch induzierten Leitungsprozess betragen $E_{A}=0,27 \mathrm{eV}\left(25 \mathrm{~nm}\right.$ LCMO) und $E_{A}=0,26 \mathrm{eV}$ (100 nm LCMO) und stimmen damit sehr gut mit Literaturwerten für LCMO-Schichten überein. [48,214.
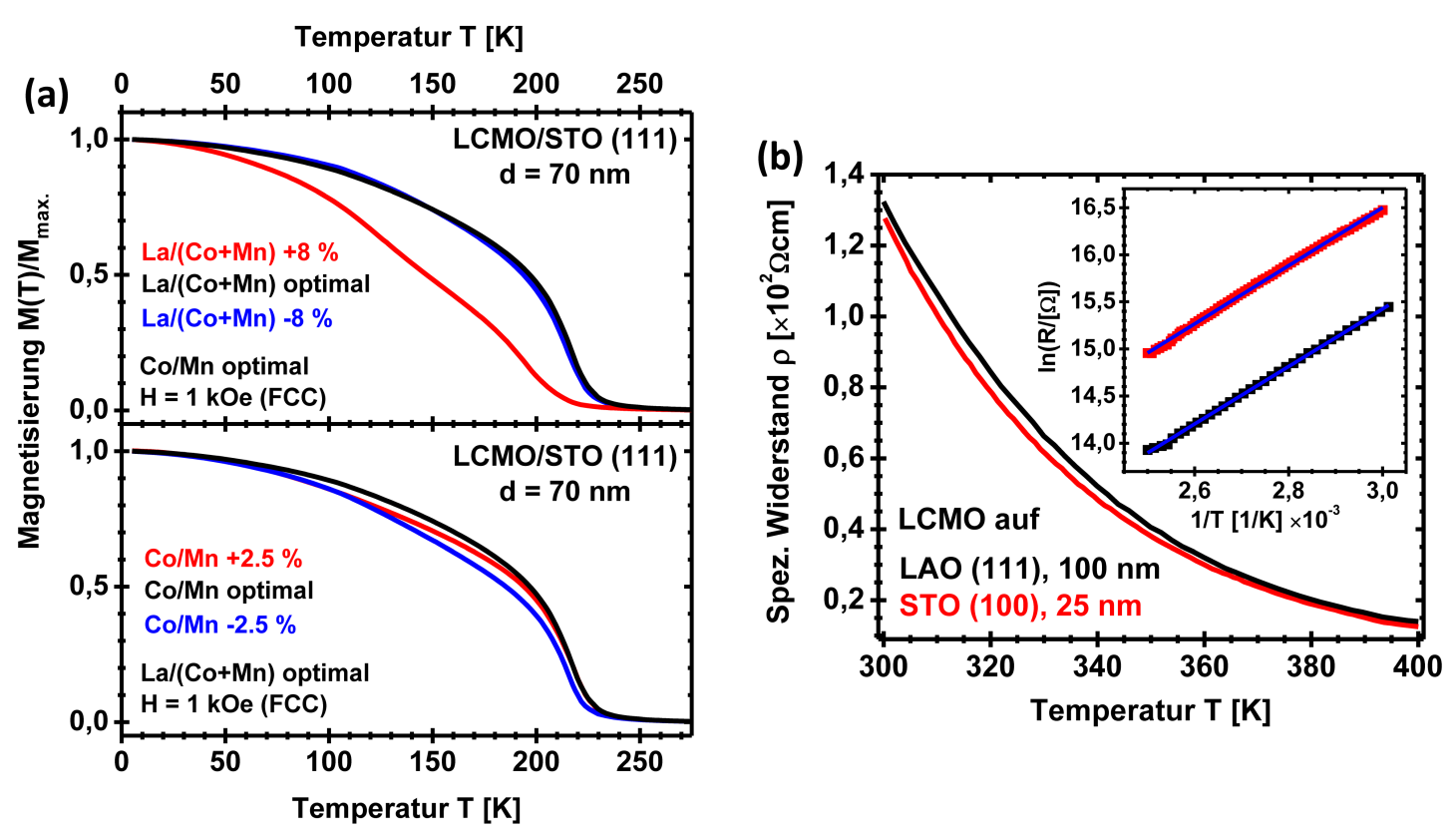

Abbildung 4.1.2: Abhängigkeit der $M(T)$-Kurven der $70 \mathrm{~nm}$ LCMO/STO (111)-Schichten vom $\mathrm{La} /(\mathrm{Co}+\mathrm{Mn})$ - und vom Co/Mn-Präkursorverhältnis (a) und der temperaturabhängige Verlauf $\rho(T)$ des spezifischer Widerstands von LCMO-Schichten auf STO $(100)(d=25 \mathrm{~nm})$ und LAO $(111)(d=100 \mathrm{~nm})(\mathrm{b})$. Die eingebettete Grafik zeigt die jeweiligen Arrhenius-Auftragungen des Widerstands. 


\section{Auswertung und Ergebnisse}

Zudem können die Doppelperowskit-Schichten mit der Raman-Spektroskopie auf ihre phononischen Eigenschaften untersucht werden. Während die $\mathrm{A}_{2} \mathrm{CoMnO}_{6}$ - und $\mathrm{A}_{2} \mathrm{NinO}_{6}$-Doppelperowskite im Fall einer B-Platz-Ordnung in der monoklinen P12/n1-Struktur kristallisieren, weisen diese für die B-Platz ungeordnete Phase eine orthorhombische PbnmStruktur auf (Abschnitte 2.3.2 und 2.3.3). Dieses hat zur Folge, dass viele der RamanModen in den beiden Phasen eine unterschiedliche Symmetrie und damit unterschiedliche Auswahlregeln besitzen, sodass in polarisationsabhängigen Raman-Messungen zwischen B-Platz geordneten und ungeordneten Doppelperowskiten differenziert werden kann [33]. Gruppentheoretische Untersuchungen zeigen für die monokline P12 $/$ n1-Raumgruppe am $\Gamma$-Punkt, dass von den 60 Phonon-Moden 24 Moden $\left(12 A_{g}+12 B_{g}\right)$ Raman-aktiv sind [33], während die weiteren Moden IR-aktiv, akustisch oder still sind. Die polarisationsabhängigen Raman-Spektren (xx-, $x^{\prime} x^{\prime}-$, xy- und $x^{\prime} y^{\prime}$-Polarisationskonfigurationen) einer $100 \mathrm{~nm}$ LCMO/MgO (100)-Schicht sind nachfolgend in den Abbildungen 4.1 .3 (a)-(c) dargestellt. Das Probenbezugssystem entspricht der in der Abbildung 3.3.8 gezeigten Orientierung mit den Richtungen x $\|$ [100], y $\|$ [010], z \| [001], x' $\|$ [15̄0] und y' $\mathrm{y}^{\prime}$ [110] (senkrechte Rückstreugeometrie mit der out-of-plane Orientierung in [001]-Richtung).
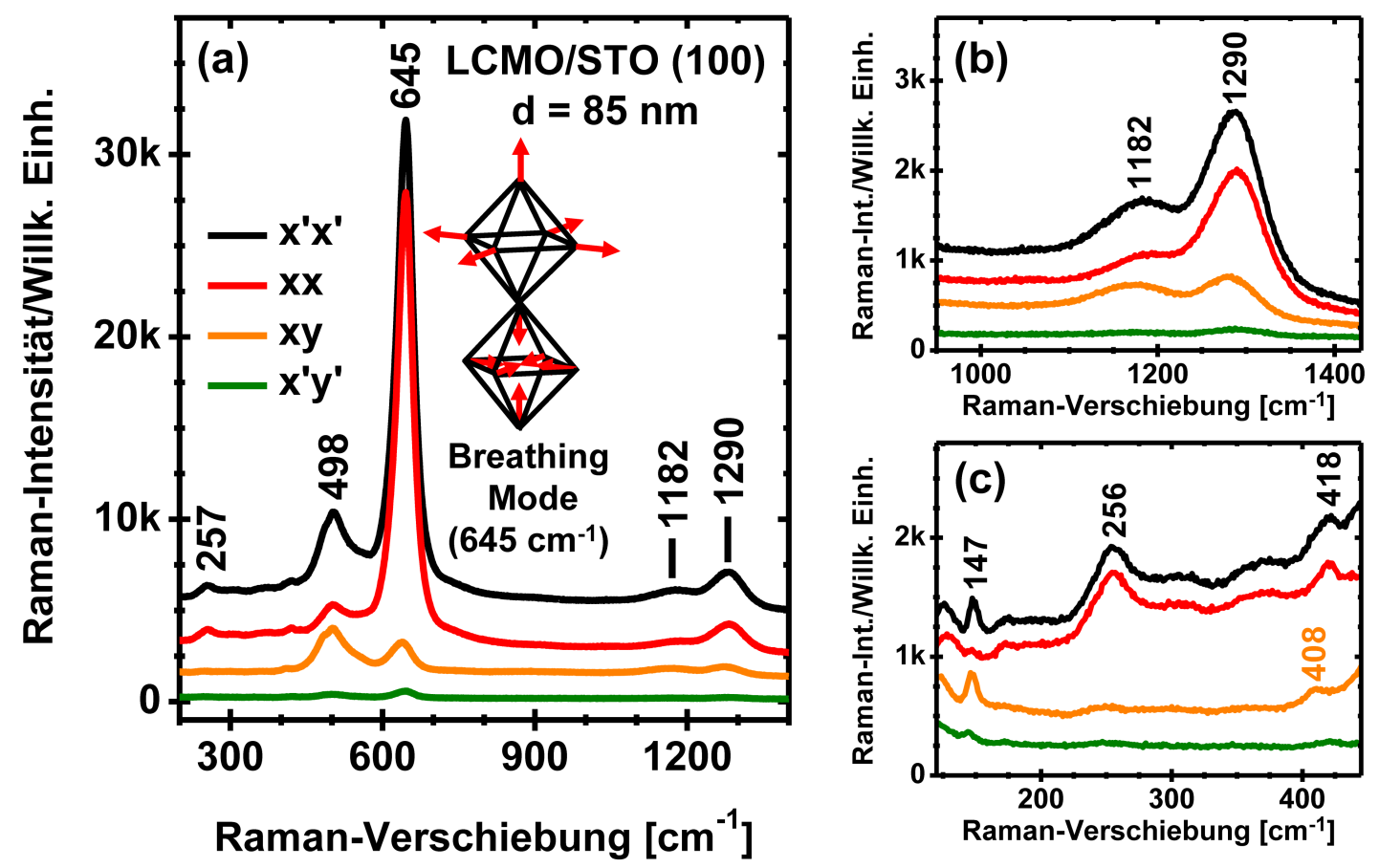

Abbildung 4.1.3: Polarisierte Raman-Spekten ( $x x-, x^{\prime} x^{\prime}-, x y-$ und $x^{\prime} y^{\prime}-$ Konfigurationen) einer $85 \mathrm{~nm} \mathrm{LCMO} / \mathrm{STO}$ (100)-Schicht (a) (mit $\lambda=532 \mathrm{~nm}, P \approx 5 \mathrm{~mW}$ und $t=3 \times 30 \mathrm{~s}$; bei Raumtemperatur) sowie mit dem Fokus auf die RamanModen in dem Bereich großer (b) und kleiner Wellenzahlen (c).

Die Raman-Spektren der LCMO-Schicht sind hierbei durch eine sehr intensive und scharfe Schwingungsmodemode bei $645 \mathrm{~cm}^{-1}$ und einem weniger intensiven und breiteren Band bei $499 \mathrm{~cm}^{-1}$ gekennzeichnet. Theoretische Berechnungen in Form von lattice dynamical calculations (LDC) zeigen [33, dass die Raman-Mode bei $645 \mathrm{~cm}^{-1}$ einer symmetrischen 
Streckschwingung der $(\mathrm{Co} / \mathrm{Mn}) \mathrm{O}_{6^{-}}$Oktaeder zugeordnet werden kann, während die Bande bei $499 \mathrm{~cm}^{-1}$ eine Mischmode aus antisymmetrischen Streckschwingungen und Biegebewegungen beschreibt. Aufgrund ihrer Charakteristik (Abbildung 4.1.3 (a)) wird die symmetrische Streckschwingung auch als Breathing Mode bezeichnet. Eine Besonderheit der Doppelperowskite ist, dass die Breathing Mode in der B-Platz geordneten monoklinen P12 1 /n1-Struktur eine $A_{g}$-Symmetrie aufweist, während in der B-Platz ungeordneten orthorhombischen Pbnm-Struktur eine $B_{1 g}$-Symmetrie vorliegt. Somit lässt sich anhand polarisationsabhängiger Raman-Messungen die Struktur des Doppelperowskits bestimmen 33]. Dabei ist die Breathing Mode für die P12 1 n1-Struktur in den beiden parallelen xx- und $\mathrm{x}^{\prime} \mathrm{x}^{\prime}$-Polarisationskonfigurationen $\mathrm{zu}$ beobachten und in den zwei gekreuzten $\mathrm{xy}-$ und $\mathrm{x}^{\prime} \mathrm{y}^{\prime}$ Polarisationskonfigurationen stark unterdrückt. Für die ungeordnete Pbnm-Struktur ist das Verhalten entsprechend umgekehrt. Für die Mischmode ist der Fall komplexer, da beide Strukturen Moden mit $A_{g^{-}}$und $B_{g^{-}}$Symmetrie (P12 $12_{1}$ n1-Struktur), beziehungsweise $B_{1 g}$-Symmetrie (Pbnm-Struktur) in diesem Wellenzahlbereich besitzen, die sich überlagern. Dennoch ist für die P12 1 n1-Struktur ein mehrheitlicher $B_{g^{-}}$Anteil und damit eine starke Präsenz in der gekreuzten xy- und der parallelen $x^{\prime} x^{\prime}$-Konfiguration sowie ein Verbot in der parallelen xx- und der gekreuzten $\mathrm{x}^{\prime} \mathrm{y}^{\prime}$-Konfiguration zu erwarten. Für die Raman-Tensoren der Moden mit $A_{g^{-}}$und $B_{g^{-}}$Symmetrie in der P12 $/$n1-Struktur folgt 39, 143:

$$
A_{g}=\left(\begin{array}{lll}
a & 0 & d \\
0 & b & 0 \\
d & 0 & c
\end{array}\right), B_{g}=\left(\begin{array}{lll}
0 & e & 0 \\
e & 0 & f \\
0 & f & 0
\end{array}\right) .
$$

Mithilfe der Gleichung 3.3.14 kann das Verhalten der Intensität der Raman-Moden in den Polarisationskonfiguration auch theoretisch bestimmt werden. Die polarisationsabhängigen Raman-Spektren der LCMO/STO (100)-Schicht in der Abbildung 4.1 .3 (a) zeigen dabei für die $A_{g}$ Breathing Mode und die $B_{g}$ Mischmode eine exzellente Übereinstimmung mit der Vorhersage für die jeweilige Modensymmetrie für die monokline P12 1 /n1-Struktur und anderen Untersuchungen an LCMO/STO (100)-Schichten 33, 49, 215, 216. Zusätzlich sind zwei Multiphonon-Moden bei $1290 \mathrm{~cm}^{-1}$ und $1182 \mathrm{~cm}^{-1}$ (Abbildung 4.1.3 (b)) zu beobachten. Die Mode bei $1290 \mathrm{~cm}^{-1}$ kann entsprechend einer Oberschwingung 2. Ordnung der $A_{g}$ Breathing Mode zugeordnet werden, während die Mode bei $1182 \mathrm{~cm}^{-1}$ eine Kombinationsschwingung der Breathing Mode und der Mischmode beschreibt 49]. Dieses wird durch die jeweilige Polarisationsabhängigkeit in den Raman-Spektren bestätigt. Das Auftreten dieser Moden dokumentiert zudem die hohe kristalline Qualität der LCMODoppelperowskit-Schicht. Ähnliche Moden 2. Ordnung zeigen sich auch in anderen Doppelperowskiten 217,218. Daneben lassen sich noch Raman-Moden bei $147 \mathrm{~cm}^{-1}\left(A_{g}\right)$, $256 \mathrm{~cm}^{-1}\left(B_{g}\right), 408 \mathrm{~cm}^{-1}\left(B_{g}\right)$ und $417 \mathrm{~cm}^{-1}\left(B_{g}\right)$ (Abbildung $4.1 .3(\mathrm{c})$ ) beobachten, die charakteristisch für die B-Platz geordnete monokline P12 /n1-Struktur sind [33, 215, 219]. 


\subsubsection{Einfluss des A-Platz- und des B-Platz-Kations}

Nach der Einstellung der optimalen MAD-Wachstumsparameter können die unter diesen Bedingungen hergestellten Schichten der $\mathrm{A}_{2} \mathrm{CoMnO}_{6}$ - und der $\mathrm{A}_{2} \mathrm{NiMnO}_{6}$-Doppelperowskit-Reihen bezüglich des Einflusses des A-Platz-Kations ( $\mathrm{A}=\mathrm{La}, \mathrm{Pr}, \mathrm{Nd}, \mathrm{Sm}, \mathrm{Gd}, \mathrm{Y}$ ) und des B-Platz-Kations $(\mathrm{B}=\mathrm{Co}, \mathrm{Ni})$ auf die strukturellen, magnetischen und phononischen Eigenschaften untersucht werden. Während in der Literatur in diesem Zusammenhang zumeist Bulk-Proben [34, 35, 38] oder einzelne dieser Doppelperowskite in Form von dünnen Schichten auf (100)-orientierten Substraten betrachtet werden [33,37,220], sind die hier vorgestellten Schichten beider Doppelperowskit-Reihen epitaktisch auf STO-Substraten mit (111)-Orientierung aufgewachsen worden. Dieses ermöglicht neben der Charakterisierung der B-Platz-Ordnung über die Sättigungsmagnetisierung $M_{S}$, elementspezifisch-chemischer Techniken (wie EELS, EDX) und der Raman-Spektroskopie eine strukturelle Abschätzung mit der Röntgendiffraktometrie (XRD). Grundlage hierfür ist die NaCl-Anordnung der BPlatz-Kationen, die in der [111]-Richtung aufgrund von abwechselnden Lagen von $\mathrm{AMnO}_{3}$ und $\mathrm{A}(\mathrm{Co} / \mathrm{Ni}) \mathrm{O}_{3}$ in einer Ordnungsüberstruktur mit einer effektiven Verdopplung der Gitterkonstante resultiert (Abbildung 2.3.3 (c)) und in den XRD-Spektren in pseudokubischer Notation zu Überstruktur-Reflexen $\frac{1}{2}$ (uuu) (mit einer ungeraden ganzen Zahl u) zusätzlich zu den Fundamentalreflexen $\frac{1}{2}(g g g)$ (mit einer geraden ganzen Zahl $g$ ) führt 30,221 . In der Abbildung 4.1.4 sind nun die XRD-Spektren der $\mathrm{A}_{2} \mathrm{CoMnO}_{6}$-Doppelperowskit-Schichtserie (A = La, Pr, Nd, Sm, Gd, Y) auf STO (111) mit einer vergleichbaren Schichtdicke $d \approx 90-100 \mathrm{~nm}$ in der Bragg-Brentano-Geometrie in der Umgebung des jeweiligen (111)Fundamentalreflexes und des $\left(\frac{1}{2} \frac{1}{2} \frac{1}{2}\right)$-Überstrukturreflexes im Vergleich dargestellt.

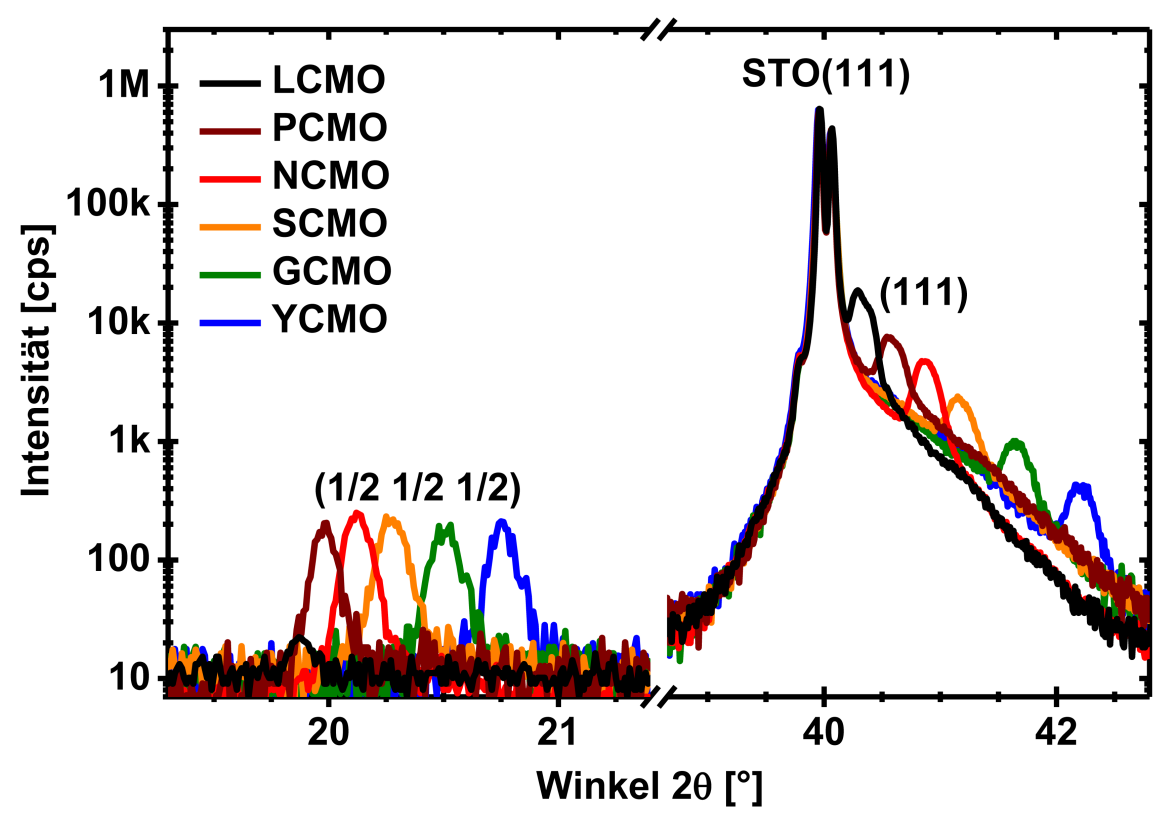

Abbildung 4.1.4: $\mathrm{XRD}$-Spektren der $\mathrm{A}_{2} \mathrm{CoMnO}_{6} / \mathrm{STO}$ (111)-Schichten mit einer Schichtdicke $d \approx 90-100 \mathrm{~nm}$ in der Bragg-Brentano-Geometrie im Vergleich. 
Die $\mathrm{A}_{2} \mathrm{CoMnO}_{6} / \mathrm{STO}$ (111)-Doppelperowskit-Schichten zeigen dabei alle neben dem (111)Fundamentalreflex auch den $\left(\frac{1}{2} \frac{1}{2} \frac{1}{2}\right)$-Überstrukturreflex in ihren Spektren (daneben ist auch der (222)-Reflex und der ( $\left.\frac{3}{2} \frac{3}{2} \frac{3}{2}\right)$-Reflex zu beobachten; hier nicht explizit gezeigt). Da in den Gesamtspektren keine anders orientierten Reflexe auftreten, weisen die Schichten damit eine vollständige out-of-plane Epitaxie auf. Daneben lässt sich der Einfluss der APlatz-Kationengröße auf die Gitterkonstante der Schichten anhand der kontinuierlichen Verschiebung der Reflexe in Richtung größerer $2 \theta$-Winkel bei einer systematischen Verkleinerung des A-Platz-Kations von $\mathrm{La}^{3+}$ (Kationenradius $r_{\mathrm{La}^{3+}}=116 \mathrm{pm}$ [222], KZ =8) bis $\mathrm{Y}^{3+}$ (Kationenradius $r_{\mathrm{Y}^{3+}}=101,9 \mathrm{pm}$ [222], KZ =8) nachvollziehen, die zu einer Abnahme der pseudokubischen out-of-plane Gitterkonstante von $c_{\mathrm{pc}, \mathrm{LCMO}}=3,875 \AA$ (LCMO) auf $c_{\mathrm{pc}, \mathrm{YCMO}}=3,707 \AA$ (YCMO) führt. In gleicher Weise kann nun die $\mathrm{A}_{2} \mathrm{NiMnO}_{6}$-Doppelperowskit-Schichtserie (A = La, Pr, Nd, Sm, Gd, Y) auf STO (111) mit einer vergleichbaren Schichtdicke $d \approx 90-100 \mathrm{~nm}$ betrachtet werden. Die dazugehörigen XRD-Spektren in der Bragg-Brentano-Geometrie sind nachfolgend in der Abbildung 4.1.5 dargestellt.

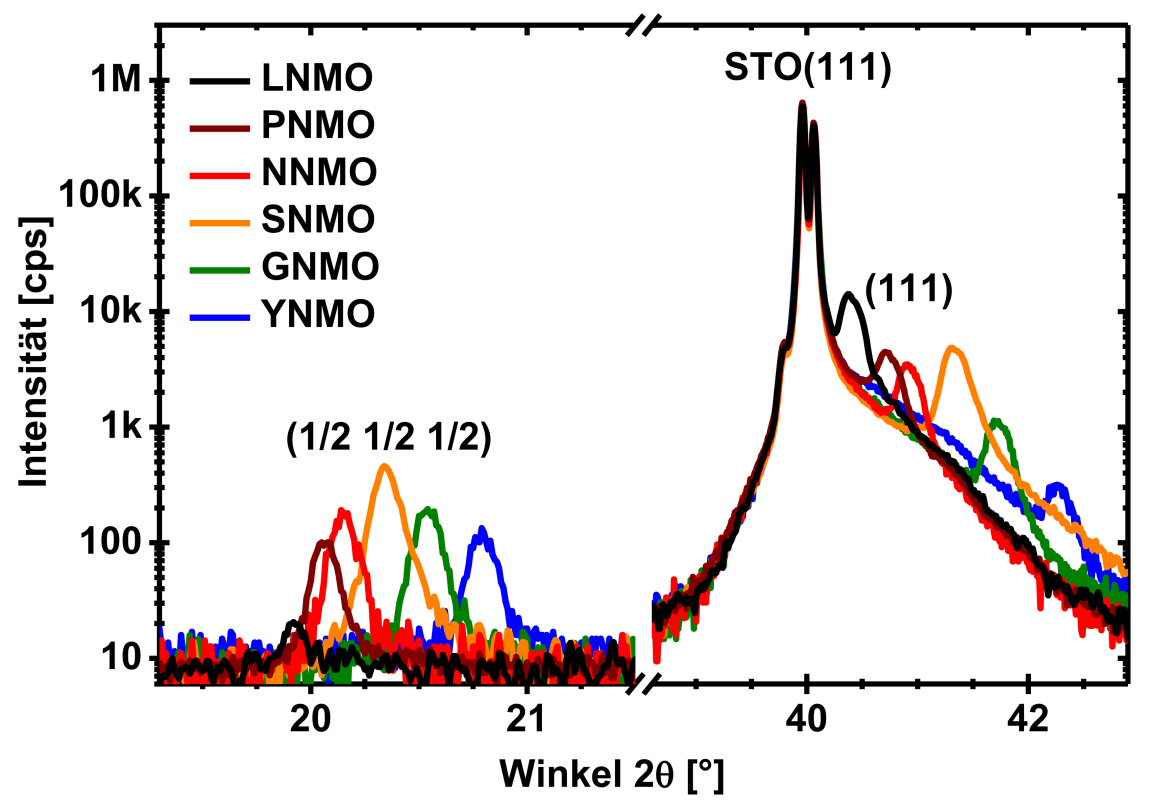

Abbildung 4.1.5: XRD-Spektren der $\mathrm{A}_{2} \mathrm{NiMnO}_{6} / \mathrm{STO}$ (111)-Schichten mit einer Schichtdicke $d \approx 90-100 \mathrm{~nm}$ in der Bragg-Brentano-Geometrie im Vergleich.

Für $\mathrm{A}_{2} \mathrm{NiMnO}_{6} / \mathrm{STO}$ (111)-Doppelperowskit-Schichten ergibt sich ein ähnliches Verhalten wie für die $\mathrm{A}_{2} \mathrm{CoMnO}_{6} / \mathrm{STO}$ (111)-Doppelperowskit-Schichten. So ist neben dem (111)Fundamentalreflex jeweils auch wieder der $\left(\frac{1}{2} \frac{1}{2} \frac{1}{2}\right)$-Überstrukturreflex in den Spektren zu beobachten. Die höheren Reflexintensitäten der SNMO-Schicht lassen sich auf eine etwas größere Schichtdicke im Vergleich zu den anderen Schichten zurückführen. Daneben ist auch hier eine systematische Abhängigkeit der Reflexpositionen und der jeweiligen out-ofplane Gitterkonstante der epitaktisch gewachsenen Schichten vom A-Platz-Kationenradius $\mathrm{zu}$ beobachten. Auffallend im Vergleich zu den anderen Schichten innerhalb ihrer Reihen ist jedoch sowohl für die LCMO- als auch die LNMO-Schicht ein in der Intensität 


\section{Auswertung und Ergebnisse}

deutlich reduzierter Überstrukturreflex. Wird berücksichtigt, dass die Intensität der Überstrukturreflexe allgemein durch die chemische Ordnung in den Doppelperowskiten auf den B-Kation-Plätzen entsteht und damit direkt von dem Unterschied der atomaren Formfaktoren der Kationen auf den B-Plätzen abhängt 30,221], sollte sich die Intensität des Reflexes innerhalb der Doppelperowskit-Reihen bei einem vergleichbaren Ordnungsgrad auf den BPlätzen hingegen nicht signifikant ändern. In einer einfachen Abschätzung lässt sich der dazugehörige Ordnungsparameter $S=I\left(\frac{1}{2} \frac{1}{2} \frac{1}{2}\right) / I(111)$ der Schichten direkt über das Verhältnis der Intensitäten von Überstruktur- und Fundamentalreflex bestimmen 36, 48,221. Die so für die zwei $\mathrm{A}_{2} \mathrm{CoMnO}_{6^{-}}$und $\mathrm{A}_{2} \mathrm{NiMnO}_{6}$-Schichtreihen auf STO (111) berechneten Ordnungsparameter $S$ sind in der Abbildung 4.1.6 in Abhängigkeit des A-Kationenradius dargestellt. Daneben ist zusätzlich die Entwicklung der pseudokubischen out-of-plane Gitterkonstanten $c_{\mathrm{pc}}$ der Schichten mit dem A-Kationenradius eingezeichnet.

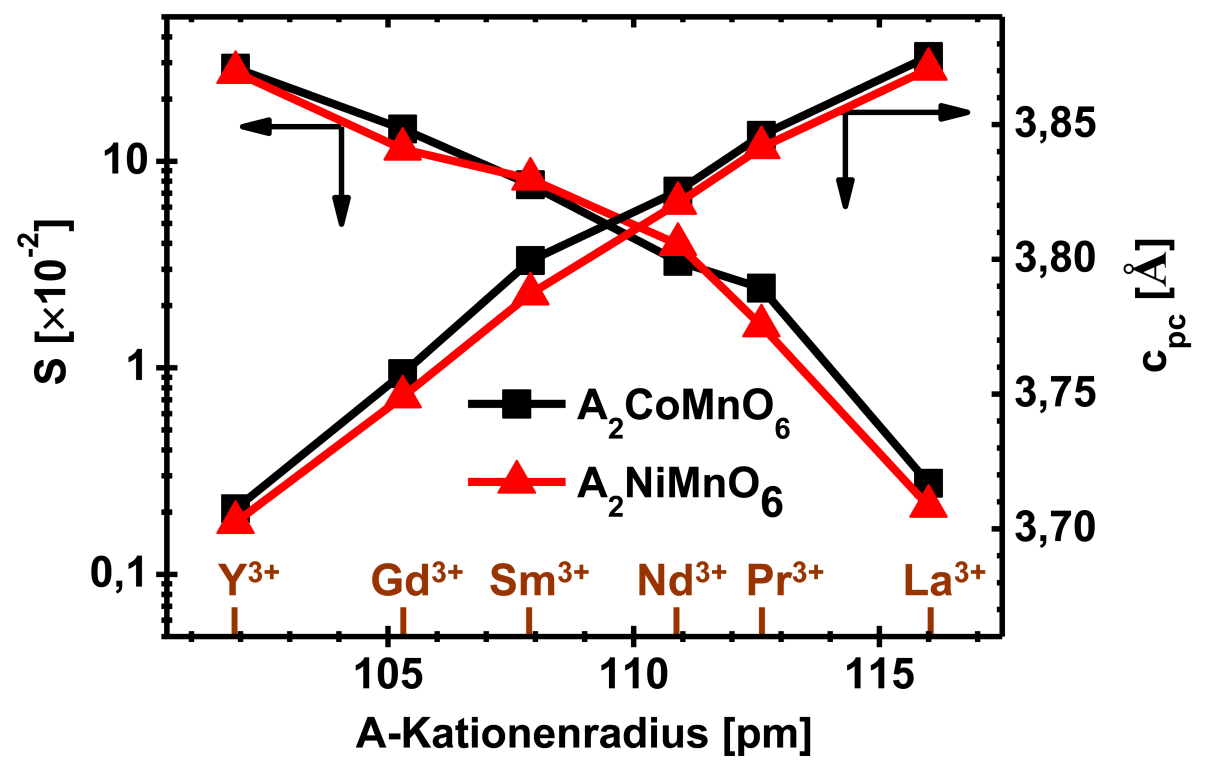

Abbildung 4.1.6: Entwicklung des Ordnungsparameters $S$ und der pseudokubischen out-ofplane Gitterkonstante $c_{\mathrm{pc}}$ der $\mathrm{A}_{2} \mathrm{CoMnO}_{6}$ - und $\mathrm{A}_{2} \mathrm{NiMnO}_{6}$-Schichtreihen auf STO (111)-Substraten mit der Größe des A-Kationenradius.

Für die pseudokubischen out-of-plane Gitterkonstanten $c_{\mathrm{pc}}$ der Schichten ergibt sich das bereits angesprochene Verhalten einer systematischen Abnahme mit der Verringerung des A-Kationenradius. Generell sind die Gitterkonstanten der $\mathrm{A}_{2} \mathrm{CoMnO}_{6}$-Schichten leicht gegenüber denen der entsprechenden $\mathrm{A}_{2} \mathrm{NiMnO}_{6}$-Schichten erhöht. Dieses lässt sich auf den leicht größeren Kationenradius $r_{\mathrm{Co}^{2+}}=74,5 \mathrm{pm}(\mathrm{KZ}=6)$ 222 von $\mathrm{HS}_{-} \mathrm{Co}^{2+}$ gegenüber dem Kationenradius $r_{\mathrm{Ni}^{2+}}=69 \mathrm{pm}(\mathrm{KZ}=6)$ von $\mathrm{Ni}^{2+}$ zurückführen. Überraschenderweise weist der Ordnungsfaktor $S$ eine genau entgegengesetzte Skalierung auf und steigt mit einer Verringerung des A-Kationenradius kontinuierlich an. Die Änderung ist dabei enorm und beträgt für beide Serien in etwa zwei Größenordnungen zwischen den kleinsten Werten für LCMO und LNMO $\left(S \approx 2,5 \cdot 10^{-3}\right)$ und den größten Werten für YCMO und YNMO $\left(S \approx 3 \cdot 10^{-1}\right)$. Um nun aus dem Ordnungsparameter den B-Platz geordneten Anteil 
in den Schichten zu bestimmen, müssen diese noch mit dem Idealwert einer vollständigen B-Platz-Ordnung in Relation gesetzt werden. Für LCMO ergeben XRD-Simulationen den theoretischen Wert für eine perfekten B-Platz-Ordnung von $S_{\text {theo. }}=10^{-2} 36,48$. Folglich unterschreitet hier die LCMO-Schicht diesen Wert mit $S=2,8(1) \cdot 10^{-3}$ signifikant. Im Vergleich mit XRD-Messungen an LCMO/STO (111)-Schichten in der Literatur, für die ein Ordnungsparameter von maximal $S=1,1 \cdot 10^{-3}$ beobachtet wurde 36, 48, ist der Wert jedoch leicht größer. Die anderen $\mathrm{A}_{2} \mathrm{CoMnO}_{6} / \mathrm{STO}$ (111)-Schichten zeigen einen Ordnungsparameter $S>10^{-2}$. Für die $\mathrm{A}_{2} \mathrm{NiMnO}_{6}$-Doppelperowskite ist aufgrund der leicht größeren Differenz in den atomaren Formfaktoren der $\mathrm{Ni}^{2+}$ - und $\mathrm{Mn}^{4+}$-Kationen 63] ein etwas größerer Wert gegenüber den $\mathrm{A}_{2} \mathrm{CoMnO}_{6}$-Doppelperowskiten zu erwarten, der sich experimentell hier jedoch nicht bestätigt. Während für die LNMO-Schicht mit $S=2,2(1) \cdot 10^{-3}$ ein ähnlich niedriger Wert wie für die LCMO-Schicht zu beobachten ist, zeigen die anderen $\mathrm{A}_{2} \mathrm{NiMnO}_{6} / \mathrm{STO}$ (111)-Schichten einen Ordnungsparameter $S>10^{-2}$. In der Literatur findet sich ein Ordnungsparameter $S \approx 1 \cdot 10^{-2}$ für $\mathrm{Bi}_{2} \mathrm{NiMnO}_{6}$ auf STO (100) [223], der in der gleichen Größenordnung der XRD-Simulationen für LCMO liegt.

Allgemein muss die Überstrukturreflexintensität im Zusammenhang mit der B-Platz-Ordnung der Doppelperowskite jedoch mit Vorsicht behandelt werden, da weitere Faktoren starken Einfluss auf diese nehmen können. So ist der Überstrukturreflex aufgrund der Ähnlichkeit der atomaren Formfaktoren der $\mathrm{Co}^{2+}-\mathrm{Ni}^{2+}-$ und $\mathrm{Mn}^{4+}$-B-Platz-Kationen nur relativ schwach und kann daher vom spektralen Hintergrund überlagert werden 224]. Eine Unterinterpretation der B-Platz-Ordnung in den Doppelperowskiten kann zudem durch die Präsenz von APG entstehen, die bei einer gegenphasigen Domänenorientierung zu einer starken Reduzierung der Überstrukturreflexintensität führen können, auch wenn die Domänen selbst eine hohe B-Platz-Nahordnung aufweisen 36, 48. Einen großen Einfluss haben zudem strukturelle Verzerrungen, Verkippungen der $\mathrm{B} / \mathrm{B}^{\prime} \mathrm{O}_{6}$-Oktaeder sowie Verschiebungen der A-Kationen, B-Kationen und $\mathrm{O}^{2-}$-Anionen innerhalb der Überstruktur der NaCl-Anordnung in der [111]-Richtung, die bei einer entsprechenden Abstandsmodulation zu einer Nachahmung der Überstruktur in den Doppelperowskiten führen können, ohne dass eine signifikante B-Platz-Kationenordnung vorliegt [4, 30, 221, 224]. Eine mögliche Ursache hierfür ist auch eine durch das Substrat in die Schichten induzierte Verspannung. Die Summe dieser Effekte kann sowohl zu einer deutlich verringerten als auch deutlich erhöhten Überstrukturreflexintensität führen, sodass die alleinige Betrachtung der Überstrukturreflexe nicht ausreichend ist, um den Grad der langreichweitigen B-Platz-Ordnung in den Doppelperowskiten zu bestimmen. Somit ist es nötig, den Ordnungsparameter in das Umfeld weiterer Untersuchungen einzufügen, um ein vollständigeres Bild von der B-Platz-Ordnung in den Doppelperowskiten zu gewinnen. Für die hergestellten $\mathrm{A}_{2} \mathrm{CoMnO}_{6} / \mathrm{STO}$ (111)- und $\mathrm{A}_{2} \mathrm{NiMnO}_{6} / \mathrm{STO}$ (111)-DoppelperowskitSchichten zeigt sich im Vergleich zu den jeweiligen Bulk-Doppelperowskiten 34, 35, 38 
eine leichte Verringerung der pseudokubischen out-of-plane Gitterkonstanten $c_{\mathrm{pc}}$ (beispielhaft $c_{\mathrm{pc}}=3,875 \AA$ anstatt $c_{\mathrm{pc} \text {, Bulk }}=3,887 \AA 34$ für LCMO), die durch eine in-plane Zugspannung des STO (111)-Substrats $(a=3,905 \AA$ 225]) und die daraus resultierende out-of-plane Druckspannung hervorgerufen wird. Da die Gitterkonstante der Doppelperowskite systematisch mit der Verringerung des A-Kationenradius abnimmt, erhöht sich gleichzeitig die in-plane durch das STO (111)-Substrat einwirkende Zugspannung. Hinweise auf den realen Ordnungsgrad der B-Platz-Kationen können die magnetischen Eigenschaften der Doppelperowskit-Schichten geben (Abschnitte 2.3.2 und 2.3.3). Nachfolgend in den Abbildungen 4.1.7 (a)-(f) sind daher entsprechend die temperaturabhängigen $M(T)$ Magnetisierungskurven (SQUID, FCC und ZFC, $H=1 \mathrm{kOe}$ ) der $\mathrm{A}_{2} \mathrm{CoMnO}_{6} / \mathrm{STO}(111)$ Doppelperowskit-Schichten (A = La, Pr, Nd, Sm, Gd, Y) dargestellt.
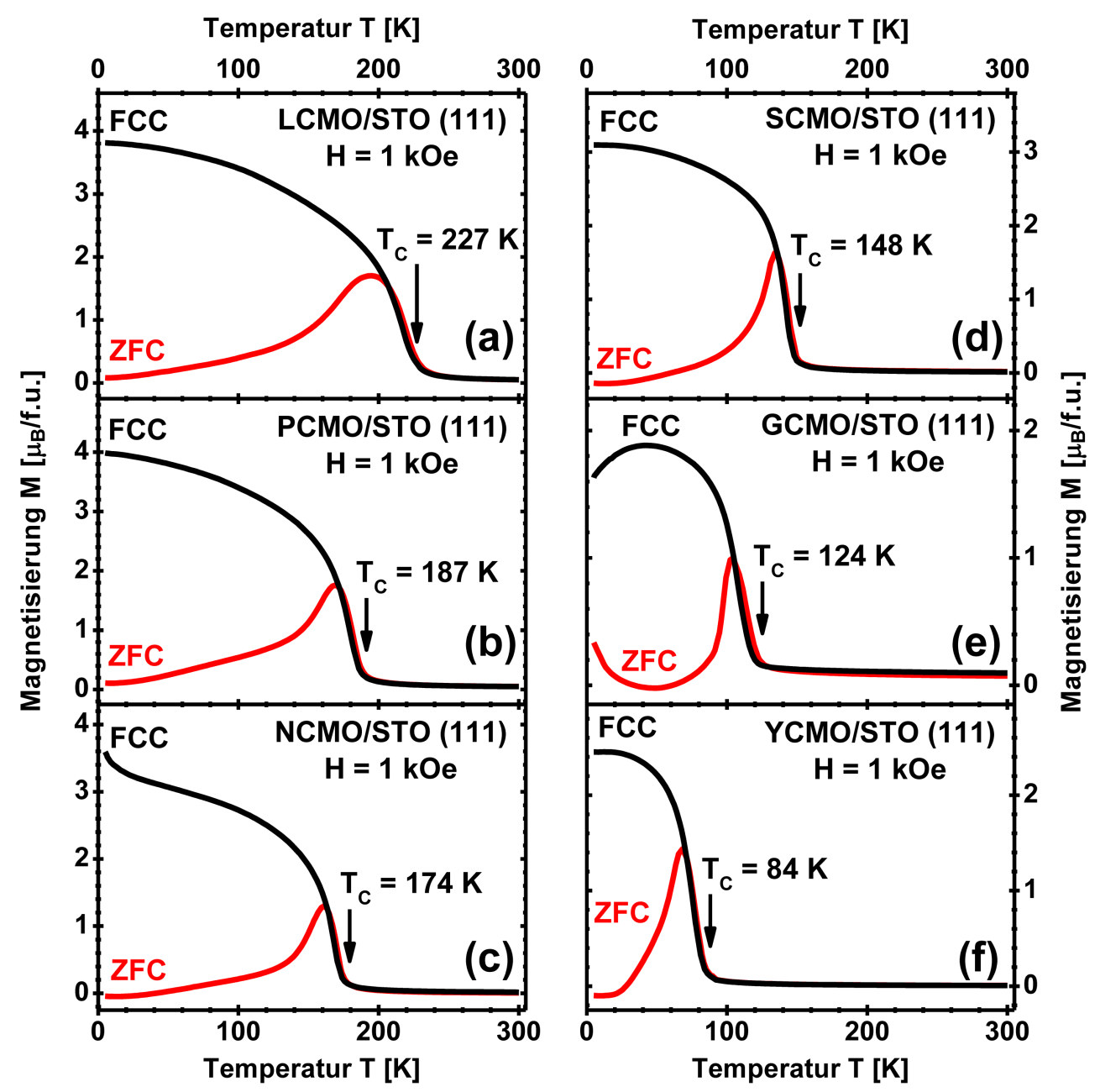

Abbildung 4.1.7: $M(T)$-Magnetisierungskurven (FCC und ZFC) von LCMO (a), PCMO (b), NCMO (c), SCMO (d), GCMO (e) und YCMO (f) auf STO (111).

Hierbei lässt sich eine systematische Abnahme der Curie-Temperatur $T_{C}$ (Minimum des Temperaturkoeffizienten TCM $=(1 / M)(\mathrm{d} M / \mathrm{d} T$ [36] $)$ mit der Verringerung des A-PlatzKationenradius von $T_{C}=227(2) \mathrm{K}(\mathrm{LCMO})$ auf $T_{C}=84(2) \mathrm{K}$ (YCMO) beobachten. Dieses Verhalten zeigt sich auch für Bulk- $\mathrm{A}_{2} \mathrm{CoMnO}_{6}$-Einkristalle 34, 35 und ist auf die 
Abnahme des $\mathrm{Co}^{2+}-\mathrm{O}^{2-}-\mathrm{M}^{4+}$-Bindungswinkels für den ferromagnetischen Superaustausch der $\mathrm{Co}^{2+}$ - und $\mathrm{Mn}^{4+}$-Kationen für kleinere A-Platz-Kationenradien zurückzuführen [34]. Die Übergangstemperaturen stimmen mit denen an Bulk-Proben überein 34,35 . Zudem lässt sich für alle $\mathrm{A}_{2} \mathrm{CoMnO}_{6} / \mathrm{STO}$ (111)-Schichten nur eine Curie-Temperatur in den FCC$M(T)$ - Kurven nachweisen. Dieses deutet auf eine mehrheitlich B-Platz geordnete Struktur hin. Gleichzeitig unterscheiden sich die FCC- und ZFC- $M(T)$-Kurven bei allen Schichten sehr deutlich. Die ZFC-Messungen zeigen ein für Spin-Gläser typisches Verhalten mit einem Maximum nahe $T_{C}$, antiferromagnetischer Kopplung für $T<T_{C}$ und thermisch aktivierten Dömanenbewegungen für $T>T_{C}$ aufgrund einer Frustration in der Spin-Anordnung sowie einer $360^{\circ}$-Dömanenwandausrichtung durch konkurrierende ferromagnetische und antiferromagnetische Wechselwirkungen in der Kristallstruktur 34,226, 227]. Somit befinden sich APG in den Dopelperowskiten, die die B-Platz geordneten Bereiche antiferromagnetisch voneinander trennen. Zur Verifizierung sind in den Abbildungen 4.1.7 (a)-(f) die $M(H)$ Hysteresekurven (SQUID, $T=5 \mathrm{~K}$, mit $H_{\text {max. }}=50 \mathrm{kOe}$ nach FCC mit $H=1 \mathrm{kOe}$ ) der $\mathrm{A}_{2} \mathrm{CoMnO}_{6} / \mathrm{STO}$ (111)-Schichten (A = La, Pr, Nd, Sm, Gd, Y) dargestellt.
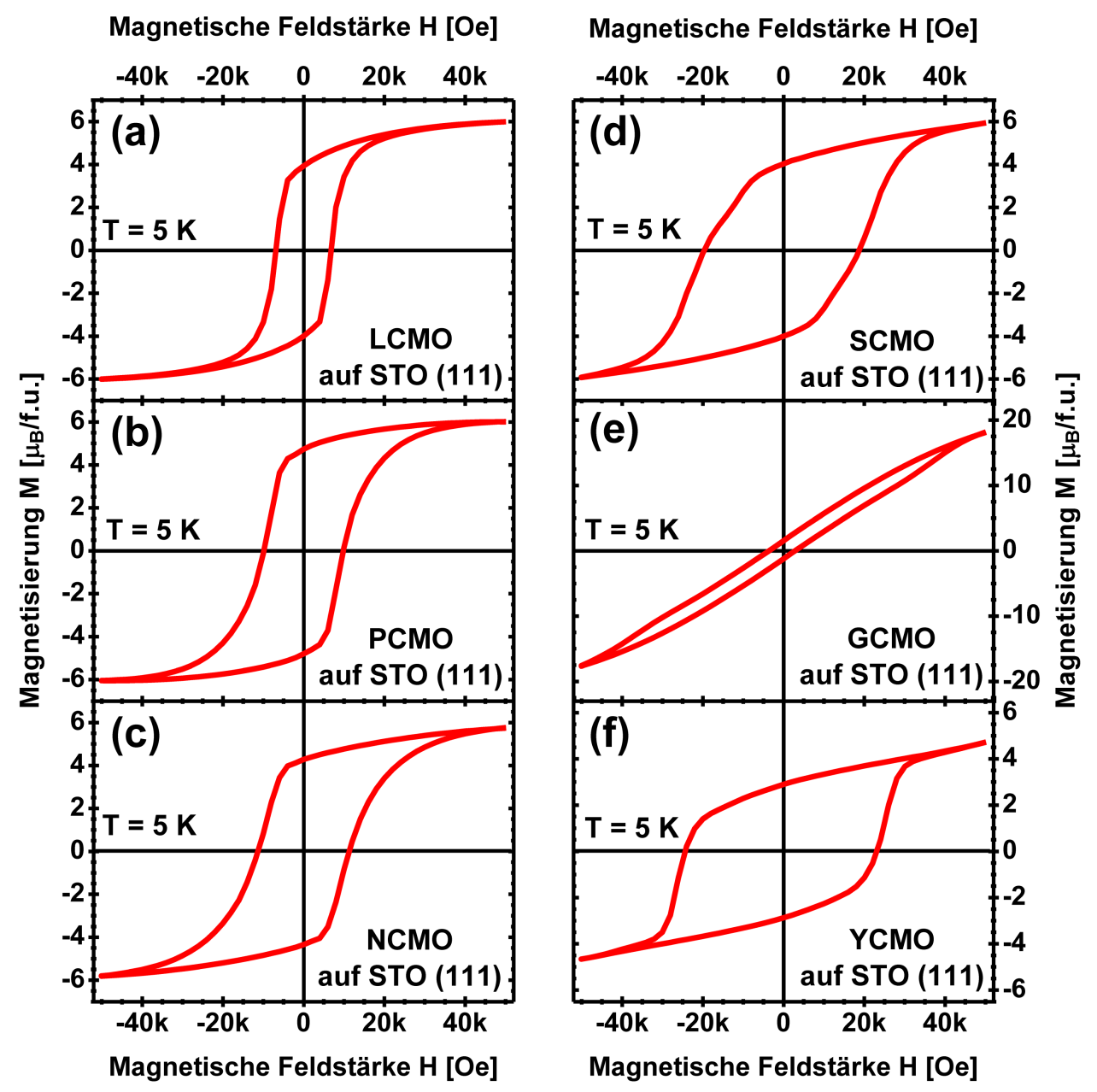

Abbildung 4.1.8: $M(H)$-Hysteresekurven von LCMO (a), PCMO (b), NCMO (c), SCMO (d), GCMO (e) und YCMO (f) auf STO (111) $(d \approx 90-100 \mathrm{~nm})$ im Vergleich $\left(T=5 \mathrm{~K}, H_{\max }=50 \mathrm{kOe}\right.$, nach FCC mit $\left.H=1 \mathrm{kOe}\right)$. 


\section{Auswertung und Ergebnisse}

Hierbei zeigt sich, dass die LCMO-, die PCMO-, die NCMO- und die SCMO-Schicht jeweils eine Sättigungsmagnetisierung $M_{S} \approx 6 \mu_{\mathrm{B}} /$ f.u. erreichen, die für vollständig B-Platz geordnete $\mathrm{A}_{2} \mathrm{CoMnO}_{6}$-Doppelperowskite durch den Spin-Beitrag der $\mathrm{Co}^{2+}$ - und $\mathrm{Mn}^{4+}$ Kationen zu erwarten ist. Dieses zeigt, dass in diesen Schichten eine hohe B-Platz-Ordnung vorhanden ist. Ein Beitrag der $\mathrm{Pr}^{3+}, \mathrm{Nd}^{3+}$ - und $\mathrm{Sm}^{3+}$-A-Kationen ist vernachlässigbar, da keine signifikanten Auffälligkeiten in den $M(T)$ - und $M(H)$-Kurven zu registrieren sind. Für die GCMO-Schicht tritt wiederum auch bei der maximalen Magnetfeldstärke $H_{\text {max. }}=50 \mathrm{kOe}$ noch keine Sättigung ein. Die erreichte Magnetisierung $M \approx 18,2 \mu_{\mathrm{B}} / \mathrm{f} . \mathrm{u}$. impliziert jedoch, dass neben den Spin-Beiträgen der B-Kationen ein starker Beitrag durch die $\mathrm{Gd}^{3+}$-Kationen präsent ist. Die theoretische Gesamtsumme der Spin-Beiträge von Aund B-Kationen mit $20 \mu_{\mathrm{B}} /$ f.u. wird jedoch nicht erreicht. Dieses wird auf eine antiferromagnetische Kopplung (3d-4f-Kopplung) der $\mathrm{Gd}^{3+}$-Kationen mit dem Co/Mn-B-Platz-Gitter für Temperaturen $T<50 \mathrm{~K}$ zurückgeführt 228]. Ein entsprechender Verlauf ist auch in der dazugehörigen FCC- $M(T)$-Kurve für $T<44 \mathrm{~K}$ in der Abbildung 4.1.7 (e) zu beobachten. Die YCMO-Schicht besitzt hingegen mit $M_{S} \approx 4,8 \mu_{\mathrm{B}} / \mathrm{f}$.u. die niedrigste Sättigungsmagnetisierung. Eine Erklärung hierfür ist nun einerseits der relativ schwache ferromagnetische $\mathrm{Co}^{2+} \mathrm{O}^{2-}-\mathrm{M}^{4+}-$ Superaustausch für YCMO (der kleinste $\mathrm{Co}^{2+} \mathrm{O}^{2-}-\mathrm{M}^{4+}$-Bindungswinkel der Serie) und andererseits die nun effektiv mehr den Gesamtmagnetismus beeinflussenden APG, die die Magnetisierung durch die antiferromagnetische Kopplung der geordneten Domänen für moderate Magnetfelder stark reduzieren [229]. Die erreichte Magnetisierung stimmt dabei mit Bulk-YCMO mit hoher B-Platz-Nahordnung sehr gut überein [229]. Die Präsenz von APG und B-Platz-Gitterfehlbesetzungen in den $\mathrm{A}_{2} \mathrm{CoMnO}_{6} / \mathrm{STO}$ (111)Schichten lässt sich nun anhand der Remanenz $M_{R}$ und der Koerzitivfeldstärke $H_{C}$ in den $M(H)$-Hysteresekurven diskutieren. Die dazugehörigen Werte sind zusammen mit der jeweiligen Sättigungsmagnetisierung $M_{S}$ nachfolgend in der Tabelle 4.1.1 aufgeführt.

\begin{tabular}{|c|c|c|c|}
\hline Doppelperowskit & $M_{S}\left[\mu_{\mathrm{B}} /\right.$ f.u. $]$ & $M_{r}\left[\mu_{\mathrm{B}} /\right.$ f.u. $]$ & $H_{C}[\mathrm{kOe}]$ \\
\hline \hline LCMO & $5,92(10)$ & $3,94(5)$ & $6,8(2)$ \\
\hline PCMO & $6,01(10)$ & $4,73(5)$ & $9,8(2)$ \\
\hline NCMO & $5,81(10)$ & $4,28(5)$ & $10,9(2)$ \\
\hline SCMO & $5,94(10)$ & $4,03(5)$ & $18,1(2)$ \\
\hline GCMO & $18,19(10)$ & $1,56(5)$ & $2,5(2)$ \\
\hline YCMO & $4,75(10)$ & $2,90(5)$ & $22,6(2)$ \\
\hline
\end{tabular}

Tabelle 4.1.1: Sättigungsmagnetisierung $M_{S}$, Remanenz $M_{R}$ und Koerzitivfeldstärke $H_{C}$ der $\mathrm{A}_{2} \mathrm{CoMnO}_{6} / \mathrm{STO}(111)$-Schichten $(d \approx 90-100 \mathrm{~nm})$ im Vergleich.

Bei einer Verringerung des A-Platz-Kations ist mit Ausnahme der GCMO-Schicht, für die die $M(H)$-Kurve durch die 3d-4f-Kopplung mit den $\mathrm{Gd}^{3+}$-Kationen geprägt ist, ein 
systematischer Anstieg der Koerzitivfeldstärke von $H_{C}=6,8(2) \mathrm{kOe}$ (für LCMO) auf $H_{C}=22,6(2) \mathrm{kOe}$ (für YCMO) zu beobachten. Ein ähnliches Verhalten ist für Bulk$\mathrm{A}_{2} \mathrm{CoMnO}_{6}$-Doppelperowskite nachzuweisen [34]. Gleichzeitig zeigt auch die Remanenz $M_{R}$ eine starke Variation, ohne eine spezifische Abhängigkeit vom A-Kationenradius aufzuweisen, mit dem höchsten Wert $M_{R}=4,73(5) \mu_{\mathrm{B}} /$ f.u. für die PCMO-Schicht und dem (abseits von GCMO) kleinsten Wert $M_{R}=2,90(5) \mu_{\mathrm{B}} /$ f.u. für die YCMO-Schicht.

Unter Berücksichtigung eines einfachen magnetischen Modellansatzes 36, 48, kann nun aus der Form der $M(H)$-Kurve die Dichte und die Größe der APG in den DoppelperowskitSchichten abgeschätzt werden. Hierzu wird angenommen, dass ein größeres Koerzitivfeld und eine geringere Remanenz mit einer höheren Dichte an APG im Gitter und einer kleineren Größe gegenphasig orientierter Domänen mit B-Platz-Nahordnung einhergehen. Folglich ist die geringste APG-Dichte in der PCMO-Schicht zu finden, während diese für die YCMO-Schicht am höchsten ist (eine Beurteilung für die GCMO-Schicht ist aufgrund der $\mathrm{Gd}^{3+}$-Kationen nicht möglich). Die Größe der von den APG umgebenden Domänen mit hoher B-Platz-Ordnung ist für die LCMO-Schicht am größten, während diese für YCMO besonders klein sind. Dieses Systematik lässt sich mithilfe von Umklappprozesses im externen Magnetfeld erklären. So zeigt sich, dass sich große Domänen mit B-Platz-Nahordnung, aber antiferromagnetischer Orientierung zu der umgebenen Matrix bereits bei kleinen Magnetfeldern ferromagnetisch ausrichten lassen, während für kleinere Domänen eine größere Feldstärke benötigt wird 36, 48]. Folglich ist die Anzahl der APG im Kristallgitter aus der Abweichung der Remanenz zur Sättigungsmagnetisierung bestimmt, während die Koerzitivfeldstärke entsprechend die mittlere Domänengröße wiedergibt.

Zudem lassen sich so die APG und Gitterfehlbesetzungen voneinander trennen. Wird die Sättigungsmagnetisierung $M_{S}$ erreicht, so sind alle Domänen ferromagnetisch zu der umgebenden Matrix ausgerichtet. Die Differenz der Remanenz zur Sättigungsmagnetisierung kennzeichnet daher den Magnetisierungsgewinn durch die Umklappprozesse. Für einzelne Gitterfehlbesetzungen ist das Umklappen auch im Maximalfeld nicht möglich [31]. Folglich gehen diese mit einer Verringerung der Sättigungsmagnetisierung einher. Somit kann der B-Platz-Nahordnungsgrad über die Abweichung zum Spin-Beitrag von $6 \mu_{\mathrm{B}} /$ f.u. der $\mathrm{Co}^{2+}$ - und $\mathrm{Mn}^{4+}$-Kationen für eine perfekte B-Platz-Ordnung abgeschätzt werden. Für die LCMO-, PCMO-, NCMO- und SCMO-Schicht ergeben sich so entsprechend Ordnungsgrade von $\approx 96-100 \%$. Einzig für die YCMO-Schicht wird durch die antiferromagnetische Kopplung kleiner Domänen-Cluster mit $\approx 80 \%$ eine geringere Nahordnung erreicht. Zudem können nun die Ordnungsparameter $S$ der $\mathrm{A}_{2} \mathrm{CoMnO}_{6} / \mathrm{STO}$ (111)-Schichten aus den XRDSpektren erklärt werden. So lässt sich der kleine Ordnungsparameter der LCMO-Schicht auf eine hohe Anzahl großer B-Platz geordneter Domänen zurückführen, die bei einer gegenphasigen Orientierung durch die APG und annähernd gleichen Volumenanteilen trotz hoher 


\section{Auswertung und Ergebnisse}

B-Platz-Nahordnung zu einer starken Reduzierung der Überstrukturreflexintensität führen, während Gitterfehlbesetzungen diesen Unterschied mehrerer Größenordnungen nicht hervorrufen können [48]. Für die anderen Doppelperowskite der $\mathrm{A}_{2} \mathrm{CoMnO}_{6}$-Reihe nimmt die Domänengröße mit der Verkleinerung des A-Kationenradius ab, wodurch die gegenphasige Auslöschung der Überstrukturreflexintensität weniger ausgeprägt ist und der Ordnungsparameter $S$ ansteigt. Gleichzeitig können jedoch auch noch Artefakte auftreten, die den Ordnungsparameter fälschlicherweise weiter erhöhen. Dieses zeigt sich an der YCMO-Schicht, für die Gitterfehlbesetzungen auftreten, die die Sättigungsmagnetisierung verringern. Der weitere Parameteranstieg ist daher nicht auf einen Ordnungszugewinn zurückführen. Vielmehr ist hier eine Abstandsmodulation durch die Verschiebung der Kationen von ihren Gitterplätzen aufgrund des kleinen A-Kations die Ursache 30,221. In gleicher Weise können nun die $\mathrm{A}_{2} \mathrm{NiMnO}_{6} / \mathrm{STO}$ (111)-Schichten (A = La, Pr, Nd, Sm, Gd, Y) betrachtet werden. Die temperaturabhängigen $M(T)$-Magnetisierungskurven (SQUID, FCC und ZFC, $H=10 \mathrm{kOe}$ ) sind in den Abbildungen 4.1 .9 (a)-(f) dargestellt.
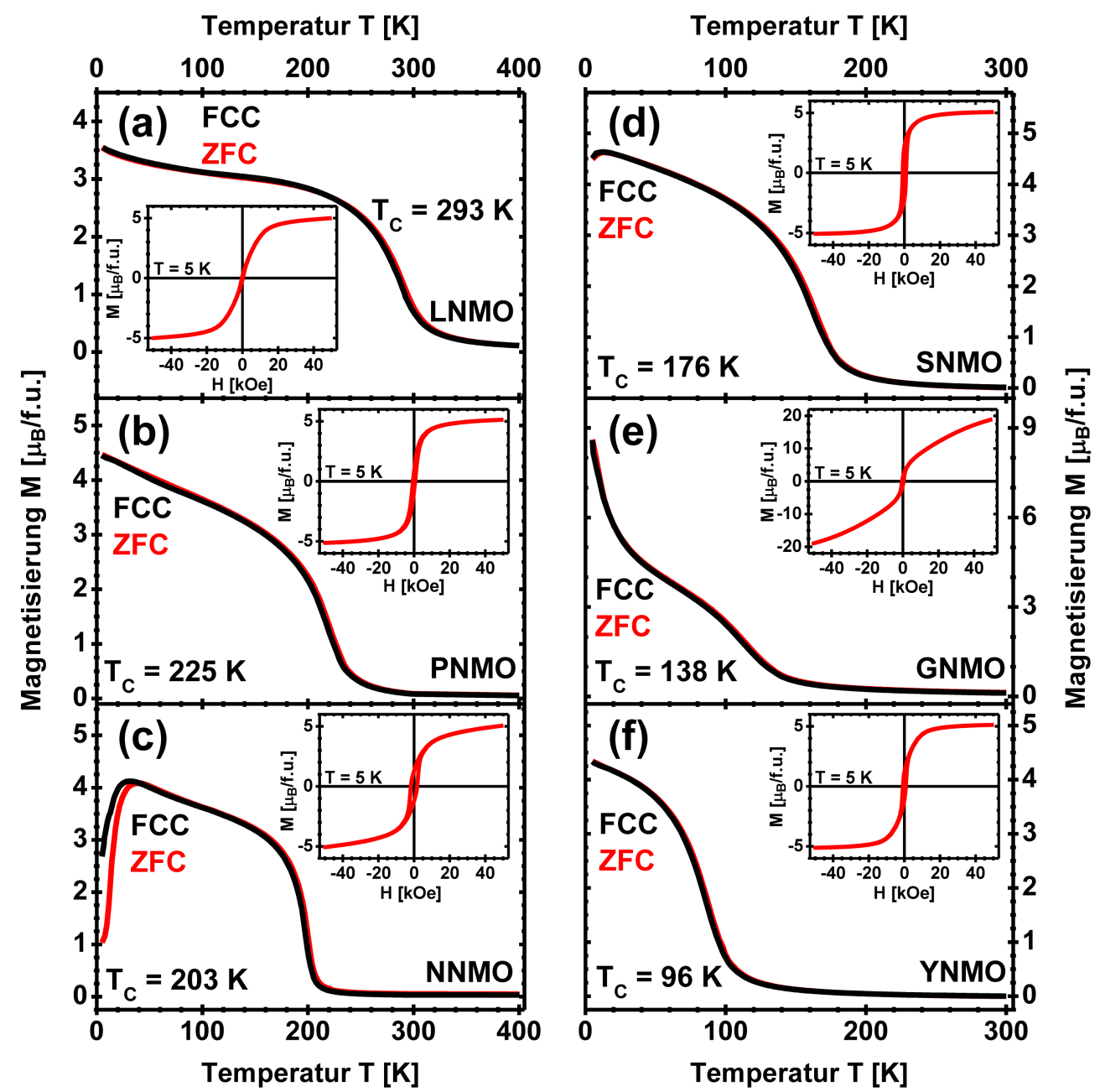

Abbildung 4.1.9: $M(T)$-Magnetisierungskurven (FCC und ZFC) von LNMO (a), PNMO (b), NNMO (c), SNMO (d), GNMO (e) und YNMO (f) auf STO (111) im Vergleich $(d \approx 90-100 \mathrm{~nm})$. Die eingebetteten Graphen zeigen die $M(H)$ Hysteresekurven $\left(T=5 \mathrm{~K}, H_{\max }=50 \mathrm{kOe}\right.$ nach FCC mit $\left.H=1 \mathrm{kOe}\right)$. 
Erneut lässt sich eine systematische Abnahme der Curie-Temperatur $T_{C}$ mit der Verringerung des A-Platz-Kationenradius von $T_{C}=293(2) \mathrm{K}(\mathrm{LNMO})$ auf $T_{C}=96(2) \mathrm{K}(\mathrm{YNMO})$ beobachten. Diese Entwicklung zeigt sich auch für Bulk- $\mathrm{A}_{2} \mathrm{NiMnO}_{6}$-Proben 35,38 und ist auf die $\mathrm{Ni}^{2+}-\mathrm{O}^{2-}-\mathrm{M}^{4+}$-Bindungswinkelabnahme für den ferromagnetischen Superaustausch der $\mathrm{Ni}^{2+}$ - und $\mathrm{Mn}^{4+}$-Kationen für kleinere A-Platz-Kationen zurückzuführen [38]. Weiterhin ist für alle Schichten nur eine Curie-Temperatur in den FCC-M(T)-Kurven nachzuweisen. Dieses bestätigt eine mehrheitlich B-Platz geordnete Struktur. Im Vergleich zu den $\mathrm{A}_{2} \mathrm{CoMnO}_{6}$-Schichten sind die Curie-Temperaturen etwas höher. Dieses weist auf eine stärkere ferromagnetische Kopplung in den $\mathrm{A}_{2} \mathrm{NiMnO}_{6}$-Schichten hin. Für die Bulk-Proben beider Reihen wird ein ähnliches Verhalten beobachtet [35]. Die FCC- und ZFC-M(T)Kurven unterscheiden sich dabei anders als bei den $\mathrm{A}_{2} \mathrm{CoMnO}_{6}$-Doppelperowskiten kaum. Ein Grund ist hier das stärkere Magnetfeld von $H=10 \mathrm{kOe}$, um den Einfluss des starken diamagnetischen Hintergrundbeitrags durch das STO (111)-Substrat bei tiefen Temperaturen abzuschwächen. Zudem ist der Unterschied zwischen FCC- und ZFC-Kurve auch bei $H=1 \mathrm{kOe}$ nur gering. Eine Ausnahme bildet die NNMO-Schicht, da die Magnetisierung für Temperaturen $T<30 \mathrm{~K}$ durch eine antiferromagnetische Spin-Bahn-Kopplung der $\mathrm{Nd}^{3+}$-Kationen mit dem B-Platz-Netzwerk abnimmt [38] und so die ZFC-Kurve dort eine etwas geringe Magnetisierung aufweist. Eine ähnliche Kopplung deutet sich für die SNMOSchicht an, der Effekt ist jedoch deutlich geringer. Auffällig ist zudem die GNMO-Schicht, die jedoch anders als GCMO für Temperaturen $T<50 \mathrm{~K}$ in beiden $M(T)$-Kurven einen starken paramagnetischen Beitrag durch die $\mathrm{Gd}^{3+}$-Kationen zeigt [230].

Werden nun die $M(H)$-Hysteresekurven der $\mathrm{A}_{2} \mathrm{NiMnO}_{6} / \mathrm{STO}$ (111)-Schichten betrachtet (eingebettete Graphen in den Abbildungen 4.1.9 (a)-(f)), so lassen sich signifikante Unterschiede zu den $\mathrm{A}_{2} \mathrm{CoMnO}_{6} / \mathrm{STO}$ (111)-Schichten zuvor beobachten. Zunächst ist der SpinBeitrag der $\mathrm{Ni}^{2+}$ - und $\mathrm{Mn}^{4+}$-Kationen durch das zusätzliche $\mathrm{Ni}^{2+}-3$ d-Elektron auf $5 \mu_{\mathrm{B}} /$ f.u. reduziert. Dieses wird für alle $\mathrm{A}_{2} \mathrm{NiMnO}_{6} / \mathrm{STO}$ (111)-Schichten mit einer Sättigungsmagnetisierung $M_{S} \approx 5 \mu_{\mathrm{B}} /$ f.u. erfüllt. Somit ist in den Schichten eine hohe B-Platz-Ordnung vorhanden. Für die GNMO-Schicht tritt jedoch ähnlich wie bei der GCMO-Schicht auch bei maximaler Magnetfeldstärke $H_{\text {max. }}=50 \mathrm{kOe}$ noch keine Sättigung ein. Die erreichte Magnetisierung $M \approx 18,9 \mu_{\mathrm{B}} /$ f.u. impliziert, dass neben den Spin-Beiträgen der B-Kationen ein starker paramagnetischer Beitrag durch die $\mathrm{Gd}^{3+}$-Kationen präsent ist. Die resultierende Kopplung führt zu einer theoretischen Magnetisierung von $19 \mu_{\mathrm{B}} /$ f.u. [228, 230], die hier nahezu erreicht wird. Auffallend sind zudem die sehr kleinen Koerzitivfeldstärken $H_{C}$, die für alle $\mathrm{A}_{2} \mathrm{NiMnO}_{6} / \mathrm{STO}$ (111)-Schichten im Vergleich zu den $\mathrm{A}_{2} \mathrm{CoMnO}_{6^{-}}$ Doppelperowskiten um mehr als eine Größenordnung reduziert sind. Eine Übersicht der Werte ist zusammen mit denen der Sättigungsmagnetisierung $M_{S}$ und der Remanenz $M_{R}$ in der Tabelle 4.1 .2 aufgeführt. Das Verhalten wird auch in der Literatur für B-Platz geordnete $\mathrm{A}_{2} \mathrm{NiMnO}_{6}$-Schichten und -Bulk-Proben beobachtet [38]. Eine Erklärung ist der 
kleinere Radius $r_{\mathrm{Ni}^{2+}}=69 \mathrm{pm}(\mathrm{KZ}=6)$ des $\mathrm{Ni}^{2+}$-Kations gegenüber dem HS-Co ${ }^{2+}$-Kation $\left(r_{\mathrm{Co}^{2+}}=74,5 \mathrm{pm}, \mathrm{KZ}=6\right)$ 222], wodurch offensichtlich die Bedingungen für die Entstehung von APG und Gitterfehlbesetzungen ebenso wie für die magnetische Kopplung modifiziert werden. Im Hinblick auf die XRD-Ordnungsparameter $S$ der $\mathrm{A}_{2} \mathrm{NiMnO}_{6}$-Schichten auf STO (111) ist eine Entwicklung wie für die $\mathrm{A}_{2} \mathrm{CoMnO}_{6} / \mathrm{STO}$ (111)-Schichten zu beobachten. Folglich ist der geringe Ordnungsparameter der LNMO-Schicht auf eine erhöhte Anzahl großer durch APG von der umgebenden Matrix getrennter Nahordnungsdomänen zurückzuführen, die aufgrund ihrer gegenphasigen Ausrichtung die Überstrukturreflexintensität stark reduzieren. Wird der A-Kationenradius verringert, so werden die Domänen kleiner und der Überstrukturreflex ist weniger stark beeinflusst. Die geringeren Werte für die Koerzitivfeldstärke und die Remanenz deuten dabei auf eine reduzierte Domänengröße in den $\mathrm{A}_{2} \mathrm{NiMnO}_{6} / \mathrm{STO}$ (111)-Schichten im Vergleich zu der $\mathrm{A}_{2} \mathrm{CoMnO}_{6}$-Reihe hin.

\begin{tabular}{|c|c|c|c|}
\hline Doppelperowskit & $M_{S}\left[\mu_{\mathrm{B}} /\right.$ f.u. $]$ & $M_{r}\left[\mu_{\mathrm{B}} /\right.$ f.u. $]$ & $H_{C}[\mathrm{kOe}]$ \\
\hline \hline LNMO & $5,00(10)$ & $0,19(5)$ & $0,25(2)$ \\
\hline PNMO & $5,13(10)$ & $0,87(5)$ & $0,55(2)$ \\
\hline NNMO & $5,07(10)$ & $1,28(5)$ & $1,76(2)$ \\
\hline SNMO & $5,07(10)$ & $2,23(5)$ & $1,05(2)$ \\
\hline GNMO & $18,94(10)$ & $1,34(5)$ & $0,45(2)$ \\
\hline YNMO & $5,12(10)$ & $1,19(5)$ & $0,79(2)$ \\
\hline
\end{tabular}

Tabelle 4.1.2: Sättigungsmagnetisierung $M_{S}$, Remanenz $M_{R}$ und Koerzitivfeldstärke $H_{C}$ der $\mathrm{A}_{2} \mathrm{NiMnO}_{6} / \mathrm{STO}(111)$-Schichten $(d \approx 90-100 \mathrm{~nm})$ im Vergleich.

Im Anschluss kann nun noch der Einfluss der A-Platz- und B-Platz-Kationen auf die phononischen Eigenschaften der $\mathrm{A}_{2} \mathrm{CoMnO}_{6^{-}}$und der $\mathrm{A}_{2} \mathrm{NiMnO}_{6}$-Doppelperowskit-Schichten auf STO (111) mithilfe der Raman-Spektroskopie charakterisiert werden. Hierzu sind in den Abbildungen 4.1.10 (a)-(d) die polarisationsabhängigen Raman-Spektren der $\mathrm{A}_{2} \mathrm{CoMnO}_{6}{ }^{-}$ und der $\mathrm{A}_{2} \mathrm{NiMnO}_{6}$-Schichten (A = La, Pr, Nd, Sm, Gd, Y) auf STO (111) mit der Schichtdicke $d \approx 90-100 \mathrm{~nm}$ für einen direkten Vergleich dargestellt. Das dazugehörige Probenbezugssystem besitzt dabei die Richtungen x $\|$ [11 $\overline{2}]$, y $\|$ [110] und z $\|$ [111]. Die Spektren selbst wurden in senkrechter Rückstreugeometrie aufgenommen. Die RamanSpektren in der parallelen xx-Konfiguration sind durch die sehr intensive $A_{g}$ Breathing Mode bei $\approx 630-675 \mathrm{~cm}^{-1}$ gekennzeichnet, die mit ihrer hohen Intensität in dieser Konfiguration und einer starken Intensitätsabnahme in der gekreuzten xy-Konfiguration die Raman-Auswahlregeln für die $A_{g}$-Symmetrie der monoklinen B-Platz geordneten P12 1 /n1Struktur erfüllt. Die umgekehrte Polarisationsabhängigkeit bestätigt wiederum gleichzeitig die mehrheitliche $B_{g}$-Symmetrie der Mischmode bei $\approx 470-530 \mathrm{~cm}^{-1}$. Daneben sind für alle Schichten sowohl eine Oberschwingung 2. Ordnung der $A_{g}$ Breathing Mode als auch eine 
Kombinationsschwingung der Breathing Mode und der Mischmode zu beobachten, wodurch die hohe kristalline Qualität beider Schichtreihen bestätigt wird. Zudem zeigt sich, dass die Positionen der Raman-Moden und ihre Anzahl stark von der A- und B-Kationengröße beeinflusst werden. So sind alle beobachtbaren Raman-Moden für die $\mathrm{A}_{2} \mathrm{NiMnO}_{6}$-Reihe gegenüber den entsprechenden $\mathrm{A}_{2} \mathrm{CoMnO}_{6}$-Schichten signifikant um etwa $20-30 \mathrm{~cm}^{-1} \mathrm{zu}$ größeren Wellenzahlen verschoben. Strukturelle Untersuchungen offenbaren jedoch, dass die mittleren Bindungslängen in beiden Reihen ebenso wie die Gitterkonstanten eine vergleichbare Größe besitzen 35. Stattdessen wird die Modenverschiebung auf einen größeren orbitalen Überlapp der $\mathrm{Ni}^{2+}$ - und $\mathrm{Mn}^{4+}$-Kationen im Vergleich zu den den $\mathrm{Co}^{2+}$ und $\mathrm{Mn}^{4+}$-Kationen und eine größere Stärke der Kationenbindungen in den $\mathrm{A}_{2} \mathrm{NiMnO}_{6}{ }^{-}$ Doppelperowskiten zurückgeführt [35]. Weiterhin zeigt sich, dass die Mischmode mit abnehmender A-Kationenradius strukturierter wird, bis sie schließlich immer weiter in verschiedene Einzelmoden aufspaltet. Zusätzlich lässt sich auch eine immer größer werdende Zahl an Moden im Bereich niedriger Wellenzahlen beobachten. Die Ursache hierfür ist dabei die zunehmende monokline Verzerrung der Doppelperowskite für kleiner werdende A-Kationen [231]. Folglich kennzeichnen die zusätzlichen Moden spezifische Biege- und Verkippungsbewegungen der die B-Kationen umgebenden Sauerstoffoktaeder [35].
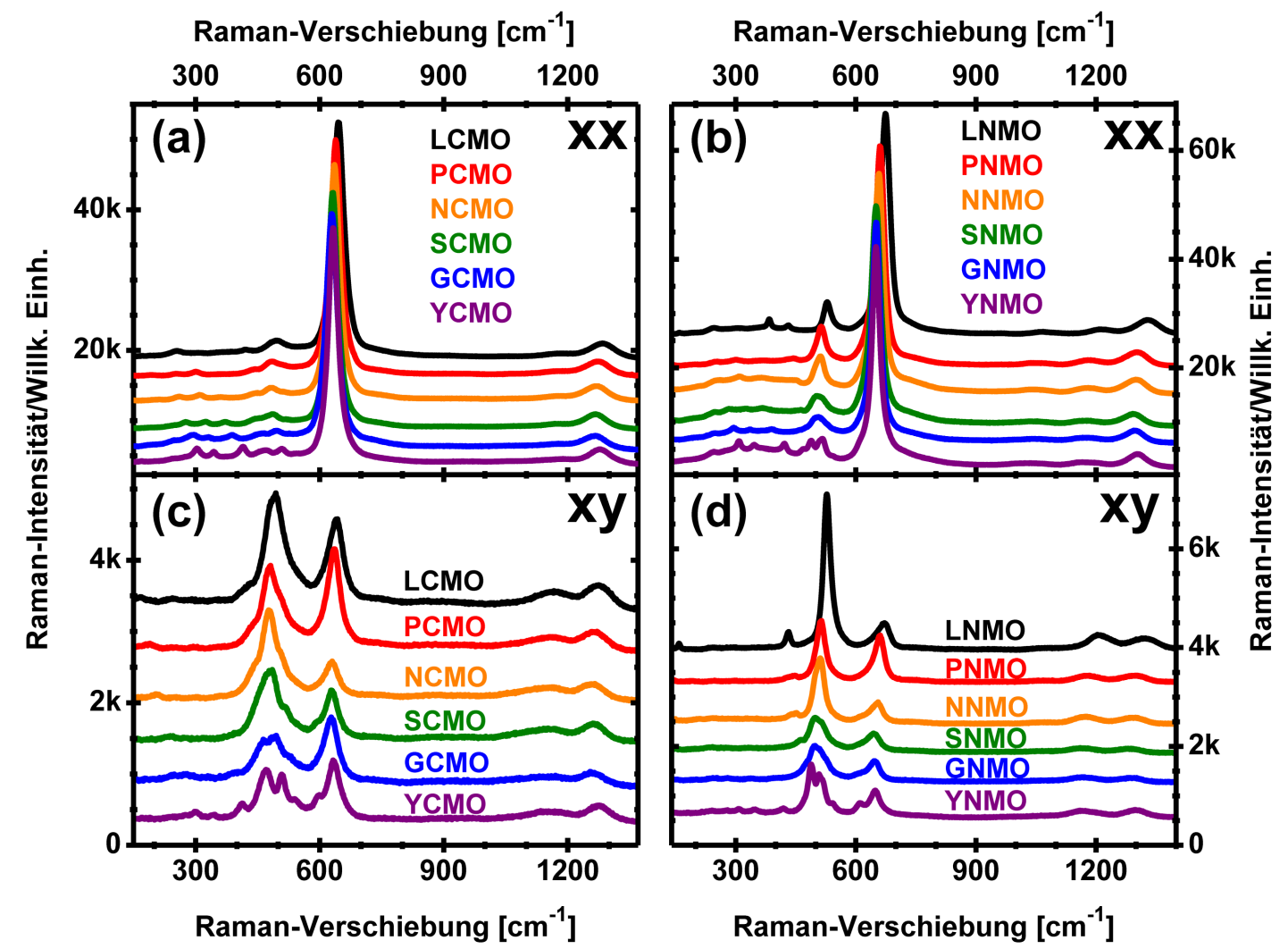

Abbildung 4.1.10: Polarisierte Raman-Spektren $(\lambda=532 \mathrm{~nm}, P \approx 5 \mathrm{~mW}, t=3 \times 30 \mathrm{~s}$; aufgenommen bei Raumtemperatur) der $\mathrm{A}_{2} \mathrm{CoMnO}_{6^{-}}$und der $\mathrm{A}_{2} \mathrm{NiMnO}_{6^{-}}$ Schichten auf STO $(111)(d \approx 90-100 \mathrm{~nm})$ in der parallelen $\mathrm{xx}-(\mathrm{a}),(\mathrm{b})$ und der gekreuzten xy-Polarisationskonfiguration (c), (d) im Vergleich. 
Die A-Kationengröße hat jedoch auch Auswirkungen auf die Modenpositionen. So lässt sich beginnend von LCMO und LNMO in beiden Doppelperowskit-Reihen eine systematische Abnahme der Wellenzahl der $A_{g}$ Breathing Mode bei Verringerung des A-Kationenradius feststellen. Diese Entwicklung hält jedoch nur bis GCMO und SNMO an, bevor sich für YCMO sowie GNMO und YNMO anschließend wieder ein Erhärten der Breathing Mode einstellt. In den Abbildungen 4.1.11 (a) und (b) ist diese Entwicklung der Position der $A_{g}$ Breathing Mode beider Doppelperowskit-Reihen mit dem A-Kationenradius zusammen mit dem Verlauf für die Curie-Temperatur $T_{C}$ dargestellt. Während die $T_{C}$-Entwicklung mit der Abnahme der A-Kationengröße ein lineares Verhalten in Richtung niedrigerer Temperaturen zeigt, ist eine deutliche Abkehr des zuvor ähnlichen Trends für die Position der $A_{g}$ Breathing Mode zu erkennen. Während die Reduzierung der Curie-Temperatur für einen kleineren A-Kationenradius auf die damit einhergehende Abnahme des für den ferromagnetischen Superaustausch relevanten $\mathrm{Co}^{2+} / \mathrm{Ni}^{2+}-\mathrm{O}^{2-}-\mathrm{Mn}^{4+}$-Bindungswinkels zurückgeführt werden kann, ist die Situation für die Position der $A_{g}$ Breathing Mode komplexer. So führt ein kleinerer A-Kationenradius zunächst zu einem Erweichen der Mode aufgrund der Zunahme der mittleren B/B'-O-Bindungslänge und der in-plane Zugspannung in den Schichten. Untersuchungen in der Literatur zeigen jedoch beispielsweise für die $\mathrm{A}_{2} \mathrm{NiMnO}_{6^{-}}$ Doppelperowskite, dass diese Bindungslängenzunahme nicht über die ganze Reihe hinweg anhält, sondern sich von SNMO auf GNMO eine Trendumkehr einstellt und die mittlere $\mathrm{Ni} / \mathrm{Mn}-\mathrm{O}-$ Bindungslänge anschließend wieder abnimmt 38, 232. Dieser Effekt wird hier mithilfe der strukturellen Sensitivität der Raman-Spektroskopie bestätigt.
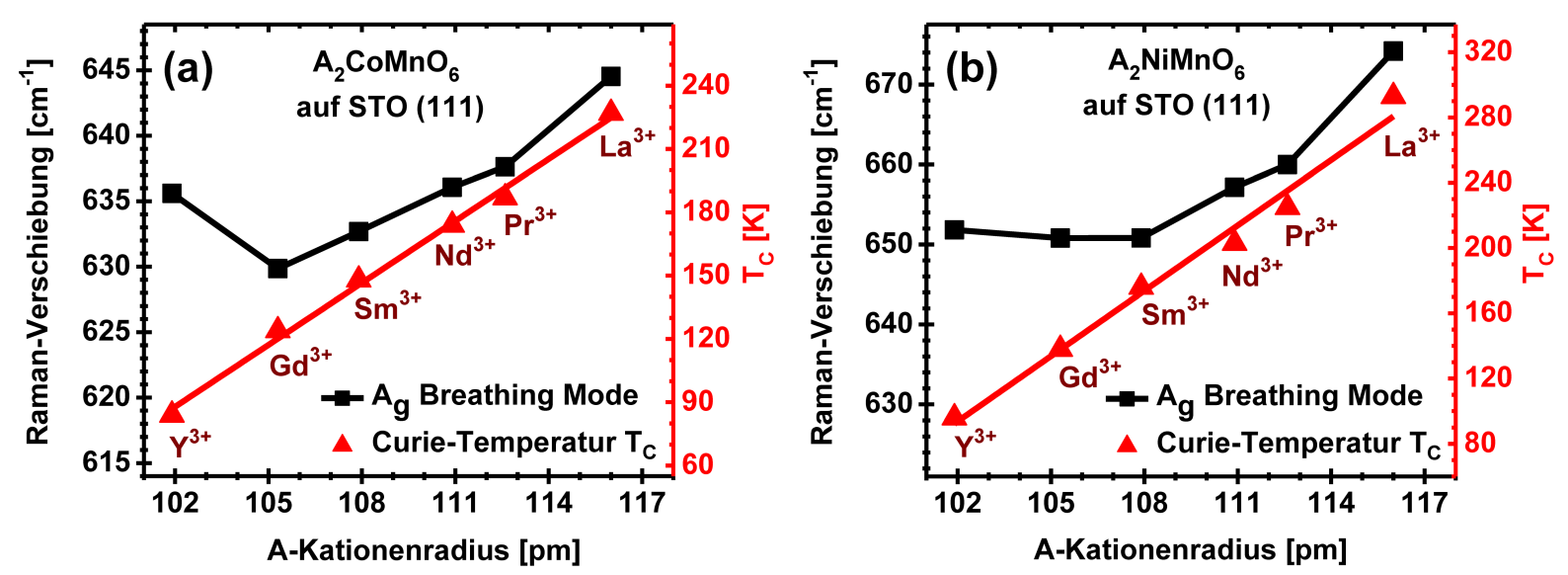

Abbildung 4.1.11: Positionen der $A_{g}$ Breathing Mode und die Curie-Temperaturen $T_{C}$ der $\mathrm{A}_{2} \mathrm{CoMnO}_{6^{-}}$(a) und der $\mathrm{A}_{2} \mathrm{NiMnO}_{6^{-}}$Schichten (b) auf STO (111) in Abhängigkeit des A-Kationenradius. Die Modenpositionen stammen aus den Raman-Spektren in den Abbildungen 4.1.10 (a) und (b). 


\subsubsection{B-Platz-Ordnung und der Einfluss der Verspannung}

Nach der Betrachtung der $\mathrm{A}_{2} \mathrm{CoMnO}_{6}$ - und $\mathrm{A}_{2} \mathrm{NiMnO}_{6}$-Doppelperowskit-Schichtreihen auf STO (111) sollen nun die B-Platz-Ordnung und der Einfluss der Schichtverspannung auf die Ordnung und die strukturellen Eigenschaften anhand von $\mathrm{La}_{2} \mathrm{CoMnO}_{6}$ betrachtet werden. Im Unterschied zum vorherigen Abschnitt, wo die Eigenschaften in Abhängigkeit der chemischen Verspannung untersucht wurden, wird die Verspannung nun extern durch das Wachstum auf Substraten mit unterschiedlichen in-plane Gitterkonstanten eingebracht. Eine Übersicht der in-plane Gitterkonstanten $a$ der verwendeten Substrate 225 und die jeweilige Gitterfehlanpassung $f$ in Relation zu Bulk-LCMO mit der pseudokubischen Gitterkonstante $a_{\mathrm{pc}, \text { Bulk }}=3,887 \AA 34$ ist in der Abbildung 4.1 .12 dargestellt.

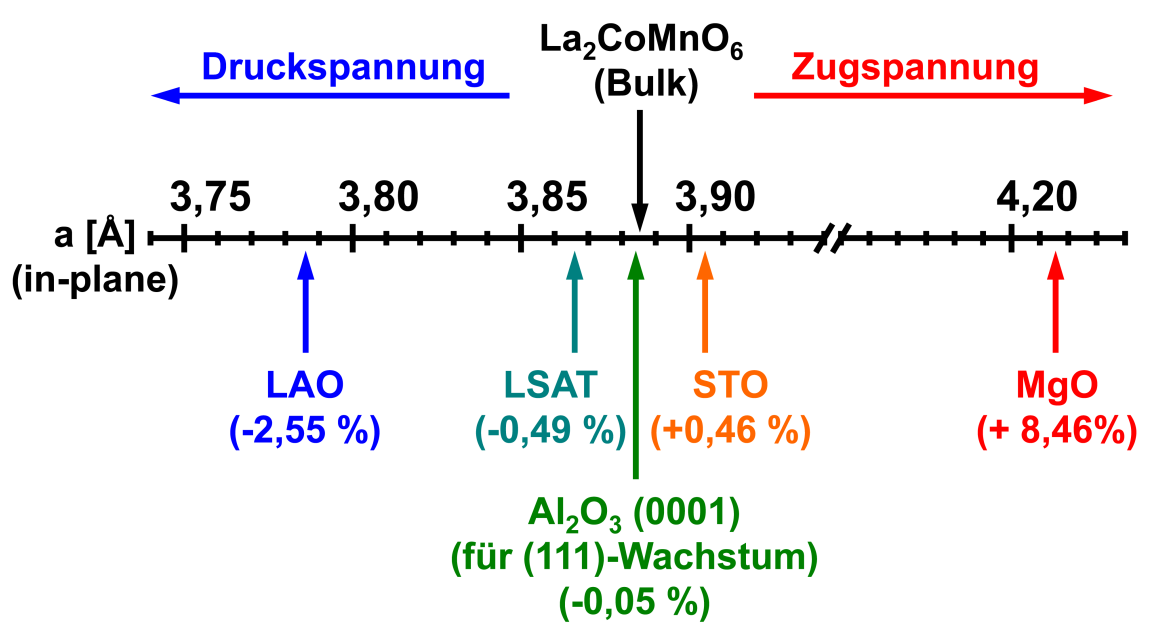

Abbildung 4.1.12: In-plane Gitterkonstanten $a$ verschiedener Substrate $[225]$ und die jeweilige
Gitterfehlanpassung $f$ in Relation zu Bulk-LCMO 34 . Für $\mathrm{Al}_{2} \mathrm{O}_{3}(0001)$
ist dabei die effektive Gitterkonstante $a_{\text {eff. für ein kubisches Wachstum von }}$
LCMO in der (111)-Orientierung angegeben.

Da der Einfluss der Verspannung auch auf den Ordnungsparameter $S$ in den XRD-Spektren bestimmt werden soll, werden LAO-, LSAT- und STO-Substrate mit einer (111)-Orientierung verwendet. Während LAO $(a=3,788 \AA$ 225]) und $\operatorname{LSAT}(a=3,868 \AA[225])$ dabei über eine kleinere in-plane Gitterkonstante als Bulk-LCMO verfügen und die LCMOSchichten somit unter dem Einfluss einer in-plane Druckspannnung aufwachsen, besitzt STO $(a=3,905 \AA 225)$ eine größere Gitterkonstante und induziert damit eine inplane Zugspannung in die LCMO-Schicht. Die Charakterisierung einer LCMO-Schicht auf STO (111) findet sich im vorherigen Abschnitt 4.1.2. Ein Vergleich mit den anderen LCMO-Schichten bezieht sich somit immer auf diese LCMO/STO (111)-Schicht. Daneben kann das Wachstum auf $\mathrm{MgO}$ mit einer besonders großen in-plane Gitterkonstante $a=4,216$ A 225 betrachtet werden, das hier jedoch nur mit einer (100)-Orientierung vorliegt. Zusätzlich soll das Wachstum von LCMO mit (111)-Orientierung auf hexagonalen $\mathrm{Al}_{2} \mathrm{O}_{3}$ (0001)-Substraten als eine neue Alternative $\mathrm{zu}$ den herkömmlich verwendeten Substraten für dünne LCMO-Schichten vorgestellt werden. Die Wachstumsart von LCMO 
ist dabei ähnlich zu der anderer Materialien mit NaCl-Struktur wie MgO mit einer (111)Orientierung auf hexagonalem $\mathrm{Al}_{2} \mathrm{O}_{3}$ (0001) 233,234 und umgekehrt zu hexagonalem $\mathrm{ZnO}$ mit einer (0001)-Orientierung auf kubischem STO (111) 235,236]. Die Ursache hierfür ist die hexagonale Symmetrie der kubischen (111)-Ebene. Somit resultiert für Schichten mit pseudokubischer Struktur out-of-plane eine (111)-Orientierung auf hexagonalen Substraten und für Schichten mit hexagonaler Struktur out-of-plane eine (0001)-Orientierung auf kubischen (111)-Substraten 233 236]. Für die (111)-Ebene von LCMO beträgt die effektive in-plane Gitterkonstante nun $a_{\mathrm{pc}} / \sqrt{2}$ und damit die Hälfte der Flächendiagonale der pseudokubischen Einheitszelle. Demnach wird eine Reduzierung der Gitterfehlanpassung durch eine $30^{\circ}$-Rotation der in-plane Orientierung von LCMO relativ zum $\mathrm{Al}_{2} \mathrm{O}_{3}$ (0001)Substrat erreicht, da die in-plane Diagonale über zwei Seiten der hexagonalen Struktur näherungsweise identisch zur dreifachen effektiven in-plane Gitterkonstante $a_{\text {eff. von LCMO }}$ in (111)-Orientierung ist. Resultierend ergibt sich daraus die Relation $\sqrt{2} a_{\text {Saphir }} \approx \sqrt{3} a_{\mathrm{pc}}$. Mit $a_{\text {Saphir }}=4,758 \AA 225$ und $a_{\mathrm{pc} \text {, Bulk }}=3,887 \AA 34$ führt dieses zu einer effektiven in-plane Gitterkonstante $a_{\text {eff. }}=3,885 \AA$ von $\mathrm{Al}_{2} \mathrm{O}_{3}$ (0001) für das Wachstum von LCMO in (111)-Orientierung mit einer Gitterfehlanpassung von nur $f=-0,05 \%$, sodass die Schichten praktisch unverspannt aufwachsen. In den Abbildungen 4.1 .13 (a)-(d) sind nun die XRD-Spektren von (111)-orientierten LCMO-Schichten auf $\mathrm{MgO}(100), \mathrm{Al}_{2} \mathrm{O}_{3}$ (0001), LSAT (111) und LAO (111) mit vergleichbaren Schichtdicken $d \approx 90-110 \mathrm{~nm}$ dargestellt.
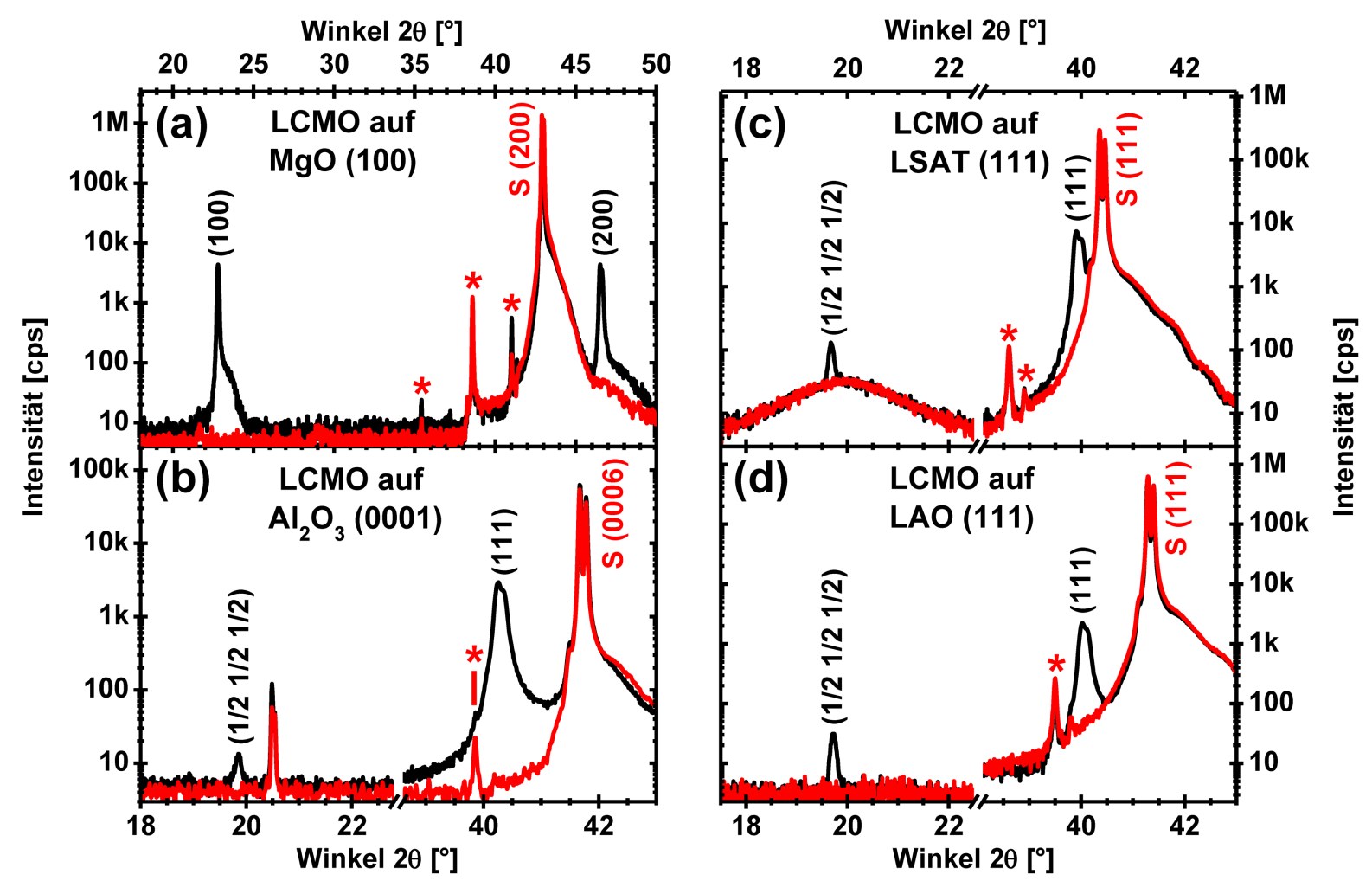

Abbildung 4.1.13: XRD-Spektren der LCMO-Schichten $(d \approx 90-110 \mathrm{~nm})$ auf $\mathrm{MgO}(100)$ (a), $\mathrm{Al}_{2} \mathrm{O}_{3}$ (0001) (b), LSAT (111) (c) und LAO (111) (d) im Vergleich. Die Substrat-Spektren (rot) dienen als Orientierung für den Hintergrund. 
Die jeweiligen Substrat-Spektren dienen als Orientierung für den Substrat-Hintergrund, wobei die mit den Sternen markierten Linien aus der nicht vollständig monochromatischen $\mathrm{Cu}_{\mathrm{K}_{\alpha}}$-Strahlung der Röntgenröhre resultieren. Alle vier LCMO-Schichten zeigen eine vollständige out-of-plane Epitaxie entsprechend der Substrat-Orientierung. Für die Fundamentalreflexe ist zudem eine $\mathrm{K}_{\alpha_{1}}-\mathrm{K}_{\alpha_{2}}$-Aufspaltung zu beobachten, die aufgrund der schmalen Linienbreite und der Scherrer-Gleichung (Gleichung (3.2.11)) die hohe kristalline Qualität der Schichten hervorhebt. Aus der Position des Fundamentalreflexes können die pseudokubischen out-of-plane Gitterkonstanten $c_{\mathrm{pc}}$ der LCMO-Schichten bestimmt werden. Diese sind zusammen mit den resultierenden Verspannungen $\varepsilon_{\perp}$ in Relation zu Bulk-LCMO in der Tabelle 4.1 .3 dargestellt. Daneben ist für die (111)-orientierten Schichten auch der für die B-Platz-Ordnung charakteristische $\left(\frac{1}{2} \frac{1}{2} \frac{1}{2}\right)$-Überstrukturreflex zu beobachten. Die dazugehörigen Ordnungsparameter $S$ der Schichten sind auch in der Tabelle 4.1 .3 aufgeführt. Für einen Vergleich sind zudem die Daten für die LCMO/STO (111)-Schicht angegeben.

\begin{tabular}{|c|c|c|c|}
\hline LCMO auf & $c_{\mathrm{pc}}[\AA]$ & $\varepsilon_{\perp}[\%]$ & $S\left[\times 10^{-2}\right]$ \\
\hline \hline $\mathrm{MgO}(100)$ & $3,902(1)$ & 0,39 & - \\
\hline $\mathrm{STO}(111)$ & $3,875(1)$ & $-0,31$ & $0,28(1)$ \\
\hline $\mathrm{Al}_{2} \mathrm{O}_{3}(0001)$ & $3,878(1)$ & $-0,23$ & $0,59(1)$ \\
\hline $\operatorname{LSAT}(111)$ & $3,909(1)$ & 0,57 & $1,17(1)$ \\
\hline LAO $(111)$ & $3,899(1)$ & 0,31 & $1,18(1)$ \\
\hline
\end{tabular}

Tabelle 4.1.3: Pseudokubische out-of-plane Gitterkonstanten $c_{\mathrm{pc}}$, die resultierenden out-ofplane Verspannungen $\varepsilon_{\perp}$ und die Ordnungsparameter $S$ der LCMO-Schichten auf verschiedenen Substraten im Vergleich.

Für die LCMO-Schichten lassen sich nun je nach Substrat und Gitterfehlanpassung zu Bulk-LCMO unterschiedliche out-of-plane Gitterkonstanten und Verspannungszustände nachweisen. Wie zu erwarten zeigen die LCMO-Schichten auf LAO (111) und LSAT (111) eine vergrößerte pseudokubische out-of-plane Gitterkonstante gegenüber Bulk-LCMO. Dieses ist auf die durch die Substrate in-plane in die Schichten induzierte Druckspannung zurückzuführen. Die Gitterkonstante und die Verspannung auf dem LAO (111)-Substrat ordnen sich hinter denen der Schicht auf LSAT (111) ein. Dieses deutet auf einen einsetzenden Relaxationsprozess in der Schicht aufgrund der größeren Gitterfehlanpassung zu Bulk-LCMO hin. Auffällig ist zudem die signifikant gegenüber Bulk-LCMO erhöhte out-of-plane Gitterkonstante für die LCMO-Schicht auf MgO (100). Dieses lässt auch hier eine in-plane Druckspannung vermuten, obwohl die große Gitterfehlanpassung zu BulkLCMO ein unverspanntes Schichtwachstum erwarten lässt. Eine mögliche Ursache zeigt sich im XRD-Spektrum. So ist neben den Schichtreflexen ein sichtbarer Untergrund mit einer deutlich reduzierten out-of-plane Gitterkonstante $c_{\mathrm{pc}}=3,814 \AA \mathrm{zu}$ beobachten. Somit wachsen offenbar die ersten Lagen der LCMO/MgO (100) mit einer durch das Substrat 


\section{Auswertung und Ergebnisse}

induzierten in-plane Zugspannung auf, bevor das Wachstum mit einer in-plane Druckspannung fortgesetzt wird. Ein weiterer Spezialfall ist die LCMO/ $\mathrm{Al}_{2} \mathrm{O}_{3}$ (0001)-Schicht, da die out-of-plane Gitterkonstante mit $c_{\mathrm{pc}}=3,878 \AA$ gegenüber Bulk-LCMO reduziert ist. Offenbar erfährt die LCMO-Schicht trotz der geringen Gitterfehlanpassung beim Wachstum auf dem hexagonalen $\mathrm{Al}_{2} \mathrm{O}_{3}$ (0001)-Substrat eine zusätzliche Verzerrung, die sich in-plane in einer Zugspannung äußert. Die LCMO-Schicht auf STO (111) verhält sich hingegen wie zu erwarten mit einer gegenüber Bulk-LCMO reduzierten out-of-plane Gitterkonstante, die sich auf die in-plane durch das Substrat induzierte Zugspannung zurückführen lässt.

Zudem zeigt sich ein mit dem Verspannungszustand der Schichten stark variierender Ordnungsparameter $S$. Dabei wird mit $S \approx 1,2 \cdot 10^{-2}$ für die LCMO-Schichten auf LAO (111) und LSAT (111) ein Wert im Bereich des theoretisch aus XRD-Simulationen bestimmten Ordnungsparameters $S_{\text {theo. }}=10^{-2}$ 36, 48 für eine perfekte B-Platz-Ordnung erreicht. Auch für die LCMO/ $\mathrm{Al}_{2} \mathrm{O}_{3}$ (0001)-Schicht ist dieser mit $S=5,9(1) \cdot 10^{-3}$ in etwa doppelt so groß wie für die LCMO/STO (111)-Schicht. Die magnetischen Untersuchungen im vorherigen Abschnitt an dieser Schicht haben jedoch gezeigt, dass auch diese über einen B-Platz-Nahordnungsgrad von > $98 \%$ verfügt. Folglich sind auch für die anderen LCMOSchichten weitere Untersuchungen notwendig, um den realen B-Platz-Ordnungsgrad abschätzen zu können. In den Abbildungen 4.1.14 (a)-(d) sind daher nun die temperaturabhängigen $M(T)$-Magnetisierungskurven (SQUID, FCC und ZFC, $H=1 \mathrm{kOe}$ ) der LCMOSchichten auf $\mathrm{MgO}$ (100), $\mathrm{Al}_{2} \mathrm{O}_{3}$ (0001), LSAT (111) und LAO (111) dargestellt.
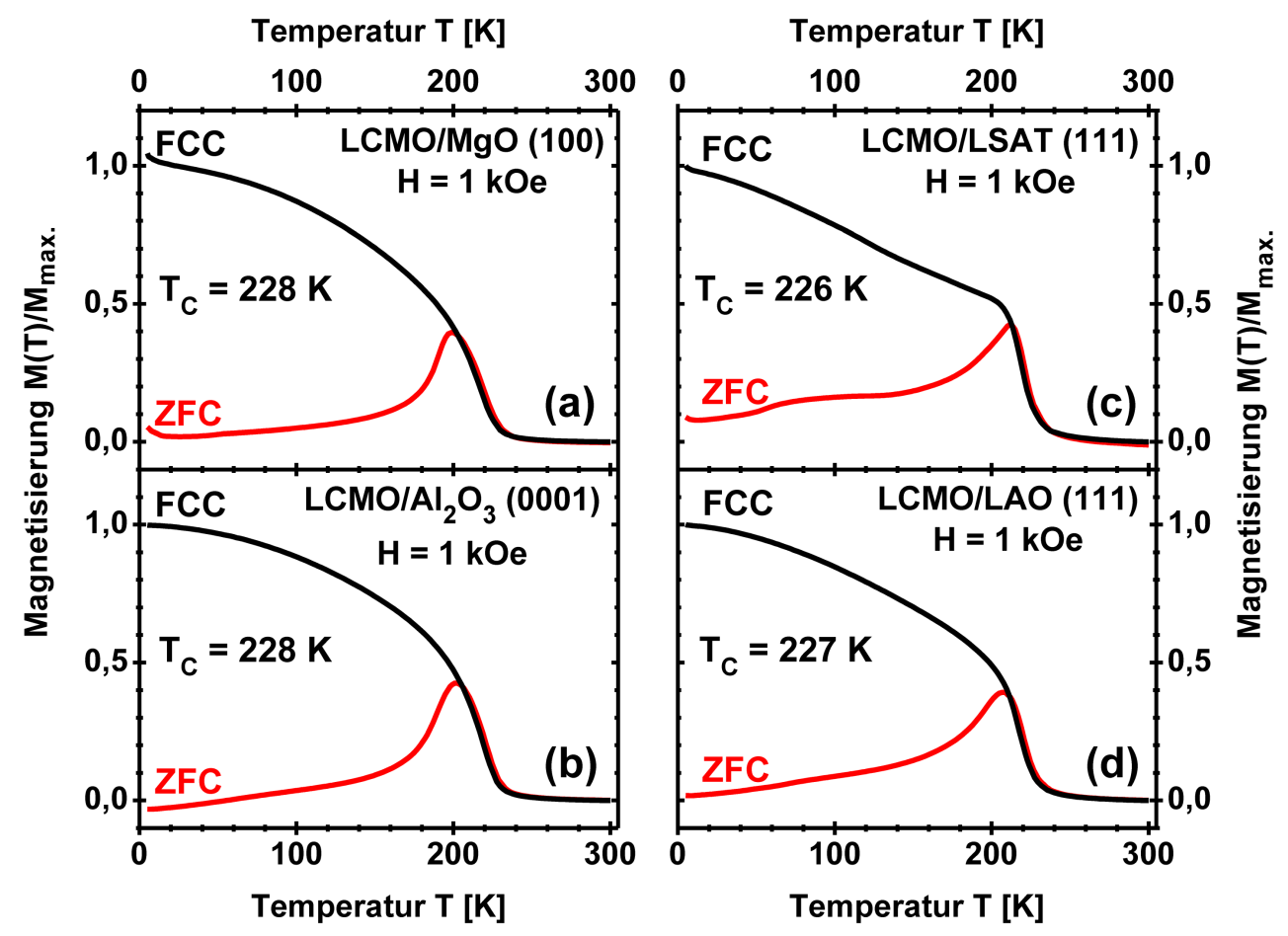

Abbildung 4.1.14: $M(T)$-Magnetisierungskurven (FCC und ZFC) der LCMO-Schichten auf $\mathrm{MgO}(100)$ (a), $\mathrm{Al}_{2} \mathrm{O}_{3}$ (0001) (b), LSAT (111) (c) und LAO (111) (d). 
Alle vier Schichten zeigen in den FCC- $M(T)$-Kurven einen ferromagnetischen Übergang bei einer Curie-Temperatur von $T_{C} \approx 230 \mathrm{~K}$ wie es für B-Platz geordnetes LCMO beobachtet wird. Für die LCMO/LSAT (111)-Schicht ist die FCC-M(T)-Kurve jedoch von einem flacheren Verlauf für Temperaturen $T<T_{C}$ gekennzeichnet. Dieses kann auf eine zweite ferromagnetische Phase aufgrund einer reduzierten B-Platz-Ordnung zurückgeführt werden. Eine weitere Ursache ist möglicherweise eine magnetische out-of-plane Anisotropie aufgrund der vom Substrat induzierten Verspannung. Ein ähnliches Verhalten ist für das Wachstum von LCMO auf LSAT (100)-Substraten bekannt, mit dem Unterschied, dass dieses zu einer magnetischen in-plane Anisotropie führt 237]. Im Vergleich dazu zeigt die LCMO/LAO (100)-Schicht trotz eines ähnlichen Verspannungszustandes diese Auffälligkeit nicht. Ein Grund hierfür ist möglicherweise der einsetzende Relaxationsprozess.

Zudem ist für alle LCMO-Schichten in den ZFC- $M(T)$-Kurven ein für Spin-Gläser typischer Verlauf zu beobachten, der deutlich von den FCC-Messungen abweicht. Somit befinden sich APG in den Schichten, die die B-Platz geordneten Domänen gegenphasig voneinander trennen. Da jedoch der Ordnungsparameter $S$ für die Schichten je nach Verspannungszustand stark variiert, ist ein Einfluss auf die Anzahl an APG und die Domänengröße zu vermuten. Um dieses zu verifizieren, sind in den Abbildungen 4.1 .15 (a)-(d) die $M(H)$ Hysteresekurven (SQUID, $T=5 \mathrm{~K}$, mit $H_{\text {max. }}=50 \mathrm{kOe}$ nach FCC mit $H=1 \mathrm{kOe}$ ) der (111)-orientierten LCMO-Schichten auf verschiedenen Substraten im Vergleich dargestellt.
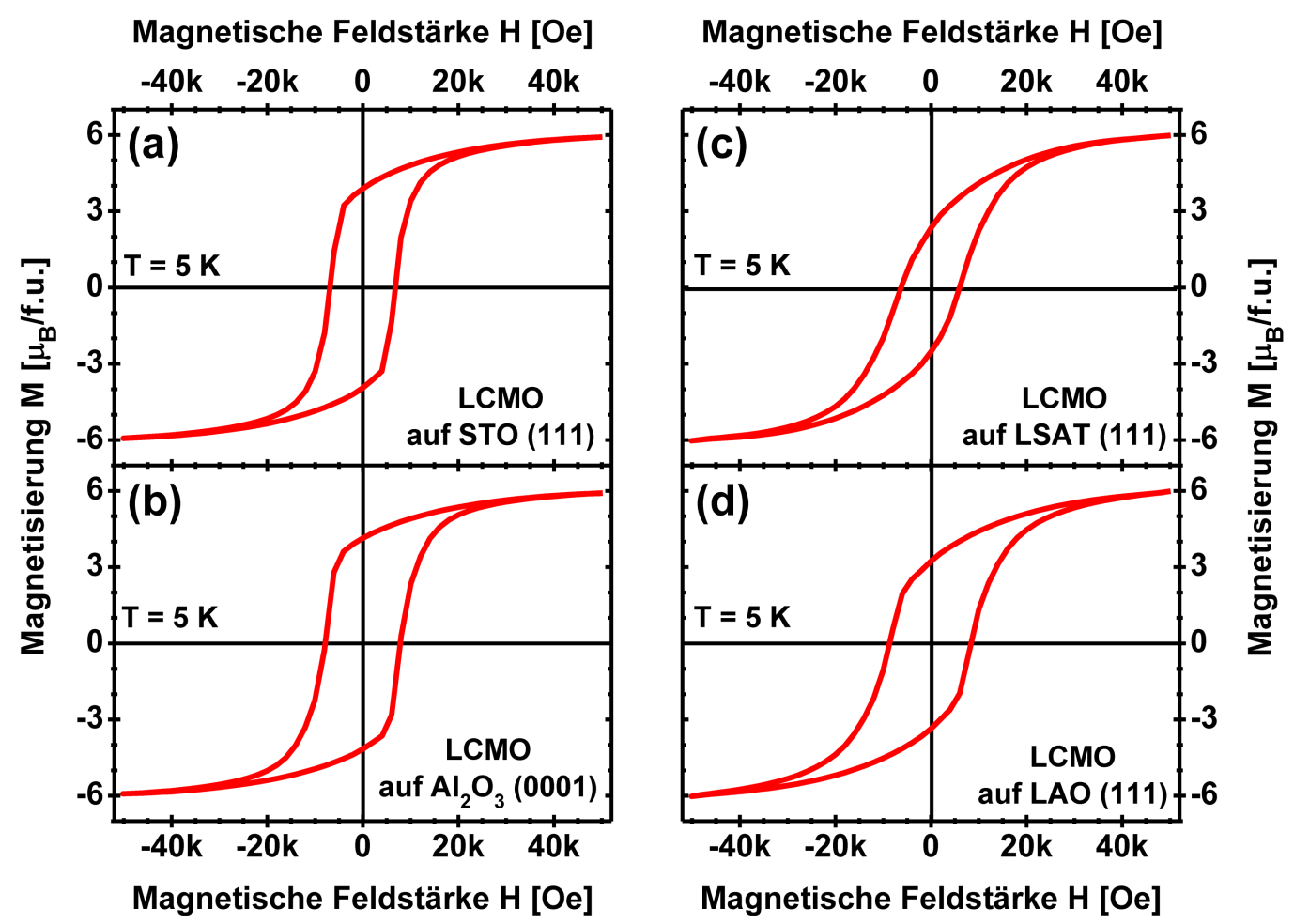

Abbildung 4.1.15: $M(H)$-Hysteresekurven $\left(T=5 \mathrm{~K}\right.$, mit $H_{\max }=50 \mathrm{kOe}$ nach FCC mit $H=1 \mathrm{kOe}$ ) der LCMO-Schichten auf STO (111) (a), $\mathrm{Al}_{2} \mathrm{O}_{3}$ (0001) (b), LSAT (111) (c) und LAO (111) (d) im Vergleich $(d \approx 90-110 \mathrm{~nm})$. 


\section{Auswertung und Ergebnisse}

Es zeigt sich, dass alle vier (111)-orientierten LCMO-Schichten eine Sättigungsmagnetisierung $M_{S}>5,90(2) \mu_{\mathrm{B}} /$ f.u. erreichen, woraus sich jeweils ein B-Platz-Nahordnungsgrad von $>98 \%$ in Relation zu $6 \mu_{\mathrm{B}} /$ f.u. für eine perfekte B-Platz-Ordnung ergibt. Dieses verdeutlicht für die LCMO/LSAT (111)-Schicht, dass die Anomalie in der FCC-M(T)-Kurve für Temperaturen $T<T_{C}$ nicht durch eine Verringerung der B-Platz-Ordnung, sondern durch eine leichte magnetische out-of-plane Anisotropie hervorgerufen wird. Dafür spricht auch die reduzierte Remanenz $M_{R}$ der in-plane $M(H)$-Hysteresekurve. Daneben lassen sich mit den $M(H)$-Kurven auch die Unterschiede im XRD-Ordnungsparameter $S$ nachvollziehen. So erhöht sich die Remanenz für die LCMO/ $\mathrm{Al}_{2} \mathrm{O}_{3}$ (0001)-Schicht gegenüber der LCMOSchicht auf STO (111) von $M_{R}=3,94(5) \mu_{\mathrm{B}} /$ f.u. auf $M_{R}=4,20(5) \mu_{\mathrm{B}} /$ f.u., während die Koerzitivfeldstärke von $H_{C}=6,8(2) \mathrm{kOe}$ auf $H_{C}=7,7(2) \mathrm{kOe}$ ansteigt. Dieses impliziert für die $\mathrm{LCMO} / \mathrm{Al}_{2} \mathrm{O}_{3}$ (0001)-Schicht eine geringere Anzahl an APG und deutlich kleinere, antiferromagnetisch gegenüber der Umgebung orientierte Domänen, sodass die langreichweitige B-Platz-Ordnung und damit der Ordnungsparameter zunimmt. Für die LCMO-Schichten auf LAO (111) und LSAT (111) nimmt dieser nochmals zu. Dieses passt zu Untersuchungen an LCMO-Schichten in der Literatur, in denen durch die Kontrolle der Verspannung in der Schicht die B-Platz-Ordnung verbessert werden kann 220, 224. Hierbei wird beobachtet, dass das Wachstum auf (111)-orientierten Substraten bei einer in-plane Druckspannung durch das Substrat zu einer Stimulation der B-Platz-Ordnung durch eine unterschiedliche Verzerrung der beiden $\mathrm{BO}_{6}$ und $\mathrm{B}^{\prime} \mathrm{O}_{6}$-Oktaeder führt 224. Dieses lässt sich auf die zwei LCMO-Schichten auf LSAT (111) und LAO (111) in dieser Arbeit übertragen. Die hohe Sättigungsmagnetisierung von nahezu $6 \mu_{\mathrm{B}} /$ f.u. bestätigt zudem, dass die großen XRD-Ordnungsparameter nicht nur durch eine Abstandsmodulation durch verschobene Kationen innerhalb der Kristallstruktur hervorgerufen werden.

Zur Verifizierung der B-Platz-Ordnung auf atomarer Skala wurden von Vladimir Roddatis BF- und HAADF-STEM-Aufnahmen von der LCMO/LAO (111)-Schicht von verschiedenen Stellen in Querschnittsrichtung zur Schicht-Substrat-Grenzfläche angefertigt. In den Abbildungen 4.1.16 (a) und (b) sind die BF- und HAADF-Aufnahmen eines repräsentativen Grenzflächenbereichs der LCMO-Schicht mit dem LAO (111)-Substrat entlang der [110]-Richtung dargestellt. Dabei wird ersichtlich, dass eine exzellente our-of-plane Epitaxie zu beobachten ist. Die Aufnahmen zeigen zudem, dass sich die Verspannung in der Schicht, die im XRD-Spektrum anhand der Vergrößerung der pseudokubischen out-ofplane Gitterkonstante auf $c_{\mathrm{pc}}=3,899 \AA\left(c_{\mathrm{pc}}\right.$, Bulk $=3,887 \AA 225$ für Bulk-LCMO $)$ durch die Gitterfehlanpassung von $f=-2,55 \%$ zwischen LCMO und LAO (111) nachgewiesen werden kann, fortsetzt, ohne dass diese durch Versetzungen oder andere Gitterfehler abgebaut wird. Folglich stellt sich out-of-plane eine Zugspannung ein. Dass diese jedoch geringer als für die LCMO-Schicht auf LSAT (111) mit kleinerer Gitterfehlanpassung ist, deutet dabei auf einen einsetzenden Relaxationsprozess mit zunehmender Schichtdicke hin. 

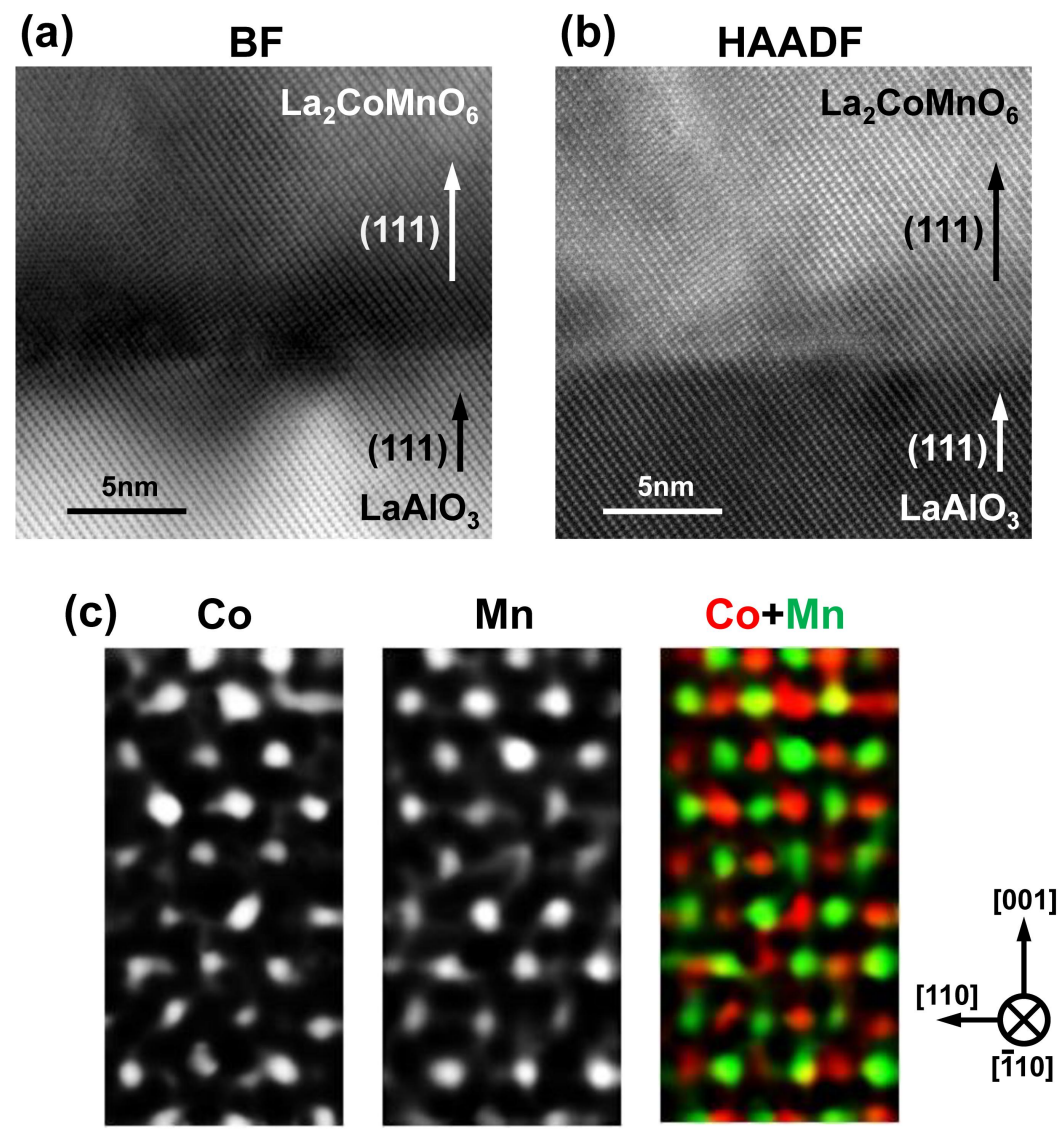
Abbildung 4.1.16: Repräsentative BF-STEM- (a) und HAADF-STEM-Aufnahmen (b) des Grenzflächenbereichs von Schicht und Substrat der B-Platz geordneten LCMO/LAO (111)-Schicht entlang der [110]-Achse zusammen mit der elementspezifischen EELS-Kartierung (c) von Co, Mn und Co $+\mathrm{Mn}$ eines für die Schicht repräsentativen Bereichs entlang der [110]-Achse.

Zur Visualisierung der B-Platz in der Schicht wurden zudem EELS-Kartierungen der Co$\mathrm{L}_{2,3}$-Kante und der Mn- $\mathrm{L}_{2,3}$-Kante von verschiedenen Bereichen der LCMO-Schicht aufgenommen. Die EELS-Kartierungen (Co, Mn, Co+Mn) einer dieser Stellen entlang der [110]-Achse sind repräsentativ in der Abbildung 4.1.16 (c) dargestellt. Die Orientierung ist so gewählt, dass die [001]-Richtung nach oben zeigt. In den EELS-Kartierungen ist dabei eine exzellente B-Platz-Ordnung in der dazugehörigen NaCl-Anordnung mit abwechselnd $\mathrm{Co}^{2+}$ - und $\mathrm{Mn}^{4+}$-Kationen auf den B-Plätzen zu beobachten. Somit bestätigt sich die bereits aus den Magnetisierungsdaten gewonnene Erkenntnis, dass der große Ordnungsparameter $S$ aus dem XRD-Spektrum für die LCMO/LAO(111)-Schicht auch real auf eine hohe B-Platz-Kationenordnung zurückzuführen ist und nicht durch Artefakte aufgrund von strukturellen Verzerrungen und Verschiebungen der Kationen im Kristall entsteht.

In der Abbildungen 4.1.22 (a) und (b) sind anschließend noch die polarisationsabhängigen Raman-Spektren der LCMO-Schichten auf den verschiedenen Substraten in der parallelen xx- und in der gekreuzten xy-Polarisationskonfiguration dargestellt. Die Spektren wurden in einem Probenbezugssystem mit den x $\|[11 \overline{2}]$, y $\|$ [110] und z $\|$ [111] in senkrechter 


\section{Auswertung und Ergebnisse}

Rückstreugeometrie aufgenommen. Die Raman-Spektren der LCMO-Schichten sind dabei in der parallelen xx-Konfiguration durch die sehr starke $A_{g}$ Breathing Mode bei $\approx 645 \mathrm{~cm}^{-1}$ geprägt, die in der xy-Konfiguration stark in der Intensität abnimmt und somit die für die monokline P12 1 n1-Struktur zu erwartende $A_{g}$-Symmetrie aufweist. Umgekehrt zeigt sich für die $B_{g}$ Mischmode bei $499 \mathrm{~cm}^{-1}$ eine entsprechend umgekehrte Polarisationsabhängigkeit. Die Raman-Spektren der LCMO-Schichten sind alle durch die Charakteristik einer BPlatz geordneten monoklinen P12/n1-Struktur gekennzeichnet. Das Auftreten der Oberschwingung 2. Ordnung der Breathing Mode bestätigt zudem die hohe kristalline Qualität der Schichten. In Abhängigkeit des Verspannungszustands ist zudem eine leichte Verschiebung der Modenpositionen zu beobachten. Für die $A_{g}$ Breathing Mode ist die Abhängigkeit der Position von der in-plane Substratgitterkonstante $a$ in der eingebetteten Grafik in der Abbildung 4.1.17 (a) dargestellt. Es ergibt sich ein systematisches Verhalten mit einem Erhärten für eine in-plane Druckspannung und ein Erweichen für eine in-plane Zugspannung. Für die LCMO-Schicht auf LAO (100) mit einer einsetzenden Relaxation erweicht die Mode dabei wieder, während wiederum auch auf die LCMO-Schicht auf MgO (100) eine in-plane Druckspannung einwirkt. Folglich lässt sich anhand des Raman-Spektrums direkt der Verspannungszustand der jeweiligen LCMO-Schicht beobachten.

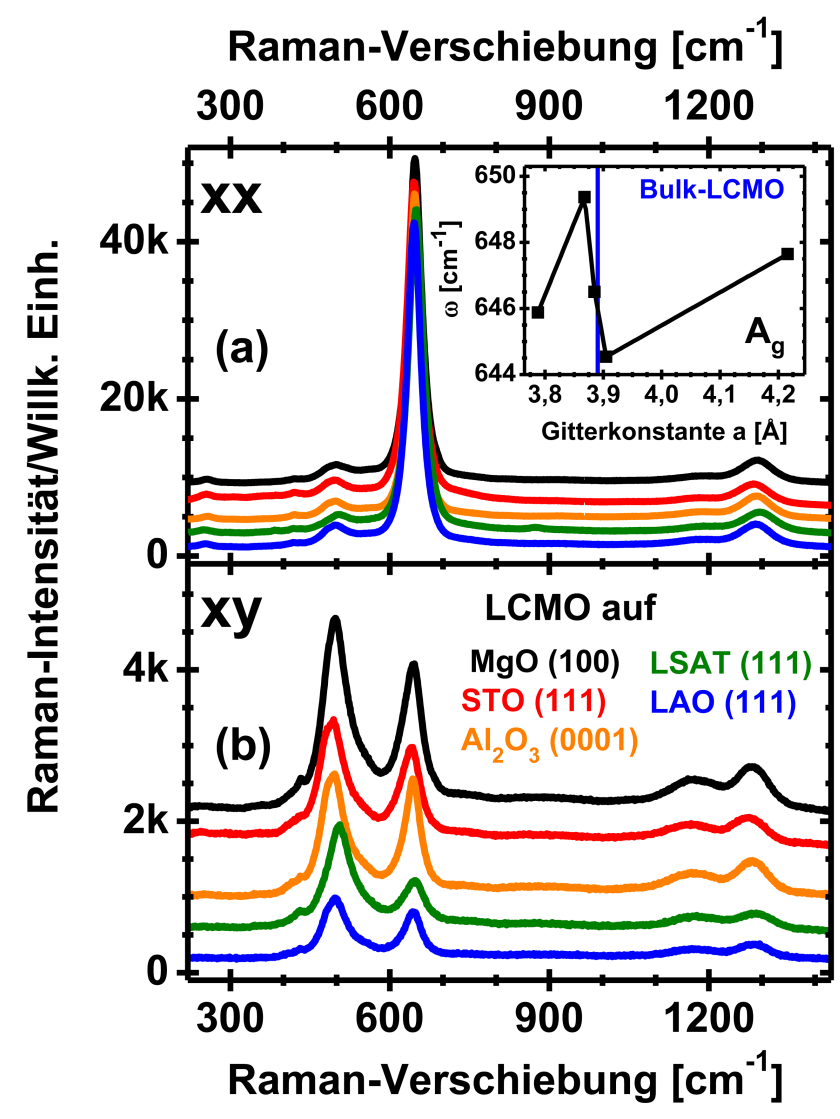

Abbildung 4.1.17: Polarisierte Raman-Spektren $(\lambda=532 \mathrm{~nm}, P \approx 2 \mathrm{~mW}, t=2 \times 60 \mathrm{~s}$, Raumtemperatur) der LCMO-Schichten auf verschiedenen Substraten in der parallelen xx- (a) und der gekreuzten xy-Konfiguration (b) im Vergleich. Die Spektren sind für die Übersicht vertikal gegeneinander verschoben. 
Anschließend können nun noch die Auswirkungen des B-Platz-Ordnungsgrads auf die strukturellen, magnetischen und phononischen Eigenschaften der LCMO-Schichten untersucht werden. In den Abbildungen 4.1 .18 (a) und (b) sind die XRD-Spektren von zwei LCMO $/ \mathrm{Al}_{2} \mathrm{O}_{3}$ (0001)-Schichten $(d \approx 100 \mathrm{~nm})$, die bei unterschiedlichen Wachstumsraten von (1) 0,1 nm/s (a) (die bereits vorgestellte LCMO-Schicht) und (2) 0,2 nm/s (b) bei ansonsten identischen Wachstumsbedingungen hergestellt wurden, in der Bragg-BrentanoGeometrie dargestellt. Das jeweils in rot gezeigte $\mathrm{Al}_{2} \mathrm{O}_{3}$ (0001)-Spektrum dient als Orientierung für den vom Substrat stammenden Beitrag. Die mit dem Stern markierte SubstratLinie resultiert dabei wieder aus der nicht vollständig monochromatischen $\mathrm{Cu}_{\mathrm{K}_{\alpha}}$-Strahlung der Röntgenröhre und kennzeichnet den nicht vollständig herausgefilterten $\mathrm{Cu}_{\mathrm{K}_{\beta}}$-Anteil.
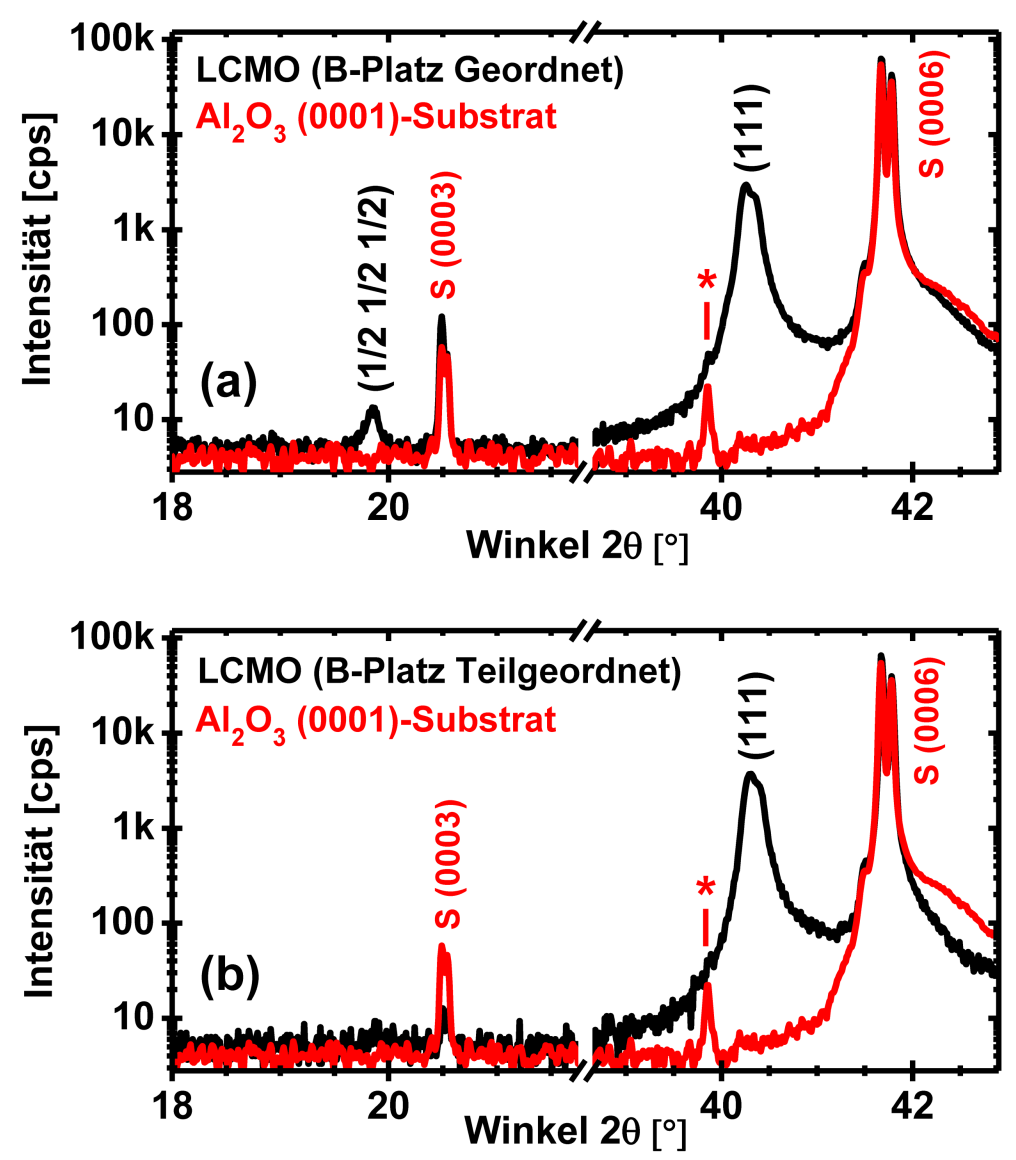

Abbildung 4.1.18: XRD-Spektren der zwei LCMO-Schichten auf $\mathrm{Al}_{2} \mathrm{O}_{3}$ (0001) mit B-PlatzOrdnung (a) und B-Platz-Teilordnung $(b)(d \approx 100 \mathrm{~nm})$ im Vergleich. Das $\mathrm{Al}_{2} \mathrm{O}_{3}$ (0001)-Spektrum dient als Orientierung für den Substratbeitrag (rot). Die Substrat-Linie mit dem Stern resultiert aus der nicht vollständig monochromatischen $\mathrm{Cu}_{\mathrm{K}_{\alpha}}$-Strahlung der Röntgenröhre.

Beide LCMO/ $/ \mathrm{Al}_{2} \mathrm{O}_{3}$ (0001)-Schichten zeigen eine vollständige out-of-plane Epitaxie in (111)-Orientierung mit den jeweiligen pseudokubischen Gitterkonstanten $c_{\mathrm{pc}}=3,878 \AA$ für die Schicht (1) und $c_{\mathrm{pc}}=3,873 \AA$ für die Schicht (2). Im Vergleich zu der dazugehörigen Gitterkonstante $c_{\mathrm{pc}}$, Bulk $=c / \sqrt{2}=3,887 \AA[34$ von Bulk-LCMO ist die Abweichung somit für beide LCMO-Schichten nur gering. Die etwas kleineren Gitterkonstanten 


\section{Auswertung und Ergebnisse}

der beiden Schichten implizieren dabei effektiv eine leichte in-plane Zugspannung durch das $\mathrm{Al}_{2} \mathrm{O}_{3}$ (0001)-Substrat, die in die out-of-plane Richtung entsprechend zu einer schwache Druckspannung von $-0,2 \%$ (1) und $-0,3 \%$ (2) führt, wobei die leicht größere Verspannung für die Schicht (2) auf die höhere Wachstumsrate zurückgeführt werden kann. Daneben ist für beide Schichten eine $\mathrm{K}_{\alpha_{1}}-\mathrm{K}_{\alpha_{2}}$-Aufspaltung des pseudokubischen (111)Fundamentalreflexes zu beobachten, die anhand der damit einhergehenden nur geringen Linienbreite des Reflexes und der Scherrer-Gleichung (Gleichung (3.2.11) die hohe kristalline Qualität der zwei LCMO-Schichten auf den $\mathrm{Al}_{2} \mathrm{O}_{3}$ (0001)-Substraten unterstreicht.

Neben dem (111)-Fundamentalreflex ist für die LCMO-Schicht (1) zusätzlich der $\left(\frac{1}{2} \frac{1}{2} \frac{1}{2}\right)$ Überstrukturreflex sehr deutlich zu beobachten. Dieses deutet auf die Präsenz einer signifikanten B-Platz-Fernordnung in der Schicht hin. Für die LCMO-Schicht (2) fehlt dieser Reflex hingegen, sodass die Ordnung im Vergleich dazu reduziert erscheint. Folglich werden die zwei Schichten im Folgenden als B-Platz geordnete LCMO-Schicht (1) und B-Platz teilgeordnete LCMO-Schicht (2) bezeichnet. Der Ordnungsparameter der B-Platz geordneten Schicht ist mit $S=5,9(1) \cdot 10^{-3}$ deutlich größer als der für die LCMO-Schicht auf STO (111) in dieser Arbeit $\left(S=2,8(1) \cdot 10^{-3}\right)$ und der Literatur $\left(S \approx 1,1 \cdot 10^{-3} 36,48\right)$, aber kleiner als die Parameter, die sich sowohl theoretisch durch XRD-Simulationen $\left(S_{\text {theo. }}=10^{-2}\right)$ als auch experimentell in dieser Arbeit auf LAO (111) und LSAT (111) (jeweils $S=1,2(1) \cdot 10^{-2}$ ) ergeben. Wie sich jedoch bei den $\mathrm{A}_{2} \mathrm{CoMnO}_{6^{-}}$ und den $\mathrm{A}_{2} \mathrm{NiMnO}_{6}$-Schichten auf STO (111) herausgestellt hat, muss der Ordnungsparameter $S$ aufgrund vieler weiterer Einflussfaktoren unabhängig der B-Platz-Ordnung stets mit Vorsicht und im Zusammenhang mit weiteren Untersuchungen betrachtet werden.

In den Abbildungen 4.1.19 (a) und (b) sind daher die temperaturabhängigen $M(T)$-Magnetisierungskurven (SQUID, FCC und ZFC, $H=1 \mathrm{kOe}$ ) der zwei LCMO-Schichten auf $\mathrm{Al}_{2} \mathrm{O}_{3}$ (0001) dargestellt. Beide Schichten zeigen dabei einen ferromagnetischen Übergang bei einer Curie-Temperatur $T_{C}=228(2) \mathrm{K}$ für die B-Platz geordnete Schicht (1) und bei $T_{C_{1}}=226(2) \mathrm{K}$ für die B-Platz teilgeordnete Schicht (2). Zusätzlich ist für die teilgeordnete Schicht ein zweiter ferromagnetischer Übergang bei einer Temperatur $T_{C_{2}}=110(2) \mathrm{K}$ nachzuweisen. Dieses entspricht Ergebnissen in dieser Arbeit und in der Literatur, in denen für B-Platz geordnetes LCMO ein scharfer Übergang bei einer Temperatur von $T \approx 230 \mathrm{~K}$ aufgrund eines ferromagnetischen $\mathrm{Co}^{2+}-\mathrm{O}^{2-}-\mathrm{Mn}^{4+}$-Superaustauschs beobachtet wird, während für eine Teilordnung ein zweiter ferromagnetischer Übergang aufgrund eines weniger stabilen vibronischen $\mathrm{Co}^{3+}{ }_{-} \mathrm{O}^{2-}{ }_{-} \mathrm{Mn}^{3+}{ }_{-}$Superaustauschs in einem Temperaturbereich $T \approx 80-130 \mathrm{~K}$ erfolgt 31,36, 37,224. Demnach ist der aus den XRD-Spektren gefolgerte Unterschied im B-Platz-Ordnungsgrad der zwei LCMO/ $\mathrm{Al}_{2} \mathrm{O}_{3}$ (0001)-Schichten auch magnetisch nachweisbar. B-Platz-Gitterfehlbesetzungen und APG führen dabei entsprechend $\mathrm{zu}$ antiferromagnetischen $\mathrm{Co}^{2+}{ }_{-} \mathrm{O}^{2-}{ }_{-} \mathrm{Co}^{2+}{ }_{-}$und $\mathrm{Mn}^{4+}-\mathrm{O}^{2-}{ }_{-} \mathrm{Mn}^{4+}{ }_{-}$Wechselwirkungen, die 
den Ferromagnetismus von B-Platz teil- oder ungeordnetem LCMO abschwächen. Dieses wird direkt anhand der zwei FCC- $M(T)$-Kurven in den Abbildungen 4.1 .19 (a) und (b) ersichtlich. Gleichzeitig zeigt sich jedoch für beide LCMO-Schichten ein deutlicher Unterschied in den FCC- und ZFC-M(T)-Kurven. Der für Spin-Gläser typische Verlauf der ZFC-Messungen lässt sich dabei wie schon zuvor bei den B-Platz geordneten $\mathrm{A}_{2} \mathrm{CoMnO}_{6}{ }^{-}$ Schichten auf STO (111) auf konkurrierende ferromagnetische und antiferromagnetische Wechselwirkungen in der Kristallstruktur zurückführen [34, 226, 227]. Dieses zeigt somit, dass auch in B-Platz geordneten LCMO-Schichten die geordneten Domänen gegenphasig durch APG getrennt sind und der XRD-Ordnungsparameter $S$ für eine alleinige Beurteilung des B-Platz-Nahordnungsgrades in den Doppelperowskiten nicht ausreichend ist.
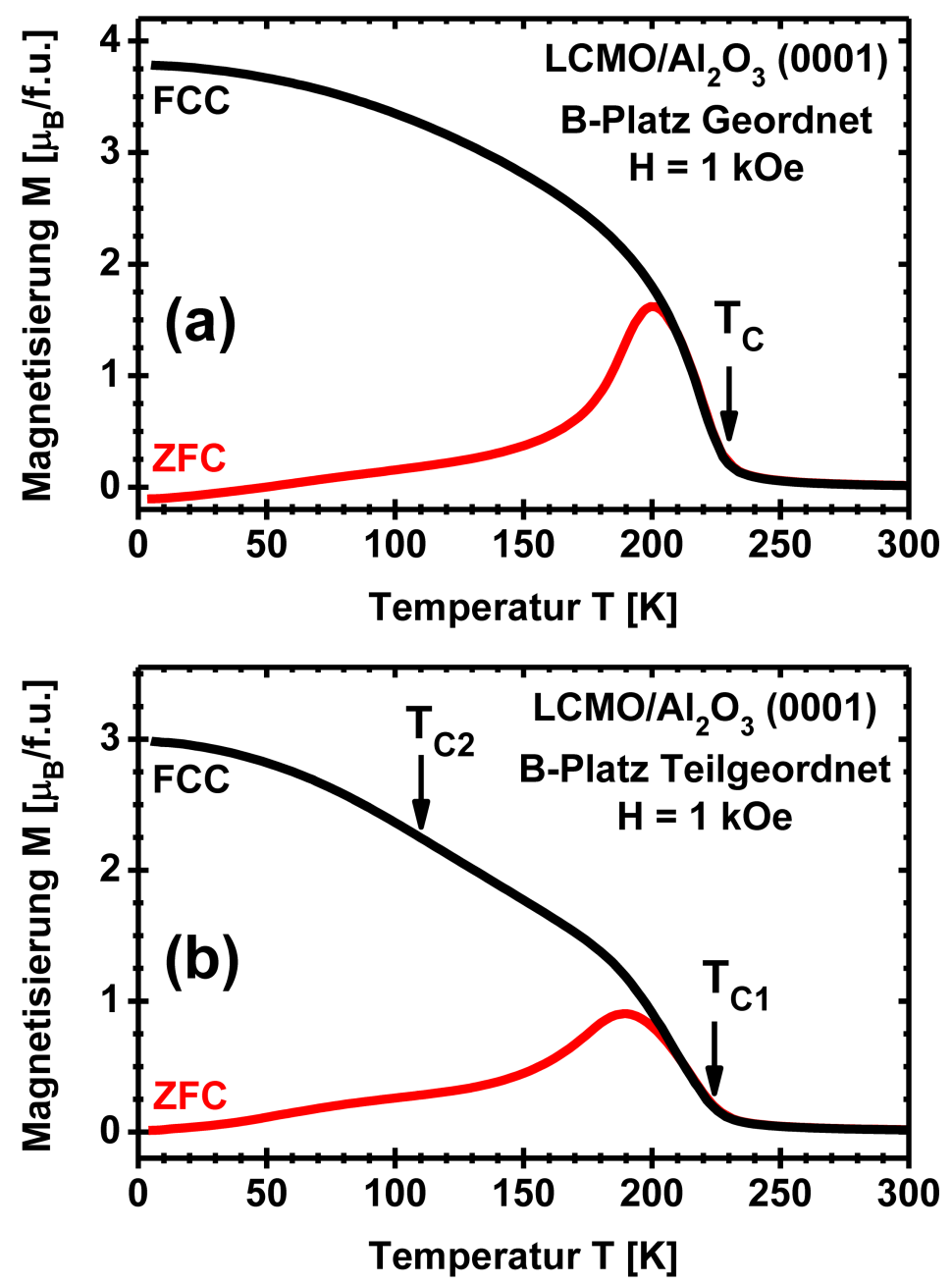

Abbildung 4.1.19: $M(T)$-Magnetisierungskurven (FCC und ZFC) der LCMO-Schichten auf $\mathrm{Al}_{2} \mathrm{O}_{3}$ (0001) mit B-Platz-Ordnung (a) und B-Platz-Teilordnung (b).

In der Abbildung 4.1.20 sind zusätzlich die $M(H)$-Hysteresekurven (SQUID, $T=5 \mathrm{~K}$, mit $H_{\text {max. }}=50 \mathrm{kOe}$ nach FCC mit $\left.H=1 \mathrm{kOe}\right)$ der beiden LCMO-Schichten auf $\mathrm{Al}_{2} \mathrm{O}_{3}(0001)$ für einen direkten Vergleich dargestellt. Hierbei ergibt sich für die B-Platz geordnete LCMO-Schicht (1) eine Sättigungsmagnetisierung $M_{S}=5,91(10) \mu_{\mathrm{B}} /$ f.u., woraus in einem Vergleich mit dem durch die $\mathrm{Co}^{2+}$ - und $\mathrm{Mn}^{4+}$-Kationen zu erwartenden Spin-Beitrag 
von $6 \mu_{\mathrm{B}} /$ f.u. ein B-Platz-Ordnungsgrad von > $98 \%$ folgt. Für die teilgeordnete LCMOSchicht (2) wird wiederum eine geringere Sättigungsmagnetisierung $M_{S}=5,43(10) \mu_{\mathrm{B}} / \mathrm{f}$.u. erreicht, woraus entsprechend ein kleinerer B-Platz-Ordnungsgrad von $\approx 90 \%$ aufgrund einer größeren Anzahl an B-Platz-Gitterfehlbesetzungen resultiert. Dieses bestätigt direkt die Beobachtungen in den XRD-Spektren. Weitere Unterschiede zeigen sich in der Koerzitivfeldstärke $H_{C}$ und der Remanenz $M_{R}$. Während nun für die B-Platz geordnete LCMO-Schicht (1) eine Koerzitivfeldstärke $H_{C}=7,7(2)$ kOe und eine Remanenz $M_{R}=4,20(5) \mu_{\mathrm{B}} /$ f.u. beobachtet werden, weist die B-Platz teilgeordnete LCMO-Schicht eine Koerzitivfeldstärke $H_{C}=10,2(2) \mathrm{kOe}$ und eine Remanenz $M_{R}=3,60(5) \mu_{\mathrm{B}} / \mathrm{f}$.u. auf. In dem Modell mit von den APG gegenphasig von der Umgebung getrennten Domänen mit B-Platz-Nahordnung 36, 48 können die größere Koerzitivfeldstärke und die verringerte Remanenz der teilgeordneten LCMO-Schicht auf eine höhere APG-Dichte und auf eine signifikante Reduzierung der mittleren Domänengröße zurückgeführt werden.

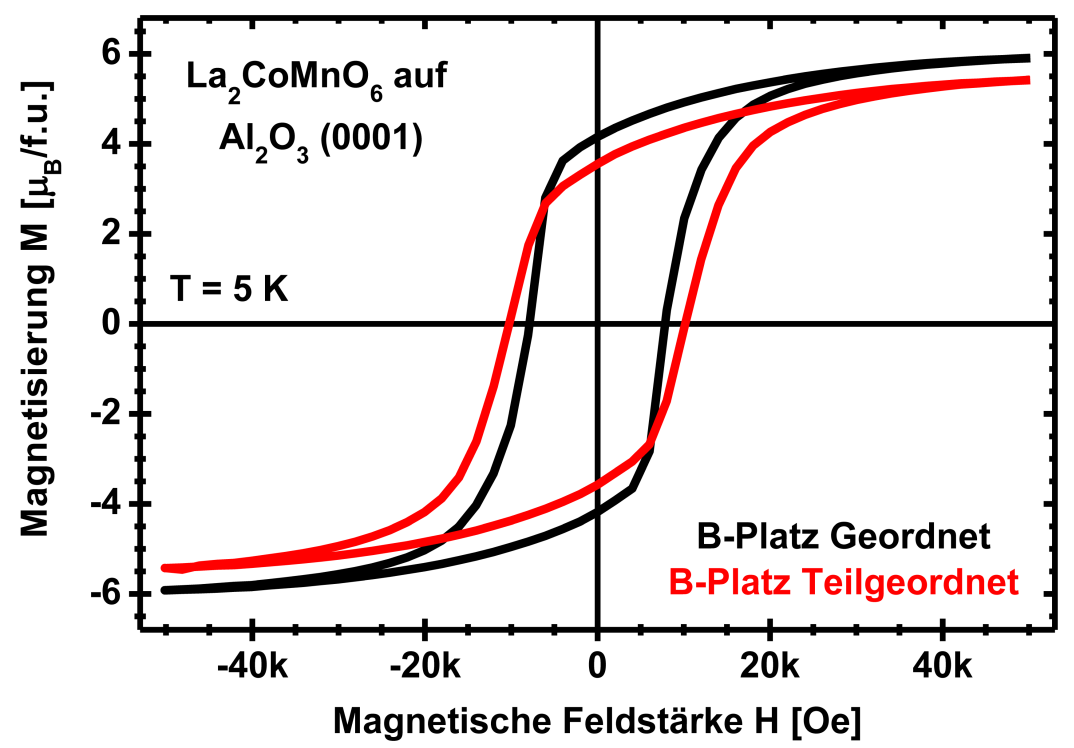

Abbildung 4.1.20: $M(H)$-Hysteresekurven $\left(T=5 \mathrm{~K}\right.$, mit $H_{\text {max. }}=50 \mathrm{kOe}$ nach FCC mit $H=1 \mathrm{kOe})$ der zwei LCMO $/ \mathrm{Al}_{2} \mathrm{O}_{3}(0001)$-Schichten $(d \approx 100 \mathrm{~nm}) \mathrm{mit}$ einem unterschiedlichen B-Platz-Ordnungsgrad im Vergleich.

Um die B-Platz-Ordnung der geordneten LCMO-Schicht (1) auf atomarer Skala weiter zu verifizieren, wurden zudem von Vladimir Roddatis BF- und HAADF-STEM-Aufnahmen von verschiedenen Stellen in Querschnittsrichtung zur LCMO/ $\mathrm{Al}_{2} \mathrm{O}_{3}$ (0001)-Grenzfläche angefertigt. In den Abbildungen 4.1.21 (a) und (b) sind die BF- und HAADF-Aufnahmen eines repräsentativen Bereichs der Schicht-Substrat-Grenzfläche entlang der [1110]-Richtung der B-Platz geordneten LCMO-Schicht gezeigt. Wie anhand der Bilder ersichtlich wird, ist eine exzellente out-of-plane Epitaxie und eine eindeutige und glatte Grenzfläche zwischen Schicht und Substrat zu beobachten. Dieses bestätigt die XRD-Spektren und kann auf die äußerst geringe Gitterfehlanpassung von nur $f=-0,05 \%$ zwischen LCMO und $\mathrm{Al}_{2} \mathrm{O}_{3}$ (0001) für das Schichtwachstum in (111)-Orientierung zurückgeführt werden. Um 
die B-Platz-Ordnung zu visualisieren, wurden zusätzlich EELS-Kartierungen der Co- $\mathrm{L}_{2,3^{-}}$ Kante und der Mn- $\mathrm{L}_{2,3}$-Kante von verschiedenen Bereichen der LCMO-Schicht aufgenommen. Die EELS-Kartierungen (Co, Mn, Co+Mn) einer dieser Stellen entlang der [110]Achse sind repräsentativ in der Abbildung 4.1.21 (c) dargestellt. Die Orientierung ist so gewählt, dass die [001]-Richtung senkrecht nach oben zeigt. In den EELS-Kartierungen ist dabei eine exzellente B-Platz-Ordnung in der $\mathrm{NaCl}$-Anordnung mit abwechselnd $\mathrm{Co}^{2+}$ und $\mathrm{Mn}^{4+}$-Kationen auf den B-Plätzen zu beobachten. Somit ist die B-Platz-Ordnung der LCMO-Schicht sowohl strukturell als auch magnetisch verifiziert. Weitere BF- und HAADF-Aufnahmen von Vladimir Roddatis mit einer geringeren Auflösung bestätigen zudem anhand von Helligkeitsunterschieden eine Domänenstruktur mit B-Platz geordneten Domänen innerhalb der Schicht. Diese Beobachtung ordnet sich dabei sehr gut in die Erkenntnisse aus den XRD-Spektren und den Magnetisierungsmessungen ein.
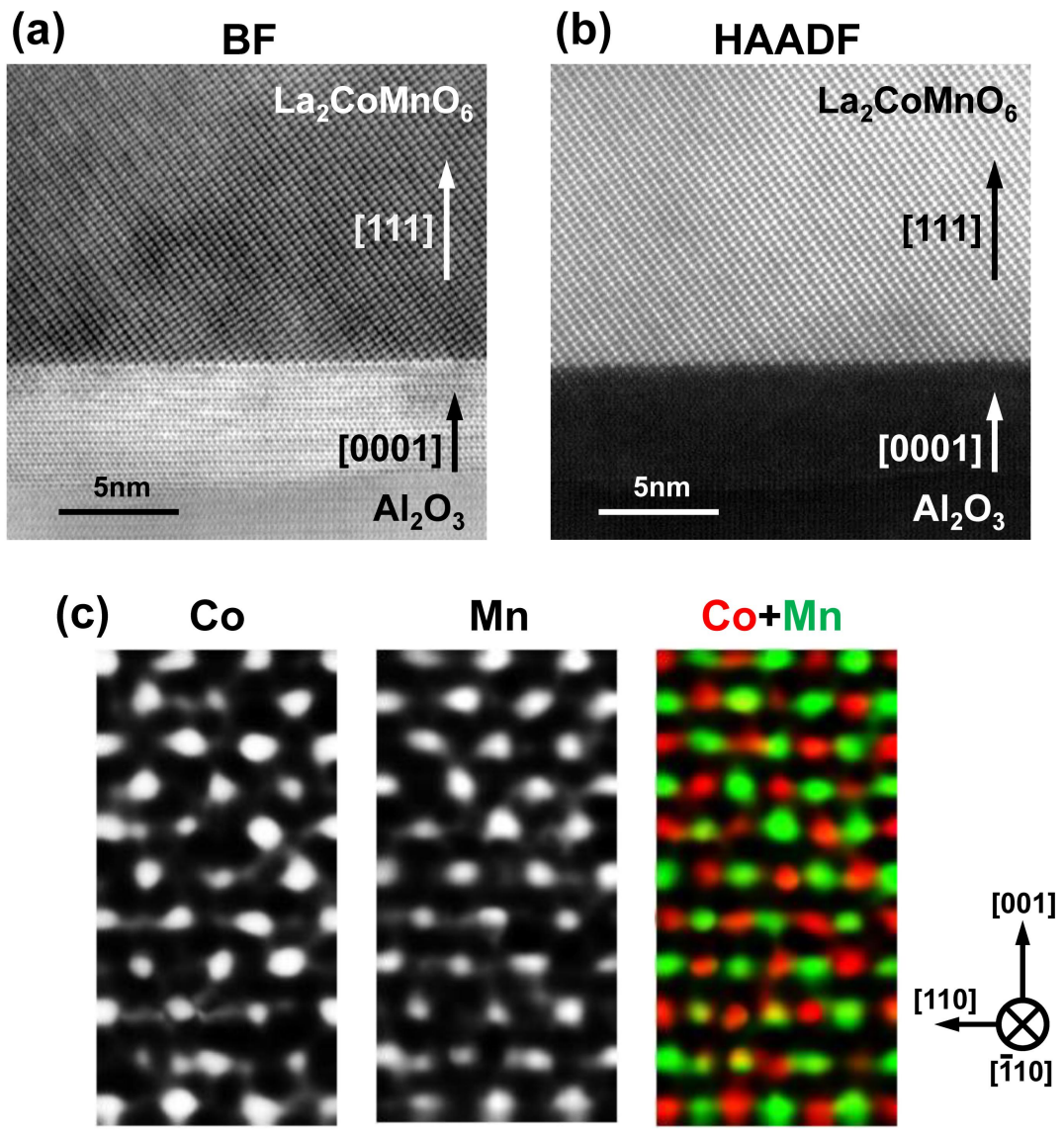

\begin{abstract}
Abbildung 4.1.21: Repräsentative BF-STEM- (a) und HAADF-STEM-Aufnahmen (b) des Grenzflächenbereichs von Schicht und Substrat der B-Platz geordneten $\mathrm{LCMO} / \mathrm{Al}_{2} \mathrm{O}_{3}$ (0001)-Schicht entlang der [110]-Achse zusammen mit der elementspezifischen EELS-Kartierung (c) von Co, Mn und Co + Mn eines für die Schicht repräsentativen Bereichs entlang der [1110]-Achse.
\end{abstract}

In der Abbildungen 4.1 .22 (a) und (b) sind nun noch die polarisationsabhängigen RamanSpektren der zwei LCMO-Schichten auf $\mathrm{Al}_{2} \mathrm{O}_{3}$ (0001) in der parallelen xx- und in der gekreuzten xy-Polarisationskonfiguration dargestellt. Das Probenbezugssystem besitzt die 
Richtungen x $\|[11 \overline{2}], \mathrm{y}\|[\overline{1} 10]$ und z $\|$ [111]. Die Spektren selbst wurden in senkrechter Rückstreugeometrie aufgenommen. Die Raman-Spektren beider LCMO-Schichten sind dabei in der parallelen xx-Konfiguration durch die sehr starke $A_{g}$ Breathing Mode bei $646 \mathrm{~cm}^{-1}$ (geordnetes LCMO), beziehungsweise bei $644 \mathrm{~cm}^{-1}$ (teilgeordnetes LCMO), gekennzeichnet, die mit ihrer hohen Intensität in dieser Konfiguration und einer starken Intensitätsabnahme in der gekreuzten xy-Konfiguration die Raman-Auswahlregeln für die $A_{g}$-Symmetrie in der monoklinen P12 $1 /$ n1-Struktur erfüllt. Umgekehrt zeigt sich für die $B_{g}$ Mischmode bei $498 \mathrm{~cm}^{-1}$ (geordnetes LCMO), beziehungsweise bei $497 \mathrm{~cm}^{-1}$ (teilgeordnetes LCMO), eine umgekehrte Polarisationsabhängigkeit, die ebenfalls den Auswahlregeln der monoklinen P12//n1-Struktur folgt. Somit sind für beide LCMO-Schichten sehr ähnliche Raman-Spektren und eine monokline P12/n1-Struktur zu beobachten. Dieses stimmt mit den Magnetisierungsmessungen überein, die auch für die teilgeordnete LCMOSchicht einen B-Platz-Ordnungsgrad von $\approx 90 \%$ zeigen. Das Auftreten der Oberschwingung 2. Ordnung der Breathing Mode bestätigt zudem die hohe kristalline Qualität der LCMO $/ \mathrm{Al}_{2} \mathrm{O}_{3}$ (0001)-Schichten, während die im Vergleich der beiden Schichten leicht verschobenen Modenpositionen auf einen unterschiedlichen Verspannungszustand hinweisen.

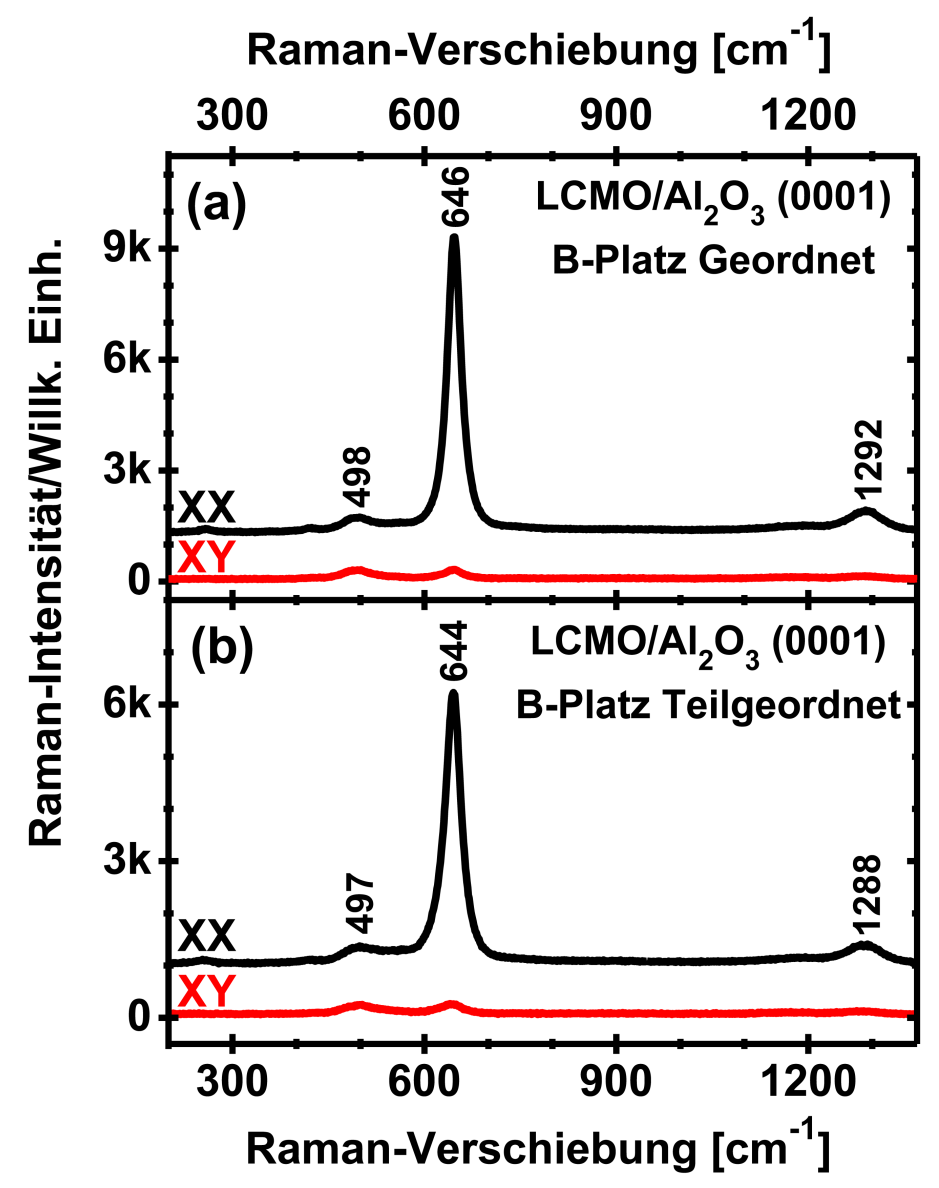

Abbildung 4.1.22: Polarisierte Raman-Spektren $(\lambda=532 \mathrm{~nm}, P \approx 2 \mathrm{~mW}, t=2 \times 60 \mathrm{~s}$, Raumtemperatur) der B-Platz geordneten (a) und der B-Platz teilgeordneten (b) LCMO-Schicht auf $\mathrm{Al}_{2} \mathrm{O}_{3}$ (0001) in der parallelen xx- und der gekreuzten xy-Polarisationskonfiguration im Vergleich. 


\subsubsection{Temperaturabhängige Raman-Messungen}

Für eine Betrachtung der Temperaturentwicklung der strukturellen und phononischen Eigenschaften der hergestellten Doppelperowskit-Schichten sind zusätzlich temperaturabhängige Raman-Spektren in einem Temperaturbereich $T=80-620 \mathrm{~K}$ (mit $\Delta T=20 \mathrm{~K}$ ) mit der in der im Abschnitt 3.3.3 vorgestellten Kühlzelle (Abbildungen 3.3.7 (a) und (b)) aufgenommen worden. Da sich besonders bei den $\mathrm{A}_{2} \mathrm{CoMnO}_{6}-$ Doppelperowskiten viele schwächere Raman-Moden überlagern (wie im Wellenzahlbereich der $B_{g}$ Mischmode), wird die Analyse zur Vermeidung von Mehrdeutigkeiten im Folgenden auf die starke und einzeln im Spektrum stehende $A_{g}$ Breathing Mode der Schichten beschränkt. Die Spektren sind mit dem grünen Laser $(\lambda=532 \mathrm{~nm}$ ) polarisationsunabhängig ohne Polarisator und Analysator gemessen worden. Die $A_{g}$ Breathing Mode wird dabei für jede angefahrene Temperatur mit einer Lorentz-Funktion angenähert und daraus die Modenposition $\omega(T)$ und die Linienbreite $\Gamma(T)$ (Halbwertsbreite) in Abhängigkeit der Temperatur bestimmt. Um einen Fehler durch eine instrumentelle Verschiebung der spektralen Position zu minimieren, wurde das Spektrometer direkt vor Messbeginn kalibriert und das Raman-Spektrum der SiliziumReferenzprobe bei Raumtemperatur nach jeweils fünf Messpunkten $(\Delta T=100 \mathrm{~K})$ gemessen. Im Fall einer signifikanten Verschiebung wurde anschließend das Raman-Spektrometer neu kalibriert und das Doppelperowskit-Raman-Spektrum bei der gleichen Temperatur erneut aufgenommen, um so instrumentelle Schwankungen in der Raman-Linienposition durch einen Vergleich zu korrigieren. Zudem wurden die Raman-Spektren erst nach einer Wartezeit von 1 min nach Erreichen der Zieltemperatur gemessen, um eine stabile Temperatur der Probe zu gewährleisten. Testmessungen bezüglich der Temperaturrichtung (Erwärmung oder Abkühlung) und dem Temperaturstartpunkt zeigten keinen Unterschied. Folglich wurden alle Messungen in Abkühlung beginnend bei $T=620 \mathrm{~K}$ mit einer Integrationszeit von jeweils $t=2 \times 60 \mathrm{~s}$ aufgenommen. Für eine Vermeidung einer lokalen Aufheizung durch den Laser wurde die Laserleistung dabei auf $P \approx 2 \mathrm{~mW}$ begrenzt.

In den Abbildungen 4.1.23 (a)-(d) ist die Temperaturentwicklung $\omega(T)$ der Position der $A_{g}$ Breathing Mode der B-Platz geordneten $\mathrm{A}_{2} \mathrm{CoMnO}_{6}$-Schichtserie (A = La, Pr, Sm, Gd) auf STO (111) mit einer Schichtdicke $d \approx 90-100 \mathrm{~nm}$ dargestellt. Die Vermessung der $\mathrm{Nd}_{2} \mathrm{CoMnO}_{6} / \mathrm{STO}$ (111)-Schicht fehlt aufgrund der Nichtverfügbarkeit der Schicht zum Zeitpunkt der Messreihe. Die $\mathrm{Y}_{2} \mathrm{CoMnO}_{6} / \mathrm{STO}$ (111)-Schicht fehlt wiederum aufgrund der Temperatureinschränkungen der Kühlzelle. Die roten Kurven kennzeichnen den an die Daten für Temperaturen $T \geq T_{C}$ angenäherten Verlauf $\omega_{\text {anh. }}(T)$ für die Position der $A_{g}$ Breathing Mode nach dem anharmonischen Modell der Gleichung (3.3.11). Für Temperaturen $T<T_{C}$ wiederum ergibt sich für alle vier Schichten eine signifikante Abweichung in der Positionsentwicklung der Breathing Mode in den Raman-Spektren mit abnehmender Temperatur in Form eines deutlichen Erweichens, das mit dem Eintreten in die ferromagnetische Phase der Doppelperowskite initiiert wird. Eine detaillierte Analyse dieses Phänomens 
und der dabei auftretenden Charakteristika wird in dem Abschnitt 4.1.5 dargestellt. Eine Sonderstellung nimmt die LCMO/STO (111)-Schicht ein. Während die anderen Schichten der $\mathrm{A}_{2} \mathrm{CoMnO}_{6}$-Serie für Temperaturen $T \geq T_{C}$ in der Positionsentwicklung $\omega(T)$ der Breathing Mode dem anharmonischen Verlauf mit einem mit zunehmender Temperatur kontinuierlichen Erweichen der Mode folgen (die leichte Wellenbewegung der experimentellen Daten bei hohen Temperaturen lässt sich durch kleine instrumentelle Schwankungen in der Linienposition begründen), ist für die LCMO/STO (111)-Schicht beginnend ab einer Temperatur $T \approx 420 \mathrm{~K}$ ein systematisches Erhärten der Position der $A_{g}$ Breathing Mode mit einem Maximum bei $T_{\mathrm{MR}}=580 \mathrm{~K}$ zu beobachten, bevor sich wieder eine Tendenz zu einem Erweichen der Mode in Richtung des anharmonischen Verlaufs $\omega_{\text {anh. }}(T)$ ergibt.
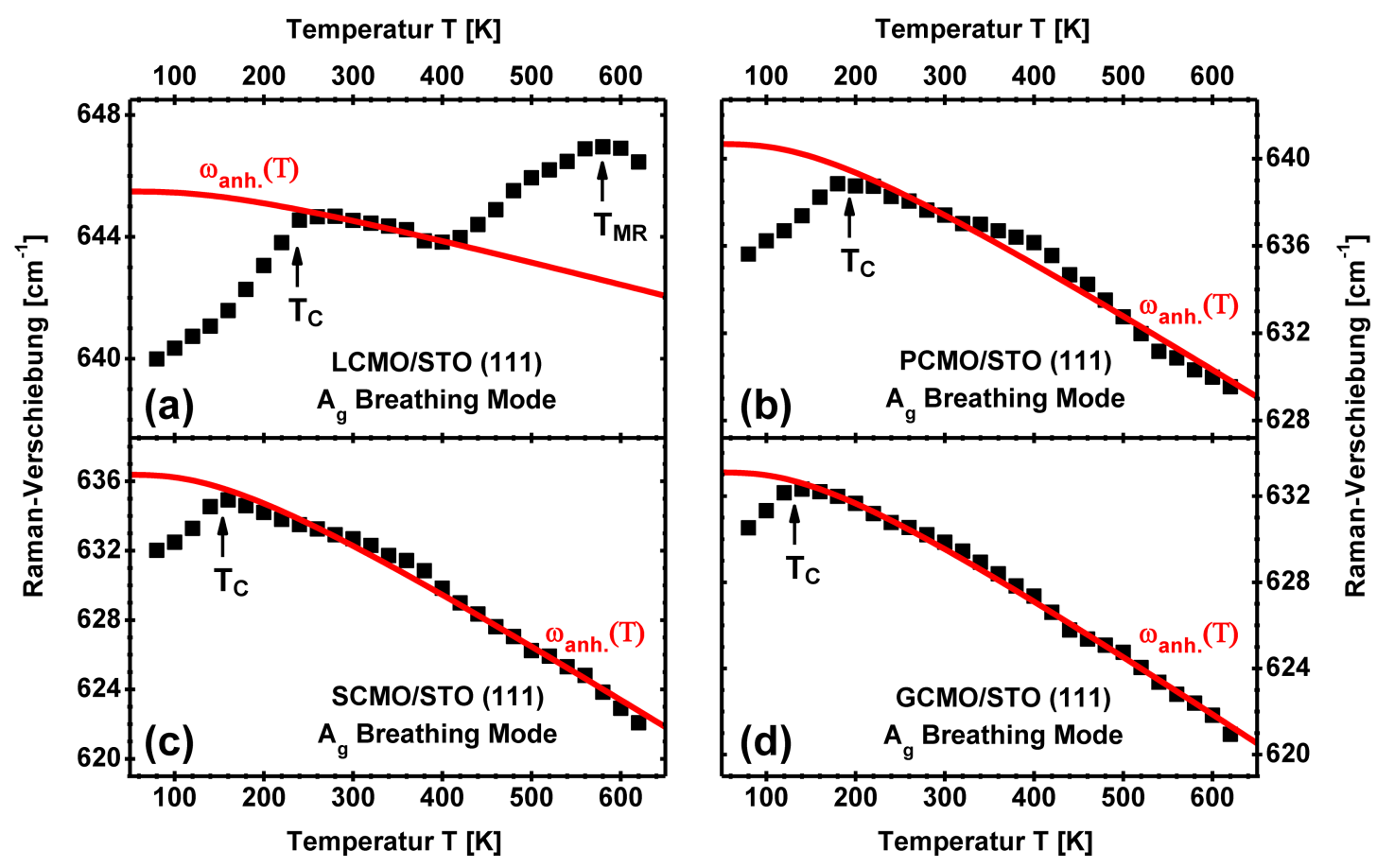

Abbildung 4.1.23: Temperaturentwicklung $\omega(T)$ der Linienposition der $A_{g}$ Breathing Mode der B-Platz geordneten Doppelperowskite LCMO (a), PCMO (b), SCMO (c) und GCMO (d) auf STO (111) im Vergleich. Die roten Kurven kennzeichnen jeweils den Verlauf nach dem anharmonischen Modell.

Die Hochtemperatur-Anomalie der LCMO-Schicht kann hierbei auf einen strukturellen Phasenübergang von der monoklinen $\mathrm{P} 12_{1} / \mathrm{n} 1$ - in die rhomboedrische R $\overline{3}$-Struktur zurückgeführt werden, der für Bulk-LCMO anhand von Neutronenstreuung bei einer Temperatur $T_{\mathrm{MR}}=598 \mathrm{~K}$ und in Raman-Messungen an Bulk-LCMO 238 und dünnen LCMOSchichten auf STO- und $\mathrm{NdGaO}_{3}$-Substraten bei einer Temperatur $T_{\mathrm{MR}} \approx 550 \mathrm{~K} 215,239$ beobachtet werden konnte. Da der Phasenübergang mit einer signifikanten Änderung der Kristallsymmetrie und der Struktur einhergeht 215, 238, sind auch die phononischen Eigenschaften und damit die Linienposition der $A_{g}$ Breathing Mode beeinflusst 33, 215,239]. Die auftretende leichte Abnahme der pseudokubischen Gitterkonstante und der mittleren Co/Mn-O-Bindunglänge 215,238 führt daher im Raman-Spektrum zu einem Erhärten der 
Breathing Mode während des Strukturübergangs, das von der anharmonischen Temperaturentwicklung $\omega_{\text {anh. }}(T)$ der Linienposition abweicht. Die Breite der Anomalie ist dabei auf den diffusen Charakter des Phasenübergangs für LCMO mit einer Koexistenz der zwei Phasen über einen großen Temperaturbereich unterhalb von $T_{\mathrm{MR}}$ zurückzuführen [215, 238], bevor sich für $T>T_{\mathrm{MR}}$ das Erweichen der Breathing Mode gemäß der anharmonischen Beschreibung fortsetzt. Für die anderen Schichten der $\mathrm{A}_{2} \mathrm{CoMnO}_{6}$-Serie fehlt diese Anomalie. Folglich verbleiben diese im untersuchten Temperaturbereich in der monoklinen P12 1 /n1Struktur. Für eine Ausweitung der Untersuchung auf die $\mathrm{A}_{2} \mathrm{NiMnO}_{6}$-Doppelperowskite ist in den Abbildungen 4.1.24 (a)-(e) in gleicher Weise die Temperaturabhängigkeit der Position der Breathing Mode der B-Platz geordneten $\mathrm{A}_{2} \mathrm{NiMnO}_{6}$-Doppelperowskit-Schichtserie $(\mathrm{A}=\mathrm{La}, \mathrm{Pr}, \mathrm{Nd}, \mathrm{Sm}, \mathrm{Gd})$ auf STO (111) mit einer Schichtdicke $d \approx 90-100 \mathrm{~nm}$ dargestellt. Die roten Kurven zeigen wieder jeweils den anharmonischen Verlauf für $T \geq T_{C}$.

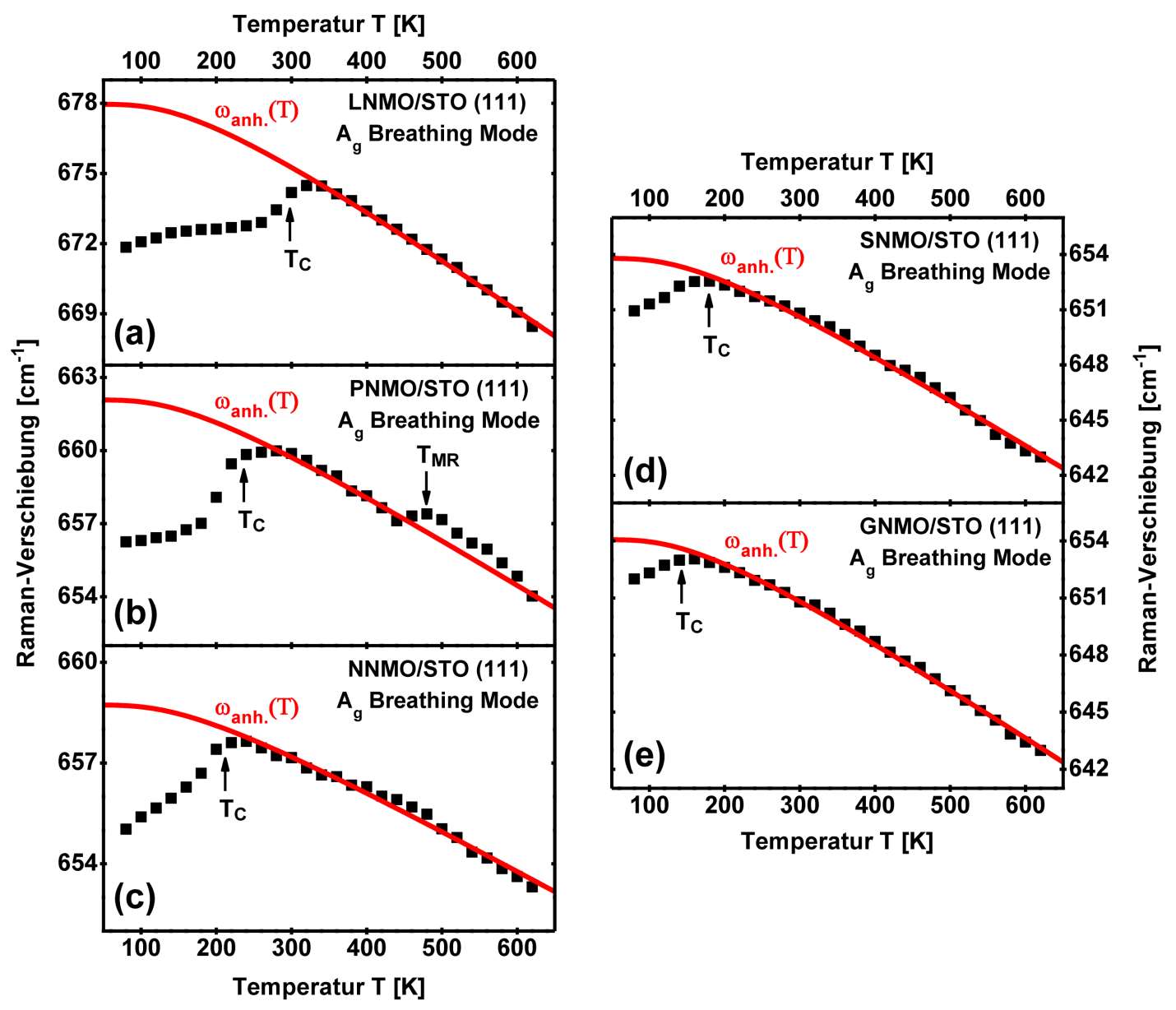

Abbildung 4.1.24: Temperaturentwicklung $\omega(T)$ der Linienposition der $A_{g}$ Breathing Mode der B-Platz geordneten Doppelperowskite LNMO (a), PNMO (b), NNMO (c), SNMO (d) und GNMO (e) auf STO (111) im Vergleich. Die roten Kurven kennzeichnen den anharmonischen Modellverlauf.

Wie bei der $\mathrm{A}_{2} \mathrm{CoMnO}_{6}$-Serie ist auch für die $\mathrm{A}_{2} \mathrm{NiMnO}_{6}$-Schichten für Temperaturen $T<T_{C}$ ein Erweichen der $A_{g}$ Breathing Mode mit abnehmender Temperatur in der ferromagnetischen Phase zu beobachten. Für $T \geq T_{C}$ folgt die Temperaturentwicklung $\omega(T)$ 
der Position der Breathing Mode mit Ausnahme der PNMO/STO (111)-Schicht einem anharmonischen Verlauf mit einem mit zunehmender Temperatur kontinuierlichen Erweichen der Mode. Für die PNMO-Schicht ist für $T>440 \mathrm{~K}$ bis zu einem Maximum bei $T_{\mathrm{MR}}=480 \mathrm{~K}$ zusätzlich ein Erhärten zu beobachten, das einen Phasenübergang in die R⿳亠丷⿵-Struktur andeutet, bevor sich das Erweichen fortsetzt. Die Literatur bietet hierzu keinen Vergleich. Ein Anhaltspunkt ist der P12, n1-R̄̄3-Phasenübergang für Bulk-LNMO, der durch Neutronenstreuung bei $T_{\mathrm{MR}}=648 \mathrm{~K}$ beobachtet wurde [238], sich jedoch für die LNMO/STO (111)-Schicht im Verlauf von $\omega(T)$ nicht nachweisen lässt. Die polarisationsabhängigen Raman-Messungen im Abschnitt 4.1.2 und in der Literatur [240] zeigen allerdings, dass bereits bei Raumtemperatur für LNMO eine Koexistenz beider Phasen vorliegt, sodass sich das Erweichen in der ferromagnetischen Phase und das Erhärten aufgrund des strukturellen Phasenübergangs überlagern. Aufgrund der strukturellen Ähnlichkeit zu LNMO kann daher auch die Anomalie der Breathing Mode bei der PNMO-Schicht bei $T_{\mathrm{MR}}=480 \mathrm{~K}$ in der Position $\omega(T)$ einem Übergang in die R $\overline{3}$-Struktur zugeordnet werden.

In den Abbildungen 4.1.25 (a) und (b) ist zusätzlich die Temperaturentwicklung $\Gamma(T)$ der Linienbreite der $A_{g}$ Breathing Mode der B-Platz geordneten $\mathrm{A}_{2} \mathrm{CoMnO}_{6^{-}}$und $\mathrm{A}_{2} \mathrm{NiMnO}_{6^{-}}$ Doppelperowskit-Schichtserien auf STO (111) dargestellt. Die Kurven repräsentieren dabei jeweils den mit der Gleichung (3.3.12) angenäherten anharmonischen Verlauf.
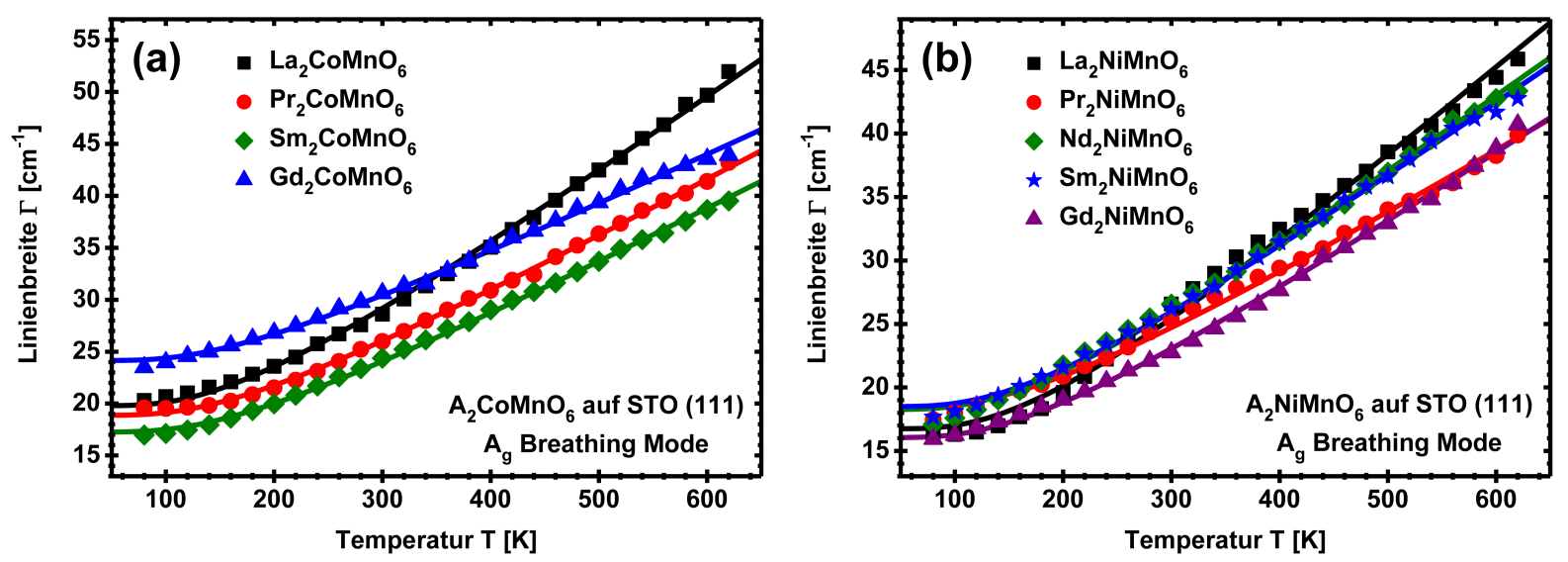

Abbildung 4.1.25: Temperaturentwicklung $\Gamma(T)$ der Linienbreite der $A_{g}$ Breathing Mode der $\mathrm{A}_{2} \mathrm{CoMnO}_{6}$-Schichten (a) und $\mathrm{A}_{2} \mathrm{NiMnO}_{6}$-Schichten (b) auf STO (111) im Vergleich. Die Kurven kennzeichnen den anharmonischen Verlauf.

Bei allen Doppelperowskit-Schichten folgt die Linienbreite $\Gamma(T)$ der $A_{g}$ Breathing Mode im gesamten Temperaturbereich dem anharmonischen Verlauf $\Gamma_{\text {anh. }}(T)$. Somit wirkt sich das in der Position $\omega(T)$ der Breathing Mode beobachtete Erweichen in der ferromagnetischen Phase nicht auf die Linienbreite aus. Ebenso bleibt für die LCMO- und die PNMO-Schicht die Linienbreite unbeeinflusst von der rhomboedrischen $\mathrm{R} \overline{3}$-Phase. Vielmehr ist die Linienbreite im Allgemeinen durch die Kristallinität, die chemische Ordnung, die Schichtverspannung und durch den Schwingungscharakter der Raman-Mode bestimmt 120, 123, 128,215]. 
Für eine Untersuchung des Einflusses der Verspannung auf die Temperaturentwicklung der Position und der Linienbreite der $A_{g}$ Breathing Mode im Allgemeinen sowie auf die für die LCMO/STO(111)-Schicht beobachtete Hochtemperatur-Anomalie bietet es sich an, die temperaturabhängigen Raman-Messungen zusätzlich an den B-Platz geordneten $\mathrm{La}_{2} \mathrm{CoMnO}_{6}$-Schichten mit vergleichbarer Schichtdicke $d \approx 90-110 \mathrm{~nm}$ auf unterschiedlichen Substraten durchzuführen. Die Temperaturentwicklung der Postion $\omega(T)$ der $A_{g}$ Breathing Mode ist für die LCMO-Schichten auf LAO (111), LSAT (111), $\mathrm{Al}_{2} \mathrm{O}_{3}$ (0001) und $\mathrm{MgO}$ (100) in den Abbildungen 4.1.26 (a)-(d) dargestellt. Die roten Kurven kennzeichnen dabei jeweils wieder den anharmonischen Verlauf nach der Gleichung (3.3.11).
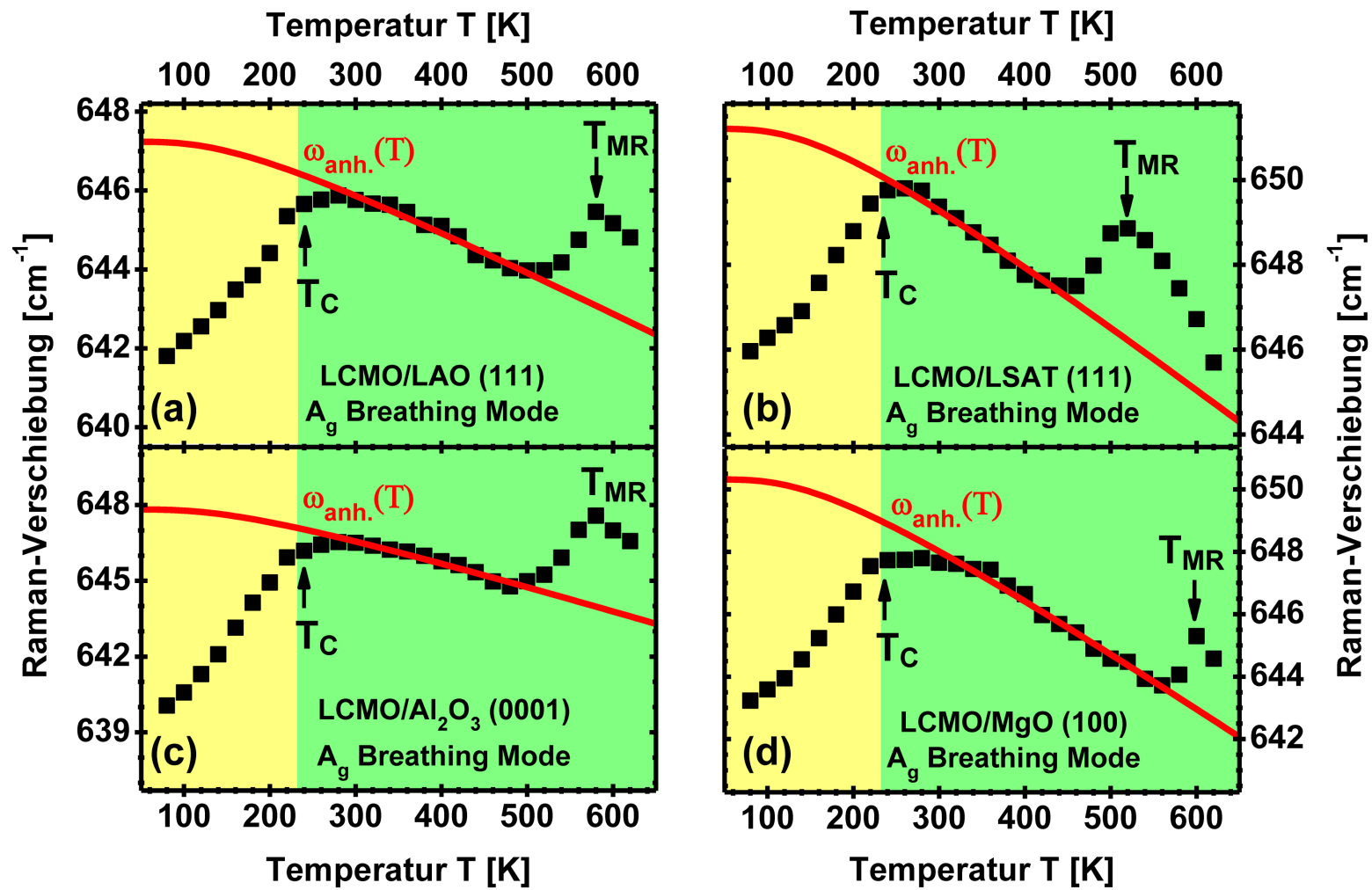

Abbildung 4.1.26: Temperaturentwicklung $\omega(T)$ der Position der $A_{g}$ Breathing Mode der BPlatz geordneten LCMO-Schichten auf LAO (111) (a), LSAT (111) (b), $\mathrm{Al}_{2} \mathrm{O}_{3}$ (0001) (c) und $\mathrm{MgO}$ (100) (d) im Vergleich. Die roten Kurven kennzeichnen jeweils den Verlauf nach dem anharmonischen Modell.

Es ergeben sich analog zur LCMO/STO (111)-Schicht zwei signifikante Abweichungen in der Entwicklung der Position der Breathing Mode mit der Temperatur vom anharmonischen Modell. Neben einem starken Erweichen der Breathing Mode für Temperaturen $T<T_{C} \approx 230 \mathrm{~K}$ beim Übergang von der paramagnetischen (grün hinterlegt) in die ferromagnetische Phase (gelbe Hintergrundfläche) ist für alle LCMO-Schichten ein Erhärten der Mode für $T>500 \mathrm{~K}$ mit einem ausgeprägten Maximum bei $T_{\mathrm{MR}} \mathrm{zu}$ beobachten, bevor mit weiter zunehmender Temperatur ein erneutes Erweichen einsetzt und sich das Verhalten wieder dem anharmonischen Verlauf annähert. Der Bereich vom einsetzenden Erhärten bis zum Maximum bei $T_{\mathrm{MR}}$ variiert dabei von $\Delta T=40 \mathrm{~K}$ für die LCMO/MgO (100)-Schicht 


\section{Auswertung und Ergebnisse}

bis zu $\Delta T=200 \mathrm{~K}$ für die LCMO/STO (111)-Schicht. Zusätzlich schwankt die Position des Maximums zwischen $T_{\mathrm{MR}}=540 \mathrm{~K}$ für die LCMO/LSAT (111)- und $T_{\mathrm{MR}}=600 \mathrm{~K}$ für die LCMO/MgO (100)-Schicht. Da die Hochtemperaturanomalie auf einen Phasenübergang von der monoklinen $\mathrm{P} 12_{1} / \mathrm{n} 1$ - in die rhomboedrische R $\overline{3}$-Struktur zurückzuführen ist und die Breite der Anomalie durch den diffusen Übergangscharakter mit einer Koexistenz beider Phasen im Umfeld des Phasenübergangs bestimmt ist 215, 238, hat die Schichtverspannung somit einen direkten Einfluss auf den Ablauf dieses Übergangs. Für eine Quantifizierung des Einflusses auf die Position der $A_{g}$ Breathing Mode der LCMO-Schichten sind in der Tabelle 4.1.5 die Parameter $\omega_{0}, C$ und $\omega_{0}+C$ für die anharmonische Entwicklung der Modenposition $\omega_{\text {anh. }}(T)$ mit den in-plane Substrat-Gitterkonstanten $a$ [225] ( $a_{\text {eff. für }}$ $\mathrm{Al}_{2} \mathrm{O}_{3}$ (0001) bei (111)-orientiertem Wachstum) und den im Abschnitt 4.1 .3 bestimmten pseudokubischen out-of-plane Gitterkonstanten $c_{\mathrm{pc}}$ der Schichten angegeben.

\begin{tabular}{|c|l|l|l|l|l|}
\hline LCMO auf & $\omega_{0}\left[\mathrm{~cm}^{-1}\right]$ & $C\left[\mathrm{~cm}^{-1}\right]$ & $\omega_{0}+C\left[\mathrm{~cm}^{-1}\right]$ & $a[\AA]$ & $c_{\mathrm{pc}}[\AA]$ \\
\hline \hline LAO $(111)$ & $649,81(32)$ & $-2,57(18)$ & $647,24(37)$ & 3,788 & 3,899 \\
\hline LSAT $(111)$ & $655,63(32)$ & $-4,11(19)$ & $651,52(37)$ & 3,868 & 3,909 \\
\hline $\mathrm{Al}_{2} \mathrm{O}_{3}(0001)$ & $650,21(30)$ & $-2,39(16)$ & $647,82(34)$ & 3,885 & 3,878 \\
\hline $\mathrm{STO}(111)$ & $647,28(32)$ & $-1,79(20)$ & $645,49(38)$ & 3,905 & 3,875 \\
\hline $\mathrm{MgO}(100)$ & $654,71(34)$ & $-4,39(17)$ & $650,32(38)$ & 4,216 & 3,902 \\
\hline
\end{tabular}

Tabelle 4.1.4: Parameter $\omega_{0}, C$ und $\omega_{0}+C$ zur anharmonischen Beschreibung der Temperaturentwicklung $\omega_{\text {anh. }}(T)$ der Position der $A_{g}$ Breathing Mode der LCMO-Schichten auf den verschiedenen Substraten zusammen mit den in-plane Gitterkonstanten $a$ der Substrate 225 und der aus den XRD-Messungen bestimmten pseudokubischen out-of-plane Gitterkonstanten $c_{\mathrm{pc}}$ der Schichten.

Die temperaturunabhängige Position $\omega_{0}+C$ der Breathing Mode bei $T=0 \mathrm{~K}$ und $\omega_{0}$ korrelieren mit der Schichtverspannung, während $C$ die anharmonische Kristallwechselwirkung und die Temperaturentwicklung der Modenposition charakterisiert. Es zeigt sich, dass eine in-plane induzierte Druckspannung zu einem in-plane Erhärten der $A_{g}$ Breathing Mode aufgrund der in-plane komprimierten Gitterstruktur führt, die sich out-of-plane durch die Zunahme von $c_{\mathrm{pc}}$ der LCMO-Schicht in Relation zu Bulk-LCMO mit $c_{\mathrm{pc} \text {, Bulk }}=3,887 \AA 34$ nachweisen lässt. Im Gegensatz dazu hat eine in-plane Zugspannung ein Erweichen zur Folge. Gleichzeitig ist der anharmonische Beitrag $C$ für eine in-plane Druckspannung oder ungleichmäßige Verspannung wie für die LCMO/MgO (100)-Schicht im Vergleich zu einer in-plane Zugspannung erhöht, was zu einem schnelleren Erweichen der $A_{g}$ Breathing Mode mit steigender Temperatur führt. Daneben wird auch der Übergang von der monoklinen P12 1 /n1- in die rhomboedrische R $\overline{3}$-Phase beeinflusst. So ist das Erhärten der $A_{g}$ Breathing Mode für die stark verspannten LCMO-Schichten auf LSAT (111) und STO (111) besonders ausgeprägt und die Phasenkoexistenz erstreckt sich daher über einen großen 
Temperaturbereich, während eine nur kleine Verspannung oder Relaxation einen schärferen Übergang begünstigt. Für den Nachweis der R $\overline{3}$-Phase können die Raman-Spektren der LCMO-Schichten bei kleinen Wellenzahlen betrachtet werden. Diese sind für $T=80 \mathrm{~K}$, $T=300 \mathrm{~K}$ und $T=T_{\mathrm{MR}}$ in den Abbildungen 4.1.27 (a)-(d) mit dem jeweiligen SubstratSpektrum $(T=300 \mathrm{~K})$ dargestellt. Auf die Spektren der LCMO/STO (111)-Schicht wurde aufgrund des starken Hintergrunds durch das Substrat bei $T=T_{\mathrm{MR}}$ verzichtet.
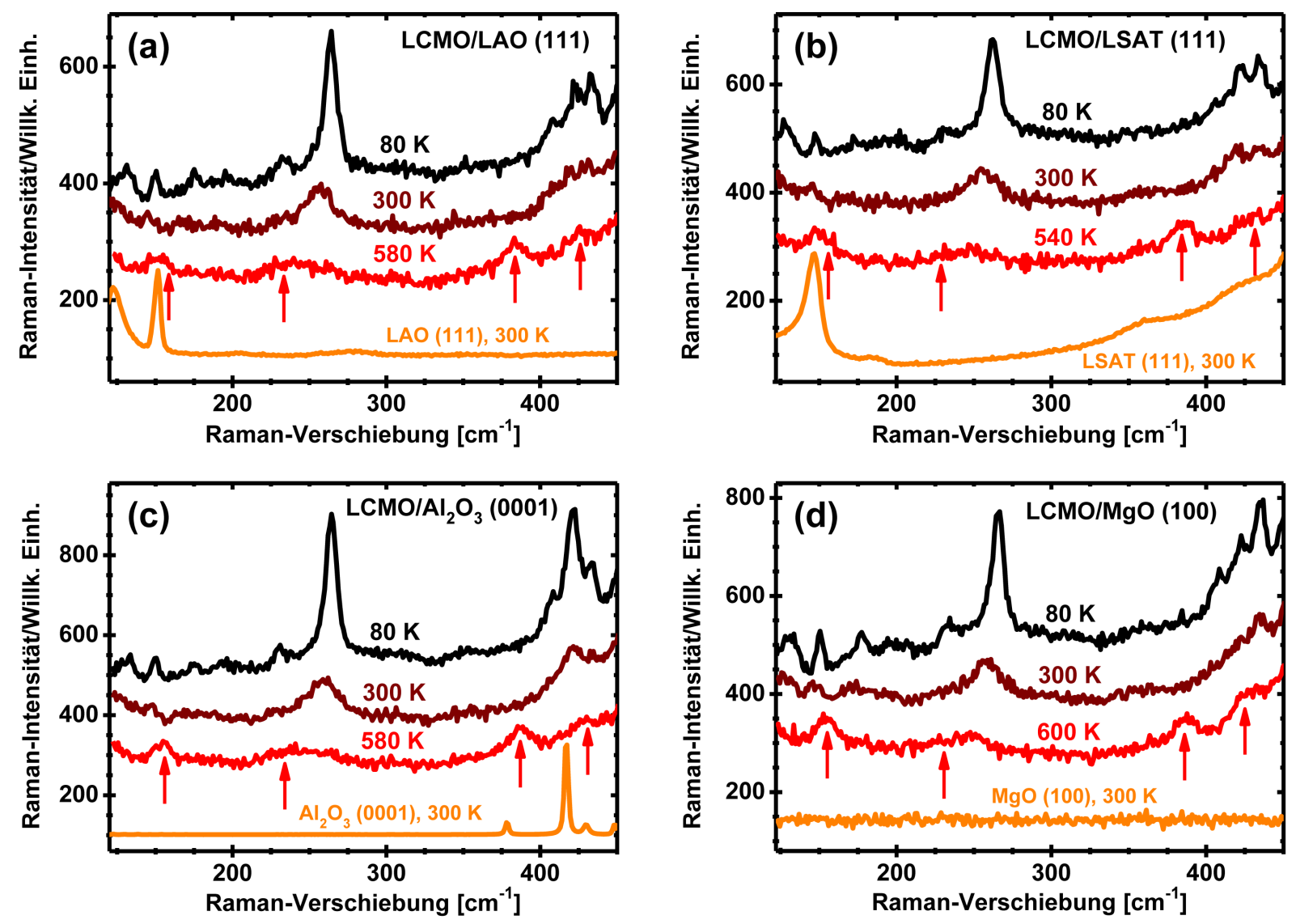

Abbildung 4.1.27: Für verschiedene Temperaturen $T$ aufgenommene, unpolarisierte RamanSpektren der $\mathrm{La}_{2} \mathrm{CoMnO}_{6}$-Schichten auf LAO (111) (a), LSAT (111) (b), $\mathrm{Al}_{2} \mathrm{O}_{3}$ (0001) (c) und $\mathrm{MgO}$ (100) (d) im Bereich kleiner Wellenzahlen. Zusätzlich sind die jeweiligen Substrat-Spektren bei Raumtemperatur dargestellt. Die roten Pfeile kennzeichnen Raman-Moden der R $\overline{3}$-Phase.

Bei $T=T_{\mathrm{MR}}$ lassen sich für alle LCMO-Schichten vier neu erscheinende Raman-Moden (rote Pfeile) bei $155 \mathrm{~cm}^{-1}\left(E_{g}\right), 235 \mathrm{~cm}^{-1}\left(E_{g}\right), 388 \mathrm{~cm}^{-1}\left(E_{g}\right)$, und $430 \mathrm{~cm}^{-1}\left(A_{g}\right)$ beobachten, die sich nach LDC-Berechnungen der R $\overline{3}$-Phase zuordnen lassen [33, 215] und den strukturellen Phasenübergang bestätigen. Die neuen Raman-Moden kennzeichnen dabei direkt die auftretende Änderung in der Kristallsymmetrie mit den entsprechend dazugehörigen Rotationen und Verkippungen der $(\mathrm{Co} / \mathrm{Mn}) \mathrm{O}_{6}$-Oktaeder [33,215]. Ein Vergleich mit den Raman-Spektren der Substrate zeigt zudem, dass die neuen Moden nicht von den Substraten stammen. Die relativ schwache Intensität und die große Verbreiterung der Moden resultiert aus der hohen Temperatur und den Gleichungen (3.3.10) und 3.3.12). 


\section{Auswertung und Ergebnisse}

In der Abbildung 4.1.28 ist zusätzlich die jeweilige Temperaturentwicklung $\Gamma(T)$ der Linienbreite der $A_{g}$ Breathing Mode der B-Platz geordneten $\mathrm{La}_{2} \mathrm{CoMnO}_{6}$-Schichten vergleichbarer Schichtdicke auf den verschiedenen Substraten in einem direkten Vergleich dargestellt.

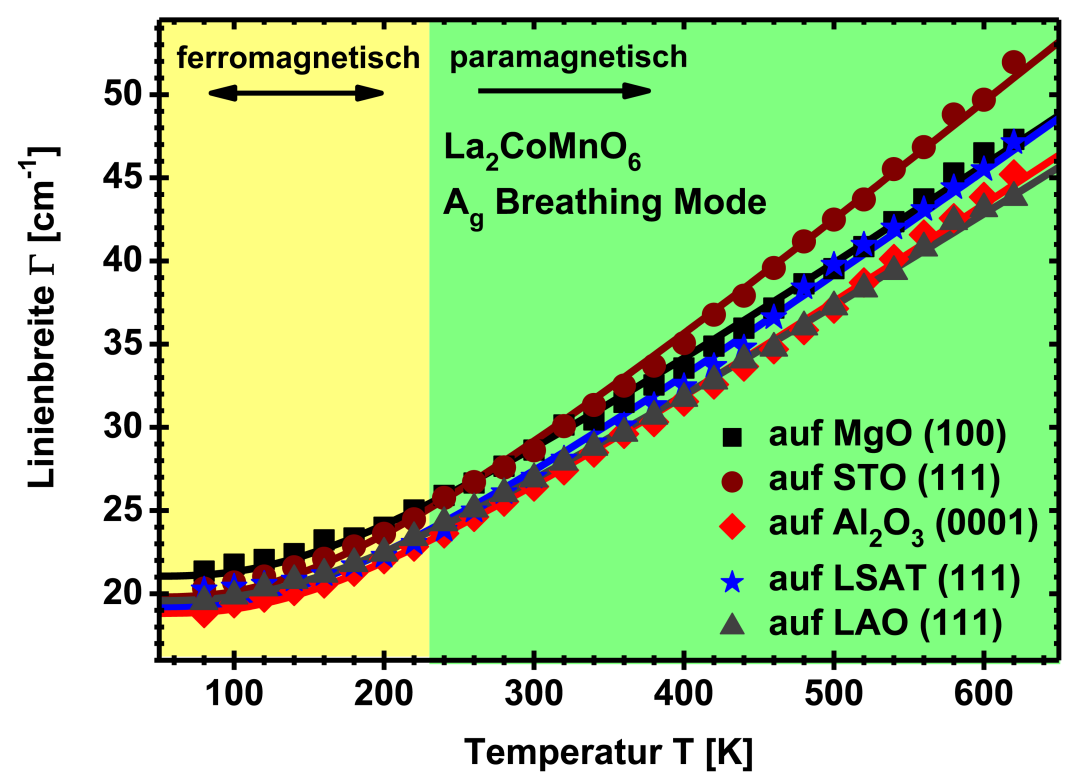

Abbildung 4.1.28: Temperaturentwicklung $\Gamma(T)$ der Linienbreite der $A_{g}$ Breathing Mode der B-Platz geordneten LCMO-Schichten auf verschiedenen Substraten im Vergleich. Die Kurven kennzeichnen den anharmonischen Modellverlauf.

Es ergibt sich für die fünf LCMO-Schichten ein ähnliches Verhalten, das sich sehr gut mit einem anharmonischen Verlauf anhand der Gleichung (3.3.12) beschreiben lässt. Die bei der Temperaturentwicklung der Position der $A_{g}$ Breathing Mode der LCMO-Schichten zu beobachtenden Unterschiede für $T>500 \mathrm{~K}$ treten somit bei der Linienbreite nicht auf. Dieses ist auf den Schwingungscharakter der Breathing Mode und auf die Art des strukturellen Phasenübergangs von der monoklinen $\mathrm{P} 12_{1} / \mathrm{n} 1$ - in die rhomboedrische R $\overline{3}$-Struktur zurückzuführen. So behält die Breathing Mode in beiden Phasen ihre $A_{g}$-Symmetrie aufgrund der in der R $\overline{3}$-Phase weiter vorhandenen B-Platz-Ordnung [238] und ihre symmetrische Streckschwingungsbewegung der $(\mathrm{Co} / \mathrm{Mn}) \mathrm{O}_{6}$-Oktaeder bei 33,215] und ist damit kaum von den Verkippungen der Sauerstoffoktaeder beim Phasenübergang beeinflusst. Im Gegensatz dazu ist der Effekt auf die $B_{g}$ Mischmode, die eine Kombination aus antisymmetrischer Streckschwingung und Biegeschwingung der Oktaeder beschreibt und eine Symmetrieänderung von der $B_{g^{-}}$zur $E_{g^{-}}$Symmetrie in der rhomboedrischen Hochtemperaturphase erfährt, entsprechend größer, sodass für die Linienbreite diese Mode in der Literatur eine Anomalie beobachtet wurde 215]. Auffällig für $T>500 \mathrm{~K}$ in der Entwicklung der Linienbreite der $A_{g}$ Breathing Mode ist jedoch eine divergierende Tendenz für die LCMO-Schichten mit der größten Linienbreite für die Schicht auf STO (111) und der kleinsten Linienbreite für die Schicht auf LAO (111), die eine Abhängigkeit von der durch das Substrat induzierten Verspannung andeutet. Für eine Quantifizierung sind in der Tabelle 4.1.5 die Parameter $\Gamma_{0}, \gamma$ und $\Gamma_{0}+\gamma$ für die anharmonische Entwicklung der Linienbreite $\Gamma_{\text {anh. }}(T)$ 
$\operatorname{der} A_{g}$ Breathing Mode für die B-Platz geordneten LCMO-Schichten auf den verschiedenen Substraten aufgelistet. Hierbei wurden die $\omega_{0}$-Parameter aus der Positionsentwicklung $\omega_{\text {anh. }}(T)$ der Breathing Mode verwendet. Zusätzlich sind in der Tabelle 4.1.5 die in-plane Gitterkonstanten $a$ der Substrate 225] angegeben. Für $\mathrm{Al}_{2} \mathrm{O}_{3}$ (0001) ist dieses die effektive in-plane Gitterkonstante $a_{\text {eff. }}$, die sich durch $\sqrt{2} a_{\text {Saphir }} \approx \sqrt{3} a_{\mathrm{pc} \text {, LCMO }}$ (Abschnitt 4.1.3) mit $a_{\text {Saphir }}=4,758 \AA 225$ ergibt. Daneben sind die aus den XRD-Spektren bestimmten pseudokubischen out-of-plane Gitterkonstanten $c_{\mathrm{pc}}$ der LCMO-Schichten aufgeführt.

\begin{tabular}{|c|l|l|l|l|l|}
\hline LCMO auf & $\Gamma_{0}\left[\mathrm{~cm}^{-1}\right]$ & $\gamma\left[\mathrm{cm}^{-1}\right]$ & $\Gamma_{0}+\gamma\left[\mathrm{cm}^{-1}\right]$ & $a[\AA]$ & $c_{\mathrm{pc}}[\AA]$ \\
\hline \hline LAO $(111)$ & $13,72(7)$ & $5,86(13)$ & $19,58(15)$ & 3,788 & 3,899 \\
\hline LSAT $(111)$ & $15,64(18)$ & $3,57(33)$ & $19,21(38)$ & 3,868 & 3,909 \\
\hline $\mathrm{Al}_{2} \mathrm{O}_{3}(0001)$ & $14,51(12)$ & $4,31(22)$ & $18,82(25)$ & 3,885 & 3,878 \\
\hline $\mathrm{STO}(111)$ & $17,47(14)$ & $2,33(26)$ & $19,80(30)$ & 3,905 & 3,875 \\
\hline $\mathrm{MgO}(100)$ & $14,73(13)$ & $6,32(25)$ & $21,05(28)$ & 4,216 & 3,902 \\
\hline
\end{tabular}

Tabelle 4.1.5: Parameter $\Gamma_{0}, \gamma$ und $\Gamma_{0}+\gamma$ zur anharmonischen Beschreibung der Temperaturentwicklung $\Gamma_{\text {anh. }}(T)$ der Linienbreite der $A_{g}$ Breathing Mode der LCMOSchichten auf den verschiedenen Substraten zusammen mit den in-plane Gitterkonstanten $a$ der Substrate [225] und der aus den XRD-Messungen bestimmten pseudokubischen out-of-plane Gitterkonstanten $c_{\mathrm{pc}}$ der Schichten.

Insbesondere die Summe $\Gamma_{0}+\gamma$, die den temperaturunabhängigen Anteil der Linienbreite bei $T=0 \mathrm{~K}$ beschreibt, charakterisiert dabei die kristalline Qualität, die chemische Ordnung sowie den Einfluss durch Verspannungen in den Schichten 120,123, 128]. Es ergibt sich eine Korrelation zwischen $\Gamma_{0}+\gamma$ und der in-plane Substrat-Gitterkonstante $a$, die in der Abbildung 4.1.29 (a) dargestellt ist. Die blaue vertikale Linie kennzeichnet die pseudokubische Gitterkonstante von Bulk-LCMO mit $c_{\mathrm{pc}}$, Bulk $=3,887 \AA$ 34. Es zeigt sich, dass der temperaturunabhängige Anteil der Linienbreite der $A_{g}$ Breathing Mode der LCMO-Schichten systematisch mit der Substrat-Gitterkonstante variiert und in Relation zu Bulk-LCMO kleinere Substrat-Gitterkonstanten tendenziell niedrigere Werte hervorrufen. Für die LCMO-Schicht auf $\mathrm{Al}_{2} \mathrm{O}_{3}$ (0001), dessen effektive in-plane Gitterkonstante für ein (111)-Wachstum nur leicht kleiner als Bulk-LCMO ist, lässt sich dabei ein Minimum für die Summe $\Gamma_{0}+\gamma$ beobachten. Anhand der strukturellen und magnetischen Eigenschaften der Schichten lässt sich dieses Verhalten mit der B-Platz-Ordnung und Ordnungsfehlern wie den APG in den Zusammenhang bringen. Folglich ist die Linienbreite auch durch die Anzahl an APG und die Größe der dadurch lokal erzeugten Domänen mit antiparalleler Ausrichtung bestimmt. Die beispielsweise für die LCMO/STO (111)-Schicht gegenüber der LCMO $/ \mathrm{Al}_{2} \mathrm{O}_{3}$ (0001)-Schicht erhöhte APG-Anzahl und -Domänengröße (Abschnitt 4.1.3) spiegelt sich somit analog zur Sättigungsmagnetisierung und der Koerzitivfeldstärke sowie der XRD-Überstrukturintensität direkt in der Linienbreite der $A_{g}$ Breathing Mode wider. 

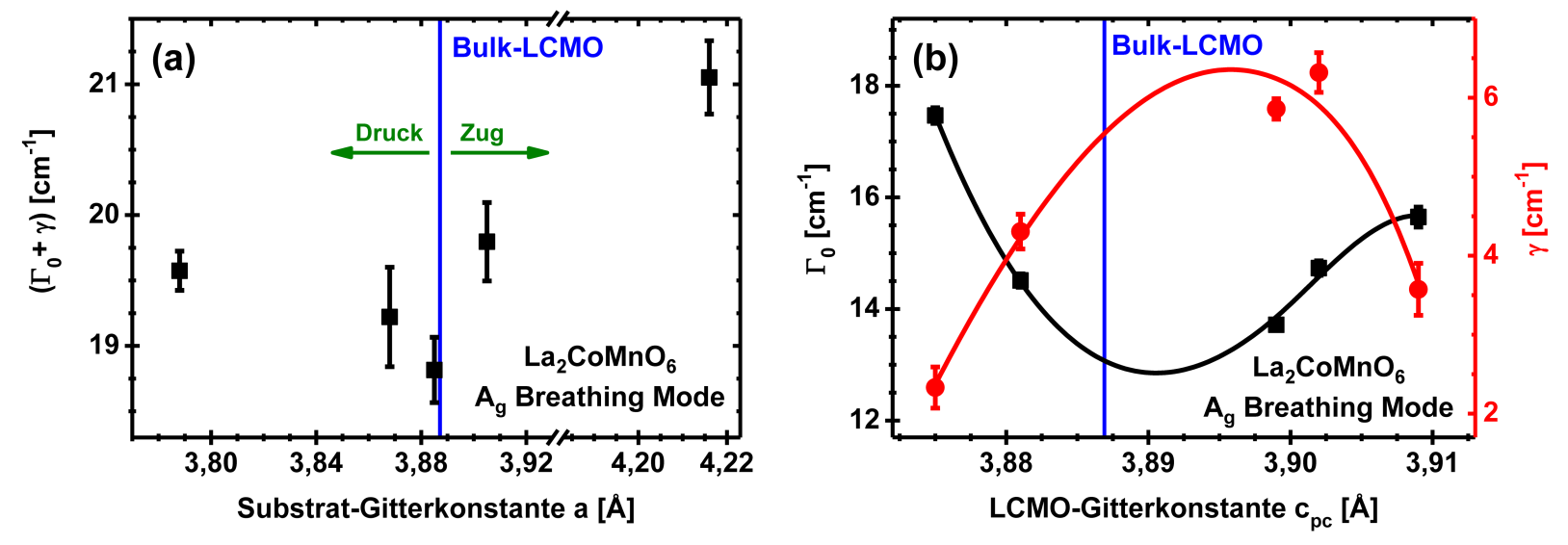

Abbildung 4.1.29: Die temperaturunabhängige Linienbreite $\Gamma_{0}+\gamma \operatorname{der} A_{g}$ Breathing Mode der LCMO-Schichten in Abhängigkeit der in-plane Gitterkonstante $a$ des Substrats (a) und die Abhängigkeit von $\Gamma_{0}$ und $\gamma$ von der pseudokubischen out-of-plane Gitterkonstante $c_{\mathrm{pc}}$ der LCMO-Schichten (b).

Die $\Gamma_{0^{-}}$und $\gamma$-Abhängigkeit von der pseudokubischen out-of-plane Gitterkonstante $c_{\mathrm{pc}}$ der LCMO-Schichten ist in der Abbildung 4.1.29 (b) dargestellt. Die blaue Linie symbolisiert erneut die pseudokubische Gitterkonstante von Bulk-LCMO. Es lässt sich eine Abnahme von $\Gamma_{0}$ sowie eine Zunahme von $\gamma$ für eine Annäherung von $c_{\mathrm{pc}}$ an $c_{\mathrm{pc}}$, Bulk beobachten, wobei die Interpolation des Minimums von $\Gamma_{0}$ und des Maximums von $\gamma$ für eine out-of-plane Gitterkonstante $c_{\mathrm{pc}}$ knapp oberhalb von Bulk-LCMO erreicht werden. Die zwei Parameter sind daher durch einen unterschiedlichen physikalischen Hintergrund bestimmt. Während $\Gamma_{0}$ den Hauptbeitrag zur temperaturabhängigen Linienbreite liefert und die kristalline Ordnung sowie die anharmonische Wechselwirkung im Kristallgitter charakterisiert, beschreibt $\gamma$ den Verspannungszustand in den LCMO-Schichten. Die Auswirkungen lassen sich in der Abbildung 4.1.28 beobachten. So verfügt die LCMO/STO (111)-Schicht über den größten $\Gamma_{0}$-Wert und die Temperaturentwicklung der Linienbreite der Breathing Mode zeigt den steilsten Anstieg. Die LCMO/LAO (111)-Schicht weist hingegen den kleinsten $\Gamma_{0}$-Wert auf und die Temperaturentwicklung der Linienbreite ist flacher. Resultierend ergibt sich eine Divergenz in der Linienbreite der Breathing Mode der Schichten für hohe Temperaturen. Der $\gamma$-Wert ist umgekehrt für große Fehlanpassungen zwischen Bulk-LCMO und Substrat erhöht. Damit führt die Relaxation der LCMO/LAO (111)-Schicht und die ungleichmäßige Verspannung der LCMO/MgO (100)-Schicht (Abschnitt 4.1.3) zu einem großen $\gamma$-Wert. Das Minimum $\Gamma_{0}+\gamma \approx 18,82 \mathrm{~cm}^{-1}$ wird für die LCMO $/ \mathrm{Al}_{2} \mathrm{O}_{3}$ (0001)-Schicht bei einer moderaten, aber gleichmäßigen Verspannung erreicht. Nach der Gleichung (3.3.13) entspricht dieses einer maximalen Lebensdauer $\tau \approx 0,28$ ps der $A_{g}$ Breathing Mode bei $T=0 \mathrm{~K}$. Diese nimmt anschließend mit steigender Temperatur aufgrund der anharmonischer Beiträge ab, ist jedoch zusätzlich durch den temperaturunabhängigen Anteil an der Linienbreite beeinflusst. Somit ist die Linienbreite und damit die Lebensdauer der Breathing Mode der LCMO-Schichten neben der Temperatur $T$ direkt von der chemisch-kristallinen Ordnung und dem jeweiligen Verspannungszustand innerhalb der Schicht bestimmt. 
Um nun noch die Temperaturentwicklung der Position $\omega(T)$ und der Linienbreite $\Gamma(T)$ $\operatorname{der} A_{g}$ Breathing Mode in Abhängigkeit der B-Platz-Ordnung zu untersuchen, sind diese für die zwei bekannten $\mathrm{La}_{2} \mathrm{CoMnO}_{6} / \mathrm{Al}_{2} \mathrm{O}_{3}$ (0001)-Schichten mit B-Platz-Ordnung und B-Platz-Teilordnung in den Abbildungen 4.1.30 (a) und (b) für einen Vergleich dargestellt.

(a)

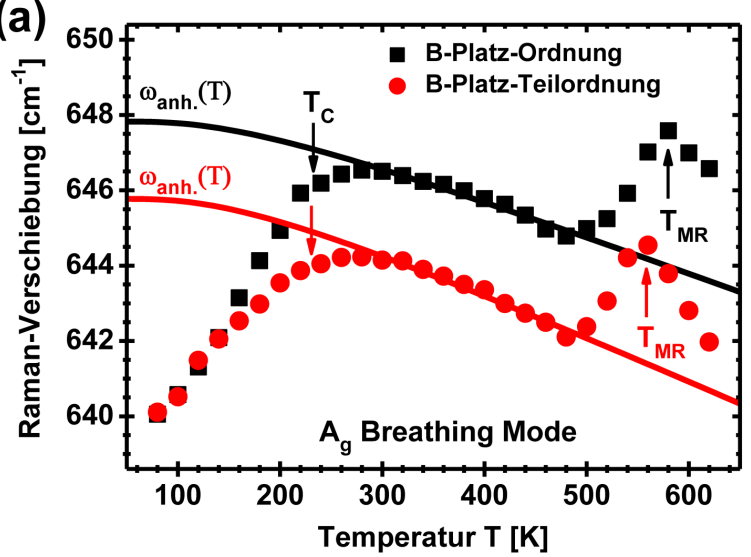

(c)

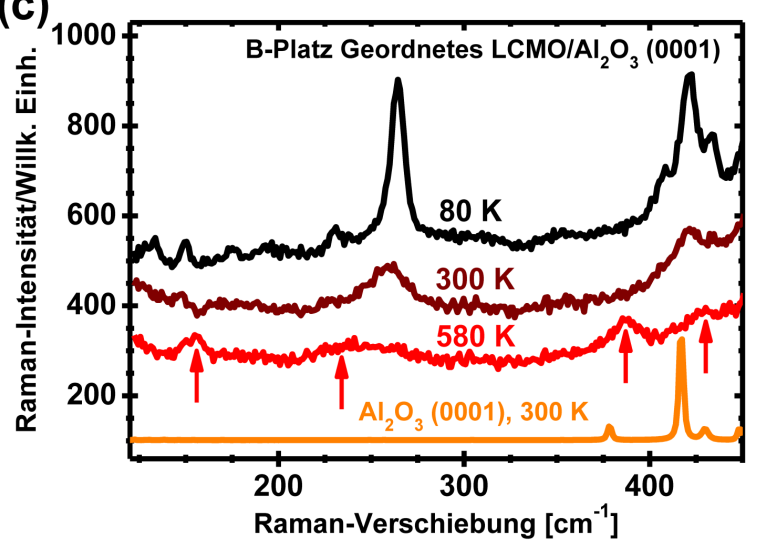

(b)

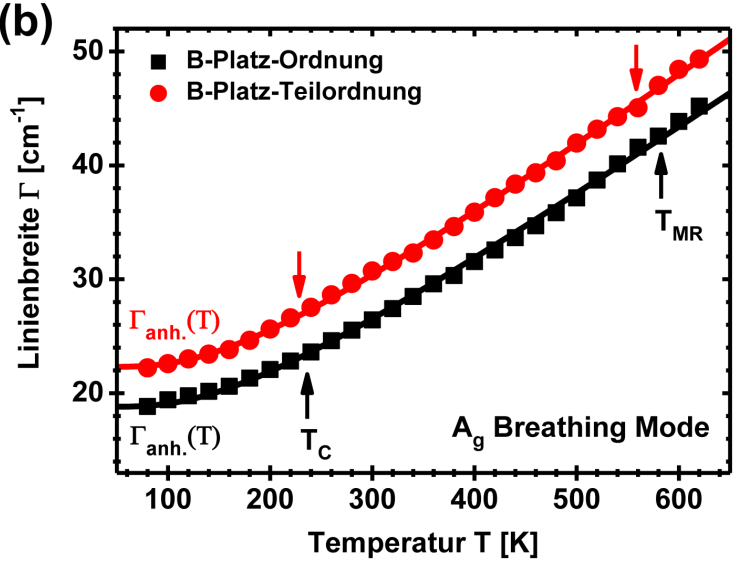

(d)

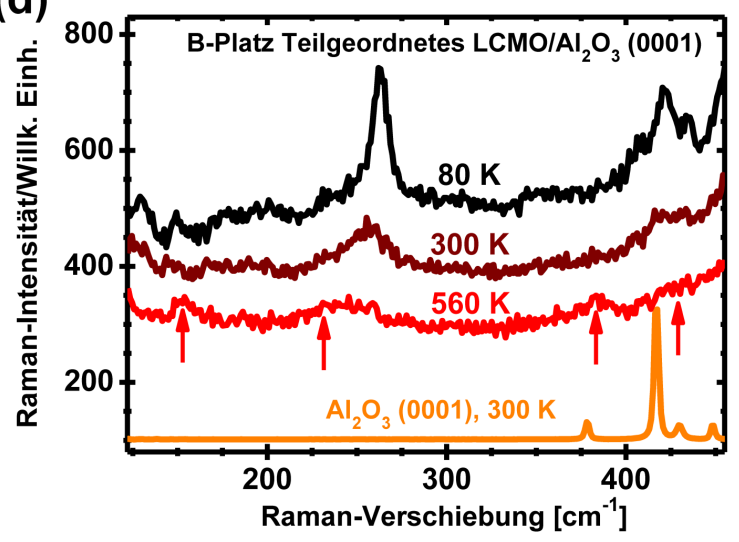

Abbildung 4.1.30: Temperaturentwicklung der Position (a) und der Linienbreite (b) der $A_{g}$ Breathing Mode der zwei LCMO $/ \mathrm{Al}_{2} \mathrm{O}_{3}$ (0001)-Schichten mit einer unterschiedlichen B-Platz-Ordnung im Vergleich. Die Kurven zeigen den anharmonischen Modellverlauf. Zusätzlich sind unpolarisierte Raman-Spektren der geordneten (c) und teilgeordneten LCMO-Schicht (d) für verschiedene Temperaturen im Bereich kleiner Wellenzahlen zusammen mit dem Spektrum eines $\mathrm{Al}_{2} \mathrm{O}_{3}$ (0001)-Substrats dargestellt. Die roten Pfeile kennzeichnen neu erscheinende Raman-Moden in der R $\overline{3}$-Phase.

Die experimentellen Ergebnisse werden wie zuvor mit der Modenposition $\omega_{\text {anh. }}(T)$ und der Linienbreite $\Gamma_{\text {anh. }}(T)$ nach dem anharmonischen Modell verglichen. Für beide LCMOSchichten ergeben sich in der Temperaturabhängigkeit der Position der $A_{g}$ Breathing Mode zwei signifikante Abweichungen vom anharmonischen Modell, die charakteristisch für alle in dieser Arbeit untersuchten LCMO-Schichten sind. So zeigt sich für die beiden Schichten ein starkes Erweichen der Breathing Mode für Temperaturen $T<T_{C}$ in der ferromagnetischen Phase und darüber hinaus ein Erhärten der Mode für Temperaturen $T>500 \mathrm{~K}$ mit einem Maximum bei $T_{M R}=580 \mathrm{~K}$ (B-Platz-Ordnung) und $T_{M R}=560 \mathrm{~K}$ (B-Platz-Teilordnung). Die Hochtemperaturanomalie kann dabei wie bereits diskutiert auf 


\section{Auswertung und Ergebnisse}

einen strukturellen Phasenübergang der LCMO-Schichten von der monoklinen P12/n1Struktur in eine rhomboedrische R⿳亠丷⿵-Struktur zurückgeführt werden 215, 238, 239. Die B-Platz-Ordnung besitzt auf diesen Übergang dabei kaum einen Einfluss, wie anhand des ähnlichen Verlaufs der Position der $A_{g}$ Breathing Mode der beiden LCMO-Schichten in diesem Temperaturbereich ersichtlich wird. Die leicht reduzierte Übergangstemperatur für die teilgeordnete LCMO-Schicht ist vielmehr durch die stärkere Schichtverspannung gegenüber der geordneten LCMO-Schicht begründet (Abschnitt 4.1.3. out-of-plane Gitterkonstanten $c_{\text {teilgeord. }}=3,873 \AA$ und $c_{\text {geord. }}=3,878 \AA$ gegenüber $c_{\mathrm{Bulk}}=3,887 \AA[34)$. Dieses lässt sich auch an der Position der $A_{g}$ Breathing Mode feststellen. So weist diese für die teilgeordnete Schicht für Temperaturen $T \geq T_{C}$ aufgrund der stärkeren in-plane Zugspannung eine etwa $2 \mathrm{~cm}^{-1}$ kleinere Wellenzahl gegenüber der geordneten LCMO-Schicht auf. Dieses Verhalten ist vergleichbar zu den LCMO-Schichten in den Abbildungen 4.1.26 (a)-(d), für die auch eine Korrelation zwischen der Schichtverspannung und der Übergangstemperatur $T_{\mathrm{MR}}$ mit einer Entwicklung hin zu kleineren Temperaturen bei einer Zunahme der Verspannung sowohl für eine in-plane Druckspannung als auch Zugspannung zu beobachten ist. Die Breite des Übergangs beträgt dabei für beide LCMO/ $\mathrm{Al}_{2} \mathrm{O}_{3}$ (0001)-Schichten vergleichbare $\approx 100 \mathrm{~K}$, bevor sich der Verhalten der Modenposition wieder dem anharmonischen Verlauf annähert, und ist damit nur auf die Koexistenz beider Phasen in diesen Temperaturbereich und auf den diffusen Übergangscharakter 215,238 zurückzuführen.

Ein Nachweis kann wie bei den anderen LCMO-Schichten mithilfe von Raman-Spektren bei kleinen Wellenzahlen erfolgen. Diese sind für die beiden LCMO/ $\mathrm{Al}_{2} \mathrm{O}_{3}$ (0001)-Schichten für $T=80 \mathrm{~K}, T=300 \mathrm{~K}$ und $T=T_{\mathrm{MR}}$ mit dem Spektrum eines $\mathrm{Al}_{2} \mathrm{O}_{3}$ (0001)-Substrats bei $T=300 \mathrm{~K}$ in den Abbildungen 4.1.30 (c) und (d) dargestellt. Hierbei lassen sich bei beiden Schichten für $T=T_{\mathrm{MR}}$ vier neue Raman-Moden bei $155 \mathrm{~cm}^{-1}, 235 \mathrm{~cm}^{-1}, 388 \mathrm{~cm}^{-1}$ und $430 \mathrm{~cm}^{-1}$ beobachten, die anhand von LDC-Berechnungen Moden der rhomboedrischen R⿳亠丷厂̈-Struktur mit $A_{g^{-}}$und $E_{g^{-}}$Symmetrie zugeordnet werden können 33, 215. Für die Linienbreite $\Gamma(T)$ der $A_{g}$ Breathing Mode wiederum zeigt sich eine vergleichbare Entwicklung mit der Temperatur $T$, die für beide $\mathrm{LCMO} / \mathrm{Al}_{2} \mathrm{O}_{3}$ (0001)-Schichten ohne Abweichungen dem anharmonischen Verlauf folgt. Der Grund hierfür ist, dass die Breathing Mode auch für die teilgeordnete LCMO-Schicht eine $A_{g}$-Symmetrie aufweist und ihren Schwingungscharakter in der ferromagnetischen Phase und in der rhomboedrischen Hochtemperaturphase beibehält 33, 215. Auffällig ist jedoch die um etwa $3,5 \mathrm{~cm}^{-1}$ kleinere Linienbreite der $A_{g}$ Breathing Mode der geordneten gegenüber der teilgeordnete LCMO-Schicht. Da diese durch die Kristallinität und die chemische Ordnung bestimmt ist [120, 123, 128], lässt sich anhand der Linienbreiten der LCMO-Schichten der Unterschied der B-Platz-Ordnung qualitativ beobachten, wobei die Breite für eine hohe Ordnung geringer ist 40,216. Das stärkere Erweichen der $A_{g}$ Breathing Mode für $T<T_{C}$ für die geordnete LCMO-Schicht (Abbildung 4.1.30 (a)) wird im nachfolgenden Abschnitt gesondert diskutiert. 


\subsubsection{Spin-Phonon-Kopplung}

Das anomale Erweichen der $A_{g}$ Breathing Mode bei den temperaturabhängigen RamanMessungen für Temperaturen $T<T_{C}$ in der ferromagnetischen Phase der DoppelperowskitSchichten ist hierbei auf eine Phonon-Renormierung induziert durch die magnetische Ordnung zurückzuführen, die auch bei anderen ferromagnetischen und antiferromagnetischen Materialsystemen wie Manganaten [241,242] und Cobaltaten [243] beobachtet wird. Resultierend ergibt sich eine Spin-Phonon-Kopplung zwischen der magnetischen Ordnung und dem Kristallgitter. Wird nur eine Nächste-Nachbarn-Wechselwirkung berücksichtigt, so ist die Phonon-Renormierung $\Delta \omega(T)=\omega(T)-\omega_{\text {anh. }}(T)$ proportional zur Spin-Spin-Korrelationsfunktion $\left\langle S_{i} \cdot S_{j}\right\rangle$ der Spins auf dem i-ten und j-ten Platz im Kristallgitter, welche in der Molekularfeldnäherung proportional zum Quadrat der normierten Magnetisierung $M^{2}(T) / M_{\text {max. }}^{2}$ pro magnetischem Kation bei der Temperatur $T$ ist 33 241 243. Da für die B-Platz geordneten $\mathrm{A}_{2} \mathrm{CoMnO}_{6}$ - und $\mathrm{A}_{2} \mathrm{NiMnO}_{6}$-Doppelperowskite die B-Platz-Kationen $\mathrm{Co}^{2+}\left(3 \mathrm{~d}^{7}, \mathrm{t}_{2 \mathrm{~g}}^{5} \mathrm{e}_{\mathrm{g}}^{2}, S=3 / 2\right)$ oder $\mathrm{Ni}^{2+}\left(3 \mathrm{~d}^{8}, \mathrm{t}_{2 \mathrm{~g}}^{6} \mathrm{e}_{\mathrm{g}}^{2}, S=1\right)$ und $\mathrm{Mn}^{4+}\left(3 \mathrm{~d}^{3}, \mathrm{t}_{2 \mathrm{~g}}^{3} \mathrm{e}_{\mathrm{g}}^{0}, S=3 / 2\right)$ in der Ebene von vier nächsten Nachbarn des anderen B-Kations umgeben sind, folgt für die Phonon-Renormierung $\Delta \omega(T)$ im Fall der B-Platz geordneten Doppelperowskite $241-243]$ :

$$
\Delta \omega(T)=\omega(t)-\omega_{\text {anh. }}(T) \approx-\lambda\left\langle S_{i} \cdot S_{j}\right\rangle \approx-4 \lambda \frac{M^{2}(T)}{M_{\max .}^{2}} .
$$

Hierbei stellt $\lambda$ die Spin-Phonon-Kopplungsstärke dar, während $M_{\max }$ die Sättigungsmagnetisierung der $M(T)$-Kurve der Probe beschreibt. Gemäß der Gleichung 4.1.2 skaliert die Abweichung $\Delta \omega(T)$ von der anharmonischen Temperaturentwicklung der Modenposition im Fall einer Spin-Phonon-Kopplung linear mit dem Quadrat der normierten Magnetisierung $M^{2}(T) / M_{\text {max. }}^{2}$. Experimentell lässt sich dieses überprüfen, indem $\Delta \omega(T)$ und $M^{2}(T) / M_{\max }^{2}$. übereinander gelegt und für $T<T_{C}$ miteinander verglichen werden. Dieses ist für die $A_{g}$ Breathing Mode der $\mathrm{A}_{2} \mathrm{CoMnO}_{6}$-Doppelperowskit-Schichtserie $(\mathrm{A}=\mathrm{La}, \mathrm{Pr}, \mathrm{Sm}, \mathrm{Gd})$ auf STO (111) mit einer Schichtdicke von $d \approx 90-100 \mathrm{~nm}$ in den Abbildungen 4.1.31 (a)-(d) dargestellt. Die Magnetisierungskurven sind mit dem SQUID beim Abkühlen mit einer magnetischen Feldstärke von $H=1 \mathrm{kOe}$ gemessen worden. Für alle vier Schichten ergibt sich dabei eine exzellente Übereinstimmung in der Entwicklung von $\Delta \omega(T)$ der $A_{g}$ Breathing Mode und $M^{2}(T) / M_{\text {max. }}^{2}$ mit der Temperatur für $T<T_{C}$. Insbesondere zeigt sich, dass das Erweichen der Breathing Mode sehr stark mit der Übergangstemperatur $T_{C}$ korreliert ist, mit dem Eintreten in die ferromagnetische Ordnung initiiert und mit weiter sinkender Temperatur signifikant verstärkt wird. Somit weisen alle vier Schichten eine signifikante Spin-Phonon-Kopplung in ihrer ferromagnetischen Phase auf. Die Schwankungen für $\Delta \omega(T)$ oberhalb von $T_{C}$ lassen sich auf die Anpassung des anharmonischen Modells an die experimentell gemessenen Positionen der $A_{g}$ Breathing Mode in diesem Temperaturbereich und instrumentelle Einflüsse des Messaufbaus zurückführen. 

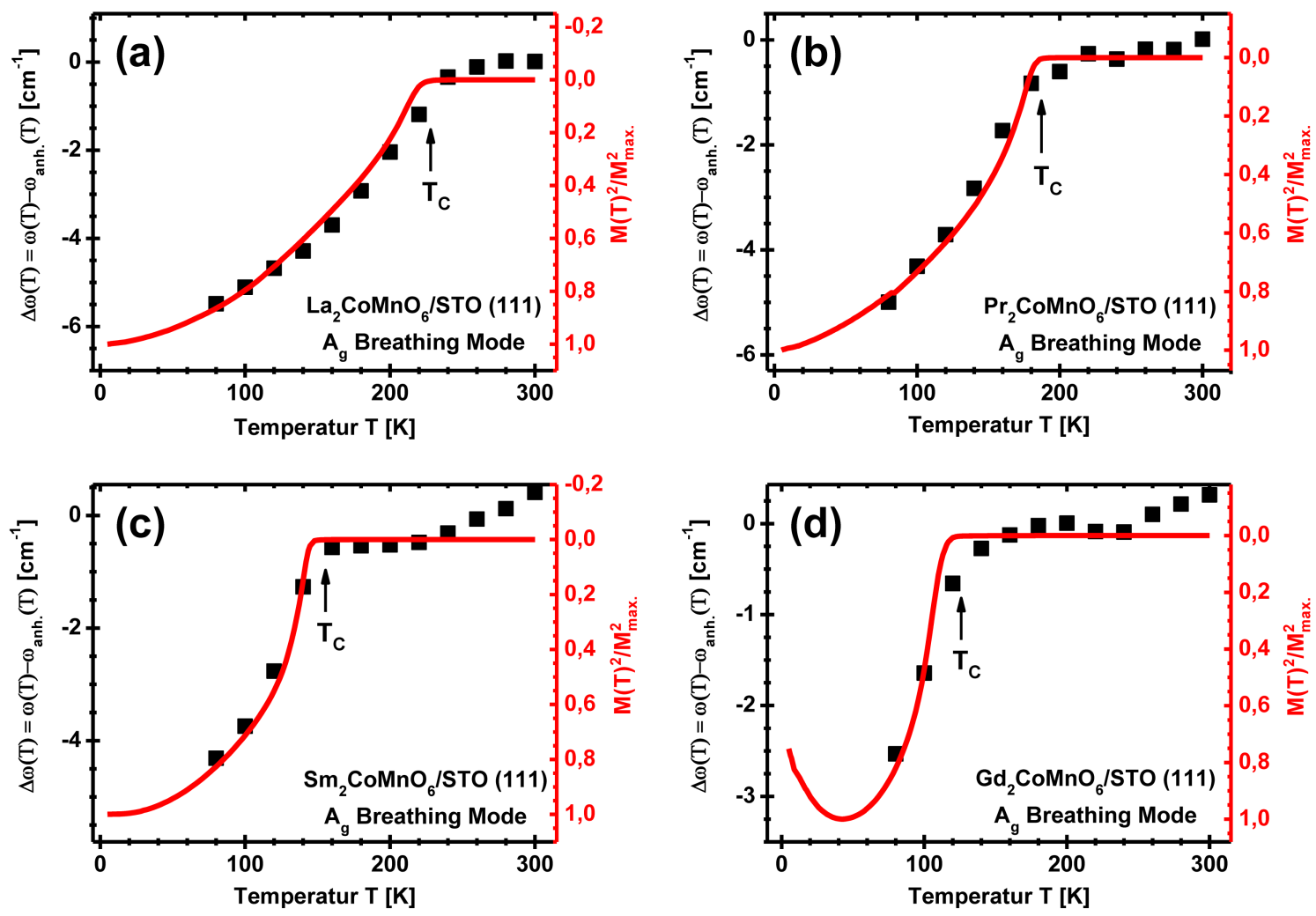

Abbildung 4.1.31: Entwicklung der Abweichung $\Delta \omega(T)$ der Position der $A_{g}$ Breathing Mode vom anharmonischen Modell und des Quadrats der Magnetisierung $M^{2}(T) / M_{\text {max. }}^{2}$ mit der Temperatur für die Doppelperowskite LCMO (a), PCMO (b), SCMO (c) und GCMO (d) auf STO (111) im Vergleich.

Durch Auftragung der experimentell gemessenen Phonon-Renormierung $\Delta \omega(T)$ in Abhängigkeit des Quadrats der normierten Magnetisierung $M^{2}(T) / M_{\max }^{2}$. ergibt sich somit eine lineare Abhängigkeit, die für die $A_{g}$ Breathing Mode der vier $\mathrm{A}_{2} \mathrm{CoMnO}_{6}$-DoppelperowskitSchichten auf STO (111) nachfolgend in der Abbildung 4.1 .32 dargestellt ist.

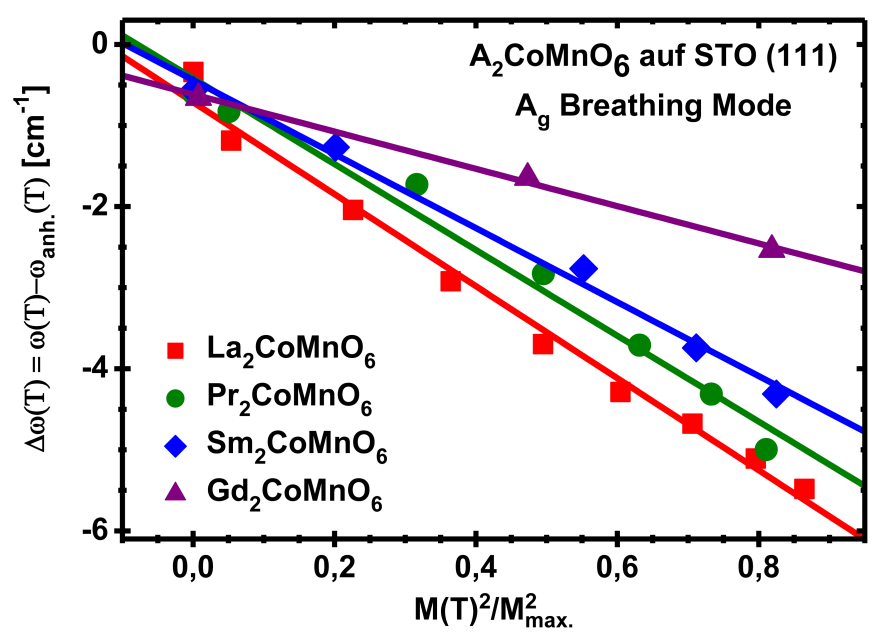

Abbildung 4.1.32: Lineare Abhängigkeit der Phonon-Renormierung $\Delta \omega(T)$ der $A_{g}$ Breathing Mode von der Magnetisierung $M^{2}(T) / M_{\text {max. }}^{2}$ für die $\mathrm{A}_{2} \mathrm{CoMnO}_{6^{-}}$ Doppelperowskit-Schichten (A = La, Pr, Sm, Gd) auf STO (111). 
Die Spin-Phonon-Kopplungsstärke $\lambda$ ergibt sich dabei nach der Gleichung 4.1.2 direkt aus der Steigung $a=4 \lambda$ des linearen Zusammenhangs von $\Delta \omega(T)$ und $M^{2}(T) / M_{\text {max. }}^{2}$. Die Werte für $\lambda$ sind für die vier $\mathrm{A}_{2} \mathrm{CoMnO}_{6} / \mathrm{STO}$ (111)-Schichten zusammen mit dem jeweiligen A-Kationenradius 222] in der Tabelle 4.1.6 dargestellt. Es ergibt sich eine systematische Abnahme der Spin-Phonon-Kopplungsstärke $\lambda$ mit der Verringerung des A-Kationenradius.

\begin{tabular}{|c|l|l|l|c|}
\hline Auf STO (111) & LCMO & PCMO & SCMO & GCMO \\
\hline \hline$\lambda\left[\mathrm{cm}^{-1}\right]$ & $1,42(6)$ & $1,32(8)$ & $1,14(6)$ & $0,58(3)$ \\
\hline$r_{\mathrm{A}^{3+}}[\mathrm{pm}](\mathrm{KZ}=8[222$ & 116 & 112,6 & 107,9 & 105,3 \\
\hline
\end{tabular}

Tabelle 4.1.6: Die aus der Steigung $a=4 \lambda$ des linearen Zusammenhangs von $\Delta \omega(T)$ und $M^{2}(T) / M_{\text {max. }}^{2}$ bestimmte Spin-Phonon-Kopplungsstärke $\lambda$ der $\mathrm{A}_{2} \mathrm{CoMnO}_{6}{ }^{-}$ Schichten auf STO (111), zusammen mit dem jeweiligen A-Kationenradius.

In gleicher Weise wird nun das Erweichen der $A_{g}$ Breathing Mode der $\mathrm{A}_{2} \mathrm{NiMnO}_{6}$-Doppelperowskit-Schichtserie (A = La, Pr, Nd, Sm, Gd) auf STO (111) mit einer Schichtdicke von jeweils $d \approx 90-100 \mathrm{~nm}$ untersucht. Der übereinander gelegte Vergleich von der Abweichung der Position $\Delta \omega(T)$ vom anharmonischen Modell und $M^{2}(T) / M_{\text {max. }}^{2}$ in Abhängigkeit der Temperatur $T$ ist für die fünf Schichten in den Abbildungen 4.1 .33 (a)-(e) dargestellt.
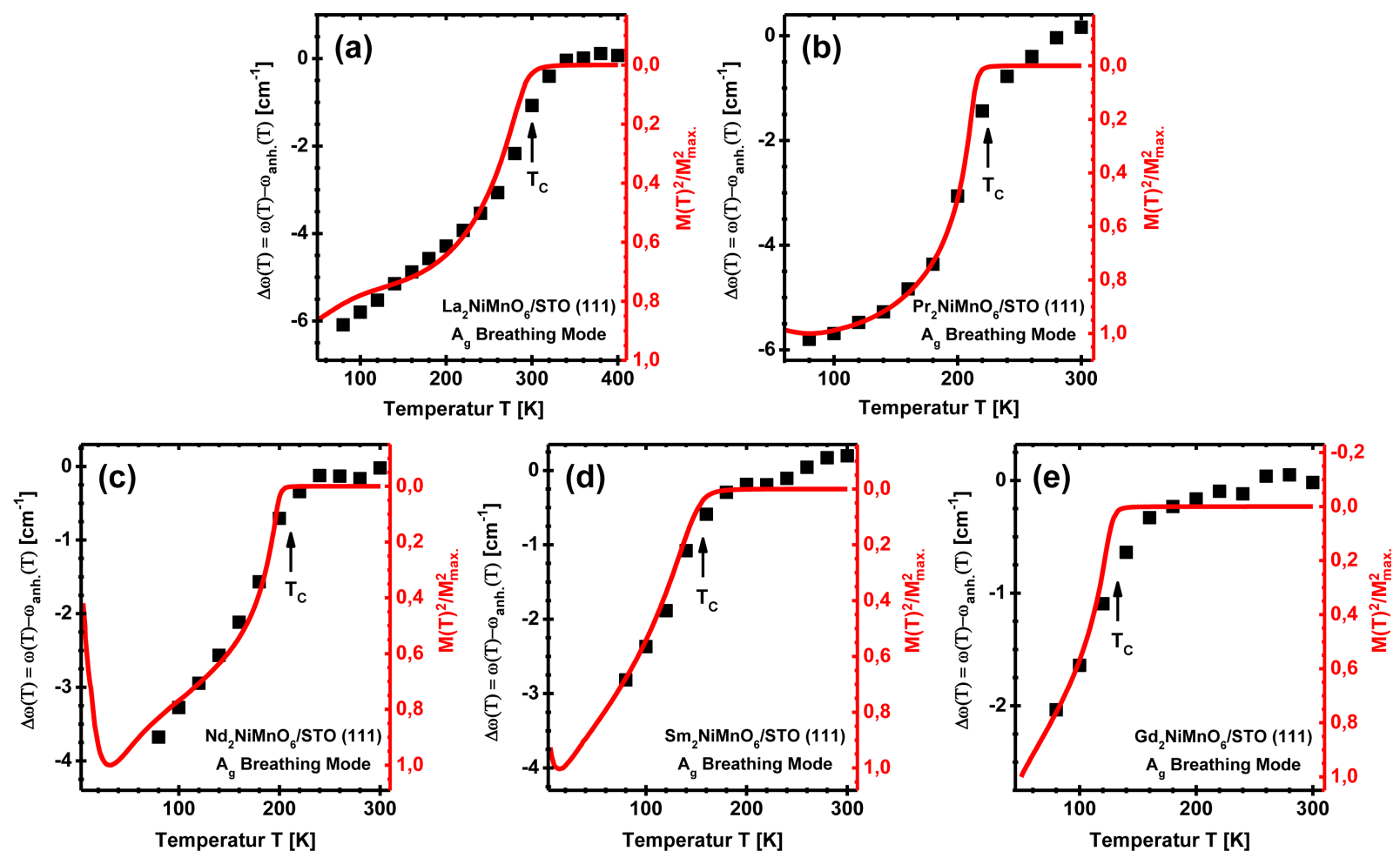

Abbildung 4.1.33: Entwicklung der Positionsabweichung $\Delta \omega(T)$ der $A_{g}$ Breathing Mode vom anharmonischen Modell und der Magnetisierung $M^{2}(T) / M_{\text {max. }}^{2}$ mit der Temperatur für die Doppelperowskite LNMO (a), PNMO (b), NNMO (c), SNMO (d) und GNMO (e) auf STO (111) im Vergleich. 
Die Magnetisierungskurven sind wieder mit dem SQUID beim Abkühlen mit einer magnetischen Feldstärke von $H=1$ kOe gemessen worden. Für die fünf $\mathrm{A}_{2} \mathrm{NiMnO}_{6}$-Schichten auf STO (111) ergibt sich erneut eine exzellente Übereinstimmung in der Entwicklung von $\Delta \omega(T)$ der $A_{g}$ Breathing Mode und $M^{2}(T) / M_{\max }^{2}$ mit der Temperatur für $T<T_{C}$. Somit zeigen alle fünf Schichten eine signifikante Spin-Phonon-Kopplung in ihrer ferromagnetischen Phase. Anzumerken sind einige Besonderheiten bezüglich der Magnetisierungskurven aus dem Abschnitt 4.1.2. So ist die Magnetisierung der GNMO-Schicht aufgrund des starken paramagnetischen Beitrags durch das $\mathrm{Gd}^{3+}$-Kation für $T<50 \mathrm{~K} 38$, 230 für eine sinnvolle $M^{2}(T) / M_{\text {max. }}^{2}$-Skalierung nur bis zu einer Temperatur $T=50 \mathrm{~K}$ dargestellt. Für die GCMO-Schicht ist dieses nicht notwendig, da eine antiferromagnetische Kopplung des $\mathrm{Gd}^{3+}$-Kations mit dem Co/Mn-B-Platz-Gitter für $T<50 \mathrm{~K} 228$ zu beobachten ist. Bei der $\mathrm{Nd}_{2} \mathrm{NiMnO}_{6}$ und der $\mathrm{Sm}_{2} \mathrm{NiMnO}_{6}$-Schicht nimmt die Magnetisierung für $T<30 \mathrm{~K}$ wiederum durch eine antiferromagnetische Spin-Bahn-Kopplung der $\mathrm{Nd}^{3+}$ - und $\mathrm{Sm}^{3+}$-Kationen mit dem B-Platz-Netzwerk wieder ab 38. Da in der Kühlzelle nur Temperaturen bis hinunter zu $T=80 \mathrm{~K}$ erreicht werden können, sind die Raman-Messungen von der konkurrierenden Koexistenz mit dem ferromagnetischen Hauptbeitrag durch den $\mathrm{Ni}^{2+}-\mathrm{O}^{2-}-\mathrm{Mn}^{4+}-$ Superaustausch nicht (SMNO) oder nur minimal (NNMO) beeinflusst. Das anomale Erweichen der $A_{g}$ Breathing Mode lässt sich somit auf das Auftreten einer Spin-Phonon-Kopplung zurückführen. Die Phonon-Renormierung $\Delta \omega(T)$ in Abhängigkeit von $M^{2}(T) / M_{\max }^{2}$ und die resultierende lineare Abhängigkeit sind für die $A_{g}$ Breathing Mode der fünf $\mathrm{A}_{2} \mathrm{NiMnO}_{6} / \mathrm{STO}$ (111)-Schichten in der Abbildung 4.1 .32 dargestellt.

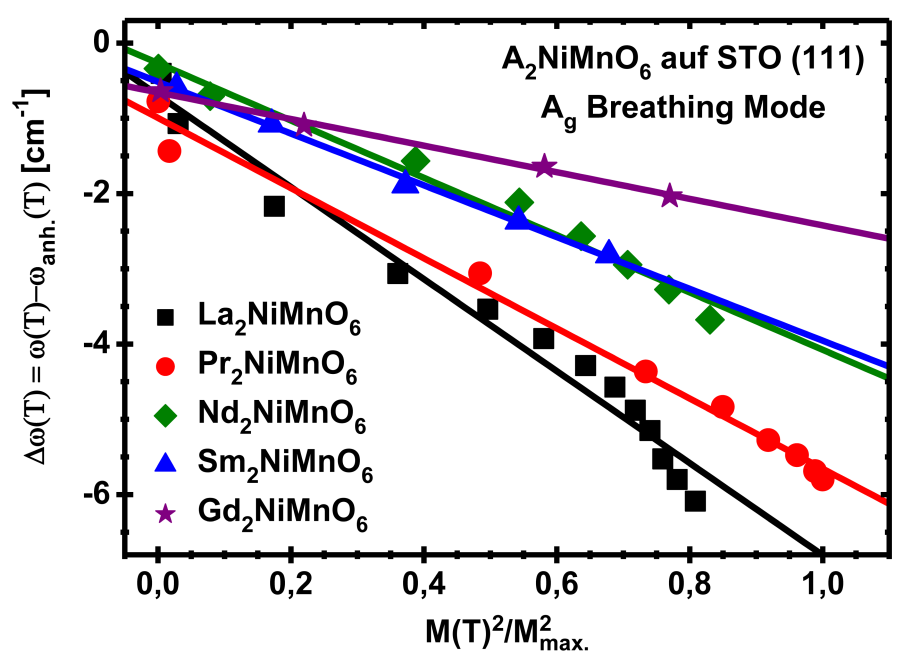

Abbildung 4.1.34: Lineare Abhängigkeit der Phonon-Renormierung $\Delta \omega(T)$ der $A_{g}$ Breathing Mode von der Magnetisierung $M^{2}(T) / M_{\text {max. }}^{2}$ für die $\mathrm{A}_{2} \mathrm{NiMnO}_{6}$ Doppelperowskit-Schichten (A = La, Pr, Nd, Sm, Gd) auf STO (111).

Die Werte für $\lambda$ sind für die fünf $\mathrm{A}_{2} \mathrm{NiMnO}_{6} / \mathrm{STO}$ (111)-Schichten zusammen mit dem jeweiligen A-Kationenradius 222 in der nachfolgenden Tabelle 4.1.7 dargestellt. Es ergibt sich erneut eine systematische Abnahme der Spin-Phonon-Kopplungsstärke $\lambda$ mit der Verringerung des A-Kationenradius wie zuvor für die $\mathrm{A}_{2} \mathrm{CoMnO}_{6} / \mathrm{STO}$ (111)-Schichten. 


\subsection{B-Platz geordnete $\mathrm{A}_{2} \mathrm{BMnO}_{6}$-Doppelperowskite}

\begin{tabular}{|c|c|c|c|c|c|}
\hline Auf STO (111) & LNMO & PNMO & NNMO & SNMO & GNMO \\
\hline \hline$\lambda\left[\mathrm{cm}^{-1}\right]$ & $1,53(8)$ & $1,17(4)$ & $0,95(5)$ & $0,86(3)$ & $0,44(2)$ \\
\hline$r_{\mathrm{A}^{3+}}[\mathrm{pm}](\mathrm{KZ}=8[222])$ & 116 & 112,6 & 110,9 & 107,9 & 105,3 \\
\hline
\end{tabular}

Tabelle 4.1.7: Die aus der Steigung $a=4 \lambda$ des linearen Zusammenhangs von $\Delta \omega(T)$ und $M^{2}(T) / M_{\max }^{2}$ bestimmte Spin-Phonon-Kopplungsstärke $\lambda$ der $\mathrm{A}_{2} \mathrm{NiMnO}_{6}$ Schichten auf STO (111), zusammen mit dem jeweiligen A-Kationenradius.

Eine Übersicht der Spin-Phonon-Kopplungsstärke $\lambda$ der $\mathrm{A}_{2} \mathrm{CoMnO}_{6^{-}}$und $\mathrm{A}_{2} \mathrm{NiMnO}_{6^{-}}$ Doppelperowskit-Schichten auf STO (111) in Abhängigkeit des A-Kationenradius ist in der Abbildung 4.1.35 dargestellt. Beide Doppelperowskit-Reihen zeigen ein vergleichbares Verhalten mit einer systematischen Abnahme von $\lambda$ mit der Verringerung des AKationenradius. Dieses entspricht Messungen an Bulk- $\mathrm{A}_{2} \mathrm{CoMnO}_{6}$-Doppelperowskiten 219 und $\mathrm{A}_{2} \mathrm{CoMnO}_{6} / \mathrm{LAO}$ (001)-Schichten [220], für die ebenfalls eine systematische Abnahme von $\lambda$ bei einer Verringerung der A-Kationengröße nachgewiesen werden konnte und widerspricht Messungen an Bulk- $\mathrm{A}_{2} \mathrm{NiMnO}_{6}$-Doppelperowskiten [231, für die eine Unabhängigkeit der Spin-Phonon-Kopplungsstärke vom A-Kationenradius beobachtet wurde.

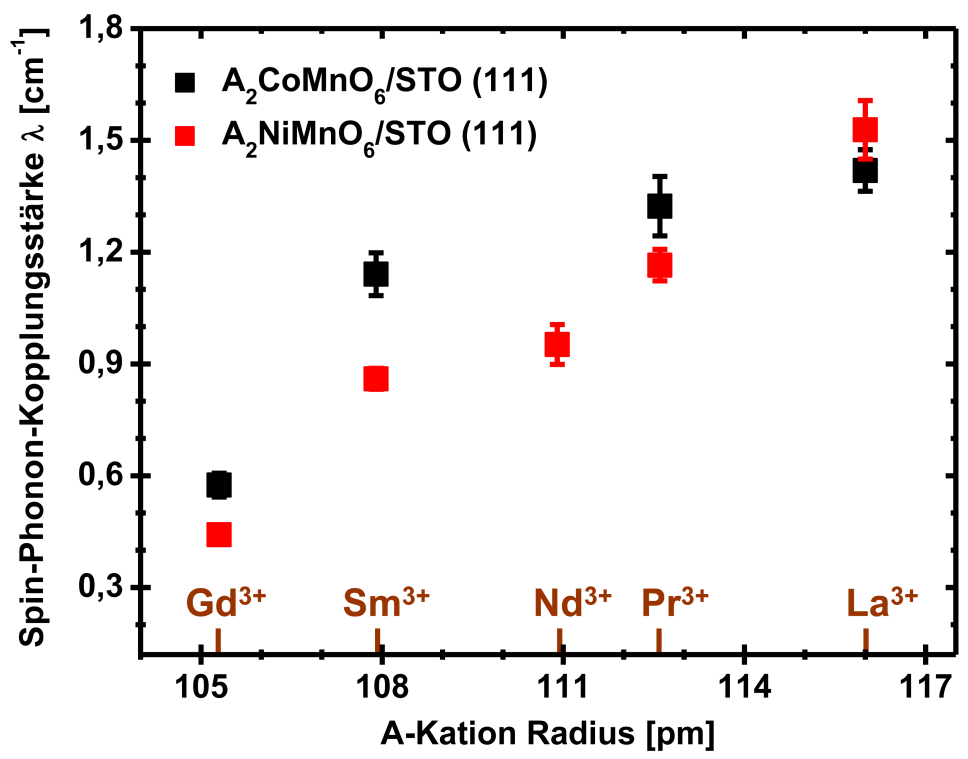

Abbildung 4.1.35: Aus der Phonon-Renormierung der $A_{g}$ Breathing Mode in der ferromagnetischen Phase der B-Platz geordneten $\mathrm{A}_{2} \mathrm{CoMnO}_{6} / \mathrm{STO}$ (111)und $\mathrm{A}_{2} \mathrm{NiMnO}_{6} / \mathrm{STO}$ (111)-Doppelperowskit-Schichten bestimmte SpinPhonon-Kopplungsstärke $\lambda$ in Abhängigkeit des A-Kationenradius.

Die Abnahme der Kopplungsstärke $\lambda$ mit der Reduzierung des A-Kationenradius wird dabei auf die Verringerung des $\mathrm{Co}^{2+} / \mathrm{Ni}^{2+}-\mathrm{O}^{2-}-\mathrm{Mn}^{4+}$-Bindungswinkels und auf eine Vergrößerung der Bindungslänge zurückgeführt, wodurch nicht nur die magnetische Wechselwirkung (Abschnitt 4.1.2) verringert, sondern auch der temperaturabhängige Einfluss des Superaustauschs auf die Kraftkonstanten des Gitters und auf die damit einhergehende Spin-PhononKopplung abgeschwächt wird 219,220]. Ein vergleichbares Verhalten zeigt sich auch für 
$\mathrm{RMnO}_{3}$-Einkristalle [242, 244] bei Verringerung des R-Kationenradius der Seltenen Erde. Mit Ausnahme von $\mathrm{A}=\mathrm{La}$ weist die $\mathrm{A}_{2} \mathrm{CoMnO}_{6}$-Reihe etwas größere Werte für $\lambda$ in der Abbildung 4.1.35 auf, obwohl die Curie-Temperaturen $T_{C}$ für die $\mathrm{A}_{2} \mathrm{NiMnO}_{6}$-Reihe höher sind. Ein Wert für NCMO fehlt mangels der Verfügbarkeit der Schicht zum Zeitpunkt der Aufnahme der Messreihe. Offenbar müssen die unterschiedlichen Spin-Beiträge von $\mathrm{Co}^{2+}$ und $\mathrm{Ni}^{2+}$ sowie die strukturellen Eigenschaften der $\mathrm{A}_{2} \mathrm{CoMnO}_{6^{-}}$und $\mathrm{A}_{2} \mathrm{NiMnO}_{6^{-}}$ Schichten mit einbezogen werden. Die Literatur finden sich nur wenige Werte für einen Vergleich. Die aus der Phonon-Renormierung der $A_{g}$ Breathing Mode bestimmten SpinPhonon-Kopplungsstärken $\lambda$ für PCMO und SCMO auf STO (111) (Tabelle 4.1.6) passen sehr gut zu den jeweiligen Werten von $\operatorname{PCMO}\left(\lambda=1,61 \mathrm{~cm}^{-1}\right)$ und $\operatorname{SCMO}\left(\lambda=1,16 \mathrm{~cm}^{-1}\right)$ auf LAO (001) 220], während der Wert für Bulk-PCMO $\left(\lambda=0,51 \mathrm{~cm}^{-1}\right)$ 219 deutlich kleiner ist. Der Unterschied zwischen Bulk-Probe und Schicht wird dabei auf die in-plane vom LAO (001)-Substrat induzierte Druckspannung zurückgeführt [220]. Da sich die Verspannung auf die B-Platz-Ordnung und die strukturelle Phase auswirkt 224,245,246], lässt sich ein Einfluss auch auf die Spin-Phonon-Kopplung ableiten. Um dieses zu untersuchen, ist in den Abbildungen 4.1.36 (a)-(d) die temperaturabhängige Entwicklung der PhononRenormierung $\Delta \omega(T)$ der $A_{g}$ Breathing Mode und der Magnetisierung $M^{2}(T) / M_{\text {max. von }}^{2}$ (111)-orientierten LCMO-Schichten auf verschiedenen Substraten dargestellt.
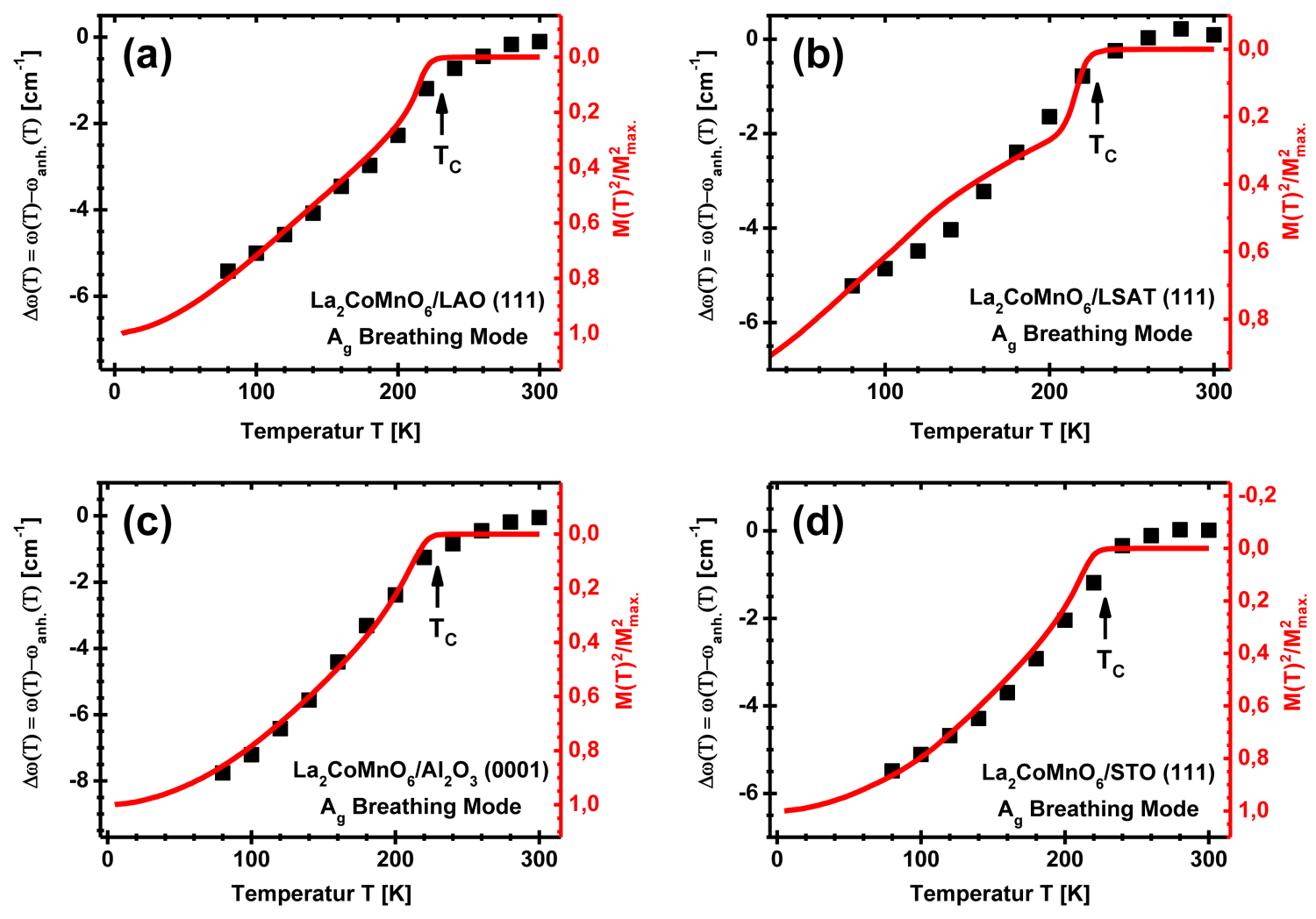

Abbildung 4.1.36: Entwicklung der Abweichung $\Delta \omega(T)$ der Position der $A_{g}$ Breathing Mode vom anharmonischen Modell und der Magnetisierung $M^{2}(T) / M_{\max }^{2}$ mit der Temperatur der $\mathrm{La}_{2} \mathrm{CoMnO}_{6}$-Schichten auf LAO (111) (a), LSAT (111) (b), $\mathrm{Al}_{2} \mathrm{O}_{3}$ (0001) (c), STO (111) (d) im Vergleich. 
Die vier LCMO-Schichten besitzen dabei alle eine Schichtdicke $d \approx 90-110 \mathrm{~nm}$. Die M(T)Kurven sind mit dem SQUID beim Abkühlen und mit Magnetfeld $H=1 \mathrm{kOe}$ gemessen worden. Für die LCMO-Schichten auf LAO (111), $\mathrm{Al}_{2} \mathrm{O}_{3}$ (0001) und STO (111) ergibt sich für die $A_{g}$ Breathing Mode eine exzellente Übereinstimmung der Entwicklung von $\Delta \omega(T)$ und $M^{2}(T) / M_{\text {max }}^{2}$ mit der Temperatur für $T<T_{C}$. Somit zeigen die drei Schichten eine signifikante Spin-Phonon-Kopplung in ihrer ferromagnetischen Phase. Für die LCMOSchicht auf LSAT (111) lässt sich ein vergleichbares Erweichen der $A_{g}$ Breathing Mode beobachten, der Verlauf von $M^{2}(T) / M_{\max }^{2}$. folgt der Phonon-Renormierung jedoch nur leicht abweichend. Eine Ursache ist die im Abschnitt 4.1.3 an der M(H)-Kurve diskutierte magnetische out-of-plane Anisotropie der LCMO-Schicht auf LSAT (111) aufgrund der vom Substrat induzierten Verspannung, die auch die in-plane M(T)-Kurve beeinflusst. Dieses Verhalten ist auch für (100)-Substrate bekannt, mit dem Unterschied, dass ein Wachstum von LCMO auf LSAT (100) zu einer magnetischen in-plane Anisotropie führt 237]. Anhand des zu den anderen LCMO-Schichten vergleichbaren Verlaufs von $\Delta \omega(T)$ kann eine stark reduzierte B-Platz-Ordnung ausgeschlossen werden. Somit ist auch für diese Schicht eine Spin-Phonon-Kopplung zu beobachten. Die Phonon-Renormierung $\Delta \omega(T)$ in Abhängigkeit von $M^{2}(T) / M_{\text {max. }}^{2}$ und die resultierende lineare Abhängigkeit sind für die $A_{g}$ Breathing Mode der vier $\mathrm{La}_{2} \mathrm{CoMnO}_{6}$-Schichten entsprechend in der Abbildung 4.1.37 dargestellt.

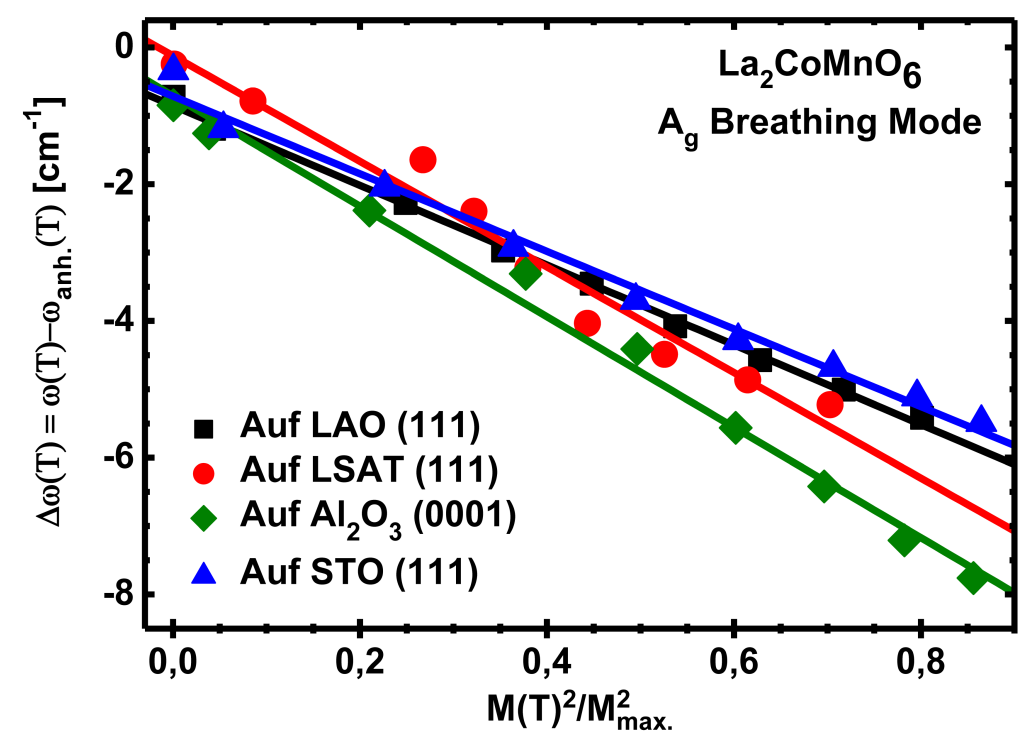

Abbildung 4.1.37: Lineare Abhängigkeit der Phonon-Renormierung $\Delta \omega(T)$ der $A_{g}$ Breathing Mode von der Magnetisierung $M^{2}(T) / M_{\text {max }}^{2}$ für die $\mathrm{La}_{2} \mathrm{CoMnO}_{6}$-Schichten auf LAO (111), LSAT (111), $\mathrm{Al}_{2} \mathrm{O}_{3}$ (0001) und STO (111).

Die jeweils aus der Steigung $a=4 \lambda$ bestimmte Spin-Phonon-Kopplungsstärke $\lambda$ für die vier (111)-orientierten $\mathrm{La}_{2} \mathrm{CoMnO}_{6}$-Schichten ist zusammen mit der Gitterkonstante $a$ des verwendeten Substrats 225] und der resultierenden in-plane Gitterfehlanpassung $f$ in Relation zum unverspannten Bulk-LCMO (mit der pseudokubischen Gitterkonstante $a_{\mathrm{pc}, \text { Bulk }} \approx b_{\mathrm{pc} \text {, Bulk }} \approx c_{\mathrm{pc} \text {, Bulk }}=3,887 \AA 34$ ) nachfolgend in der Tabelle 4.1 .8 aufgeführt. 
4 Auswertung und Ergebnisse

\begin{tabular}{|c|c|c|c|c|}
\hline LCMO auf & LAO (111) & LSAT (111) & $\mathrm{Al}_{2} \mathrm{O}_{3}(0001)$ & STO (111) \\
\hline \hline$\lambda\left[\mathrm{cm}^{-1}\right]$ & $1,46(3)$ & $1,93(13)$ & $2,02(7)$ & $1,42(6)$ \\
\hline$a[\AA]$ & 3,788 & 3,868 & 3,885 & 3,905 \\
\hline$f[\%]$ & $-2,55$ & $-0,49$ & $-0,05$ & $+0,46$ \\
\hline
\end{tabular}

Tabelle 4.1.8: Die aus der Steigung $a=4 \lambda$ des linearen Zusammenhangs von $\Delta \omega(T)$ und $M^{2}(T) / M_{\max }^{2}$. bestimmte Spin-Phonon-Kopplungsstärke $\lambda$ der (111)-orientierten $\mathrm{La}_{2} \mathrm{CoMnO}_{6}$-Schichten, zusammen mit der jeweiligen in-plane Gitterkonstante $a$ der Substrate 225] für das (111)-Wachstum von LCMO und die dazugehörige Gitterfehlanpassung $f$ zu unverspanntem Bulk-LCMO.

Für das hexagonale $\mathrm{Al}_{2} \mathrm{O}_{3}$ (0001)-Substrat ist hierbei die effektive in-plane Gitterkonstante

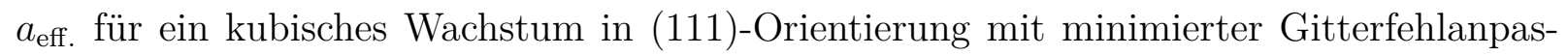
sung angegeben, die sich durch eine in-plane Rotation der Orientierung der LCMO-Schicht in Relation zum Substrat über $\sqrt{2} a_{\text {Saphir }} \approx \sqrt{3} a_{\mathrm{pc}}$ mit $a_{\text {Saphir }}=4,758 \AA 225$ ergibt. Für die LCMO-Schichten mit (111)-Orientierung zeigt sich eine starke Abhängigkeit der Spin-Phonon-Kopplungsstärke $\lambda$ vom Substrat und der resultierenden in-plane Gitterfehlanpassung $f$, die graphisch in der nachfolgenden Abbildung 4.1.38 dargestellt ist. Die blaue vertikale Linie kennzeichnet hierbei die Gitterkonstante $a_{\mathrm{pc}}$, Bulk von Bulk-LCMO.

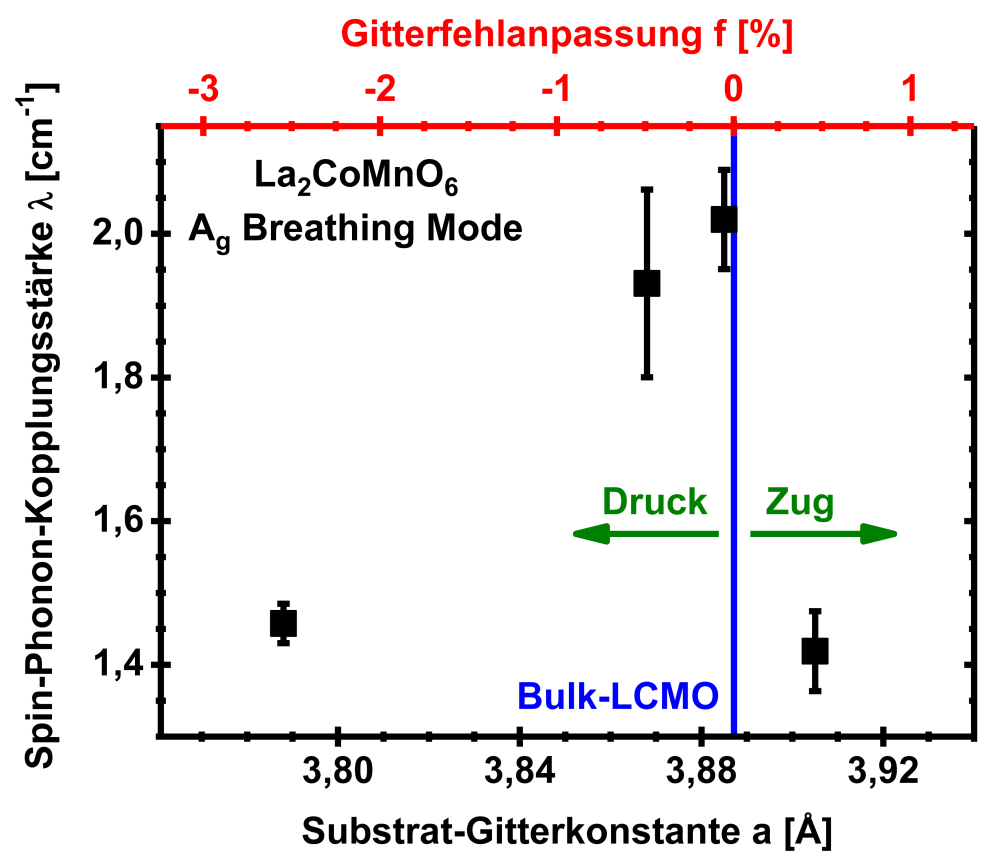

Abbildung 4.1.38: Spin-Phonon-Kopplungsstärke $\lambda$ der $\mathrm{La}_{2} \mathrm{CoMnO}_{6}$-Schichten mit einer (111)Orientierung in Abhängigkeit der Gitterkonstante $a$ der verwendeten Substrate und der jeweils resultierenden in-plane Gitterfehlanpassung $f$.

Hierbei zeigt sich, dass die Spin-Phonon-Kopplungsstärke $\lambda$ für die LCMO-Schichten auf $\mathrm{Al}_{2} \mathrm{O}_{3}$ (0001) und LSAT (111) im Vergleich zu den LCMO-Schichten auf LAO (111) und STO (111) signifikant erhöht ist. Neben der chemischen Verspannung durch das A-Kation 
hat somit auch die vom Substrat in die Schicht induzierte Verspannung einen großen Einfluss auf die Spin-Phonon-Kopplung. $\mathrm{Da}_{\mathrm{Al}_{2} \mathrm{O}_{3}}$ (0001) und LSAT (111) eine etwas kleinere Gitterkonstante als Bulk-LCMO aufweisen, erscheint eine moderate in-plane Druckspannung eine starke Spin-Phonon-Kopplung der Doppelperowskite zu begünstigen. Der größere Fehlerbalken für die LCMO/LSAT (111)-Schicht resultiert aus der magnetischen out-ofplane Anisotropie. Die out-of-plane Gitterkonstanten ergeben sich aus XRD-Messungen (Abschnitt 4.1.3). So zeigt die LCMO/LSAT (111)-Schicht mit $c_{\mathrm{pc}}=3,909 \AA$ die größte out-of-plane Gitterkonstante und erfährt durch die in-plane Substrat-Druckspannung eine out-of-plane Zugspannung, während die Gitterkonstante mit $c_{\mathrm{pc}}=3,899 \AA$ für die Schicht auf LAO (111) durch eine begonnene Relaxation aufgrund der größeren in-plane Gitterfehlanpassung verringert ist. Die LCMO/STO (111)-Schicht erfährt hingegen eine inplane Substrat-Zugspannung und entsprechend mit $c_{\mathrm{pc}}=3,875 \AA$ eine out-of-plane Druckspannung. Somit korreliert die Spin-Phonon-Kopplungsstärke $\lambda$ der LCMO-Schichten in der Abbildung 4.1 .38 direkt mit der in-plane Schichtverspannung. Ein Spezialfall ist die $\mathrm{LCMO} / \mathrm{Al}_{2} \mathrm{O}_{3}$ (0001)-Schicht. In den XRD-Messungen ist die out-of-plane Gitterkonstante mit $c_{\mathrm{pc}}=3,878 \AA$ sogar minimal kleiner als für Bulk-LCMO, sodass die LCMO-Schicht offenbar durch das Wachstum auf dem hexagonalen $\mathrm{Al}_{2} \mathrm{O}_{3}$ (0001)-Substrat eine zusätzliche Verzerrung erfährt, die out-of-plane in einer leichten Druckspannung resultiert, die zu einer besonders starken Spin-Phonon-Kopplung führt. Zur Ergänzung soll mit LCMO auf $\mathrm{MgO}(100)$ daher eine Schicht auf einem Substrat mit besonders großer Fehlanpassung (Gitterkonstante $a_{\mathrm{MgO}}=4,216 \AA 225$, Gitterfehlanpassung von $+8,46 \%$ zu Bulk-LCMO) betrachtet werden. In der Abbildung 4.1 .39 ist die Entwicklung der Phonon-Renormierung $\Delta \omega(T)$ der $A_{g}$ Breathing Mode und der Magnetisierung $M^{2}(T) / M_{\text {max. }}^{2}$ der LCMO-Schicht auf $\mathrm{MgO}$ (100) und die resultierende lineare Abhängigkeit der beiden Größen dargestellt.

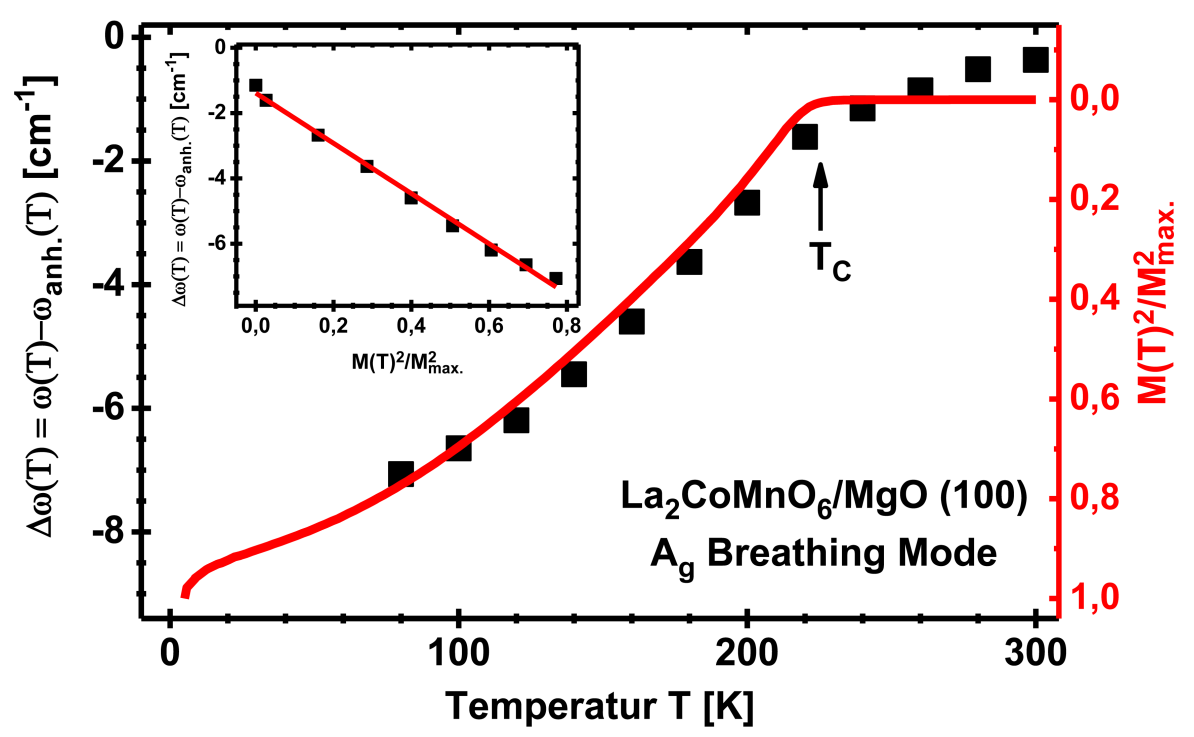

Abbildung 4.1.39: Abweichung $\Delta \omega(T)$ der $A_{g}$ Breathing Mode von $\omega_{\text {anh. }}(T)$ und die Magnetisierung $M^{2}(T) / M_{\text {max. }}^{2}$ der $\mathrm{La}_{2} \mathrm{CoMnO}_{6} / \mathrm{MgO}(100)$-Schicht $(d \approx 100 \mathrm{~nm})$ sowie die resultierende lineare Abhängigkeit für $\Delta \omega\left(M^{2}(T) / M_{\text {max. }}^{2}\right)$. 


\section{Auswertung und Ergebnisse}

Hierbei zeigt sich auch für die LCMO/MgO (100)-Schicht eine vergleichbare Entwicklung in der Abweichung von $\Delta \omega(T)$ der $A_{g}$ Breathing Mode vom anharmonischen Modell sowie der Magnetisierung $M^{2}(T) / M_{\max }^{2}$ (FCC, $H=1 \mathrm{kOe}$ ) für $T<T_{C}$ und damit ebenfalls eine signifikante Spin-Phonon-Kopplung in der ferromagnetischen Phase. Aus der Steigung $a=4 \lambda$ der linearen Abhängigkeit $\Delta \omega\left(M^{2}(T) / M_{\text {max. }}^{2}\right)$ resultiert eine Spin-PhononKopplungsstärke $\lambda=1,92(5) \mathrm{cm}^{-1}$. Somit ergibt sich auch für die LCMO/MgO (100)Schicht mit der großen Gitterfehlanpassung eine starke Spin-Phonon-Kopplung, die im Bereich von LCMO/LSAT (111) liegt (Abbildung 4.1.38) und die Verspannungsabhängigkeit von $\lambda$ für die LCMO-Schichten auf (111)-Substraten zu widerlegen scheint. Bei der out-of-plane Gitterkonstante zeigt sich jedoch, dass diese mit $c_{\mathrm{pc}}=3,902 \AA$ signifikant zu Bulk-LCMO vergrößert ist. Dieses lässt eine in-plane Druckspannung vermuten. Eine Erklärung liefert das XRD-Spektrum (Abschnitt 4.1.3) anhand eines sichtbaren Untergrunds mit einer reduzierten out-of-plane Gitterkonstanten $c_{\mathrm{pc} \text {, red. }}=3,814 \AA$. Offenbar wachsen die ersten Lagen der LCMO/MgO (100)-Schicht mit einer durch das Substrat induzierten in-plane Zugspannung auf, bevor das Wachstum daraufhin mit einer in-plane Druckspannung fortgeführt und somit eine starke Spin-Phonon-Kopplung begünstigt wird. Die Schichtorientierung scheint dagegen anders als bei der Stimulation der B-Platz-Ordnung von LCMO durch eine Schichtverspannung durch das Substrat 224 keinen Einfluss auf die Spin-Phonon-Kopplungsstärke zu haben, wenn die Schicht selbst B-Platz geordnet ist.

Da bisher die auf die Spin-Phonon-Kopplung untersuchten LCMO-Schichten alle eine optimierte B-Platz-Ordnung aufweisen, bietet sich ein Vergleich einer geordneten Schicht mit einer nur teilgeordneten Schicht an. Hierfür können erneut die zwei aus den vorherigen Abschnitten bekannten LCMO-Schichten auf $\mathrm{Al}_{2} \mathrm{O}_{3}$ (0001) mit B-Platz-Ordnung und B-Platz-Teilordnung betrachtet werden. In der Abbildung 4.1.39 (a) ist daher die Entwicklung der Phonon-Renormierung $\Delta \omega(T)$ der $A_{g}$ Breathing Mode und der Magnetisierung $M^{2}(T) / M_{\text {max. }}^{2}$ der beiden $\mathrm{LCMO} / \mathrm{Al}_{2} \mathrm{O}_{3}$ (0001)-Schichten im Vergleich dargestellt. Hierbei ist für beide Schichten eine exzellente Korrelation zwischen der PhononRenormierung der $A_{g}$ Breathing Mode und der Magnetisierung für $T<T_{C}$ sowie eine damit einhergehende Spin-Phonon-Kopplung erkennbar. Zudem lässt sich in der Temperaturentwicklung und in der Ausprägung des Erweichens der $A_{g}$ Breathing Mode ein signifikanter Unterschied für beide Schichten beobachten. Folglich besitzt auch die BPlatz-Ordnung in den Doppelperowskiten einen großen Einfluss auf die Spin-PhononKopplung. Für eine Quantifizierung sind in der Abbildung 4.1.39 (b) die linearen Abhängigkeiten $\Delta \omega\left(M^{2}(T) / M_{\text {max. }}^{2}\right)$ der $A_{g}$ Breathing Mode der beiden LCMO $/ \mathrm{Al}_{2} \mathrm{O}_{3}(0001)$ Schichten im direkten Vergleich dargestellt. Aus der Steigung $a=4 \lambda$ resultiert eine SpinPhonon-Kopplungsstärke $\lambda_{\text {geord. }}=2,02(7) \mathrm{cm}^{-1}$ für die B-Platz geordnete Schicht und $\lambda_{\text {teilgeord. }}=1,59(4) \mathrm{cm}^{-1}$ für die B-Platz teilgeordnete Schicht. Der B-Platz-Ordnungsgrad wirkt sich somit nicht nur auf die magnetischen Eigenschaften aus, sondern beeinflusst 
auch direkt die phononischen Eigenschaften und die Spin-Phonon-Kopplung der Doppelperowskite. Umgekehrt spiegelt damit die Spin-Phonon-Kopplungsstärke $\lambda$ wiederum die B-Platz-Ordnung der Doppelperowskite wider und kann daher als eine weitere Methode für eine qualitative Abschätzung der vorhandenen Ordnung in Kombination mit den Magnetisierungsmessungen oder den EELS-Kartierungen betrachtet werden.
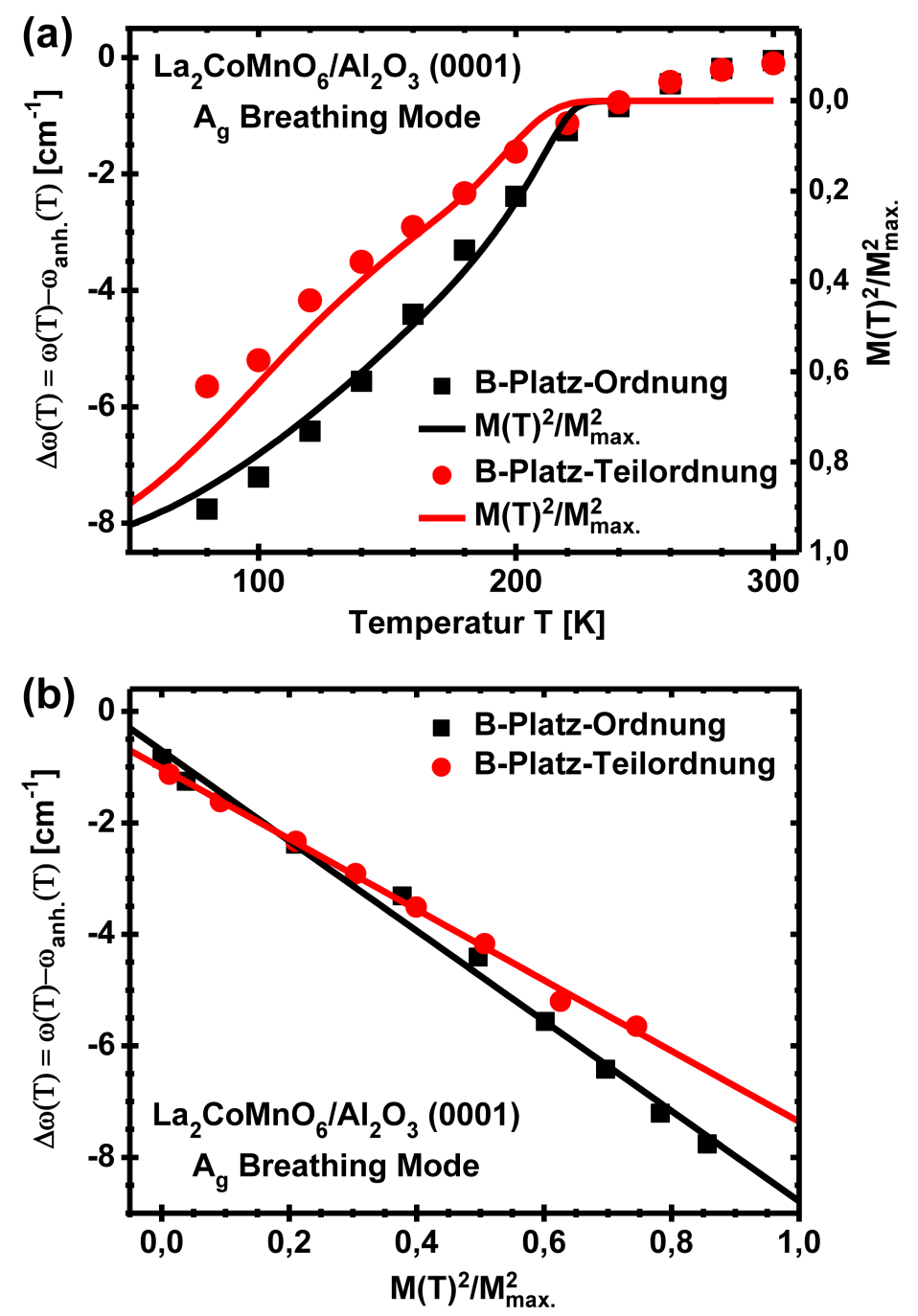

Abbildung 4.1.40: Abweichung $\Delta \omega(T)$ der $A_{g}$ Breathing Mode vom $\omega_{\text {anh. }}(T)$-Verlauf und die Magnetisierung $M^{2}(T) / M_{\max }^{2}$. (a) sowie die jeweilige lineare Abhängigkeit für $\Delta \omega\left(M^{2}(T) / M_{\text {max. }}^{2}\right)$ (b) der zwei $\mathrm{La}_{2} \mathrm{CoMnO}_{6} / \mathrm{Al}_{2} \mathrm{O}_{3}$ (0001)-Schichten mit B-Platz-Ordnung und -Teilordnung im Vergleich.

Die Spin-Phonon-Kopplungsstärke $\lambda$, untersucht anhand der Abweichung der Modenposition $\Delta \omega(T)$ der $A_{g}$ Breathing Mode von der Temperaturentwicklung $\omega_{\text {anh. }}(T)$ des anharmonischen Modells und des normierten Quadrats der Magnetisierung $M^{2}(T) / M_{\text {max. }}^{2}$, zeigt somit eine hohe Sensitivität sowohl bezüglich der chemischen Verspannung als auch von der durch das Substrat induzierten Spannung sowie der chemisch-strukturellen B-PlatzOrdnung. Folglich stellt die Spin-Phonon-Kopplung eine Kenngröße dar, die die strukturellen, magnetischen und phononischen Eigenschaften der Doppelperowskite umfasst. 


\subsection{Tip-Enhanced Raman Spectroscopy (TERS)}

Nach der allgemeinen Untersuchung und Charakterisierung der mit der MAD-Technik hergestellten $\mathrm{A}_{2} \mathrm{BB}^{\prime} \mathrm{O}_{6}$-Doppelperowskit-Schichten $\left(\mathrm{A}=\right.$ Seltene Erde; $\mathrm{B}=\mathrm{Co}, \mathrm{Ni} ; \mathrm{B}^{\prime}=\mathrm{Mn}$ ) bezüglich ihrer strukturellen, magnetischen und phononischen Eigenschaften im Zusammenhang mit ihrer B-Platz Ordnung und des Einflusses von Verspannungen in der Schicht soll nun in diesem Kapitel ein Weg zu der erfolgreichen Etablierung von TERS-Messungen an diesem Materialsystem aufgezeigt werden. Hierzu werden zunächst mit den beiden Farbstoffen Azobenzol und Malachitgrün Isothiocynat (MGITC) sowie mit kristallinem Silizium bekannte TERS-Testmaterialien zur Verifizierung und Quantifizierung der TERSVerstärkung für das hier verwendete STM-TERS-System betrachtet. Im Anschluss da$\mathrm{zu}$ werden dann polarisationsabhängige TERS-Untersuchungen an dünnen $\mathrm{La}_{2} \mathrm{CoMnO}_{6^{-}}$ Schichten, die auf STO (100)-Substrate aufgewachsen wurden, vorgestellt und die Ergebnisse für die verschiedenen Polarisationskonfigurationen mithilfe theoretischer Berechnungen unter Berücksichtigung eines kombinierten Verstärkungs- und Depolarisationsmodells für das TERS-Nahfeld verglichen. Zum Abschluss wird dann noch der Einfluss der TERSSpitze, der STM-Parameter (Tunnelstrom, Spannung), der Doppelperowskit-Schichtdicke, des verwendeten Substrats, der A-und B-Platz-Kationen und der Schichtverspannung auf die TERS-Spektren und die jeweils zu erzielende Verstärkung untersucht.

\subsubsection{Erste Testmessungen an Azobenzol und Malachit-Grün}

Der erste Schritt für die nachfolgenden TERS-Messungen ist zunächst die Überprüfung der Funktionalität und die Quantifizierung der TERS-Verstärkung gegenüber den FernfeldRaman-Spektren. Hierzu werden mit den Farbstoffen Azobenzol $\left(\mathrm{C}_{12} \mathrm{H}_{10} \mathrm{~N}_{2}\right)$ und Malachitgrün Isothiocynat (MGITC, $\mathrm{C}_{24} \mathrm{H}_{24} \mathrm{ClN}_{3} \mathrm{O}_{4} \mathrm{~S}$ ) zwei charakteristische TERS-Testproben betrachtet, die für ihre Sensitivität bezüglich einer Nahfeldverstärkung und für ihr großes Verstärkungspotential bekannt sind [171, 173, 187, 198]. MGITC verfügt zudem aufgrund resonanter Raman-Streuung für den roten HeNe-Laser $(\lambda=632,8 \mathrm{~nm})$ über einen besonders großen Raman-Wirkungsquerschnitt 171, 173]. Folglich werden die Farbstoffe auch von TERS-Systemherstellern als Testproben verwendet. Die hier betrachteten Proben stammen von Bruker. Für die TERS-Messungen sind die Farbstoffmoleküle auf einer Goldoberfläche in (111)-Orientierung, die auf einem Glassubstrat aufgewachsen wurde, adsorbiert, sodass sich einzelne Moleküllagen des Farbstoffs bilden. Das Fernfeld-Raman-Signal ist daher nur äußerst schwach ausgeprägt, sodass sich eine erfolgreiche TERS-Messung sofort an der großen Verstärkung erkennen lässt. Das Substrat dient dabei einer besseren Handhabung der Proben, während die Goldoberfläche mit der TERS-Goldspitze im gap mode plasmonisch koppelt (resonante Nahfeldverstärkung). Zudem werden die Fluoreszenzbeiträge durch das metallische Substrat effektiv gedämpft [171. Die Strukturformeldarstellungen beider Farbstoffe sind nachfolgend in den Abbildungen 4.2.1 (a) und (b) gezeigt. 
(a)<smiles>[Al]=Nc1ccccc1</smiles><smiles>Nc1ccccc1</smiles>

Z)<smiles>Cc1ccccc1</smiles>

(b)<smiles>CN(C)c1ccc(C(=C2C=CC(=[N+](C)C)C=C2)c2ccc(N=C=S)cc2)cc1</smiles>

Abbildung 4.2.1: Darstellung der Strukturformel von Azobenzol mit seiner cis-trans-Isomerie ((Z)-(E)-Isomerie) (a) und von Malachitgrün Isothiocynat (MGITC) (b).

Azobenzol weist dabei eine cis-trans-Isomerie (auch als (Z)-(E)-Isomerie bezeichnet) auf. In der cis-Anordnung ((Z)-Anordnung) befinden sich beide Phenylringe auf der gleichen Seite der Referenzebene des Azobenzolmoleküls, während die zwei Phenylringe in der transAnordnung ((E)-Anordnung) des Azobenzols auf den entgegengesetzten Seiten der Referenzebene liegen. Beide Isomere können durch Photoisomerisierung in die andere Form überführt werden [247]. Wird trans-Azobenzol mit ultraviolettem Licht bestrahlt, so geht es in die cis-Anordnung über, während die Bestrahlung von cis-Azobenzol mit blauem Licht wiederum zu einem Übergang in die trans-Anordnung führt [247, 248]. Zudem tritt eine spontane Relaxation von cis-Azobenzol in das thermisch stabile trans-Azobenol auf [247]. Die Isomerie von Azobenzol hat auch Auswirkungen auf das Raman-Spektrum von Azobenzol, welches sich für die beiden Anordnungen stark unterscheidet. Eine ausführliche Darstellung findet sich in der Literatur 248 250]. In der Abbildung 4.2.2 ist nun das TERS- und Fernfeld-Raman-Spektrum der Azobenzol-Testprobe auf Au (111) dargestellt.

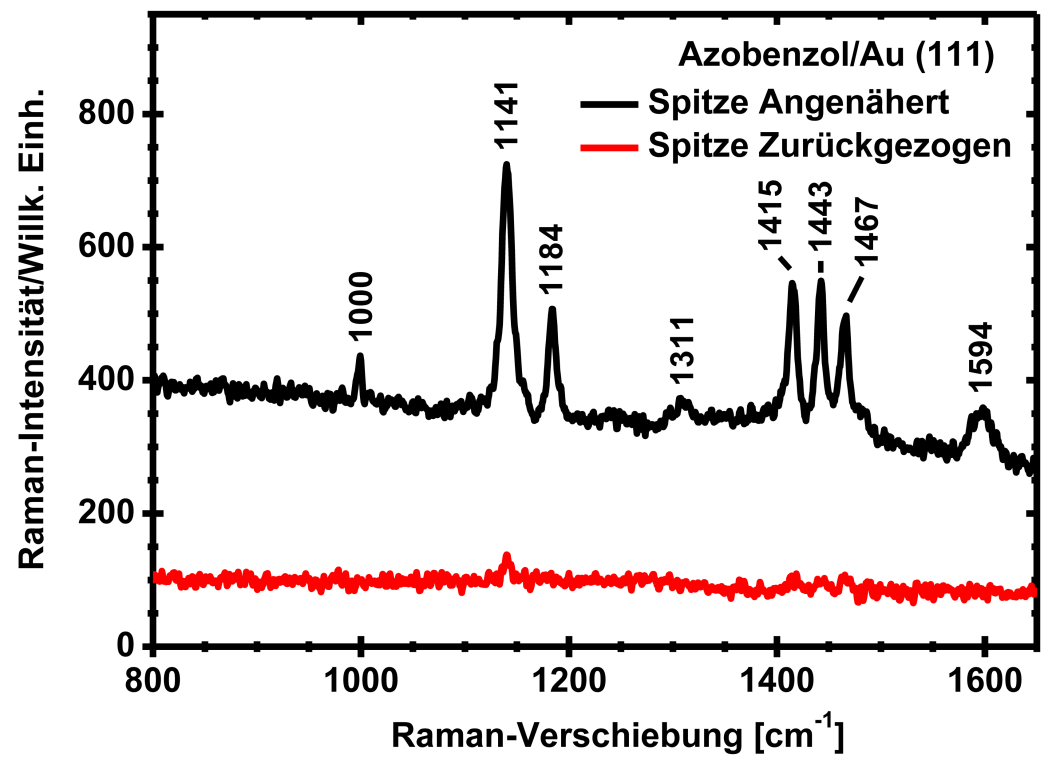

Abbildung 4.2.2: TERS- und Fernfeld-Raman-Spektrum von Azobenzol auf Au (111) mit einer einfallenden P-Laserpolarisation und ohne Analysator. 


\section{Auswertung und Ergebnisse}

Die Spektren wurden zur Vermeidung von Heiz- und Photobleichungseffekten 171, 173, 198 bei einer Laserleistung von nur $P \approx 0,5 \mathrm{~mW}(\lambda=632,8 \mathrm{~nm}, t=2 \times 60 \mathrm{~s})$ in seitlicher Einstrahlungsgeometrie (Abbildung 3.4.6) mit einer Laserpolarisation parallel (P) zur TERS-Spitzenachse und ohne Analysator vor dem Detektor (unpolarisiert) aufgenommen. Das Fernfeld-Raman-Spektrum (Spitze mehrere Mikrometer von der Probenoberfläche zurückgezogen) weist dabei eine äußerst geringe Intensität mit einem kontinuierlichen Hintergrund, der durch inelastische Streuung an der Substratgoldoberfläche entsteht, auf. Die TERS-Messung (Spitze in Tunnelposition an die Probenoberfläche angenähert) erfolgte im Konstant-Strom-Modus bei einem Tunnelstrom von $j=0,2 \mathrm{nA}$, einer angelegten Spannung von $U_{\text {Bias }}=0,4 \mathrm{~V}$ und einem Abstand zwischen Spitze (Radius $R_{\text {Spitze }} \approx 25 \mathrm{~nm}$; REMBild) und Probenoberfläche von $\delta z \approx 1 \mathrm{~nm}$ (Strom-Abstands-Kurve). Im TERS-Modus ergibt sich dabei eine große Verstärkung des gesamten Azobenzol-Raman-Spektrums (sowie des kontinuierlichen Hintergrunds durch inelastische Streuprozesse an der Goldspitze). Insgesamt lassen sich acht Raman-Moden beobachten, die alle dem trans-Isomer von Azobenzol zugeordnet werden können und eine in-plane $A_{g}$-Symmetrie aufweisen 249,250 . Bei senkrechter Ausrichtung der Azobenzol-Moleküle im Nahfeld zur Goldoberfläche koppeln die Moden somit sehr stark an die bevorzugte Komponente des Nahfeldes parallel zur Spitzenachse bei Anregung mit in gleicher Weise polarisiertem Laserlicht 176,251], sodass sich resultierend eine große TERS-Verstärkung beobachten lässt. Die stärkste Mode bei $1141 \mathrm{~cm}^{-1}$ beschreibt hierbei eine symmetrische Streckschwingung der beiden C-NBindungen des Azobenzols, während die Mode bei $1443 \mathrm{~cm}^{-1}$ eine Streckschwingung der N-N-Bindung kennzeichnet. Die anderen Moden charakterisieren wiederum verschiedene in-plane Deformationen der Phenylringe des Azobenzols 249, 250]. Für eine Quantifizierung der Verstärkung kann der TERS-Kontrast $C$ (Gleichung (3.4.3) bestimmt werden. Hierzu wird der spektrale Hintergrund abgezogen und die integrierte Raman-Intensität der jeweiligen Mode im TERS-Gesamtfeld und im Fernfeld berechnet. Für die stärkste Raman-Mode bei $1141 \mathrm{~cm}^{-1}$ ergibt sich dabei ein TERS-Kontrast $C_{1141}=18,75$. Mithilfe der Gleichung (3.4.5) lässt sich daraus der TERS-Verstärkungsfaktor auf $\mathrm{EF}_{\mathrm{TERS}} \approx 5 \cdot 10^{4}$ (mit $R_{\text {Fokus }} \approx 1 \mu \mathrm{m}, R_{\text {Spitze }} \approx 25 \mathrm{~nm}, L_{\mathrm{nf}} \approx L_{\mathrm{ff}}$ und $\beta=60^{\circ}$ ) abschätzen. Dieses ist in guter Übereinstimmung mit anderen TERS-Messungen an Azobenzol [187, 198].

In der Abbildung 4.2.2 ist nachfolgend das TERS- und Fernfeld-Raman-Spektrum der MGITC-Testprobe auf Au (111) dargestellt. Die Spektren wurden zur Vermeidung von Heiz- und Photobleichungseffekten bei einer Laserleistung von ebenfalls $P \approx 0,5 \mathrm{~mW}$ $(\lambda=632,8 \mathrm{~nm}, t=2 \times 30 \mathrm{~s})$ in Seiteneinstrahlung mit einer Laserpolarisation parallel $(\mathrm{P})$ zur TERS-Spitzenachse und ohne Analysator aufgenommen. Die TERS-Messung (Spitze in Tunnelposition an die Probenoberfläche angenähert) erfolgte dabei mit der gleichen TERS-Goldspitze wie zuvor für die Azobenzol-Probe im Konstant-Strom-Modus bei einem Tunnelstrom von $j=1 \mathrm{nA}$ und einer angelegten Spannung von $U_{\text {Bias }}=1 \mathrm{~V}$. 


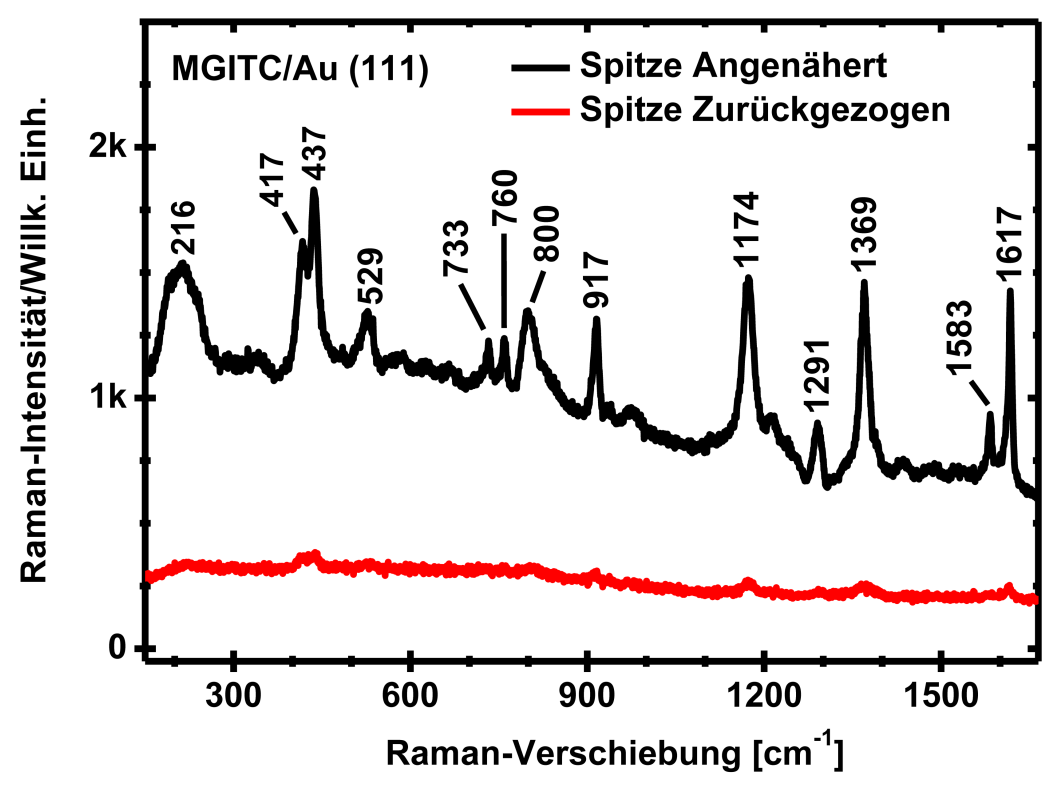

Abbildung 4.2.3: TERS- und Fernfeld-Raman-Spektrum von MGITC auf Au (111) mit einer einfallenden P-Laserpolarisation und ohne Analysator.

Im Fernfeld-Raman-Spektrum (Spitze mehrere Mikrometer zurückgezogen) der MGITCProbe zeigt sich wie bei der Azobenzol-Probe eine nur geringe Intensität zusammen mit einem kontinuierlichen Hintergrund, der durch die Goldoberfläche des Substrats hervorgerufen wird. Im TERS-Spektrum (Spitze in Tunnelposition an die Probenoberfläche angenähert) ergibt sich eine signifikante Verstärkung des gesamten Spektrums der MGITC-Probe durch das Spitzennahfeld und eine Verstärkung des kontinuierlichen Hintergrunds durch inelastische Streuprozesse an der Spitze. Im TERS-Spektrum lassen sich 13 Raman-Moden beobachten, die alle dem MGITC-Molekül zugeordnet werden können 252,253. Die starke Mode bei $1174 \mathrm{~cm}^{-1}$ beschreibt eine in-plane Biegeschwingung der C-H-Bindungen, während die intensive Mode bei $1359 \mathrm{~cm}^{-1}$ eine Streckschwingung der N-Phenylring-Bindung und die ebenfalls intensive Mode bei $1617 \mathrm{~cm}^{-1}$ eine kombinierte Streckschwingung der N-Phenylring-Bindung und der C-C-Bindungen kennzeichnen. Die anderen schwächeren Moden lassen sich verschiedenen Deformationen der Phenylringe und von weiteren Bindungen im MGITC-Molekül zuordnen 252,253. Der leichte Unterschied in der relativen Verstärkung der Moden im TERS-Spektrum ist dabei auf Variationen der jeweiligen Raman-Auswahlregeln im Nahfeld zurückzuführen [171. Für eine Quantifizierung der TERS-Verstärkung kann auch hier mithilfe der Gleichungen (3.4.3) und 3.4.5) der TERSKontrast $C$ und der TERS-Verstärkungsfaktor $\mathrm{EF}_{\text {TERS }}$ bestimmt werden. Für die besonders intensive Mode bei $1174 \mathrm{~cm}^{-1}$ ergibt sich ein TERS-Kontrast $C_{1174}=12,79$ und ein TERS-Verstärkungsfaktor $\mathrm{EF}_{\mathrm{TERS}} \approx 4 \cdot 10^{4}$. Gegenüber der Azobenzol-Probe sind beide Werte aufgrund des stärkeren Fernfeldbeitrags leicht reduziert, lassen sich aber erneut in guter Übereinstimmung mit anderen TERS-Messungen an MGITC vergleichen 171, 173. Folglich ist für beide Farbstoff-Proben eine signifikante Nahfeldverstärkung in den TERSSpektren gegenüber den jeweiligen Fernfeld-Raman-Spektren zu beobachten. 


\subsubsection{Polarisationsabhängige TERS-Messungen an Silizium}

Nach den TERS-Messungen an Azobenzol und MGITC soll der Fokus nun auf kristalline Proben gelegt werden. In der Literatur untersuchte Materialien sind diesbezüglich vor allem Halbleitereinkristalle und -Schichten wie Silizium 169, 170, 192, 194, 197, 210, 254, GaAs 193, CdS 172 oder GaN 209,255]. Da hier in dieser Arbeit ein STM-TERS-System verwendet wird und die Proben somit einen moderaten elektrischen Widerstand aufweisen müssen, um die TERS-Spitze in eine stabile Tunnelposition an die Probe anzunähern, bietet es sich entsprechend an, mit einem kristallinen Halbleiter zu beginnen. Folglich soll nun ein einkristallines Silizium-Substrat mit einer (100)-Orientierung (p-Dotierung mit Bor; von Crystal) betrachtet werden. Silizium kristallisiert in der kubischen DiamantStruktur (Raumgruppe 227, Fd $\overline{3} \mathrm{~m} 256$ ) mit einer Gitterkonstante $a=5,431 \AA 257$. Eine Darstellung der Diamant-Struktur von Silizium ist in der Abbildung 4.2.4 (a) gegeben.
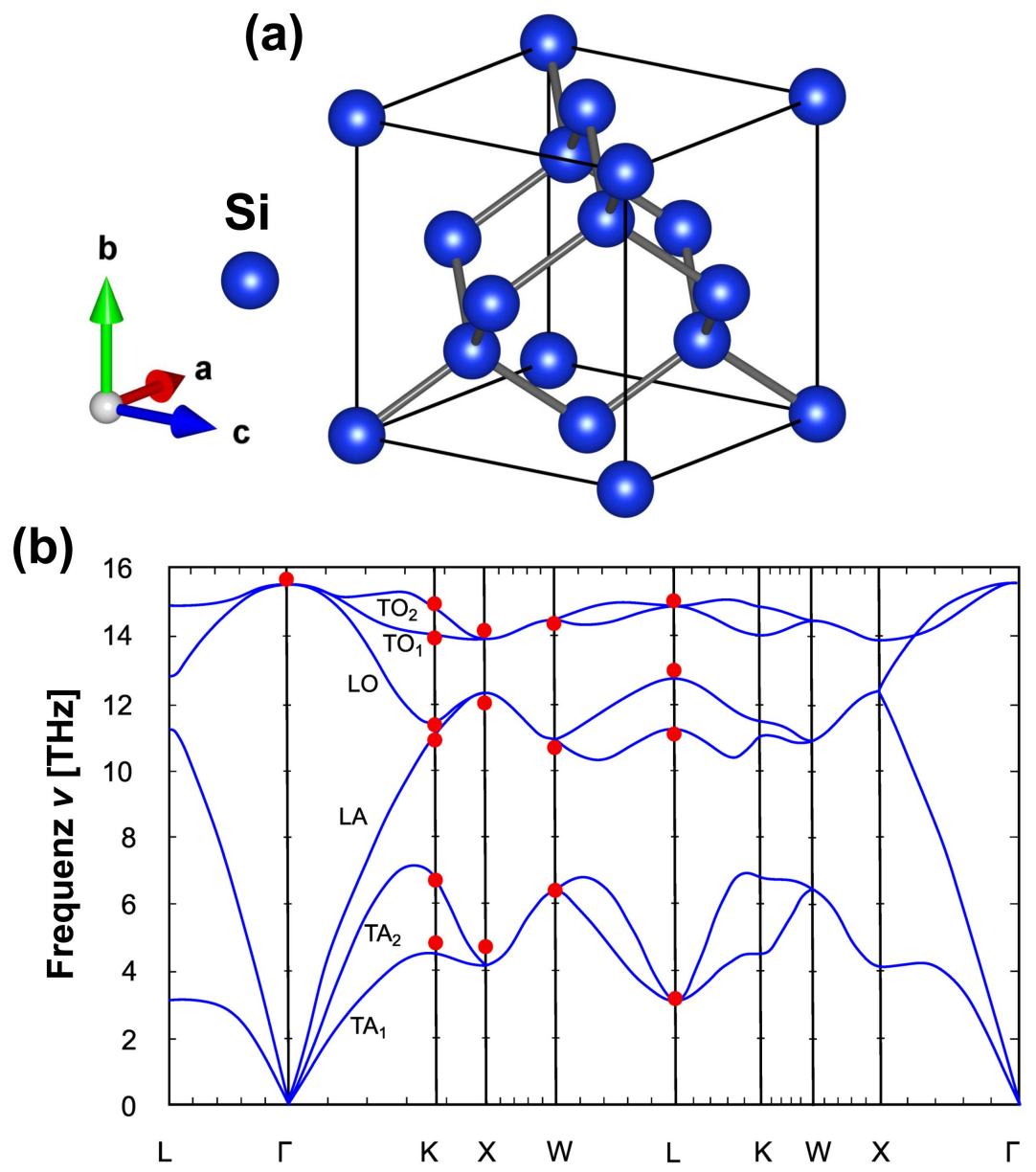
Abbildung 4.2.4: Diamantstruktur (a) (gezeichnet mit VESTA 10) und die Phononen-Disper- sionskurven von Silizium (b) [258, 259]. Die roten Punkte zeigen Phononen- frequenzen an den kritischen Punkten der 1. Brillouin-Zone.

Die Kristallstruktur von Silizium bestimmt nun zusammen mit den Dispersionskurven der Phononen in der 1. Brillouin-Zone die in 1. Ordnung beobachtbaren Raman-Moden und durch die zu der Raumgruppe gehörenden Raman-Tensoren die Auswahlregeln für die 
polarisationsabhängigen Raman-Messungen. Da die primitive Einheitszelle von Silizium aufgrund der zweiatomigen Basis der Diamantstruktur $p=2$ Atome beinhaltet, ergeben sich 3 akustische Phononenzweige (LA, $\mathrm{TA}_{1}, \mathrm{TA}_{2}$ ) und $3 p-3=3$ optische Phononenzweige $\left(\mathrm{LO}, \mathrm{TO}_{1}, \mathrm{TO}_{2}\right)$ 118. Phononen sind akustisch (A), wenn jeweils benachbarte Atome in Phase schwingen, und optisch $(\mathrm{O})$, wenn sich eine gegenphasige Bewegung der benachbarten Atome ergibt. Es wird zudem zwischen longitudinalen (L) und transversalen (T) Moden unterschieden, wobei longitudinale Moden in Richtung des Ausbreitungsvektors $\vec{k}$ und transversale Moden senkrecht zu $\vec{k}$ schwingen. Der Verlauf der Dispersionskurven von Silizium in der 1. Brillouin-Zone ist in der Abbildung 4.2.4 (b) [258, 259] dargestellt. Die roten Punkte kennzeichnen die Phononenfrequenzen an den kritischen Punkten der 1. BrillouinZone. Da Photonen für Licht im sichtbaren Wellenlängenbereich einen Wellenvektor mit nur einem kleinen Betrag $|\vec{k}|=\frac{2 \pi}{\lambda}$ im Vergleich zu denen der Wellenvektoren der Phononen und der Ausdehnung der Brillouin-Zone von $\frac{2 \pi}{a}$ (Gitterkonste $a$ ) besitzen, ist die RamanStreuung 1. Ordnung auf den Bereich um den $\Gamma$-Punkt mit $|\vec{q}| \approx 0$ beschränkt 60,118 . Für Silizium in der Diamantstruktur ergibt sich aufgrund der Fd̄̄m-Raumgruppe und der kovalenten Bindung der gleichatomigen Basis eine dreifache Entartung der optischen Phononen am $\Gamma$-Punkt (Abbildung 4.2.4 (b)) [59, 118,261, , sodass sich im Raman-Spektrum nur eine scharfe Mode 1 . Ordnung mit einer $T_{2 g}$-Symmetrie (wird auch oft als $F_{2 g}$-Symmetrie bezeichnet) und hoher Intensität bei einer Wellenzahl von $520 \mathrm{~cm}^{-1}(\nu \approx 15,6 \mathrm{Thz})$ beobachten lässt [139, 140]. Für die Raman-Tensoren der drei Moden folgt dabei 143,261]:

$$
\mathcal{R}_{1}=\left(\begin{array}{lll}
0 & 0 & 0 \\
0 & 0 & d \\
0 & d & 0
\end{array}\right), \mathcal{R}_{2}=\left(\begin{array}{lll}
0 & 0 & d \\
0 & 0 & 0 \\
d & 0 & 0
\end{array}\right), \mathcal{R}_{3}=\left(\begin{array}{lll}
0 & d & 0 \\
d & 0 & 0 \\
0 & 0 & 0
\end{array}\right)
$$

Im Probenbezugssystem des TERS-Aufbaus (Abbildung 3.4.6) lässt sich $\mathcal{R}_{1}$ dem LOPhononenzweig von Silizium zuordnen, während $\mathcal{R}_{2}$ und $\mathcal{R}_{3}$ dem $\mathrm{TO}_{1}$ - und dem $\mathrm{TO}_{2}$-Zweig zugewiesen werden können. Aufgrund der Seiteneinstrahlung müssen die drei Tensoren mithilfe der Gleichung (3.4.9) aus dem Proben- in das Laborbezugssystem durch Rotation um einen Winkel $\beta=90^{\circ}-\alpha=60^{\circ}$ im mathematisch positiven Sinn um die y-Achse überführt werden. Eine mögliche in-plane Rotation des Substrats um einen Winkel $\phi$ um die Spitzenachse herum wird durch eine Rotation um die x-Achse mithilfe der Gleichung 3.4.8 berücksichtigt. In den Abbildungen 4.2.5 (a) und (b) sind nun die polarisationsabhängigen TERS- und Fernfeld-Raman-Spektren eines Silizium (100)-Substrats in den parallelen $\mathrm{P}^{\prime} \mathrm{P}^{\prime}$ und $\mathrm{S}^{\prime} \mathrm{S}^{\prime}$ - sowie den gekreuzten $\mathrm{P}^{\prime} \mathrm{S}^{\prime}$ - und $\mathrm{S}^{\prime} \mathrm{P}^{\prime}$-Polarisationskonfigurationen für eine in-plane Rotation der Probe um einen Winkel $\phi=45^{\circ}$ um die Spitzenachse herum dargestellt. Die Spektren wurden bei einer Laserleistung von $P \approx 10 \mathrm{~mW}(\lambda=532 \mathrm{~nm}, t=3 \times 20 \mathrm{~s})$ aufgenommen. Die TERS-Messung erfolgte im Konstant-Strom-Modus ( $\left.j=1 \mathrm{nA}, U_{\text {Bias }}=1 \mathrm{~V}\right)$ mit der gleichen TERS-Spitze $\left(R_{\text {Spitze }} \approx 25 \mathrm{~nm}, \delta z \approx 1 \mathrm{~nm}\right)$ wie zuvor bei den Farbstoffen. 

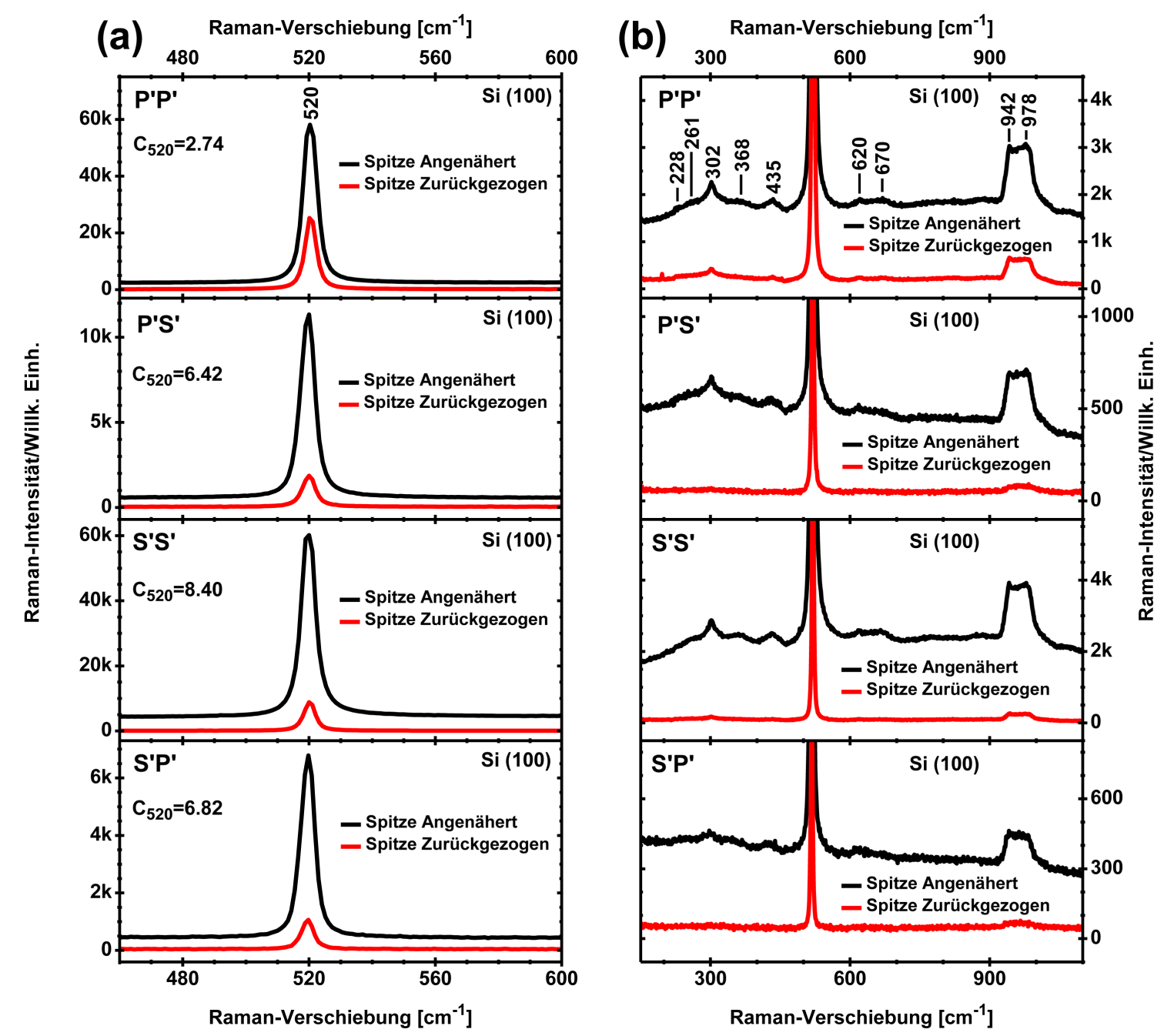

Abbildung 4.2.5: Polarisierte TERS- und Fernfeld-Raman-Spektren eines Silizium (100)Substrats, aufgenommen in den parallelen $\mathrm{P}^{\prime} \mathrm{P}^{\prime}$ - und $\mathrm{S}^{\prime} \mathrm{S}^{\prime}$ - sowie den gekreuzten $\mathrm{P}^{\prime} \mathrm{S}^{\prime}$ - und $\mathrm{S}^{\prime} \mathrm{P}^{\prime}$-Polarisationskonfigurationen (in-plane Rotation um $\phi=45^{\circ}$ ), mit dem Fokus auf die intensive Raman-Mode 1. Ordnung bei $520 \mathrm{~cm}^{-1}$ (a) und die verschiedenen Raman-Moden 2. Ordnung (b).

Die Fernfeld-Raman-Spektren (Spitze mehrere Mikrometer von der Probenoberfläche zurückgezogen) in den Abbildungen 4.2.5 (a) und (b) zeigen dabei die für Silizium zu erwartende dreifach entartete LTO(Г)-Raman-Mode 1. Ordnung bei $520 \mathrm{~cm}^{-1}$, die entsprechend der $T_{2 g}$-Symmetrie und der Raman-Auswahlregeln in der betrachteten Seiteneinstrahlung und der in-plane Rotation um $\phi=45^{\circ}$ in den parallelen $\mathrm{P}^{\prime} \mathrm{P}^{\prime}$ - und $\mathrm{S}^{\prime} \mathrm{S}^{\prime}$-Polarisationskonfigurationen durch die Anregung der $\mathrm{LO}(\Gamma)$-Phononmode entsteht, während in den gekreuzten $\mathrm{P}^{\prime} \mathrm{S}^{\prime}$ - und $\mathrm{S}^{\prime} \mathrm{P}^{\prime}$-Konfigurationen einzig die zwei $\mathrm{TO}(\Gamma)$-Phononmoden aktiv sind und zu einem im Vergleich zur LO-Mode reduzierten Raman-Signal beitragen. In den TERS-Spektren (Spitze in Tunnelposition an die Probenoberfläche angenähert) ergibt sich wiederum eine signifikante Verstärkung der LTO $(\Gamma)$-Mode in allen Polarisationskonfigurationen (und des kontinuierlichen Hintergrundes durch die TERS-Goldspitze). Zur Quantifizierung der Verstärkung kann der TERS-Kontrast $C_{520}$ der LTO(Г)-Mode bei $520 \mathrm{~cm}^{-1}$ bestimmt werden. Die resultierenden Werte sind in der Abbildung 4.2.5 (a) angegeben und 
zeigen eine starke Abhängigkeit des TERS-Kontrastes von der Polarisationskonfiguration mit $C_{520, \mathrm{P}^{\prime} \mathrm{P}^{\prime}}=2,74, C_{520, \mathrm{P}^{\prime} \mathrm{S}^{\prime}}=6,42, C_{520, \mathrm{~S}^{\prime} \mathrm{S}^{\prime}}=8,40$ und $C_{520, \mathrm{~S}^{\prime} \mathrm{P}^{\prime}}=6,82$. Ein ähnliches Verhalten wird auch in der Literatur bei TERS-Messungen an Silizium und GaAs beobachtet und auf einen neben der plasmonischen Nahfeldverstärkung auftretenden Depolarisationseffekt des Nahfeldes zurückgeführt, der die Raman-Auswahlregeln bricht, die in der Polarisationskonfiguration verbotenen Raman-Moden wieder erlaubt und somit eine besonders große Verstärkung gegenüber dem Fernfeld ermöglicht 169, 170, 192, 194, 254. Dieses Verhalten zeigt sich insbesondere in den gekreuzten $\mathrm{P}^{\prime} \mathrm{S}^{\prime}$ - und $\mathrm{S}^{\prime} \mathrm{P}^{\prime}$-Konfigurationen, in denen der LO-Beitrag zur Gesamtintensität der $\operatorname{LTO}(\Gamma)$-Phononmode verboten ist, in den TERS-Spektren aber zu einem Teil beiträgt. Bei Silizium ist jedoch die Trennung der LO- und TO-Anteile an der $\operatorname{LTO}(\Gamma)$-Phononmode in seitlicher Einstrahlung schwierig, da diese sich durch die Dreifachentartung überlagern. Folglich eignen sich Materialsysteme wie das $\mathrm{La}_{2} \mathrm{CoMnO}_{6}$-Doppelperowskit mit seiner intensiven und nicht-entarteten $A_{g}$ Breathing Mode besser für eine Betrachtung des TERS-Nahfelddepolarisationseffekts [49], wie im nächsten Abschnitt 4.2 .3 dieser Arbeit am Beispiel dünner $\mathrm{La}_{2} \mathrm{CoMnO}_{6}$-Schichten auf STO (100) gezeigt werden soll. Der größere TERS-Kontrast für die LTO $(\Gamma)-$ Mode bei $520 \mathrm{~cm}^{-1}$ in der $\mathrm{S}^{\prime} \mathrm{S}^{\prime}$ - im Vergleich zur $\mathrm{P}^{\prime} \mathrm{P}^{\prime}$-Konfigurationen kann für die verwendete Seiteneinstrahlung auf einen erhöhten Reflexionsgrad des Laserlichts mit S'-Polarisation im Fernfeld zurückgeführt werden [194,197], während das Nahfeld der TERS-Spitze davon unbeeinflusst bleibt, sodass der TERS-Kontrast für die $\mathrm{S}^{\prime} \mathrm{S}^{\prime}$-Konfigurationen erhöht ist.

Neben der LTO(Г)-Mode 1. Ordnung bei $520 \mathrm{~cm}^{-1}$ lassen sich in den TERS- und FernfeldRaman-Spektren des Silizium-Substrats Moden 2. Ordnung beobachten, die Ober- und Kombinationsschwingungen von Phononen auch abseits des $\Gamma$-Punkts der 1 . Brillouin-Zone beschreiben, da für Zwei-Phonon-Raman-Streuprozesse die Bedingung der Wellenvektoren der zwei Phononen mit $\vec{q}_{1} \approx-\vec{q}_{2}$ für die Quasiimpulserhaltung nicht auf den Bereich des $\Gamma$-Punkts beschränkt, sondern auf die ganze 1. Brillouin-Zone ausgeweitet ist [59, 261]. Die Intensität dieser Moden ist aufgrund der geringeren Anregungswahrscheinlichkeit gegenüber der LTO $(\Gamma)$-Mode um den Faktor $\approx 10^{2}$ reduziert. Folglich sind die Spektren in der Abbildung 4.2.5 (b) für die Zwei-Phonon-Raman-Moden auf einen Bereich niedrigerer Intensität skaliert. Die Mode bei $228 \mathrm{~cm}^{-1}$ lässt sich einer 2TA(L)-Oberschwingung zuordnen, während die Mode bei $302 \mathrm{~cm}^{-1}$ einer 2TA(X)-Oberschwingung und die Mode bei $435 \mathrm{~cm}^{-1}$ einer 2TA(W)-Oberschwingung entspricht 262, 263. Zudem lassen sich eine $\mathrm{TA}(\mathrm{X})+\mathrm{TO}(\mathrm{X})$-Kombinationsschwingung bei $620 \mathrm{~cm}^{-1}$, eine $2 \mathrm{LA}(\mathrm{L}, \mathrm{K})$-Oberschwingung bei $670 \mathrm{~cm}^{-1}$, eine $2 \mathrm{TO}(\mathrm{W})$-Oberschwingung bei $942 \mathrm{~cm}^{-1}$ sowie eine $2 \mathrm{TO}(\mathrm{L})$ Oberschwingung bei $978 \mathrm{~cm}^{-1}$ beobachten 262,263. Weitere Moden, die auf mögliche Oberflächenrekonstruktionen des Silizium-Substrats zurückzuführen sind 264], deuten sich bei $261 \mathrm{~cm}^{-1}$ und $368 \mathrm{~cm}^{-1}$ an. Die Moden 2. Ordnung zeigen dabei eine vergleichbare Verstärkung in den TERS-Spektren wie die LTO(Г)-Mode 1. Ordnung bei $520 \mathrm{~cm}^{-1}$. 


\subsubsection{Polarisationsabhängige TERS-Messungen an $\mathrm{La}_{2} \mathrm{CoMnO}_{6}$}

Im Folgenden soll nun nach der TERS-Einführung an Farbstoff-Testproben und dem in der Literatur verbreiteten kristallinen Beispiel von polarisationsabhängigen Messungen an Silizium die TERS-Spektren dünner $\mathrm{La}_{2} \mathrm{CoMnO}_{6}$-Doppelperowskit-Schichten, die mithilfe der MAD-Technik auf STO (100)-Substraten aufgewachsen wurden, untersucht werden. Hierzu müssen zunächst die STM-TERS-Parameter an das neue Probensystem angepasst werden. Dabei sind die TERS-STM-Goldspitzen zuerst mit dem REM bezüglich ihrer Qualität zu untersuchen und anschließend geeignete Werte für den Tunnelstrom $j$ und die angelegte Spannung $U_{\text {Bias }}$ für einen stabilen Konstant-Strom-Betrieb des STM zu ermitteln. Da diese von der elektrischen Leitfähigkeit der Schichten abhängen, wurden Strom-Abstands- und Strom-Spannungs-Kennlinien des Tunnelkontakts von TERS-Goldspitze und Probenoberfläche aufgenommen. Der Betrieb erfolgt wie zuvor unter Umgebungsluft bei Raumtemperatur $(T \approx 295 \mathrm{~K})$ in einem klimatisierten Labor. In den Abbildungen 4.2.6 (a)-(c) ist die REM-Aufnahme der verwendeten TERS-STM-Goldspitze zusammen mit den dazugehörigen Strom-Spannungs- und Strom-Abstands-Kurven, gemessen mit dem TERS-STM an einer $\mathrm{La}_{2} \mathrm{CoMnO}_{6} / \mathrm{STO}$ (100)-Schicht mit einer Schichtdicke von $d=25 \mathrm{~nm}$, dargestellt.
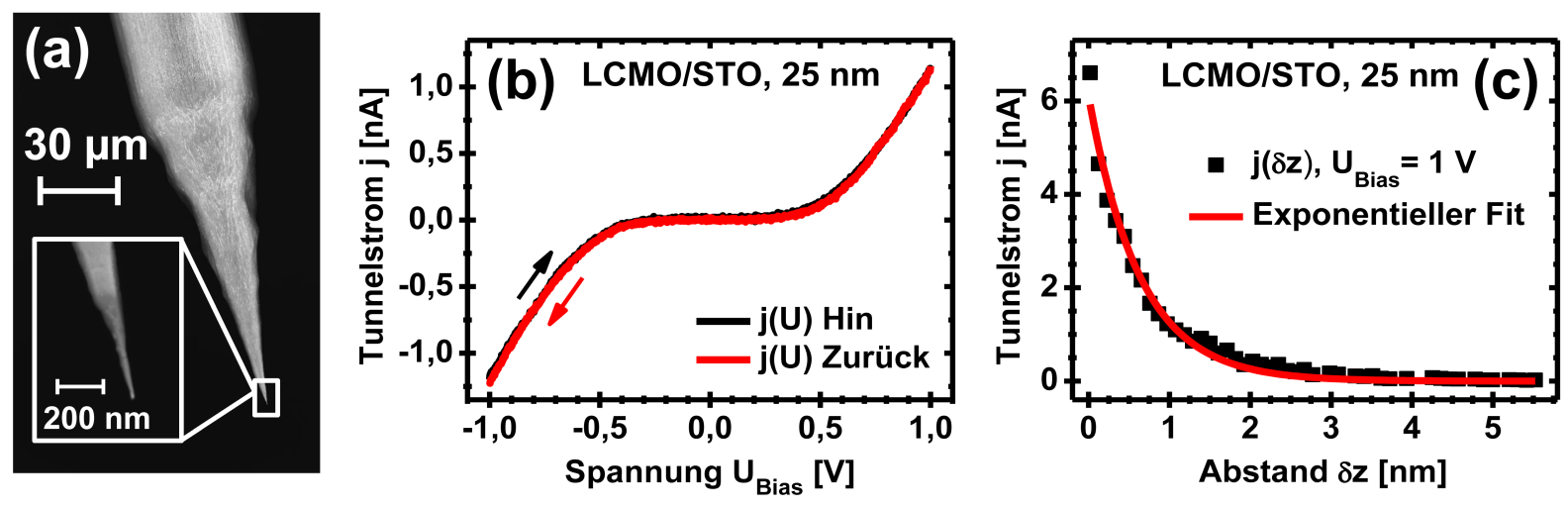

Abbildung 4.2.6: REM-Aufnahme der verwendeten TERS-STM-Goldspitze mit einem abgeschätzten Spitzenradius $R_{\text {Spitze }} \approx 20 \mathrm{~nm}$ (a) und die dazugehörigen Strom-Spannungs- (b) und Strom-Abstands-Kurven (c), gemessen an einer $\mathrm{La}_{2} \mathrm{CoMnO}_{6} / \mathrm{STO}(100)$-Schicht mit einer Schichtdicke $d=25 \mathrm{~nm}$.

Die REM-Aufnahme der TERS-Goldspitze zeigt hierbei mit einer gleichmäßigen Spitzenform, einem hohen Aspektverhältnis und einem scharfen Spitzenbereich mit einem abgeschätzten Spitzenradius von etwa $R \approx 20 \mathrm{~nm}$ eine gute Qualität. Die dazugehörige StromSpannungs-Kurve $j\left(U_{\text {Bias }}\right)$ (Hin- und Rückkurve) an der 25 nm LCMO/STO (100)-Schicht weist wiederum den für den Isolator LCMO zu erwartenden Verlauf mit einem Plateau für kleine Spannungen auf, das der LCMO-Bandlücke $E_{g} \approx 0,75 \mathrm{eV} 35$ zugeordnet werden kann (siehe Abbildung 3.2.7 (b)). Für die Strom-Spannungs-Kennlinie $j(\delta z)$ ergibt sich das nach der Gleichung (3.2.16) vorhergesagte exponentielle Abklingen des Tunnelstroms $j$ bei Vergrößerung des relativen Abstandes $\delta z$ der Spitze von der Probenoberfläche. Im Vergleich zu den vorherigen Proben (Farbstoffe auf Gold-Substrat und Silizium) führt die 
reduzierte elektrische Leitfähigkeit jedoch zu kleineren Einschränkungen bei der STMParameterwahl. So sollte ein Strom-Spannungs-Wertepaar in der $j\left(U_{\text {Bias }}\right)$-Kurve gewählt werden, für das ein stabiler Tunnelstrom und eine Position der TERS-Spitze nahe über der Probe erreicht werden kann. Gleichzeitig darf der Tunnelstrom nicht zu groß gewählt werden, um einen Kontakt mit der Probe beim Annäherungsvorgang und eine damit einhergehende Beschädigung der Spitze zu vermeiden. Eine ausführliche Darstellung dieser Thematik findet sich im Abschnitt 4.2.4 dieser Arbeit. Für die TERS-Messungen an den LCMO/STO (100)-Schichten wurde ein Konstant-Strom-Betrieb bei einem Tunnelstrom von $j=1 \mathrm{nA}$ bei einer Spannung $U_{\text {Bias }}=1 \mathrm{~V}$ (Tunnelstrom von der Spitze in Richtung Probe) gewählt. Dieses gewährleistet sowohl einen nur geringen Abstand der Spitze von der Probenoberfläche $(\delta z \leq 1 \mathrm{~nm}$, Abbildung 4.2.6 (c)) als auch einen stabilen Tunnelstrom in der Spitzenposition (Abbildung 4.2.6(b)). Die unter diesen Bedingungen in den parallelen PP- und SS-Polarisationskonfigurationen sowie in den gekreuzten PS- und SPPolarisationskonfigurationen aufgenommenen TERS- und Fernfeld-Raman-Spektren des $25 \mathrm{~nm}$ LCMO-Films auf STO (100) sind nachfolgend in der Abbildung 4.2.7 dargestellt.

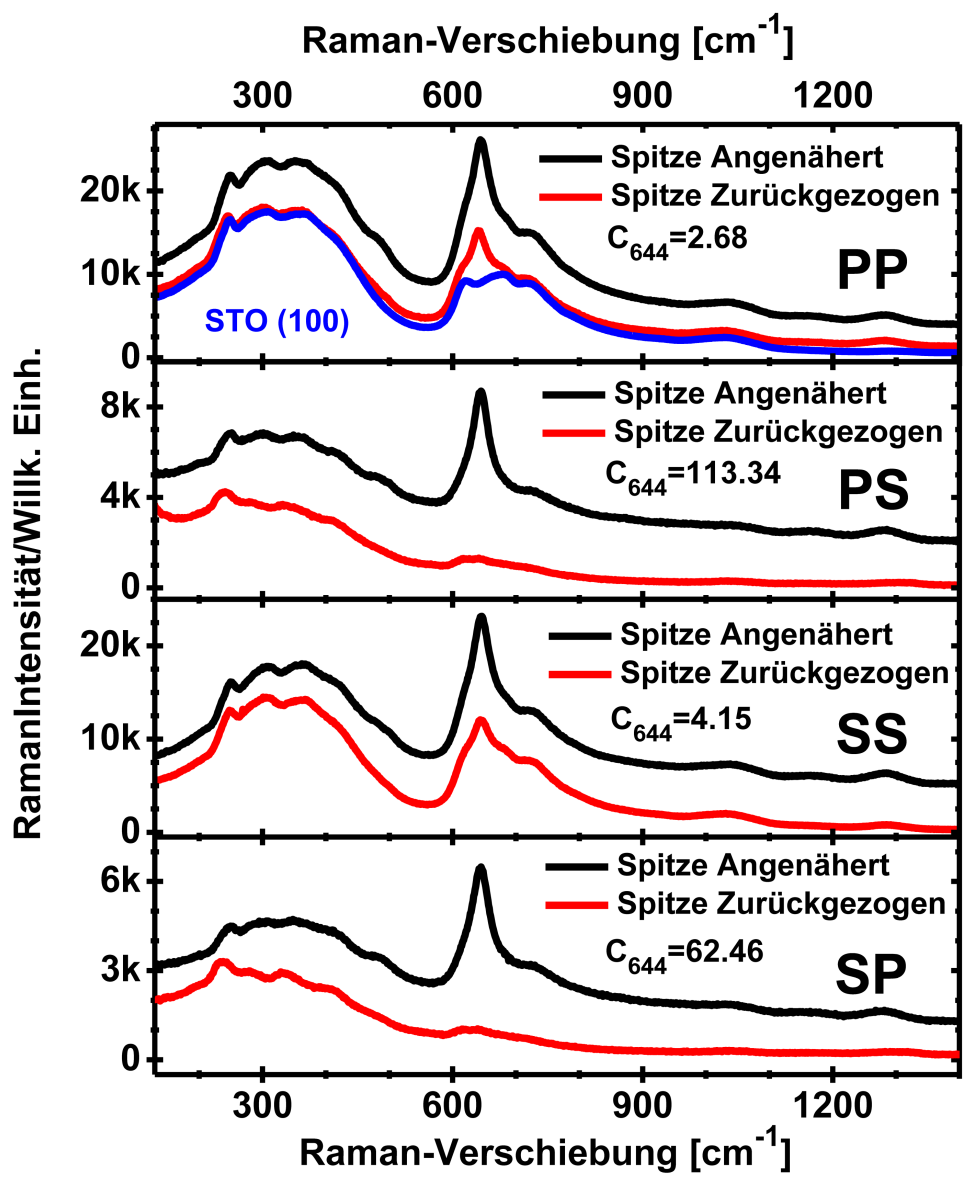

Abbildung 4.2.7: Polarisierte TERS- und Fernfeld-Raman-Spektren einer $\mathrm{La}_{2} \mathrm{CoMnO}_{6}$ - Schicht mit einer Schichtdicke $d=25 \mathrm{~nm}$ auf einem STO (100)-Substrat, aufgenommen in den parallelen PP- und SS-Polarisationskonfigurationen und in den gekreuzten PS- und SP-Polarisationskonfigurationen. Das STO (100)Raman-Spektrum zeigt die Hintergrund-Überlagerung durch das Substrat. 


\section{Auswertung und Ergebnisse}

Die Spektren wurden bei einer Laserleistung von $P \approx 10 \mathrm{~mW}(\lambda=532 \mathrm{~nm}, t=2 \times 60 \mathrm{~s})$ in einer seitlichen Einstrahlung (mit der dazugehörigen Definition des Proben- und Laborbezugssystems wie in [49] und in der Abbildung 3.4.6) aufgenommen. Charakteristisch für die Fernfeld-Raman-Spektren (Spitze mehrere Mikrometer von der Probe zurückgezogen) in der Abbildung 4.2.7 ist zunächst, dass diese den Raman-Auswahlregeln für die monokline P12/n1-Struktur der B-Platz geordnetem LCMO-Schicht folgen. Ersichtlich wird dieses an der markanten Breathing Mode bei einer Wellenzahl von $644 \mathrm{~cm}^{-1}$, die mit einer signifikanten Intensität in den parallelen PP- und SS-Polarisationskonfigurationen und einem fast vollständigen Fehlen in den gekreuzten PS- und SP-Polarisationskonfigurationen eine für geordnete LCMO-Filme zu erwartende $A_{g}$-Symmetrie aufweist und damit eine gute Übereinstimmung mit den im Kapitel 4.1 aufgenommenen polarisationsabhängigen Raman-Spektren an anderen geordneten LCMO-Filmen zeigt. Daneben ist ein starker Untergrund zu beobachten, der durch das STO-Substrat (Abbildung 4.2.7) hervorgerufen wird. Folglich ist für die $25 \mathrm{~nm}$ Schicht die Analyse auf die $A_{g}$ Breathing Mode beschränkt, da sich die anderen schwächeren Moden der Schicht nicht vom Substrat abheben können.

Erkennbar bei den spitzenverstärkten Spektren (Spitze in Tunnelposition an die Probe angenähert) ist eine deutliche Verstärkung der $A_{g}$ Breathing Mode bei $644 \mathrm{~cm}^{-1}$ in allen Polarisationskonfigurationen und insbesondere in den gekreuzten Konfigurationen sowie ein starker kontinuierlicher Untergrund, generiert durch inelastische Streuprozesse an der Goldspitze (siehe Abschnitt 3.4.6), der zusätzlich vom STO-Hintergrund überlagert ist. Für eine Quantifizierung der Verstärkung kann der TERS-Kontrast $C_{644}$ (Gleichung (3.4.3) ) für die $A_{g}$ Breathing Mode bei $644 \mathrm{~cm}^{-1}$ bestimmt werden. Die Werte sind für die verschiedenen Polarisationskonfigurationen in den Spektren in der Abbildung 4.2.7 angegeben. Es zeigt sich, dass der TERS-Kontrast $C_{644}$ eine starke Polarisationsabhängigkeit aufweist, mit einem Maximum für die gekreuzten PS- und SP-Konfigurationen, für die die $A_{g}$ Mode in der monoklinen P12/n1-Struktur verboten und damit in den Fernfeld-Spektren unterdrückt ist (für die $25 \mathrm{~nm}$ LCMO-Schicht ist fast nur der STO-Hintergrund registrierbar). Im Gegensatz dazu ist die $A_{g}$ Mode in den TERS-Spektren auch in den gekreuzten Polarisationskonfigurationen mit einer signifikanten Intensität zu beobachten, sodass sich ein sehr großer Kontrast $C_{644} \approx 60-110$ ergibt. Dieser liegt um eine Größenordnung über den Werten für die parallelen PP- und SS-Konfigurationen, für die sich mit einem Kontrast von $C_{644} \approx 2-5$ ebenfalls eine signifikante Verstärkung der $A_{g}$ Breathing Mode in den TERSMessungen ergibt, für die die $A_{g}$ Mode jedoch auch in den Fernfeld-Spektren beobachtbar ist, sodass der Kontrast gegenüber den gekreuzten Konfigurationen verringert ist [49].

Um die Überlagerung des Hintergrunds durch das STO-Substrat zu reduzieren, wurden anschließend die gleichen polarisationsabhängigen TERS- und Fernfeld-Raman-Messungen an einer 120 nm LCMO-Schicht auf STO (100), die unter den gleichen Bedingungen 
wie die $25 \mathrm{~nm}$ Schicht mithilfe der MAD-Technik hergestellt wurde, durchgeführt. Zusätzlich wurden entsprechende Spektren für eine in-plane Rotation der Probe um einen Winkel $\phi=45^{\circ}$ um die Spitzenachse herum (Abbildung 3.4.6) mit den sich ergebenden parallelen $\mathrm{P}^{\prime} \mathrm{P}^{\prime}$ - und $\mathrm{S}^{\prime} \mathrm{S}^{\prime}$-Polarisationskonfigurationen und den gekreuzten $\mathrm{P}^{\prime} \mathrm{S}^{\prime}$ - und $\mathrm{S}^{\prime} \mathrm{P}^{\prime}$ Polarisationskonfigurationen aufgenommen. Die resultierenden Spektren in den jeweiligen Polarisationskonfigurationen sind nachfolgend in der Abbildung 4.2.8 dargestellt.
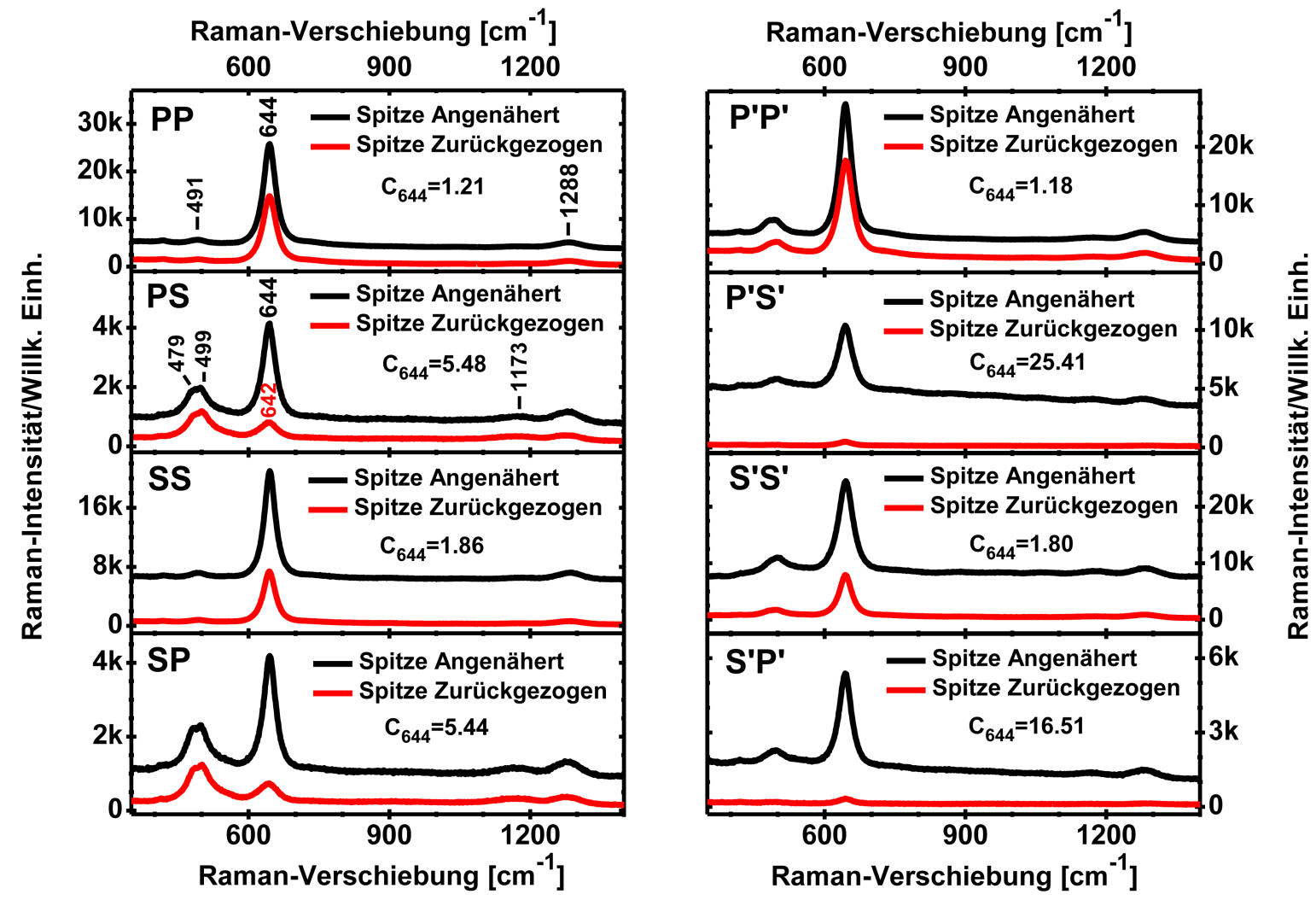

Abbildung 4.2.8: Polarisierte TERS- und Fernfeld-Raman-Spektren einer $\mathrm{La}_{2} \mathrm{CoMnO}_{6}$ - Schicht mit einer Schichtdicke $d=120 \mathrm{~nm}$ auf einem STO (100)-Substrat. Gezeigt sind die parallelen PP- und SS-Konfigurationen, die gekreuzten PS- und SPKonfigurationen sowie die $\mathrm{P}^{\prime} \mathrm{P}^{\prime}-, \mathrm{S}^{\prime} \mathrm{S}^{\prime}-, \mathrm{P}^{\prime} \mathrm{S}^{\prime}$ - und $\mathrm{S}^{\prime} \mathrm{P}^{\prime}$-Konfigurationen für eine in-plane Rotation der Schicht um den Winkel $\phi=45^{\circ}$.

Für die 120 nm LCMO-Schicht zeigt sich dabei eine vergleichbare Charakteristik für die $A_{g}$ Breathing Mode bei $644 \mathrm{~cm}^{-1}$ wie zuvor bei $25 \mathrm{~nm}$ LCMO-Schicht, jedoch mit einem reduzierten TERS-Kontrast $C_{644}$. Dieses deutet auf eine Kontrastabhängigkeit von der Schichtdicke hin und kann mit der räumlichen Ausdehnung des lokalen TERS-Nahfeldes und dem schnellen Abklingen (siehe Gleichung (3.4.6) bei Vergrößerung des Abstandes von der TERS-Spitze erklärt werden 162,174, 176 179]. So zeigt sich, dass die räumliche Ausdehnung des Nahfeldes von der Spitze in Richtung der Probe typischerweise $\approx 30 \mathrm{~nm}$ beträgt, einhergehend mit einer entsprechend hohen Oberflächensensitivität 150,172,181]. Im Gegensatz dazu besitzt das Fernfeld eine viel größere Eindringtiefe, die hauptsächlich durch die Laserwellenlänge und den Extinktionskoeffizient der Probe beeinflusst ist [266], sodass auch das vom Fernfeld erfasste Probenvolumen sehr viel mehr mit der Schichtdicke 


\section{Auswertung und Ergebnisse}

skaliert. Umgekehrt ist der Fernfeldbeitrag zum TERS-Signal für eine dünnere Schichtdicke aufgrund des verringerten Streuvolumens reduziert und ein höherer TERS-Kontrast ist zu beobachten. Eine detaillierte Untersuchung der Abhängigkeit des TERS-Signals von der Schichtdicke findet sich im Abschnitt 4.2.4 dieser Arbeit. Da eine signifikante TERS-Verstärkung der $A_{g}$ Breathing Mode sowohl in den parallelen als auch in den gekreuzten Polarisationskonfigurationen zu beobachten ist, ist der TERS-Verstärkungseffekt folglich eine Kombination einer durch die TERS-Goldspitze plasmonisch induzierten Nahfeldverstärkung und eines Nahfelddepolarisationseffektes, der die polarisationsabhängigen Raman-Auswahlregeln im Nahfeld bricht. Für die 120 nm LCMO-Schicht kann zudem das Verhalten der zweitstärksten Mode im LCMO-Spektrum, die $B_{g}$ Mischmode bei $499 \mathrm{~cm}^{-1}$, in den polarisationsabhängigen TERS-Messungen untersucht werden. Diese zeigt in den Konfigurationen mit $\phi=0^{\circ}$ eine nur geringe Verstärkung (Abbildung 4.2.8). Nach den Raman-Auswahlregeln für die monokline P12 1 /n1-Struktur ist die Mischmode mit einer $B_{g^{-}}$Symmetrie in den parallelen Konfigurationen verboten und in den gekreuzten Konfigurationen erlaubt. Ein Vergleich mit den aufgenommenen Spektren bestätigt dieses Verhalten für das Fernfeld und ohne ein Anzeichen einer auftretenden Depolarisation auch für die TERS-Messungen. Der Grund hierfür findet sich in der Charakteristik der Mischmode. So liegen in diesem Wellenzahlbereich mehrere Moden mit $A_{g^{-}}$und $B_{g^{-}}$Symmetrie sehr nah beieinander und überlagern sich, was zu Folge hat, dass sie aufgrund ihrer entgegengesetzter Polarisationsabhängigkeit der Nahfelddepolarisation entgegenwirken und diese aufheben. Da die Mischmode nur für einen Teil der Intensität in diesem Spektralbereich verantwortlich ist, ergibt sich resultierend eine geringe TERS-Verstärkung von nur $\approx 10 \%$.

Stattdessen kann für die monokline P12//n1-Struktur eine in-plane Rotation der $120 \mathrm{~nm}$ LCMO-Schicht um einen Winkel $\phi=45^{\circ}$ nicht nur genutzt werden, um Informationen über die Epitaxie der Schicht und koexistierende Phasen zu gewinnen [33], sondern auch, um die Nahfeldbeiträge zur TERS-Verstärkung für die Raman-Moden abseits der signifikanten $A_{g}$ Breathing Mode voneinander zu trennen. So sind in den parallelen $\mathrm{P}^{\prime} \mathrm{P}^{\prime}$ - und $\mathrm{S}^{\prime} \mathrm{S}^{\prime}$-Polarisationskonfigurationen sowohl die $A_{g}$ Breathing Mode bei $644 \mathrm{~cm}^{-1}$ als auch die $B_{g}$ Mischmode bei $499 \mathrm{~cm}^{-1}$ nach den Auswahlregeln für die monokline P12 $/$ n1-Struktur erlaubt, während beide in den gekreuzten $\mathrm{P}^{\prime} \mathrm{S}^{\prime}$ - und $\mathrm{S}^{\prime} \mathrm{P}^{\prime}$-Konfigurationen verboten sind [33]. In Bezug auf die Fernfeldspektren in der Abbildung 4.2 .8 zeigt sich, dass die Moden im Fernfeld diese theoretische Vorhersage bestätigen. Die schwache Intensität der $A_{g}$ Mode in den $\mathrm{P}^{\prime} \mathrm{S}^{\prime}$ - und $\mathrm{S}^{\prime} \mathrm{P}^{\prime}$-Konfigurationen lässt sich auf eine leichte Abweichung bei der in-plane Rotation der Probe zurückführen. Daneben können Verspannungen und strukturelle Verzerrungen in der Schicht zu kleinen Abweichungen der Raman-Auswahlregeln führen. Für die LCMO- und LNMO-Doppelperowskite ist zusätzlich eine Koexistenz mit der ebenfalls B-Platz geordneten rhomboedrischen R3̄-Phase möglich [33, 215, 240]. In den TERS-Spektren wiederum ist eine signifikante Verstärkung des gesamten Spektrums für 
die parallelen $\mathrm{P}^{\prime} \mathrm{P}^{\prime}$ - und $\mathrm{S}^{\prime} \mathrm{S}^{\prime}$-Polarisationskonfigurationen zu beobachten, während in den gekreuzten $\mathrm{P}^{\prime} \mathrm{S}^{\prime}$ - und $\mathrm{S}^{\prime} \mathrm{P}^{\prime}$-Konfigurationen eine starke Depolarisation für alle sichtbaren Moden im Spektrum auftritt, die mit einem großen TERS-Kontrast $C_{644} \approx 15-25$ einhergeht. Dieses Verhalten kann erneut mit der unterschiedlichen Polarisationsabhängigkeit der Raman-Moden im Fernfeld und TERS-Nahfeld und der Überlagerung mehrerer Raman-Moden mit $A_{g^{-}}$und $B_{g^{-}}$-Symmetrie im Falle der $B_{g}$ Mischmode in dem für diese Mode relevanten Wellenzahlbereich erklärt werden. In den gekreuzten $\mathrm{P}^{\prime} \mathrm{S}^{\prime}$ - und $\mathrm{S}^{\prime} \mathrm{P}^{\prime}$ Polarisationskonfigurationen sind nun nach den Raman-Auswahlregeln sowohl die Moden mit $A_{g^{-}}$als auch mit $B_{g^{-}}$Symmetrie verboten, während in den PP-, SS- PS- und SPKonfigurationen einige dieser Moden permanent zum Fernfeld-Signal beitragen. Folglich ist das Fernfeld in den $\mathrm{P}^{\prime} \mathrm{S}^{\prime}$ - und $\mathrm{S}^{\prime} \mathrm{P}^{\prime}$-Polarisationskonfigurationen nahezu vollständig unterdrückt und einzig das TERS-Nahfeld liefert einen Intensitätsbeitrag zum Gesamtfeld.

Induziert von der Wechselwirkung mit der TERS-Spitze, weist das Nahfeld nun zu einem gewissen Grad eine Depolarisation auf, wodurch die Polarisationskonfiguration für das Nahfeld zu einem Teil effektiv in eine parallele $\mathrm{P}^{\prime} \mathrm{P}^{\prime}$ - oder $\mathrm{S}^{\prime} \mathrm{S}^{\prime}$-Konfiguration umgeschaltet wird, sodass die eigentlich verbotenen Raman-Moden im TERS-Spektrum erscheinen. Dabei ist ersichtlich, dass der Depolarisationseffekt des Nahfeldes nur für gekreuzte Polarisationskonfigurationen mit einem signifikanten Intensitätsbeitrag der Raman-Moden im TERS-Gesamtfeld auftreten kann, während in den parallelen Konfigurationen das partielle Drehen in eine effektiv gekreuzte Konfiguration kein zusätzliches Signal für die $A_{g}$ und $B_{g}$ Moden im TERS-Gesamtfeld liefert. Folglich trägt in den parallelen Polarisationskonfigurationen nur der nicht-depolarisierte Anteil des plasmonischen Spitzennahfeldes zur TERS-Signalverstärkung in den Spektren bei. Ein Vergleich mit den TERS-Messungen an dem Silizium-Substrat mit (100)-Orientierung in dem Abschnitt 4.2 .2 und in der Literatur an kristallinem Silizium $169,170,192,194$ und GaAS 193 zeigt hierbei eine gute Übereinstimmung mit einer ebenso starken Polarisationsabhängigkeit des TERS-Kontrastes der Raman-Moden mit einem in gleicher Art beobachteten Nahfelddepolarisationseffekt.

Für eine quantitative Bestimmung und Trennung der durch die Spitze induzierten plasmonischen Nahfeldverstärkung und der Nahfelddepolarisation können nun die Beiträge der zwei Komponenten zum TERS-Gesamtfeld in Abhängigkeit der Orientierung der linearen Polarisation des einfallenden Laserlichts in Bezug zur TERS-Spitzenachse betrachtet werden, indem das als Polarisator zum Einsatz kommende $\lambda / 2$-Plättchen systematisch um einen Winkel $\theta$ (hier $\Delta \theta=15^{\circ}$ in einem Bereich von $\theta=0-180^{\circ}$ ) relativ zur ursprünglichen Laserpolarisation (S-Polarisation im verwendeten TERS-Messaufbau) gedreht wird. Die Analyse der Polarisationsabhängigkeit soll nun zunächst mit der 25 nm LCMO-Schicht auf STO (100) und der $A_{g}$ Breathing Mode bei $644 \mathrm{~cm}^{-1}$ begonnen werden, da die Mode für diese Schicht einen sehr großen TERS-Kontrast bei einer gleichzeitig hohen Intensität 
aufweist. In den Abbildungen 4.2.9 (a) und (b) sind die integrierten Raman-Intensitäten (Flächenintegral) der $A_{g}$ Breathing Mode aus den TERS-Spektren (Spitze im Tunnelmodus an die Probe angenähert) und Fernfeld-Raman-Spektren (Spitze mehrere Mikrometer zurückgezogen) der 25 nm LCMO-Schicht nach der Korrektur um das Hintergrund-Signal vom STO (100)-Substrat (siehe Abbildung 4.2.7) in Abhängigkeit des eingestellten Polarisatorwinkels $\theta$ mit dem Analysator in der P- sowie in der S-Konfiguration dargestellt.

(a)

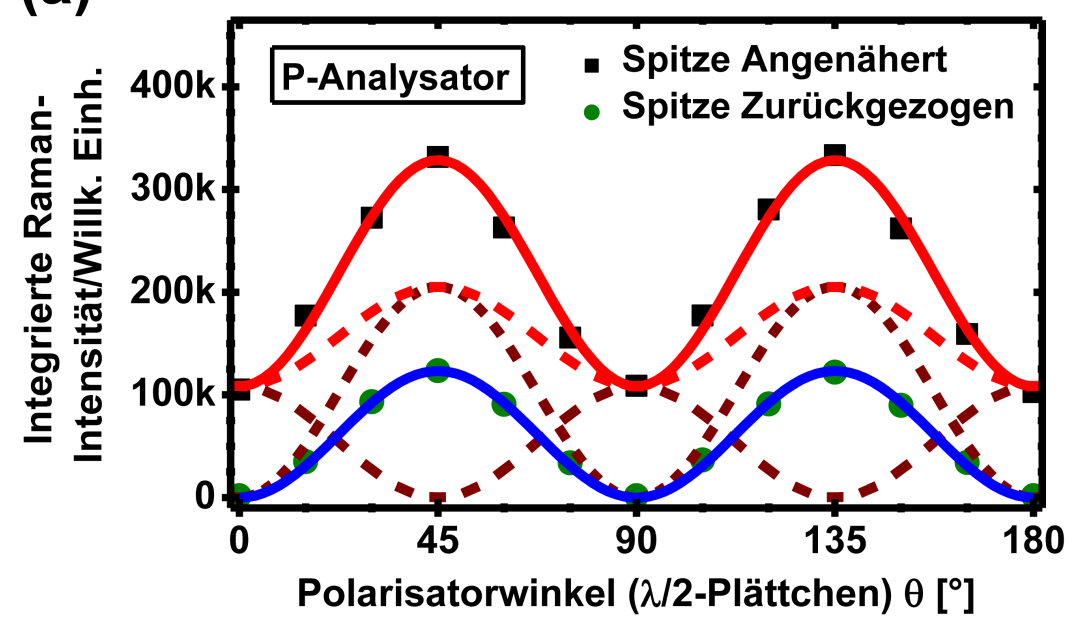

(b)

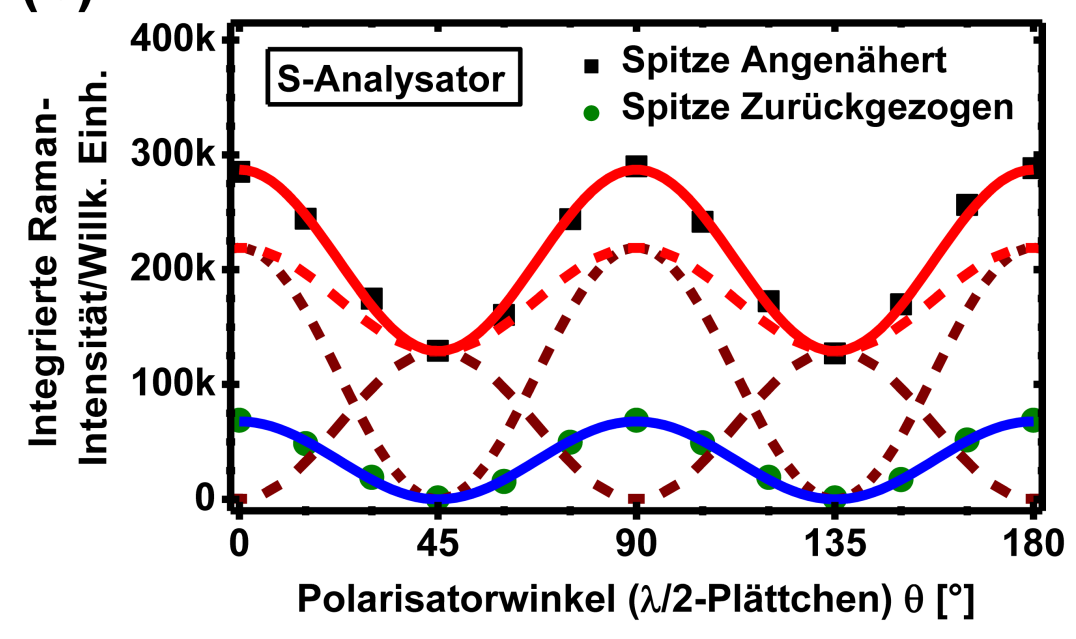

Abbildung 4.2.9: Integrierte TERS- und Raman-Intensität der $A_{g}$ Breathing Mode bei $644 \mathrm{~cm}^{-1}$ der $d=25 \mathrm{~nm}$ dicken $\mathrm{La}_{2} \mathrm{CoMnO}_{6}$-Schicht auf STO (100) in Abhängigkeit des Polarisatorwinkels $\theta$ ( $\lambda / 2$-Plättchen) für den Analysator in P-Konfiguration (a) und in S-Konfiguration (b). Die Linien zeigen das berechnete Gesamtfeld (rot) und Fernfeld (blau), während die gestrichelten Linien das jeweils bestimmte Nahfeld (rot) mit den feldverstärkenden und depolarisierenden Komponenten (braun) kennzeichnen.

Die Fernfeldintensität der $A_{g}$ Breathing Mode ist dabei durch die Raman-Auswahlregeln (Gleichung (3.3.14) ) und den Raman-Tensor für Moden mit $A_{g}$-Symmetrie in der monoklinen P12//n1-Struktur (Gleichung 4.1.1) ) der B-Platz geordnetem LCMO-Schicht vorgegeben. Die Überführung des Probenbezugssystems in das Laborbezugssystem ergibt sich 
nach der Abbildung 3.4.6 durch eine Rotation um den Winkel $\beta=90^{\circ}-\alpha=60^{\circ}$ um die $\mathrm{y}$-Achse im mathematisch positiven Sinn, wobei $\beta$ den Winkel zwischen der Richtung der einfallenden Strahlung und der Spitzenachse, die entlang der Normalen der Probenoberfläche orientiert ist, beschreibt. Folglich kann die Gleichung 3.4.9) für die Überführung des $A_{g}$-Symmetrie-Raman-Tensors in das Laborbezugssystem verwendet werden. Anhand des gewählten Probenbezugssystems und der c-Achsenorientierung der LCMO-Schichten auf STO (100) und der senkrechten Orientierung der TERS-Spitze zur Probenoberfläche folgt für die einfallenden und gestreuten elektrischen Feldvektoren $\hat{e}_{i}$ und $\hat{e}_{s}$ entsprechend:

$$
\hat{e}_{i}=\left(\begin{array}{c}
0 \\
\cos (2 \theta) \\
\sin (2 \theta)
\end{array}\right), \quad \hat{e}_{s}^{\mathrm{P}}=\left(\begin{array}{l}
0 \\
0 \\
1
\end{array}\right) \quad \text { (P-Analysator), } \quad \hat{e}_{s}^{\mathrm{S}}=\left(\begin{array}{l}
0 \\
1 \\
0
\end{array}\right) \text { (S-Analysator) }
$$

Für die Fernfeldintensität der $A_{g}$ Breathing Mode der B-Platz geordneten LCMO-Filme mit monokliner P12 $/$ n1-Struktur ergibt sich daraus mithilfe der Gleichung (3.3.14) die Abhängigkeit vom Polarisatorwinkel $\theta$ für den Analysator jeweils in P- und S-Konfiguration:

$$
\begin{aligned}
& I_{\mathrm{ff}}^{\mathrm{P}}(\theta) \propto \sin ^{2}(2 \theta), \quad\left(\mathrm{A}_{\mathrm{g}}, \mathrm{P} \text {-Analysator }\right) \\
& I_{\mathrm{ff}}^{\mathrm{S}}(\theta) \propto \cos ^{2}(2 \theta), \quad\left(\mathrm{A}_{\mathrm{g}}, \text { S-Analysator }\right)
\end{aligned}
$$

Die Gleichungen 4.2.3 und 4.2.4 geben dabei direkt die Raman-Auswahlregeln der $A_{g}$ Breathing Mode mit einer maximalen Intensität in den parallelen Polarisationskonfigurationen und einem Verbot in den gekreuzten Polarisationskonfigurationen wieder (für Moden mit $B_{g}$-Symmetrie ergibt sich entsprechend $I_{\mathrm{ff}}^{\mathrm{P}}(\theta) \propto \cos ^{2}(2 \theta)$ und $\left.I_{\mathrm{ff}}^{\mathrm{S}}(\theta) \propto \sin ^{2}(2 \theta)\right)$. Im Vergleich der vorhergesagten Abhängigkeit der Fernfeldintensität $I_{\mathrm{ff}}(\theta)$ vom eingestellten Polarisatorwinkel $\theta$ (Abbildungen 4.2.9 (a) und (b); die jeweils blaue durchgezogene Linie) mit den gemessen Fernfeldintensitäten (Spitze zurückgezogen) zeigt sich eine exzellente Übereinstimmung von Messung und Vorhersage. Für die TERS-Messungen muss nun noch das plasmonisch generierte TERS-Nahfeld und der Einfluss der TERS-Spitze auf die Nahfeldintensität und die Nahfeldpolarisation einbezogen werden. Dieses kann mit dem in den Abschnitten 3.4.5 und 3.4.6 vorgestellten phänomenologischen Modell entsprechend über den Spitzenverstärkungstensor $A$ realisiert werden, der in seiner diagonalisierten Form (Gleichung (3.4.13) ) erfolgreich für die Beschreibung polarisationsabhängiger TERS-Messungen an kristallinem Silizium 169, 170 und dem Farbstoff Azobenzol [198 angewandt wurde. Um reale Spitzenformen und die Polarisationseigenschaften des Nahfelds zu berücksichtigen, muss jedoch die tensorielle Antwort über die finiten Öffnungswinkel der Spitze gemittelt werden [169]. Allgemein kann auch die nichtdiagonalisierte 


\section{Auswertung und Ergebnisse}

Form des TERS-Verstärkungstensors $A_{\text {TERS }}$ (Gleichung (3.4.15) ) verwendet werden und die einzelnen Tensoreinträge auf die Streugeometrie und das verwendete Probenbezugssystem angepasst werden 190, 199]. Ein Beispiel hierfür sind TERS-Messungen an $\mathrm{LiNbO}_{3}-$ Nanokristallen [190]. Im Zuge dieser Arbeit entstanden [49], soll nun ein darauf aufbauender Ansatz diskutiert werden, der eine genauere Betrachtung der Polarisationseigenschaften des Nahfeldes ermöglicht, indem der TERS-Tensor $A_{\text {TERS }}=A+B$ in einen diagonalisierten Spitzenverstärkungstensor $A$ für die plasmonische Nahfeldverstärkung und einen Depolarisationstensor $B$ für die Nahfelddepolarisation in der Art aufgeteilt wird, dass der Depolarisationstensor $B$ nur Nichtdiagonalelemente beinhaltet und in einer allgemeinen Form unabhängig von der Symmetrie der betrachteten Raman-Moden ist [49]:

$$
B=\left(\begin{array}{ccc}
0 & b_{1} & b_{3} \\
b_{1} & 0 & b_{2} \\
b_{3} & b_{2} & 0
\end{array}\right) .
$$

Hierbei sind die Koeffizienten $b_{1}, b_{2}$ und $b_{3}$ phänomenologische Spitzendepolarisationsfaktoren, deren Größe wie bei den Spitzenverstärkungsfaktoren von den Spitzeneigenschaften sowie von der Streugeometrie und der Polarisation der einfallenden Laserstrahlung abhängt. Mithilfe der Definition des Labor- und Probenbezugssystems können die Faktoren charakterisiert werden [49]. Für den verwendeten Messaufbau (Abbildung 3.4.6) wird ersichtlich, dass anhand des Probenbezugssystems $b_{3}=0$ gesetzt werden kann, da die dazugehörige Depolarisationskomponente in der x-z-Ebene liegt und somit keinen effektiven Depolarisationsbeitrag am Analysator mit y-z-Konfiguration im Laborbezugssystem liefert, während ein zusätzlich auftretender Beitrag zur Nahfeldverstärkung innerhalb der Streuebene bereits mithilfe des Spitzenverstärkungstensors $A$ beschrieben wird. In gleicher Weise lässt sich $b_{1}=0$ setzen, da die entsprechende Depolarisationskomponente in der x-y-Ebene bereits als Projektion auf die y-z-Ebene im Koeffizient $b_{2}$ berücksichtigt wird. Die Nahfeldintensität $I_{\text {nf }}$ lässt sich anschließend über die Summe der feldverstärkenden Beiträge mit der Intensität $I_{\mathrm{FE}}$ und der Depolarisationsbeiträge mit der Intensität $I_{\mathrm{DP}}$ bestimmen. Mithilfe der Gleichungen (3.3.14) und (3.4.14) ergibt sich für die Nahfeldintensität $I_{\mathrm{nf}}$ [49]:

$$
I_{\mathrm{nf}}=I_{\mathrm{FE}}+I_{\mathrm{DP}} \propto \sum_{j, l=1}^{2}\left|\sum_{k=1}^{n} \hat{e}_{s}^{T} A_{j}^{\prime T} \mathcal{R}_{k}^{\prime} A_{l}^{\prime} \hat{e}_{i}\right|^{2} .
$$

Dabei ist $A_{1}=A^{\prime}$ der mithilfe der Gleichung 3.4 .9 in das Laborbezugssystem überführte Spitzenverstärkungstensor $A$ und $A_{2}=B^{\prime}$ der entsprechend überführte Depolarisationstensor $B$. Da beide Tensoren auch nach einer y-Rotation in das Laborbezugssystem symmetrisch sind, folgt $A^{\prime} T=A^{\prime}$ und $B^{\prime T}=B^{\prime}$. Die Wechselwirkung des einfallenden und gestreuten Lichts mit der TERS-Spitze ergibt sich direkt aus der Gleichung 4.2.6). Nach 
Auswertung der äußeren Doppelsumme ergeben sich vier Summanden. Der erste Ausdruck beschreibt mit $A^{\prime T} \mathcal{R}_{k}^{\prime} A^{\prime}$ eine polarisationserhaltende Feldverstärkung durch das Nahfeld mit dem Spitzenverstärkungsfaktor $A$ wie in der Gleichung 3.4.14), während $B^{\prime T} \mathcal{R}_{k}^{\prime} A^{\prime}$ und $A^{\prime} T \mathcal{R}_{k}^{\prime} B^{\prime}$ die spitzeninduzierte Nahfelddepolarisation mit einer Depolarisation des einfallenden Lichts mit anschließender Verstärkung des gestreuten Signals sowie umgekehrt mit einer Verstärkung des einfallenden Lichts mit darauffolgender Depolarisation des gestreuten Lichts charakterisieren. Der letzte Summand $B^{\prime} \mathcal{R}_{k}^{\prime} B^{\prime}$ wiederum beschreibt den Fall einer spitzeninduzierten Depolarisation sowohl des einfallenden Lichts als auch des gestreuten Signals und trägt damit zum feldverstärkenden Teil des Nahfeldes bei [49]. Für die Intensität des Nahfeldes der $A_{g}$ Breathing Mode der geordneten LCMO-Filme mit monokliner P12 1 /n1-Struktur (für LCMO ist $n=1$, da die Raman-Moden nicht entartet sind) folgt mithilfe der Gleichung (4.2.6) für den Analysator in der P- und in der S-Konfiguration [49]:

$$
\begin{aligned}
& I_{\mathrm{nf}}^{\mathrm{P}}(\theta)=k_{\mathrm{FE}}^{\mathrm{P}} \sin ^{2}(2 \theta)+k_{\mathrm{DP}}^{\mathrm{P}} \cos ^{2}(2 \theta), \quad\left(\mathrm{A}_{\mathrm{g}}, \text { P-Analysator }\right) \\
& I_{\mathrm{nf}}^{\mathrm{S}}(\theta)=k_{\mathrm{FE}}^{\mathrm{S}} \cos ^{2}(2 \theta)+k_{\mathrm{DP}}^{\mathrm{S}} \sin ^{2}(2 \theta), \quad\left(\mathrm{A}_{\mathrm{g}}, \mathrm{S} \text {-Analysator }\right)
\end{aligned}
$$

Die Konstanten $k_{\mathrm{FE} / \mathrm{DP}}^{\mathrm{P} / \mathrm{S}}$ hängen hierbei von der Spitzen-Proben-Wechselwirkung ab und charakterisieren die Stärke des feldverstärkenden und depolarisierenden Anteils am Nahfeld in der betrachteten Polarisationskonfiguration der TERS-Messung (für Moden mit $B_{g^{-}}$ Symmetrie ist die Polarisationsabhängigkeit der Nahfeldbeiträge entsprechend vertauscht). Durch einen Vergleich der Gleichungen (4.2.7) und 4.2.8 mit der Abhängigkeit der jeweiligen Intensität des Fernfelds vom Polarisationswinkel $\theta$ (Gleichungen (4.2.3) und 4.2.4) zeigt sich hierbei, dass die jeweils erste Komponente die gleiche Polarisatorabhängigkeit wie im Fernfeld aufweist und damit als die plasmonisch durch die TERS-Spitze induzierte Nahfeldverstärkung identifiziert werden kann. Folglich lässt sich die zweite Komponente mit einer dazu entgegengesetzten trigonometrischen Abhängigkeit vom Polarisatorwinkel dem Depolarisationsbeitrag zuordnen. Resultierend ergibt sich für das Gesamtnahfeld $I_{\mathrm{nf}}^{\mathrm{P} / \mathrm{S}}(\theta)$ am Beispiel der $A_{g}$ Breathing Mode der B-Platz geordneten LCMO-Schichten in Abhängigkeit des Polarisatorwinkels $\theta$ eine Überlagerung beider Anteile. Dieses ist am Beispiel für die $d=25 \mathrm{~nm}$ dicke LCMO-Schicht auf STO (100) in den Abbildungen 4.2.9 (a) und (b) für beide möglichen Analysatorstellungen gezeigt (die jeweils gestrichelte rote Linie), mit einem Verschwinden des Depolarisationsanteils und einem maximalen feldverstärkenden Beitrag in den parallelen Konfigurationen sowie mit einer signifikanten Depolarisation und einem fehlenden feldverstärkenden Beitrag in den gekreuzten Polarisationskonfigurationen (gestrichelte braune Linien). Für die $25 \mathrm{~nm}$ LCMO-Schicht überwiegt in der Gesamtintensität beider Anteile der feldverstärkende Beitrag, sodass somit $k_{\mathrm{FE}}^{\mathrm{P} / \mathrm{S}}>k_{\mathrm{DP}}^{\mathrm{P} / \mathrm{S}}$ ist. Die Intensität $I_{\mathrm{tef}}=I_{\mathrm{nf}}+I_{\mathrm{ff}}$ des resultierenden spitzenverstärkten Gesamtfeldes (die jeweils 
rote durchgezogene Linie in den Abbildungen 4.2.9 (a) und (b)) ergibt sich aus der Summe beider Nahfeldbeiträge und dem dazugehörigen Fernfeld. Ein Vergleich mit den TERSMessungen an der $25 \mathrm{~nm}$ LCMO-Schicht zeigt für die Intensitätsabhängigkeit $I_{\text {tef }}(\theta)$ der $A_{g}$ Breathing Mode eine exzellente Übereinstimmung der experimentellen Daten mit dem Modell. Zudem wird die große Variation des TERS-Kontrastes $C_{644}$ (Gleichung (3.4.3) der $A_{g}$-Mode bei $644 \mathrm{~cm}^{-1}$ für die einzelnen Polarisationskonfigurationen ersichtlich. Die aus den Spektren resultierenden TERS-Kontrastwerte der $A_{g}$ Breathing Mode sind für die $25 \mathrm{~nm}$ LCMO-Schicht in Abhängigkeit des Polarisatorwinkels $\theta$ für den Analysator in der $\mathrm{P}$ - und in der S-Konfiguration in der nachfolgenden Abbildung 4.2 .10 dargestellt.

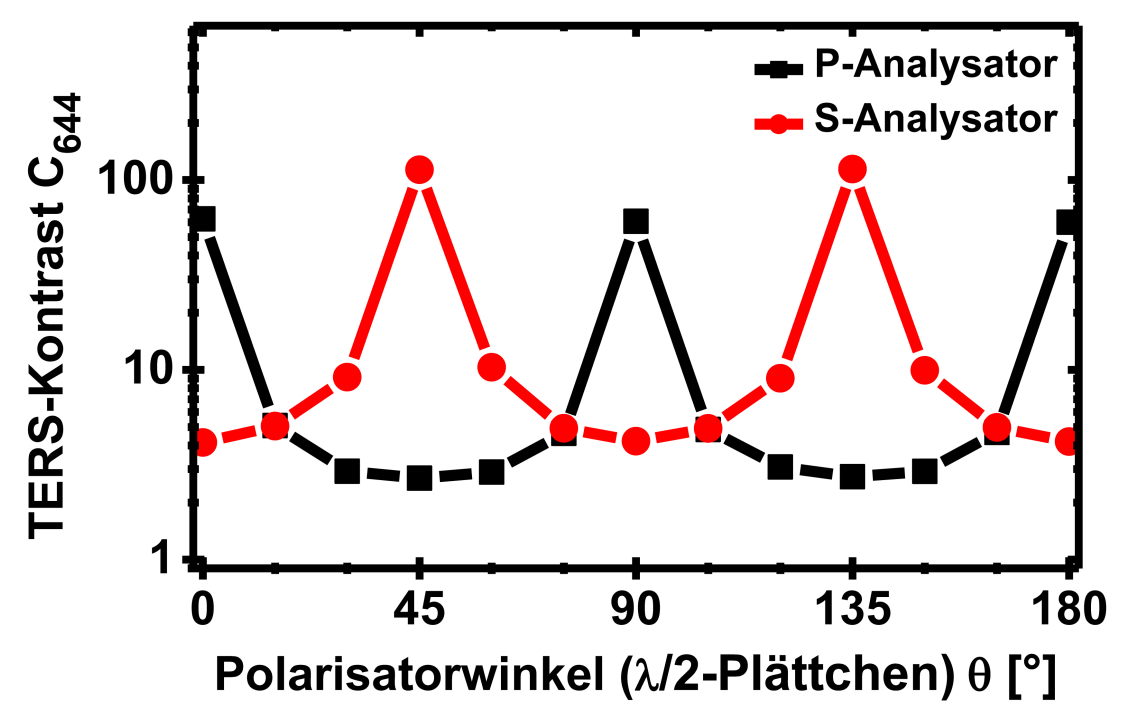

Abbildung 4.2.10: Der resultierende TERS-Kontrast $C_{644}$ für die $A_{g}$ Breathing Mode bei $644 \mathrm{~cm}^{-1}$ der $d=25 \mathrm{~nm}$ dicken $\mathrm{La}_{2} \mathrm{CoMnO}_{6} / \mathrm{STO}$ (100)-Schicht in Abhängigkeit des eingestellten Polarisatorwinkels $\theta(\lambda / 2$-Plättchen) für den Analysator in der P-Konfiguration und in der S-Konfiguration.

So führt das Verbot der $A_{g}$ Mode in den gekreuzten Polarisationskonfigurationen zu einer Unterdrückung der Intensität der Mode im Fernfeld, während im Nahfeld und damit im TERS-Gesamtfeld durch den Depolarisationseffekt durch die TERS-Spitze eine signifikante Intensität der Breathing Mode zu beobachten ist, sodass sich insgesamt ein stark erhöhter TERS-Kontrast von bis zu $C_{\text {TERS, PS }} \approx 113$ in der PS-Konfiguration ergibt. In den parallelen Konfigurationen ist die $A_{g}$ Mode hingegen aufgrund der Raman-Auswahlregeln erlaubt und mit einer deutlichen Intensität im Fernfeld-Raman-Spektrum beobachtbar, sodass die TERS-Verstärkung durch den feldverstärkenden Anteil des Nahfeldes zu einem moderaterem Kontrast von $C_{\text {TERS, SS }} \approx 4$ für die SS-Konfiguration führt. Im Allgemeinen zeigt sich der TERS-Kontrast für den Analysator in S-Konfiguration im Vergleich zu der P-Konfiguration erhöht. Die Ursache hierfür ist einerseits die unterschiedliche Intensität des Fernfeldes für die beiden Analysatorstellungen in der verwendeten Seiteneinstrahlung. Dieses kann durch einen erhöhten Reflexionsgrad des einfallenden Laserlichts mit SPolarisation an der Probenoberfläche im Vergleich zu einer Einstrahlung mit P-Polarisation 
erklärt werden. Folglich ist der an der Raman-Streuung in der Probe beteiligte Anteil der Laserleistung für eine P-Laserpolarisation größer. Detaillierte TERS-Untersuchungen in seitlicher Einstrahlungsgeometrie an Silizium-Proben mit einer quantitativen Betrachtung der Reflexions- und Transmissionsanteile für P- und S-polarisiertes Laserlicht unter Berücksichtigung des Snelliusschen Brechungsgesetzes und der Fresnelschen Formeln zeigen hierbei ebenfalls eine im Vergleich zu einer P-Polarisation geringere Raman-Intensität für die S-Polarisation [194, 197]. Da das Fernfeld frei von Depolarisationseffekten ist, zeigt sich die registrierte Fernfeld-Raman-Intensität in der SS-Konfiguration im Vergleich zur PPKonfiguration reduziert. Das Nahfeld hingegen ist aufgrund der Anregung durch die Spitze nicht von dieser Systematik betroffen, sodass der TERS-Kontrast für die SS-Konfiguration erhöht ist. Für die PS-Konfiguration wiederum ist der Nahfeldanteil gegenüber einer SPKonfiguration durch die Tendenz einer stärkeren Nahfeldanregung für eine P-Polarisation parallel zur Spitzenachse erhöht, woraus sich auch ein stärkerer Depolarisationsanteil ergibt. Insgesamt lässt sich somit ein erhöhter TERS-Kontrast für einen Analysator in SStellung sowohl in paralleler als auch in gekreuzter Polarisationskonfiguration beobachten.

Aufgrund des starken Hintergrundsignals durch das STO (100)-Substrat für die $25 \mathrm{~nm}$ LCMO-Schicht ist eine Analyse der Abhängigkeit der TERS- und Fernfeldintensitäten von der einfallenden Laserpolarisation auf die besonders starke $A_{g}$ Mode beschränkt. Für eine Analyse der $B_{g}$ Mischmode, die zusätzlich von den Anteilen weiterer schwächerer Moden mit $A_{g^{-}}$und $B_{g^{-}}$-Symmetrie überlagert ist, muss daher die $120 \mathrm{~nm}$ dicke LCMO-Schicht auf STO (100) betrachtet werden, da die Spektren bei dieser Schichtdicke nicht mehr vom STO-Hintergrund überlagert sind. Von besonderem Interesse ist für die monokline $\mathrm{P} 12_{1} /$ n1-Struktur die in-plane Rotation der LCMO-Schicht um einen Winkel $\phi=45^{\circ}$ um die Spitzenachse herum, da in diesem Fall für die $A_{g}$ und $B_{g}$ Moden im Fernfeld nach den Raman-Auswahlregeln die gleiche Polarisationsabhängigkeit vorhergesagt wird und die im Wellenzahlbereich der $B_{g}$ Mischmode nah beieinander liegenden Moden mit $A_{g^{-}}$und $B_{g}$-Symmetrie sich durch die Nahfelddepolarisation nicht mehr gegensätzlich überlagern.

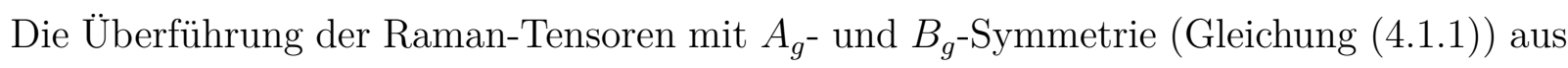
dem Probenbezugssystem in das Laborbezugssystem (Abbildung 3.4.6) kann dabei wie bei der $25 \mathrm{~nm}$ LCMO-Schicht erfolgen, mit dem Unterschied, dass die in-plane Rotation um $\phi=45^{\circ}$ um die x-Achse im mathematisch negativen Sinn mithilfe der Gleichung 3.4.9 zusätzlich berücksichtigt werden muss. Alternativ lässt sich dieses durch eine Rotation der Polarisationsvektoren realisieren. Für die rotierten Polarisationsvektoren $\hat{e}_{i}^{\prime}$ und $\hat{e}_{s}^{\prime}$ folgt:

$$
\hat{e}_{i}^{\prime}=\left(\begin{array}{c}
0 \\
\cos \left(2 \theta-45^{\circ}\right) \\
\sin \left(2 \theta-45^{\circ}\right)
\end{array}\right), \quad \hat{e}_{s}^{\mathrm{P}^{\prime}}=\frac{1}{\sqrt{2}}\left(\begin{array}{l}
0 \\
1 \\
1
\end{array}\right), \quad \hat{e}_{s}^{\mathrm{S}^{\prime}}=\frac{1}{\sqrt{2}}\left(\begin{array}{l}
0 \\
1 \\
\overline{1}
\end{array}\right)
$$




\section{Auswertung und Ergebnisse}

Für die Fernfeldintensität der Raman-Moden mit $A_{g^{-}}$und $B_{g^{-S y m m e t r i e ~}}$ der B-Platz geordneten LCMO-Filme mit monokliner P12 1 n1-Struktur folgt damit für die Abhängigkeit vom Polarisatorwinkel $\theta$ für den Analysator in der $\mathrm{P}^{\prime}$ - und in der $\mathrm{S}^{\prime}$-Konfiguration:

$$
\begin{aligned}
& I_{\mathrm{ff}}^{\mathrm{P}^{\prime}}(\theta) \propto \sin ^{2}(2 \theta), \quad\left(\mathrm{A}_{\mathrm{g}} / \mathrm{B}_{\mathrm{g}}, \mathrm{P}^{\prime} \text {-Analysator }\right), \\
& I_{\mathrm{ff}}^{\mathrm{S}^{\prime}}(\theta) \propto \cos ^{2}(2 \theta), \quad\left(\mathrm{A}_{\mathrm{g}} / \mathrm{B}_{\mathrm{g}}, \mathrm{S}^{\prime} \text {-Analysator }\right) .
\end{aligned}
$$

Die gleiche Abhängigkeit der Fernfeldintensität vom Polarisatorwinkel $\theta$ für beide Symmetrien wird dabei von den Spektren in der Abbildung 4.2.8 bestätigt, in denen die $A_{g}$ Breathing Mode und die $B_{g}$ Mischmode in den parallelen $\mathrm{P}^{\prime} \mathrm{P}^{\prime}$ - und $\mathrm{S}^{\prime} \mathrm{S}^{\prime}$-Polarisationskonfigurationen stark ausgeprägt und in den gekreuzten $\mathrm{P}^{\prime} \mathrm{S}^{\prime}$ - und $\mathrm{S}^{\prime} \mathrm{P}^{\prime}$-Konfigurationen stark unterdrückt sind. In den Abbildungen 4.2.11 (a)-(d) sind nun die integrierten RamanIntensitäten der $A_{g}$ Breathing Mode bei $644 \mathrm{~cm}^{-1}$ und der $B_{g}$ Mischmode bei $499 \mathrm{~cm}^{-1}$ aus den dazugehörigen TERS-Spektren (Spitze an die Probe angenähert) und FernfeldRaman-Spektren (Spitze zurückgezogen) der $120 \mathrm{~nm}$ LCMO-Schicht in Abhängigkeit des Polarisatorwinkels $\theta$ mit dem Analysator in der $\mathrm{P}^{\prime}$ - und in der $\mathrm{S}^{\prime}$-Konfiguration dargestellt.
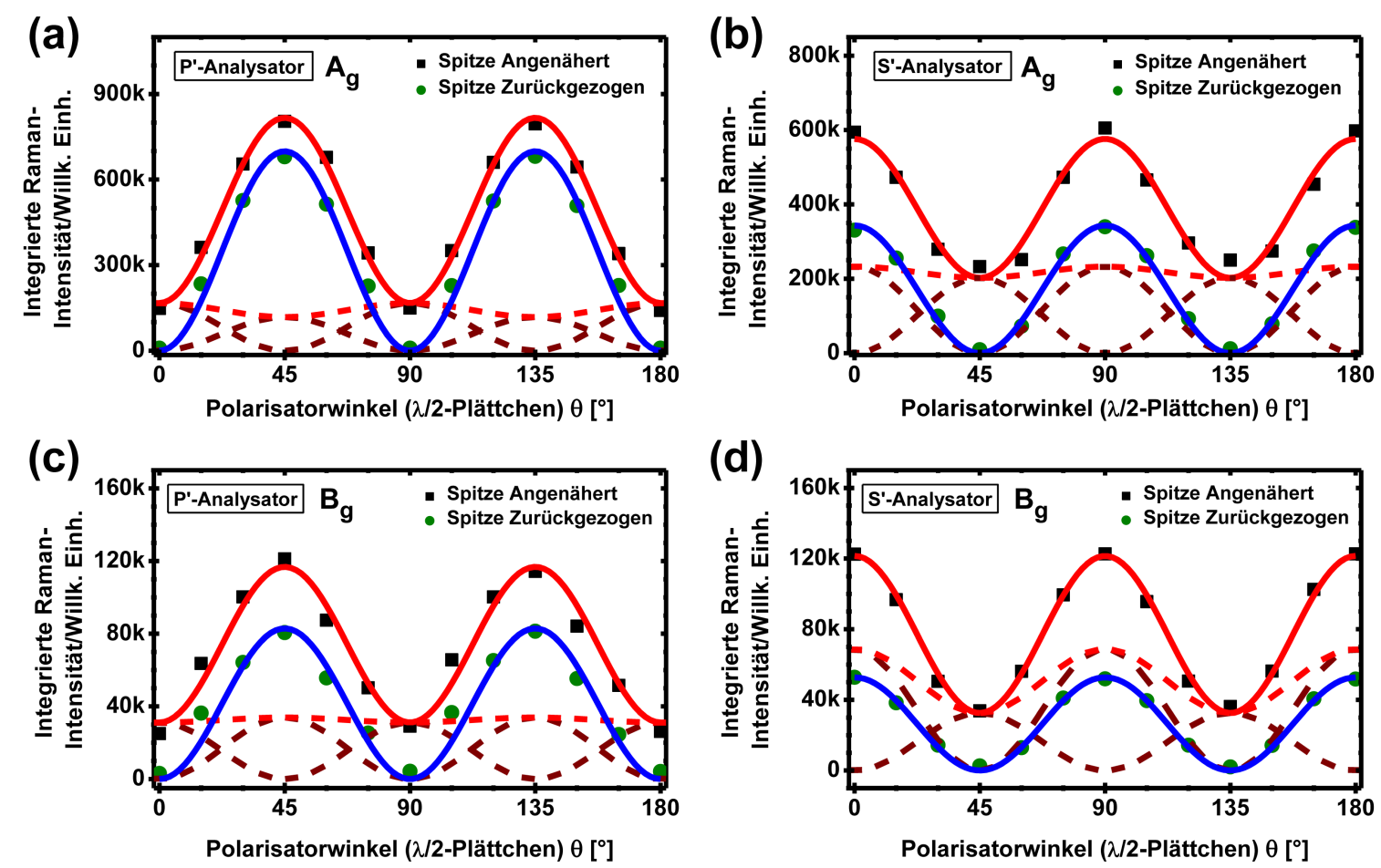

Abbildung 4.2.11: Integrierte TERS- und Raman-Intensität der $A_{g}$ Breathing Mode bei $644 \mathrm{~cm}^{-1}$ und der $B_{g}$ Mischmode bei $499 \mathrm{~cm}^{-1}$ der $120 \mathrm{~nm} \mathrm{La} \mathrm{CoMnO}_{6}$ Schicht auf STO (100) in Abhängigkeit des Polarisatorwinkels $\theta$ für den Analysator in $\mathrm{P}^{\prime}-((\mathrm{a}),(\mathrm{c}))$ und in $\mathrm{S}^{\prime}$-Konfiguration ((b), (d)). Die Linien zeigen das Gesamtfeld (rot) und Fernfeld (blau). Die gestrichelten Linien kennzeichnen das Nahfeld (rot) mit den zwei Komponenten (braun). 
Im Vergleich der vorhergesagten Abhängigkeit der Fernfeldintensität $I_{\mathrm{ff}}(\theta)$ vom eingestellten Polarisatorwinkel $\theta$ (Abbildungen 4.2.11 (a)-(d); die jeweils blaue durchgezogene Linie) mit den gemessen Fernfeldintensitäten (Spitze zurückgezogen) zeigt sich dabei für die $120 \mathrm{~nm}$ LCMO-Schicht sowohl für die $A_{g}$ Breathing Mode als als auch für die $B_{g}$ Mischmode eine exzellente Übereinstimmung der Messung mit der Vorhersage. Die Bestimmung der Abhängigkeit der Nahfeldintensität $I_{\mathrm{nf}}(\theta)$ beider Moden erfolgt hingegen wie schon zuvor bei der $25 \mathrm{~nm}$ LCMO-Schicht mithilfe der Gleichung (4.2.6) unter der Berücksichtigung der zusätzlichen in-plane Rotation um $\phi=45^{\circ}$. Für die Intensität des Nahfeldes der beiden Moden ergibt sich somit für den Analysator in der $\mathrm{P}^{\prime}$ - und in der $\mathrm{S}^{\prime}$ - Konfiguration [49]:

$$
\begin{aligned}
& I_{\mathrm{nf}}^{\mathrm{P}^{\prime}}(\theta)=k_{\mathrm{FE}}^{\mathrm{P}^{\prime}} \sin ^{2}(2 \theta)+k_{\mathrm{DP}}^{\mathrm{P}^{\prime}} \cos ^{2}(2 \theta), \quad\left(\mathrm{A}_{\mathrm{g}} / \mathrm{B}_{\mathrm{g}}, \mathrm{P}^{\prime} \text {-Analysator }\right), \\
& I_{\mathrm{nf}}^{\mathrm{S}^{\prime}}(\theta)=k_{\mathrm{FE}}^{\mathrm{S}^{\prime}} \cos ^{2}(2 \theta)+k_{\mathrm{DP}}^{\mathrm{S}^{\prime}} \sin ^{2}(2 \theta), \quad\left(\mathrm{A}_{\mathrm{g}} / \mathrm{B}_{\mathrm{g}}, \mathrm{S}^{\prime} \text {-Analysator }\right) .
\end{aligned}
$$

Die jeweils für die Stärke des feldverstärkenden und des depolarisierenden Anteils am Nahfeld charakteristischen Konstanten $k_{\mathrm{FE} / \mathrm{DP}}^{\mathrm{P}^{\prime} / \mathrm{S}^{\prime}}$ unterscheiden sich dabei im Allgemeinen für die $A_{g}$ Breathing Mode und die $B_{g}$ Mischmode sowie von denjenigen Konstanten für den Fall ohne betrachtete in-plane Rotation in den Gleichungen 4.2.7 und 4.2.8. Da bereits im Fernfeld die Intensitätsabhängigkeit vom Polarisatorwinkel $\theta$ für die $A_{g}$ und $B_{g}$ Moden für eine in-plane Rotation der LCMO-Schicht um $\phi=45^{\circ}$ gleich ist, ergibt sich eine gleiche Abhängigkeit vom Polarisatorwinkel $\theta$ für beide Symmetrien auch für das TERS-Nahfeld. Ein Vergleich der Gleichungen 4.2.12 und 4.2.13 mit der jeweiligen Intensitätsabhängigkeit des Fernfeldes vom Polarisationswinkel $\theta$ (Gleichungen 4.2.10 und 4.2.11) zeigt hierbei, dass die jeweils erste Komponente die gleiche Polarisatorabhängigkeit wie im Fernfeld aufweist und daher entsprechend als die plasmonisch durch die TERS-Spitze induzierte Nahfeldverstärkung identifiziert werden kann, während die zweite Komponente mit einer dazu entgegengesetzten trigonometrischen Abhängigkeit vom Polarisatorwinkel sich folglich dem Nahfelddepolarisationsbeitrag zuordnen lässt. Der Unterschied ist nun jedoch, dass für eine in-plane Rotation der LCMO-Schicht um $\phi=45^{\circ}$ die Nahfelddepolarisation auch bei der $B_{g}$ Mischmode in den gekreuzten $\mathrm{P}^{\prime} \mathrm{S}^{\prime}$ - und $\mathrm{S}^{\prime} \mathrm{P}^{\prime}$-Polarisationskonfigurationen eine Verstärkung im TERS-Gesamtfeld bewirkt, da die Polarisationsabhängigkeit der $B_{g}$ Moden in diesem Fall der der $A_{g}$ Moden gleicht und somit beide Symmetrien in den gekreuzten Konfigurationen verboten sind. Dieses wird durch die Fernfeld-Raman-Spektren in der Abbildung 4.2 .8 bestätigt, während in den TERS-Spektren signifikante Intensitäten der Moden zu beobachten sind. Da sowohl die $A_{g}$ als auch die $B_{g}$ Moden zudem in den parallelen $\mathrm{P}^{\prime} \mathrm{P}^{\prime}$ - und $\mathrm{S}^{\prime} \mathrm{S}^{\prime}$-Polarisationskonfigurationen verstärkt werden, lässt sich die Verstärkung durch das Nahfeld erneut als Kombination einer plasmonischen Verstärkung und eines Depolarisationseffektes beschreiben. Das resultierende Gesamtnahfeld ist in den 
Abbildungen 4.2.11(a)-(d) für beide Symmetrien und beide mögliche Analysatorstellungen gezeigt (die jeweils gestrichelte rote Linie), mit einem Verschwinden des Depolarisationsanteils und einem maximalen feldverstärkenden Beitrag in den parallelen Konfigurationen sowie mit einer signifikanten Depolarisation und einem Fehlen des feldverstärkenden Beitrags in den gekreuzten Polarisationskonfigurationen (gestrichelte braune Linien). Die Intensität $I_{\text {tef }}=I_{\mathrm{nf}}+I_{\mathrm{ff}}$ des spitzenverstärkten Gesamtfeldes (die jeweils rote durchgezogene Linie in den Abbildungen 4.2.11 (a)-(d)) ergibt sich auch hier aus der Summe beider Nahfeldbeiträge und dem jeweiligen Fernfeld. Ein Vergleich mit den TERS-Messungen an der 120 $\mathrm{nm}$ LCMO-Schicht mit der in-plane Rotation der Schicht um $\phi=45^{\circ}$ zeigt hierbei für die Intensitätsabhängigkeit $I_{\text {tef }}(\theta)$ sowohl der $A_{g}$ Breathing Mode als auch der $B_{g}$ Mischmode eine exzellente Übereinstimmung der experimentellen Daten mit dem Modell. Auch hier lässt sich an dem Verlauf der Intensitäten des TERS-Gesamtfeldes und des Fernfeldes eine große Variation des TERS-Kontrastes beider Moden für die einzelnen Polarisationskonfigurationen beobachten. Die resultierenden TERS-Kontrastwerte $C_{644}$ der $A_{g}$ Breathing Mode bei $644 \mathrm{~cm}^{-1}$ und $C_{499}$ der $B_{g}$ Mischmode bei $499 \mathrm{~cm}^{-1}$ sind für die $120 \mathrm{~nm}$ LCMO-Schicht in Abhängigkeit des Polarisatorwinkels $\theta$ für den Analysator in der $\mathrm{P}^{\prime}$-Konfiguration und in der $\mathrm{S}^{\prime}$ - Konfiguration in den Abbildungen 4.2 .12 (a) und (b) dargestellt.
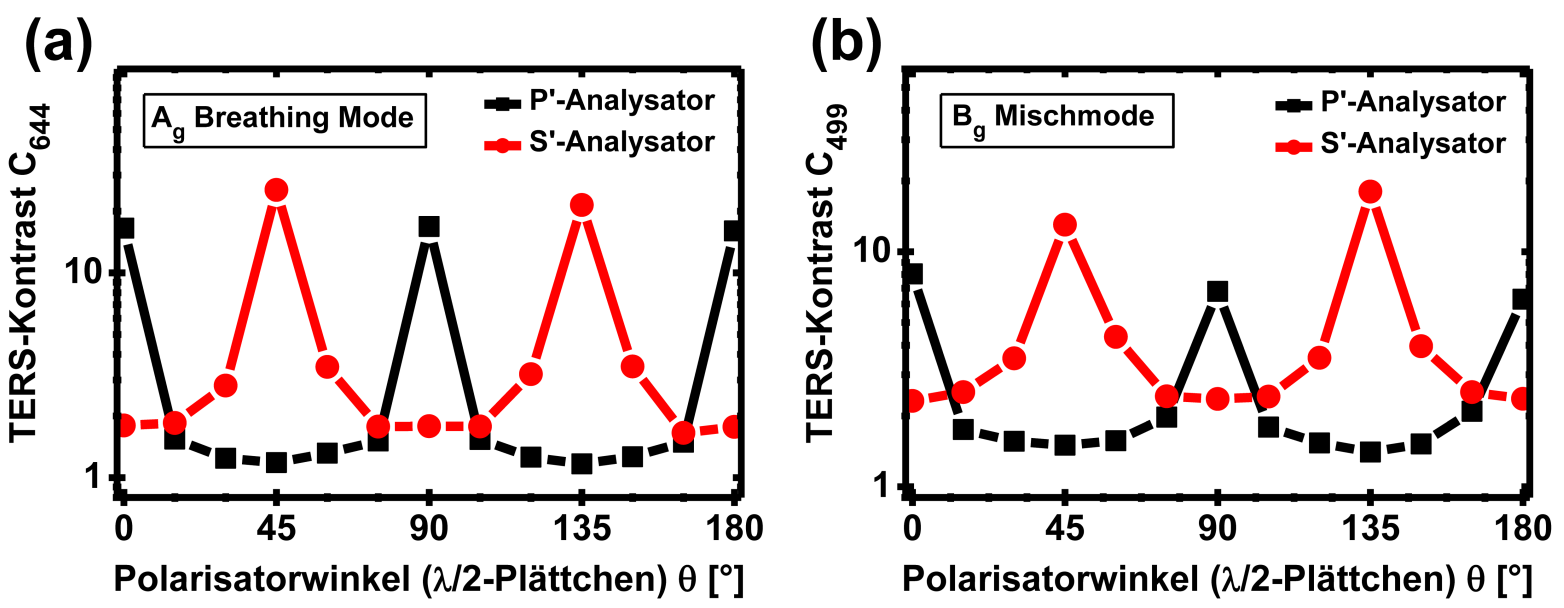

Abbildung 4.2.12: Der resultierende TERS-Kontrast $C_{644}$ für die $A_{g}$ Breathing Mode bei $644 \mathrm{~cm}^{-1}$ (a) und der jeweilige TERS-Kontrast $C_{499}$ der $B_{g}$ Mischmode bei $499 \mathrm{~cm}^{-1}$ (b) der $d=120 \mathrm{~nm}$ dicken $\mathrm{La}_{2} \mathrm{CoMnO}_{6} / \mathrm{STO}$ (100)-Schicht in Abhängigkeit des eingestellten Polarisatorwinkels $\theta(\lambda / 2$-Plättchen) für den Analysator in der $\mathrm{P}^{\prime}$-Konfiguration und in der $\mathrm{S}^{\prime}$-Konfiguration.

Dabei führt das Verbot der $A_{g}$ Mode und der $B_{g}$ Mode in den gekreuzten Polarisationskonfigurationen zu einer nahezu vollständigen Unterdrückung der Moden im Fernfeld, während im TERS-Gesamtfeld durch den Depolarisationseffekt des Spitzennahfeldes eine signifikante Intensität für beide Moden und somit ein hoher Kontrast von bis zu $C_{644, \mathrm{P}^{\prime} \mathrm{S}^{\prime}} \approx 25$,

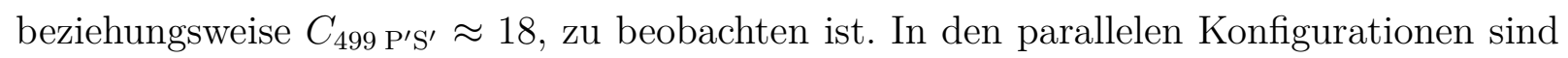
beide Moden hingegen im Fernfeld erlaubt, sodass sich durch die Nahfeldverstärkung ein moderater TERS-Kontrast $\left(C_{644, \mathrm{P}^{\prime} \mathrm{P}^{\prime}} \approx 1,2, C_{499, \mathrm{P}^{\prime} \mathrm{P}^{\prime}} \approx 1,5\right)$ für beide Moden ergibt. 


\subsubsection{Einfluss der STM-Parameter und TERS-Oberflächensensitivität}

Anschließend soll der Fokus auf einige der Rahmenparameter zur spitzenverstärkten RamanSpektroskopie gelegt werden, die bei der Verwendung der TERS-Technik auftreten und zu beachten sind. Ein Großteil der nachfolgend präsentierten Messungen ist dabei im Zuge der im vorherigen Abschnitt gezeigten polarisationsabhängigen TERS- und Raman-Messungen an dünnen $\mathrm{La}_{2} \mathrm{CoMnO}_{6}$-Schichten auf STO (100) und der damit einhergehenden Veröffentlichung in [49] entstanden und soll an dieser Stelle einen Einblick darauf geben, welchen Einfluss sowohl die TERS-Spitzen und die STM-Parameter im TERS-STM-Betrieb als auch die Schichtdicke der Schichten auf die resultierende TERS-Verstärkung und den zu erzielenden TERS-Kontrast haben. Eine Übersicht hierzu findet sich auch in [267]. Eine zentrale Rolle bei der TERS-Technik kommt dabei der Spitze zu, da diese für die Generierung des Nahfeldes und damit für die TERS-Verstärkung verantwortlich ist. Entsprechend wichtig ist daher die Bestimmung des Einflusses der Spitze und eine Optimierung der STMParameter für den jeweils gebildeten Tunnelkontakt von Spitze und Probe. Dieses soll hier für drei Goldspitzen anhand von Messungen an der $25 \mathrm{~nm}$ LCMO-Schicht auf STO (100) aus dem vorherigen Abschnitt 4.2.3 gezeigt werden. Die Form und der Spitzenradius werden dazu zunächst mithilfe eines Rasterelektronenmikroskops bestimmt. Die dazugehörigen Aufnahmen der Spitzen sind nachfolgend in der Abbildung 4.2.13 (a) dargestellt.

(a)

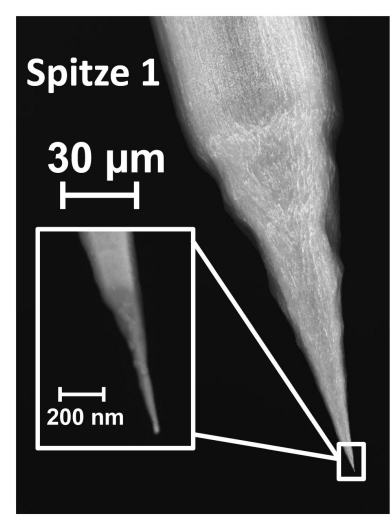

(b)

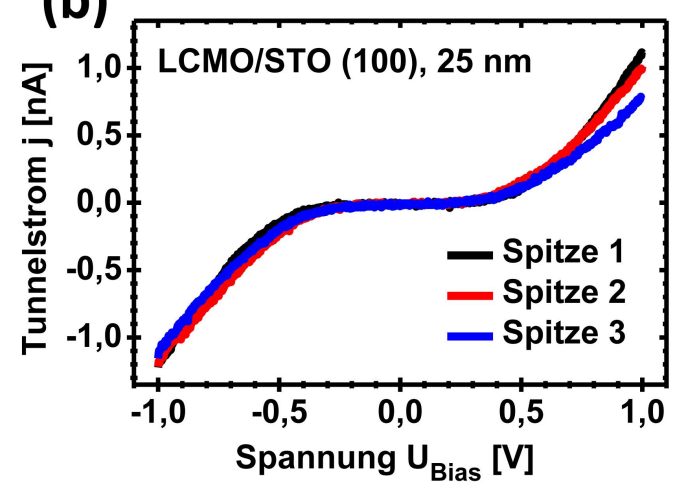

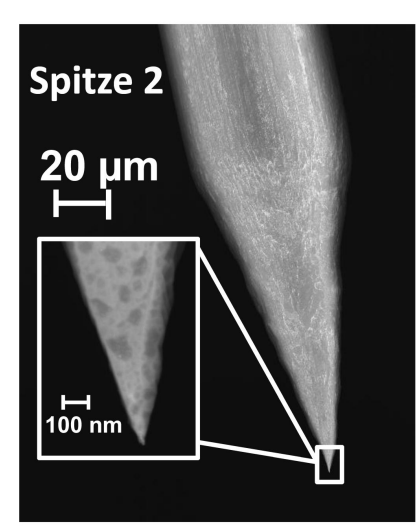

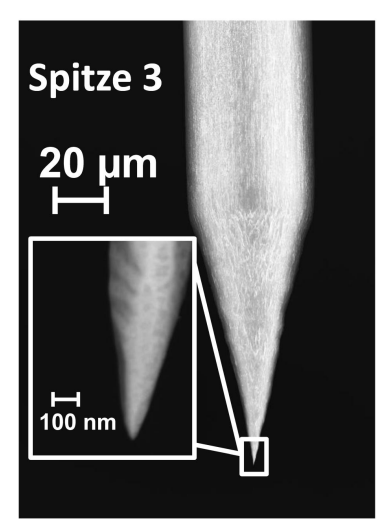

(c)

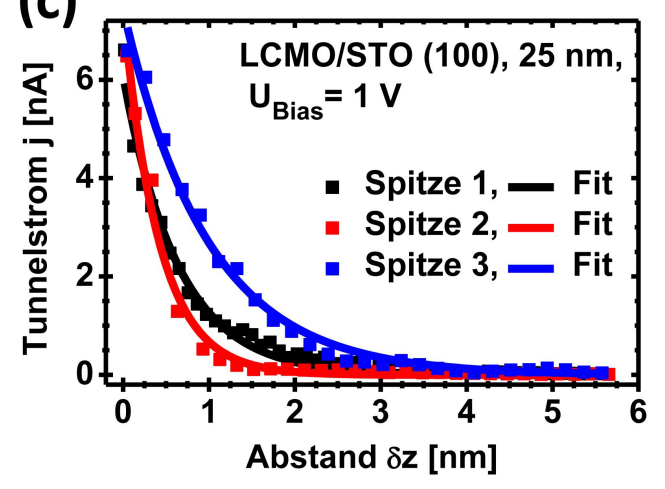

Abbildung 4.2.13: REM-Aufnahmen von drei repräsentativen TERS-STM-Goldspitzen (a) und die Strom-Spannungs- (b) und Strom-Abstands-Kurven (c), gemessen an einer $d=25 \mathrm{~nm}$ dicken $\mathrm{La}_{2} \mathrm{CoMnO}_{6} / \mathrm{STO}$ (100)-Schicht. 


\section{Auswertung und Ergebnisse}

Die Bilder des Rasterelektronenmikroskops dienen dabei einer Spitzenvorselektion. Kriterien für eine gute Spitzenqualität sind insbesondere ein kleiner Spitzenradius, ein hohes Aspektverhältnis und eine gleichmäßige Form. Diese Bedingungen treffen auf alle drei der in der Abbildung 4.2.13 (a) gezeigten Spitzen zu. Der jeweilige Spitzenradius kann auf etwa $R_{\text {Spitze }} \approx 20-25 \mathrm{~nm}$ abgeschätzt werden. Anschließend werden die drei Spitzen im STM-Betrieb charakterisiert, indem Strom-Spannungs- und Strom-Abstands-Kurven an der LCMO-Schicht aufgenommen werden (bei Raumtemperatur unter Umgebungsluft). Diese sind in den Abbildungen 4.2.13 (b) und (c) dargestellt. Hierbei zeigt sich für die drei Spitzen sehr ähnliche Strom-Spannungs-Kurven $j\left(U_{\text {Bias }}\right)$, deren Verlauf durch den Tunnelkontakt mit der LCMO-Schicht bestimmt ist. Das für kleine Spannungen auftretende Plateau kann der Bandlücke von LCMO zugeordnet werden, die mit $E_{g} \approx 0,75 \mathrm{eV}$ in guter Übereinstimmung mit elektrischen Transportmessungen an Bulk-LCMO 35 ist. Die Strom-Abstands-Kurven $j(\delta z)$ der drei Spitzen weisen zudem ein exponentielles Abfallen des Tunnelstroms mit einer Abklinglänge (Abnahme des Tunnelstroms um die Hälfte) von nur $\approx 0,5-1,0 \mathrm{~nm}$ bei Vergrößerung des relativen Abstandes $\delta z$ der Spitze von der Probenoberfläche auf, wie es für eine hohe Spitzenqualität zu erwarten ist 268. Nach dieser allgemeinen Charakterisierung können die Spitzen auf ihr Verhalten in den TERS-Messungen untersucht werden. Für die drei vorgestellten Spitzen wurden diese an der bekannten $25 \mathrm{~nm}$ LCMO-Schicht auf STO (100) in der parallelen PP-Polarisationskonfiguration und der gekreuzten PS-Polarisationskonfiguration unter identischen Bedingungen $(j=1 \mathrm{nA}$, $U_{\text {Bias }}=1 \mathrm{~V}, \lambda=532 \mathrm{~nm}, P \approx 10 \mathrm{~mW}$ und $t=2 \times 60 \mathrm{~s}$ ) durchgeführt. Die resultierenden TERS-Spektren sind mit dem dazugehörigen Fernfeld-Raman-Referenzspektrum (Spitze mehrere Mikrometer zurückgezogen) in den Abbildungen 4.2.14 (a) und (b) dargestellt.
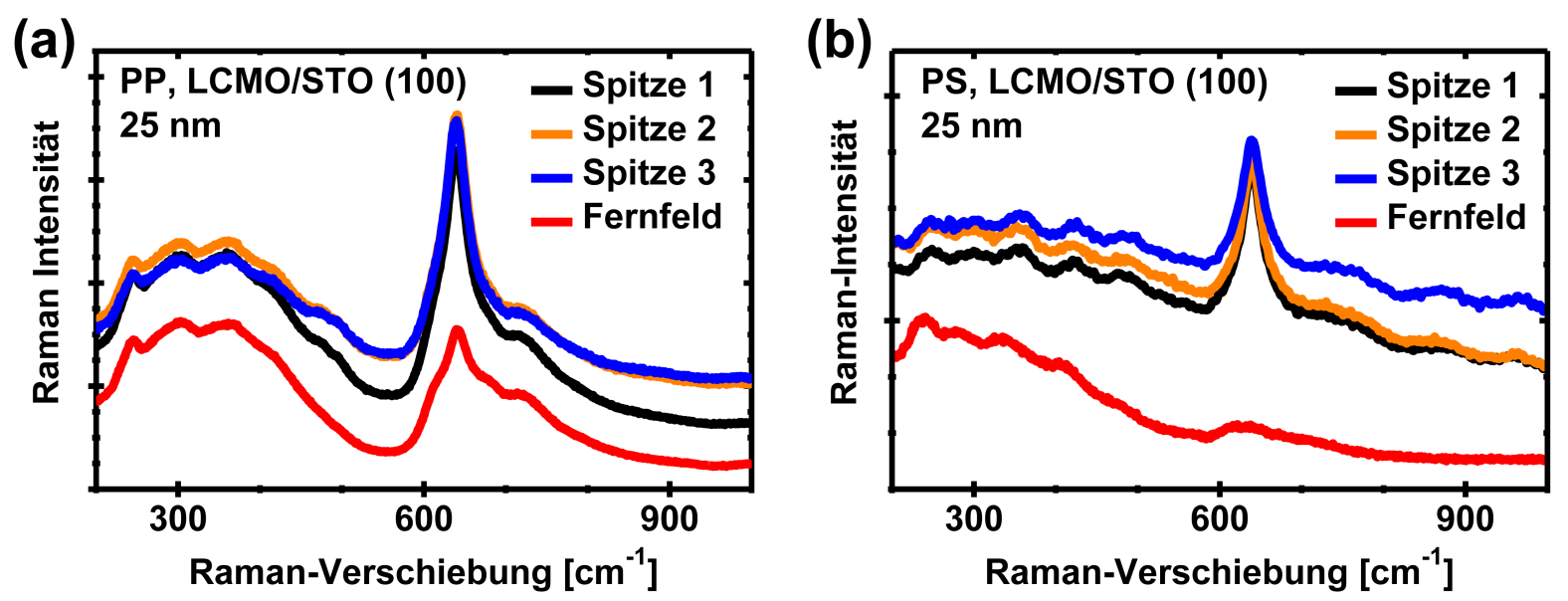

Abbildung 4.2.14: Polarisationsabhängige TERS-Messungen mit den drei zuvor charakterisierten TERS-Goldspitzen an der $25 \mathrm{~nm}$ LCMO-Schicht auf STO (100) in der parallelen PP- (a) und in der gekreuzten PS-Polarisationskonfiguration (b).

Hierbei ergibt sich für alle drei Spitzen ein sehr ähnliches Bild in beiden Polarisationskonfigurationen mit einer deutlichen Verstärkung der $A_{g}$ Breathing Mode bei $644 \mathrm{~cm}^{-1}$ in 
der parallelen PP-Konfiguration und einer ebenfalls signifikanten Intensität dieser Mode in der gekreuzten PS-Konfiguration, für die die $A_{g}$ Mode im Fernfeld verboten und damit im entsprechenden Fernfeld-Raman-Spektrum stark unterdrückt ist. Folglich ist die bereits im vorherigen Abschnitt 4.2.3 mit der Spitze 1 in den TERS-Spektren der LCMO-Filme in gekreuzter Polarisationskonfiguration beobachtete Nahfelddepolarisation auch für die anderen beiden TERS-Spitzen völlig unabhängig voneinander reproduzierbar. Ein vergleichbarer Effekt tritt zudem bei den TERS-Messungen an dem kristallinem Silizium-Substrat auf (Abschnitt 4.2.2). Die leichte Variation im kontinuierlichen Hintergrundsignal der Spektren, das durch inelastische Streuprozesse an den Spitzen selbst entsteht, kann dabei durch die leicht unterschiedlichen Spitzenformen und Spitzenabstände erklärt werden.

Nach dem Einfluss der TERS-Spitze soll nun die Wirkung der STM-Kontrollparameter (Tunnelstrom $j$, angelegte Spannung $U_{\text {Bias }}$ ) auf die TERS-Spektren untersucht werden. Hierfür ist nachfolgend in den Abbildungen 4.2.15 (a) und (b) die Abhängigkeit der TERSSpektren von den beiden STM-Parametern dargestellt. Die Messungen erfolgten hierbei mit der Spitze 1 aus der Abbildung 4.2.13 (a) an der $25 \mathrm{~nm}$ LCMO-Schicht auf STO (100) bei einer Laserpolarisation parallel (P) zur Spitzenachse und ohne Analysator.
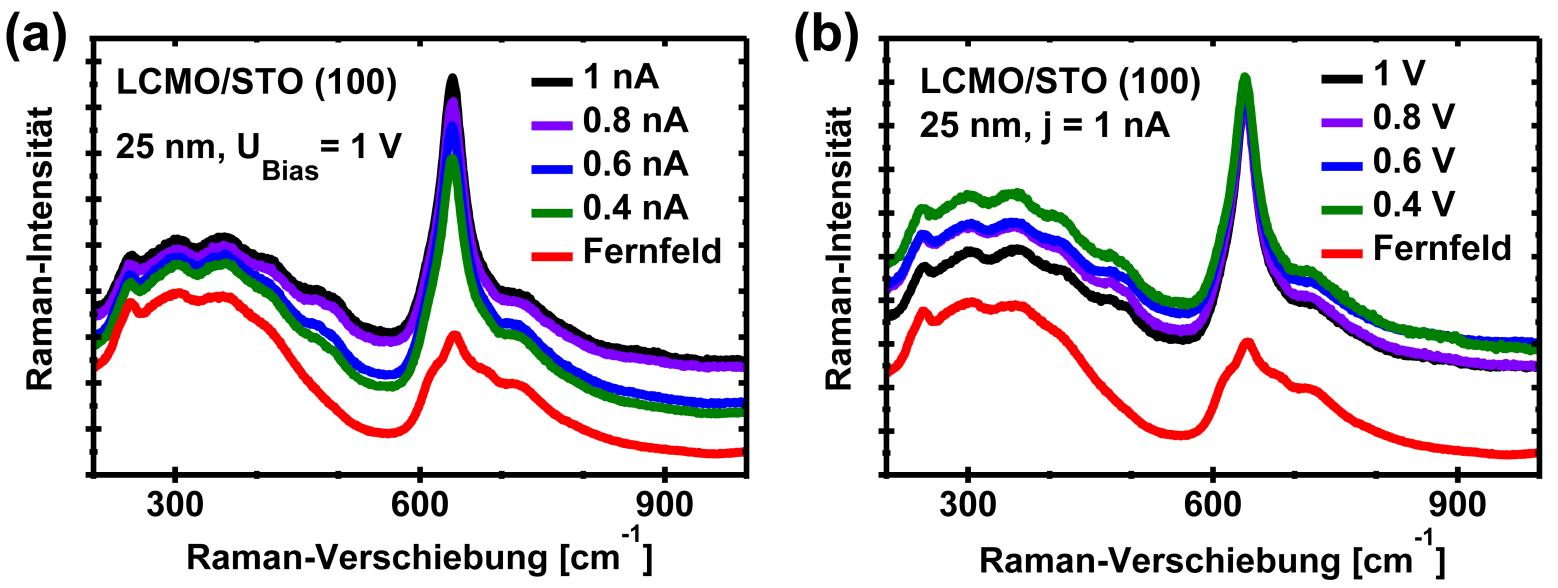

Abbildung 4.2.15: Mit der Spitze 1 aufgenommene TERS-Messungen (P-Polarisation, ohne Analysator) an der $25 \mathrm{~nm}$ LCMO-Schicht auf STO (100) in Abhängigkeit des Tunnelstroms $j$ (a) und der angelegten Spannung $U_{\text {Bias }}(\mathrm{b})$.

Der Tunnelstrom und die angelegte Spannung sind jedoch nicht völlig frei wählbar. Der jeweilige Bereich ist vielmehr durch die elektrischen Transporteigenschaften der zu untersuchenden Schicht und die dazugehörige Strom-Spannungs-Kurve (Abbildung 4.2.13 (b)) vorgegeben. So zeigte sich für die $25 \mathrm{~nm}$ LCMO-Schicht im Allgemeinen ein Tunnelstrom $j<0,3 \mathrm{nA}$ als sehr instabil, sodass in der Abbildung 4.2.15 (a) die Tunnelstromabhängigkeit des TERS-Signals lediglich in einem Bereich $j=0,4-1 \mathrm{nA}$ untersucht wurde. Gleichzeitig wurde hier der maximale Tunnelstrom auf $j=1 \mathrm{nA}$ begrenzt, um Beschädigungen an der TERS-Spitze durch einen Kontakt mit der Probe aufgrund eines zu schnellen Annäherns an die Probenoberfläche zu vermeiden. Entsprechend erfolgt die Annäherung 


\section{Auswertung und Ergebnisse}

der TERS-Spitze an die Probe zunächst bei einem geringeren Tunnelstrom. Anschließend wird dieser schrittweise auf den Zielwert erhöht. Ein Tunnelstrom von $j=1 \mathrm{nA}$ entspricht nach der Abbildung 4.2.13 (c) einem relativen Abstand der Spitze zur Schichtoberfläche von $\delta z \approx 1 \mathrm{~nm}$. Für die angelegte Spannung ergeben sich vergleichbare Begrenzungen. So führt eine Spannung $U_{\text {Bias }}<0,25 \mathrm{~V}$ für die untersuchte LCMO-Schicht zu keiner stabilen Tunnelposition, sodass ein Bereich $U_{\text {Bias }}=0,4-1 \mathrm{~V}$ betrachtet wurde. Zu berücksichtigen ist zudem die aktive Rückkopplung im Konstant-Strom-Modus und der damit einhergehende Einfluss der Spannung auf den Abstand $\delta z$ zwischen Spitze und Probenoberfläche. Weiterhin wurde auf eine Umkehr der Tunnelstromrichtung (negatives Spannungsvorzeichen) verzichtet, da diese auf die TERS-Messungen an der LCMO-Schicht keinen Einfluss hatte. Wird der Tunnelstrom variiert, so zeigt sich für einen kleineren Tunnelstrom sowohl eine leichte Abnahme des TERS-Signals der LCMO-Schicht als auch des kontinuierlichen TERS-Hintergrundsignals der inelastischen Streuung an der Spitze. Dieses lässt sich durch den vergrößerten Abstand der Spitze zur Schichtoberfläche erklären. So ergibt sich nach der Abbildung 4.2.13 (c) für eine Verringerung des Tunnelstroms von $1 \mathrm{nA}$ auf 0,4 nA eine Vergrößerung des relativen Spitzenabstandes $\delta z$ zur Probe von $1 \mathrm{~nm}$ auf etwa $3-4 \mathrm{~nm}$.

Ein umgekehrter Effekt kann für die angelegte Spannung $U_{\text {Bias }}$ beobachtet werden. Wird diese reduziert, so verringert sich auch der Tunnelstrom $j$, sodass das STM-System die Spitze im Konstant-Tunnel-Modus durch die Rückkopplung näher an die Probe heranbringt, um den ursprünglichen Tunnelstrom beizubehalten. Folglich führt die Spannungsreduzierung zu einem geringeren Abstand der Spitze zur Probe und damit im TERS-Signal zu einem leicht erhöhten kontinuierlichen Hintergrundsignal durch eine stärkere inelastische Streuung des einfallenden Lichts an der Spitze sowie einen erhöhten Hintergrundbeitrag durch das Substrat. Dieser Effekt ist jedoch nur schwach ausgeprägt und der Einfluss der Spannung auf das TERS-Signal der LCMO-Schicht gering. Insgesamt ergibt sich für die LCMO-Schichten das größte TERS-Signal in Kombination mit einer stabilen Tunnelposition und einem optimierten Signal-Rausch-Verhältnis für einen Tunnelstrom von $j=1 \mathrm{nA}$ bei einer angelegten Spannung von $U_{\text {Bias }}=1 \mathrm{~V}$, sodass die weiteren TERS-Messungen an den LCMO/STO (100)-Schichten mit diesen Werten durchgeführt wurden. Allgemein müssen die STM-Parameter jedoch individuell an das Probensystem angepasst werden.

Eine weitere charakteristische Eigenschaft der TERS-Technik ist die Oberflächensensitivität durch das schnelle Abklingen des Nahfeldes bei Vergrößerung des Abstandes von der TERS-Spitze, die sich sowohl in experimentellen Beobachtungen 150, 172, 177, 178, 184 als auch in theoretischen Betrachtungen zeigt 162, 174, 181, 183. Im Fall des LCMODoppelperowskits auf STO (100) kann diese mithilfe einer LCMO-Schichtdickenserie untersucht werden. Hierfür wurden die LCMO-Schichten mit einer systematischen Variation der Schichtdicke $d=25-175 \mathrm{~nm}$ mithilfe der MAD-Technik unter gleichen Bedingungen 
auf STO (100)-Substraten aufgewachsen. Anschließend wurden von jeder LCMO-Schicht die jeweiligen TERS- und Fernfeld-Raman-Spektren unter ebenfalls gleichen Bedingungen $\left(j=1 \mathrm{nA}, U_{\text {Bias }}=1 \mathrm{~V}, \lambda=532 \mathrm{~nm}, P \approx 10 \mathrm{~mW}\right.$ und $\left.t=2 \times 60 \mathrm{~s}\right)$ mit einer einfallenden Laserpolarisation parallel (P) zur TERS-Spitzenachse und ohne Analysator aufgenommen. Die Spektren sind nachfolgend mit den jeweiligen Werten für den TERS-Kontrast $C_{644}$ der $A_{g}$ Breathing Mode bei $644 \mathrm{~cm}^{-1}$ in der Abbildung 4.2 .16 dargestellt.

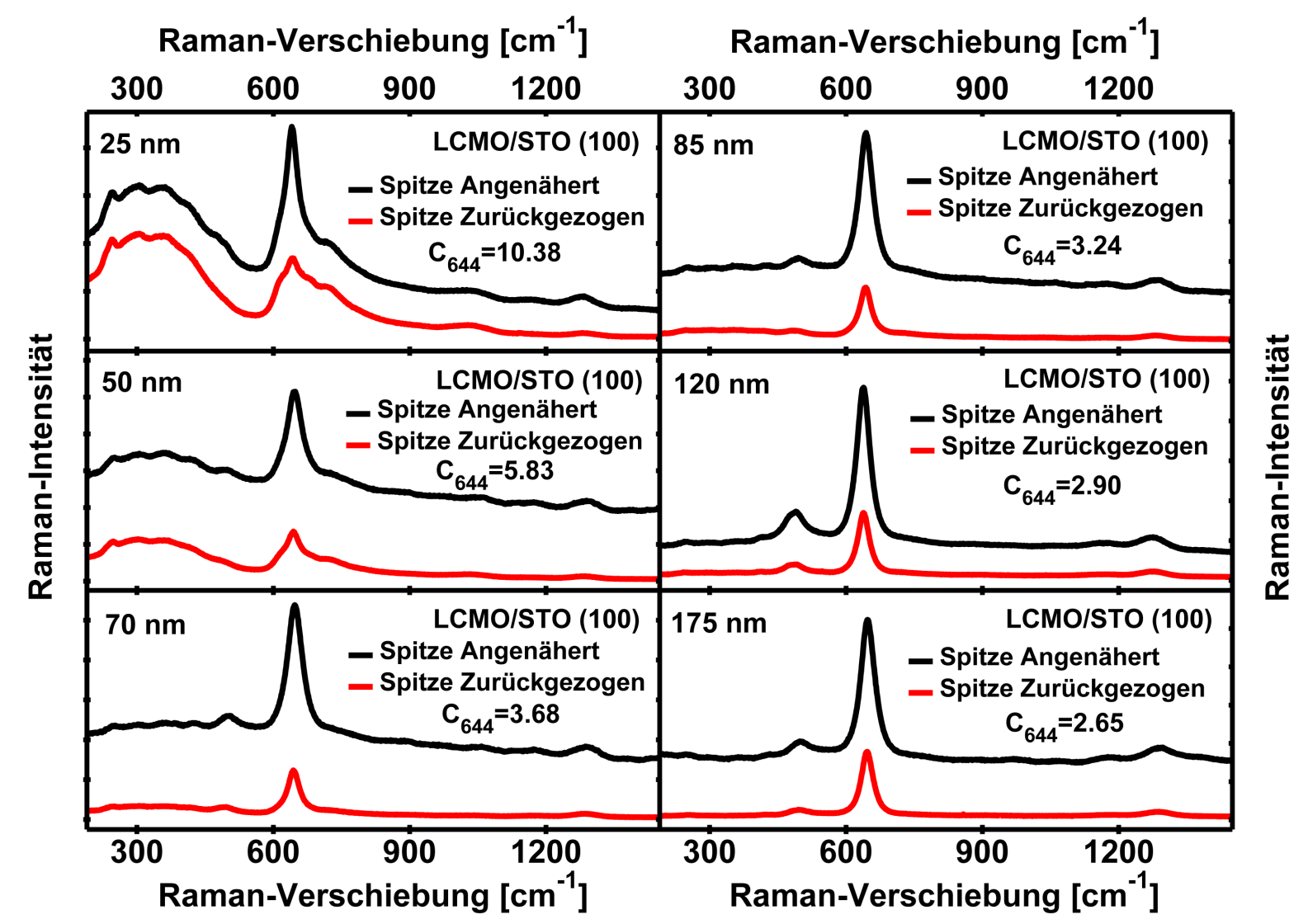

Abbildung 4.2.16: TERS- $\left(U_{\text {Bias }}=1 \mathrm{~V}, j=1 \mathrm{nA}\right)$ und Fernfeld-Raman-Spektren dünner $\mathrm{La}_{2} \mathrm{CoMnO}_{6} / \mathrm{STO}$ (100)-Schichten mit einer unterschiedlichen Schichtdicke $d=25-175 \mathrm{~nm}$, aufgenommen bei einer Laserpolarisation parallel $(\mathrm{P})$ zur Spitzenachse und ohne Analysator. Der TERS-Kontrast $C_{644}$ für die $A_{g}$ Breathing Mode bei $644 \mathrm{~cm}^{-1}$ ist jeweils angegeben.

In den TERS-Spektren zeigt sich hierbei eine starke Abnahme des TERS-Kontrastes $C_{644}$ $\operatorname{der} A_{g}$ Breathing Mode bei $644 \mathrm{~cm}^{-1}$ von $C \approx 10$ auf etwa $C \approx 2,5$ mit der Vergrößerung der Schichtdicke $d$ der LCMO-Schichten von $25 \mathrm{~nm}$ auf $175 \mathrm{~nm}$. Eine Auftragung des TERS-Kontrastes $C_{644}$ der $A_{g}$ Breathing Mode in Abhängigkeit der Schichtdicke $d$ der LCMO-Filme auf STO (100) ist in der Abbildung 4.2.17 (a) dargestellt. Es ergibt sich in guter Näherung eine exponentielle Abnahme des Kontrastes bei Vergrößerung der LCMOSchichtdicke $d$. Ein vergleichbares Verhalten wurde in der Literatur bei TERS-Messungen an dünnen CdS-Schichten beobachtet [172]. Der Grund für die Abhängigkeit des TERSKontrastes von der Schichtdicke ist die unterschiedliche Eindringtiefe von Nahfeld und Fernfeld in die Schichten und damit einhergehend die Erfassung unterschiedlich großer 
Anregungsvolumen in der Schicht. Ein möglicher Polarisationseffekt durch das Nahfeld kann durch die unpolarisierte Streukonfiguration ohne Analysator ausgeschlossen werden. Die Eindringtiefe $L_{\mathrm{ff}}$ des Fernfeldes lässt sich nun für die LCMO-Filme auf STO (100) mithilfe des STO-Substrats abschätzen, indem das Hintergrundsignal des Subtrats in den Fernfeld-Raman-Spektren in Abhängigkeit der Schichtdicke $d$ der LCMO-Schichten betrachtet wird (Abbildung 4.2.16). Aufgrund dass der STO-Hintergrund für die dünneren Schichten deutlicher in Erscheinung tritt, lässt sich die Eindringtiefe des Fernfeldes auf etwa $L_{\mathrm{ff}} \approx 85 \mathrm{~nm}$ bestimmen. Eine weitere Möglichkeit ist die Betrachtung der integralen Raman-Intensität der $A_{g}$ Breathing Mode bei $644 \mathrm{~cm}^{-1}$ der LCMO-Schichten in den TERS- und Fernfeld-Raman-Spektren in Abhängigkeit der Schichtdicke $d$. Diese sind (nach Substrat- und Hintergrundkorrektur) für das TERS-Gesamtfeld, Fernfeld und Nahfeld (Differenz aus TERS-Gesamtfeld und Fernfeld) in der Abbildung 4.2.17 (b) dargestellt.

(a)

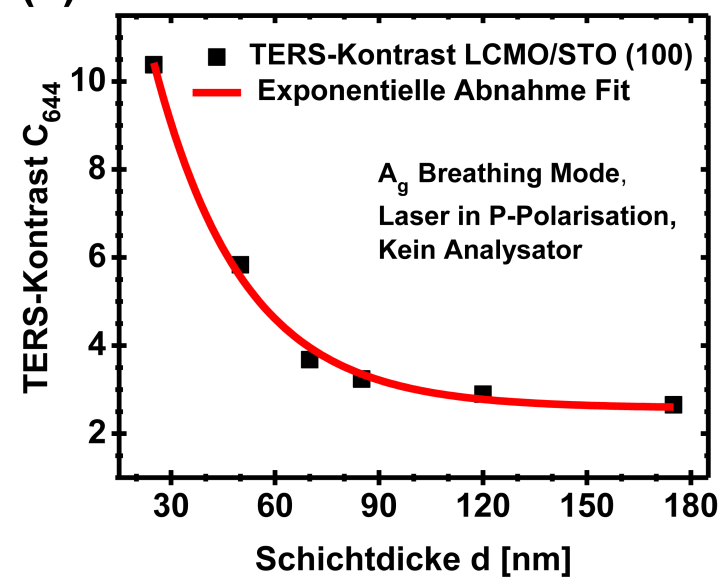

(b)

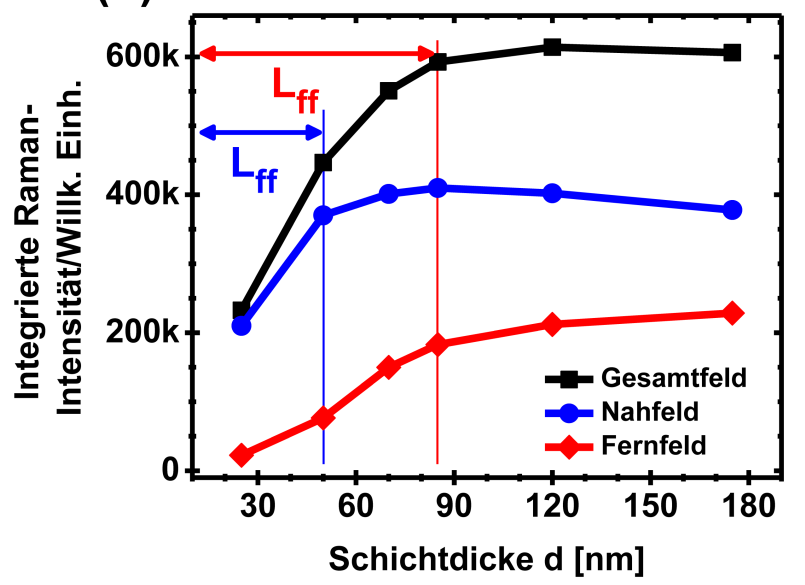

Abbildung 4.2.17: Abhängigkeit des TERS-Kontrastes $C_{644}$ (a) und der integrierten RamanIntensität des Gesamtfeldes (angenäherte Spitze), des Fernfeldes (zurückgezogene Spitze) und des Nahfeldes (die Differenz aus dem Gesamtfeld und dem Fernfeld) (b) der $A_{g}$ Breathing Mode bei $644 \mathrm{~cm}^{-1}$ von der Schichtdicke $d$ der $\mathrm{La}_{2} \mathrm{CoMnO}_{6} / \mathrm{STO}$ (100)-Schichten, deren TERS- und FernfeldRaman-Spektren in der Abbildung 4.2.16 dargestellt sind.

Anhand des Verlaufs der integrierten Raman-Intensität der $A_{g}$ Breathing Mode mit der Schichtdicke $d$ der LCMO-Schichten im TERS-Gesamtfeld, Fernfeld und Nahfeld können nun die Eindringtiefen von Nahfeld und Fernfeld bestimmt werden. Hierfür wird die einsetzende Sättigung der integrierten Raman-Intensitäten in den beiden Feldern betrachtet (vertikale Linien in der Abbildung 4.2.17 (b)). Es ergibt sich eine Eindringtiefe des Nahfeldes von $L_{\mathrm{nf}} \approx 50 \mathrm{~nm}$, während für das Fernfeld eine Eindringtiefe von $L_{\mathrm{ff}} \approx 85 \mathrm{~nm}$ abschätzt werden kann. Zudem lässt sich mit der Abbildung 4.2.17 (b) die Abnahme des TERS-Kontrasts $C_{644}$ der $A_{g}$ Breathing Mode mit der Schichtdicke $d$ der LCMO-Schichten (Abbildung 4.2.17 (a)) nachvollziehen. So ist für kleine Schichtdicken der Intensitätsanteil des Nahfeldes am TERS-Gesamtfeld aufgrund der lokalen Konzentration im Spitzenumfeld sehr groß, während das Fernfeld wiederum mit seiner größeren Eindringtiefe aufgrund der 
begrenzten Schichtdicke nicht in dem Maß zum TERS-Gesamtfeld beitragen kann wie für größere Schichtdicken. Wird die Schichtdicke nun erhöht, so beginnt das Nahfeld aufgrund seiner Oberflächensensitivität im Beitrag zum TERS-Gesamtfeld früher zu sättigen als das Fernfeld, sodass der TERS-Kontrast aufgrund seiner Definition (Gleichung (3.4.3)) in diesem Schichtdickenbereich schnell mit der Schichtdicke abnimmt. Wird diese weiter erhöht, so beginnt auch der Beitrag des Fernfeldes zum TERS-Gesamtfeld zu sättigen, da auch die Eindringtiefe des Fernfeldes erreicht ist. Folglich stagniert auch das TERS-Gesamtfeld $I_{\text {tef }}=I_{\mathrm{nf}}+I_{\mathrm{ff}}$ und der TERS-Kontrast konvergiert gegen einen festen Wert, der durch den maximalen Nahfeldbeitrag zum TERS-Gesamtfeld bestimmt ist. Zu beachten ist, dass der TERS-Kontrast von Spitzeneffekten beeinflusst wird. Während nicht-plasmonische Verstärkungen aufgrund von Reflexionen und Mehrfachstreuungen an der Spitze durch das verwendete konfokale Raman-Mikroskop vernachlässigt werden können (Abschnitt 3.4.6) und keine Heiz- und Photobleichungsprozesse an den LCMO-Schichten auftreten, bleibt ein kleiner Abschattungseffekt durch die TERS-Spitze und damit eine leichte Unterschätzung des Fernfeldbeitrags zum Gesamtfeld $I_{\text {tef }}=I_{\text {nf }}+I_{\text {ff }}$ für die TERS-Spektren gegenüber dem Fernfeld der Raman-Spektren ohne angenäherte Spitze. Der Effekt lässt sich geometrisch auf etwa $5 \%$ abschätzen (Abschnitt 3.4.6) und ist direkt in der Abbildung 4.2.17 (b) sichtbar. So zeigt sich, dass das TERS-Gesamtfeld und das Nahfeld nach eintretender Sättigung für eine darüber hinausgehende LCMO-Schichtdicke um 2-8\% abnehmen, obwohl das erfasste Probenvolumen vergleichbar groß wie für die dünneren LCMO-Schichten ist. Dieses ist auf den Abschattungseffekt durch die TERS-Spitze zurückzuführen, da das Fernfeld für eine zurückgezogene Spitze bei einer Vergrößerung der LCMO-Schichtdicke von $120 \mathrm{~nm}$ auf $175 \mathrm{~nm}$ gleichzeitig um $7 \%$ zunimmt und dieser Zuwachs in etwa dem geometrischen Abschattungseffekt entspricht. Folglich wird der TERS-Kontrast leicht unterschätzt.

Mit den Eindringtiefen $L_{\mathrm{nf}} \approx 50 \mathrm{~nm}$ und $L_{\mathrm{ff}} \approx 85 \mathrm{~nm}$ sowie des TERS-Kontrastes $C_{644}$ der $A_{g}$ Breathing Mode bei $644 \mathrm{~cm}^{-1}$ kann zudem mithilfe der Gleichung 3.4.5 der TERS-Verstärkungsfaktor EF TERS für die LCMO-Schichten auf STO (100) abgeschätzt werden. So ergibt sich mit den TERS-Kontrasten $C_{644}$ aus der Abbildung 4.2.16, einem TERS-Spitzenradius $R_{\text {Spitze }} \approx 20-25 \mathrm{~nm}$, einem Laserfokuspunkt mit $R_{\text {Fokus }} \approx 1 \mu \mathrm{m}$ und $\beta=60^{\circ}$ ein TERS-Verstärkungsfaktor $\mathrm{EF}_{\text {TERS }} \approx 10^{4}$. Dieses ist in guter Übereinstimmung mit TERS-Messungen an Silizium und CdS-Schichten [167, 172, 266. Werden zusätzlich polarisationsabhängige TERS-Spektren betrachtet, so kann der TERS-Verstärkungsfaktor mit $\mathrm{EF}_{\mathrm{TERS}} \approx 10^{5}$ (für die $25 \mathrm{~nm}$ LCMO-Schicht auf STO mit $L_{\mathrm{nf}} \approx L_{\mathrm{nf}} \approx 25 \mathrm{~nm}$ und $C_{644}=113,34$, siehe Abbildung 4.2.7 in der gekreuzten PS-Konfiguration um eine Größenordnung erhöht werden. Zu berücksichtigen ist, dass dieses durch den Depolarisationseffekt des Nahfeldes und nicht durch eine stärkere Nahfeldanregung hervorgerufen wird. 


\subsubsection{TERS an weiteren Doppelperowskit-Dünnschichten}

Im Folgenden soll nun eine Betrachtung von TERS-Messungen an weiteren Doppelperowskit-Dünnschichten erfolgen. Wie bereits gezeigt, ist eine Messung an den LCMO-Doppelperowskiten trotz eines spezifischen Widerstands von $\rho \approx 10^{2} \Omega \mathrm{cm}$ bei Raumtemperatur (Abbildung 4.1.2 (b), $\rho(T)$-Kurve eine $25 \mathrm{~nm} \mathrm{La}_{2} \mathrm{CoMnO}_{6}$-Schicht auf STO (100)) durch eine geeignete Wahl der STM-Parameter (Tunnelstrom, Spannung) ohne Probleme möglich. Entsprechend lassen sich daher TERS-Messungen auch an den anderen Doppelperowskiten der $\mathrm{A}_{2} \mathrm{CoMnO}_{6}$ - und der $\mathrm{A}_{2} \mathrm{NiMnO}_{6}$-Reihe mit ihren ähnlichen elektrischen Transporteigenschaften 35] durchführen. In der Abbildung 3.4.6 sind nun die polarisationsabhängigen TERS- und Fernfeld-Raman-Spektren einer $\operatorname{Pr}_{2} \mathrm{CoMnO}_{6}$-Doppelperowskit-Schicht auf STO (111) mit einer Schichtdicke $d=65 \mathrm{~nm}$ dargestellt. Für die TERS-Messungen wurde dabei wie für die LCMO-Schichten (gleiche TERS-Spitze) im Konstant-Strom-Betrieb ein Tunnelstrom $j=1 \mathrm{nA}$ bei einer angelegten Spannung $U_{\text {Bias }}=1 \mathrm{~V}$ gewählt.

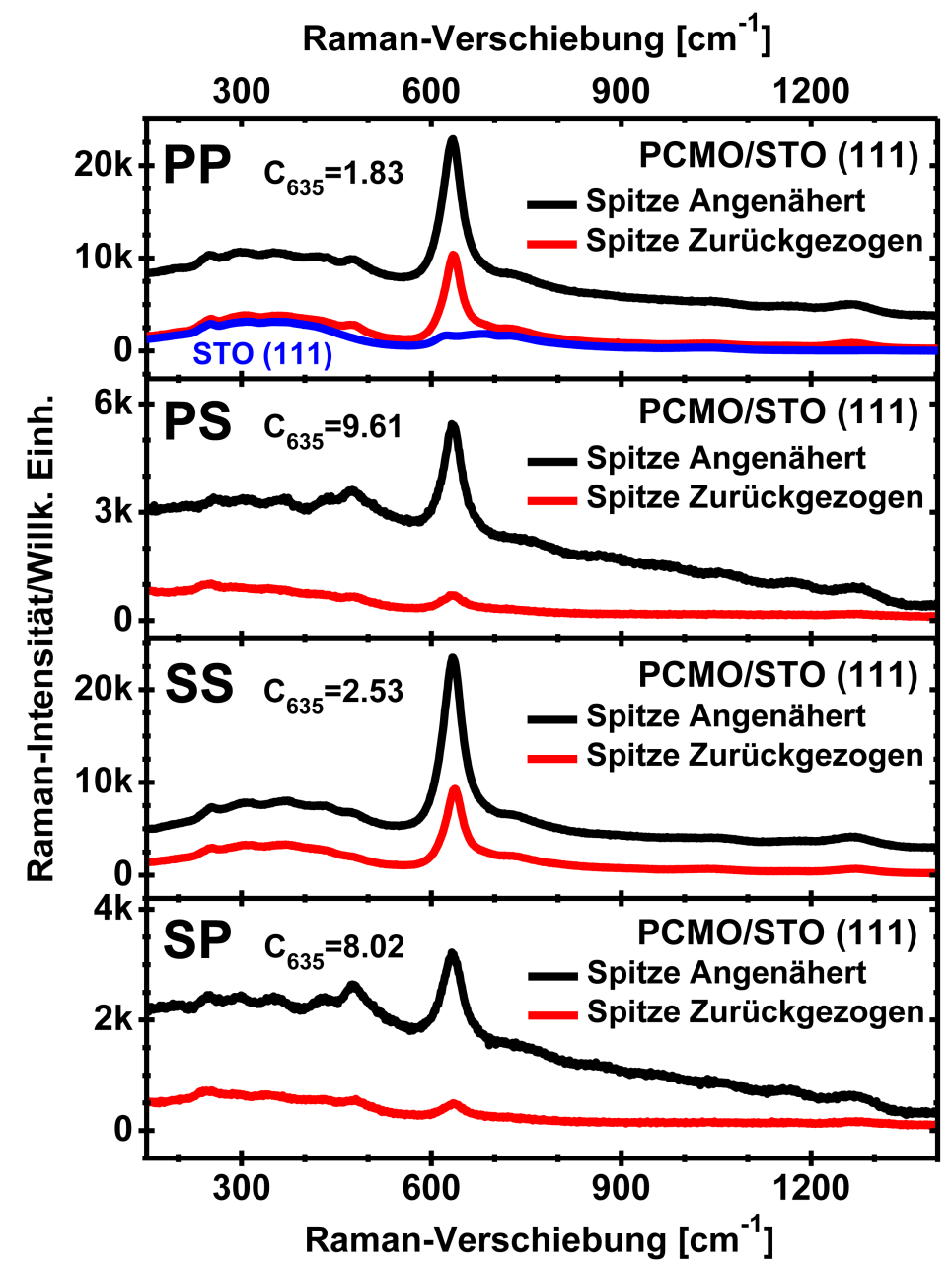

Abbildung 4.2.18: Polarisierte TERS- und Fernfeld-Raman-Spektren von einer $\mathrm{Pr}_{2} \mathrm{CoMnO}_{6}{ }^{-}$ Schicht mit einer Schichtdicke $d=65 \mathrm{~nm}$ auf einem STO (111)-Substrat, aufgenommen in den parallelen PP- und SS-Konfigurationen und in den gekreuzten PS- und SP-Konfigurationen. Das STO (111)-Raman-Spektrum zeigt die Hintergrund-Überlagerung durch das Substrat. 
Die Spektren wurden bei einer Laserleistung $P \approx 10 \mathrm{~mW}(\lambda=532 \mathrm{~nm}, t=2 \times 60 \mathrm{~s})$ aufgenommen. Aufgrund der (111)-Schicht- und Substratorientierung muss für die Betrachtung der Polarisationsabhängigkeit der Spektren und der Raman-Auswahlregeln das Probenbezugssystem zunächst mithilfe der Gleichung (3.4.12) aus der (100)-Orientierung in die (111)-Orientierung gedreht werden. Anschließend muss die Geometrie der seitlichen Einstrahlung (Abbildung 3.4.6) berücksichtigt werden. Die Fernfeld-Raman-Spektren (Spitze mehrere Mikrometer zurückgezogen) in der Abbildung 4.2 .18 weisen dabei die für ein B-Platz geordnetes Doppelperowskit mit monokliner P12/n1-Struktur zu erwartende Charakteristik mit einer starken $A_{g}$ Breathing Mode in den parallelen PP- und SSPolarisationskonfigurationen, die für die gekreuzten PS- und SP-Konfigurationen wiederum nach den Raman-Auswahlregeln fast vollständig unterdrückt ist, und mit einer breiten $B_{g}$ Mischmode, die von weiteren $A_{g}$ und $B_{g}$ Moden überlagert ist, auf. Im Vergleich zu den LCMO-Schichten sind die Modenpositionen mit $635 \mathrm{~cm}^{-1}$ für die $A_{g}$ Breathing Mode und $476 \mathrm{~cm}^{-1}$ für die $B_{g}$ Mischmode aufgrund des kleineren Radius des $\operatorname{Pr}^{3+}$-Kations $\left(r_{\mathrm{Pr}^{3+}}=112,6\right.$ pm gegenüber $r_{\mathrm{La}^{3+}}=116$ pm für eine Koordinationszahl $\mathrm{N}=8$ [222] $)$ und der damit einhergehenden Vergrößerung der mittleren CO/Mn-O-Bindungslänge leicht zu geringeren Wellenzahlen verschoben. Weitere Raman-Moden der Schicht heben sich vom ebenfalls zu beobachtenden Hintergrundsignal des STO (111)-Substrats nicht ab. In den TERS-Spektren (Spitze in Tunnelposition angenähert) der PCMO-Schicht wiederum zeigt sich wie für die LCMO-Schichten eine signifikante Verstärkung der $A_{g}$ Breathing Mode in allen Polarisationskonfigurationen mit einer besonders deutlichen Verstärkung in den gekreuzten PS- und SP-Konfigurationen sowie ein starker kontinuierlicher Hintergrund, der auch hier inelastischen Streuprozessen an der TERS-Spitze zugeordnet werden kann.

Zusätzlich ist in der Abbildung 4.2.18 der TERS-Kontrast $C_{635} \operatorname{der} A_{g}$ Breathing Mode für die einzelnen Polarisationskonfigurationen angeben. Wie bei den LCMO-Schichten zeigt sich auch für die PCMO-Schicht aufgrund der Raman-Auswahlregeln für das Fernfeld und der Nahfelddepolarisation eine starke Polarisationsabhängigkeit des TERS-Kontrastes $C_{635}$ von der Polarisationskonfiguration mit einem Kontrast in der Größenordnung $C_{635} \approx 8-10$ in den gekreuzten PS- und SP-Polarisationskonfigurationen und einem Kontrast in der GröBenordnung $C_{635} \approx 1,8-2,6$ in den parallelen PP- und SS-Konfigurationen. Wird zusätzlich die Schichtdicke $d=65 \mathrm{~nm}$ berücksichtigt, so ergibt ein Vergleich mit den beiden $25 \mathrm{~nm}$ und $120 \mathrm{~nm}$ LCMO-Schichten auf STO (100) in Abschnitt 4.2.3, dass sich die erreichten TERS-Kontrastwerte für die $A_{g}$ Breathing Mode sehr gut zwischen denen der beiden LCMO-Schichten in den jeweiligen Polarisationskonfigurationen einordnen lassen. Dieses entspricht der in dem Abschnitt 4.2.4 beobachteten Abhängigkeit des TERS-Kontrastes von der Schichtdicke. Folglich ist die TERS-Verstärkung hauptsächlich durch die Schichtdicke des Doppelperowskits und die Polarisationskonfiguration bestimmt mit einer für unterschiedliche A-Kationen und STO-Substratorientierungen vergleichbaren Ausprägung. 


\section{Auswertung und Ergebnisse}

In der Abbildung 4.2.19 sind nun die polarisationsabhängigen TERS- und Fernfeld-RamanSpektren einer $\mathrm{La}_{2} \mathrm{NiMnO}_{6}$-Doppelperowskit-Schicht auf $\mathrm{MgO}$ (100) mit einer Schichtdicke $d=100 \mathrm{~nm}$ für eine in-plane Rotation um einen Winkel $\phi=45^{\circ} \mathrm{um}$ die Spitzenachse herum dargestellt. Im Gegensatz zu den LCMO-Schichten erfolgten die TERS-Messungen aufgrund einer besseren elektrischen Leitfähigkeit der LNMO-Schicht mit einem reduzierten Tunnelstrom von $j=0,3 \mathrm{nA}$ bei einer angelegten Spannung von nur $U_{\text {Bias }}=0,1 \mathrm{~V}$. Der Abstand zur Probenoberfläche ist folglich mit $\delta z \approx 3 \mathrm{~nm}$ minimal größer, die Annäherung der Spitze an die Probe dabei jedoch schonender. Zudem wird eine neue TERS-Spitze mit einem vergleichbaren Spitzenradius $R_{\text {Spitze }} \approx 25 \mathrm{~nm}$ wie zuvor verwendet. Die Spektren wurden bei einer Laserleistung $P \approx 10 \mathrm{~mW}(\lambda=532 \mathrm{~nm}, t=2 \times 60 \mathrm{~s})$ aufgenommen.

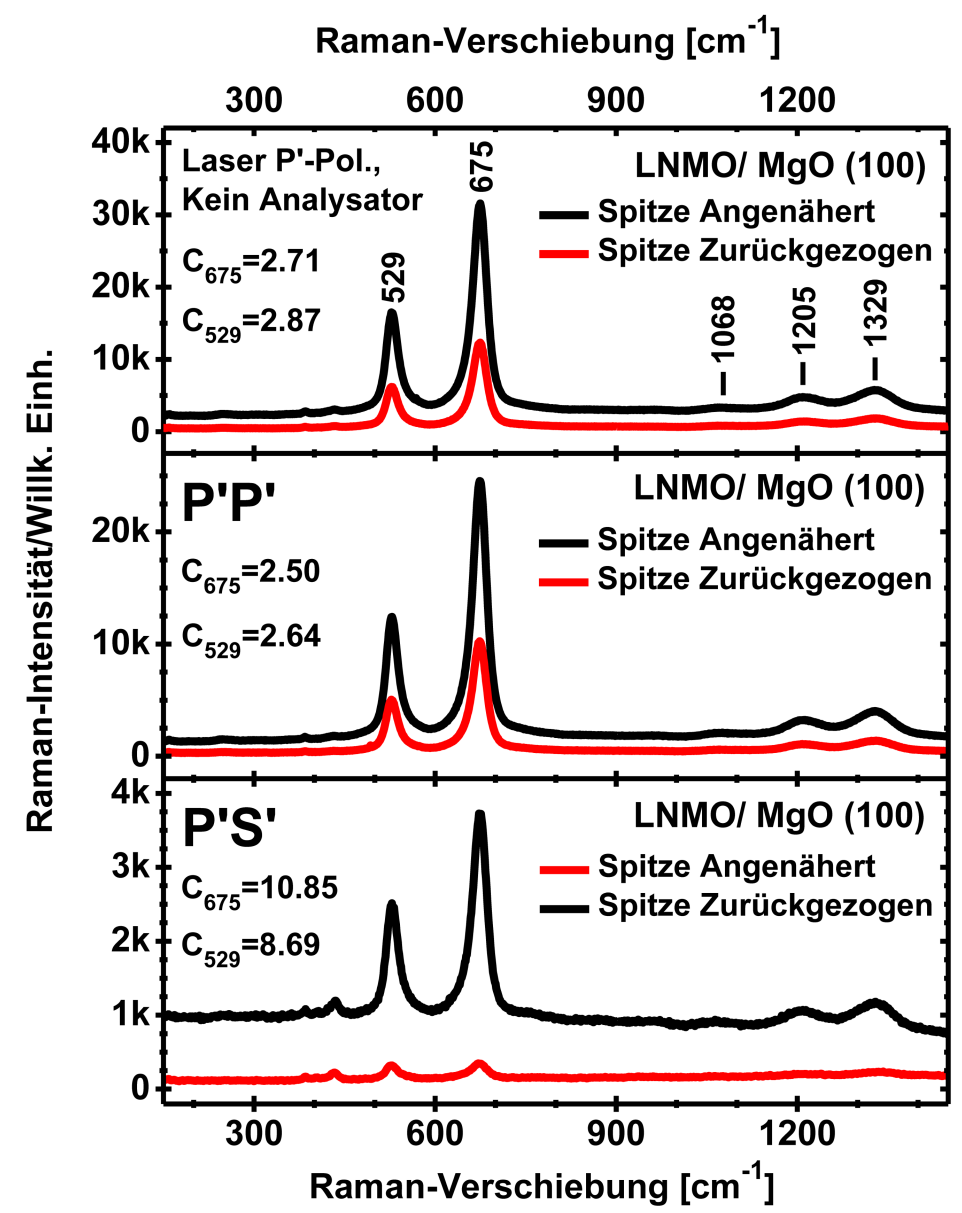

Abbildung 4.2.19: Polarisierte TERS- und Fernfeld-Raman-Spektren von einer $\mathrm{La}_{2} \mathrm{NiMnO}_{6}{ }^{-}$ Schicht mit einer Schichtdicke $d=100 \mathrm{~nm}$ auf einem MgO (100)-Substrat, aufgenommen für eine in-plane Rotation um $\phi=45^{\circ}$ in der parallelen $\mathrm{P}^{\prime} \mathrm{P}^{\prime}-$ und in der gekreuzten $\mathrm{P}^{\prime} \mathrm{S}^{\prime}$-Polarisationskonfiguration sowie mit einer einfallenden $\mathrm{P}^{\prime}$-Laserpolarisation ohne Analysator.

Da das MgO (100)-Substrat mit seiner kubischen NaCl-Struktur keine Raman-Aktivität und damit nur einen flachen Hintergrund aufweist [265], stammen alle in den TERS- und Fernfeld-Raman-Spektren zu beobachtenden Raman-Moden von der LNMO-Schicht selbst. Aufgrund der in-plane Rotation der LNMO-Schicht um $\phi=45^{\circ} \mathrm{um}$ die Spitzenachse ist in 
den Fernfeld-Raman-Spektren (Spitze mehrere Mikrometer zurückgezogen) die Polarisationsabhängigkeit der $A_{g}$ Breathing Mode bei $675 \mathrm{~cm}^{-1}$ und der $B_{g}$ Mischmode bei $529 \mathrm{~cm}^{-1}$ in der B-Platz geordneten monoklinen P12 1 n1-Struktur nach den Raman-Auswahlregeln mit einer starken Intensität in der parallelen $\mathrm{P}^{\prime} \mathrm{P}^{\prime}$-Polarisationskonfiguration und einer Unterdrückung in der gekreuzten $\mathrm{P}^{\prime} \mathrm{S}^{\prime}$-Konfiguration identisch. Im Vergleich zu den LCMOSchichten ist die $B_{g}$ Mischmode der LNMO-Schicht dabei schärfer und intensiver ausgeprägt. In den TERS-Spektren (Spitze in Tunnelposition angenähert) der LNMO-Schicht in der Abbildung 4.2.19 zeigt sich wiederum eine signifikante Verstärkung des Spektrums in allen Polarisationskonfigurationen mit einer besonders großen Verstärkung für die $A_{g}$ Breathing Mode und die $B_{g}$ Mischmode durch die Nahfelddepolarisation in der gekreuzten $\mathrm{P}^{\prime} \mathrm{S}^{\prime}$-Konfiguration. Die schwache Intensität der zwei Moden im Fernfeld in der gekreuzten $\mathrm{P}^{\prime} \mathrm{S}^{\prime}$-Konfiguration ist auf Abweichungen bei der in-plane Rotation und eine Koexistenz der monoklinen P12 1 n1- mit der ebenfalls B-Platz geordneten rhomboedrischen R $\overline{3}$-Struktur zurückzuführen, die für LNMO bereits bei Raumtemperatur auftreten kann [240,269].

So haben die Raman-Moden in der R $\overline{3}$-Struktur eine $A_{g}$ oder $E_{g}$-Symmetrie, wobei die $A_{g}$-Symmetrie in der gekreuzten $\mathrm{P}^{\prime} \mathrm{S}^{\prime}$-Konfiguration verboten und die $E_{g}$-Symmetrie erlaubt ist. Während nun die Breathing Mode und die Mischmode in der $\mathrm{P}^{\prime} \mathrm{S}^{\prime}$-Konfiguration vergleichbar an Intensität gewinnen, kann ein nur geringer Intensitätsanstieg für die schwachen Moden bei $384 \mathrm{~cm}^{-1}$ und $435 \mathrm{~cm}^{-1}$ beobachtet werden, der sich auf eine mögliche $E_{g}$-Symmetrie zurückführen lässt. Da diese in der $\mathrm{P}^{\prime} \mathrm{P}^{\prime}$ - und in der $\mathrm{P}^{\prime} \mathrm{S}^{\prime}$-Polarisationskonfiguration erlaubt sind, ist die Nahfelddepolarisation für diese Moden nicht wirksam. Die Breathing Mode hat wiederum auch in der R $\overline{3}$-Struktur eine $A_{g^{-}}$Symmetrie [33,240], sodass die Nahfelddepolarisation in der gekreuzten $\mathrm{P}^{\prime} \mathrm{S}^{\prime}$-Konfiguration zu einer großen Verstärkung führt. Die Mischmode hat eine $E_{g}$-Symmetrie in der R $\overline{3}$-Struktur, koexistiert jedoch mit einer $B_{g}$ Symmetrie in der monoklinen P12 $/$ n1-Struktur, sodass sich durch die Nahfelddepolarisation ebenfalls eine große TERS-Verstärkung in der $\mathrm{P}^{\prime} \mathrm{S}^{\prime}$-Konfiguration ergibt.

Zusätzlich sind in der Abbildung 4.2.19 der TERS-Kontrast $C_{675}$ der $A_{g}$ Breathing Mode und der TERS-Kontrast $C_{529}$ der $B_{g}$ Mischmode für die jeweiligen Polarisationskonfigurationen angegeben. Es zeigt sich dabei eine starke Polarisationsabhängigkeit des TERSKontrastes beider Moden mit $C_{675, \mathrm{P}^{\prime} \mathrm{S}^{\prime}} \approx 11$ und $C_{529 \mathrm{P}^{\prime} \mathrm{S}^{\prime}} \approx 9$ in der gekreuzten $\mathrm{P}^{\prime} \mathrm{S}^{\prime}$ Polarisationskonfiguration und $C_{675} \approx 2,5$ und $C_{529} \approx 2,6$ in der parallelen $\mathrm{P}^{\prime} \mathrm{P}^{\prime}$-Konfiguration. Für eine Laserpolarisation parallel $\left(\mathrm{P}^{\prime}\right)$ zur Spitzenachse ohne Analysator ergibt sich wiederum $C_{675} \approx 2,7$ und $C_{529} \approx 2,9$. Bezogen auf die Schichtdicke $d=100 \mathrm{~nm}$ der LNMO-Schicht ordnet sich der erreichte TERS-Kontrast für die $A_{g}$ Breathing Mode wieder sehr gut in die in der Abbildung 4.2.17 (a) beobachteten Abhängigkeit des TERSKontrastes von der Schichtdicke ein, sodass sich für die LNMO-Schicht auf MgO (100) ein vergleichbares TERS-Verhalten wie für die LCMO-Schichten auf STO (100) ergibt. 


\section{Auswertung und Ergebnisse}

Anschließend sollen TERS-Messungen an verspannten Doppelperowskit-Schichten betrachtet werden. Hierfür werden diese auf LAO-Substraten (Gitterkonstante $a_{\mathrm{pc}}=3,788 \AA 225$ ) aufgewachsen, da diese in-plane eine Druckspannung in den Doppelperowskit-Schichten induzieren, die out-of-plane zu einer erhöhten Gitterkonstante der Doppelperowskit-Struktur führt (Abschnitt 4.1.3). In der Abbildung 4.2.20 sind die TERS- und Fernfeld-RamanSpektren einer $12 \mathrm{~nm} \mathrm{La} \mathrm{CoMnO}_{6} / \mathrm{LAO}$ (100)-Schicht mit einer P-Laserpolarisation ohne Analysator, in der parallelen PP-Polarisationskonfiguration und in der gekreuzten $\mathrm{P}^{\prime} \mathrm{S}^{\prime}$ Konfiguration für eine in-plane Rotation der Schicht um $\phi=45^{\circ}$ dargestellt. Die TERSMessungen erfolgten mit der gleichen Spitze wie bei den LCMO/STO (100)-Schichten bei einem Tunnelstrom $j=0,5 \mathrm{nA}$ und einer Spannung von $U_{\text {Bias }}=0,5 \mathrm{~V}$. Die Spektren wurden bei einer Laserleistung $P \approx 10 \mathrm{~mW}(\lambda=532 \mathrm{~nm}, t=2 \times 30 \mathrm{~s})$ aufgenommen.

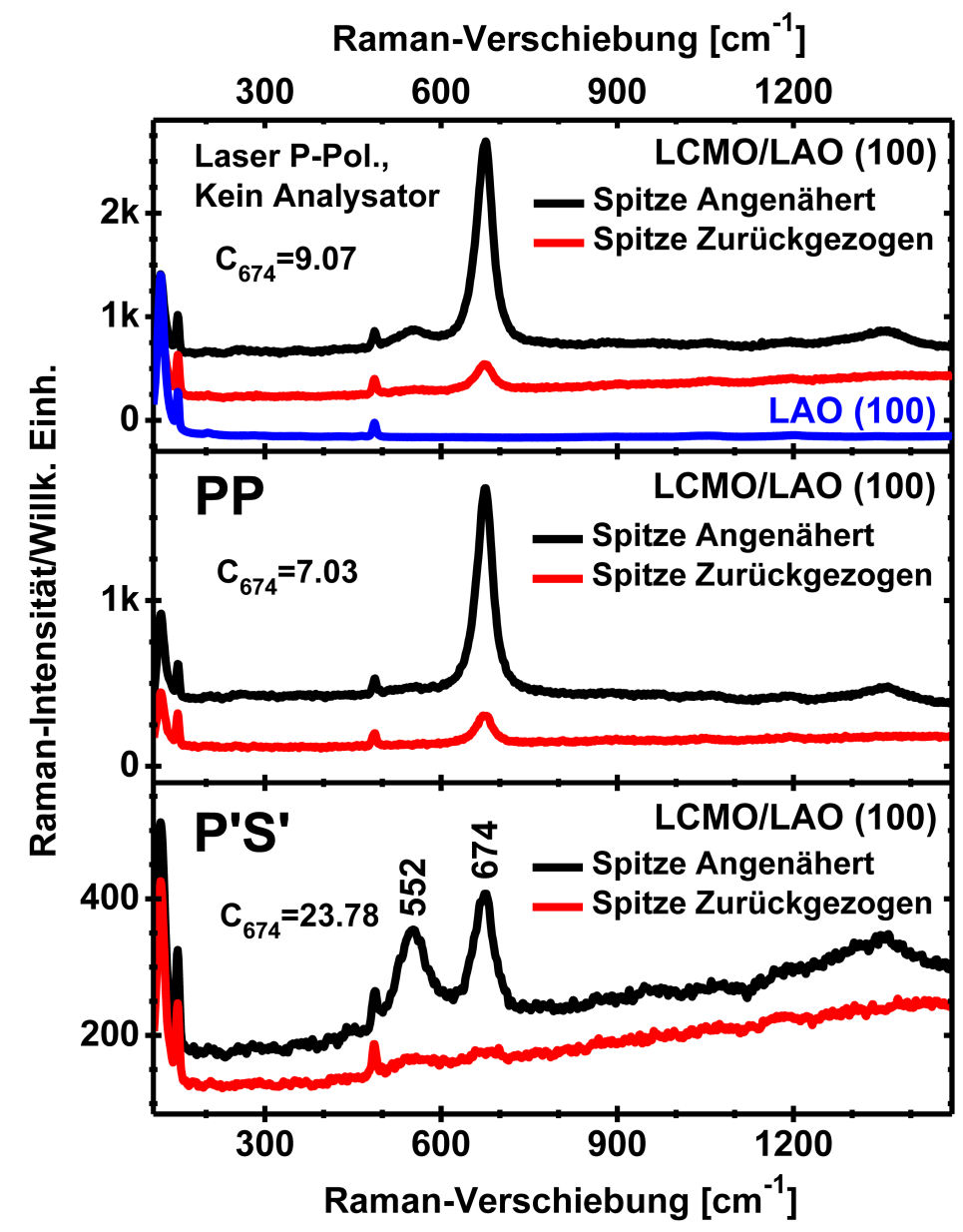

Abbildung 4.2.20: TERS- und Fernfeld-Raman-Spektren einer $12 \mathrm{~nm} \mathrm{La} \mathrm{CoMnO}_{6}$ - Schicht auf LAO (100) mit einer einfallenden P-Laserpolarisation ohne Analysator, in der parallelen PP-Konfiguration und in der gekreuzten $\mathrm{P}^{\prime} \mathrm{S}^{\prime}$-Konfiguration (in-plane Schichtrotation um $\phi=45^{\circ}$ ). Das LAO (100)-Raman-Spektrum zeigt für die Orientierung den Hintergrund durch das Substrat.

Die Fernfeld-Raman-Spektren in der Abbildung 4.2.20 (Spitze mehrere Mikrometer zurückgezogen) weisen die für eine monokline P12 $/ 2$ n1-Struktur zu erwartende Charakteristik mit einer starken $A_{g}$ Breathing Mode und einer unterdrückten $B_{g}$ Mischmode in der parallelen 
PP-Polarisationskonfigurationen sowie einem nahezu vollständigen Fehlen beider Moden in der gekreuzten $\mathrm{P}^{\prime} \mathrm{S}^{\prime}$-Konfiguration (bei einer in-plane Schichtrotation um $\phi=45^{\circ} \mathrm{um}$ die Spitzenachse herum) auf und folgen damit den Raman-Auswahlregeln beider Moden. In den TERS-Spektren (Spitze in Tunnelposition angenähert) ergibt sich eine signifikante Verstärkung des gesamten Spektrums für eine Laserpolarisation parallel zur Spitzenachse ohne Analysator, die für die parallele Konfiguration auf die $A_{g}$ Breathing Mode begrenzt ist, da in diesem Fall nur die feldverstärkende Komponente des Nahfelds, nicht aber die Nahfelddepolarisation, beiträgt. In der $\mathrm{P}^{\prime} \mathrm{S}^{\prime}$-Konfiguration lässt sich hingegen aufgrund der Nahfelddepolarisation eine sehr große Verstärkung beider Moden beobachten. Folglich weist der TERS-Kontrast eine starke Polarisationsabhängigkeit auf. In der Abbildung 4.2.20 ist der TERS-Kontrast $C_{674}$ der $A_{g}$ Breathing Mode der $12 \mathrm{~nm}$ LCMO-Schicht auf LAO (100) für die jeweilige Polarisationskonfiguration angegeben. Mit $C_{674, \mathrm{P}^{\prime} \mathrm{S}^{\prime}} \approx 24, C_{674, \mathrm{PP}} \approx 7$ und $C_{674} \approx 9$ für eine Laserpolarisation parallel (P) zur Spitzenachse ohne Analysator ordnet sich die $12 \mathrm{~nm}$ LCMO-Schicht bezüglich ihrer Schichtdicke sehr gut in die Werte der anderen untersuchten LCMO-Schichten ein. Im Hintergrund ist zusätzlich das rhomboedrische LAO-Substrat mit scharfen Raman-Moden bei $123 \mathrm{~cm}^{-1}\left(A_{1 g}\right), 152 \mathrm{~cm}^{-1}\left(E_{g}\right)$ und $487 \mathrm{~cm}^{-1}\left(E_{g}\right) 270$ 271 sichtbar. Die Substrat-Moden können in den TERS-Spektren als Indikator für die Oberflächensensitivität der plasmonischen Nahfeldverstärkung betrachtet werden, da diese in der Intensität im Gegensatz zu den Raman-Moden der LCMO-Schicht in allen Polarisationskonfigurationen nicht gegenüber dem Fernfeld verstärkt werden.

Die Schichtverspannung beeinflusst hingegen die Position der Raman-Moden. Aufgrund der in-plane Druckspannung durch das LAO-Substrat (die out-of-plane Gitterkonstante der $12 \mathrm{~nm}$ LCMO-Schicht beträgt $c_{\mathrm{pc}}=3,959 \AA$ gegenüber $c_{\mathrm{pc}}$, Bulk $=3,887 \AA 31,34$ für BPlatz geordnetes Bulk-LCMO) und der damit einhergehenden Verkleinerung der mittleren

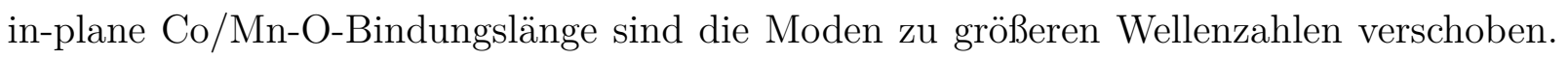
So ist die $A_{g}$ Breathing Mode der $12 \mathrm{~nm}$ LCMO-Schicht bei $674 \mathrm{~cm}^{-1}\left(644-647 \mathrm{~cm}^{-1}\right.$ für unverspanntes LCMO $33,49,215,216,219$ ) und die $B_{g}$ Mischmode bei $552 \mathrm{~cm}^{-1}$ $\left(493-500 \mathrm{~cm}^{-1}\right.$ für unverspanntes LCMO [33, 49, 215, 216, 219]) positioniert. Folglich ist die $B_{g}$ Mischmode stärker von der Verspannung in der Schicht beeinflusst. Ein Vergleich der TERS- und Fernfeld-Raman-Spektren zeigt dabei keinen Unterschied in den Raman-Modenpositionen. Dieses deutet aufgrund der Oberflächensensitivität des TERSNahfeldes auf eine einheitliche Verspannung der Schicht bis zur Oberfläche hin, da die LCMO-Schichtdicke von $d=12 \mathrm{~nm}$ noch nicht für eine Schichtrelaxation ausreicht. In der Abbildung 4.2.21 (a) ist daher das TERS- und Fernfeld-Raman-Spektrum einer $35 \mathrm{~nm}$ $\mathrm{La}_{2} \mathrm{NiMnO}_{6}$-Schicht auf LAO (100) mit einer Laserpolarisation parallel (P) zur Spitze und ohne Analysator dargestellt. Die TERS-Messungen erfolgten mit der gleichen Spitze bei einem Tunnelstrom $j=0,3 \mathrm{nA}$ und einer Spannung $U_{\text {Bias }}=0,5 \mathrm{~V}$. Die Spektren wurden bei einer Laserleistung $P \approx 10 \mathrm{~mW}(\lambda=633 \mathrm{~nm}, t=2 \times 60 \mathrm{~s})$ aufgenommen. 

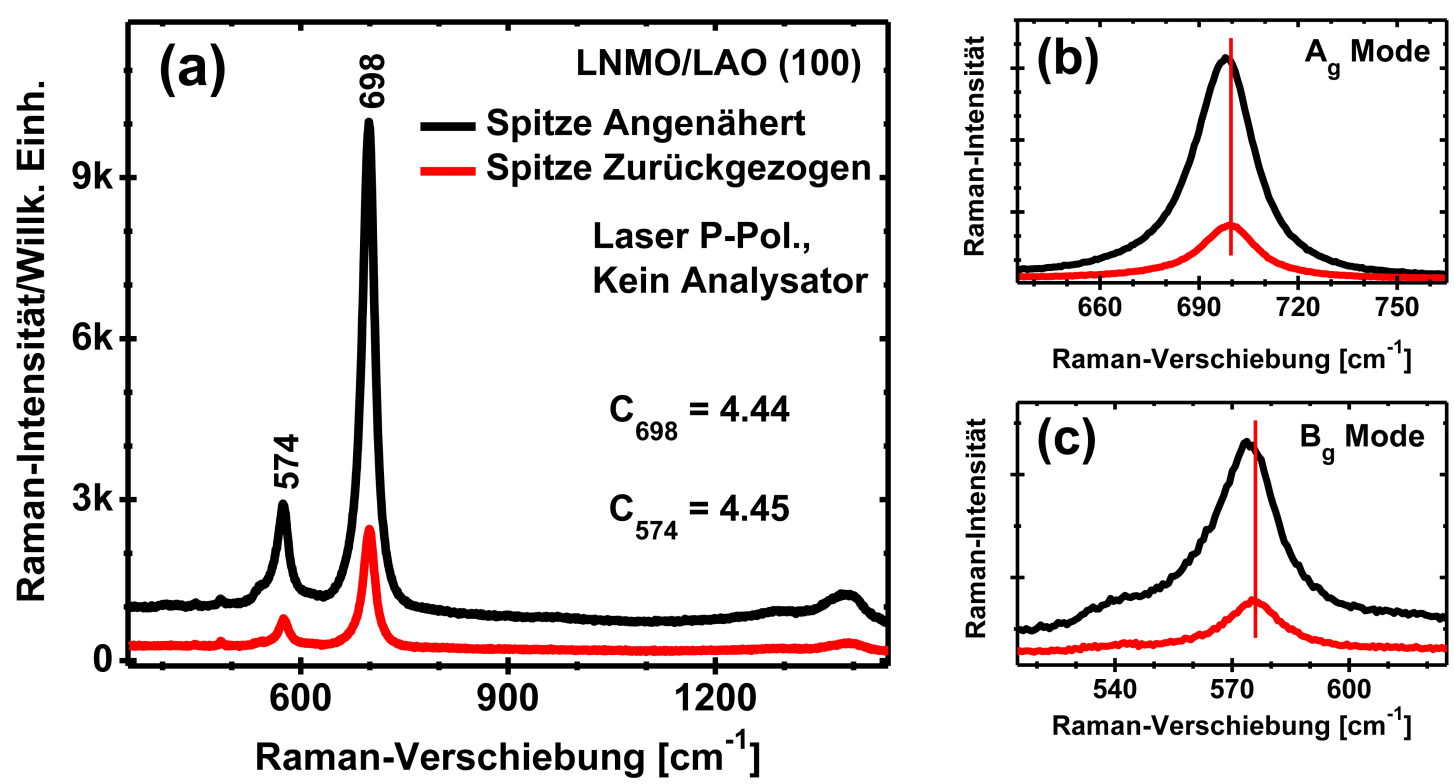

Abbildung 4.2.21: TERS- und Fernfeld-Raman-Spektrum einer $35 \mathrm{~nm} \mathrm{La} \mathrm{NiMnO}_{6}$-Schicht auf LAO (100) mit einer einfallenden P-Laserpolarisation parallel zur TERSSpitzenachse und ohne Analysator, mit den relevanten Bereichen für die $A_{g}$ Breathing Mode (b) und die $B_{g}$ Mischmode (c). Die vertikale rote Linie markiert jeweils die Modenposition im Fernfeld-Raman-Spektrum.

Ohne Analysator ist für die $35 \mathrm{~nm}$ LNMO/LAO (100)-Schicht eine gleichmäßige Verstärkung im TERS-Spektrum gegenüber dem Fernfeld zu beobachten. Der TERS-Kontrast der $A_{g}$ Breathing Mode und der $B_{g}$ Mischmode ist mit $C \approx 4,5$ identisch. Der TERS-Nahfeldcharakter wird anhand der Raman-Moden des LAO (100)-Substrats sichtbar, die im Gegensatz zu den Schichtmoden nicht verstärkt werden. Aufgrund der Schichtverspannung (die out-of-plane Gitterkonstante der $35 \mathrm{~nm}$ LNMO-Schicht beträgt $c_{\mathrm{pc}}=3,940 \AA$ gegenüber $c_{\mathrm{pc} \text {, Bulk }}=3,876 \AA$ 238 für B-Platz geordnetes Bulk-LNMO) sind die Schicht-Moden zu größeren Wellenzahlen verschoben. Ein Vergleich von TERS- und Fernfeld-RamanSpektrum zeigt zudem im Gegensatz zur 12 nm LCMO/LAO (100)-Schicht einen kleinen Unterschied in der Position der $A_{g}$ Breathing Mode und der $B_{g}$ Mischmode. Dieses ist in den Abbildungen 4.2.21 (b) und (c) dargestellt. Während die $A_{g}$ Breathing Mode im TERSSpektrum bei $698 \mathrm{~cm}^{-1}$ positioniert ist, liegt sie im Fernfeld bei $699 \mathrm{~cm}^{-1}\left(675-678 \mathrm{~cm}^{-1}\right.$ für unverspanntes LNMO 39,240,272). Die $B_{g}$ Mischmode hat im TERS-Spektrum eine Position bei $574 \mathrm{~cm}^{-1}$, während sie im Fernfeld bei $576 \mathrm{~cm}^{-1}\left(529-534 \mathrm{~cm}^{-1}\right.$ für unverspanntes LNMO [39,240,272]) liegt. Die Differenz lässt sich durch eine beginnende Relaxation der LNMO-Schicht in Richtung Oberfläche erklären. Aufgrund der Oberflächensensitivität des Nahfeldes wird diese in den TERS-Spektren stärker erfasst und die $A_{g}$ Breathing Mode und die $B_{g}$ Mischmode leicht zu kleineren Wellenzahlen verschoben. Dieses stimmt mit Raman-Messungen von LNMO auf LAO (100) überein, bei denen sich bei Vergrößerung der Schichtdicke ebenfalls eine Verschiebung der Raman-Modenpositionen zu kleineren Wellenzahlen und eine Annäherung an die Bulk-LNMO-Positionen ergibt 272, 273]. 


\section{3 $\mathrm{La}_{1-\mathrm{x}} \mathrm{Sr}_{\mathrm{x}} \mathrm{MnO}_{3}$-Schichten $(0 \leq x \leq 0,3)$}

Im Anschluss sollen nun noch dünne $\mathrm{La}_{1-\mathrm{x}} \mathrm{Sr}_{\mathrm{x}} \mathrm{MnO}_{3}$-Schichten (LSMO) mit einer moderaten Strontium-Dotierung $(0 \leq x \leq 0,3)$ bezüglich ihrer Vielfalt an strukturellen, elektrischen und magnetischen Phasen in diesem Bereich des $T$ - $x$-Phasendiagramms betrachtet werden. Hierbei dient erneut die Raman-Spektroskopie aufgrund der hohen Sensitivität für strukturelle Änderungen als zentrale Charakterisierungsmethode. Neben der Entwicklung der Raman-Spektren der LSMO-Schichten mit der Dotierung $x$ und der Temperatur $T$ soll dabei insbesondere ein möglicher Einfluss des eingestrahlten Laserlichts auf die strukturelle Phase der Schichten und damit einhergehende Ordnungsphänomene diskutiert werden.

\subsubsection{Schichtwachstum und allgemeine Charakterisierung}

Der Ausgangspunkt für das Wachstum von LSMO-Schichten von hoher Qualität mit der MAD ist zu Beginn erneut das Einstellen und Optimieren der MAD-Wachstumsparameter. Hierbei kann zunächst mit dem undotierten LMO $(x=0)$ begonnen werden, da die optimale LMO-Zusammensetzung der Präkursoren bereits von der Herstellung der LCMOund LNMO-Doppelperowskite bekannt ist. Das stöchiometrische La/Mn-Verhältnis des Laund Mn-Präkursors (Korrekturfaktor $k_{\mathrm{La} / \mathrm{Mn}}$, Gleichung (3.1.5) ) lässt sich anschließend aufgrund der sehr ähnlichen Effektivität von La- und Sr-Präkursor beim Schichtwachstum für die dotierten LSMO-Schichten auf das $\mathrm{Sr} / \mathrm{Mn}$-Verhältnis $\left(k_{\mathrm{La} / \mathrm{Sr}} \approx 1\right)$ übertragen, indem ein gemeinsames A/B-Verhältnis mit demselben Korrekturfaktor $k_{\mathrm{A} / \mathrm{B}}$ der beiden Präkursoren in Relation zum Mangan-Präkursor verwendet wird. Die Dotierung selbst lässt sich dann direkt über das stöchiometrische La/Sr-Verhältnis $x_{\mathrm{La} / \mathrm{Sr}}$ bestimmen und anpassen. Ebenso wichtig für das Schichtwachstum ist die Wahl des Substrats, da die Schichten später mithilfe der Raman-Spektroskopie untersucht werden sollen und die Substrate demnach im für LSMO relevanten Wellenzahlbereich keine hohe Raman-Aktivität aufweisen dürfen. Dieses ist insbesondere notwendig, da der jeweilige Raman-Wirkungsquerschnitt und damit die Intensität der bei LSMO auftretenden Raman-Moden im Vergleich zu der $A_{g}$ Breathing Mode der Doppelperowskite sehr viel geringer ist. Zur Reduzierung von Heizeffekten bei der Untersuchung des Effekts des eingestrahlten Laserlichtintensität auf die strukturellen Eigenschaften der LSMO-Schichten ist es zudem erforderlich, die Schichtdicke zu begrenzen, sodass das Streuvolumen für die Raman-Anregung weiter verringert wird. Folglich werden die LSMO-Schichten im Folgenden sowohl auf MgO (100)-Substrate, die aufgrund der kubischen NaCl-Struktur von $\mathrm{MgO}$ in einkristalliner Substratform im Allgemeinen keine Raman-Aktivität, sondern nur einen flachen Hintergrund zeigen [265], als auch auf rhomboedrisch verzerrte LAO (100)-Substrate, mit drei scharfen Raman-Moden bei $123 \mathrm{~cm}^{-1}\left(A_{1 g}\right), 152 \mathrm{~cm}^{-1}\left(E_{g}\right)$ und $487 \mathrm{~cm}^{-1}\left(E_{g}\right)$ 270, 271, aufgewachsen. Die Schichtdicke $d \approx 30 \mathrm{~nm}$ der LSMO-Schichten wurde dabei über die Menge des Lösungsvolumens (bei gleicher Lösungskonzentration und Substrattemperatur $T \approx 1000{ }^{\circ} \mathrm{C}$ ) mithilfe 


\section{Auswertung und Ergebnisse}

der Röntgenreflektometrie und einer entsprechenden Schichtdickenserie eingestellt. In der Abbildung 4.3.1 sind nun die XRD-Spektren der $\mathrm{La}_{1-\mathrm{x}} \mathrm{Sr}_{\mathrm{x}} \mathrm{MnO}_{3} / \mathrm{MgO}$ (100)-Schichten für verschiedene Sr-Dotierungen $x$ in der Bragg-Brentano-Geometrie im Vergleich dargestellt. Das in grau gezeigte MgO (100)-Spektrum dient hierbei als eine direkte Orientierung für den vom Substrat stammenden Beitrag. Die drei mit den Sternen markierten SubstratLinien links des (200)-Reflexes des MgO-Substrats resultieren hingegen auch hier von der nicht vollständig monochromatischen $\mathrm{Cu}_{\mathrm{K}_{\alpha}}$-Strahlung der Röntgenröhre und können charakteristischer $\mathrm{Cu}_{\mathrm{K}_{\beta}-}, \mathrm{W}_{\mathrm{L}_{\alpha}-}$ und $\mathrm{W}_{\mathrm{L}_{\beta}}$-Röntgenstrahlung zugeordnet werden. Die LSMOSchichten auf MgO (100) zeigen wiederum mit dem (100)-Reflex und dem (200)-Reflex in dem betrachteten 2 $\theta$-Winkelbereich lediglich Schichtreflexe mit einer ( $h 00$ )-Orientierung. Entsprechend weisen die Schichten somit alle eine vollständige out-of-plane Epitaxie auf.

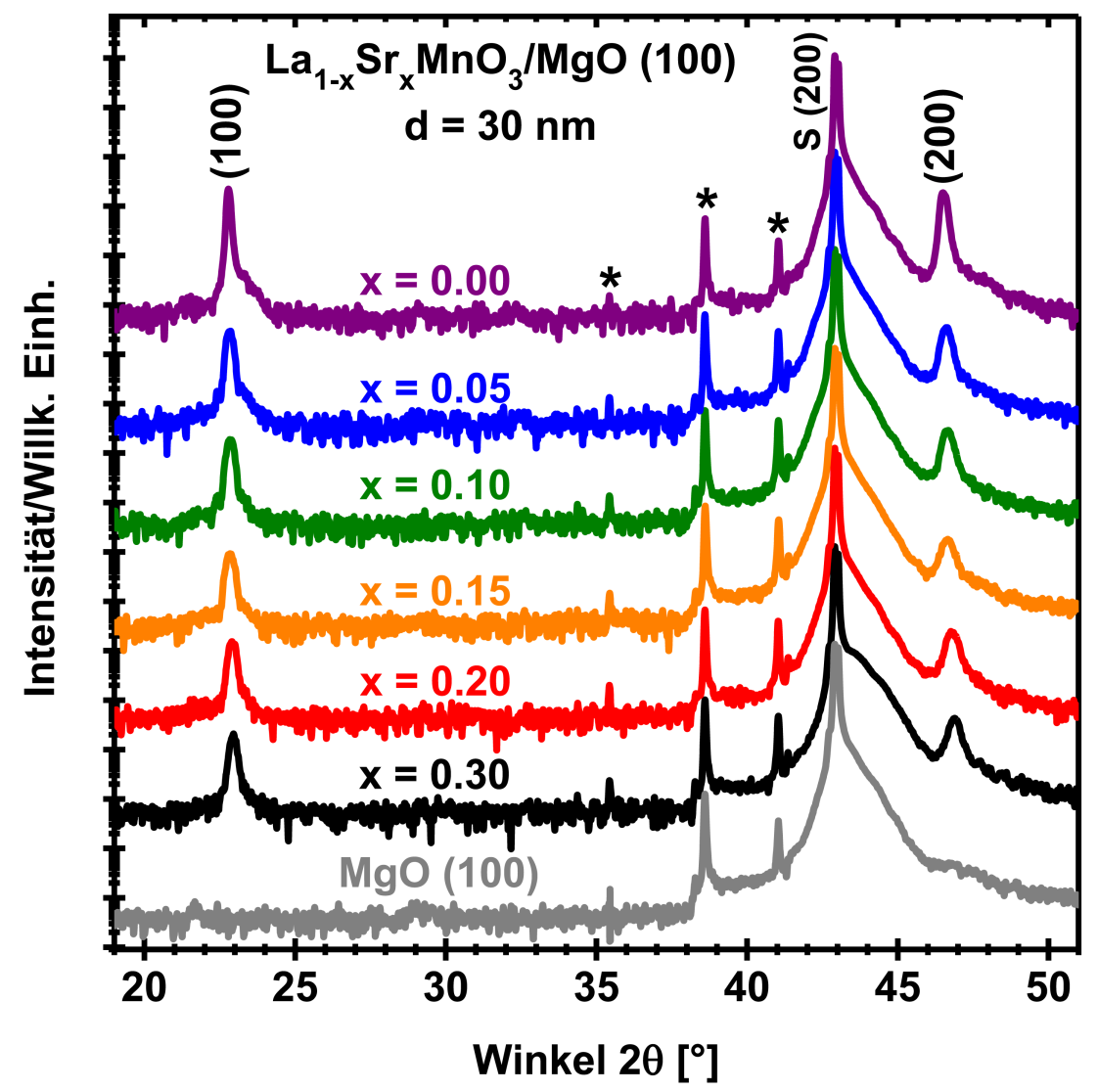

Abbildung 4.3.1: XRD-Spektren der $\mathrm{La}_{1-\mathrm{x}} \mathrm{Sr}_{\mathrm{x}} \mathrm{MnO}_{3} / \mathrm{MgO}$ (100)-Schichten mit einer vergleichbaren Schichtdicke $d \approx 30 \mathrm{~nm}$ für verschiedene Sr-Dotierungen $x$ in der Bragg-Brentano-Geometrie im Vergleich. Das MgO (100)-Spektrum dient als Orientierung für den Substratbeitrag (grau). Die jeweils mit den Sternen gekennzeichneten Substrat-Linien resultieren auch hier aus der nicht völlig monochromatischen $\mathrm{Cu}_{\mathrm{K}_{\alpha}}$-Strahlung der Röntgenröhre.

Die aus der Position der Schichtreflexe bestimmten pseudokubischen Gitterkonstanten $a_{\mathrm{pc}}$ der LSMO/MgO (100)-Schichten sind nun in Abhängigkeit der jeweiligen Sr-Konzentration in der Abbildung 4.3.2 dargestellt. Hierbei ergibt sich eine systematische Abnahme von $a_{\mathrm{pc}}$ mit ansteigender Sr-Konzentration, die auf den unterschiedlichen Kationenradius von 
HS-Mn ${ }^{3+}$ und $\mathrm{Mn}^{4+}\left(\right.$ mit $r_{\mathrm{Mn}^{3+}}=0,645 \AA$ und $r_{\mathrm{Mn}^{4+}}=0,53 \AA$ für $\left.\mathrm{KZ}=6\right)[222]$ zurückzuführen ist und mit einem linearen Verlauf angenähert werden kann. Ein Vergleich mit den pseudokubischen Gitterkonstanten $a_{\mathrm{pc}}$, Bulk $=3,90 \AA$ für unverspanntes Bulk-LMO 6 und $a_{\mathrm{pc}, \text { Bulk }}=3,875 \AA$ für unverspanntes Bulk-LSMO $(x=0,30) 225,274$ (die jeweils rot gestrichelten Linien in der Abbildung 4.3.2 lässt aufgrund der erzielten Übereinstimmung mit den jeweiligen Schichtwerten $a_{\mathrm{pc}}=3,902(2) \AA(x=0)$ und $a_{\mathrm{pc}}=3,875(2) \AA(x=0,30)$ zudem darauf schließen, dass die LSMO-Schichten trotz der nur geringen Schichtdicke von $d=30 \mathrm{~nm}$ nahezu unverspannt auf den MgO (100)-Substraten aufwachsen. Dieses kann mit der großen in-plane Gitterfehlanpassung von 7,5-8,1 \% je nach Sr-Dotierung $x$ von LSMO zur Gitterkonstante $a_{\mathrm{MgO}}=4,216 \AA$ 225] von MgO (100) begründet werden. Diese Fehlanpassung führt in den ersten Lagen der Schicht zu Versetzungen, bevor die Schicht anschließend epitaktisch und völlig unverspannt weiter aufwächst. Dieses Verhalten tritt insbesondere dann auf, wenn die jeweiligen in-plane Substrat- und Schichtgitterkonstanten in einem passenden Verhältnis $n \cdot a_{\text {Substrat }} \approx m \cdot a_{\text {Schicht }}$ zueinander stehen und wird daher als Domain Matching Epitaxy (DME) [275] bezeichnet. Die Verspannung ist bei LSMO (mit $x=0.3)$ auf $\mathrm{MgO}(100)$ gerade für ein Verhältnis $\frac{m}{n}=\frac{12}{11}$ und bei LMO auf $\mathrm{MgO}(100)$ für ein Verhältnis $\frac{m}{n}=\frac{13}{12}$ minimiert. Eine ähnliches Verhalten zeigt sich auch bei dem epitaktischen Wachstum von $\mathrm{La}_{2} \mathrm{CoMnO}_{6}$-Schichten auf $\mathrm{Al}_{2} \mathrm{O} 3$ (0001) (Abschnitt 4.1.3).

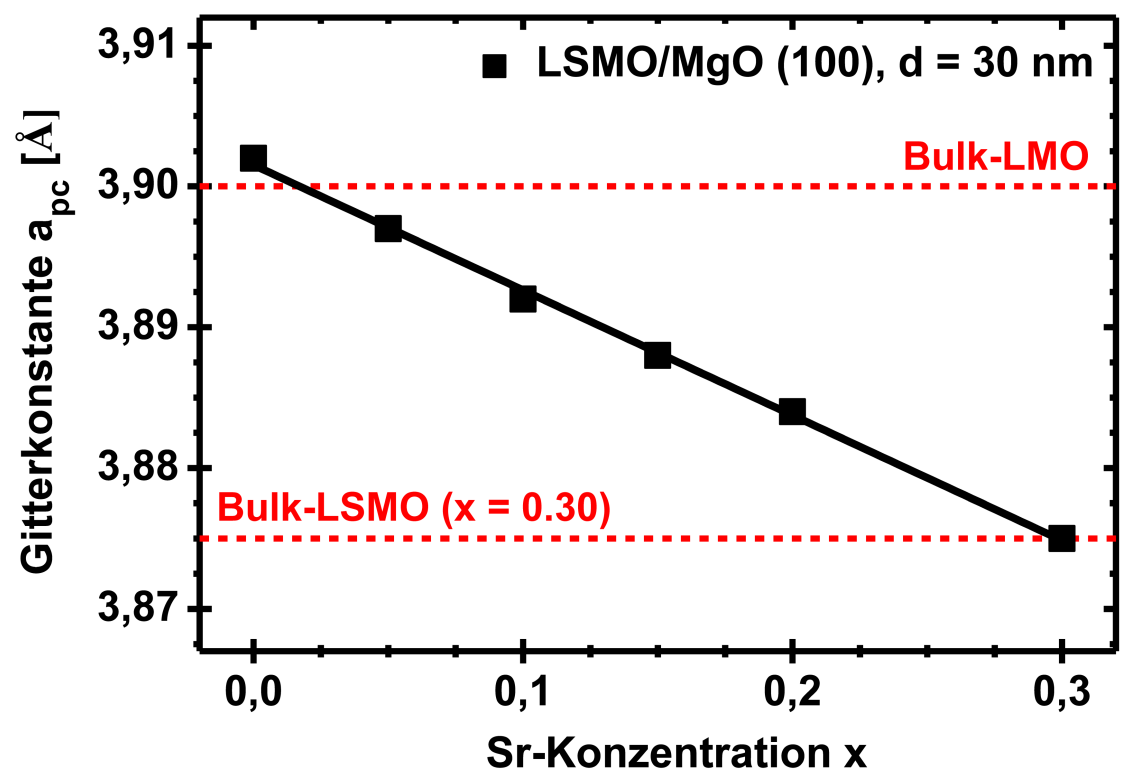

Abbildung 4.3.2: Aus den XRD-Spektren bestimmte pseudokubische out-of-plane Gitterkonstanten $a_{\mathrm{pc}}$ der LSMO/MgO (100)-Schichten $(d=30 \mathrm{~nm})$ in Abhängigkeit der Sr-Konzentration. Es ergibt sich einer lineare Abnahme von $a_{\mathrm{pc}}$ mit ansteigender Sr-Dotierung. Die rot gestrichelten Linien kennzeichnen die jeweiligen pseudokubischen Bulk-Werte für LMO und LSMO mit $x=0,30$.

In gleicher Weise können nun die LSMO-Schichten mit einer Schichtdicke von ebenfalls $d \approx 30 \mathrm{~nm}$, die mit den Sr-Konzentrationen $x=0,05$ und $x=0,10$ auf LAO (100)Substraten aufgewachsen wurden, betrachtet werden. Bis auf eine leichte Erhöhung des 
A/B-Verhältnisses der Präkursoren (Korrekturfaktor $k_{\mathrm{A} / \mathrm{B}}$ ) und einer Reduzierung der Substrattemperatur auf $T \approx 800{ }^{\circ} \mathrm{C}$ erfolgte das Wachstum auf LAO (100) unter den gleichen Bedingungen (bezüglich Lösungskonzentration und Wachstumsgeschwindigkeit) wie auf MgO (100). Die XRD-Spektren der Schichten sind in der Abbildung 4.3.3 dargestellt. Das in grau gezeigte LAO (100)-Spektrum dient erneut zur Orientierung des vom Substrat stammenden Beitrags. Die aus der nicht vollständig monochromatischen $\mathrm{Cu}_{\mathrm{K}_{\alpha}}$-Strahlung resultierenden Substrat-Zusatzlinien sind wieder mit Sternen gekennzeichnet. Die Spektren der LSMO/LAO (100)-Schichten zeigen mit dem (100)-Reflex und dem (200)-Reflex in dem betrachteten 2 $\theta$-Winkelbereich ebenfalls nur Schichtreflexe mit einer ( $h 00)$-Orientierung. Folglich besitzen auch diese Schichten eine vollständige out-of-plane Epitaxie.

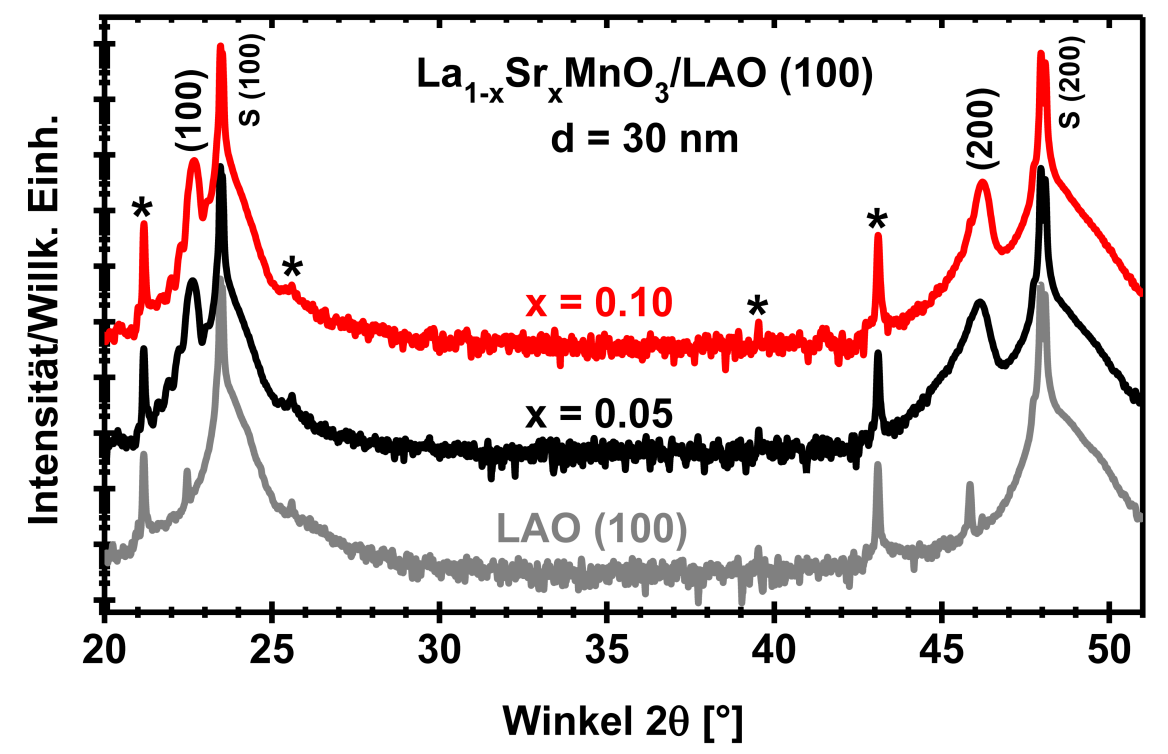

Abbildung 4.3.3: XRD-Spektren der $\mathrm{La}_{1-\mathrm{x}} \mathrm{Sr}_{\mathrm{x}} \mathrm{MnO}_{3} / \mathrm{LAO}$ (100)-Schichten mit einer vergleichbaren Schichtdicke $d \approx 30 \mathrm{~nm}$ für die Sr-Dotierungen $x=0,05$ und $x=0,10$ in der Bragg-Brentano-Geometrie. Das LAO (100)-Spektrum dient auch hier als Orientierung für den Substratbeitrag (grau), während die mit den Sternen markierten Reflexe wieder von der nicht vollständig monochromatischen $\mathrm{Cu}_{\mathrm{K}_{\alpha}}$-Strahlung der zum Einsatz kommenden Röntgenröhre stammen.

Im Vergleich zu den LSMO-Schichten auf MgO (100) mit gleicher Sr-Konzentration sind die Schichtreflexe der LSMO/LAO (100)-Schichten jedoch signifikant zu kleineren 2 $\theta$-Winkeln verschoben. Die aus den Positionen der Schichtreflexe bestimmten pseudokubischen Gitterkonstanten sind daher mit $a_{\mathrm{pc}}=3,930(2) \AA(x=0,05)$ und $a_{\mathrm{pc}}=3,921(2) \AA(x=0,10)$ deutlich gegenüber denen der LSMO-Schichten auf $\mathrm{MgO}$ (100) und dem Wert für BulkLMO erhöht. Folglich sind die LSMO-Schichten verspannt auf den LAO (100)-Substraten aufgewachsen. Aufgrund der kleineren Gitterkonstante von LAO in Relation zu LMO $\left(a_{\mathrm{MgO}}=3,788 \AA 225\right.$; die Gitterfehlanpassung zu Bulk-LMO beträgt somit $\left.-3 \%\right)$ wird dabei durch das Substrat eine in-plane Druckspannung in die Schichten induziert, die outof-plane durch eine Zugspannung mit der Zunahme der dazugehörigen Gitterkonstante kompensiert wird. Da die Schichtdicke mit nur $d \approx 30 \mathrm{~nm}$ für einen Relaxationsprozess 
noch nicht ausreicht, ergibt sich ein starker Verspannungszustand in der gesamten Schicht. Dieser führt anhand der Gleichungen (3.2.9) und 3.2.10 zu einer Verschiebung der Schichtreflexe zu kleineren $2 \theta$-Winkeln. Daneben lässt sich aus der Breite $b_{h k l}$ der Schichtreflexe mit der Scherrer-Gleichung (Gleichung (3.2.11) die Kristallitgröße $D_{h k l}$ senkrecht zu der betrachteten Reflexgitterebene bestimmen. Bezogen auf den (100)-Reflex ergibt sich für beide Schichten auf LAO (100) eine Halbwertsbreite von $b_{100} \approx 0,22^{\circ}$, woraus eine Kristallitgröße $D_{100}=36,8(5) \mathrm{nm}$ folgt. Die Kristallitgröße ist somit in der Größenordnung der eigentlichen Schichtdicke der beiden Schichten. Folglich besitzen die zwei LSMO-Schichten auf LAO (100) eine sehr hohe Schichtqualität und Kristallinität, die von der starken Verspannung unbeeinträchtigt ist. Dieses äußert sich auch darin, dass die LSMO-Schichten auf $\mathrm{MgO}$ (100) mit gleicher Sr-Konzentration eine nahezu identische Reflexbreite und Kristallitgröße aufweisen. Ein Kennzeichen der hohen kristallinen Qualität der LSMO-Schichten auf LAO (100) ist zudem das Auftreten von Laue-Oszillationen in der Umgebung der Schichtreflexe. In den Abbildungen 4.3.4 (a) und (b) ist daher jeweils der (100)-Reflex der beiden LSMO/LAO (100)-Schichten mit seinem spektralen Umfeld dargestellt.
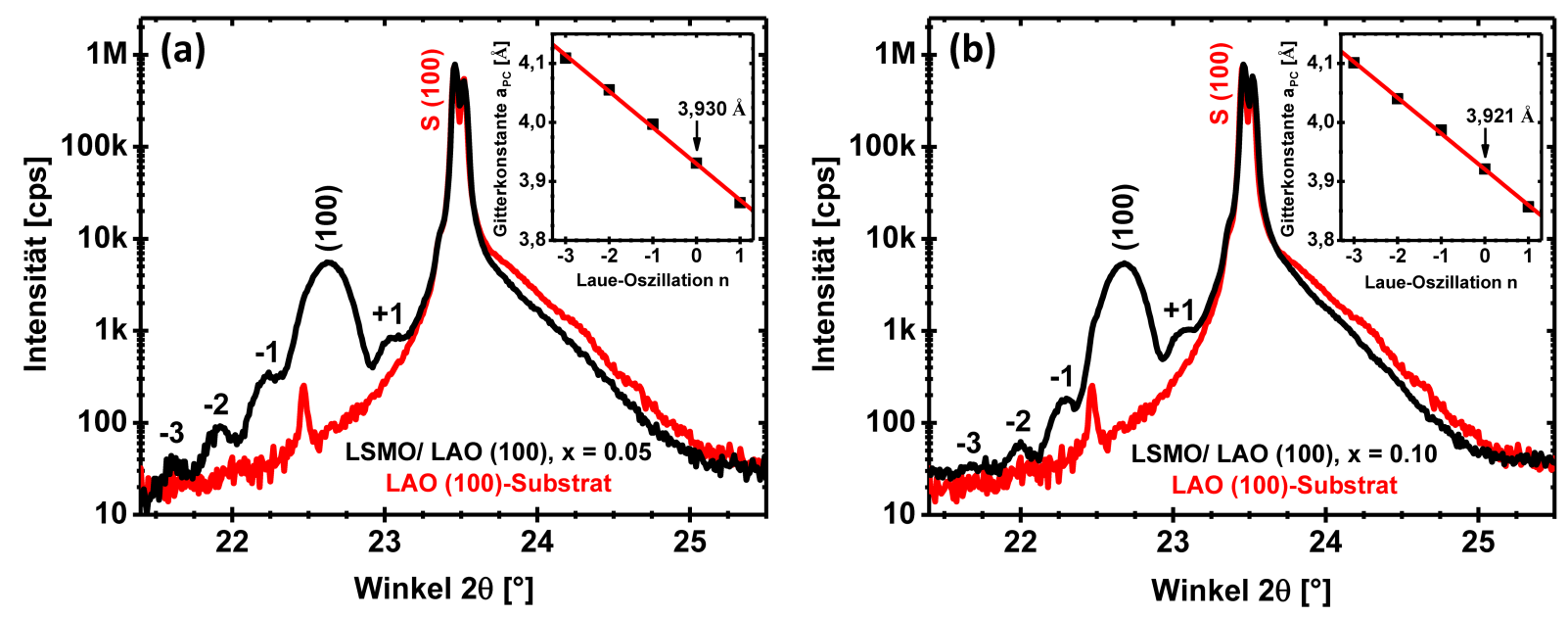

Abbildung 4.3.4: XRD-Spektren der beiden $30 \mathrm{~nm}$ LSMO/LAO (100)-Schichten mit der SrKonzentrierung $x=0,05$ (a) und $x=0,10$ (b) im Umfeld des (100)Schichtreflexes. Aus der linearen Positionsabhängigkeit der Maxima der LaueOszillationen mit der Ordnung $n$ lässt sich direkt die out-of-plane Gitterkonstante $a_{\mathrm{pc}}$ der Schichten bestimmen (eingebettete Graphen).

Aus der linearen Abhängigkeit der Positionen der Maxima der Laue-Oszillationen mit der Ordnung $n$ um den (100)-Schichtreflex herum ergibt sich bei $n=0$ die out-of-plane Gitterkonstante der $a_{\mathrm{pc}}$ der LSMO/LAO (100)-Schichten. Die Ergebnisse für die beiden Schichten sind in den Abbildungen 4.3.4 (a) und (b) eingebettet. Zusätzlich kann mithilfe der Gleichung (3.2.13) aus dem Abstand zweier Laue-Oszillationen $\Delta_{(n+1)-n}$ die jeweilige Schichtdicke $d$ bestimmt werden. Es ergibt sich eine nahezu identische Schichtdicke von $d=29,1(5) \mathrm{nm}$ für beide LSMO/LAO (100)-Schichten. Dieser Wert passt sehr gut zu dem aus den XRR-Messungen (Kiesig-Oszillationen) ermittelten Wert von $d=29,5(1) \mathrm{nm}$. Da 


\section{Auswertung und Ergebnisse}

die aus den Abständen der Laue-Oszillationen bestimmte Schichtdicke den kristallin geordneten Teil der Schicht beschreibt [67], wird für die beiden LSMO-Schichten aufgrund der Übereinstimmung mit der Schichtdicke aus den XRR-Messungen die besonders hohe Schichtqualität erneut bestätigt. Die Oberflächen der LSMO-Schichten können wiederum mit dem AFM betrachtet werden. In den Abbildungen 4.3.5 (a) und (b) sind nun die AFMBilder von den Schichtoberflächen der zwei LSMO-Schichten mit $x=0,05$ auf MgO (100) und LAO (100) mit einer Schichtdicke $d=30 \mathrm{~nm}$ dargestellt. Beide Schichten zeigen hierbei eine glatte Oberflächenmorphologie mit RMS-Rauigkeitswerten von nur RMS =0,69 nm auf $\mathrm{MgO}$ (100) und RMS = 0,33 nm auf LAO (100). Die Korngröße an der Oberfläche ist jedoch für die LSMO-Schicht auf MgO (100) gegenüber der auf LAO (100) deutlich erhöht. Dieses lässt sich auf die Wachstumsart der Schichten und die unterschiedlichen Keimbildungsbedingungen beim Schichtwachstum zurückführen. Während die Schichten auf MgO durch ein unverspanntes DME-Wachstum mit großen Körnern gekennzeichnet sind, wachsen die Schichten auf LAO in einem starken Verspannungszustand mit einer feineren Struktur auf. Die AFM-Bilder sind dabei repräsentativ für die anderen LSMO-Schichten, für die sich auf den jeweiligen Substraten eine vergleichbare Oberflächenmorphologie ergibt.
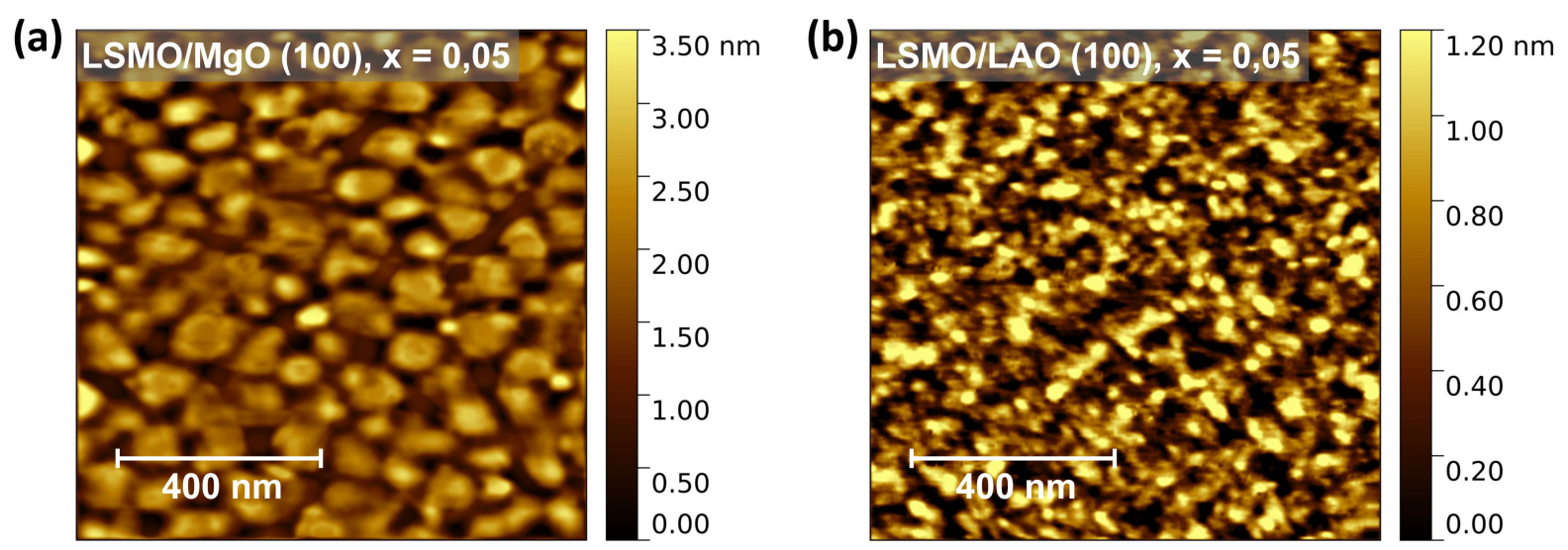

Abbildung 4.3.5: AFM-Aufnahmen von den $30 \mathrm{~nm}$ LSMO-Schichten mit einer Sr-Dotierung $x=0,05$ auf $\mathrm{MgO}$ (100) (a) und LAO (100) (b) im Vergleich.

Daneben müssen für eine Einordnung in das T-x-LSMO-Phasendiagramm (Abschnitt 2.2.3) auch die elektrischen und magnetischen Eigenschaften der hergestellten LSMO-Schichten betrachtet werden. Für LMO kann dadurch insbesondere das korrekte La/Mn-Verhältnis und der Sauerstoffgehalt überprüft werden. So ist stöchiometrisches Bulk-LMO ein antiferromagnetischer (A-Typ) Mott-Hubbard-Isolator $\left(T_{N}=140 \mathrm{~K}\right.$ [16, 26]). Ein Sauerstoffüberschuss $\left(\mathrm{LaMnO}_{3+\delta}, \delta>0\right)$, der allgemein jedoch nicht durch ein entsprechendes Einbringen auf die Zwischengitterplätze, sondern durch das Erzeugen einer äquivalenten Anzahl von Kationenleerstellen auf den La- und Mn-Plätzen $\left(\mathrm{La}_{1-\xi} \mathrm{Mn}_{1-\varepsilon} \mathrm{O}_{3}\right.$ mit $\varepsilon+\xi \approx 2 \delta / 3$ und $\xi>\varepsilon)$ realisiert wird [276, 277], oder ein Lanthan-Defizit $\left(\mathrm{La}_{1-\mathrm{z}} \mathrm{MnO}_{3}, z>0\right)$ 8, 278] führen zu einem nicht-stöchiometrischen LMO mit einer $\mathrm{Mn}^{3+} / \mathrm{Mn}^{4+}-$ Mischvalenz. Dieses hat große Auswirkungen auf die strukturellen, elektrischen und magnetischen Eigenschaften. 
So lässt sich für einen Sauerstoffüberschuss $\delta>0,05$ ein Übergang von LMO von der orthorhombischen Pbnm- in die rhomboedrische R $\overline{3}$ c-Struktur beobachten [276, 277, 279], der mit einem ferromagnetischen Grundzustand einhergeht und für $\delta>0,13$ zusätzlich einen Metall-Isolator-Übergang (engl.: metal-insulator transition, MIT) zeigt [276, 277, 279]. In gleicher Weise führt ein Lanthan-Defizit $z>0$ zu einem ferromagnetischen Grundzustand, der von einem MIT begleitet wird [8, 278. Die Curie-Temperatur nimmt dabei mit der Nichtstöchiometrie bis auf $T_{C} \approx 240 \mathrm{~K}$ für $\delta=0,18$ oder $z=0,25 \mathrm{zu} 276$ 278. Für dünne Schichten ist zudem der Einfluss der in-plane durch das Substrat induzierten Verspannung zu beachten. Diese kann je nach Ausprägung die orbitale Ordnung im LMO derart verändern, dass eine ferromagnetische Ordnung bevorzugt wird 280,281]. Folglich ist für stöchiometrische LMO-Schichten ein ferromagnetischer Grundzustand mit einer je nach Herstellung, Sauerstoffpartialdruck und Verspannung variierenden Curie-Temperatur von $T_{C} \approx 85-175 \mathrm{~K}$ zu beobachten [48, 278, 282,284]. Die temperaturabhängigen $M(T)$ Magnetisierungskurven (SQUID, FCC mit $H=100$ Oe) der $30 \mathrm{~nm}$ LSMO-Schichten auf $\mathrm{MgO}$ (100) und LAO (100) sind in den Abbildungen 4.3.6 (a) und (b) dargestellt. Für einen Vergleich sind diese auf die maximal gemessene Magnetisierung $M_{\max }$ normiert.
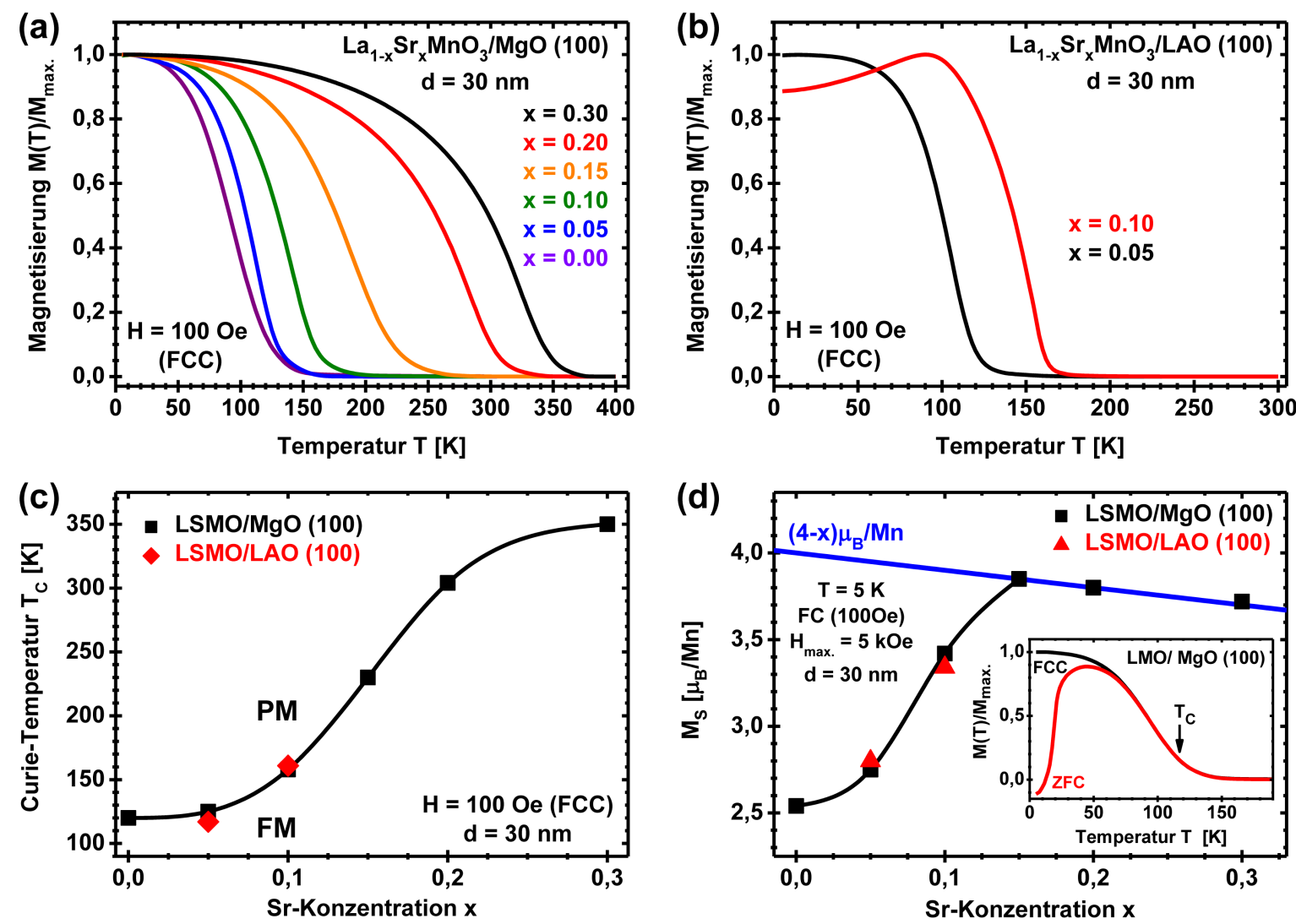

Abbildung 4.3.6: Temperaturabhängige Magnetisierungskurven $M(T)$ von den $30 \mathrm{~nm}$ LSMOSchichten auf $\mathrm{MgO}$ (100) (a) und LAO (100) (b) (FCC, $H=100$ Oe) sowie die Entwicklung der Curie-Temperatur $T_{C}(\mathrm{c})$ und der jeweiligen Sättigungsmagnetisierung $M_{S}(\mathrm{~d})\left(T=5 \mathrm{~K}, H_{\max .}=5 \mathrm{kOe}\right)$ in Abhängigkeit der Sr-Dotierung $x$. Die eingebettete Grafik zeigt einen Vergleich der FCC- und der ZFC- $M(T)$-Kurve $(H=100$ Oe) der LMO/MgO (100)-Schicht. 


\section{Auswertung und Ergebnisse}

Es zeigt sich, dass alle LSMO-Schichten unabhängig vom Substrat und der Sr-Dotierung einen ferromagnetischen Grundzustand aufweisen. Für die Curie-Temperatur $T_{C}$ lässt sich ein systematischer Anstieg mit der Erhöhung der Sr-Konzentration von $T_{C}=120(2) \mathrm{K}$ für $x=0,00$ auf $T_{C}=350(2) \mathrm{K}$ für $x=0,30$ beobachten. Der entsprechende $T_{C}(x)$-Verlauf ist in der Abbildung 4.3.6 (c) dargestellt (wobei die $T_{C}$-Werte aus dem Minimum der Funktion des Temperaturkoeffizienten TCM $=(1 / M)(\mathrm{d} M / \mathrm{d} T)$ bestimmt wurden [36]). Der in dem $T$ - $x$-Phasendiagramm für Bulk-LSMO (Abbildung 2.2.4 beobachtete antiferromagnetische Grundzustand für Sr-Dotierungen $x \leq 0,10$ fehlt somit. Ein Vergleich mit dünnen LMO-Schichten in der Literatur bestätigt wiederum einen ferromagnetischen Grundzustand als Folge einer durch das Substrat induzierten Verspannung 280 284], der in seiner Ausprägung zusätzlich von der Stöchiometrie [278], vom Sauerstoffpartialdruck während und nach der Schichtherstellung 282,283, sowie von der Schichtdicke 284 beeinflusst ist. Für die LSMO-Schichten mit $x \geq 0,15$ stimmt der ferromagnetische Grundzustand und der $T_{C}(x)$-Verlauf mit Bulk-LSMO überein. Die im Vergleich dazu leicht reduzierten CurieTemperaturen (beispielsweise $T_{C}=350(2) \mathrm{K}$ gegenüber $T_{C} \approx 370 \mathrm{~K} 16$, 27 für $x=0,3$ ) lassen sich dabei auf den Einfluss von Verspannungs- und Grenzflächeneffekten zwischen dem Substrat und der nur $30 \mathrm{~nm}$ dicken LSMO-Schicht zurückführen [50,285].

Im Vergleich zu mit der MAD hergestellten LMO-Schichten mit einer ähnlichen Schichtdicke auf STO (100), die eine Curie-Temperatur von $T_{C} \approx 175 \mathrm{~K}$ aufweisen 48, 50, sind die $T_{C}$-Werte der unverspannten LSMO-Schichten auf $\mathrm{MgO}(100)$ mit $x \leq 0,10$ deutlich reduziert. Andererseits bestätigt dieses den großen Einfluss der Verspannung auf den ferromagnetischen Grundzustand niedrig-dotierter LSMO-Schichten. Durch die fehlende Verspannung nehmen diese auf $\mathrm{MgO}$ (100) dabei offenbar einen weniger stabilen ferromagnetischen Grundzustand ein, der zwischen dem antiferromagnetischen Grundzustand von Bulk-LMO und dem ferromagnetischen Grundzustand von LMO/STO (100) eingeordnet werden kann. Dieses zeigt auch die jeweilige Sättigungsmagnetisierung $M_{S}$ der Schichten. Diese ist für die LSMO-Schichten auf MgO (100) und LAO (100) in Abhängigkeit der Sr-Dotierung $x$ in der Abbildung 4.3.6 (d) dargestellt (gemessen bei $T=5 \mathrm{~K}$ und $H_{\text {max. }}=5 \mathrm{kOe}$, nach FCC mit $H=100$ Oe). Die blaue Gerade mit $M_{S}=(4-x) \mu_{\mathrm{B}} / \mathrm{Mn}$ kennzeichnet hierbei den theoretischen Verlauf der maximalen Sättigungsmagnetisierung durch die Spin-Beiträge der $\mathrm{Mn}^{3+}$ - und $\mathrm{Mn}^{4+}$-Kationen. Während die LSMO-Schichten auf $\mathrm{MgO}(100)$ mit $x \geq 0,15$ diese erreichen, zeigt sich für $x \leq 0,10$ sowohl auf $\mathrm{MgO}$ (100) als auch auf LAO (100) eine gegenüber diesem Idealwert deutlich reduzierte Sättigungsmagnetisierung, die bis auf $M_{S} \approx 2,5 \mu_{\mathrm{B}} / \mathrm{Mn}$ für die LMO/MgO (100)-Schicht zurückgeht. Dieses Verhalten ist ähnlich zu Bulk-LSMO-Kristallen, für die eine drastisch verringerte Sättigungsmagnetisierung für Dotierungen $x \leq 0,10$ bis auf $M_{S} \approx 0,5 \mu_{\mathrm{B}} / \mathrm{Mn}$ 276,286 für reines LMO aufgrund eines gekantet-antiferromagnetischen Grundzustands zu beobachten ist. Da zudem für LMO/STO (100)-MAD-Schichten je nach Stöchiometrie eine signifikant 
höhere Sättigungsmagnetisierung $M_{S} \approx 3,4-4,0 \mu_{\mathrm{B}} / \mathrm{Mn}$ [48,50] erreicht wird, lassen sich die LSMO-Schichten auf MgO (100) und LAO (100) mit $x \leq 0,10$ genau dazwischen einordnen. Dieses zeigt zudem, dass eine in-plane durch das LAO (100)-Substrat induzierte Druckspannung nicht in dem Maße wie die in-plane durch das STO (100)-Substrat induzierte Zugspannung den ferromagnetischen Grundzustand der niedrig-dotierten LSMOSchichten beeinflusst. LMO/LAO (100)-Schichten in der Literatur bestätigen mit CurieTemperaturen $T_{C} \approx 85-130 \mathrm{~K}$ 283, 284,287] je nach Schichtdicke und Herstellung die reduzierten $T_{C}$-Werte der zwei niedrig-dotierten LSMO/LAO (100)-Schichten gegenüber LMO-Schichten auf STO (100). Einen Hinweis auf konkurrierende magnetische Wechselwirkungen in den Schichten mit $x \leq 0,10$ kann ein Vergleich der FCC- $M(T)$-und der ZFC$M(T)$-Kurven geben, der repräsentativ für die LMO/MgO (100)-Schicht ( $H=100$ Oe) in der eingebetteten Grafik in der Abbildung 4.3.6 (d) dargestellt ist. Die beobachtbaren Unterschiede eines ferromagnetischen Verhaltens in der FCC- $M(T)$-Kurve und eines SpinGlas-Verhaltens in der ZFC- $M(T)$-Kurve mit einem Maximum nahe $T_{C}$ lassen sich dabei direkt auf ein konkurrierendes Zusammenspiel von ferromagnetisch geordneten Clustern in einer antiferromagnetisch ordnenden Matrix in der Schicht zurückführen 283, 284,287]. In Bezug auf das T-x-Phasendiagramm für Bulk-LSMO stellt der magnetische Grundzustand der LSMO-Schichten auf MgO (100) und LAO (100) mit $x \leq 0,10$ somit eine Kombination aus einer ferromagnetischen und einer gekantet-antiferromagnetischer Phase dar, die für Bulk-LMO/-LSMO mit $x \leq 0,10$ beide elektrisch isolierend sind. Dieses soll nun für die LSMO-Schichten auf MgO (100) und LAO (100) überprüft werden. In der Abbildung 4.3.7 ist daher die Temperaturabhängigkeit des spezifischen elektrischen Widerstands $\rho(T)$ der $30 \mathrm{~nm}$ LSMO/MgO (100)-Schichten $(0 \leq x \leq 0,3)$ dargestellt (PPMS, ohne Magnetfeld).

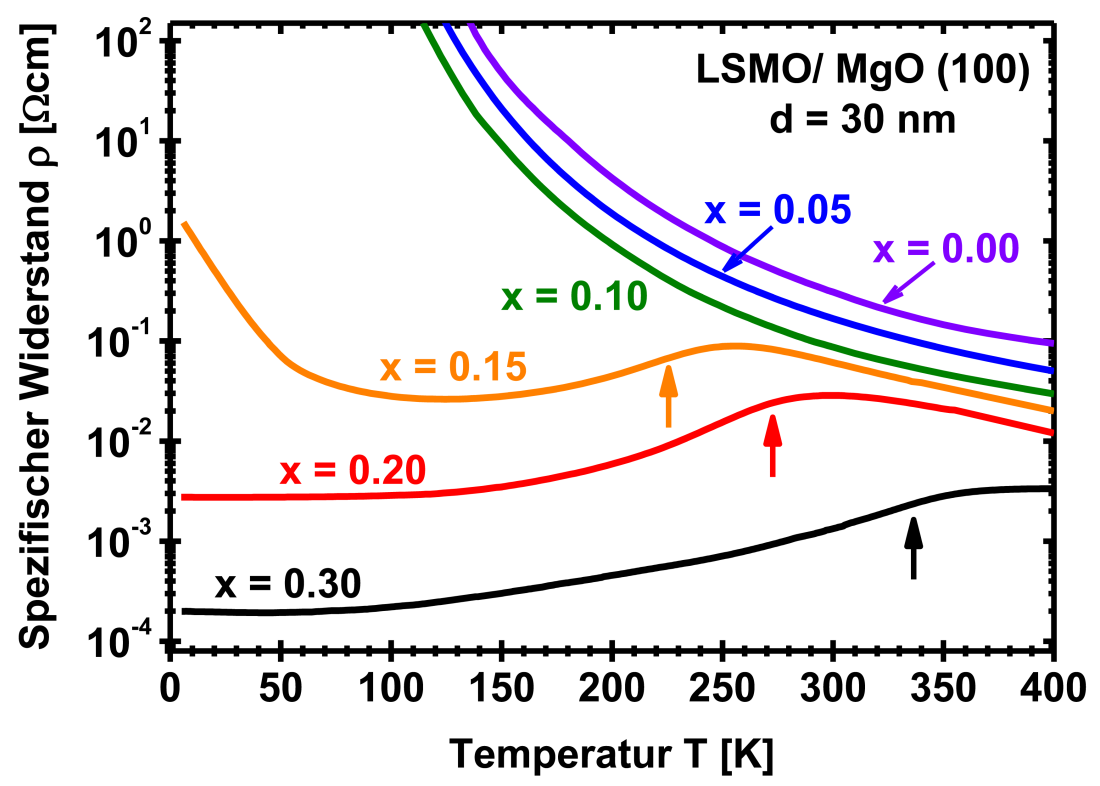

Abbildung 4.3.7: Temperaturabhängigkeit des spezifischen elektrischen Widerstands $\rho(T)$ der $30 \mathrm{~nm}$ LSMO-Schichten auf MgO (100) $(0 \leq x \leq 0,3)$. Die Pfeile kennzeichnen jeweils die MIT-Temperatur $T_{M I}$ (falls vorhanden). 
Für die LSMO/MgO (100)-Schichten mit $x \leq 0,10$ zeigt sich ein durchgängig elektrisch isolierendes Verhalten $(\mathrm{d} \rho / \mathrm{d} T<0)$, wobei der spezifische Widerstand für die LMO-Schicht am größten ist und systematisch mit der Sr-Dotierung abnimmt. Dieses ist konsistent mit Bulk-LSMO und LSMO-Schichten mit $x<0,10$ in der Literatur, für die sich im stöchiometrischen Fall ebenfalls ein stets isolierendes Verhalten ergibt 16, 276, 278, 286, 288, während ein Lanthan-Defizit oder ein Sauerstoffüberschuss bereits bei undotiertem LMO zu einer signifikanten $\mathrm{Mn}^{3+} / \mathrm{Mn}^{4+}$-Mischvalenz führt, die mit dem Auftreten eines MetallIsolator-Übergangs einhergeht 276 278. Dieses kann für die LSMO/ MgO (100)-Schichten aufgrund des stets isolierenden Charakters für $x \leq 0,10$ ausgeschlossen werden. Ab einer Sr-Dotierung $x \geq 0,15$ lässt sich eine Änderung in der Entwicklung $\rho(T)$ des spezifischen Widerstands beobachten, indem ein Metall-Isolator-Übergang bei der Temperatur $T_{M I}$ auftritt, unterhalb der die LSMO-Schichten metallisch sind $(\mathrm{d} \rho / \mathrm{d} T>0)$. Die Temperatur des Übergangs (Maximum des Temperaturkoeffizienten TCR $=(1 / R)(\mathrm{d} R / \mathrm{d} T)[50]$ ) steigt mit zunehmender Sr-Dotierung von $T_{M I}=228(2) \mathrm{K}(x=0,15)$ auf $T_{M I}=265(2) \mathrm{K}(x=0,20)$ an, bis sich für $x=0,30$ ein durchgängig metallisches Verhalten mit einem Metall-MetallÜbergang bei $T_{M M}=337(2) \mathrm{K}$ beobachten lässt. Für $x=0,15$ wird die LSMO-Schicht zudem für $T<120 \mathrm{~K}$ wieder elektrisch isolierend. Dieses Verhalten stimmt mit BulkLSMO überein 16, 286, 288, die $T_{M I^{-}}$Werte der LSMO/MgO (100)-Schichten sind jedoch im Vergleich zu den Bulk-Werten und den eigenen $T_{C}$-Werten $\left(T_{M I}<T_{C}\right)$ etwas reduziert. Neben Grenzflächeneffekten zwischen Substrat und Schicht ist dieses möglicherweise auf das DME-Wachstum und damit auf Versetzungen, die die elektrischen Transporteigenschaften beeinflussen, zurückzuführen. Für die zwei LSMO-Schichten auf LAO (100) $(x=0,05$ und $x=0,10)$ ist die Temperaturabhängigkeit des spezifischen elektrischen Widerstands $\rho(T)$ zum Vergleich in der Abbildung 4.3.8 dargestellt (PPMS, ohne Magnetfeld).

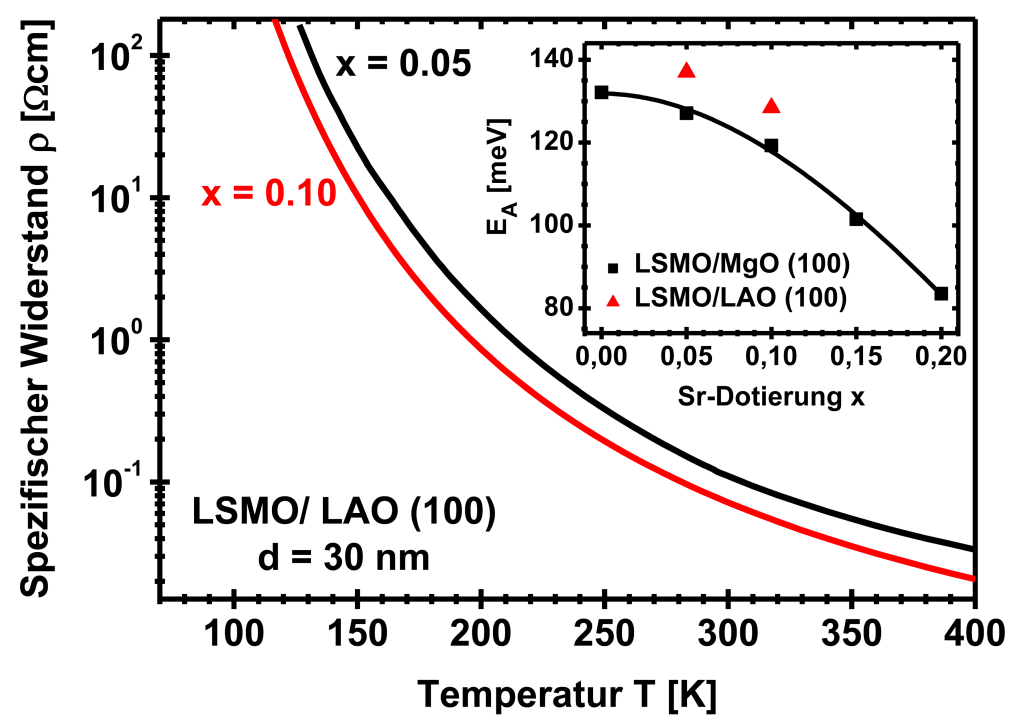

Abbildung 4.3.8: Temperaturabhängigkeit des spezifischen Widerstands $\rho(T)$ der zwei $30 \mathrm{~nm}$ LSMO/LAO (100)-Schichten und die Aktivierungsenergie $E_{A}(x)$ der beiden LSMO-Schichtreihen für den Bereich $\mathrm{d} \rho / \mathrm{d} T<0$ (eingebettete Grafik). 
Für die zwei LSMO/LAO (100)-Schichten zeigt sich ein durchgängig isolierendes Verhalten $(\mathrm{d} \rho / \mathrm{d} T<0)$, sodass erneut sowohl ein Lanthan-Defizit als auch ein signifikanter Sauerstoffüberschuss ausgeschlossen werden kann. Der spezifische Widerstand ist dabei auch hier mit größerer Sr-Konzentration niedriger. Im Vergleich mit den LSMO/MgO (100)-Schichten mit $x \leq 0,10$ ergibt sich wiederum ein ähnlicher spezifischer Widerstand $\left(\rho \approx 10^{-1} \Omega \mathrm{cm}\right.$ bei $T \approx 300 \mathrm{~K})$. Die isolierenden Bereiche mit $\mathrm{d} \rho / \mathrm{d} T<0$ können zudem mithilfe einer Arrhenius-Gleichung $\rho(T)=\rho_{0} \mathrm{e}^{E_{A} / k_{B} T}$ für thermisch aktivierte Ladungsträger, die in Form eines Hüpfmodells kleiner Polaronen zum elektrischen Ladungstransport beitragen, charakterisiert werden 8,278 . Hierbei bezeichnet $E_{A}$ die Aktivierungsenergie und $\rho_{0}$ einen temperaturabhängigen spezifischen Widerstandskoeffizienten. Die aus den $\rho(T)$-Verläufen bestimmte Aktivierungsenergie $E_{A}$ der LSMO-Schichten auf MgO (100) und LAO (100) ist in Abhängigkeit der Sr-Konzentration in der eingebetteten Grafik in der Abbildung 4.3.8 dargestellt. Es ergibt sich eine systematische Abnahme mit ansteigender Sr-Dotierung. Dieses kann auf die erhöhte elektrische Leitfähigkeit aufgrund der Zunahme an $\mathrm{Mn}^{4+}$-Kationen zurückgeführt werden, wodurch der Doppelaustausch verstärkt aktiviert und die Ladungsträger delokalisiert werden. Die Größenordnung der Aktivierungsenergie von $E_{A} \approx 0,1 \mathrm{eV}$ passt dabei sehr gut zu Messungen in der Literatur an Einkristallen und dünnen Schichten (LMO und niedrig-dotiertes LSMO) 278,289]. Für die LSMO/MgO (100)-Schicht mit $x=0,30$, die durchgängig metallisch ist, verschwindet die Aktivierungsenergie hingegen.

\subsubsection{Dotierungs- und temperaturabhängige Raman-Messungen}

Anschließend können die LSMO-Schichten auf MgO (100) und LAO (100) nun mithilfe der Raman-Spektroskopie auch auf ihre phononischen Eigenschaften untersucht werden. Undotiertes, stöchiometrisches Bulk-LMO kristallisiert dabei in der orthorhombischen PnmaStruktur. Gruppentheoretische Untersuchungen zeigen für diese Raumgruppe am $\Gamma$-Punkt der 1. Brillouin-Zone, dass von maximal 60 Phonon-Moden 24 Moden Raman-aktiv sind $\left(7 A_{g}+5 B_{1 g}+7 B_{2 g}+5 B_{3 g}\right)$, während die weiteren Moden entweder IR-aktiv, akustisch oder still sind 270, 290 292. Nicht-stöchiometrisches LMO und LSMO mit einer Dotierung $x>0,17$ zeigen hingegen einen strukturellen Phasenübergang und kristallisieren in der rhomboedrischen R $\overline{3}$ c-Struktur $276,277,279,288$. Anhand gruppentheoretischer Untersuchungen ergeben sich für diese Raumgruppe 20 Phonon-Moden am $\Gamma$-Punkt, von denen fünf Moden Raman-aktiv sind $\left(A_{1 g}+4 E_{g}\right)$ 270,293. Um diesen Unterschied für die LSMO-Schichten zu überprüfen, sind nun in den Abbildungen 4.3.9 (a) und (b) die polarisationsabhängigen Raman-Spektren (xx-, $\mathrm{x}^{\prime} \mathrm{x}^{\prime}-$, xy- und $\mathrm{x}^{\prime} \mathrm{y}^{\prime}$-Polarisationskonfigurationen) der $30 \mathrm{~nm}$ LMO/MgO (100)-Schicht und der $30 \mathrm{~nm}$ LSMO/MgO (100)-Schicht $(x=0,30)$ dargestellt. Das Probenbezugssystem entspricht der in der Abbildung 3.3.8 gezeigten Ausrichtung mit den Richtungen $\mathrm{x} \|$ [100], y || [010], z \| [001], $\mathrm{x}^{\prime} \|$ [110] und $\mathrm{y}^{\prime}$ || [110] (senkrechte Rückstreugeometrie mit der definierten out-of-plane Orientierung in [001]-Richtung). Die Spektren wurden für eine bessere Übersicht vertikal gegeneinander verschoben. 

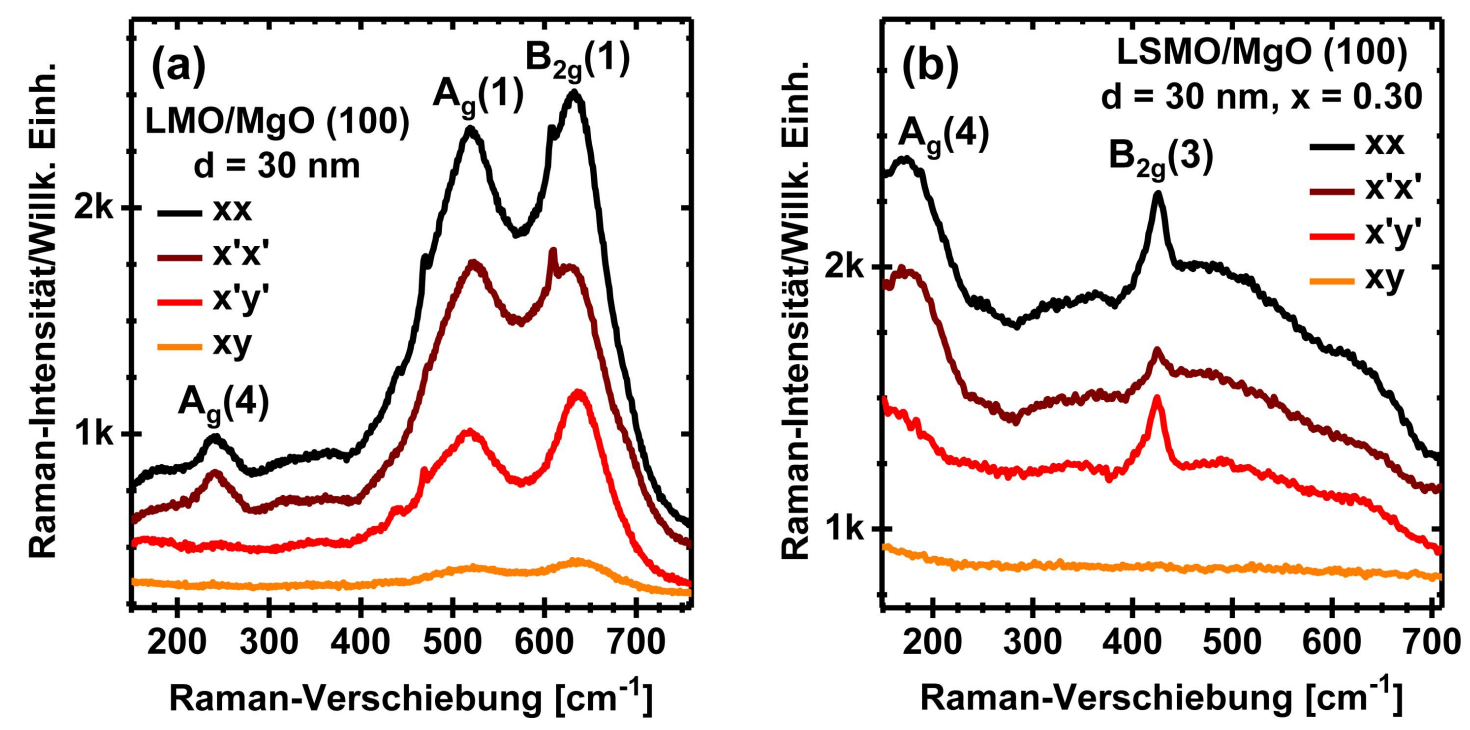

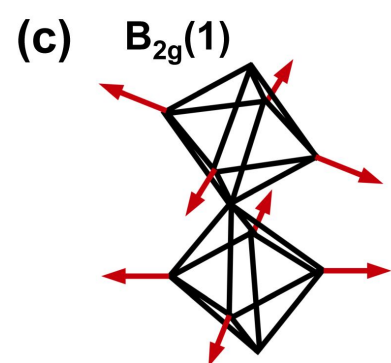

$634 \mathrm{~cm}^{-1}$

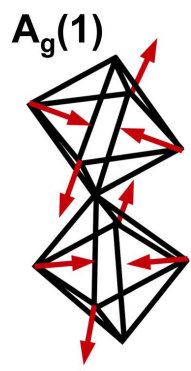

$514 \mathrm{~cm}^{-1}$

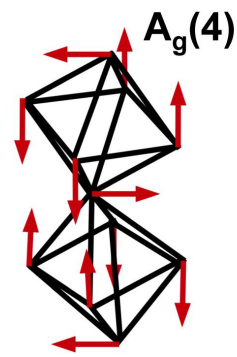

$235 \mathrm{~cm}^{-1}$ (LMO) $171 \mathrm{~cm}^{-1}(\mathrm{x}=0.30)$

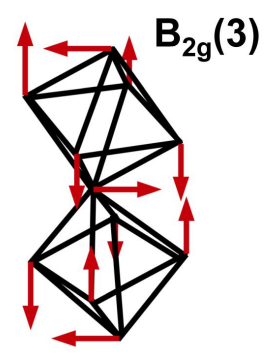

$426 \mathrm{~cm}^{-1}$

Abbildung 4.3.9: Polarisierte Raman-Spektren ( $x x-, x^{\prime} x^{\prime}-, x y-$ und $x^{\prime} y^{\prime}-$ Konfigurationen) der $30 \mathrm{~nm} \mathrm{LMO} / \mathrm{MgO}$ (100)- (a) und der $30 \mathrm{~nm} \mathrm{LSMO} / \mathrm{MgO}$ (100)-Schicht $(x=0,30)(\mathrm{b})(\lambda=532 \mathrm{~nm}, P \approx 2 \mathrm{~mW}, t=3 \times 120 \mathrm{~s}$, Raumtemperatur $)$ zusammen mit der Darstellung der vier besonders charakteristischen RamanModen und der Zuordnung der jeweiligen Symmetrie (c) (nach [290, 291]).

Der Ausgangspunkt für eine Analyse ist zunächst das Raman-Spektrum von orthorhombischem Bulk-LMO. Dieses ist in der Literatur durch zwei sehr intensive Moden geprägt, die sich durch einen Abgleich von experimentellen Messungen und LDC-Berechnungen für die orthorhombische Pnma-Struktur einer symmetrischen Streckschwingung (SS) der $\mathrm{MnO}_{6^{-}}$ Sauerstoffoktaeder mit $B_{2 g}$-Symmetrie bei $\approx 610 \mathrm{~cm}^{-1}\left(B_{2 g}(1)\right.$ Mode 290 292]) und einer antisymmetrischen Streckschwingung (AS) bei $\approx 490 \mathrm{~cm}^{-1}\left(A_{g}(1)\right.$ Mode 290 292]) zuordnen lassen. Da diese die kohärenten (kooperativ vorhandenen) Jahn-Teller-Verzerrungen im LMO repräsentieren, werden diese zwei Moden als Jahn-Teller-Moden (JT-Moden) bezeichnet. Daneben lässt sich mit einer gegenphasigen Kipp-Rotation (KR) der $\mathrm{MnO}_{6^{-}}$ Oktaeder mit $A_{g}$-Symmetrie bei $\approx 285 \mathrm{~cm}^{-1}\left(A_{g}(4)\right.$ Mode 290292$)$ eine weitere Mode geringerer Intensität unabhängig von den JT-Verzerrungen beobachten. Ein Vergleich mit den Raman-Spektren der $30 \mathrm{~nm} \mathrm{LMO} / \mathrm{MgO}$ (100)-Schicht in der Abbildung 4.3.9 (a) zeigt jedoch, dass die zwei JT-Moden mit $\approx 634 \mathrm{~cm}^{-1}$ (SS-JT) und $\approx 514 \mathrm{~cm}^{-1}$ (AS-JT) zu größeren Wellenzahlen verschoben sind und deutlich verbreitert erscheinen, während die 
KR-Mode mit $\approx 235 \mathrm{~cm}^{-1}$ eine Verschiebung zu kleineren Wellenzahlen aufweist. Dieses Verhalten ist typisch für dünne LMO-Schichten und kann auf die Problematik des Sauerstoffgehalts und Verspannungseinflüsse des Substrats zurückgeführt werden [294 297]. Vergleichbare Spektren werden für rhomboedrisches Bulk-LMO beobachtet 270, 293, 298]. Die R $\overline{3}$ c-Struktur ist jedoch inkompatibel zu intrinsischen Jahn-Teller-Verzerrungen, da alle $\mathrm{Mn}^{3+}{ }_{-} \mathrm{O}^{2-}$-Bindungen die gleiche Länge besitzen, sodass diese entsprechend in einer inkohärenten (dynamischen) Form auftreten und mit einer Unordnung des SauerstoffKristalluntergitters einhergehen 270,293,298. Die JT-Moden folgen somit nicht der durch LDC-Berechnungen vorhergesagten $E_{g}$-Symmetrie, sondern weisen eine Verhaltensähnlichkeit zu den JT-Moden der orthorhombischen Pnma-Struktur auf und repräsentieren die Ein-Phonon-Sauerstoff-Zustandsdichte. Die Verbreiterung kann dabei direkt auf die strukturelle Unordnung zurückgeführt werden und und ist durch die energetische Dispersion der Sauerstoffphononenzweige bestimmt, während die Intensität entsprechend mit dem Grad der Unordnung korreliert 270,293. Da die JT-Verzerrungen gleichzeitig stark an das $\mathrm{Mn}^{4+} / \mathrm{Mn}^{3+}$-Kationenverhältnis, die elektrische Leitfähigkeit und magnetische Korrelationen in der Schicht gekoppelt sind, ist eine starke Abhängigkeit der JT-Modenausprägung von der Position im T-x-Phasendiagramm zu erwarten. Dieses wird für LSMO direkt anhand der polarisationsabhängigen Raman-Spektren der metallischen LSMO-Schicht auf $\mathrm{MgO}(100)(x=0,30)$ in der Abbildung 4.3.9 (b) ersichtlich, für die beide JT-Moden stark unterdrückt sind, da die Unordnung des Sauerstoff-Kristalluntergitters durch die Ladungsträgerdelokalisierung in der metallisch-ferromagnetischen Phase zusammenbricht [270,293.

Mit einer gegenphasigen Biegemode mit $B_{2 g}(3)$-Symmetrie 290292 bei $\approx 426 \mathrm{~cm}^{-1}$ erscheint zudem neben der KR-Mode eine weitere JT-unabhängige Raman-Bande, die charakteristisch für LSMO mit $x>0,17$ 296 ist und durch ihre Schärfe direkt den Übergang in eine höhere Ordnung in der Schicht widerspiegelt, auch wenn sie ebenfalls mit der $E_{g}$-Symmetrie der R $\overline{3}$ c-Struktur inkompatibel bleibt, während die zu $\approx 171 \mathrm{~cm}^{-1}$ verschobene KR-Mode in dieser eine $A_{1 g^{-}}$Symmetrie aufweist. Eine Darstellung der diskutierten Raman-Moden ist in der Abbildung 4.3.9 (c) (nach 290, 291]) gegeben. Der metallische Charakter der LSMO-Schicht mit $x=0,30$ wird zudem an dem elektronischen Hintergrund in den Raman-Spektren sichtbar, der für die undotierte LMO-Schicht fehlt. Die Präsenz der KR-Mode und der Biegemode kennzeichnet wiederum eine Annäherung an das RamanSpektrum von rhomboedrischen LAO, das keine JT-Aktivität aufweist [270]. Anhand des $T$ - $x$-Phasendiagramms von LSMO ist es nun möglich durch eine horizontale (Sr-Dotierung) oder vertikale Verschiebung (Temperatur) den Ordnungszustand der LSMO-Schichten direkt zu beeinflussen und mit der Raman-Spektroskopie zu beobachten. Um den Einfluss der Sr-Dotierung auf das Raman-Spektrum von LSMO weiter zu charakterisieren, sind nun in der Abbildung 4.3 .10 die unpolarisierten Raman-Spektren der $30 \mathrm{~nm}$ LSMO-Schichten auf $\mathrm{MgO}(100)$ mit den Sr-Dotierungen $0 \leq x \leq 0,30$ in einem Vergleich dargestellt. 


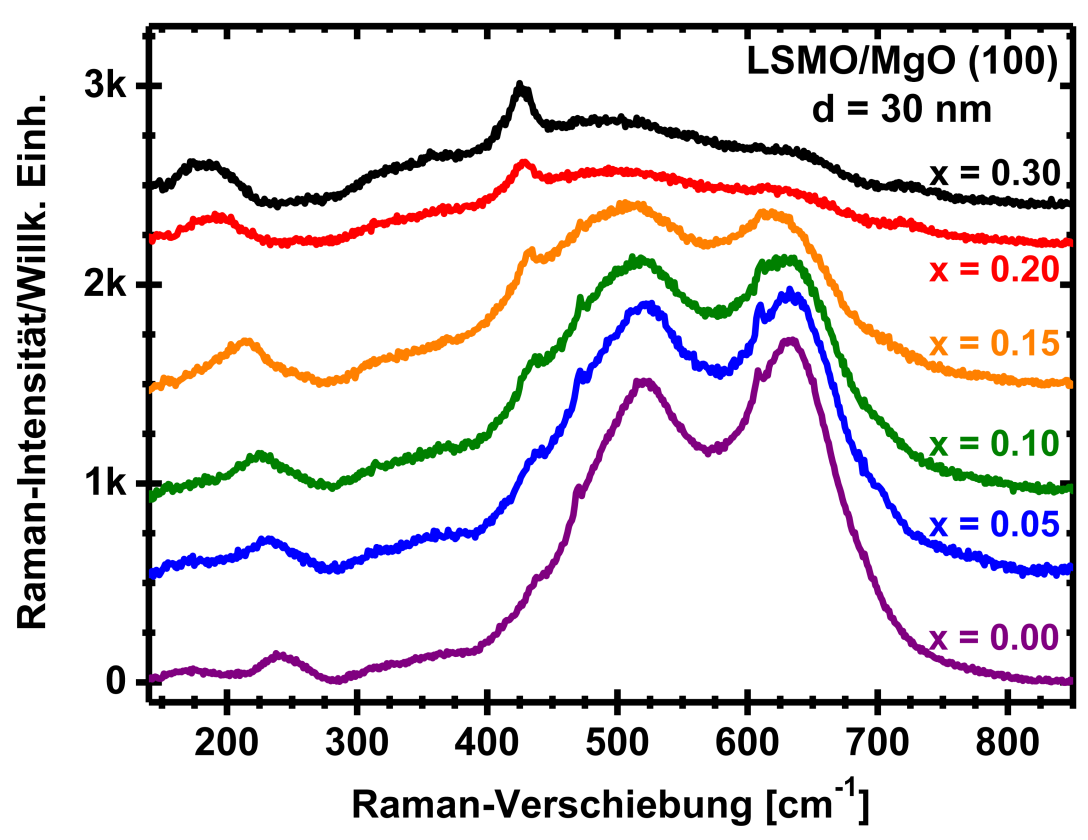

Abbildung 4.3.10: Unpolarisierte Raman-Spektren der 30 nm LSMO/MgO (100)-Schichten für die verschiedenen Sr-Dotierungen $x$ im Vergleich, aufgenommen bei Raumtemperatur $(\lambda=532 \mathrm{~nm}, P \approx 2 \mathrm{~mW}, t=3 \times 120 \mathrm{~s})$. Für eine bessere Übersicht sind die Spektren vertikal gegeneinander verschoben.

Hierfür wurde jeweils der spektrale Hintergrund (elektronischer Hintergrund, Bose-EinsteinKorrektur) abgezogen und die Spektren vertikal gegeneinander verschoben. Anhand der Raman-Spektren der LSMO-Schichten können so die Entwicklungen der einzelnen RamanModen mit der Sr-Dotierung nachvollzogen werden. Es ergibt sich eine systematische Abnahme der JT-Intensität mit ansteigender Sr-Dotierung, die an das größere $\mathrm{Mn}^{4+} / \mathrm{Mn}^{3+}{ }_{-}$ Kationenverhältnis, das mit einer erhöhten elektrischen Leitfähigkeit und magnetischen Korrelationen in der Struktur einhergeht, gekoppelt ist. Ausgehend von undotiertem LMO werden mit der Sr-Dotierung $\mathrm{Mn}^{4+}$-Kationen in die Schicht eingebracht, die die kooperativ geordnete JT-Verzerrung stören und zunächst das relative Volumen der ungeordneten Struktur der nicht-kohärenten JT-Verzerrungen vergrößern. Dieses lässt sich direkt anhand der Verbreiterung der JT-Moden beobachten. Wird die Dotierung anschließend darüber hinaus bis in die metallisch-ferromagnetische Phase von LSMO für $x>0,17$ erhöht, so führt die durch den Doppelaustausch initiierte Ladungsträgerdelokalisation zu einem Zusammenbruch dieser Unordnung und die Intensität der JT-Moden reduziert sich drastisch.

Die Entwicklung der JT-Linienbreiten mit der Sr-Dotierung ist in der Abbildung 4.3.11(a) dargestellt. Hierfür wurden beide Banden mithilfe von Lorentz-Funktionen angenähert und aus diesen die Linienposition, die Linienbreite und die Linienintensität (integrierte Fläche) bestimmt. Es ergibt sich für beide Moden ein starker Anstieg in der Linienbreite zwischen den Sr-Konzentrationen $x=0,15$ und $x=0,20$ und damit im Bereich des strukturellen Unordnung-Ordnung-Übergangs einer zusammenbrechenden inkohärenten JT-Verzerrung. Für Bulk-LSMO ist dieser an einen strukturellen Phasenübergang in die rhomboedrische 
$\mathrm{R} \overline{3} \mathrm{c}-$ Struktur für $x>0,17$ gekoppelt, während dünne LSMO-Schichten bereits undotiert eine rhomboedrische Verzerrung aufweisen. Hinweise auf den strukturellen Phasenübergang können dabei die von den JT-Verzerrungen unabhängige Kipp-Rotationsmode und die für die metallisch-geordnete LSMO-Phase charakteristische $B_{2 g}(3)$ Biegemode geben. Die Entwicklung der Linienpositionen der beiden Moden mit der Sr-Dotierung ist für die $30 \mathrm{~nm}$ LSMO/MgO (100)-Schichten in der Abbildung 4.3.11(b) dargestellt. Für beide Moden lässt sich ein systematisches Erweichen mit ansteigender Sr-Dotierung beobachten (die Biegemode fehlt für undotiertes LMO), das im Bereich des Ordnungsübergangs besonders ausgeprägt ist und direkt auf strukturelle Änderungen beim Einritt in die geordnete R $\overline{3}$ cStruktur zurückzuführen ist 292, 298, 299. Die Modenposition der KR-Mode kann daher auch als Indikator für die im LSMO vorhandene Sr-Dotierung verwendet werden [299].
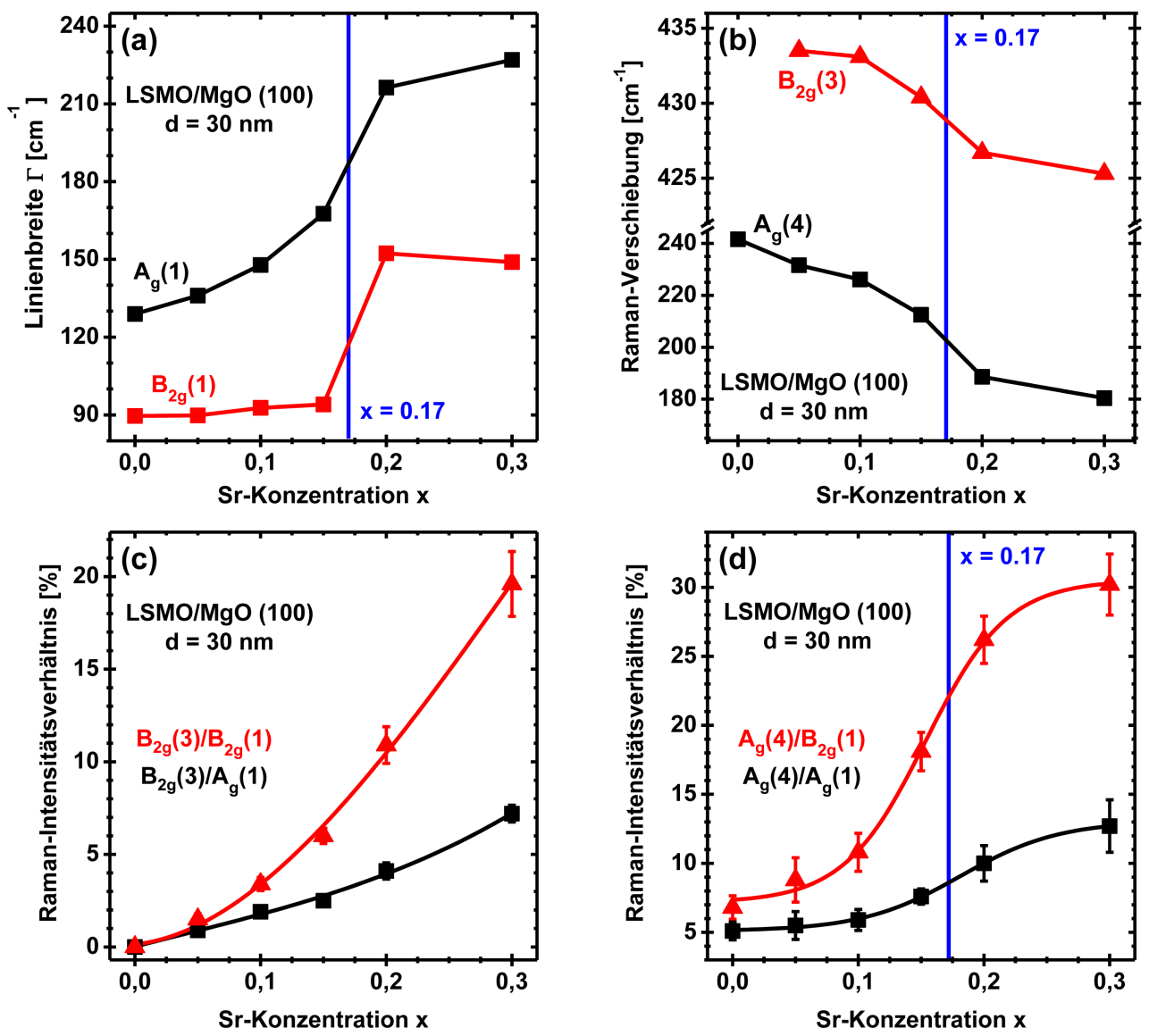

Abbildung 4.3.11: Charakterisierung von dem Einfluss der Sr-Konzentration auf die RamanSpektren der $30 \mathrm{~nm}$ LSMO/MgO (100)-Schichten mit der Entwicklung der JT-Linienbreiten (a), der Linienpositionen der KR-Mode und der $B_{2 g}(3)$ Biegemode (b) sowie der relativen Intensitätsverhältnisse der Biegemode zu den JT-Moden (c) und der KR-Mode zu den JT-Moden (d).

In den Abbildungen 4.3.11 (c) und (d) ist zudem die Intensitätsentwicklung dieser beiden Moden in Relation zu den zwei JT-Moden dargestellt. Anhand der Verläufe wird dabei der unterschiedliche Charakter deutlich. So ist die Biegemode repräsentativ für die geordnete und JT-freie Struktur von LSMO und nimmt in in der Intensität daher kontinuierlich 


\section{Auswertung und Ergebnisse}

mit der Sr-Dotierung zu, wobei sich die Zunahme in der geordneten Phase verstärkt. Da gleichzeitig die Intensität der JT-Moden mit der Sr-Dotierung drastisch zurückgeht, ergibt sich ein starker Anstieg im Intensitätsverhältnis im Bereich des Ordnungsübergangs und darüber hinaus. Die KR-Mode wiederum ist unabhängig von der Sr-Dotierung immer im Raman-Spektrum mit nahezu konstanter Intensität zu beobachten, sodass das relative Verhältnis zu den JT-Moden nur durch deren Intensitätsabnahme bestimmt ist. Folglich ergibt sich im strukturellen Übergangsbereich der größte Anstieg im Intensitätsverhältnis. Für Sr-Dotierungen darüber hinaus stellt sich daher eine stagnierende Tendenz ein. Es zeigt sich zudem, dass die SS-JT-Mode $\left(B_{2 g}(1)\right.$ Mode) in der Intensitätsabnahme stärker als die AS-JT-Mode $\left(A_{g}(1)\right.$ Mode) durch den Ordnungsübergang betroffen ist. Dieses kann auf die Entwicklung der spektralen Verteilung der durch die JT-Moden repräsentierten EinPhonon-Zustandsdichte beim Übergang in eine rhomboedrische Struktur zurückgeführt werden 293. Dieses wird experimentell für rhomboedrisches LMO bestätigt 270,298.

In gleicher Weise soll nun der Einfluss der Temperatur $T$ auf die LSMO-Raman-Spektren diskutiert werden. Hierzu sind in der Abbildung 4.3 .12 die unpolarisierten Raman-Spektren der $30 \mathrm{~nm}$ LSMO/LAO (100)-Schicht mit $x=0,05$ im Bereich $T=80-550 \mathrm{~K}$ dargestellt.

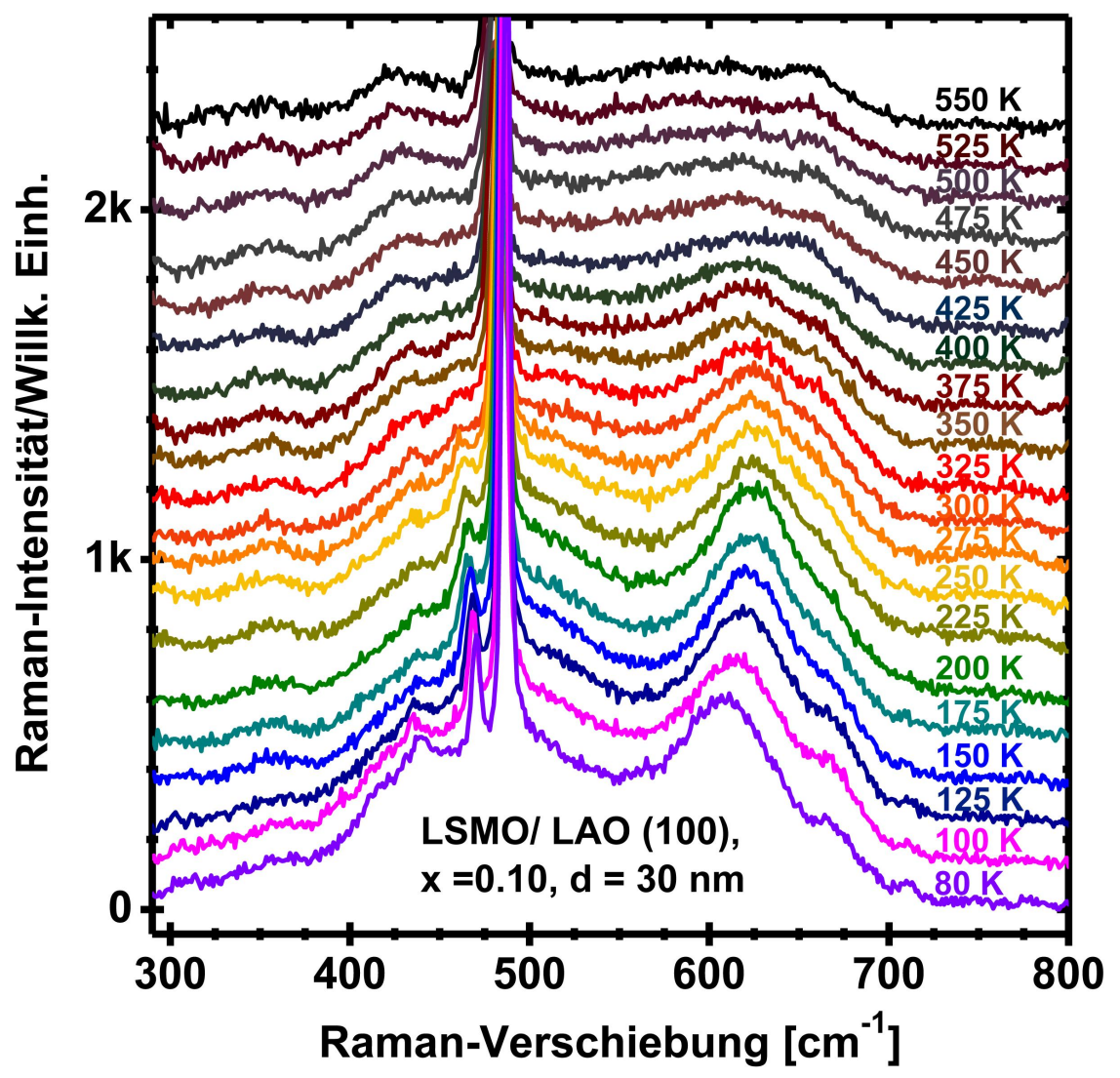

Abbildung 4.3.12: Unpolarisierte Raman-Spektren der $30 \mathrm{~nm}$ LSMO/LAO (100)-Schicht mit einer Sr-Konzentration $x=0,10$, aufgenommen in dem Temperaturbereich $T=80-550 \mathrm{~K}(\lambda=532 \mathrm{~nm}, P \approx 2 \mathrm{~mW}, t=2 \times 360 \mathrm{~s})$. Für eine bessere Übersicht sind die Spektren jeweils vertikal gegeneinander verschoben. 
Dabei wurde wieder jeweils der spektrale Hintergrund (elektronischer Hintergrund, BoseEinstein-Korrektur) abgezogen und die Spektren vertikal gegeneinander verschoben. Zudem wurde eine LSMO-Schicht auf LAO (100) gewählt, um die Entwicklung der Linienbreite der $E_{g}$ Biegemode des LAO-Substrats $\left(\approx 485 \mathrm{~cm}^{-1}\right.$ bei $\left.T=300 \mathrm{~K}\right)$ mit der Temperatur für eine Abschätzung auftretender Heizeffekte später bei den nachfolgenden Raman-Messungen an der gleichen LSMO-Schicht in Abhängigkeit der eingestrahlten Laserleistung (Abschnitt 4.3.3) verwenden zu können. Zunächst soll der Fokus jedoch auf der Entwicklung der Raman-Spektren der LSMO-Schicht mit der Temperatur liegen. Hierfür werden sowohl die $B_{2 g}(3)$ Biegemode als auch die SS-JT-Mode $\left(B_{2 g}(1)\right.$ Mode) betrachtet, da anhand dieser direkt der Verlauf des strukturellen Ordnungsgrads und der Phasen, die LSMO für eine Sr-Dotierung $x=0,10$ vertikal bei Änderung der Temperatur im $T$ - $x$ Phasendiagramm durchschreitet, nachvollzogen werden kann. So lässt sich für die SS-JTMode bei einer Reduzierung der Temperatur $(T<300 \mathrm{~K})$ ein Anstieg in der Intensität bis zu einem Maximum bei einer Temperatur $T \approx T_{C}=161(2) \mathrm{K}$ beobachten, der mit einer signifikanten Verringerung der Linienbreite einhergeht, bevor anschließend für $T<T_{C}$ die Intensität bei gleichzeitiger Zunahme der Linienbreite wieder leicht zurückgeht. Zusätzlich zeigt sich ein Erweichen der SS-JT-Mode für $T<T_{C}$. Die Entwicklung der Linienposition mit der Temperatur ist zusammen mit der Annäherung mit dem anharmonischen Modell (Gleichung (3.3.11) ) für Temperaturen $T \geq T_{C}$ in der Abbildung 4.3.13 (a) dargestellt. Das Erweichen für $T<T_{C}$ ähnelt stark der bei den Doppelperowskiten in der ferromagnetischen Phase beobachteten Spin-Phonon-Kopplung. In der Literatur zeigt sich für LMO-Schichten und Bulk-LMO ein ähnliches Verhalten für die SS-JT-Mode 241,294,295. Das Erweichen wird dabei ebenfalls auf das Eintreten einer Spin-Phonon-Kopplung zurückgeführt 241.
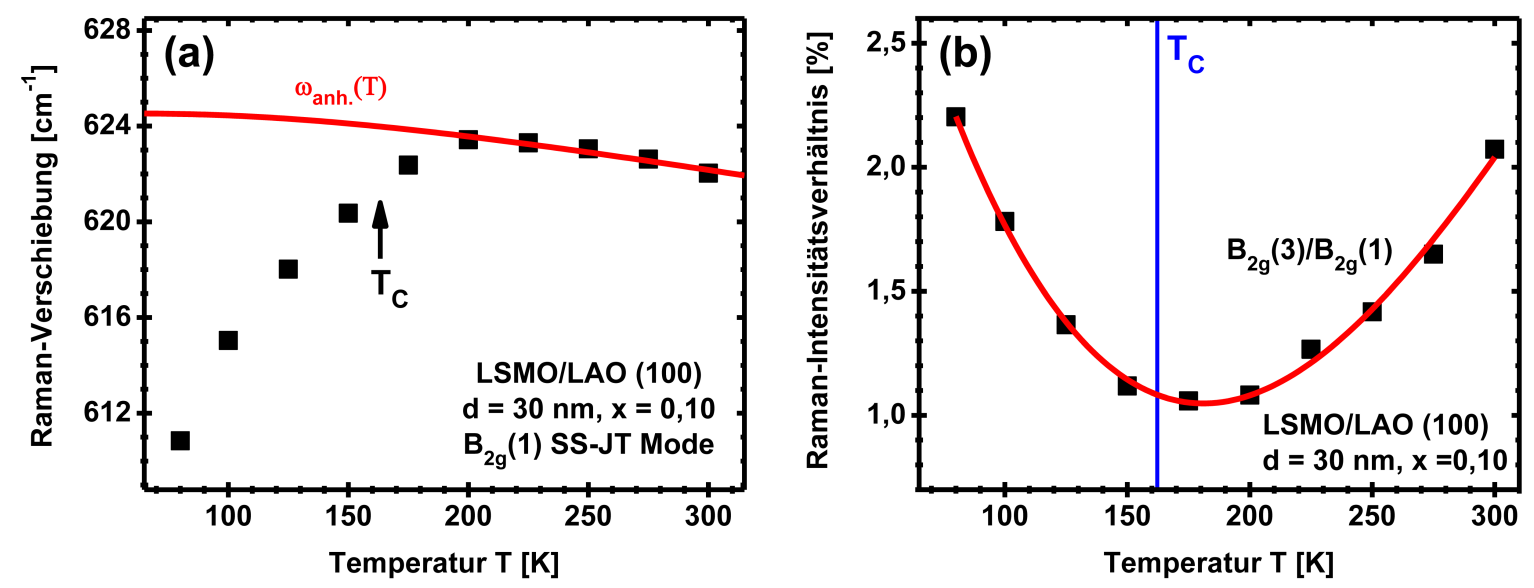

Abbildung 4.3.13: Temperaturentwicklung $\omega(T)$ der SS-JT-Mode der LSMO/LAO (100)Schicht $(x=0,10)$ (a) und das relative Intensitätsverhältnis der $B_{2 g}(3)$ Biegemode zur SS-JT-Mode im Temperaturbereich $T=80-300 \mathrm{~K}$ (b).

Die Entwicklung der Intensität der Biegemode verläuft gegenüber der JT-Mode genau entgegengesetzt. So nimmt die Intensität mit der Abkühlung zunächst bis zu einem Minimum bei $T_{C}$ ab, bevor sich die Mode für Temperaturen $T<T_{C}$ wieder intensiviert. 


\section{Auswertung und Ergebnisse}

Die Entwicklung der Intensität der Biegemode in Relation zur SS-JT-Mode (jeweils durch eine Lorentz-Funktion angenähert) ist für den Temperaturbereich $T=80-300 \mathrm{~K}$ in der Abbildung 4.3.13 (b) dargestellt. Hierbei lässt sich ein Minimum in der Umgebung der Curie-Temperatur $T_{C}$ beobachten, während das Verhältnis für Temperaturen darunter und darüber ansteigt. Der Grund ist das Zusammenspiel der JT-Verzerrungen, des strukturellen Ordnungsgrads und der ferromagnetischen Phase. So nimmt mit abnehmender Temperatur zunächst die Stabilität und damit die Intensität der inkohärenten JT-Moden zu, bis bei $T_{C}$ die ferromagnetische Phase erreicht wird und durch die ferromagnetische Ordnung die JT-Verzerrungen unterdrückt werden 270]. Der nur moderate Rückgang der JT-Moden für $T<T_{C}$ kennzeichnet dabei gleichzeitig den in diesem Sr-Dotierungsbereich weiterhin elektrisch isolierenden Charakter in der LSMO-Schicht (Abbildung 4.3.8), wodurch die JT-Verzerrungen nicht vollständig zusammenbrechen. Zudem deuten die Magnetisierungsmessungen (Abbildung 4.3.6 (d)) für die LSMO/LAO (100)-Schicht mit $x=0,10$ auf einen Grundzustand mit ferromagnetischen Clustern in einer antiferromagnetischen Matrix hin.

Wird die LSMO-Schicht über die Raumtemperatur $T>300 \mathrm{~K}$ hinaus aufgeheizt, so setzt für alle Raman-Moden mit steigender Temperatur entsprechend der anharmonischen Entwicklung eine Verbreiterung ein. Diese fällt jedoch für die beiden JT-Moden im Vergleich zu der Biegemode sehr viel stärker aus. Zudem zeigt sich für Temperaturen $T>400 \mathrm{~K}$ eine drastische Abnahme der Intensität der JT-Moden bei gleichzeitiger Ausweitung der Linienbreiten auf über $\Gamma>150 \mathrm{~cm}^{-1}$, während die Biegemode immer ausgeprägter wird. Anhand des $T$ - $x$-Phasendiagramms (Abbildung 2.2.4) lässt sich die spektrale Verschiebung in Richtung der JT-unabhängigen Biegemode erneut auf einen strukturellen Phasenübergang zurückführen, der für stöchiometrisches Bulk-LMO bei der Annäherung an die Übergangstemperatur $T_{\mathrm{JT}}$ zu einem Aufschmelzen der kooperativen JT-Verzerrungen und der damit einhergehenden orbitalen Ordnung führt 292,300,301]. Die JT-Restintensität in den Spektren wird im System verbleibenden dynamischen JT-Verzerrungen zugeordnet, die durch orbitale Fluktuationen einer thermisch angeregten Unordnung der $e_{g}$-Orbitale der $\mathrm{Mn}^{3+}$-Kationen entstehen [300]. Die LSMO/LAO (100)-Schicht besitzt jedoch eine Sr-Dotierung $x=0,10$ und eine rhomboedrisch verzerrte Struktur, wodurch das System durch die inkohärenten JT-Verzerrungen bereits eine orbitale Unordnung erfährt. Folglich bewirkt die Fluktuation eine Verringerung der inkohärenten JT-Verzerrung und damit eine Abnahme der strukturellen Unordnung. Ein Anzeichen hierfür ist die Präsenz der Biegemode, deren Intensität mit dem strukturellen Ordnungsgrad in der Schicht skaliert. Der Prozess beginnt bei einer Temperatur $T_{\mathrm{JT}} \approx 350 \mathrm{~K}$ und setzt sich darüber hinaus fort. Somit ist der Übergang ähnlich diffus wie der strukturelle P12 $1 /$ n1-R $\overline{3}$ c-Übergang bei den LCMO-Doppelperowskiten (Abschnitt 4.1.4). Das Raman-Spektrum der Schicht nähert sich dabei mit ansteigender Temperatur immer mehr dem Spektrum von LSMO mit einer metallisch-geordneten R $\overline{3}$ c-Struktur an (Übergangstemperatur $T_{O} \geq 500 \mathrm{~K}$ für $x=0,10$ ). 


\subsubsection{Laserleistungsabhängige Raman-Messungen und TERS}

Anschließend soll nun der Einfluss des Laserlichts auf die strukturelle Phase der LSMOSchichten und mögliche damit einhergehende lichtinduzierte Anregungen mit der RamanSpektroskopie betrachtet werden. In der Literatur wird dabei für leicht dotiertes BulkLSMO mit $x=0,05$ und $x=0,10$ eine spektrale Entwicklung in Richtung eines metallischgeordnet geprägten Raman-Spektrums, wie es für LSMO mit einer Sr-Dotierung $x>0,17$ charakteristisch ist, durch ein systematisches Erhöhen der Laserleistung beobachtet [302]. Folglich soll auch hier der Effekt der Laserleistung $P$ untersucht werden. Hierfür wird die $30 \mathrm{~nm}$ LSMO/LAO (100)-Schicht mit $x=0,10$ betrachtet, um so eine Ausgangsposition zwischen undotiertem LMO mit lokalisierten Ladungsträgern und metallisch-geordnetem LSMO mit $x>0,17$ zu ermöglichen. Zudem kann anhand der temperaturabhängigen Raman-Spektren in der Abbildung 4.3.12 und des Beitrags des LAO-Substrats ein auftretender Heizeffekt abgeschätzt und von dem lichtinduzierten Effekt separiert werden. In den Abbildungen 4.3 .14 (a)-(d) sind nun die polarisationsabhängigen Raman-Spektren $\left(\mathrm{xx}-, \mathrm{x}^{\prime} \mathrm{x}^{\prime}-, \mathrm{xy}-\right.$ und $\mathrm{x}^{\prime} \mathrm{y}^{\prime}$-Polarisationskonfigurationen) der $30 \mathrm{~nm}$ LSMO/LAO (100)-Schicht mit $x=0,10$ in Abhängigkeit der eingestrahlten Laserleistung $P(\lambda=532 \mathrm{~nm})$ dargestellt.
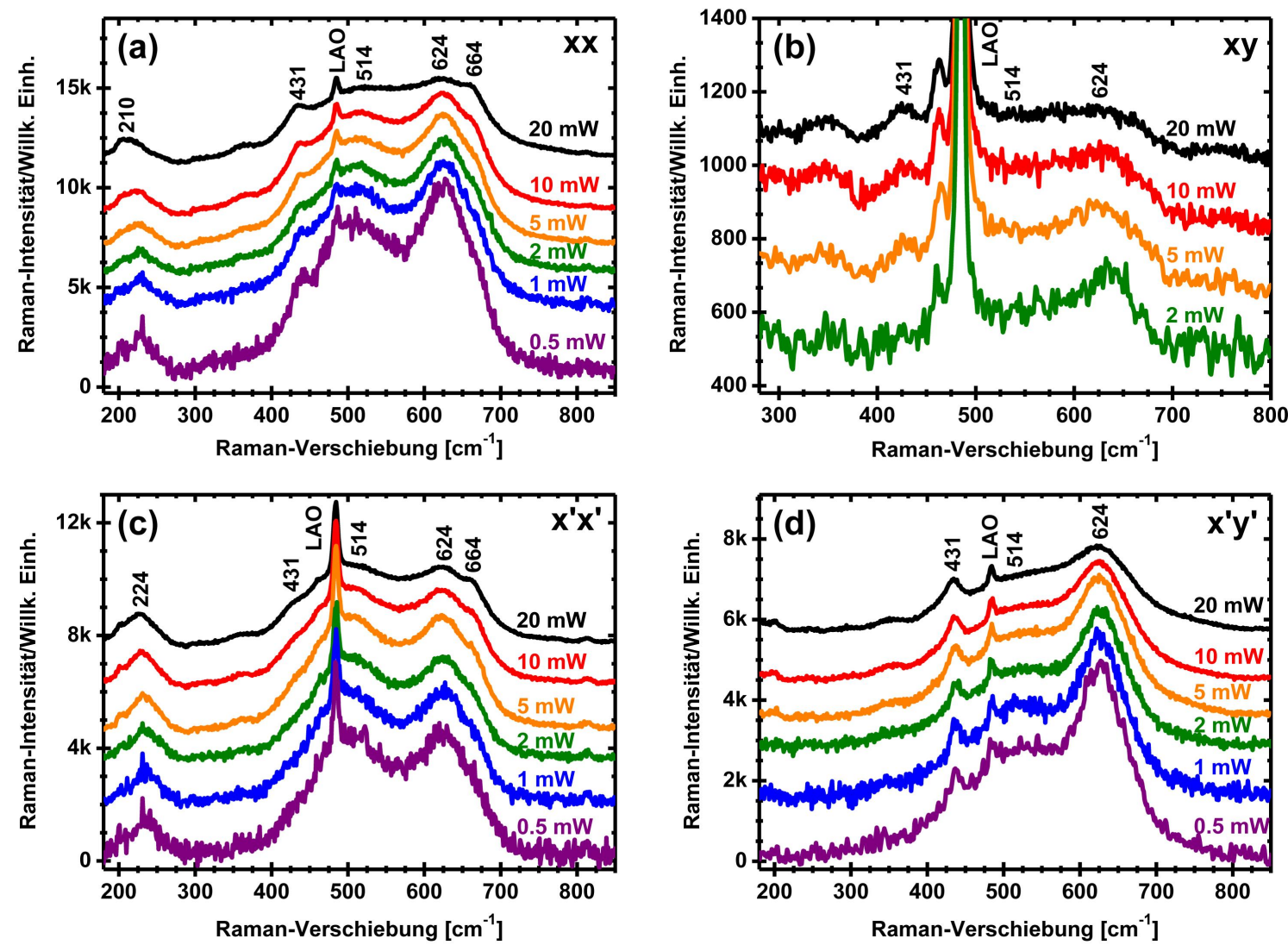

Abbildung 4.3.14: Polarisierte Raman-Spektren ( $x x-, x^{\prime} x^{\prime}-$, xy- und $x^{\prime} y^{\prime}-$ Konfigurationen) der $30 \mathrm{~nm}$ LSMO/LAO (100)-Schicht mit einer Sr-Dotierung $x=0,10$ in Abhängigkeit der eingestrahlten Laserleistung $P$, aufgenommen bei Raumtemperatur $(\lambda=532 \mathrm{~nm}, t=3 \times 120 \mathrm{~s})$. Für eine bessere Übersicht sind die Spektren dabei jeweils vertikal gegeneinander verschoben. 


\section{Auswertung und Ergebnisse}

Wie bei den LSMO-Spektren zuvor wurde auch hier der spektrale Hintergrund abgezogen. Die Polarisationskonfigurationen sind dabei in gleicher Weiser wie für die Spektren in den Abbildungen 4.3 .9 (a) und (b) definiert. Die eingestrahlte Laserleistung des grünen Laser wird über einen Neutraldichtefilter in den Abstufungen $100 \%, 50 \%, 25 \%, 10 \%, 5 \%$ und 2,5 \% eingestellt. Die real bei der LSMO-Schicht ankommende Laserleistung wurde mithilfe eines optischen Leistungsmessgerätes bestimmt. Für die zum Zeitpunkt der Aufnahme der Spektren unter Verwendung des Objektivs mit 100× Vergrößerung (der resultierende Laserfokuspunkt hat eine Fläche $A \approx 1 \mu \mathrm{m}^{2}$ ) verwendete Konfiguration des Messaufbaus konnte für die 100 \%-Stellung (kein Absorptionsfilter eingesetzt) eine Maximalleistung von $P_{\text {max. }} \approx 20 \mathrm{~mW}$ gemessen werden. Eine Überprüfung der Abstufungen des Neutraldichtefilters ergab keine Auffälligkeiten, sodass eine Leistung entsprechend der Filterangabe zur Schicht gelangt. Die Leistungsangabe in den Spektren ist somit von 0,5 $\mathrm{mW}(2,5 \%)$ bis $20 \mathrm{~mW}(100 \%)$ angegeben. Für einen direkten Vergleich wurden die Spektren dabei entsprechend der Laserleistung skaliert und anschließend vertikal gegeneinander verschoben.

In den laserleistungsabhängigen Raman-Spektren in den Abbildungen 4.3.14 (a)-(d) lässt sich für alle vier Polarisationskonfigurationen eine systematische Abnahme der JT-Modenintensitäten mit ansteigender Laserleistung beobachten, die zudem mit einer starken Zunahme der dazugehörigen Linienbreiten einhergeht. Gleichzeitig zeigt sich jedoch für die $B_{2 g}(3)$ Biegemode ein entgegengesetztes Verhalten. So ist diese in der gekreuzten xyKonfiguration erst ab einer Laserleistung $P \geq 5 \mathrm{~mW}$ sichtbar und gewinnt mit ansteigender Laserleistung kontinuierlich an spektralem Gewicht gegenüber den JT-Moden. In gleicher Weise kann die gekreuzte $x^{\prime} y^{\prime}$-Konfiguration betrachtet werden, für die die Biegemode schon bei niedrigeren Laserleistungen aufgrund der stärkeren Unterdrückung der AS-JT-Mode mit $A_{g}$-Symmetrie zu beobachten ist. Während die beiden JT-Moden jedoch mit ansteigender Laserleistung an Intensität verlieren und sich deutlich verbreitern, verbleibt die Biegemode bei einer nahezu konstanten Intensität und Linienbreite. Auch in den parallelen $\mathrm{xx}$ - und $\mathrm{x}^{\prime} \mathrm{x}^{\prime}$-Konfigurationen ist ein ähnliches Verhalten sichtbar, mit dem Unterschied, dass aufgrund der parallelen Konfigurationen die zwei JT-Moden eine sehr hohe Intensität aufweisen, sodass die Biegemode insbesondere in der $\mathrm{x}^{\prime} \mathrm{x}^{\prime}$-Konfiguration für geringe Laserleistungen stark von der AS-JT-Mode überlagert ist und sich erst mit ansteigender Laserleistung von dieser abheben kann. Gleichzeitig bedeutet jedoch auch diese Entwicklung einen Zugewinn der Intensität der Biegemode gegenüber den zwei JT-Moden. Ein weiterer Hinweis ist das Auftreten einer zusätzlichen Raman-Mode bei $\approx 664 \mathrm{~cm}^{-1}$ für hohe Laserleistungen in den parallelen Konfigurationen, die auch in der Literatur für Bulk-LSMO und LSMO-Schichten beobachtet und einem Mehrphononenprozess 2. Ordnung zugeordnet wird 299,303]. Möglich ist in diesem Fall eine Kombinationsschwingung zwischen der Biegemode und der $A_{g}(4)$ KR-Mode, insbesondere da diese Mode nur in den parallelen Konfiguration erscheint, in denen die KR-Mode erlaubt ist. Die Präsenz dieser 
Mode ist daher ebenfalls ein Indikator für eine sich gegenüber den JT-Moden mit ansteigender Laserleistung immer weiter verstärkende Biegemode. Dieses Verhalten in allen vier Polarisationskonfigurationen ist dabei ähnlich dem Effekt einer Erhöhung der Sr-Dotierung mit einer Annäherung in Richtung eines Dotierungsgrades $x \approx 0,17$. Diese Tendenz wird auch in der Literatur an Bulk-LSMO beobachtet [302], ohne den Effekt weiter zu quantifizieren. Um dieses zu ermöglichen, muss zunächst der Einfluss der Laserleistung auf die Temperatur der LSMO-Schicht und möglicher Heizeffekte untersucht werden. Hierfür können die Raman-Linien des LAO-Substrats ein Indikator sein. In Abbildung 4.3.15ist die aus den temperaturabhängigen Raman-Spektren der Abbildung 4.3.12 bestimmte Entwicklung der Linienbreite der $E_{g}$ Biegemode des LAO-Substrats bei $\approx 485 \mathrm{~cm}^{-1}$ mit der Temperatur $T$ mit dem angenäherten Verlauf nach dem anharmonischen Modell dargestellt.

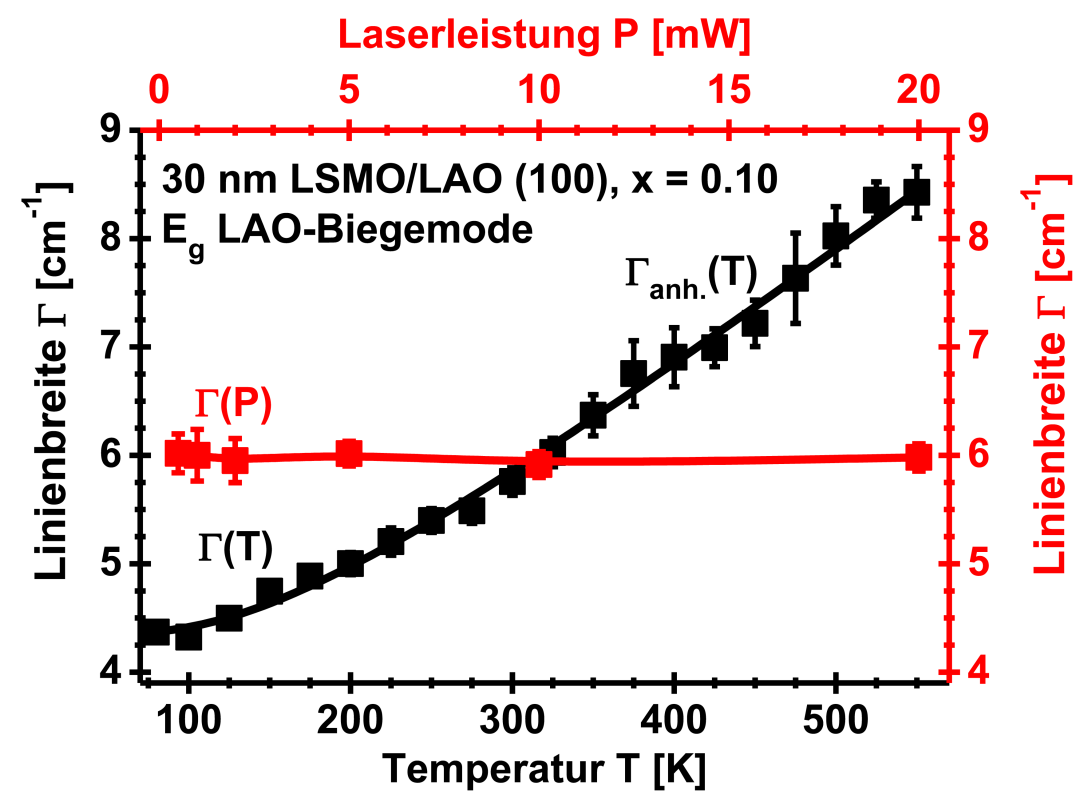

Abbildung 4.3.15: Entwicklung der Linienbreite $\Gamma$ der $E_{g}$ Biegemode des LAO (100)-Substrats
der $30 \mathrm{~nm}$ LSMO-Schicht $(x=0,10)$ mit der Temperatur $T$ (schwarz) und
der eingestrahlten Laserleistung $P$ (rot) im Vergleich. Die schwarze Kurve
kennzeichnet entsprechend den anharmonischen Verlauf $\Gamma_{\text {anh. }}(T)$.

Die Linienbreite folgt dabei über den gesamten Temperaturbereich dem anharmonischen Verlauf. Dieser kann für die Abschätzung der LSMO-Schichttemperatur während der laserleistungsabhängigen Raman-Messungen verwendet werden. Hierfür wurden unpolarisierte Raman-Spektren der LSMO-Schicht in Abhängigkeit der Laserleistung aufgenommen, um ein Signal vom Substrat ohne Polarisationseinfluss zu erhalten, und dann jeweils die Linienbreite der LAO-Biegemode extrahiert. Diese Werte sind in Abhängigkeit der eingestrahlten Laserleistung ebenfalls in der Abbildung 4.3 .15 dargestellt. Ein Vergleich mit der Temperaturentwicklung der LAO-Linienbreite zeigt, dass die Linienbreite der LAO-Biegemode völlig unabhängig von der Laserleistung bleibt und sich auch für die höchste Laserleistung nicht signifikant ändert. Ein Vergleich mit der Temperaturabhängigkeit der Linienbreite dieser Mode offenbart zudem, dass die aus den laserleistungsabhängigen Raman-Spektren 


\section{Auswertung und Ergebnisse}

bestimmten Linienbreiten sehr gut zu Linienbreiten nahe Raumtemperatur passen. Folglich ist die $30 \mathrm{~nm}$ LSMO-Schicht dünn genug, um ein Aufheizen des Substrats zu verhindern. Jedoch muss davon ausgegangen werden, dass sich die LSMO-Schicht moderat mit der ansteigenden Laserleistung aufheizt. Für eine Abschätzung können die Spektren in der $\mathrm{x}^{\prime} \mathrm{y}^{\prime}$-Konfiguration betrachtet werden, da die $B_{2 g}(3)$ Biegemode in dieser Konfiguration sehr präsent ist. Es ergibt sich eine maximale Linienverbreiterung von $\Delta \Gamma \approx 5 \mathrm{~cm}^{-1}$ für eine Erhöhung der Laserleistung von $P \approx 0,5 \mathrm{~mW}$ auf $P_{\text {max. }} \approx 20 \mathrm{~mW}$. Unter Berücksichtigung der Zunahme der $B_{2 g}(3)$-Linienbreite in den temperaturabhängigen Raman-Messungen kann damit der maximale Temperaturanstieg der LSMO-Schicht im Laserfokus bei Erhöhung der Laserleistung auf $100 \%$ auf maximal $\Delta T \approx 50 \mathrm{~K}$ abgeschätzt werden. Folglich geht der lichtinduzierte Einfluss auf die Raman-Spektren immer mit einem kleinen Temperatureffekt einher. Um die Änderung in den Raman-Spektren in Abhängigkeit der eingestrahlten Laserleistung zu quantifizieren, kann nun erneut die Intensitätsentwicklung der Biegemode in Relation zu den zwei JT-Moden betrachtet werden. Die Verläufe für die xx-, xy- und $\mathrm{x}^{\prime} \mathrm{y}^{\prime}$-Polarisationskonfigurationen sind in den Abbildungen 4.3.16 (a)-(c) dargestellt.
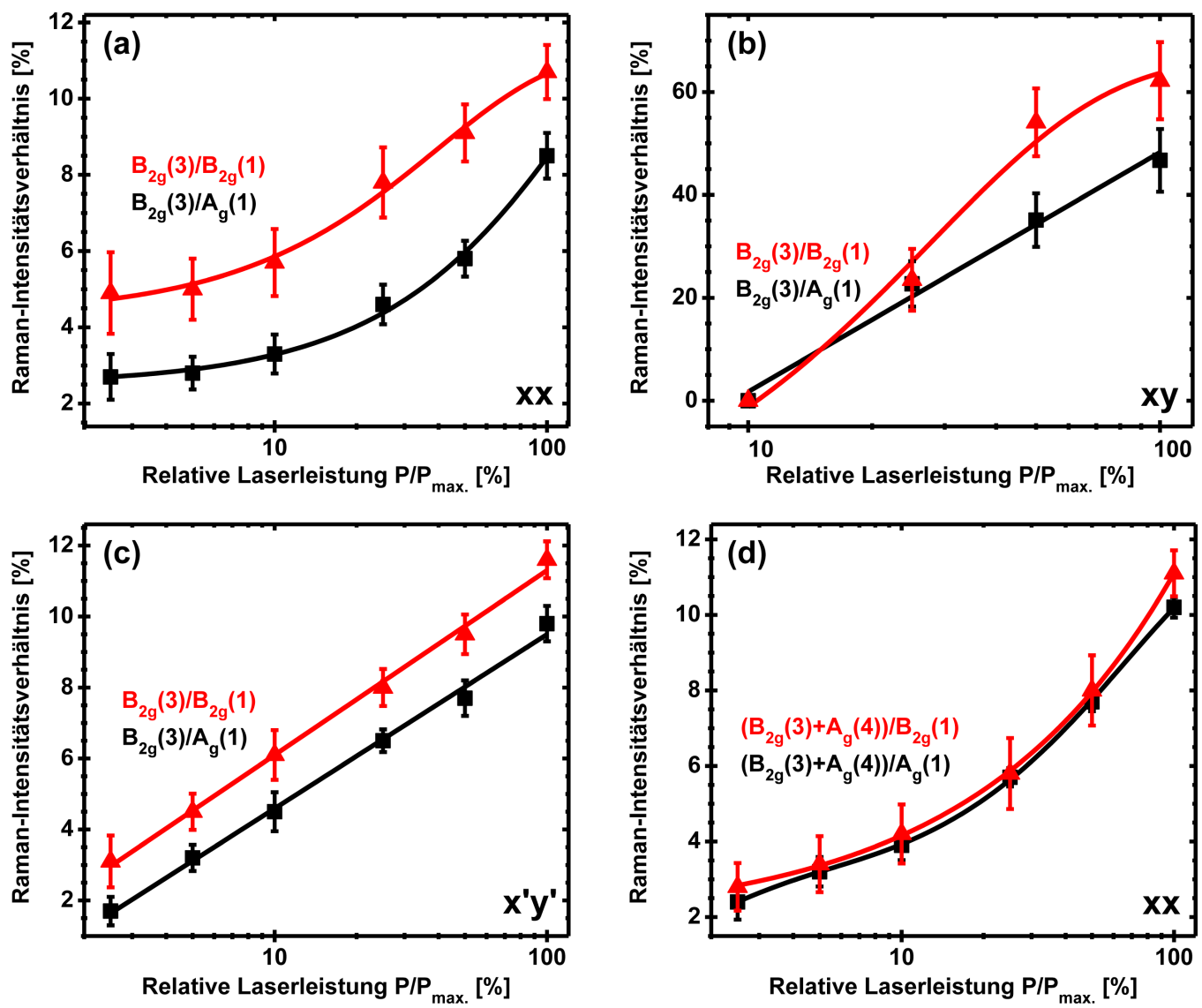

Abbildung 4.3.16: Charakterisierung des Einflusses der Laserleistung $(\lambda=532 \mathrm{~nm})$ auf die Raman-Spektren der $30 \mathrm{~nm}$ LSMO/LAO (100)-Schicht $(x=0,10)$ anhand der relativen Intensitätsverhältnisse der $B_{2 g}(3)$ Biegemode zu den JT-Moden in der xx- (a), in der xy- (b) und in der $x^{\prime} y^{\prime}$-Konfiguration (c). Zusätzlich ist die Kombinationsschwingung der KR- und der Biegemode in Relation zu den JT-Moden in der xx-Konfiguration dargestellt (d). 
Nach der Gleichung (3.3.6) (wenn die Auswirkungen auf die Raman-Tensoren vernachlässigbar sind und die Wellenlängenverschiebung der Moden klein gegenüber der Laserwellenlänge ist) ist eine lineare Skalierung des gesamten Raman-Spektrums mit der eingestrahlten Laserintensität zu erwarten. Somit sollten auch die Intensitäten der $B_{2 g}(3)$ Biegemode und der zwei JT-Moden ohne weitere Einflussfaktoren nahezu gleich skalieren. In allen drei Polarisationskonfigurationen zeigt sich stattdessen ein signifikanter Anstieg der Intensität der $B_{2 g}(3)$ Biegemode in Relation zu den zwei JT-Moden und damit eine Verschiebung des spektralen Gewichts in Richtung der Biegemode (auf die x' $\mathrm{x}^{\prime}$-Konfiguration wurde aufgrund der starken Überlagerung der Biegemode durch die AS-JT-Mode verzichtet), der einen lichtinduzierten Effekt einer Verschiebung der LSMO-Schicht in Richtung einer metallischgeordneten Struktur bestätigt. Die Entwicklung in den Polarisationskonfigurationen sind dabei leicht unterschiedlich. Der größte Effekt wird in der gekreuzten xy-Konfiguration beobachtet, wo die Biegemode für niedrige Laserleistungen fehlt, um dann für die höchste Laserleistung in etwa die Hälfte der Intensitäten der beiden JT-Moden zu erreichen.

Ein Vergleich zu den unpolarisierten Raman-Spektren in der Abbildung 4.3.11 (c) kann gezogen werden, wenn die Modenintensitäten der xx- und der xy-Konfiguration addiert werden. Es ergeben sich die Relationen $B_{2 g}(3) / A_{g}(1) \approx 9,3 \%$ und $B_{2 g}(3) / B_{2 g}(1) \approx 11,7 \%$. Das $B_{2 g}(3) / B_{2 g}(1)$-Verhältnis stimmt damit nahezu mit dem für die LSMO/MgO (100)Schicht mit $x=0,20$ überein. Folglich wird durch den lichtinduzierten Effekt ein sich im Raman-Spektrum widerspiegelnder Zustand in der LSMO/LAO (100)-Schicht mit $x=0,10$ erreicht, der einer metallisch-geordneten LSMO-Schicht mit doppelt so hoher Sr-Dotierung $x=0,20$ nahekommt. Zusätzlich ist in der Abbildung 4.3.16 (d) die laserleistungsabhängige Entwicklung der Kombinationsschwingung $B_{2 g}(3)+A_{g}(4)$ der Biege- und der KR-Mode in Relation zu den zwei JT-Moden in der xx-Polarisationskonfiguration dargestellt. Der Verlauf ähnelt dem der Biegemode. Die Unterschiede für die beiden JT-Moden reduzieren sich jedoch drastisch, da die KR-Mode ein größeres spektrales Gewicht als die Biegemode einbringt. Dieses lässt sich auch in der Abbildung 4.3.11(d) beobachten.

Eine Erklärung für den lichtinduzierten Effekt kann anhand optischer Leitfähigkeitsmessungen an undotiertem Bulk-LMO in der Literatur gegeben werden 304,305, die sich auch auf niedrig-dotiertes LSMO übertragen lassen [302. Der Ausgangspunkt ist der JahnTeller-Effekt, der in LMO mit seinen $\mathrm{Mn}^{3+}{ }$-Kationen zu der Aufspaltung des $e_{g}$-Orbitals in zwei Teilbänder $e_{g}^{1}$ und $e_{g}^{2}$ führt. Das $e_{g}^{1}$-Orbital besitzt aufgrund einer starken Hybridisierung einen großen Überlapp mit dem 2p-Orbital der $\mathrm{O}^{2-}$-Anionen, während das $e_{g}^{2}$-Orbital das energetisch niedrigste leere Leitungsband formt. Diese Bandlücke ist für den elektrisch isolierenden Charakter von LMO verantwortlich [302, während das Einbringen von $\mathrm{Mn}^{4+}$-Kationen mit unbesetztem $e_{g}$-Orbital zu einem Bandlückenzusammenbruch und zu einem metallischen Verhalten führt. Ein möglicher lichtinduzierter Prozess 
zur Anregung eines metallisch-geprägten Zustands niedrig-dotierter LSMO-Schichten ist schematisch in der Abbildung 4.3.17 (a) (nach [302]) dargestellt. So zeigen optische LMOLeitfähigkeitsspektren einen Anregungskanal bei einer Energie $E \approx 1,9 \mathrm{eV}$ [304,305, der sich einem Übergang zwischen den aufgespaltenen $e_{g}$-Bändern zuschreiben lässt und über die $2 \mathrm{p}$-Orbitale der $\mathrm{O}^{2-}$-Anionen vermittelt wird, da der direkte $e_{g}$-Orbitalübergang durch die Dipolauswahlregel verboten ist. Folglich hat der grüne Laser $(\lambda=532 \mathrm{~nm}, E=2,3 \mathrm{eV})$ eine passende Energie. Die lichtinduzierte Anregung durch den Laser erfolgt nun in Form eines Zweielektron-Hüpfprozesses, indem das $e_{g}^{2}$-Band durch den Übergang von Elektronen aus dem 2p-Orbital der $\mathrm{O}^{2-}$-Anionen besetzt wird und gleichzeitig Elektronen aus den $e_{g}^{1}$ - in die $2 \mathrm{p}$-Orbitale übergehen. Die in das $e_{g}^{2}$-Band angeregten Elektronen agieren nun als Leitungsbandelektronen. Dieses führt zu einer Erhöhung der elektrischen Leitfähigkeit und zu einer Ladungsträgerdelokalisation, die in einer temporär metallisch-geordneteren Phase resultiert und die inkohärenten JT-Verzerrungen in der niedrig-dotierten LSMOSchicht reduzieren. Diese Strukturänderung wird direkt in den Raman-Spektren anhand der Intensitätszunahme der Biegemode und Intensitätsabnahme der JT-Moden sichtbar.
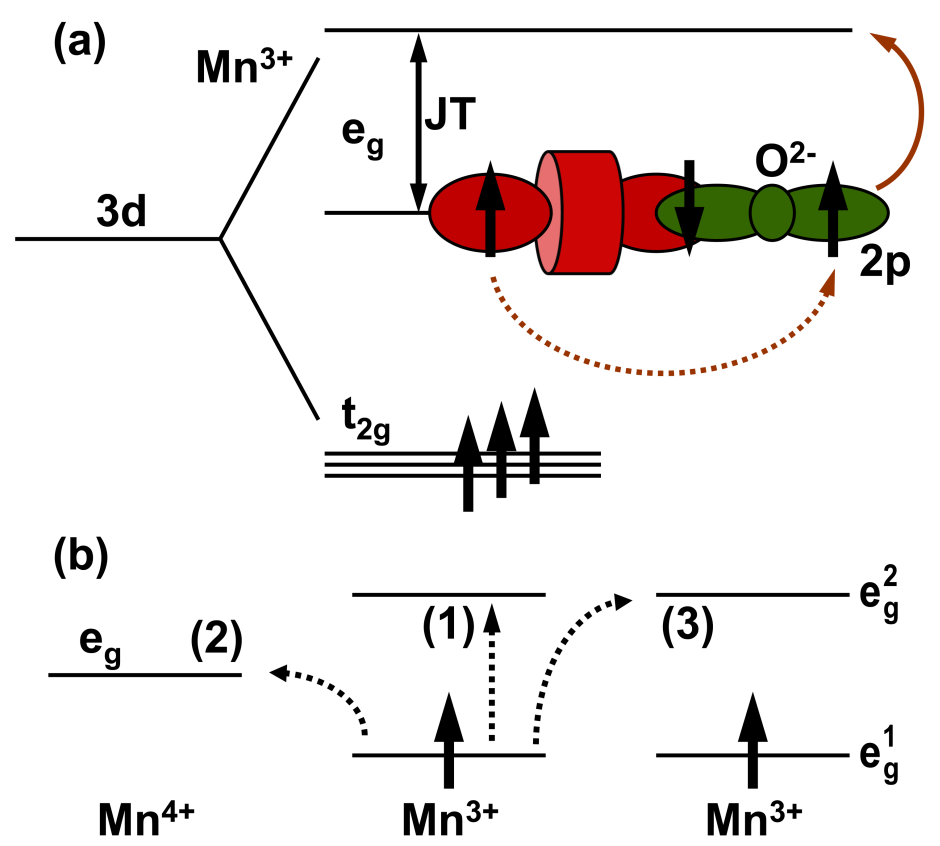

Abbildung 4.3.17: Lichtinduzierte Anregung des $e_{g}$-Elektrons der $\mathrm{Mn}^{3+}-$ Kationen in das obere $e_{g}$-Orbital durch einen Zweielektron-Hüpfprozess vermittelt über das $2 \mathrm{p}$ Orbital der $\mathrm{O}^{2-}$-Anionen (a) (nach [302]) und eine Übersicht (b) der möglichen Übergangskanäle des $e_{g}$-Elektrons mit einem inneratomaren Übergang $\mathrm{Mn}^{3+} \rightarrow \mathrm{Mn}^{3+}$ (1) und den beiden zwischenatomaren Übergängen $\mathrm{Mn}^{3+} \rightarrow \mathrm{Mn}^{4+}(2)$ und $\mathrm{Mn}^{3+} \rightarrow \mathrm{Mn}^{3+}$ (3) (nach 305).

Eine Übersicht weiterer Übergangskanäle ist in der Abbildung 4.3.17 (b) dargestellt. Neben dem inneratomaren Übergang $\mathrm{Mn}^{3+} \rightarrow \mathrm{Mn}^{3+}(1)$ ist auch ein zwischenatomarer Übergang $\mathrm{Mn}^{3+} \rightarrow \mathrm{Mn}^{4+}(2)$ und ein zwischenatomarer Übergang $\mathrm{Mn}^{3+} \rightarrow \mathrm{Mn}^{3+}$ (3) möglich. Aufgrund der nur leichten Sr-Dotierung der LSMO-Schicht kann jedoch eine Beteiligung des Kanals (2) im Vergleich zu den Kanälen (1) und (3) vernachlässigt werden. 
Mit der TERS-Technik lässt sich nun noch untersuchen, inwieweit die lichtinduzierte Verschiebung in Richtung eines metallisch-geordneten Zustands der niedrig-dotierten und elektrisch isolierenden LSMO/LAO (100)-Schicht $(x=0,10)$ auch lokal an der Schichtoberfläche erzeugt werden kann. In den Abbildungen 4.3.18 (a) und (b) sind daher die TERSund Fernfeld-Raman-Spektren der LSMO-Schicht auf LAO (100) mit $x=0.10$ mit einer Schichtdicke von $d=30 \mathrm{~nm}$ für die zwei Laser $(\lambda=532 \mathrm{~nm}$ und $\lambda=632,8 \mathrm{~nm})$ dargestellt. Die Spektren wurden für eine Vermeidung von Heizeffekten bei einer Laserleistung von $P \approx 5 \mathrm{~mW}(t=2 \times 360 \mathrm{~s})$ mit einer Laserpolarisation parallel $(\mathrm{P})$ zur Spitzenachse ohne Analysator aufgenommen. Die TERS-Messungen erfolgten mit der gleichen Goldspitze (Radius $R_{\text {TERS }}=25 \mathrm{~nm}$ ) im Konstant-Strom-Modus $\left(j=0,25 \mathrm{nA}, U_{\text {Bias }}=0,3 \mathrm{~V}\right)$.

(a)
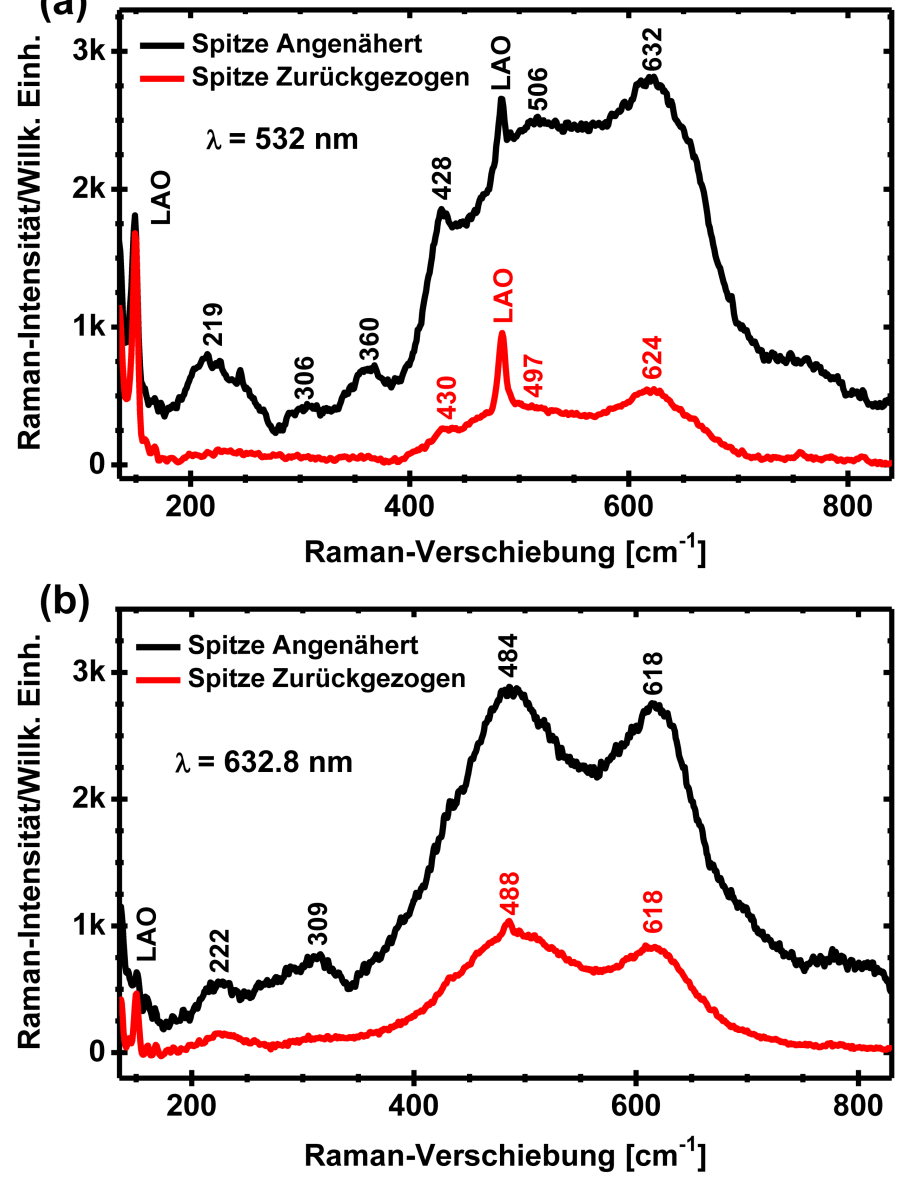

Abbildung 4.3.18: TERS- und Fernfeld-Raman-Spektren einer $30 \mathrm{~nm}$ LSMO/LAO (100)Schicht $(x=0.10)$ mit einer einfallenden P-Laserpolarisation parallel zur TERS-Spitzenachse und ohne Analysator, aufgenommen mit dem grünen $(\lambda=532 \mathrm{~nm})$ (a) und dem roten Laser $(\lambda=632,8 \mathrm{~nm})(\mathrm{b})$.

Für eine bessere Vergleichbarkeit wurde der jeweilige Hintergrund (TERS-Hintergrundsignal, elektronischer Hintergrund, Bose-Einstein-Korrektur) abgezogen. Zusätzlich sind die TERS-Spektren aus Übersichtsgründen vertikal leicht nach oben verschoben worden. Die Raman-Moden von Schicht und Substrat wurden auch hier mit Lorentz-Funktionen angenähert und so die Linienposition, die Linienbreite und die Linienintensität (integrierte 
Fläche) bestimmt. Beide Fernfeld-Raman-Spektren sind durch die zwei Jahn-Teller-Schwingungsbanden (SS-JT und AS-JT) geprägt. Darüber hinaus sind diese mit dem grünen Laser zu höheren Wellenzahlen verschoben und es ist auch die $B_{2 g}(3)$ Biegemode (B) zu beobachten, während diese für den roten Laser nahezu fehlt. Signifikant ist zudem der stärkere Beitrag des LAO-Substrats für den grünen Laser im Vergleich zu dem roten Laser mit einer eigentlich größeren Eindringtiefe. Gleichzeitig ist die Intensität der JT-Moden für den grünen Laser geringer, sodass sich die $E_{g}$ Biegeschwingung (B) des LAO-Substrats stärker aus dem Hintergrund erhebt. In den TERS-Spektren lässt sich eine Verstärkung aller LSMO-Raman-Moden beobachten. Gleichzeitig setzen sich die Unterschiede zwischen den Lasern fort. So sind die JT-Moden für den grünen Laser noch weiter gegenüber dem roten Laser zu größeren Wellenzahlen verschoben. Zudem ist auch eine deutliche Verschiebung der JT-Moden gegenüber dem Fernfeld zu beobachten, während die $B_{2 g}(3)$ Biegemode nur moderat zu kleineren Wellenzahlen verschoben ist. Ein Vergleich mit der $E_{g}$ Biegemode des LAO-Substrats zeigt zudem, dass ein instrumenteller Einfluss ausgeschlossen werden kann. Für den roten Laser lässt sich nur eine leichte Verschiebung der AS-JT-Mode im TERS-Spektrum beobachten, während die $B_{2 g}(3)$ Biegemode weiterhin fehlt. Eine Übersicht der Positionen und Linienbreiten der diskutierten Raman-Moden in den TERS- und Fernfeld-Spektren der $30 \mathrm{~nm}$ LSMO/LAO (100)-Schicht mit $x=0,10$ ist jeweils in der Tabelle $4.3 .1(\lambda=532 \mathrm{~nm})$ und in der Tabelle $4.3 .2(\lambda=632,8 \mathrm{~nm})$ zusammengefasst.

\begin{tabular}{|c|c|c|c|c|}
\hline Raman-Mode & $B_{2 g}(3)(\mathrm{B})$ & $A_{g}(1)(\mathrm{AS}-\mathrm{JT})$ & $B_{2 g}(1)(\mathrm{SS}-\mathrm{JT})$ & $\mathrm{E}_{\mathrm{g}}(\mathrm{B} ; \mathrm{LAO})$ \\
\hline \hline$\omega\left[\mathrm{cm}^{-1}\right]($ TERS $)$ & $428,5(3)$ & $506,5(8)$ & $631,9(5)$ & $483,7(2)$ \\
\hline$\omega\left[\mathrm{cm}^{-1}\right]($ Fernfeld$)$ & $430.5(5)$ & $497,4(6)$ & $624,2(3)$ & $484,2(1)$ \\
\hline$\Gamma\left[\mathrm{cm}^{-1}\right]($ TERS $)$ & $24,1(12)$ & $194,2(38)$ & $135,3(16)$ & $6,4(6)$ \\
\hline$\Gamma\left[\mathrm{cm}^{-1}\right]($ Fernfeld) & $21,4(24)$ & $154,9(30)$ & $101,4(14)$ & $7,0(2)$ \\
\hline
\end{tabular}

Tabelle 4.3.1: Position $\omega$ und Breite $\Gamma$ verschiedener Schwingungsmoden der mit dem grünen Laser $(\lambda=532 \mathrm{~nm})$ aufgenommenen TERS- und Fernfeld-Raman-Spektren der $30 \mathrm{~nm}$ LSMO/LAO (100)-Schicht $(x=0,10)$ im Vergleich.

\begin{tabular}{|c|c|c|}
\hline Raman-Mode & $A_{g}(1)(\mathrm{AS}-\mathrm{JT})$ & $B_{2 g}(1)(\mathrm{SS}-\mathrm{JT})$ \\
\hline \hline$\omega\left[\mathrm{cm}^{-1}\right]($ TERS $)$ & $484,2(3)$ & $618,0(3)$ \\
\hline$\omega\left[\mathrm{cm}^{-1}\right]$ (Fernfeld) & $488,3(2)$ & $618,2(2)$ \\
\hline$\Gamma\left[\mathrm{cm}^{-1}\right]($ TERS $)$ & $134,2(13)$ & $114,3(12)$ \\
\hline$\Gamma\left[\mathrm{cm}^{-1}\right]$ (Fernfeld) & $141,6(9)$ & $90,9(8)$ \\
\hline
\end{tabular}

Tabelle 4.3.2: Position $\omega$ und Breite $\Gamma$ der zwei JT-Schwingungsbanden der mit dem roten Laser $(\lambda=632,8 \mathrm{~nm})$ aufgenommenen TERS- und Fernfeld-Raman-Spektren der $30 \mathrm{~nm}$ LSMO/LAO (100)-Schicht $(x=0,10)$ im Vergleich. 
Während die AS-JT-Mode dabei im TERS-Spektrum des roten Lasers schmaler und die SS-JT-Mode etwas breiter wird, lässt sich für den grünen Laser eine Verbreiterung beider JT-Moden $\mathrm{um} \approx 35-40 \mathrm{~cm}^{-1}$ beobachten. Im Gegensatz dazu nimmt die Linienbreite der $B_{2 g}(3)$ Biegemode für den grünen Laser nur um $\approx 3 \mathrm{~cm}^{-1}$ zu und die der $E_{g}$ Biegemode des LAO-Substrats sogar leicht ab. Die Substrat-Linienbreite kann wie bei den laserleistungsabhängigen Raman-Spektren für eine Einschätzung der Temperaturerhöhung im TERS-Nahfeld herangezogen werden. Die sich nicht ändernde Linienbreite deutet dabei auf eine konstante Substrattemperatur hin. Der starke LAO-Linienbeitrag zeigt zudem, dass die Nahfeldabsorption in der $30 \mathrm{~nm}$ LSMO-Schicht begrenzt ist. Ein lokaler Temperaturanstieg an der Schichtoberfläche im TERS-Nahfeld kann jedoch nicht ausgeschlossen werden. Die Zunahme der Linienbreite der $B_{2 g}(3)$ Biegemode ist aber nur moderat. Für eine anharmonische Temperaturentwicklung (Gleichungen (3.3.11) und 3.3.12) lässt sich der Temperaturanstieg auf $\Delta T<50 \mathrm{~K}$ eingrenzen. Die entgegengesetzte Verschiebung der JT-Moden im TERS-Spektrum zu größeren Wellenzahlen lässt sich nicht durch eine Temperaturerhöhung erklären. Die Verspannung der Schicht ist nicht die Ursache, da die $30 \mathrm{~nm}$ LSMO/LAO (100)-Schichten keine Relaxation zeigen (Abschnitt 4.3.1). Außerdem müsste ein Nachlassen der in-plane wirkenden Druckspannung im oberflächensensitiven TERS-Nahfeld mit einem Erweichen der JT-Moden einhergehen, wie es bei RamanMessungen an relaxierenden LMO-Schichten mit zunehmender Schichtdicke auf LAO beobachtet wird 294,295. Vielmehr zeigt sich, dass ein leichter Sauerstoffüberschuss $\delta>0$ zu einem Erhärten der JT-Moden führt 241,296, der hier aufgrund der Oberflächensensitivität des TERS-Nahfeldes verstärkt an der Oberfläche auftritt. Ein Anzeichen für den Sauerstoffüberschuss stellt auch die Verbreiterung der JT-Moden dar, die typischerweise für dünne LMO- und niedrig-dotierte LSMO-Schichten gegenüber Messungen an stöchiometrischem Bulk-LMO in der orthorhombischen Pnma-Struktur beobachtet wird [294 297].

Für eine Quantifizierung der TERS-Verstärkung der Raman-Spektren der LSMO-Schicht kann wieder der TERS-Kontrast (Gleichung (3.4.3) ) der Raman-Moden über die integrierte Raman-Intensität (Lorentz-Funktion) im TERS-Gesamtfeld und im Fernfeld bestimmt werden. Die TERS-Kontrastwerte sind für die zwei JT-Moden (für beide Laser) und für die $\mathrm{B}_{2 \mathrm{~g}}(3)$ LSMO- und die $E_{g}$ LAO-Biegemode (grüner Laser) in der Tabelle 4.3 .3 dargestellt.

\begin{tabular}{|c|c|c|c|c|}
\hline TERS-Kontrast & $C_{B_{2 g}(3)}(\mathrm{B})$ & $C_{A_{g}(1)}(\mathrm{AS}-\mathrm{JT})$ & $C_{B_{2 g}(1)}(\mathrm{SS}-\mathrm{JT})$ & $C_{\mathrm{E}_{\mathrm{g}}}(\mathrm{B} ; \mathrm{LAO})$ \\
\hline \hline$\lambda=532 \mathrm{~nm}$ & 11,17 & 6,81 & 6,18 & 0,73 \\
\hline$\lambda=632,8 \mathrm{~nm}$ & - & 2,77 & 3,68 & - \\
\hline
\end{tabular}

Tabelle 4.3.3: Übersicht der mit den beiden Lasern für verschiedene Raman-Moden der $30 \mathrm{~nm}$ LSMO/LAO (100)-Schicht $(x=0,10)$ aus den TERS- und Fernfeld-RamanSpektren erzielten TERS-Kontrast-Werte $C$. Für den roten Laser fehlen die $\mathrm{B}_{2 \mathrm{~g}}(3)$ LSMO- und die $E_{g}$ LAO-Biegemode in den Spektren. 


\section{Auswertung und Ergebnisse}

Auffallend sind die größeren TERS-Kontrastwerte $C_{\mathrm{JT}}>6$ der zwei JT-Moden für den grünen Laser, die etwa doppelt so groß wie für den roten Laser sind. Der Grund ist das stärkere Fernfeld (Resonanzeffekt [302]) für den roten Laser bei ähnlicher Gesamtfeldintensität. Für den grünen Laser kann die $E_{g}$ LAO-Biegemode als Indikator für die Oberflächensensitivität der plasmonischen TERS-Nahfeldverstärkung betrachtet werden. Der TERS-Kontrast $C_{\text {LAO }}<1$ bestätigt den TERS-Nahfeldcharakter. Den größten TERS-Kontrast der LSMOSchicht für den grünen Laser zeigt die $\mathrm{B}_{2 \mathrm{~g}}(3)$ Biegemode mit $C_{\mathrm{B}_{2 \mathrm{~g}}(3)} \approx 11$. Im Vergleich zu den JT-Moden ist dieses fast eine Verdopplung und deutet auf eine Abnahme der JTVerzerrungen hin, wie sie auch für die LSMO-Schichten mit höheren Sr-Dotierungen in der Abbildung 4.3.10 und in den laserleistungsabhängigen Raman-Spektren bei einer Erhöhung der Laserleistung in den Abbildungen 4.3.14 (a)-(d) und 4.3.16 (a)-(d) an der gleichen LSMO-Schicht $(x=0,10)$ zu beobachten ist. Somit kann der Kontrastunterschied auch hier als eine lichtinduzierte Verschiebung der LSMO-Schicht im $T$-x-Phasendiagramm in Richtung einer temporär höheren Sr-Dotierung interpretiert werden, die lokal durch das TERS-Spitzennahfeld initiiert wird. Ein Anzeichen für diese spektrale Veränderung zeigt sich auch, indem die integralen Raman-Intensitätsverhältnisse der Raman-Moden für das TERS-Gesamtfeld und das Fernfeld bestimmt und verglichen werden. Diese sind für die $\mathrm{B}_{2 \mathrm{~g}}(3)$ Biegemode und die beiden JT-Moden nachfolgend in der Tabelle 4.3 .4 aufgeführt.

\begin{tabular}{|c|c|c|c|c|}
\hline$\lambda=532 \mathrm{~nm}$ & $\frac{I_{B_{2 g}(3)}}{I_{A_{g}(1)}}[\%]$ & $\frac{I_{B_{2 g}(3)}}{I_{B_{2 g}(1)}}[\%]$ & $\frac{I_{B_{2 g}(3)}}{I_{A_{g}(1)}+I_{B_{2 g}(1)}}[\%]$ & $\frac{I_{A_{g}(1)}}{I_{B_{2 g}(1)}}[\%]$ \\
\hline \hline TERS & 3,80 & 5,65 & 2,27 & 148,68 \\
\hline Fernfeld & 2,32 & 3,13 & 1,33 & 135,04 \\
\hline
\end{tabular}

Tabelle 4.3.4: Relative Verhältnisse der Raman-Intensität verschiedener Raman-Moden der $30 \mathrm{~nm}$ LSMO/LAO (100)-Schicht $(x=0,10)$ der mit dem grünen Laser aufgenommenen TERS- und Fernfeld-Raman-Spektren im Vergleich.

Es ergibt sich eine Erhöhung des Intensität der $B_{2 g}(3)$ Biegemode im TERS- gegenüber dem Fernfeld-Raman-Spektrum um $64 \%$ in Relation zur AS-JT-Mode $\left(A_{g}\right.$-Symmetrie) und um $81 \%$ in Relation zur SS-JT-Mode $\left(B_{2 g}\right.$-Symmetrie). Bezüglich der Summe beider JT-Moden nimmt die relative Intensität um $71 \%$ zu. Im Gegensatz dazu ändert sich die relative Intensität der beiden JT-Moden im TERS-Gesamtfeld nur um etwa $10 \%$, wobei ein Einfluss der Schichtoberfläche berücksichtigt werden muss. Messungen an LMO-Schichten mit unterschiedlicher Schichtdicke zeigen jedoch keine Systematik 294,295]. Im Vergleich zu den laserleistungsabhängigen Raman-Messungen entspricht die relative Intensitätssteigerung der $\mathrm{B}_{2 \mathrm{~g}}(3)$ Biegemode in etwa einer Verdopplung der eingestrahlten Laserleistung. Das für die $30 \mathrm{~nm}$ LSMO-Schicht um den Faktor $>10^{3}$ größere Anregungsvolumen des Fernfeldes (Gleichung (3.4.5), $L_{\mathrm{nf}} \approx L_{\mathrm{ff}}$ ) verdeutlicht zudem die lokale Feldstärke, die das plasmonische TERS-Nahfeld erreicht, um den lichtinduzierten Effekt zu initiieren. Der TERS-Verstärkungsfaktor für die $\mathrm{B}_{2 \mathrm{~g}}(3)$ Biegemode beträgt dabei $\mathrm{EF}_{\text {TERS }} \approx 10^{4}$. 


\section{Zusammenfassung und Ausblick}

In dieser Arbeit wurden nun die Ordnungs- und Unordnungsphänomene dünner $\mathrm{A}_{2} \mathrm{BMnO}_{6}$ Doppelperowskit-Schichten ( $\mathrm{A}=\mathrm{La}, \mathrm{Pr}, \mathrm{Nd}, \mathrm{Sm}, \mathrm{Gd}, \mathrm{Y}, \mathrm{B}=\mathrm{Co}, \mathrm{Ni})$ und niedrig-dotierter $\mathrm{La}_{1-\mathrm{x}} \mathrm{Sr}_{\mathrm{x}} \mathrm{MnO}_{3}$-Dünnschichten (LSMO) $(0 \leq x \leq 0,3)$, die mithilfe der metallorganischen Aerosol Deposition (MAD) hergestellt wurden, anhand der strukturellen, magnetischen und phononischen Eigenschaften untersucht. Während der Fokus bei den Doppelperowskiten auf die B-Platz-Kationenordnung in Abhängigkeit chemischer (A-/B-Platz-Kation) und externer Verspannungen (Substrat) gesetzt wurde, lag der Schwerpunkt bei den niedrigdotierten LSMO-Schichten auf den Einfluss der Sr-Dotierung $x$, der Temperatur $T$ und der Einstrahlung von Licht auf die JT-Verzerrungen und die strukturelle Ordnung. Dabei nimmt die Raman-Spektroskopie aufgrund ihrer Sensitivität für strukturelle Änderungen eine zentrale Position ein. Zudem wird mit der TERS-Technik eine erweiterte Methode der Raman-Spektroskopie erstmalig an beiden Materialsystemen angewandt. Im nachfolgenden Abschnitt werden die zentralen Ergebnisse der Arbeit zusammengefasst, bevor im Anschluss ein Ausblick auf weitere Möglichkeiten im Kontext der Arbeit gegeben wird.

\subsection{Zusammenfassung der Ergebnisse}

Der erste Teil der Arbeit befasst sich mit den B-Platz geordneten $\mathrm{A}_{2} \mathrm{BMnO}_{6}$-Doppelperowskiten. Nach der Einführung in das Wachstum der Doppelperowskit-Schichten mit der MAD-Technik und der einzustellenden Wachstumsparameter wurden in der Folge Doppelperowskit-Schichten der $\mathrm{A}_{2} \mathrm{CoMnO}_{6^{-}}$und der $\mathrm{A}_{2} \mathrm{NiMnO}_{6}$-Reihe auf STO (111)Substraten hergestellt, um so den Einfluss des A-Kations (A = La, Pr, Nd, Sm, Gd, Y) und des B-Kations auf die strukturellen, magnetischen und phononischen Eigenschaften sowie die zu erreichende B-Platz-Ordnung zu bestimmen. Hierzu wurden die Schichten umfangreich charakterisiert (XRD, Magnetisierung, Raman-Spektroskopie). Dabei konnte eine systematische Abnahme der Curie-Temperatur $T_{C}$ bei einer Verringerung des AKationenradius beobachtet werden, die sich auf die Abnahme des $\mathrm{Co}^{2+} \mathrm{O}^{2-}-\mathrm{Mn}^{4+}$-Bindungswinkels für den ferromagnetischen Superaustausch zurückführen lässt. Gleichzeitig nimmt in gleicher Weise die out-of-plane Gitterkonstante ab. Über einen Vergleich des Ordnungsparameters $S=I\left(\frac{1}{2} \frac{1}{2} \frac{1}{2}\right) / I(111)$, der das Verhältnis der Intensitäten von Überstruktur- und Fundamentalreflex angibt 36, 48,221, mit dem theoretischen Idealwert für eine perfekte B-Platz-Ordnung, konnte zudem der langreichweitig geordnete Anteil der 


\section{Zusammenfassung und Ausblick}

Schichten abgeschätzt werden. Dabei zeigte sich, dass dieses Verfahren nur mit Vorsicht $\mathrm{zu}$ verwenden ist, da viele weitere Faktoren neben der B-Platz-Ordnung Einfluss auf die Intensität des Überstrukturreflexes nehmen (APG, strukturelle Verzerrungen, Kationenverschiebungen oder Abstandsmodulationen [4, 30, 36, 48, 221, 224]). Während die LCMOSchicht mit $S=2,8(1) \cdot 10^{-3}$ und die LNMO-Schicht mit $S=2,2(1) \cdot 10^{-3} \mathrm{im}$ Vergleich zum theoretischen Wert von $S_{\text {theo. }}=10^{-2}$ für LCMO [36, 48 einen deutlich geringeren Wert erreichten, konnten für alle anderen Doppelperowskit-Schichten Ordnungsparameter $S>10^{-2}$, die zudem systematisch mit der Verringerung des A-Kationenradius ansteigen, beobachtet werden. Mit $M(H)$-Magnetisierungsmessungen (Sättigungsmagnetisierung, Remanenz, Koerzitivfeldstärke) konnte anschließend jedoch nachgewiesen werden, dass auch LCMO und LNMO auf STO (111) eine hohe B-Platz-Nahordnung aufweisen und der geringe XRD-Ordnungsparameter auf die höhere Präsenz von APG zurückzuführen ist, während der weitere Anstieg über den theoretischen Wert $S_{\text {theo. }}$ hinaus für sehr viel kleinere A-Kationen durch eine zusätzliche Abstandsmodulation der Überstruktur entsteht.

Um den Einfluss externer Verspannungen in den Doppelperowskit-Schichten auf die BPlatz-Ordnung zu analysieren, wurden zudem LCMO-Schichten auf LAO, LSAT, STOSubstraten mit (111)-Orientierung, auf $\mathrm{MgO}$ (100) sowie auf hexagonalem $\mathrm{Al}_{2} \mathrm{O}_{3}(0001)$ mit einer jeweils unterschiedlichen Gitterfehlanpassung $f$ zu Bulk-LCMO aufgewachsen. Dabei konnte gezeigt werden, dass insbesondere eine in-plane durch das Substrat induzierte Druckspannung für LCMO auf LAO (111) und LSAT (111) zu einem besonders hohen XRD-Ordnungsparameter $S \approx 1,2 \cdot 10^{-2}$ im Bereich von $S_{\text {theo. }}$ für eine vollständige B-PlatzOrdnung führt. Magnetisierungsmessungen, Raman-Messungen und EELS-Aufnahmen bestätigten die Ordnung dabei auch unabhängig der XRD-Spektren. Dieses passt zu LCMOSchichten in der Literatur mit einer Verbesserung der B-Platz-Ordnung 220, 224, indem eine in-plane Druckspannung zu einer Stimulation der B-Platz-Ordnung durch eine unterschiedliche Verzerrung der beiden $\mathrm{BO}_{6}$ und $\mathrm{B}^{\prime} \mathrm{O}_{6}$-Oktaeder führt 224. Dieses lässt sich auf die zwei LCMO-Schichten auf LSAT (111) und LAO (111) übertragen. Die hohe Sättigungsmagnetisierung $M_{S} \approx 6 \mu_{\mathrm{B}} /$ f.u. bestätigt, dass die großen XRD-Ordnungsparameter nicht nur durch eine Abstandsmodulation in der Kristallstruktur hervorgerufen werden.

Mit dem Wachstum von LCMO auf hexagonalem $\mathrm{Al}_{2} \mathrm{O}_{3}$ (0001) mit einem B-Platz-Ordnungsgrad von $>98 \%$ und einer Gitterfehlanpassung von nur $f=-0,05 \%$ konnte außerdem eine exzellente Alternative zu den herkömmlichen kubischen Substraten wie STO, LAO und LSAT für das Wachstum in (111)-Orientierung aufgezeigt werden. Dieses bestätigen die Magnetisierungsdaten, Raman-Spektren und EELS-Aufnahmen sowie der XRD-Ordnungsparameter mit $S=5,9(1) \cdot 10^{-3}$, der etwa doppelt so groß wie für die LCMO/STO (111)-Schicht ist und auf eine verringerte Anzahl an APG zurückzuführen ist. 


\subsection{Zusammenfassung der Ergebnisse}

Anhand von temperaturabhängigen Raman-Messungen konnte über die Entwicklung der Position und der Linienbreite der $A_{g}$ Breathing Mode außerdem gezeigt werden, dass die Verspannung und der B-Platz-Ordnungsgrad auch die phononischen Eigenschaften der Doppelperowskite stark beeinflussen. Im Vergleich zu der anharmonischen Modellbeschreibung der Temperaturabhängigkeit der Modenposition konnte für alle DoppelperowskitSchichten ein signifikantes Erweichen der Breathing Mode für Temperaturen $T<T_{C}$ beobachtet werden, das mit dem Eintritt in die ferromagnetische Ordnung initiiert wird und auf die Präsenz einer ausgeprägten Spin-Phonon-Kopplung zurückzuführen ist. Für die resultierende Spin-Phon-Kopplungsstärke $\lambda$ der $\mathrm{A}_{2} \mathrm{CoMnO}_{6^{-}}$und der $\mathrm{A}_{2} \mathrm{NiMnO}_{6}$-DoppelperowskitReihen auf STO (111)-Substraten konnte dabei jeweils eine systematische Abnahme mit der Verringerung des A-Kationenradius nachgewiesen werden. Dieses lässt sich erneut auf die Verkleinerung des $\mathrm{Co}^{2+} / \mathrm{Ni}^{2+}-\mathrm{O}^{2-}-\mathrm{Mn}^{4+}$-Bindungswinkels und auf eine Vergrößerung der Bindungslängen zurückführen, wodurch nicht nur die magnetische Kopplung, sondern auch die Kraftkonstanten des Kristallgitters signifikant abgeschwächt werden [219,220].

Für die LCMO-Schichten auf den verschiedenen Substraten konnte zudem eine systematische Abhängigkeit der Spin-Phonon-Kopplungsstärke $\lambda$ von dem Verspannungszustand in den Schichten mit den größten Werten für eine moderate in-plane durch das Substrat induzierten Druckspannung beobachtet werden. So erreicht die B-Platz geordnete LCMO-Schicht auf $\mathrm{Al}_{2} \mathrm{O}_{3}$ (0001) eine Kopplungsstärke $\lambda=2,02(7) \mathrm{cm}^{-1}$, während sich für die geordnete LCMO-Schicht auf STO (111) eine geringere Kopplungsstärke von $\lambda=1,42(6) \mathrm{cm}^{-1}$ ergibt. In gleicher Weise konnte gezeigt werden, dass das Erhärten der $A_{g}$ Breathing Mode für Temperaturen $T>500 \mathrm{~K}$, das auf einen Phasenübergang von der monoklinen P12 /n1- in die rhomboedrische R⿳亠丷-Struktur zurückzuführen ist 215,238,239], in der Ausprägung und dem Ablauf ebenfalls sehr stark von dem Verspannungszustand in den LCMO-Schichten beeinflusst ist. So ist der strukturelle Übergang für stark verspannte Schichten sehr ausgeprägt und eine Phasenkoexistenz über einen großen Temperaturbereich vorhanden, während die nur moderat verspannten oder relaxierten LCMO-Schichten einen deutlich schärferen Übergang aufweisen. Dieses bestätigt den in den XRD- und Magnetisierungsmessungen bereits beobachteten großen Einfluss der Verspannung in den LCMODoppelperowskit-Schichten auf die kristallinen Eigenschaften und die B-Platz-Ordnung.

In einem Vergleich zweier LCMO-Schichten auf $\mathrm{Al}_{2} \mathrm{O}_{3}$ (0001) mit den B-Platz-Ordnungsgraden $>98 \%$ und $\approx 90 \%$ konnte anschließend der Unterschied in der B-Platz-Ordnung auch anhand der Spin-Phon-Kopplungsstärke mit $\lambda=2,02(7) \mathrm{cm}^{-1}$ (B-Platz geordnet) und $\lambda=1,59(4) \mathrm{cm}^{-1}$ (B-Platz teilgeordnet) verifiziert werden. Somit kann die SpinPhonon-Kopplungsstärke $\lambda$ neben ihrer Sensitivität bezüglich der chemischen Verspannung und der durch das Substrat in-plane induzierten Verspannung als ein weiterer Parameter zur Abschätzung der B-Platz-Kationenordnung der Doppelperowskite verwendet werden. 


\section{Zusammenfassung und Ausblick}

In dem zweiten Teil wurden anschließend TERS-Messungen an verschiedenen Materialsystemen betrachtet. Nach einer Erprobung an den zwei TERS-Testfarbstoffen Azobenzol und Malachitgrün Isothiocynat (MITC) auf einer Goldoberfläche, für die die TERSKontrastwerte $C \approx 19$ (Azobenzol) und $C \approx 13$ (MGITC) und damit die TERS-Verstärkungsfaktoren $\mathrm{EF}_{\mathrm{TERS}} \approx 5 \cdot 10^{4}$ (Azobenzol) und $\mathrm{EF}_{\mathrm{TERS}} \approx 4 \cdot 10^{4}$ (MGITC) in guter Übereinstimmung mit der Literatur beobachtet werden konnten 171, 173, 187, 198, wurden polarisationsabhängige TERS-Messungen an einem Silizium-Substrat (p-Dotierung mit Bor) mit einer (100)-Orientierung durchgeführt, das für TERS-Untersuchungen ebenfalls als Refenrenzmaterial verwendet wird. Dabei konnte eine starke Abhängigkeit des TERS-Kontrastes der dreifach-entarteten LTO(Г)-Phonomode bei $520 \mathrm{~cm}^{-1}$ von der Polarisationskonfiguration mit einer Variation $C_{520} \approx 2,8-8,4$ nachgewiesen werden. Dieses Verhalten wird auch in der Literatur beobachtet und auf einen neben der plasmonischen Nahfeldverstärkung auftretenden Depolarisationseffekt des Nahfeldes zurückgeführt, der die Raman-Auswahlregeln bricht, wodurch die in bestimmten Polarisationskonfigurationen verbotenen Raman-Moden wieder erlaubt sind und so eine besonders große Verstärkung gegenüber dem stark reduzierten Fernfeld ermöglicht wird $169,170,192,194,254$.

Eine Trennung der LO- und TO-Anteile der LTO $(\Gamma)$-Phonomode von Silizium ist jedoch in der Streugeometrie mit Seiteneinstrahlung schwierig. Folglich wurde mit dem zuvor charakterisierten B-Platz geordneten $\mathrm{La}_{2} \mathrm{CoMnO}_{6}$-Doppelperowskit ein System gewählt, dass sich aufgrund des großen Raman-Wirkungsquerschnitts und der Nicht-Entartung der RamanModen in der monoklinen P12/n1-Struktur hervorragend für eine Analyse der Beiträge zur TERS-Verstärkung durch die plasmonische Nahfeldverstärkung und die Nahfelddepolarisation eignet. Hierfür wurden an zwei LCMO-Schichten auf STO (100) mit den Schichtdicken $d=25 \mathrm{~nm}$ und $d=120 \mathrm{~nm}$ polarisationsabhängige TERS-Messungen durchgeführt und die Ergebnisse mit theoretischen Berechnungen für die Raman-Auswahlregeln unter Berücksichtigung eines kombinierten Verstärkungs- und Depolarisationsmodells des TERSSpitzennahfeldes verglichen. Hierbei konnte eine exzellente Übereinstimmung zwischen den aufgenommenen TERS-Spektren und den Berechnungen für die einzelnen Polarisationskonfigurationen erzielt werden. Für die $A_{g}$ Breathing Mode bei $644 \mathrm{~cm}^{-1}$, die eine symmetrische Streckschwingung der $(\mathrm{Co} / \mathrm{Mn}) \mathrm{O}_{6}$-Oktaeder in den Doppelperowskiten beschreibt, konnte dabei eine besonders große Verstärkung der Intensität in den gekreuzten Polarisationskonfigurationen für die $25 \mathrm{~nm}$ LCMO-Schicht mit dem Kontrast $C_{644} \approx 60-110$ beobachtet werden, die direkt auf die Nahfelddepolarisation und die damit einhergehende Aufhebung der Raman-Auswahlregeln für die Mode zurückgeführt werden kann. Anhand der Verstärkung mit einem Kontrast $C_{644} \approx 2-5$ in den parallelen Konfigurationen aufgrund des plasmonischen Nahfeldes ist es möglich beide Effekte zu separieren. Mit einer in-plane Drehung konnte zudem für die 120 nm LCMO-Schicht gezeigt werden, dass die Depolarisation unabhängig von der Symmetrie auch für andere Raman-Moden auftritt. 


\subsection{Zusammenfassung der Ergebnisse}

Dieses führt zu der nach wie vor diskutierten Frage [148, 169, 190, 199, 200, 209], wodurch die Polarisationseigenschaften des TERS-Nahfeldes bestimmt sind. So wird zunächst experimentell wie auch theoretisch eine Bevorzugung der TERS-Verstärkung für eine Polarisation parallel zur Spitzenachse beobachtet [169, 170, 190, 191, 199, 200]. Diese Affinität wird hauptsächlich auf die Spitzengeometrie der TERS-Spitzen, die üblicherweise über ein großes Aspektverhältnis verfügen, zurückgeführt. Gleichzeitig dokumentieren einfache Dipol-Modelle für STM-TERS-Systeme im gap mode, dass sich abseits von der Spitzenachse durch die Feldlinienkrümmung eine Depolarisation mit Komponenten sowohl parallel (P) als auch senkrecht (S) zur TERS-Spitzenachse einstellt [199]. Zudem wird ein Einfluss der Spitzenbeschaffenheit (wie Qualität, Form, Oberfläche und Radius) und von verschiedenen Streuartefakten diskutiert 190,200, 206, 207]. Die Ausprägung des Nahfelddepolarisation und die Reproduzierbarkeit mit unterschiedlichen TERS-Systemen (AFM, STM), Probensystemen (Silizium [169, 170,192, 194], GaAs [193], GaN [209], Graphen [209] oder wie hier $\mathrm{La}_{2} \mathrm{CoMnO}_{6}$ ) und Spitzen (AFM, STM) in der Literatur zeigt aber, dass die genannten Effekte nicht die Hauptursache für die Polarisationseigenschaften des Nahfeldes sein können. Vielmehr stimmen die TERS-Messungen an den LCMO-Schichten mit den Vorhersagen eines neuen quantenmechanischen Modellvorschlags 209 überein, nach dem die Nahfeldpolarisation völlig unabhängig von der Fernfeldpolarisation ist und das Nahfeld eigene TERSAuswahlregeln besitzt. Messungen an weiteren $\mathrm{A}_{2} \mathrm{BMnO}_{6}$-Schichten, mit anderen TERSSpitzen und in Abhängigkeit der STM-Parameter (Tunnelstrom $j$, Spannung $U_{\text {Bias }}$ ) bestätigen zudem, dass die TERS-Verstärkung eine Kombination aus einer plasmonischen Nahfeldverstärkung und einer Nahfelddepolarisation ist. Mit einer LCMO-Schichtdickenserie auf STO (100) konnte außerdem gezeigt werden, dass die TERS-Verstärkung aufgrund der unterschiedlichen Anregungsvolumina von Nahfeld und Fernfeld von der Schichtdicke, mit einem entsprechend größeren TERS-Kontrast für dünnere Schichten, abhängt.

Im dritten Teil wurden schließlich dünne niedrig-dotierte LSMO-Schichten $(0 \leq x \leq 0,3)$ mit einer Schichtdicke von $d \approx 30 \mathrm{~nm}$ auf ihre strukturellen, elektrischen und magnetischen Phasen untersucht und der Einfluss der Sr-Dotierung, der Temperatur $T$ und der eingestrahlten Laserleistung auf die strukturelle Ordnung diskutiert. Der Ausgangspunkt ist dabei die Herstellung der Schichten mit der MAD-Technik. Über eine umfangreiche strukturelle, magnetische und elektrische Charakterisierung (XRD, AFM, Magnetisierung, elektrische Transporteigenschaften) konnte sowohl für für das Wachstum auf MgO (100)- als auch auf LAO (100)-Substraten eine hohe Schichtqualität bestätigt werden. Im Vergleich zu dem $T$ - $x$-Phasendiagramm von Bulk-LSMO ergeben sich jedoch spezifische Unterschiede. So zeigte sich, dass die LSMO-Schichten unabhängig von der Sr-Konzentration und dem Substrat in dem betrachteten Bereich $0 \leq x \leq 0,3$ stets einen ferromagnetischen Grundzustand aufweisen, während Bulk-LMO/-LSMO bis zu einer Dotierung von $x \leq 0,10$ von einem (gekanntet)-antiferromagnetischen Grundzustand gekennzeichnet ist [16, 17, 25 27]. 


\section{Zusammenfassung und Ausblick}

Dieses abweichende Verhalten ist für LSMO-Schichten mit niedriger Sr-Dotierung charakteristisch und wird auf eine durch das Substrat induzierte Verspannung [280 284] sowie auf weitere Faktoren wie die Stöchiometrie [278], den Sauerstoffpartialdruck während und nach der Schichtherstellung 282,283 und die Schichtdicke 284 zurückgeführt. Die elektrischen Transportmessungen und Magnetisierungsmessungen an den hergestellten LSMOSchichten zeigen jedoch, dass ein signifikanter Sauerstoffüberschuss $\delta$ in den Schichten wie auch eine Abweichung in der Stöchiometrie (insbesondere ein Lanthan-Defizit) ausgeschlossen werden können. Die im Vergleich zu LMO-Schichten auf STO (100) reduzierten Curie-Temperaturen $T_{C}$ und Sättigungsmagnetisierungen $M_{S}$ der LSMO-Schichten mit $x \leq 0,10$ kennzeichnen stattdessen einen Grundzustand mit ferromagnetischen Clustern in einer antiferromagnetisch ordnenden Matrix [283, 284, 287]. Dieses lässt sich auf die unterschiedlichen Verspannungszustände (unverspanntes Wachstum auf MgO (100), in-plane Druckspannung auf LAO (100), in-plane Zugspannung auf STO (100)) zurückführen.

In den Raman-Messungen bestätigt sich dieses mit signifikanten Unterschieden zu dem Raman-Spektrum von orthorhombischem Bulk-LMO. So weisen die Spektren der niedrigdotierten LSMO-Schichten eine Verschiebung der für LMO charakteristischen JT-Schwingungsbanden zu größeren Wellenzahlen auf und die JT-Moden sind deutlich verbreitert. Diese Merkmale werden auch für rhomboedrisches Bulk-LMO beobachtet und kennzeichnen aufgrund der Inkompatibilität der dazugehörigen R $\overline{3}$ c-Struktur zu intrinsischen JTVerzerrungen die Inkohärenz der beobachtbaren JT-Banden, die mit einer strukturellen Unordnung des Sauerstoff-Kristalluntergitters einhergehen (energetische Dispersion der Ein-Phonon-Sauerstoff-Zustandsdichte) 270, 293, 298. Anhand der Raman-Spektren in Abhängigkeit der Sr-Dotierung $x$ und der Temperatur $T$ zeigt sich zudem, dass die JT-Modenausprägung stark an das $\mathrm{Mn}^{3+} / \mathrm{Mn}^{4+}$-Kationenverhältnis, die elektrische Leitfähigkeit und magnetische Korrelationen in der Schicht gekoppelt ist sowie durch thermische Fluktuationen beeinflusst wird. Während die JT-Moden dabei durch die zunehmende Ladungsträgerdelokalisation mit steigender Sr-Dotierung oder in der ferromagnetischen Phase unterdrückt werden, lässt sich gleichzeitig eine Intensitätszunahme der JTunabhängigen, gegenphasigen $B_{2 g}(3)$ Biegemode beobachten, die für LSMO insbesondere für Sr-Konzentrationen $x>0,17$ charakteristisch ist und sich dem Übergang in eine mehr geordnete Struktur zuordnen lässt. Am Beispiel der LSMO-Schicht auf LAO (100) mit $x=0,10$ konnte zusätzlich anhand von laserleistungsabhängigen Raman-Messungen und mit TERS-Messungen lokal durch das Spitzennahfeld gezeigt werden, dass sich der Ordnungszustand von niedrig-dotiertem LSMO mit einer effektiven Verschiebung in Richtung einer mehr metallisch-geordneten Schicht auch durch eine lichtinduzierte Ladungsträgerdelokalisation beeinflussen lässt. Im Vergleich zu den Doppelperowskiten wird für die niedrigdotierten LSMO-Schichten hier somit nicht die Anordnung der Kationen im Kristallgitter, sondern der Charakter der auftretenden JT-Verzerrungen extern beeinflusst. 


\subsection{Ausblick}

Die Raman-Spektroskopie kann aufgrund der strukturellen Sensitivität und der SpinPhonon-Kopplung als eine Größe, die das Zusammenspiel der magnetischen und phononischen Eigenschaften charakterisiert, auch für andere als die in dieser Arbeit gezeigten Doppelperowskite für eine Abschätzung der B-Platz-Ordnung und für die Entwicklung von Strategien zur Erhöhung des Ordnungsgrades verwendet werden. Eine Erweiterungsmöglichkeit für die Vermessung der Spin-Phonon-Kopplung wäre dabei der Einsatz eines mit flüssigem Helium $\left(T_{\mathrm{He}}=4,2 \mathrm{~K}\right)$ gekühlten Kryostaten mit integriertem supraleitendem Magneten. So könnte zudem die Temperaturentwicklung der Spin-Phonon-Kopplungsstärke der $\mathrm{A}_{2} \mathrm{CoMnO}_{6^{-}}$und der $\mathrm{A}_{2} \mathrm{NiMnO}_{6}$-Doppelperowskit-Reihen bis hinunter zu YCMO und YNMO mit dem besonders kleinen $\mathrm{Y}^{3+}$-A-Kation bestimmt werden. Außerdem wäre es möglich den Einfluss der 3d-4f-Kopplung der A- und B-Kationen, die für einige der Doppelperowskite für Temperaturen $T<50 \mathrm{~K}$ auftritt [38, 228, 230], zu untersuchen. Einen zusätzlichen Einblick in die B-Platz-Ordnung und die Wirkung der APG in den Doppelperowskiten könnten wiederum magnetfeldabhängige Raman-Messungen geben, wenn diese wie die $M(T)$-Kurven sowohl beim Abkühlen mit Magnetfeld (FCC) als auch bei der Erwärmung mit Magnetfeld nach einer vorherigen Abkühlung ohne Magnetfeld (FCW) aufgenommen und die jeweiligen Phonon-Renormierungen der $A_{g}$ Breathing Mode für Temperaturen $T<T_{C}$ miteinander und mit den $M(T)$-Kurven verglichen werden.

Daneben kann die Spin-Phonon-Kopplungsstärke $\lambda$ als Maß für die B-Platz-Ordnung auch bei anderen Doppelperowskit-Systemen verwendet werden. Insbesondere für solche Doppelperowskite mit Cr-, Fe-, Mo-, Re- oder W-Kationen auf den B-/B'-Plätzen, die aufgrund ihrer hohen Curie-Temperaturen $T_{C}>400 \mathrm{~K}[4,29]$ sehr interessant für technologische Anwendungen sind, deren magnetische Eigenschaften aber empfindlich von den Wachstumsbedingungen und von dem B-Platz-Ordnungsgrad abhängen, stellt die Raman-Spektroskopie neben der Magnetisierung, elementspezifisch-chemischer Techniken (EDX, EELS) und dem nur mit Sorgfalt zu betrachtenden XRD-Ordnungsparameter $S$ [4,30,221] eine sehr attraktive Möglichkeit zur qualitativen und quantitativen Verifizierung des B-Platz-Ordnungsgrades dar. Bezogen auf diese Arbeit konnte zudem ein Einfluss der in-plane durch das Substrat induzierten Verspannung auf die B-Platz-Ordnung und die phononischen Eigenschaften von LCMO mit der Raman-Spektroskopie nachgewiesen werden. Dieses Verfahren bietet folglich auch für die anderen $\mathrm{A}_{2} \mathrm{CoMnO}_{6}$ - und der $\mathrm{A}_{2} \mathrm{NiMnO}_{6}$-Doppelperowskite das Potential für eine weitere Verbesserung der B-Platz-Ordnung. Interessante Kandidaten sind sowohl LNMO mit einem zu LCMO sehr ähnlichen Verhalten auf STO (111) bezüglich des XRD-Ordnungsparameters als auch YCMO mit seiner nur schwachen ferromagnetischen Kopplung aufgrund des kleinen A-Kationenradius und einer auf STO (111) nur reduzierten Sättigungsmagnetisierung bei einer gleichzeitig großen Koerzitivfeldstärke. Für LNMOSchichten ist zudem in der Literatur eine Abhängigkeit der strukturellen Phase von der 


\section{Zusammenfassung und Ausblick}

in-plane Verspannung des Substrats mit einer monoklinen P12 1 n1-Struktur für in-plane wirkende Zugspannungen und einer rhomboedrischen $\mathrm{R} \overline{3}$-Struktur für in-plane induzierte Druckspannungen zu beobachten 245,246. Der Übergang von der monoklinen P12 $1 /$ n1in die R $\overline{3}$-Phase könnte hierbei ähnlich wie für LCMO mithilfe temperaturabhängiger und polarisationsabhängiger Raman-Messungen direkt beobachtet und charakterisiert werden.

Die TERS-Technik wiederum bietet die Möglichkeit der lokalen und oberflächennahen Betrachtung der phononischen Eigenschaften bei einer gleichzeitigen Verstärkung des RamanSignals. Entsprechend ist diese insbesondere für sehr dünne Schichten geeignet, zumal hier in dieser Arbeit gezeigt werden konnte, dass der erzielte TERS-Kontrast mit einer Verringerung der Schichtdicke stark zunimmt. Ein Schwierigkeit kann jedoch ein starker Hintergrundbeitrag des Substrats im Spektrum sein. Wie an den Doppelperowskit-Schichten nachgewiesen, kann dieses Problem jedoch mithilfe polarisationsabhängiger TERS-Messungen und des Depolarisationseffektes des TERS-Nahfeldes umgangen werden, wenn die Raman-Moden der zu untersuchenden Schicht eine dafür geeignete Symmetrie aufweisen. In Kombination mit der TERS-Oberflächensensitivität ist es so möglich auch sehr dünne Schichten mit Schichtdicken $d<10 \mathrm{~nm}$ problemlos zu vermessen. Mögliche Anwendungen hierfür sind die Untersuchung des Schichtwachstum der ersten atomaren Lagen auf verschiedenen Substraten oder die Entwicklung des Verspannungszustandes der Schichten in Abhängigkeit der Schichtdicke. Daneben bietet sich die Technik auch für die Analyse von Oberflächenzuständen, der lokalen Stöchiometrie oder von Grenzflächeneffekten an.

Weiterhin zeigt sich an den niedrig-dotierten LSMO-Schichten, dass eine lichtinduzierte Anregung in Richtung eines metallisch-geordneten Zustands sowohl durch eine Erhöhung der eingestrahlten Laserleistung als auch lokal mithilfe des TERS-Nahfeldes möglich ist. Hier könnten weitere Messungen an niedrig-dotierten LSMO-Schichten auf unterschiedlichen Substraten durchgeführt werden, um einen möglichen Einfluss der Schichtverspannung zu charakterisieren. In gleicher Weise kann zudem eine Ausweitung der Messungen auf niedrig-dotierte $\mathrm{Pr}_{1-\mathrm{x}} \mathrm{Sr}_{\mathrm{x}} \mathrm{MnO}_{3}$ - oder $\mathrm{Nd}_{1-\mathrm{x}} \mathrm{Sr}_{\mathrm{x}} \mathrm{MnO}_{3}$-Schichten realisiert werden, um so zusätzlich den Einfluss des A-Kations und die damit einhergehenden Änderungen der strukturellen Eigenschaften mit einzubeziehen. Ein möglicher lichtinduzierter Effekt lässt sich dann wieder anhand eines entsprechenden Vergleichs mit temperaturabhängigen und polarisationsabhängigen Raman-Messungen quantitativ abschätzen und einordnen. 


\section{Literaturverzeichnis}

[1] Richard J. D. Tilley: Perovskites, John Wiley \& Sons Verlag, 1. Auflage 2016.

[2] H. Zhang, N. Li, K. Li, D. Xue: Structural stability and formability of $\mathrm{ABO}_{3}$-type perovskite compounds, Acta Crystallogr. B 63, 812-818 (2007).

[3] G. King, P. M. Woodward: Cation ordering in perovskites, J. Mater. Chem. 20, 5785 (2010).

[4] S. Vasala, M. Karppinen: $\mathrm{A}_{2} \mathrm{~B}^{\prime} \mathrm{B}^{\prime \prime} \mathrm{O}_{6}$ perovskites: A review, Prog. Solid State Chem. 43, 1-36 (2015).

[5] Rudolf Gross, Achim Marx: Der Kolossale Magnetowiderstandseffekt, Kapitel 4 aus dem Vorlesungsskript: Spinelektronik, Walther-Meissner-Institut, 2004.

[6] G. H. Jonker, J. H. Van Santen: Ferromagnetic compounds of manganese with perovskite structure, Physica 16, 337-349 (1950).

[7] Y. Tokura, Y. Tomioka: Colossal magnetoresitive manganites, J. Magn. Magn. Mater 200, 1 (1999).

[8] J. M. D. Coey, M. Viret, S. von Molnár: Mixed-valence manganites, Adv. Phys. 48, 167-293 (1999).

[9] P. K. Davies, H. Wu, A. Y. Borisevich, I. E. Molodetsky, L. Farber: Crystal Chemistry of Complex Perovskites: New Cation-Ordered Dielectric Oxides, Annu. Rev. Mater. Res. 38, 369-401 (2008).

[10] K. Momma, F. Izumi: VESTA: a three-dimensional visualization system for electronic and structural analysis, Journal of Applied Crystallography 41, 653-658 (2008).

[11] D. I. Khomskii: Transition Metal Compounds, Cambridge University Press, 1. Auflage 2014.

[12] V. M. Goldschmidt: Die Gesetze der Krystallographie, Naturwissenschaften 14, 477-485 (1926).

[13] S. Middey, J. Chakhalin, P. Mahadevan, J. W. Freeland, A. J. Millis, D. D. Sarma: Physics of Ultrathin Films and Heterostructures of Rare-Earth Nickelates, Annual Review of Materials Research 46, 11.1-11.30 (2016).

[14] Bernard Raveau, Md. Motin Seikh: Cobalt Oxides, Wiley-VCH Verlag, 1. Auflage 2012.

[15] H. A. Jahn, E. Teller: Stability of Polyatomic Molecules in Degenerate Electronic States. I. Orbital Degeneracy, Proc. R. Soc. Lond. A 161, 220-235 (1937).

[16] A. A. Muhkin, V. Yu Ivanov, V. D. Travkin, S. P. Lebedev, A. Pimenov, A. Loidl, A. M. Balbashov: Magnetic and structural transitions in $\mathrm{La}_{1-\mathrm{x}} \mathrm{Sr}_{\mathrm{x}} \mathrm{MnO}_{3}$ : T-x phase diagram, Journal of Experimental and Theoretical Physics Letters 68, 356 (1998).

[17] M. Paraskevopoulos, F. Mayr, C. Hartinger, A. Pimenov, J. Hemberger, P. Lunkenheimer, A. Loidl, A. A. Muhkin, V. Yu Ivanov, A. M. Balbashov: The phase diagram and optical properties of $\mathrm{La}_{1-\mathrm{x}} \mathrm{Sr}_{\mathrm{x}} \mathrm{MnO}_{3}$ for $x \leq 0.2$, J. Magn. Magn. Mater 211, 118-127 (2000). 


\section{Literaturverzeichnis}

[18] H. A. Kramers: L'interaction Entre les Atomes Magnétogènes dans un Cristal Paramagnétique, Physica 1, 182 (1934).

[19] P. W. Anderson: Antiferromagnetism. Theory of Superexchange Interaction, Phys. Rev. 79, 350 (1950).

[20] C. Zener: Interaction between the d-Shells in the Transition Metals. II. Ferromagnetic Compounds of Manganese with Perovskite Structure, Phys. Rev. 82, 403 (1951).

[21] P. W. Anderson, H. Hasegawa: Considerations on Double Exchange, Phys. Rev. 100, 675 (1955).

[22] A. Bhattacharya, S. J. May: Magnetic Oxide Heterostructures, Annual Review of Materials Research 44, 65-90 (2014).

[23] J. B. Goodenough: Theory of the Role of Covalence in the Perovskite-Type Manganites $[\mathrm{La}, \mathrm{M}(\mathrm{II})] \mathrm{MnO}_{3}$, Phys. Rev. 100, 564 (1950).

[24] J. Kanamori: Superexchange interaction and symmetry properties of electron orbitals, J. Phys. Chem. Solids 10, 87-98 (1959).

[25] M. Paraskevopoulos, F. Mayr, J. Hemberger, A. Loidl, R. Heichele, A. A. Muhkin, A. M. Balbashov: Magnetic properties and the phase diagram of $\mathrm{La}_{1-\mathrm{x}} \mathrm{Sr}_{\mathrm{x}} \mathrm{MnO}_{3}$ for $x \leq 0.2$, Journal of Physics: Condensed Matter 12, 3993-4001 (2000).

[26] J. Geck, P. Wochner, S. Kiele, R. Klingeler, A. Revcolevschi, M. von Zimmermann, B. Büchner, P. Reutler: Orbital order induced ferromagnetic insulating properties, New J. Phys. 6, 152 (2004).

[27] J. Hemberger, A. Krimmel, T. Kurz, H.-A. Krug von Nidda, V. Yu. Ivanov, A. A. Muhkin, A. M. Balbashov, A. Loidl: Structural, magnetic, and electrical properties of single-crystalline $\mathrm{La}_{1-\mathrm{x}} \mathrm{Sr}_{\mathrm{x}} \mathrm{MnO}_{3}$ $(0.4<x<0.85)$, Phys. Rev. B 66, 094410 (2002).

[28] C. J. Howard, B. J. Kennedy, P. M. Woodward: Ordered double perovskites - a group-theoretical analysis, Acta Crystallogr. B 59, 463-471 (2003).

[29] D. Serrate, J. M. De Teresa, M. R. Ibarra: Double perovskites with ferromagnetism above room temperature, Journal of Physics: Condensed Matter 19, 023201 (2007).

[30] S. Chakraverty, A. Ohtomo, D. Okuyama, M. Saito, M. Okude, R. Kumai, T. Arima, Y. Tokura, S. Tsukimoto, Y. Ikuhara, M. Kawasaki: Ferrimagnetism and spontaneous ordering of transition metals in double perovskite $\mathrm{La}_{2} \mathrm{CrFeO}_{6}$ films, Phys. Rev. B 84, 064436 (2011).

[31] R. I. Dass, J. B. Goodenough: Multiple magnetic phases of $\mathrm{La}_{2} \mathrm{CoMnO}_{6-\delta}(0 \leq \delta \leq 0.05)$, Physical Review B 67, 014401 (2003).

[32] R. I. Dass, J.-Q. Yan, J. B. Goodenough: Oxygen stoichiometry, ferromagnetism, and transport properties of $\mathrm{La}_{2-\mathrm{x}} \mathrm{NiMnO}_{6+\delta}$, Physical Review B 68, 064415 (2003).

[33] M. N. Iliev, M. V. Abrashev, A. P. Litvinchuk, V. G. Hadjiev, H. Guo, A. Gupta: Raman spectroscopy of ordered double perovskite $\mathrm{La}_{2} \mathrm{CoMnO}_{6}$ thin films, Physical Review B 75, 104118 (2007).

[34] M. K. Kim, J. Y. Moon, H. Y. Choi, S. H. Oh, N. Lee, Y. J. Choi: Investigation of the magnetic properties in double perovskite $\mathrm{R}_{2} \mathrm{CoMnO}_{6}$ single crystals $(\mathrm{R}=$ rare earth: $\mathrm{La}$ to $\mathrm{Lu})$, Journal of Physics: Condensed Matter 27, 426002 (2015). 


\section{Literaturverzeichnis}

[35] C. L. Bull, P. F. McMillan: Raman scattering study and electrical properties characterization of elpasolite perovskites $\mathrm{Ln}_{2}\left(\mathrm{BB}^{\prime}\right) \mathrm{O}_{6}\left(\mathrm{Ln}=\mathrm{La}, \mathrm{Sm} \ldots \mathrm{Gd}\right.$ and $\mathrm{B}, \mathrm{B}^{\prime}=\mathrm{Ni}$, Co, Mn), Journal of Solid State Chemistry 177, 2323-2328 (2004).

[36] R. Egoavil, S. Hühn, M. Jungbauer, N. Gauquelin, A. Bécheé, G. Van Tendeloo, J. Verbeeck: Phase problem in the B-site ordering of $\mathrm{La}_{2} \mathrm{CoMnO}_{6}$ : impact on structure and magnetism, Nanoscale $\mathbf{7}$, 9835 (2015).

[37] H. Z. Guo, A. Gupta, J. Zhang, M. Varela, S. J. Pennycook: Effect of oxygen concentration on the magnetic properties of $\mathrm{La}_{2} \mathrm{CoMnO}_{6}$ thin films, Applid Physics Letters 91, 202509 (2007).

[38] R. J. Booth, R. Fillmann, H. Whitaker, A. Nag, R. M. Tiwari, K. V. Ramanujachary, J. Gopalakrishnan, S. E. Lofland: An investigation of structural, magnetic and dielectric properties of $\mathrm{R}_{2} \mathrm{NiMNO}_{6}$ ( $\mathrm{R}=$ rare earth, Y), Mat. Res. Bull. 44, 1559-1564 (2009).

[39] M. N. Iliev, M. M. Gospodinov, M. P. Singh, J. Meen, K. D. Truong, P. Fournier, S. Jandl: Growth, magnetic properties, and Raman scattering of $\mathrm{La}_{2} \mathrm{NiMnO}_{6}$ single crystals, Journal of Applied Physics 106, 023515 (2009).

[40] K. D. Truong, M. P. Singh, S. Jandl, P. Fournier: Influence of Ni/Mn cation order on the spinphonon coupling on multifunctional $\mathrm{La}_{2} \mathrm{NiMnO}_{6}$ epitaxial films by polarized Raman spectroscopy, Physical Review B 80, 134424 (2009).

[41] M. P. Singh, K. D. Truong, S. Jandl, P. Fournier: Long-range Ni/Mn structural order in epitaxial double perovskite $\mathrm{La}_{2} \mathrm{NiMnO}_{6}$ epitaxial films, Physical Review B 79, 224421 (2009).

[42] V. Moshnyaga, I. Khoroshun, A. Sidorenko, P. Petrenko, A. Weidinger, M. Zeitler, B. Rauschenbach, R. Tidecks, K. Samwer: Preparation of rare-earth manganite-oxide thin films by metalorganic aerosol deposition technique, Applied Physics Letters 74, 2842 (1999).

[43] V. Moshnyaga: Metal-Insulator Transition and Magnetoresistance in Manganite Thin Films: Lattice Strain and Disorder Effects, Kapitel 15 aus: A.V. Narlikar: Frontiers in Magnetic Materials, Springer Verlag, 1. Auflage 2005.

[44] V. Moshnyaga, A. Belenchuk, S. Hühn, C. Kalkert, M. Jungbauer, O. I. Lebedev, S. Merten, K.Y. Choi, P. Lemmens, B. Damaschke, K. Samwer: Intrinsic antiferromagnetic coupling underlies colossal magnetoresistance effect: Role of correlated polarons, Physical Review B 89, 024420 (2014).

[45] M. Jungbauer, S. Hühn, R. Egoavil, H. Tan, J. Verbeeck, G. Van Tendeloo, V. Moshnyaga: Atomic layer epitaxy of Ruddlesden-Popper $\mathrm{SrO}\left(\mathrm{SrTiO}_{3}\right)_{\mathrm{n}}$ films by means of metalorganic aerosol deposition, Applied Physics Letters 105, 251603 (2014).

[46] I. V. Khoroshun, E. V. Karyaev, V. T. Moshnyaga, G. A. Kiosse, M. A. Krachun, V. M. Zakosarenko, V. Yu Davydov: Characteristics of epitaxial $\mathrm{Y}-\mathrm{Ba}-\mathrm{Cu}-\mathrm{O}$ thin films grown by aerosol MOCVD technique, Supercond. Sci. Technol. 3, 493-496 (1990).

[47] Christoph Meyer: Strukturelle und optische Eigenschaften dünner mithilfe von metallorganischer Aerosol Deposition hergestellter ZnO-Schichten, Masterarbeit, Georg-August-Universität Göttingen (2013).

[48] Sebastian Hühn: Kationen-Ordnung in ferri/ferromagnetischen perowskitischen Dünnfilmen, Dissertation, Georg-August-Universität Göttingen (2015). 


\section{Literaturverzeichnis}

[49] C. Meyer, S. Hühn, M. Jungbauer, S. Merten, B. Damaschke, K. Samwer, V. Moshnyaga: Tipenhanced Raman spectroscopy (TERS) on double perovskite $\mathrm{La}_{2} \mathrm{CoMnO}_{6}$ thin films: field enhancement and depolarization effects, J. Raman Spectrosc. 48, 46-52 (2017).

[50] Markus Jungbauer: Gestaltung der elektronischen Korrelationen in Perowskit-Heterostrukturen auf atomarer Skala, Dissertation, Georg-August-Universität Göttingen (2015).

[51] HiTec Zang GmbH: SyrDos-Präzisions-Spritzendosierer-Familie, Datenblatt (2017).

[52] Fryderyk Lyzwa: Wachstum und Charakterisierung von Ruddlesden-Popper $\mathrm{SrO}\left(\mathrm{SrTiO}_{3}\right)_{\mathrm{n}=4}$ Schichten mittels Atomlagenepitaxie, Masterarbeit, Georg-August-Universität Göttingen (2016).

[53] Sven Esser: Untersuchung der Elektronen-Gitter Korrelation in $\mathrm{VO}_{2}$ Dünnfilmen, Masterarbeit, Georg-August-Universität Göttingen (2016).

[54] Victor Pfahl: Sauerstoffarme MAD: Struktur und Magnetotransport von Magnetitschichten, Masterarbeit, Georg-August-Universität Göttingen (2014).

[55] Philipp Ksoll: Wachstum von oxidischen Mikrostrukturen mittels Metallorganischer Aerosol Deposition, Masterarbeit, Georg-August-Universität Göttingen (2017).

[56] A. Biswas, P. B. Rossen, C.-H. Yang, W. Siemons, M.-H. Jung, I. K. Yang, R. Ramesh, Y. H. Jeong: Universal Ti-rich termination of atomically flat $\mathrm{SrTiO}_{3}$ (001), (110), and (111) surfaces, Applied Physics Letters 98, 051904 (2011).

[57] Neil W. Ashcroft, David N. Mermin: Festkörperphysik, Oldenbourg Verlag, 3. verbesserte Aufl. 2007.

[58] Siegfried Hunklinger: Festkörperphysik, Oldenbourg Verlag, 2. verbesserte Auflage 2009.

[59] Konrad Kopitzki, Peter Herzog: Einführung in die Festkörperphysik, Vieweg+Teubner Verlag, 6. Auflage (unverändert) 2009.

[60] Rudolf Gross, Achim Marx: Festkörperphysik, Oldenbourg Verlag, 1. Auflage 2012.

[61] Charles Kittel: Einführung in die Festkörperphysik, Oldenbourg Verlag, 15. Auflage 2013.

[62] B. D. Cullity, S. R. Stock: Elements of X-Ray Diffraction, Pearson Education, Addison-Wesley Publishing Company, 3. Auflage 1956.

[63] P. J. Brown, A. G. Fox, E. N. Maslen, M. A. O'Keefe, B. T. M. Willis: Intensity of diffracted intensities, International Tables for Crystallography Vol. C 6.1, 554-595 (2006).

[64] M. De Graef, M. E. McHenry: Structure of Materials: An Introduction to Crystallography, Diffraction, and Symmetry, Cambridge University Press, 1. Auflage 2007.

[65] Peter Debye: Interferenz von Röntgenstrahlen und Wärmebewegung, Ann. d. Phys. 348, 49 (1913).

[66] Ivar Waller: Zur Frage der Einwirkung der Wärmebewegung auf die Interferenz von Röntgenstrahlen, Zeitschrift für Physik 17, 398-408 (1923).

[67] Mario Birkholz: Thin Film Analysis by X-Ray Scattering, Wiley-VCH Verlag, 1. Aufl. 2006.

[68] Brent Fultz, James M. Howe: Transmission Electron Microscopy and Diffractometry of Materials, Springer Verlag, 3. Auflage 2008. 
[69] Paul Scherrer: Bestimmung der Größe und der inneren Struktur von Kolloidteilchen mittels Röntgenstrahlen, Nachrichten von der Gesellschaft der Wissenschaften zu Göttingen, MathematischPhysikalische Klasse 1918, 98-100 (1918).

[70] J. I. Langford, A. J. C. Wilson: Scherrer after Sixty Years: A Survey and Some New Results in the Determination of Crystallite Size, J. Appl. Cryst. 11, 102-113 (1978).

[71] U. Holzwarth, N. Gibson: The Scherrer equation versus the 'Debye-Scherrer equation', Nature Nanotechnology 6, 534 (2011).

[72] B. E. Warren: X-Ray Diffraction, Dover Publications, 1. korrigierte Wiederveröffentlichung 1990.

[73] A. Guinier: X-Ray Diffraction In Crystals, Imperfect Crystals and Amorphous Bodies, W. H. Freeman And Company, 1. Auflage 1963.

[74] H. Kiessig: Untersuchungen zur Totalreflexion von Röntgenstrahlen, Ann. d. Phys. 402, 715 (1931).

[75] H. Kiessig: Interferenz von Röntgenstrahlen an dünnen Schichten, Ann. d. Phys. 402, 769 (1931).

[76] M. Krumrey, M. Hoffmann, M. Kolbe: Schichtdickenbestimmung mit Röntgenreflektometrie, PTBMitteilungen 115, Heft 3, Kapitel 38 (2005).

[77] A. Segmüller: Observation of X-ray interferences on thin films of amorphous silicon, Thin Solid Films 18, 287-294 (1973).

[78] G. Binnig, C. F. Quate, Ch. Gerber: Atomic Force Microscope, Phys. Rev. Lett. 56, 930 (1986).

[79] Bruker: Innova - Superior AFM Research Performance and Versatility, Broschüre (2016).

[80] Thomas Fauster, Lutz Hammer, Klaus Heinz, M. Alexander Schneider: Oberflächenphysik: Grundlagen und Methoden, Oldenbourg Verlag, 1. Auflage 2013.

[81] V. Shahin, Y. Ludwig, C. Schafer, D. Nikova, H. Oberleithner: Glucocorticoids remodel nuclear envelope structure and permeability, Journal of Cell Science 118, 2881-2889 (2005).

[82] Peter Eaton, Paul West: Atomic Force Microscopy, Oxford University Press, 1. Aufl. 2010.

[83] S. N. Magonov, M.-H. Whangbo: Surface Analysis with STM and AFM, VCH Verlag, 1. Aufl. 1996.

[84] H. Hölscher, J. Falter, A. Schirmeisen: Atomic Force Microscopy And Spectroscopy, Characterization of Materials, Second Edition, 2290-2302 (2012).

[85] Petr Klapetek, David Necas, Christopher Anderson: Gwyddion user guide, Anleitung (2016).

[86] Digital Instruments: Scanning Probe Microscopy Training Notebook, Version 3.0 (1999).

[87] V. Kanniah, E. A. Grulke, T. Druffel: The effects of surface roughness on low haze ultrathin nanocomposite films, Thin Solid Films 539, 170-180 (2013).

[88] G. Binnig, H. Rohrer, Ch. Gerber, E. Weibel: Surface Studies by Scanning Tunneling Microscopy, Physical Review Letters 49, 57 (1982).

[89] G. Binnig, H. Rohrer: Scanning tunneling microscopy - from birth to adolescence, Reviews of Modern Physics 59, 615 (1987). 


\section{Literaturverzeichnis}

[90] J. Y. Park: Scanning Tunneling Microscopy, Characterization of Materials, 2nd Edition, 1-10 (2012).

[91] C. J. Chen: Introduction to Scanning Tunneling Microscopy, Oxford University Press, 1. Aufl. 1993.

[92] S. Lounis: Theory of Scanning Tunneling Microscopy, Lecture Notes, 45th IFF Spring School (2014).

[93] L. Tersoff, D. R. Hamann: Theory and Application for the Scanning Tunneling Microscope, Physical Review Letters 50, 1998 (1983).

[94] L. Tersoff, D. R. Hamann: Theory of the scanning tunneling microscope, Physical Review B 31, 805 (1985).

[95] Mike McElfresh: Fundamentals of Magnetism and Magnetic Measurements: Featuring Quantum Design's Magnetic Property Measurement System (MPMS), Quantum Design (1994).

[96] Quantum Design: Magnetic Property Measurement System: SQUID VSM User's Manual, 2009.

[97] J. Clarke, A. I. Braginski: The SQUID Handbook, Vol. I: Fundamentals and Technology of SQUIDS and SQUID Systems, Wiley-VCH Verlag, 1. Auflage 2004.

[98] R. L. Fagaly: Supercdonducting quantum interference device instruments and applications, Review of Scientific Instruments 77, 101101 (2006).

[99] R. P. Guertin, S. Foner: Magnetometry, Characterization of Materials, 2nd Edition, 1 (2012).

[100] J. M. D. Coey: Magnetism and Magnetic Materials, Cambridge Univ. Press, 1. Aufl. 2009.

[101] M. Khalid, A. Setzer, M. Ziese, P. Esquinazi, D. Spemann, A. Pöppl, E. Goering: Ubiquity of ferromagnetic signals in common diamagnetic oxide crystals, Physical Review B 81, 214414 (2010).

[102] M. Venkatesan, P. Kavle, S. B. Porter, K. Ackland, J. M. D. Coey: Magnetic Analysis of Polar and Nonpolar Oxide Substrates, IEEE Trans. Magn. 50, 2201704 (2014).

[103] M. Sawicki, W. Stefanowicz, A. Ney: Sensitive SQUID magnetometry for studying nanomagnetism, Semicond. Sci. Technol. 26, 064006 (2011).

[104] M. A. Garcia, E. Fernandez Pinel, J. de la Venta, A. Quesada, V. Bouzas, J. F. Fernández, J. J. Romero, M. S. Martín González, J. L. Costa-Krämer: Sources of experimental errors in the observation of nanoscale magnetism, Journal of Applied Physics 105, 013925 (2009).

[105] J. M. D. Coey: $d^{0}$ ferromagnetism, Solid State Sciences 7, 660-667 (2005).

[106] A. Le Febvrier, J. Jensen, P. Eklund: Wet-cleaning of MgO(001): Modification of surface chemistry and effects on thin film growth investigated by $x$-ray photoelectron spectroscopy and time-of-flight secondary ion mass spectroscopy, J. Vac. Sci. Technol. A 35, 021407 (2017).

[107] Quantum Design: Physical Property Measurement System: Resistivity Option User's Manual, 1999.

[108] Ray F. Egerton: Physical Principles of Electron Microscopy, Springer Verlag, 1. Aufl. 2005.

[109] D. N. Leonhard, G. W. Chandler, S. Seraphin: Scanning Electron Microscopy, Characterization of Materials, Second Edition, 1721-1736 (2012).

[110] K. D. Vernon-Perry: Scanning Electron Microscopy: an introduction, III-Vs Review 13, 40-44 (2000). 
[111] Carl Zeiss: Supra Series: Ultra High Resolution FE-SEM for Versatile Analytics, Broschüre (2010).

[112] L. Reimer, H. Kohl: Transmission Electron Microscopy, Springer Verlag, 5. Auflage 2008.

[113] C. V. Raman: A new radiation, Indian J. Phys. 2, 387-398 (1928).

[114] C. V. Raman, K. S. Krishnan: A New Type of Secondary Radiation, Nature 121, 501 (1928).

[115] C. V. Raman: The Molecular Scattering of Light, Nobel Lecture 1930.

[116] American Chemical Society and the Indian Association for the Cultivation of Science: The Raman Effect, An International Historic Chemical Landmark (1998).

[117] Ewen Smith, Geoffrey Dent: Modern Raman Spectroscopy - A Practical Approach, John Wiley \& Sons Verlag, 1. Auflage 2005.

[118] Mischa Nicklaus: Tip-Enhanced Raman Spectroscopy For Nanoelectronics, Books on Demand Verlag, 1. Auflage 2014 .

[119] E. Le Ru, P. Etchegoin: Principles of Surface Enhanced Raman Spectroscopy, Elsevier Verlag, 1. Auflage 2014.

[120] Peter Vandenabeele: Practical Raman Spectroscopy - An Introduction, John Wiley \& Sons Verlag, 1. Auflage 2013.

[121] Wolfgang Demtröder: Laserspektroskopie 2 - Experimentelle Techniken, Springer Verlag, 6., neu bearbeitete und aktualisierte Auflage 2013.

[122] J. M. Atkin, S. Berweger, A. C. Jones, M. B. Raschke: Nano-optical imaging and spectroscopy of order, phases, and domains im complex solids, Adv. Phys. 61, 745-842 (2012).

[123] B. Dietzek, D. Cialla, M. Schmitt, J. Popp: Introduction of the Fundamentals of Raman Spectroscopy, Kapitel 2 aus: T. Dieing, O. Hollrichter, J. Toporski: Confocal Raman Microscopy, Springer Verlag, 1. Auflage 2010.

[124] Derek A. Long: The Raman Effect, John Wiley \& Sons Verlag, 1. Auflage 2002.

[125] Peter F. Bernath: Spectra of Atoms and Molecules, Oxford University Press, 1. Auflage 1995.

[126] T. Jawhari, P. J. Hendra, H. A. Willis, M. Judkins: Quantitative analysis using Raman methods, Spectrochimica Acta Part A: Molecular Spectroscopy 46, 161-170 (1990).

[127] Rainer Engelbrecht: Nichtlineare Faseroptik, Springer Vieweg Verlag, 1. Auflage 2014.

[128] G. Gouadec, L. Bellot-Gurlet, D. Baron, Ph. Colomban: Raman Mapping fo the Investigation of Nano-phased Materials, Kapitel 3 aus: Arnaud Zoubir: Raman Imaging - Techniques and Applications, Springer Verlag, 1. Auflage 2012.

[129] M. Balkanski, R. F. Wallis, E. Haro: Anharmonic effects in light scattering due to optical phonons in silicon, Physical Review B 28, 1928 (1983).

[130] P. G. Klemmens: Anharmonic Decay of Optical Phonons, Physical Review 148, 845 (1966).

[131] Y. Chen, B. Peng, B. Wang: Raman Spectra and Temperature-Dependent Raman Scattering of Silicon Nanowires, J. Phys. Chem. C 111, 5855-5858 (2007). 


\section{Literaturverzeichnis}

[132] R. Cuscó, E. Alarcón-Lladó, J. Ibáñez, L. Artús, J. Jiménez, B. Wang, M. J. Callahan: Temperature dependence of Raman scattering in ZnO, Physical Review B 75, 165202 (2007).

[133] Horiba Jobin Yvon: Horiba LabRAM HR Evolution, Broschüre (2016).

[134] K. M. Abramski, E. F. Plinski: Other Gas Lasers, Kapitel 11 aus: M. Endo, R. F. Walter: Gas Lasers, CRC Press, 1. Auflage 2007.

[135] J. Eichler, H. J. Eichler: Laser - Bauformen, Strahlführung, Anwendungen, Springer Verlag, 6. Auflage 2006.

[136] Märzhäuser Wetzlar: Scanning Stage SCAN $100 \times 100$, Broschüre (2016).

[137] F. Adar: Considerations of Grating Selection in Optimizing a Raman Spectrograph, Spectroscopy 28, $10(2013)$

[138] J. Dubbessy, M.-C. Caumon, F. Rull, S. Sharma: Instrumentation in Raman spectroscopy: elementary theory and practice, Kapitel 3 aus: J. Dubessy, M.-C. Caumon, F. Rull: Raman Spectroscopy applied to Earth Sciences and Cultural Heritage, EMU Notes Vol. 12, 2012.

[139] P. A. Temple, C. E. Hathaway: Multiphonon Raman Spectrum of Silicon, Physical Review B 7, 3685 (1973).

[140] A. Zwick, R. Charles: Multiple-order Raman scattering in crystalline and amorphous silicon, Physical Review B 48, 6024 (1993).

[141] S. Fukura, T. Mizukami, S. Odake, H. Kagi: Factors Determining the Stability, Resolution, and Precision of a Conventional Raman Spectrometer, Applied Spectroscopy 60, 946 (2006).

[142] Linkam Scientific Instruments: THMS350V Vacuum System, Broschüre (2017).

[143] R. Loudon: The Raman effect in crystals, Advances in Physics 13, 423 (1964).

[144] M. I. Aroyo, J. M. Perez-Mato, D. Orobengoa, E. Tasci, G. de la Flor, A. Kirov: Crystallography online: Bilbao Crystallographic Server, Bulg. Chem. Commun. 743, 183 (2011).

[145] R. S. Mulliken: Report on Notation for the Spectra of Polyatomic Molecules, Journal of Chemical Physics 23, 1997 (1955).

[146] D. Tuschel: Practical Group Theory and Raman Spectroscopy, Part I: Normal Vibrational Modes, Spectroscopy 29(2), 14 (2014).

[147] T. C. Damen, S. P. S. Porto, B. Tell: Raman Effect in Zinc Oxide, Physical Review 142, 570 (1966).

[148] P. G. Gucciardi: Polarization-Sensitive Tip-Enhanced Raman Scattering, Kapitel 3 aus: B. Bhushan: Scanning Probe Microscopy in Nanoscience and Nanotechnology, Springer Verlag, 1. Auflage 2010.

[149] N. C. Lindquist, P. Nagpal, K. M. McPeak, D. J. Norris, S.-H. Oh: Engineering metallic nanostructures for plasmonics and nanophotonics, Rep. Prog. Phys. 75, 036501 (2012).

[150] J. Stadler, T. Schmid, R. Zenobi: Developments in and practical guidelines for tip-enhanced Raman spectroscopy, Nanoscale 4, 1856 (2012). 


\section{Literaturverzeichnis}

[151] B. Pettinger, G. Picardi, R. Schuster, G. Ertl: Surface-enhanced and STM-tip-enhanced Raman Spectroscopy at Metal Surfaces, Single Mol. 3, 285-294 (2002).

[152] D. W. Pohl, W. Denk, M. Lanz: Optical stethoscopy: Image recording with resolution $\lambda / 20$, Applied Physics Letters 44, 651 (1984).

[153] A. Lewis, M. Isaacson, A. Harootunian, A. Muray: Development of a $500 \AA$ Å spatial resolution light microscope: I. light is efficiently transmitted through $\lambda / 16$ diameter apertures, Ultramicroscopy $\mathbf{1 3}$, 227-232 (1984).

[154] John Wessel: Surface-enhanced optical microscopy, J. Opt. Soc. Am. B 2, 1538 (1985).

[155] Y. Inouye, P. Verma, T. Ichimura, S. Kawata: Near-field effects in tip-enhanced Raman scattering, Kapitel 3 aus: S. Kawata, V. Shalaev: Tip Enhancement, Elsevier Verlag, 1. Auflage 2007.

[156] M. Asghari-Khiavi, B. R. Wood, O. Hojati-Talemi, A. Downes, D. McNaughton, A. Mehler: Exploring the origin of tip-enhanced Raman scattering; preparation of efficient TERS probes with high yield, J. Raman Spectrosc. 43, 173-180 (2012).

[157] F. Demming, J. Jersch, K. Dickmann, P. I. Geshev: Calculation of the field enhancement on laserilluminated scanning probe tips by the boundary element method, Appl. Phys. B 66, 593-598 (1998).

[158] P. I Geshev, S. Klein, T. Witting, K. Dickmann, M. Hietschold: Calculation of the electric-field enhancement at nanoparticles of arbitrary shape in close proximity to a metallic surface, Physical Review B 70, 075402 (2004).

[159] C. Sönnichsen, S. Geier, N. E. Hecker, G. von Plessen, J. Feldmann, H. Ditlbacher, B. Lamprecht, J. R. Krenn, F. R. Aussenegg, V. Z.-H. Chan, J. P. Spatz, M. Möller: Spectroscopy of single nanoparticles using total internal reflection microscopy, Applied Physics Letters 77, 2949 (2000).

[160] L. Meng, T. Huang, X. Wang, S. Chen, Z. Yang, B. Ren: Gold-coated AFM tips for tip-enhanced Raman spectroscopy: theoretical calculation and experimental demonstration, Optics Express 23, 13804 (2015).

[161] C. C. Neascu, G. A. Steudle, M. B. Raschke: Plasmonic light scattering from nanoscopic metal tips, Applied Physics B 80, 295-300 (2005).

[162] Z. Yang, J. Aizpurua, H. Xu: Electromagnetic field enhancement in TERS configurations, J. Raman Spectrosc. 40, 1343-1348 (2009).

[163] P. L. Stiles, J. A. Dieringer, N. C. Shah, R. P. Van Duyne: Surface-Enhanced Raman Spectroscopy, Annu. Rev. Anal. Chem. 1, 601 (2008).

[164] J. A. Creighton, D. G. Eadon: Ultraviolet-Visible Absorption Spectra of the Colloidal Metallic Elements, J. Chem. Soc., Farady Trans. 87, 3881-3891 (1991).

[165] P. K. Jain, X. Huang, I. H. El-Sayed, M. A. El-Sayed: Noble Metals on the Nanoscale: Optical and Photothermal Properties and Some Applications in Imaging, Sensing, Biology, and Medicine, Accounts of Chemical Research 41, 1578-1586 (2008).

[166] S. Link, M. A. El-Sayed: Optical Properties and Ultrafast Dynamics of Metallic Nanocrystals, Annu. Rev. Chem. Phys. 41, 1578-1586 (2008). 
[167] B. Pettinger, P. Schambach, C. J. Villagómez, N. Scott: Tip-Enhanced Raman Spectroscopy: NearFields Acting on a Few Molecules, Annu. Rev. Phys. Chem. 63, 379-399 (2012).

[168] T. Schmid, B.-S. Yeo, W. Zhang, R. Zenobi: Use of tip-enhanced vibrational spectroscopy for analytical applications in chemistry, biology, and materials science, Kapitel 4 aus: S. Kawata, V. Shalaev: Tip Enhancement, Elsevier Verlag, 1. Auflage 2007.

[169] R. Ossikovski, Q. Nguyen, G. Picardi: Simple model for the polarization effects in tip-enhanced Raman spectroscopy, Physical Review B 75, 045412 (2007).

[170] Q. Nguyen, R. Ossikovski, J. Schreiber: Contrast enhancement on crystalline silicon in polarized reflection mode tip-enhanced Raman spectroscopy, Opt. Commun. 274, 231 (2007).

[171] B. Pettinger, B. Ren, G. Picardo, R. Schuster, G. Ertl: Nanoscale Probing of Adsorbed Species by Tip-Enhanced Raman Spectroscopy, Physical Review Letters 92, 096101 (2004).

[172] D. Mehtani, N. Lee, R. D. Hartschuh, A. Kisliuk, M. D. Foster, A. P. Sokolov, J. F. Maguire: NanoRaman spectroscopy with side-illumination optics, J. Raman Spectrosc. 36, 1068-1075 (2005).

[173] B. Pettinger, B. Ren, G. Picardo, R. Schuster, G. Ertl: Tip-enhanced Raman spectroscopy (TERS) of malachite green isothiocyanate at Au(111): bleaching behavior under the influence of high electromagnetic fields, J. Raman Spectrosc. 36, 541-550 (2005).

[174] F. Festy, A. Demming, D. Richards: Resonant excitation of tip plasmons for tip-enhanced Raman SNOM, Ultramicroscopy 100, 437-441 (2004).

[175] M. D. Sonntag, J. M. Klingsporn, A. B. Zrimsek, B. Sharma, L. K. Ruvuna, R. P. Van Duyne: Molecular plasmonics for nanoscale spectroscopy, Chem. Soc. Rev. 43, 1230 (2014).

[176] C. Toccafondi, G. Picardi, R. Ossikovski: Molecular Bending at the Nanoscale Evidenced by TipEnhanced Raman Spectroscopy in Tunneling Mode on Thiol Self-Assembled Monolayers, J. Phys. Chem. C 120, 18209-18219 (2016).

[177] B. Pettinger, K. F. Domke, D. Zhang, R. Schuster, G. Ertl: Direct monitoring of plasmon resonances in a tip-surface gap of varying width, Physical Review B 76, 113409 (2007).

[178] T.-A. Yano, T. Ichimura, A. Taguchi, N. Hayazawa, P. Verma, Y. Inouye, S. Kawata: Confinement of enhanced field investigated by tip-sample gap regulation in tapping-mode tip-enhanced Raman microscopy, Applied Physics Letters 91, 121101 (2007).

[179] A. Hartschuh, E. J. Sánchez, X. S. Xie, L. Novotny: High-Resolution Near-Field Raman Microscopy of Single-Walled Carbon Nanotubes, Physical Review Letters 90, 095503 (2003).

[180] G. Picardi, Q. Nguyen, J. Schreiber, R. Ossikovski: Comparative study of atomic force mode and tunneling mode tip-enhanced Raman spectroscopy, Eur. Phys. J. Appl. Phys. 40, 197-201 (2007).

[181] B. Pettinger, K. F. Domke, D. Zhang, G. Picardi, R. Schuster: Tip-enhanced Raman scattering: Influence of the tip-surface geometry on optical resonance and enhancement, Surface Science 603, 1335-1341 (2009).

[182] L. G. Cançado, A. Jorio, A. Ismach, E. Joselevich, A. Hartschuh, L. Novotny: Mechanism of NearField Raman Enhancement in One-Dimensional Systems, Phys. Rev. Lett. 103, 186101 (2009). 
[183] R. V. Maximiano, R. Beams, L. Novotny, A. Jorio, L. G. Cançado: Tip-enhanced Raman scattering: Influence of the tip-surface geometry on optical resonance and enhancement, Physical Review B 85, 235434 (2012).

[184] N. M. Sabanés, A. Elizabeth, J. H. K. Pfisterer, K. F. Domke: The effect of STM parameters on tip-enhanced Raman spectra, Faraday Discussions 205, 233 (2017).

[185] N. M. Sabanés, L. M. A. Driessen, K. F. Domke: Versatile Side-Illumination Geometry for TipEnhanced Raman Spectroscopy at Solid/Liquid Interfaces, Anal. Chem. 88, 7108-7114 (2016).

[186] Y. Fujita, N. N. Horimoto, S. Kajimoto, H. Fukumura: Bias voltage-dependent STM-tip-enhanced Raman spectroscopy of benzenethiol-modified gold nanoplates, Chem. Phys. Lett. 582, 110 (2013).

[187] G. Picardo, M. Chaigneau, R. Ossikovski, C. Licitra, G. Delapierre: Tip enhanced Raman spectroscopy on azobenzene thiol self-assembled monolayers on Au(111), J. Raman Spectrosc. 40, 1407-1412 (2009).

[188] Bruker Nano Inc.: TERS-STM TERS Probe, Product Description (2017).

[189] J. Freund, J. Halbritter, J. K. H. Hörber: How Dry Are Dried Samples? Water Adsorption Measured By STM, Microscopy Research And Technique 44, 327-338 (1999).

[190] S. Berweger, M. B. Raschke: Polar phonon mode selection rules in tip-enhanced Raman scattering, J. Raman Spectrosc. 40, 1413-1419 (2009).

[191] A. L. Demming, F. Festy, D. Richards: Plasmon resonances on metal tips: Understanding tipenhanced Raman scattering, J. Chem. Phys. 122, 184716 (2005).

[192] P. G. Gucciardi, M. Lopes, R. Déturche, C. Julien, D. Barchiesi, M. Lamy de la Chapelle: Light depolarization induced by metallic tips in apertureless near-field optical microscopy and tip-enhanced Raman spectroscopy, Nanotechnology 19, 215702 (2008).

[193] P. G. Gucciardi, J.-C. Valmalette: Different longitudinal optical-transverse optical mode amplificationin tip enhanced Raman spectroscopy of GaAs(001), Applied Physics Letters 97, 263104 (2010).

[194] M. Motohashi, N. Hayazawa, A. Tarun, S. Kawata: Depolarization effect in reflection-mode tipenhanced Raman scattering for Raman active crystals, J. Appl. Phys. 103, 034309 (2008).

[195] I. N. Bronstein, K. A. Semendjajew, G. Musiol, H. Mühlig: Taschenbuch der Mathematik, Verlag Harri Deutsch, 7., vollständig überarbeitete und ergänzte Auflage (2008).

[196] E. Piña: Rotations with Rodrigues' vector, Eur. J. Phys. 32, 1171-1178 (2011).

[197] A. Tarun, N. Hayazawa, M. Motohashi, S. Kawata: Highly efficient tip-enhanced Raman spectroscopy and microscopy of strained silicon, Rev. Sci. Instrum. 79, 013706 (2008).

[198] M. Chaigneau, G. Picardi, R. Ossikovski: Molecular Arrangement in Self-Assembled AzobenzeneContaining Thiol Monolayers at the Individual Domain Level Studied through Polarized Near-Field Raman Spectroscopy, Int. J. Mol. Sci. 12, 1245-1258 (2011).

[199] P. J. Schuck, W. Bao, N. J. Borys: A polarizing situation: Taking an in-plane perspective for nextgeneration near-field studies, Front. Phys. 11, 117804 (2016). 


\section{Literaturverzeichnis}

[200] T. Mino, Y. Saito, P. Verma: Quantitative Analysis of Polarization-controlled Tip-Enhanced Raman Imaging through the Evaluation of the Tip Dipole, ACS Nano 8, 10187 (2014).

[201] A. Downes, D. Salter, A. Elfick: Heating effects in tip-enhanced optical microscopy, Optics Express 14, 5216 (2006).

[202] W. Zhang, T. Schmid, B.-S. Yeo, R. Zenobi: Near-Field Heating, Annealing, and Signal Loss in Tip-Enhanced Raman Spectroscopy, J. Phys. Chem. C 112, 2104-2108 (2008).

[203] M. Mochizuki, G. Lkhamsuren, K. Suthiwanich, E. A. Mondarte, T. Yano, M. Hara, T. Hayashi: Damage-free tip-enhanced Raman spectroscopy for heat-sensitive materials, The Journal of Physical Chemistry C 112, 2104-2108 (2008).

[204] W. Zhang, B. S. Yeo, T. Schmid, R. Zenobi: Single Molecule Tip-Enhanced Raman Spectroscopy with Silver Tips, The Journal of Physical Chemistry C 111, 1733 (2007).

[205] T. Hartmann, C. S. Wondergem, N. Kumar, A. van den Berg, B. M. Weckhuysen: Surface- and Tip-Enhanced Raman Spectroscopy in Catalysis, J. Phys. Chem. Lett. 7, 1570 (2016).

[206] R. Ramos, M. J. Gordon: Near-field artifacts in tip-enhanced Raman spectroscopy, Applied Physics Letters 100, 213111 (2012).

[207] C. Georgi, M. Hecker, E. Zschech: Raman intensity enhancement in silicon-on-insulator substrates by laser deflection at atomic force microscopy tips and particles, Appl. Phys. Lett. 90, 171102 (2007).

[208] J. Sun, P. S. Carney, J. C. Schotland: Strong tip effects in near-field scanning optical tomography, Journal of Applied Physics 102, 103103 (2007).

[209] E. Poliani, M. R. Wagner, A. Vierck, F. Herziger, C. Nenstiel, F. Gannott, M. Schweiger, S. Fritze, A. Dadgar, J. Zaumseil, A. Krost, A. Hoffmann, J. Maultzsch: Breakdown of Far-Field Raman Selection Rules by Light-Plasmon Coupling Demonstrated by Tip-Enhanced Raman Scattering, J. Phys. Chem. Lett. 8, 5462-5471 (2017).

[210] L. Aigouy, A. Lahrech, S. Grésillon, H. Cory, A. C. Boccarra, J. C. Rivoal: Polarization effects in apertureless scanning near-field optical microscopy: an experimental study, Optics Letters 24, 187 (1999).

[211] A. Passian, A. Wig, A. L. Lereu, F. Meriaudeau, T. Thundat, T. L. Ferell: Photon tunneling via surface plasmon coupling, Applied Physics Letters 85, 3420 (2004).

[212] W.-C. Liu, D. P. Tsai: Optical tunneling effect of surface plasmon polaritons and localized surface plasmon resonance, Physical Review B 65, 155423 (2002).

[213] I. I. Smolyaninov, A. V. Zayats, A. Gungor, C. C. Davis: Single-Photon Tunneling via Localized Surface Plasmons, Physical Review Letters 88, 187402 (2002).

[214] M. P. Singh, S. Carpenter, K. D. Truong, P. Fournier: Evidence of bidomain structure in doubleperovskite $\mathrm{La}_{2} \mathrm{CoMnO}_{6}$ thin films, Applid Physics Letters 90, 211915 (2007).

[215] D. Kumar, V. G. Sathe: Raman spectroscopic study of structural transformation in ordered double perovskites $\mathrm{La}_{2} \mathrm{CoMnO}_{6}$ bulk and epitaxial film, Solid State Commun. 224, 10 (2015). 
[216] K. D. Truong, J. Laverdière, M. P. Singh, S. Jandl, P. Fournier: Impact of Co/Mn cation ordering on phonon anomalies in $\mathrm{La}_{2} \mathrm{CoMnO}_{6}$ double perovskites: Raman spectroscopy, Physical Review B 76, 132413 (2007).

[217] H. Guo, J. Burgess, S. Street, A. Gupta, T. G. Calvarese, M. A. Subramanian: Growth of epitaxial thin films of the ordered double perovskite $\mathrm{La}_{2} \mathrm{NiMnO}_{6}$ on different substrates, Appl. Phys. Lett. 89, 022509 (2006).

[218] P. Kumar, S. Ghara, B. Rajeswaran, D. V. S. Muthu, A. Sundaresan, A. K. Sood: Temperature dependent magnetic, dielectric and Raman studies of partially disorderd $\mathrm{La}_{2} \mathrm{NiMnO}_{6}$, Solid State Commun. 184, 47-51 (2014).

[219] D. Kumar, S. Kumar, V. G. Sathe: Spin-phonon coupling in ordered double perovskites $\mathrm{A}_{2} \mathrm{CoMnO}_{6}$ $(\mathrm{A}=\mathrm{La}, \mathrm{Pr}, \mathrm{Nd})$ probed by micro-Raman spectroscopy, Solid State Commun. 194, 59-64 (2014).

[220] C. Xie, L. Shi, J. Zhao, S. Zhou, Y. Li, X. Yuan: Spin-phonon coupling in $\mathrm{R}_{2} \mathrm{CoMnO}_{6}$ $(\mathrm{R}=\mathrm{Pr}, \mathrm{Nd}, \mathrm{Sm})$ thin films under biaxial compressive strain, J. Appl. Phys. 120, 155302 (2016).

[221] V. Shabadi, M. Major, P. Komissinsky, M. Vafaee, A. Radetinac, M. Baghaie Yazdi: Origin of superstructures in (double) perovskite thin films, J. Appl. Phys. 116, 114901 (2014).

[222] R. D. Shannon: Revised Effective Ionic Radii and Systematic Studies of Interatomic Distances in Halides and Chalcogenides, Acta Cryst. A 32, 751 (1976).

[223] Y. Shimakawa, M. Azuma, N. Ichikawa: Multiferroic Compounds with Double-Perovskite Structures, Materials 4, 153-168 (2011).

[224] J. E. Kleibeuker, E.-M. Choi, E. D. Jones, T.-M. Yu, B. Sala, B. A MacLaren, D. Kepaptsoglou, D. Hernandez-Maldonado, Q. M. Ramasse, L. Jones, J. Barthel, I. MacLaren, J. L. MacManusDriscoll: Route to achieving perfect B-site ordering in double perovskite perovskite thin films, NPG Aisa Materials 9, e406 (2017).

[225] D. P. Norton: Synthesis and properties of epitaxial electronic oxide thin-film materials, Materials Science and Engineering R 43, 139-247 (2004).

[226] Y. Bai, Y. Xia, H. Li, L. Han, Z. Wang, X. Wu, S. Lv, X. Liu, J. Meng: A-Site-Doping Enhanced $B$-Site Ordering and Correlated Magnetic Property in $\mathrm{La}_{2-\mathrm{x}} \mathrm{Bi}_{\mathrm{x}} \mathrm{CoMnO}_{6}, \mathrm{~J}$. Phys. Chem. C 116, 16841-16847 (2012).

[227] X. L. Wang, M. James, J. Horvat, S. X. Dou: Spin glass behaviour in ferromagnetic $\mathrm{La}_{2} \mathrm{CoMnO}_{6}$ perovskite manganite, Supercond. Sci. Technol. 15, 427-430 (2002).

[228] J. Krishna Murthy, K. Devi Chandrasekhar, S. Mahana, D. Topwal, A. Venimadhav: Giant magnetocaloric effect in $\mathrm{Gd}_{2} \mathrm{NiMnO}_{6}$ and $\mathrm{Gd}_{2} \mathrm{CoMnO}_{6}$ ferromagnetic insulators, J. Phys. D: Appl. Phys. 48, 355001 (2015).

[229] J. Blasco, J. García, G. Subías, J. Stankiewicz, J. A. Rodríguez-Velamazán, C. Ritter, J. L. GarcíaMuñoz, F. Fauth: Magnetoelectric and structural properties of Y2CoMnO6: The role of antisite defects, Physical Review B 93, 214401 (2016).

[230] S. H. Oh, H. Y. Choi, J. Y. Moon, M. K. Kim, Y. Jo, N. Lee, Y. J. Choi: Nonlinear magnetodielectric effect in double-perovskite $\mathrm{Gd}_{2} \mathrm{NiMnO}_{6}$, J. Phys. D: Appl. Phys. 48, 445001 (2015). 
[231] R. B. Macedo Filho, D. A. B. Barbosa, H. Reichlova, X. Marti, A. S. de Menezes, A. P. Ayala, C. W. A. Paschoal: Role of rare-earth ionic radii on the spin-phonon coupling in multiferroic ordered double perovskites, Mater. Res. Express 2, 075201 (2015).

[232] H. J. Zhao, X. Q. Liu, X. M. Chen, L. Bellaiche: Effects of chemical and hydrostatic pressures on structural, magnetic, and electronic properties of $\mathrm{R}_{2} \mathrm{NiMnO}_{6}(\mathrm{R}=$ rare-earth ion $)$ double perovskites, Physical Review B 90, 195147 (2014).

[233] P. A. Stampe, M. Bullock, W. P. Tucker, R. J. Kennedy: Growth of MgO thin films on M-, A-, Cand R-plane sapphire by laser ablation, J. Phys. D: Appl. Phys. 32, 1778-1787 (1999).

[234] Z.-X. Mei, X.-L. Du, Z.-Q. Zeng, Y. Guo, J. Wang, J.-F. Jia, Q.-K. Xue: Two-Step Growth of MgO Films on Sapphire (0001) Substrates by Radio Frequency Plasma-Assisted Molecular Beam Epitaxy, Chin. Phys. Lett. 21, 410-413 (1999).

[235] C. H. Jia, Y. H. Chen, G. H. Liu, X. L. Liu, S. Y. Yang, Z. G. Wang: Structural and optical properties of $Z n O$ films on $\mathrm{SrTiO}_{3}$ substrates by MOCVD, J. Phys. D: Appl. Phys. 42, 015415 (2009)

[236] C. H. Jia, Y. H. Chen, G. H. Liu, X. L. Liu, S. Y. Yang, Z. F. Zhang, Z. G. Wang: Control of epitaxial relationships of $\mathrm{ZnO} / \mathrm{SrTiO}_{3}$ heterointerfaces by etching the substrate surface, Nanoscale Research Letters 8, 23 (2013).

[237] R. Galceran, L. López-Mir, B. Bozzo, J. Cisneros-Fernández, J. Santiso, L. Balcells, C. Frontera, B. Martínez: Strain-induced perpendicular magnetic anisotropy in $\mathrm{La}_{2} \mathrm{CoMnO}_{6-\epsilon}$ thin films and its dependence on film thickness, Physical Review B 93, 144417 (2016).

[238] C. L. Bull, D. Gleeson, K. S. Knight: Determination of B-site ordering and structural transformations in the mixed transition metal perovskites $\mathrm{La}_{2} \mathrm{CoMnO}_{6}$ and $\mathrm{La}_{2} \mathrm{NiMnO}_{6}$, J. Phys.: Condens. Matter 15, 4927 (2003).

[239] D. Kumar, S. Kumar, V. G. Sathe: Raman Studies of Ordered Double Perovskite Thin Film at High Temperatures, AIP Conference Proceedings 1665, 140030 (2015).

[240] M. N. Iliev, H. Guo, A. Gupta: Raman spectroscopy evidence of strong spin-phonon coupling in epitaxial thin films of the double perovskite $\mathrm{La}_{2} \mathrm{NiMnO}_{6}$, Applied Physics Letters 80, 151914 (2007).

[241] E. Granado, A. García, J. A. Sanjuro, C. Rettori, I. Torriani, F. Prado, R. D. Sánchez, A. Caneiro, S. B. Oseroff: Magnetic ordering effects in the Raman spectra of $\mathrm{La}_{1-\mathrm{x}} \mathrm{Mn}_{1-\mathrm{x}} \mathrm{O}_{3}$, Physical Review B 60, 11879 (1999).

[242] J. Laverdière, S. Jandl, A. A. Mukhin, V. Yu. Ivanov, V. G. Ivanov, M. N. Iliev: Spin-phonon coupling in orthorhombic $\mathrm{RMnO}_{3}(\mathrm{R}=\mathrm{Pr}, \mathrm{Nd}, \mathrm{Sm}, \mathrm{Eu}, \mathrm{Gd}, \mathrm{Tb}, \mathrm{Dy}, \mathrm{Ho}, \mathrm{Y})$ : A Raman study, Physical Review B 73, 214301 (2006).

[243] P. K. Pandey, R. J. Choudhary, D. H. Mishra, V. G. Sathe, D. M. Phase: Signature of spin-phonon coupling in $\mathrm{Sr}_{2} \mathrm{CoO}_{4}$ thin film: A Raman spectroscopic study, Apppl. Phys. Lett. 102, 142401 (2013).

[244] S. Issing, A. Pimenov, Y. Vu. Ivanonv, A. A. Muhkin, J. Geurts: Spin-phonon coupling in multiferroic manganites $\mathrm{RMnO}_{3}$ : comparison of pure $(\mathrm{R}=\mathrm{Eu}, \mathrm{Gd}, \mathrm{Tb})$ and substituted $\left(\mathrm{R}=\mathrm{Eu}_{1-\mathrm{x}} \mathrm{Y}_{\mathrm{x}}\right)$ compounds, Eur. Phys. J. B 78, 367-372 (2010).

[245] X.-W. Jin, L. Lu, S.-B. Mi, M. Liu, C.-L. Jia: Phase stability and B-site ordering in $\mathrm{La}_{2} \mathrm{NiMnO}_{6}$ thin films, Applied Physics Letters 109, 031904 (2016). 


\section{Literaturverzeichnis}

[246] S.-Q. Wu, S. Cheng, L. Lu, M. Liu, X.-W. Jin, S.-D. Cheng, S.-B. Mi: B-site ordering and straininduced phase transition in double-perovskite $\mathrm{La}_{2} \mathrm{NiMnO}_{6}$ films, Sci. Rep. 8, 2516 (2018).

[247] H. M. D. Bandara, S. C. Burdette: Photoisomerization in different classes of azobenzene, Chemical Society Reviews 41, 1809-1825 (2012).

[248] Y. B. Zheng, J. L. Payton, C.-.H. Chung, R. Liu, S. Cheunkar, B. K. Pathem, Y. Yang, L. Jensen, P. S. Weiss: Surface-Enhanced Raman Spectroscopy to Probe Reversibly Photoswitchable Azobenzene in Controlled Nanoscale Environments, Nano Lett. 11, 3447 (2011).

[249] C. M. Stuart, R. R. Frontiera, R. A. Mathies: Excited-State Structure and Dynamics of cis-and trans-Azobenzene from Resonance Raman Intensity Analysis, J. Phys. Chem. A 111, 12072 (2007).

[250] D. R. Armstrong, J. Clarkson, W. E. Smith: Vibrational Analysis of trans-Azobenzene, J. Phys. Chem. 99, 17825-17831 (1995).

[251] M. Moskovits, J. S. Suh: Surface Selection Rules for Surface-Enhanced Raman Spectroscopy: Calculations and Application to the Surface-Enhanced Raman Spectrum of Phthalazine on Silver, J. Phys. Chem. 88, 5526-5530 (1984).

[252] H. B. Lueck, D. C. Daniel, J. L. McHale: Resonance Raman Study of Solvent Effects on a Series of Triarylmethane Dyes, J. Raman Spectrosc. 24, 363-370 (1993).

[253] A. Kamińska, I. Dzięcielewski, J. L. Weyher, J. Waluk, S. Gawinkowski, V. Sashuk, M. Fiałkowski, M. Sawicka, T. Suski, S. Porowski, R. Hołyst: Highly reproducible, stable and multiply regenerated surface-enhanced Raman scattering substrate for biomedical applications, J. Mater. Chem. 21, 8662 (2011).

[254] A. Merlen, J. C. Valmalette, P. G. Gucciardi, M. Lamy de la Chapelle, A. Frigout, R. Ossikovski: Depolarization effects in tip-enhanced Raman spectroscopy, J. Raman Spectrosc. 40, 1361 (2009).

[255] R. Matsui, P. Verma, T. Ichimura, Y. Inouye, S. Kawata: Nanoanalysis of crystalline properties of GaN thin film using tip-enhanced Raman spectroscopy, Applied Physics Letters 90, 06196 (2007).

[256] Marius Grundmann: The Physics of Semiconductors - An Introduction Including Nanophysics and Applications, Springe Verlag, De Gruyter Verlag, 3. Auflage 2016.

[257] C. R. Hubbard, H. E. Swanson, F. A. Mauer: A Silicon Powder Diffraction Standard Reference Material, Journal of Applied Crystallography 8, 45 (1975).

[258] S. Wei, M. Y. Chou: Phonon dispersions of silicon and germanium from first-principles calculations, Physical Review B 50, 2221 (1994).

[259] D. Franta, D. Nečas, L. Zajíčková, D. Ohlídal: Dispersion model of two-phonon absorption: application to c-Si, Opt. Mater. Express 4, 1641-1656 (2014).

[260] D. R. Neuville, D. de Ligny, G. S. Henderson: Advances in Raman Spectroscopy Applied to Earth and Material Sciences, Kapitel 13 aus: G. S. Henderson, D. R. Neuville, R. T. Downs: Spectroscopic Methods in Mineralogy and Materials Sciences, Reviews in Mineralogy and Geochemistry 78, De Gruyter Verlag, 1. Auflage 2014.

[261] P. Y. Yu, M. Cardona: Fundamentals of Semiconductors, Springer Verlag, 4. Auflage 2010. 
[262] K. Uchinokura, T. Sekine, E. Matsura: Critical-point analysis of the two-phonon Raman spectrum of silicon, J. Phys. Chem. Solids 35, 171 (1973).

[263] C. S. Wang, J. M. Chen, R. Becker, A. Zdetsis: Second order Raman spectrum and phonon density of states of silicon, Physics Letters A 44, 517 (1973).

[264] M. Liebhaber, U. Bass, P. Bayersdorfer, J. Gurts, E. Speiser, J. Räthel, A. Baumann, S. Chandola, N. Esser: Surface phonons of the $\operatorname{Si}(111)-(7 \times 7)$ reconstruction observed by Raman spectroscopy, Physical Review B 89, 045313 (2014).

[265] L. Gasdparov, T. Jegorel, L. Loetgering, S. Middey, J. Chakhalian: Thin film substrates from the Raman spectroscopy point of view, J. Raman Spectrosc. 45, 465-469 (2014).

[266] D. Mehtani, N. Lee, R. D. Hartschuh, A. Kisliuk, M. D. Foster, A. P. Sokolov, F. C̆ajko, I. Tsukerman: Optical properties and enhancement factors of the tips for apertureless near-field optics, J. Opt. A: Pure Appl. Opt. 8, 183-190 (2006).

[267] C. Meyer, S. Hühn, M. Jungbauer, S. Merten, B. Damaschke, K. Samwer, V. Moshnyaga: Supporting Information For: Tip-enhanced Raman spectroscopy (TERS) on double perovskite $\mathrm{La}_{2} \mathrm{CoMnO}_{6}$ thin films: field enhancement and depolarization effects, J. Raman Spectrosc. 48, 46-52 (2017).

[268] NT-MDT SI: "Observed” Physical Quantities in STM, Application Notes (2018).

[269] R. Takahashi, I. Ohkubo, K. Yamauchi, M. Kitamura, Y. Sakurai, M. Oshima, T. Oguchi, Y. Cho, M. Lippmaa: A-site driven ferroelectricity in strained ferromagnetic $\mathrm{La}_{2} \mathrm{NiMnO}_{6}$ thin films, Physical Review B 91, 134107 (2015).

[270] M. N. Iliev, M. V. Abrashev: Raman phonons and Raman Jahn-Teller bands in perovskite-like manganites, J. Raman Spectrosc. 32, 805-811 (2001).

[271] V. G. Sathe, A. Dubey: Broken symmetry in $\mathrm{LaAlO}_{3}$ single crystal probed by resonant Raman spectroscopy, J. Phys.: Condens. Matter 19, 382201 (2007).

[272] D. Kumar, D. Kaur: Structural and magnetic properties of $\mathrm{La}_{2} \mathrm{NiMnO}_{6}$ thin films on $\mathrm{LaAlO}_{3}$ substrate with varying thickness, J. Alloy. Comp. 554, 277-283 (2013).

[273] J. Burgess, H. Guo, A. Gupta, S. Street: Raman spectroscopy of $\mathrm{La}_{2} \mathrm{NiMnO}_{6}$ films on $\mathrm{SrTiO}_{3}(100)$ and $\mathrm{LaAlO}_{3}$ (100) substrates: Observation of epitaxial strain, Vib. Spectrosc. 48, 113-117 (2008).

[274] J. M. D. Coey, M. Viret, L. Ranno, K. Ounadjela: Electron Localization in Mixed-Valence Manganites, Physical Review Letters 75, 3910 (1995).

[275] J. Narayan, B. C. Larson: Domain epitaxy: A unified paradigm fo thin film growth, Journal of Applied Physics 93, 278 (2003).

[276] John B. Goodenough: Rare Earth-Manganese Perovskites, Kapitel 214 aus: K. A. Gschneidner, J.C. G. Bünzli, V. K. Pecharsky: Handbook on the Physics and Chemitry of Rare Earths Vol. 33, Elsevier Verlag, 1. Auflage 2003.

[277] J. Töpfer, J. B. Goodenough: $\mathrm{La}_{\mathrm{x}} \mathrm{MnO}_{3+\delta}$ Revisited, J. Solid State Chem. 130, 117-128 (1997).

[278] A. Gupta, T. R. McGuire, P. R. Duncombe, M. Rupp, J. Z. Sun, W. J. Gallagher, G. Xiao: Growth and giant magnetoresistance properties of La-deficient $\mathrm{La}_{\mathrm{x}} \mathrm{MnO}_{3-\delta}(0.67 \leq x \leq 1)$ films, Applied Physics Letters 67, 3494 (1995). 
[279] C. Ritter, M. R. Ibarra, J. M. De Teresa, P. A. Algarabel, C. Marquina, J. Blasco, J. García, S. Oseroff, S.-W. Cheong: Influence of oxygen content on the structural, magnetotransport, and magnetic properties of $\mathrm{La}_{\mathrm{x}} \mathrm{MnO}_{3+\delta}$, Phys. Rev. B. 56, 8902 (1997).

[280] Y. S. Hou, H. J. Xiang, Y. G. Gong: Intrinsic insulating ferromagnetism in manganese oxide thin films, Physical Review B 89, 064415 (2014).

[281] Z. Fang, I. V. Solovyev, K. Terakura: Phase Diagram of Tetragonal Manganites, Physical Review Letters 84, 3169 (2000).

[282] J. Roqueta, A. Pomar, L. Balcells, C. Frontera, S. Valencia, R. Abrudan, B. Bozzo, Z. Konstantinović, J. Santiso, B. Martínez: Strain-Engineered Ferromagnetism in $\mathrm{LaMnO}_{3}$ Thin Films, Crystal Growth \& Design 15, 5332-5337 (2015).

[283] A. M. Zhang, W. C. Zhang, X. S. Wu, J. G. Lin: Abnormal enhancement of ferromagnetism for $\mathrm{LaMnO}_{3+\delta}$ thin films with deacreasing oxygen pressure, AIP Advances 7, 055837 (2017).

[284] A. M. Zhang, S. L. Cheng, J. G. Lin, X. S. Wu: Strain controlled orbital state and magnetization in insulating $\mathrm{LaMnO}_{3+\delta}$ films, Journal of Applied Physics 117, 17B325 (2015).

[285] A. K. Pradhan, D. Hunter, T. Williams, B. Lasley-Hunter, R. Bah, H. Mustafa, R. Rakhimov, J. Zhang, D. J. Sellmyer, E. E. Carpenter, D. R. Sahu, J.-L. Huang: Magnetic properties of $\mathrm{La}_{0.6} \mathrm{Sr}_{0.4} \mathrm{MnO}_{3}$ thin films on $\mathrm{SrTiO}_{3}$ and buffered Si substrates with varying thickness, Journal of Applied Physics 103, 023914 (2008).

[286] G.-L. Liu, J.-S. Zhou, J. N. Goodenough: Interplay between charge, orbital, and magnetic ordering in $\mathrm{La}_{1-\mathrm{x}} \mathrm{Sr}_{\mathrm{x}} \mathrm{MnO}_{3}$, Physical Review B 64, 144414 (2001).

[287] C. Aruta, M. Angeloni, G. Balestrino, N. G. Boggio, P. G. Medaglia, A. Tebano, B. Davidson, M. Baldini, D. Di Castro, P. Postorino, P. Dore, A. Sidorenko, G. Allodi, R. De Renzi: Preparation and characterization of $\mathrm{LaMnO}_{3}$ thin films grown by pulsed laser deposition, Journal of Applied Physics 100, 023910 (2006).

[288] A. Urushibara, Y. Moritomo, T. Arima, A. Asamitsu, G. Kido, Y. Tokura: Insulator-metal transition and giant magnetoresistance in $\mathrm{La}_{1-\mathrm{x}} \mathrm{Sr}_{\mathrm{x}} \mathrm{MnO}_{3}$, Physical Review B 51, 14103 (1995).

[289] A. de Andrés, N. Biškup, M. Garcia-Hernández, Y. M. Mukovskii: Anisotropy, orbital order, and colossal electroresistance in untwinned $\mathrm{La}_{0.9} \mathrm{Sr}_{0.1} \mathrm{MnO}_{3}$ single crystals, Physical Review B 79, 014437 (2009).

[290] M. N. Iliev, M. V. Abrashev, H.-G. Lee, V. N. Popov, Y. Y. Sun, C. Thomsen, R. L. Meng, C. W. Chu: Raman spectroscopy of orthorhombic perovskitelike $\mathrm{YMnO}_{3}$ and $\mathrm{LaMnO}_{3}, \mathrm{Physical}^{\mathrm{Review}} \mathrm{B}$ 57, 2872 (1998).

[291] M. N. Iliev, M. V. Abrashev, J. Laverdière, S. Jandl, M. M. Gospodinov, Y.-Q. Wang, Y.-Y. Sun: Distortion-dependent Raman spectra and mode mixing in $\mathrm{RMnO}_{3}$ perovskites $(\mathrm{R}=\mathrm{La}, \mathrm{Pr}, \mathrm{Nd}, \mathrm{Sm}, \mathrm{Eu}, \mathrm{Gd}, \mathrm{Tb}, \mathrm{Dy}, \mathrm{Ho}, \mathrm{Y})$, Physical Review B 73, 064302 (2006).

[292] L. Martín-Carrón, A. de Andrés, M. J. Martínez-Lope, M. T. Casais, J. A. Alonso: Raman phonons as a probe of disorder, fluctuations, and local structure in doped and undoped orthorhombic and rhombohedral manganites, Physical Review B 66, 174303 (2002). 


\section{Literaturverzeichnis}

[293] M. V. Abrashev, A. P. Litvinchuk, M. N. Iliev, R. L. Meng, V. N. Popov, V. G. Ivanov, R. A. Chakalov, C. Thomsen: Comparative study of optical phonons in the rhombohedrally distorted perovskites $\mathrm{LaAlO}_{3}$ and $\mathrm{LaMnO}_{3}$, Physical Review B 59, 4146 (1999)

[294] A. Dubey, V. G. Sathe: The effect of magnetic order and thickness in the Raman spectra of oriented thin films of $\mathrm{LaMnO}_{3}$, J. Phys.: Condens. Matter 19, 346232 (2007).

[295] A. Chaturvedi, V. Sathe: Thickness dependent Raman study of epitaxial $\mathrm{LaMnO}_{3}$ thin films, Thin Solid Films 548, 75-80 (2013).

[296] A. Venimadhav, K. A. Yates, M. G. Blamire: Scanning Raman Spectroscopy for Characterizing Compositionally Spread Films, J. Comb. Chem. 7, 85-89 (2005).

[297] A. Dubey, V. G. Sathe, R. Rawat: Signature of Jahn-Teller distortion and oxygen stoichiometry in Raman spectra of epitaxial $\mathrm{LaMnO}_{3+\delta}$ thin films, J. Appl. Phys. 104, 113530 (2008).

[298] M. N. Iliev, M. V. Abrashev, V. N. Popov, V. G. Hadjiev: Role of Jahn-Teller disorder in Raman scattering of mixed-valence manganites, Physical Review B 67, 212301 (2003).

[299] V. B. Podobedov, A. Weber, D. B. Romero, J. P. Rice, H. D. Drew: Raman scattering in $\mathrm{La}_{1-\mathrm{x}} \mathrm{Sr}_{\mathrm{x}} \mathrm{MnO}_{3}$ single crystals $(x=0,0.1,0.2,0.3)$, Solid State Commun.105, 589-593 (1998).

[300] L. Martín-Carrón, A. de Andrés: Melting of the cooperative Jahn-Teller distortion in $\mathrm{LaMnO}_{3}$ single crystal studied by Raman spectroscopy, Eur. Phys. J. B 22, 11-16 (2001).

[301] E. Granado, A. Sanjurjo, C. Rettori, J. J. Neumeier, S. B. Oseroff: Order-disorder in the Jahn-Teller transition of $\mathrm{LaMnO}_{3}$ : A Raman scattering study, Physical Review B 62, 11304 (2001).

[302] V. G. Sathe, R. Rawat, A. Dubey, A. V. Narlikar, D. Prabhakaran: Photo-induced insulator-metal transition probed by Raman spectroscopy, J. Phys.: Condens. Matter 21, 075603 (2009).

[303] V. A. Dediu, J. López, F. C. Matacotta, P. Nozar, G. Ruani, R. Zamboni, C. Taliani: MicroRaman and Resistance Measurements of Epitaxial $\mathrm{La}_{0.7} \mathrm{Sr}_{0.3} \mathrm{MnO}_{3}$ Films, Phys. Stat. Sol. B 215, 625 (1999).

[304] J. H. Jung, K. H. Kim, D. J. Eom, T. W. Noh, E. J. Choi, J. Yu, Y. S. Kwon, Y. Chung: Determination of electronic band structures of $\mathrm{CaMnO}_{3}$ and $\mathrm{LaMnO}_{3}$ using optical-conductivity analyses, Physical Review B 55, 15489 (1997).

[305] J. H. Jung, K. H. Kim, T. W. Noh, E. J. Choi, J. Yu: Midgap states of $\mathrm{La}_{1-\mathrm{x}} \mathrm{Ca}_{\mathrm{x}} \mathrm{MnO}_{3}$ : Dopingdependent optical conductivity studies, Physical Review B 57, R11043 (1998). 


\section{Publikationen}

1. C. Meyer, S. Hühn, M. Jungbauer, S. Merten, B. Damaschke, K. Samwer, V. Moshnyaga, Journal of Raman Spectroscopy 48, 46-52 (2017):

"Tip-enhanced Raman spectroscopy (TERS) on double perovskite $\mathrm{La}_{2} \mathrm{CoMnO}_{6}$ thin films: field enhancement and depolarization effects"

2. C. Meyer, V. Roddatis, P. Ksoll, B. Damaschke, V. Moshnyaga, eingereicht bei Physical Review B (2018):

„Structure, magnetism and spin-phonon coupling in heteroepitaxial $\mathrm{La}_{2} \mathrm{CoMnO}_{6} / \mathrm{Al}_{2} \mathrm{O} 3$ (0001) films" 


\section{Beiträge auf Konferenzen}

- Frühjahrstagung der Deutschen Physikalischen Gesellschaft, Regensburg, 2013 "Optical properties of ZnO thin films grown by metalorganic aerosol deposition" (Poster, Beitrag im Rahmen der Master-Arbeit)

- Frühjahrstagung der Deutschen Physikalischen Gesellschaft, Berlin, 2015:

„Tip-enhanced Raman spectroscopy (TERS) on $\mathrm{La}_{2} \mathrm{CoMnO}_{6}$ thin films: field enhancement and polarization effects" (Vortrag)

- Microscopy Conference, Deutsche Gesellschaft für Elektronenmikroskopie, Göttingen, 2015:

„Far-field Raman and tip-enhanced Raman spectroscopy (TERS) on B-site ordered double perovskite $A_{2} B B^{\prime} O_{6}\left(A=L a, P r, N d, S m ; B=C o, N i ; B^{\prime}=M n\right)$ thin films" (Poster)

- Frühjahrstagung der Deutschen Physikalischen Gesellschaft, Regensburg, 2016

„Phonon, structural and magnetic properties of ordered double perovskite $A_{2} \mathrm{BMnO}_{6}$ ( $A=L a, \operatorname{Pr}, N d, S m, G d, Y ; B=C o, N i)$ thin films" (Poster)

- Frühjahrstagung der Deutschen Physikalischen Gesellschaft, Dresden, 2017

"Photo-induced effects in hole-doped $\mathrm{La}_{1-x} \mathrm{Sr}_{x} \mathrm{MnO}_{3},(0<x \leq 0.1)$ films probed by Raman spectroscopy" (Poster)

- Raman Scattering in Advanced Materials Symposium, Braunschweig, 2017:

„Tip-Enhanced Raman Spectroscopy (TERS) in the Real World" (Vortrag)

\section{Teilnahme an wissenschaftlichen Workshops}

- WITec Academy, Advanced instrument operation training for Confocal Raman Imaging, WITec Wissenschaftliche Instrumente und Technologie GmbH, Ulm, 2014 


\section{Danksagung}

Zum Abschluss möchte ich mich nun an dieser Stelle bei den vielen Menschen bedanken, die mich bei der Anfertigung meiner Doktor-Arbeit unterstützt haben, mir in der ganzen Zeit in vielfältiger Weise hilfsbereit und mit Rat und Tat zur Seite standen und es mir ermöglicht haben, die vorliegende Arbeit in dieser Art und in diesem Umfang fertigzustellen.

Der größte Dank geht in diesem Zusammenhang an meinen Doktorvater, Betreuer und Erstgutachter Herrn Prof. Dr. Vasily Moshnyaga, der mir die Möglichkeit, gegeben hat diese Arbeit in seiner Arbeitsgruppe anfertigen zu können und mir schon beginnend bei der Bachelor-Arbeit, über die Master-Arbeit bis zum Ende meiner Doktor-Arbeit als Wegbegleiter bei Fragen oder Problemen stets mit seinem Fachwissen zur Seite stand, immer Zeit für die Diskussion der neusten Messergebnisse oder neue Ideen hatte und auch abseits der Welt der Physik immer Zeit für ein Gespräch oder die ein oder andere Anekdote hatte.

Daneben möchte ich auch Herrn Prof. Dr. Peter Lemmens für die Bereitschaft danken, sich die Zeit zu nehmen, meine Arbeit als Korreferent zu begutachten. Ein großer Dank gilt zudem Herrn Dr. Bernd Damaschke für die Betreuung und die unterstützenden Hilfestellungen im Rahmen des Teilprojekts B04 des Sonderforschungsbereichs 1073 der Deutschen Forschungsgemeinschaft, Herrn Prof. Dr. Claus Ropers für die Betreuung im Rahmen des Betreuungsausschusses und Herrn Prof. Dr. Konrad Samwer für die gegebene Möglichkeit, diese Arbeit innerhalb des I. Physikalischen Instituts anfertigen zu können.

Ein weiteres großes Danke geht zudem an die Mitglieder der Manganatgruppe, in der die wissenschaftliche Arbeit und der Austausch untereinander stets in freundschaftlicher Atmosphäre und mit viel Spaß auch abseits der Physik verbunden war. Die vielen Gesprächsund Diskussionsrunden samt der vielen Anregungen zu Messergebnissen, Fortschritten in der Arbeit und neuer kreativer Ideen wie auch die gute Zusammenarbeit der einzelnen Mitglieder in der Arbeitsgruppe werden mir immer in Erinnerung bleiben.

Ein großer Dank geht außerdem an meinen Büro-Kollegen Philipp Ksoll für die freundschaftliche Atmosphäre in unserem Büro, die gute Zusammenarbeit und die vielen Gespräche über physikalische und nicht-physikalische Themen, an Vitaly Bruchmann-Bamberg, der stets eine gute Idee für die Lösung eines anfallenden Problems hatte und immer ohne zu zögern seine Hilfe angeboten hat, und an Sebastian Merten für die guten Raman-Gespräche, die gute Zusammenarbeit im Raman-Labor und die vielen Hinweise und Anregungen bei der Interpretation und Einordnung der Ergebnisse der Raman- und TERS-Messungen.

Daneben möchte ich dem ganzen I. Physikalischen Institut für die freundliche und herzliche Arbeitsatmosphäre, die vielen wissenschaftlichen Gespräche und die außerordentliche Hilfsbereitschaft während meiner ganzen Zeit im Institut, begonnen bei der Bachelor-Arbeit, über die Master-Arbeit bis zu der Anfertigung der Doktor-Arbeit danken. Ein großer Dank 


\section{Literaturverzeichnis}

ist dabei insbesondere den Technikern Carsten Mahn, Uta Filippich, Kai Dörner, Simon Stoldt, Dennis Kohl und den Mitgliedern der Feinmechanik-Werkstatt für ihre schnelle und tatkräftige Hilfe bei Problemstellungen aller Art auszusprechen, sowie auch Frau Michaela Mecke aus dem Sekretariat für die vielen Hilfestellungen bei Bestellungen und für die Bearbeitung der ganzen Bestellformulare, Anträge und Abrechnungen, die während der ganzen Zeit angefallen sind. Dennis Kohl möchte ich zudem noch einmal gesondert für die Hilfe bei der Aufnahme der REM-Bilder der TERS-STM-Goldspitzen danken.

Ein weiterer Dank gilt außerdem Herrn Dr. Vladimir Roddatis für die Aufnahme der in dieser Arbeit gezeigten TEM-Messungen, für die anschließende Diskussion der Bilder und Daten sowie für die vielen hilfreichen Erklärungen in diesem Zusammenhang.

Ein großer Dank ist zudem dem Sonderforschungsbereich 1073 (Kontrolle von Energiewandlung auf atomaren Skalen) der Deutschen Forschungsgemeinschaft (DFG) für die Finanzierung des Teilprojekts B04 (Steuerung der Konversion von Lichtenergie in Manganaten durch externe Felder) auszusprechen, in dessen Rahmen diese Arbeit entstanden ist.

Zu guter Letzt möchte ich insbesondere meinen Eltern für den vielen Zuspruch, die Unterstützung sowie das Ermöglichen des Studiums der Physik an der Universität, meiner Familie im Ganzen für die Unterstützung während der Zeit meines Studiums und meinen Freunden für die vielfältigen Aktivitäten, den Rückhalt, die Freizeitgestaltung und die schönen Stunden außerhalb der Arbeit an der Universität und der Physik danken. 